BNL - 52624

Formal Report

\title{
Proceeding of Workshop on Photo-injector For Energy Recovery Linac
}

\author{
X.J. Wang \\ National Synchrotron Light Source \\ Brookhaven National Laboratory \\ Upton, New York 11973
}

January 2001

\section{National Synchrotron Light Source}

\author{
Brookhaven National Laboratory \\ Operated by \\ Brookhaven Science Associates \\ Upton, NY 11973 \\ Under Contract with the United States Department of Energy \\ Contract Number DE-AC02-98CH10886
}




\section{DISCLAIMER}

This report was prepared as an account of work sponsored by an agency of the United States Government. Neither the United States Government nor any agency thereof, nor any of their employees, nor any of their contractors, subcontractors or their employees, makes any warranty, express or implied, or assumes any legal liability or responsibility for the accuracy, completeness, or any third party's use or the results of such use of any information, apparatus, product, or process disclosed, or represents that its use would not infringe privately owned rights. Reference herein to any specific commercial product, process, or service by trade name, trademark, manufacturer, or otherwise, does not necessary constitute or imply its endorsement, recommendation, or favoring by the United States Government or any agency thereof or its contractors or subcontractors. The views and opinions of authors expresses herein do not necessarily state to reflect those of the United States Government or any agency thereof. 


\section{National Synchrotron Light Source}

\section{Proceeding of Workshop on Photo- injector For Energy Recovery Linac}

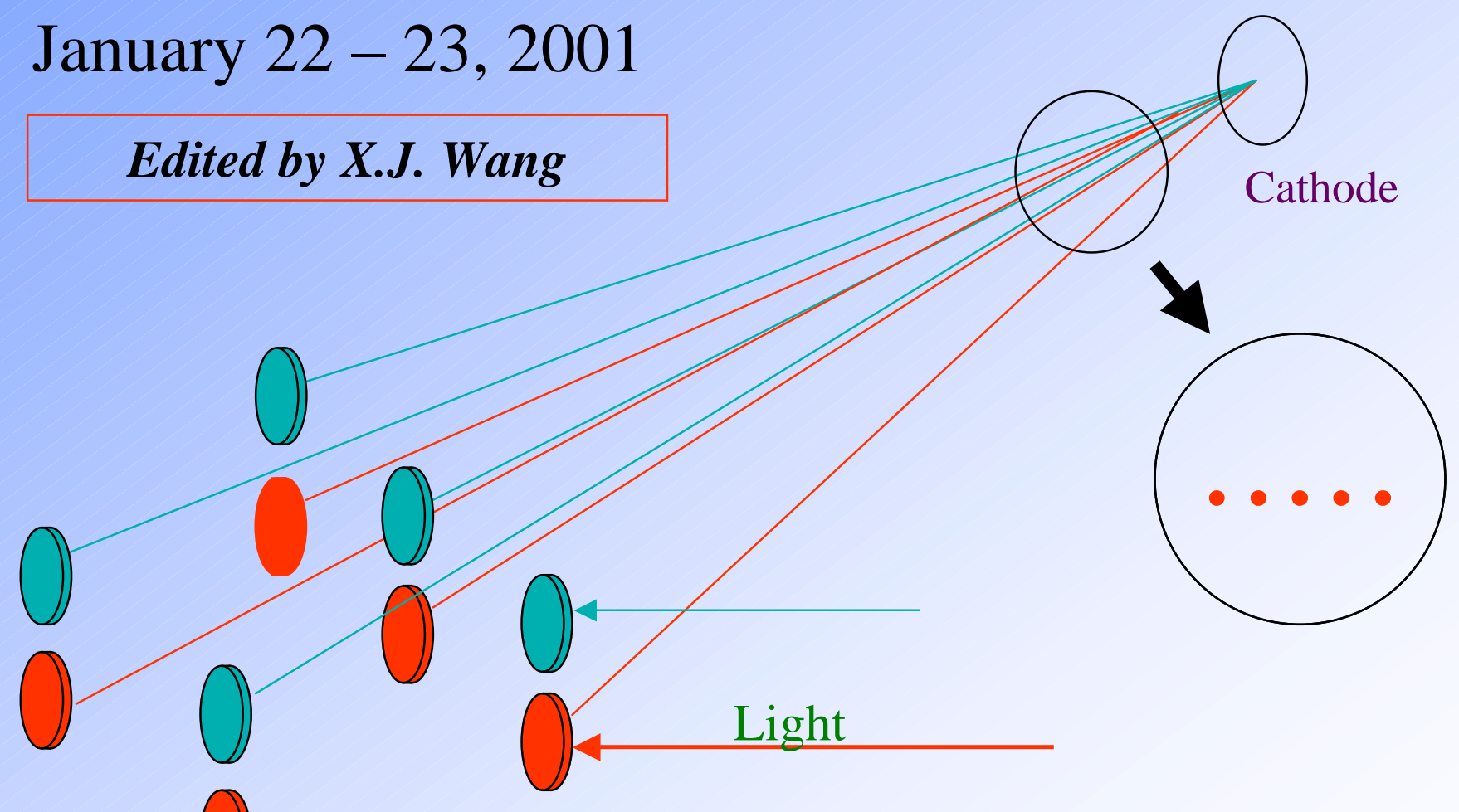


Almost 50 people attended the workshop, it is the first time a workshop covered all major aspects of photoinjector, from DC to RF.

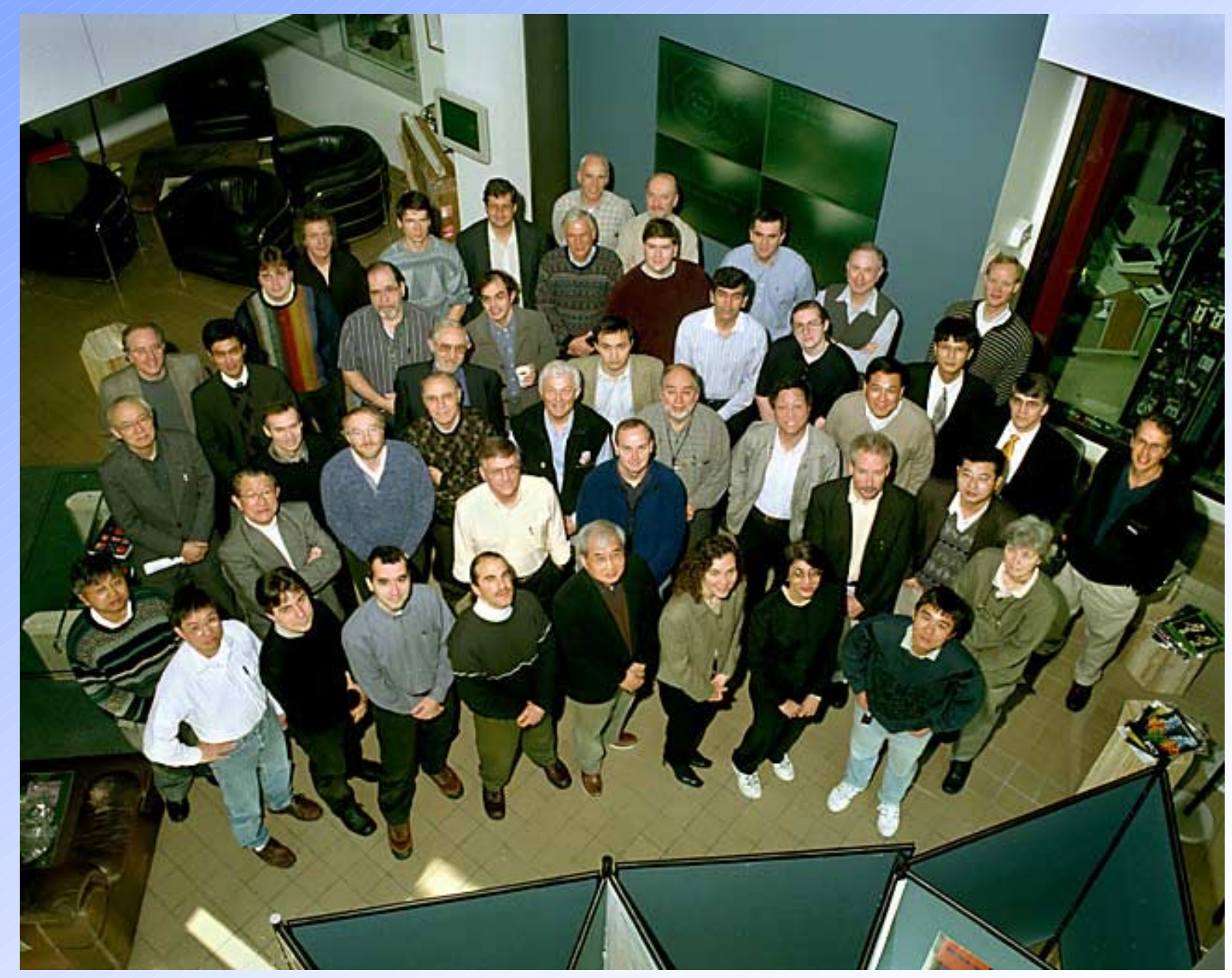




\title{
Introduction
}

Workshop on Photo-injectors for Energy Recovery Linac was held at National Synchrotron Light Source (NSLS) of Brookhaven National Laboratory (BNL) on January 22 and 23, 2001. Fifty people attended the workshop; they came from three countries, representing universities, industries and national laboratories.

This is the first workshop ever held on photo-injectors for CW operation, and for the first time, both DC and RF photo-injectors were discussed at the workshop. Workshop covered almost all major issues of photo-injectors, photocathode, laser system, vacuum, DC, $433 \mathrm{MHz} / \mathrm{B}$-factory cavities based RF gun, $1.3 \mathrm{GHz} \mathrm{RF}$ gun and beam instrumentation. High quantum efficiency and long live time photocathode is the issue discussed during the workshop. Four working group leaders have done great jobs summarizing the workshop discussion, and identifying the major issues for future R\&D. The support provided by BNL and NSLS made it possible for this workshop. Service and help from A. Bowden, K. Loverro and E. Morello made this workshop successful. I would like to express my gratitude to Drs J. Marburger, R. Osgood and S. Krinsky for their support. The workshop attendee and all my BNL colleague, who made this workshop productive, thank you.

\author{
X.J. Wang \\ Workshop Chairman
}


Workshop On Photo-injector For Energy Recovery Linac

Monday, January 22, 2001 NSLS Seminar Room

8:00 - 8:30 Continental breakfast

Chair

J.B.Murphy/BNL

8:30 - 8:40 AM: Welcome

S. Krinsky / BNL

8:40 - 9:00 AM: Overview of PERL

I. Ben-Zvi / BNL

9:00 - 9:20 AM: Workshop Charge

9:20 - 9:40 AM: Beam Dynamics issues in Energy Recovery Linac

W. Graves/BNL

9:40-10:00 AM: Coffee Break

Chair

V. Yakimenko / BNL

10:00 - 10:45 AM: Issues in Low Emittance, High Average Current DC

Photoemission Guns

C. Sinclair / JLAB

10:45-11:30 AM: Laser Systems for DC and RF guns

T. Rao / BNL

11:45-12:15 Recent results of the high-current Advanced FEL photoinjector

operating with surface-passivated cesium telluride photocathodes

12:15 - 1:15 PM: Working lunch provided by NSLS

Dinh Nguyen /LANL

Afternoon Session NSLS Seminar Room

Chair

D. Robin /ALS

1:15 PM - 2:00PM: Boeing High Duty Photocathode RF Gun Experiments

2:00 - 2:15: The new L-band 1 1/2 cell photocathode RF gun at Argonne

D. Dowell / Boeing

Manoel Conde / ANL

2:15- 2:30: Ultra-stable Laser system for Photocathode RF gun Applications

Katsuyuki Kobayashi / SHI

2:30 - 2:45 A regenerative electron source for a high average current energy recovery linacs

Alexander Zholents /LBNL

2:45- 3:05: Working groups Organization Babzien, Sinclair, Dowell, Gai

3:05-3:20 Coffee Break

3:20 - 6:00 Working group presentation and discussion

1. Laser system for PERL (NSLS Conference room A) Chair: M. Babzien /BNL

A. Femto-second and Picosecond lasers for accelerator applications

Katsuyuki Kobayashi / SHI

2. DC Gun working group (NSLS Chairman's office)

Chair: C. Sinclair / JLAB

A. DC Photoinjector for PERL

T. Shaftan and F. Zhou/ BNL

3. $433 \mathrm{~Hz}$ RF gun working group (NSLS Conference room B) Chair: D. Dowell / Boeing A. $433 \mathrm{MHz}$ Cavity based photocathode RF Gun Injector for PERL

D. Dowell/Boeing

B. Possible use of PEP-II cavity technology for a photocathode RF gun 
R. Rimmer of LBNL

4. L-band RF gun working group (NSLS Seminar Room)

Chair: W. Gai /ANL

A. Results of the Fermilab High-Brightness Photo-Injecto Jean-Paul Carneiro of Fermilab

B. TESLA X-Ray FEL and TTF Injector

P. Piot/DESY

C. L-band $\mathrm{LN}_{2}$ Cooled Photoinjector for PERL

X.Y.Chang/BNL

D. Beam Dynamics Issues at PERL Injector

X.J. Wang/BNL

6:30 PM Working Dinner (Berkner Hall Room A.) Courtesy of BNL director office

Tuesday, January 23, 2001

8:00 AM-8:30 AM Continental Breakfast at NSLS Seminar room

8:30 AM - 9:30 AM: NSLS Seminar room

Chair: H. Edward/FNAL

1. 8:30 - 8:50 Beam instrumentation Consideration for PERL Injector X.J. Wang

2. 8:50 - 9:00 Energy Compression Optimization at JLab IR-DEMO

Recirc. FEL

P. Piot/DESY

2. 8:50-9:30 Discussion on beam instrumentation and PERL injector configuration

9:30 - 9:45 Coffee Break

9:45 - 12:15 Working group discussions and Report Preparation

12:15 - 1:30 PM Lunch

1:30 - 3:30 PM Working group report and Discussion at NSLS Seminar Room Chair: M. Cornacchia / SSRL

1. Laser system for PERL

M. Babzien

2. DC Gun option for PERL

C. Sinclair

3. $433 \mathrm{MHz} / \mathrm{B}$-factory cavity based RF gun

D. Dowell

4. L-band RF gun for PERL

W. Gai

4:00 PM Adjourn 


\section{Photoinjected Energy Recovery Linac R\&D at Brookhaven National Laboratory}

- PERL - Promising technology: High-brightness, high-power electron beams.

- Three PERL projects at BNL:

- Electron cooler for RHIC

- Electron Ion Collider (eRHIC)

- PERL based light source

- Work started nearly two years ago on eRHIC

- Workshop on NSLS upgrade, October 23, 2000.

- Essential element: The photoinjector 


\section{Electron Cooling of lons}

Electron cooling is established at low energy, but:

- High-energy - New technology

- Operation in a collider

- Bunched beam
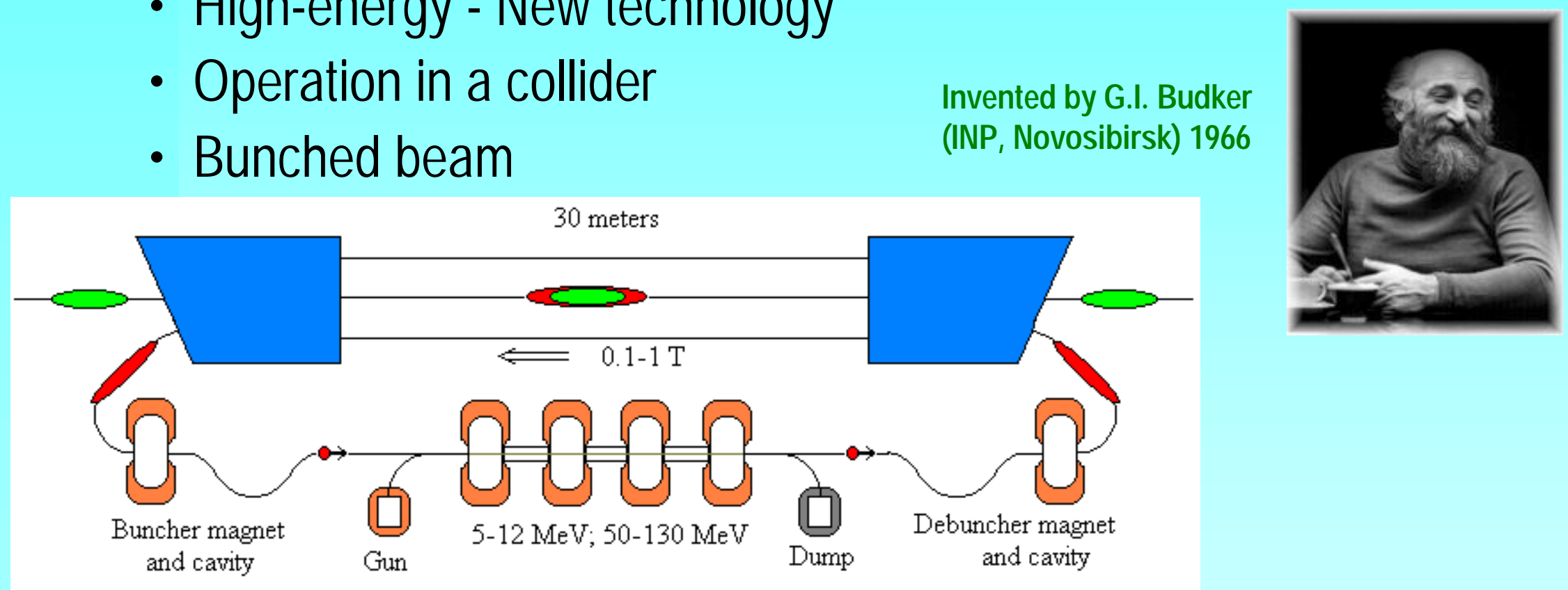

Electron cooling will provide a factor of 10 increase in the integrated luminosity of RHIC, as well as better accumulation of rare species.

Ilan Ben-Zvi

PERL Photoinjector Workshop,

BNL, January 22-23, 2001 


\section{eRHIC - a Polarized Electron on Ion or Polarized Proton in RHIC}

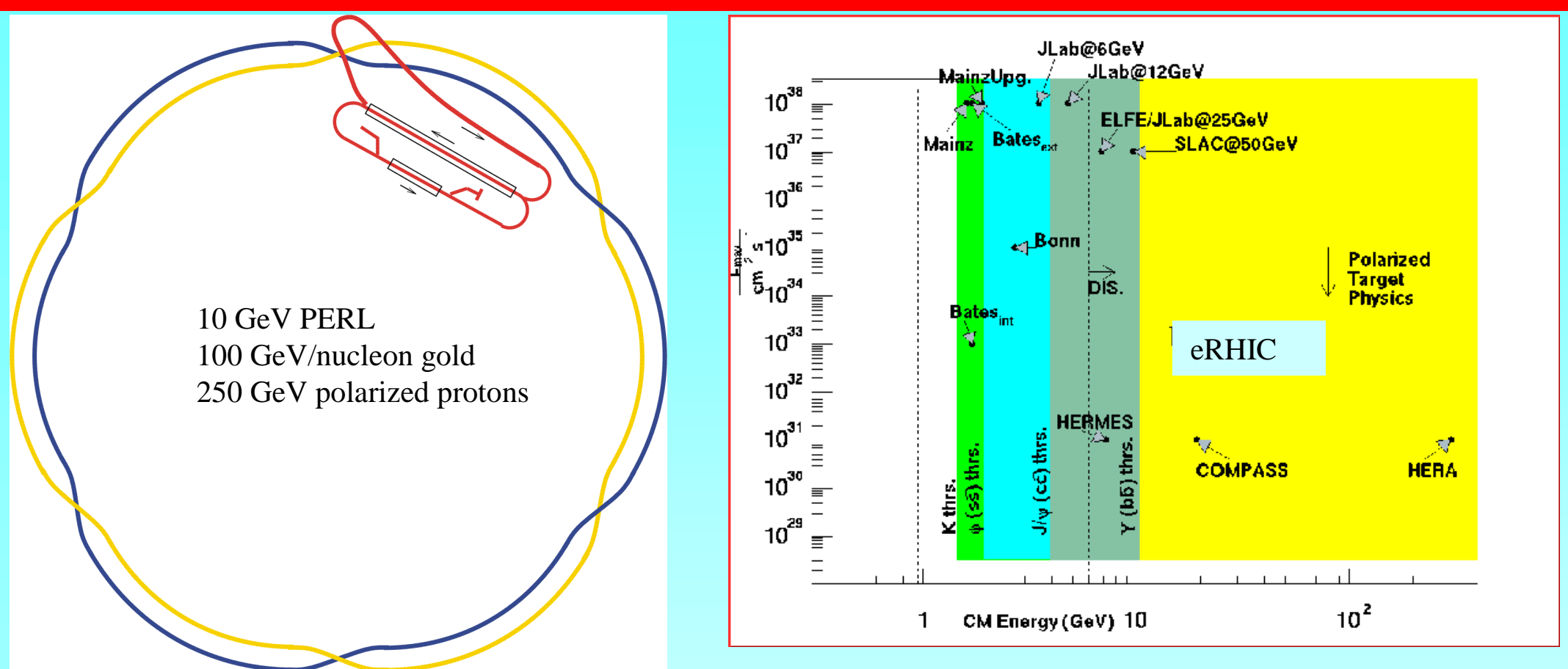

The Electron-Ion Collider is proposed as an essential tool for research into the fundamental structure of matter:

-What is the structure of hadrons in terms of their quark and gluon constituents?

- How do quarks and gluons evolve into hadrons via the dynamics of confinement?

-How do the quarks and gluons reveal themselves in the structure of atomic nuclei?

Ilan Ben-Zvi

PERL Photoinjector Workshop,

BNL, January 22-23, 2001
BFADDERHARTER WATODAL LABORATORE 


\section{Synchrotron Radiation: Where are we going?}

추을 Laser revolutions:

- High resolution spectroscopy

- Short pulse (dynamics)

- X-rays due next!

- How do we do it? The answer may be linac based sources.
BRIGHTNESS [Photons per sec - (mm mrad) $)^{2} 0.1 \%$ BW]

Large Ribosomal Subunit at $2.4 \AA$ resolution,

N. Ban et al.

Science, 289, 905 (2000)

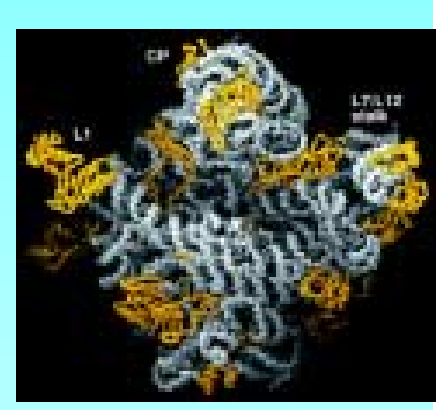

18
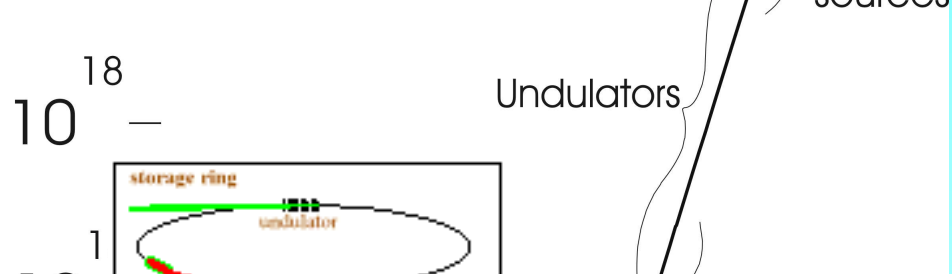

10

$10^{12}$

-Ultrafast Structural Dynamics

-Ultrafast Processes \& Time-Dependent Measurements

-Ultrahigh Spatial Resolution

-Microscopy

-Coherent X-ray Scattering

-Ultrasmall Membrane Proteins
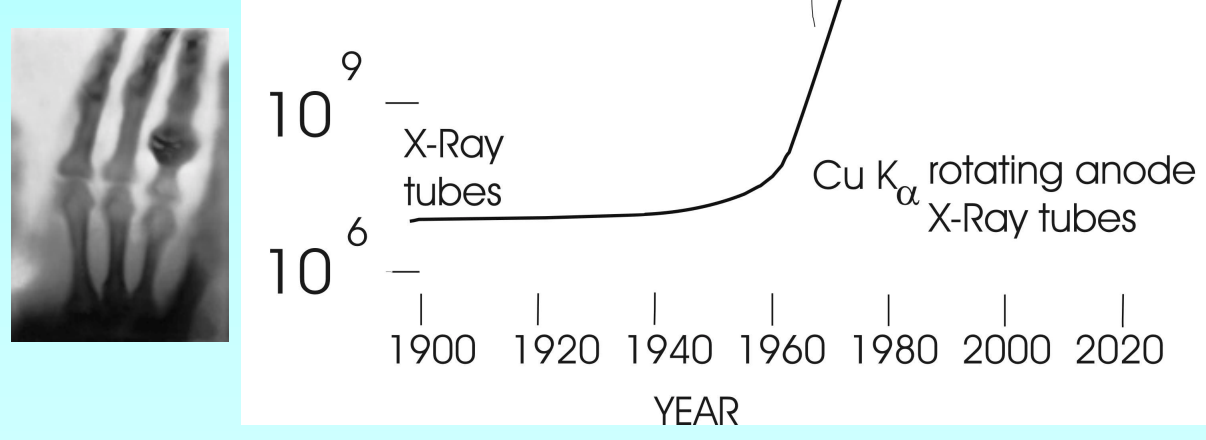

Ilan Ben-Zvi

PERL Photoinjector Workshop,

BNL, January 22-23, 2001 


\section{Electron beam brightness}

$$
B_{\text {und }} \cong \frac{2 \times 10^{8}}{\varepsilon_{x} \varepsilon_{y}} \frac{N_{W} K^{2}}{1+K^{2} / 2} \quad \varepsilon \text { in } \mathrm{mm} \mathrm{mrac}
$$

- In a storage ring emittance is the result of an equilibrium:

(Chasman-Green Lattice)

$$
\varepsilon_{x}=\left(7.7 \times 10^{-4} n m-r a d\right) \frac{\gamma^{2}}{N_{c e l l}^{3}}
$$

- In a linac, no equilibrium: "Normalized Emittance" is a conserved quantity.

$$
\varepsilon_{x}=\frac{\varepsilon_{n}}{\gamma}
$$




\section{Short pulses, peak brightness:}

Storage rings can not produce a very short pulse due to Touschek lifetime!

\begin{tabular}{|l|c|c|}
\hline Ring & $\mathrm{E}[\mathrm{GeV}]$ & $\sigma_{\mathrm{L}}[\mathrm{ps}]$ \\
\hline ALS & 1.5 & 14 \\
\hline SLS & 2.4 & 13 \\
\hline SOLEIL & 2.5 & 12 \\
\hline DIAMOND & 3 & 10 \\
\hline ESRF & 6 & 16 \\
\hline LCLS (Linac) & 15 & 0.28 \\
\hline
\end{tabular}

Ilan Ben-Zvi

PERL Photoinjector Workshop,

BNL, January 22-23, 2001 


\section{Various linac tools:}

- Photoinjector

- Small emittance

- High charge

- SRF linac

- CW operation

- Preserve emittance

- Energy recovery

- High current

- Bunch compression

- Short pulse

JLAB high power

FEL PERL

TESLA $1.3 \mathrm{GHz}$

$\mathrm{Nb}$ structure

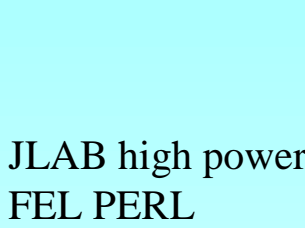

Ilan Ben-Zvi

PERL Photoinjector Workshop,

BNL, January 22-23, 2001

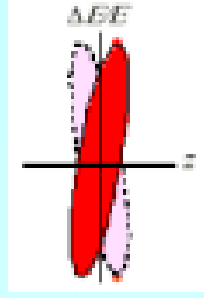

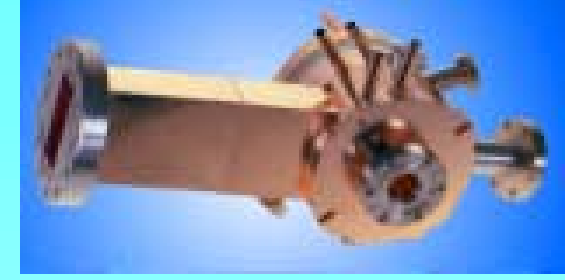
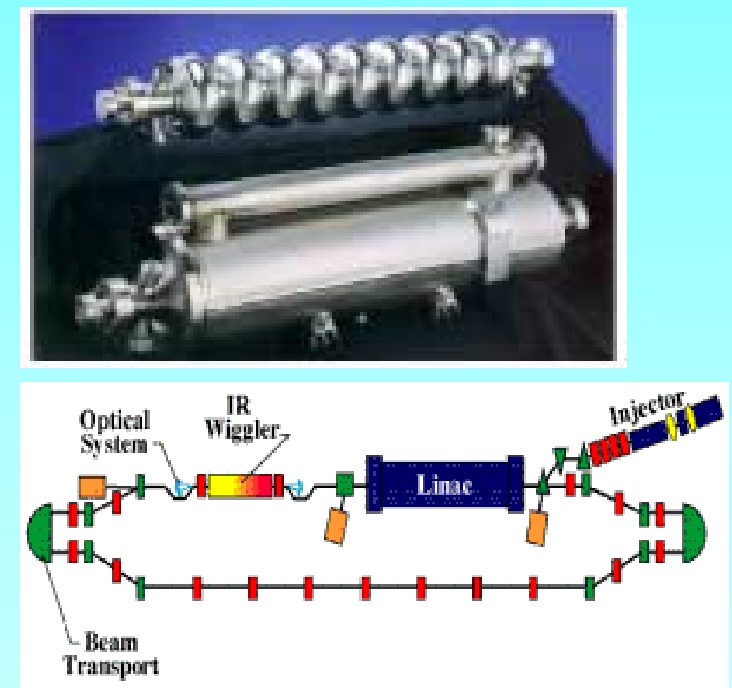

$\vdash 10 \mathrm{~m} \rightarrow$ 


\section{Linac Beam Parameters}

\begin{tabular}{|c|c|c|}
\hline Parameter & Units & Value \\
\hline Energy, single pass & $\mathrm{GeV}$ & 3 \\
\hline Average current & Amperes & 0.2 \\
\hline Bunch repetition frequency & $\mathrm{GHz}$ & $0.433 / 1.3$ \\
\hline Bunch charge & $\mathrm{nC}$ & $0.45 / 0.15$ \\
& & \\
\hline rms emittance: & & $1 / 0.5$ \\
Normalized - & $\mu \mathrm{m}$ & $1.7 / 0.8$ \\
Geometrical - & $\AA$ & \\
\hline
\end{tabular}




\section{PERL Brightness}

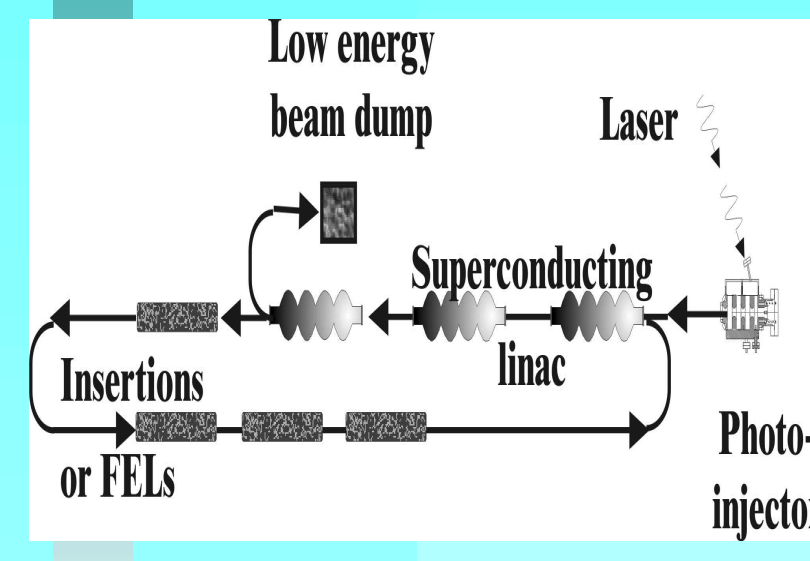

Even the spontaneous emission is outstanding!

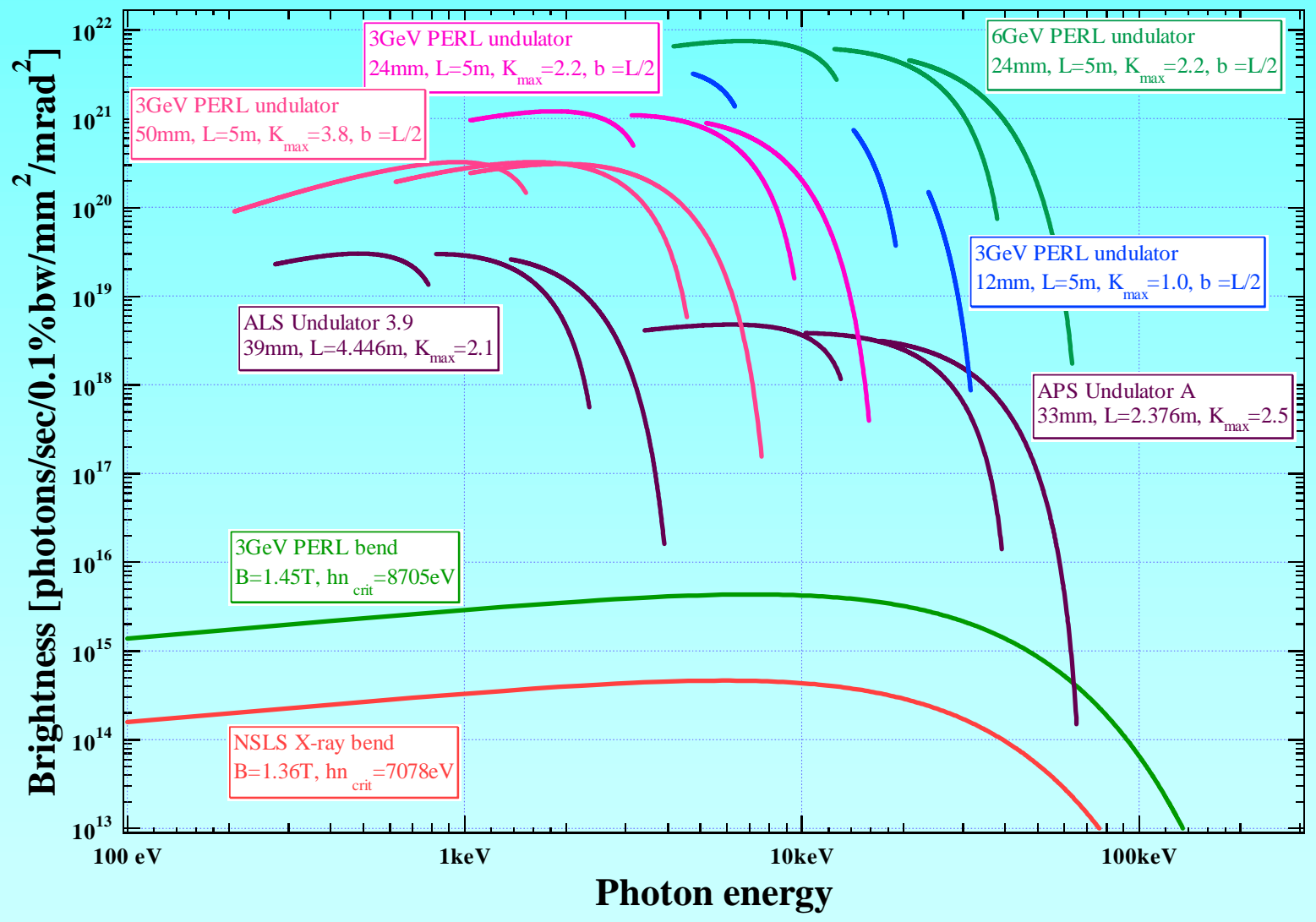

Ilan Ben-Zvi

PERL Photoinjector Workshop,

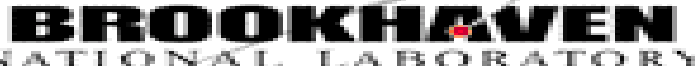
BNL, January 22-23, 2001 


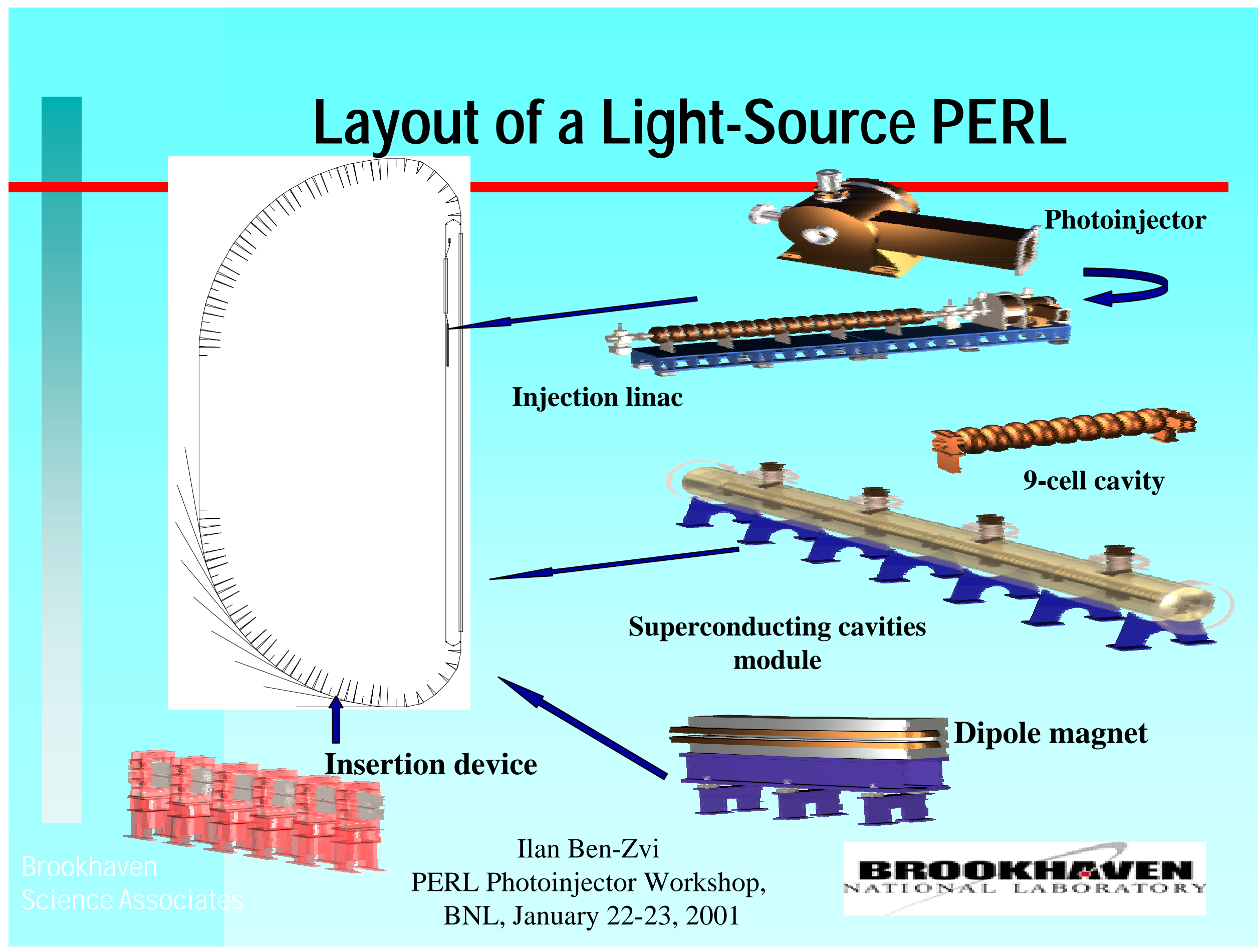




\section{Promise of a PERL Light Source}

- Diffraction-limited source to:

- $\quad 10 \sim 20 \AA$ in BOTH planes, $\varepsilon_{x} \varepsilon_{y} \sim(1-3 \AA)^{2}$ at 3-GeV.

- $\quad 5 \sim 10 \AA$ in BOTH planes, $\varepsilon_{x} \varepsilon_{y} \sim(0.25-0.75 \AA)^{2}$ at $6-\mathrm{GeV}$.

- Variable $\varepsilon_{\mathrm{x}} / \varepsilon_{\mathrm{y}}$ emittance ratio, (at a constant product $\varepsilon_{\mathrm{x}} \varepsilon_{\mathrm{y}}$ ) allowing an extremely small vertical emittance (with larger horizontal emittance) on demand.

- Sub-picosecond in the range of $100 \mathrm{fs}$.

- Virtual 'top-off' yielding a constant heat load on chambers, optics - high long-term stability.

- Electro-optical control of the pulse-format through the laser (spacing, pseudorandom sequences of arbitrary length, variable charge, variable pulse length). 


\section{Performance Goals for the PERL Photoinjector}

Presented by William S. Graves at the PERL Photoinjector Workshop

NSLS/BNL

Jan. 22, 2001 


\section{Overview}

-Workshop goals and key issues

-Desired parameters at injector end

-Laser system

-Cathode

-Gun: DC, $433 \mathrm{MHz}, 1.3 \mathrm{GHz}$

-Booster accelerator to $25 \mathrm{MeV}$

-Beam diagnostics 


\section{Key Issues for PERL Injector}

- Compare all approaches at $25 \mathrm{MeV}$ final energy.

-Charge per bunch: $0.15 \mathrm{nC}$ or $0.45 \mathrm{nC}$

-High rep rate: 1300 or $433 \mathrm{MHz}$

•Normalized RMS emittance: 1 mm-mrad

-Longitudinal RMS emittance: 3 ps*23.2 KeV @ $25 \mathrm{MeV}$

-Uptime: 24 hrs/day, 25 days/month, 11 months/year 


\section{Beam Parameters at end of Photoinjector}

- Average current $\sim 200 \mathrm{~mA}$

- RMS Pulse length $<3$ ps.

- Normalized RMS emittance $\sim 1 \mathrm{~mm}$-mrad

- Energy $25 \mathrm{MeV}$

- Uncorrelated RMS energy spread $<23 \mathrm{keV}$ at $25 \mathrm{MeV}$

DC, $433 \mathrm{MHz}$,

or $1.3 \mathrm{GHz}$ gun

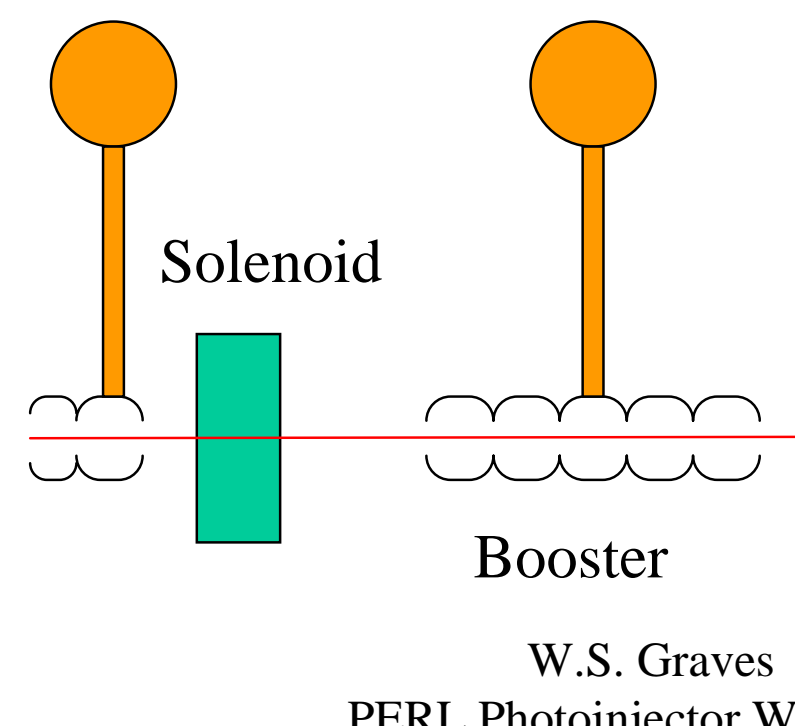

PERL Photoinjector Workshop

Chicane (if needed)

To SC

linac 


\section{Laser System}

Laser system and cathode are closely related and must be considered together.

Laser must operate at either $433 \mathrm{MHz}$ or $1.3 \mathrm{GHz}$.

\section{Laser}

- Performance goals are:

- Amplitude stability: $\quad 1.0 \mathrm{e}-3$ peak-to-peak at $10 \mathrm{MHz}$

- Pointing stability: 1.0e-3 RMS

- Timing stability: $\quad 0.2$ ps RMS at frequencies greater than $1 \mathrm{MHz}$

- Uniformity: $\quad 5 \%$ peak-to-peak (including cathode emission)

- Show block diagram of system from oscillator through transport to cathode.

- Estimate power losses in transverse and longitudinal pulse shaping and mode cleanup.

- Average power required in IR, visible, UV. Show assumptions for losses and Q.E.

- Address heating and peak power effects.

- Identify measured results for existing components. Compare with requirements.

- Strategy for 24 hour uninterrupted operation.

- Identify major technical challenges to meet PERL requirements. 


\section{Cathode Material}

\section{Cathode}

- Identify material.

- Plot quantum efficiency vs time for life of cathode.

- Strategy for 24 hour operation including redundancy/changeout requirements for planned and unplanned cathode replacement.

- Address reproducibility of performance: Q.E., uniformity, lifetime.

- Limitations in bunch length (prompt emission?) and average charge emitted.

- Cathode production and handling equipment required.

- Describe existing performance and enhancements needed.

- Identify major technical challenges.

- If considering cryogenic cooling, identify special requirements, e.g. load-lock mechanism, and cathode change procedure. 


\section{Gun Performance}

\section{RF System}

- Average beam current required is $200 \mathrm{~mA}(0.45 \mathrm{nC}$ at $433 \mathrm{MHz}$ or $0.15 \mathrm{nC}$ at $1.3 \mathrm{GHz})$.

- Address average power requirements: CW power source, thermal loading, acceleration.

- System must run 24 hours/day, 7 days/week.

- Phase and amplitude stability.

- State existing measured results for high average power operation.

- Limitations in average current (variable beam loading)?

- Identify major technical challenges.

\section{Beam Dynamics}

- Simulations should indicate optimized performance including effects of space-charge, $\mathrm{RF}$, thermal emittance, and emittance correction. Indicate peak current, bunch length, energy, energy spread, emittance, charge. Note correlated and uncorrelated quantities.

- Show scaling of performance with charge per bunch.

- State self-consistent measured results for energy gain, emittance, bunch length, charge, average current, energy spread.

- Indicate performance tradeoffs used in optimization.

- Identify major technical challenges to achieving predicted performance.

- Provide phase space plots for each plane at final energy of $25 \mathrm{MeV}$. 


\section{Booster Accelerator Performance}

\section{RF System}

- Average beam current required is $200 \mathrm{~mA}$.

- Address average power requirements: CW power source, thermal loading, acceleration.

- $\quad$ Phase and amplitude stability.

- State existing measured results for high average power operation.

- Limitations in average current (variable beam loading)?

- Identify major technical challenges.

\section{Beam Dynamics}

- Simulations should indicate optimized performance including effects of space-charge, wakefields, CSR, and emittance correction. Indicate peak current, bunch length, energy, energy spread, emittance, charge. Note correlated and uncorrelated quantities.

- State self-consistent measured results for energy gain, emittance, bunch length, charge, average current, energy spread.

- Address bunch compression if necessary.

- $\quad$ State energy spread, emittance, charge, pulse length at final energy.

- Indicate performance tradeoffs used in optimization.

- Identify major technical challenges.

- $\quad$ Provide phase space plots for each plane at final energy of $25 \mathrm{MeV}$. 


\section{Electron Beam Diagnostics}

For high average current beams, identify methods to measure:

- Vertical and horizontal emittance.

- Energy and energy spread.

- Bunch length.

- Charge.

- Peak and average current.

- Bunch-to-bunch timing jitter.

Identify feedback systems required for laser and RF. 


\title{
Beam Dynamics Issues in \\ Photoinjected Energy Recovery Linac
}

\author{
Vitaly Yakimenko \\ PERL Beam Dynamics \& Optics Working Group
}

January 22, 2001 


\section{Beam Dynamics Issues}

Key Question: Can we preserve the "bright" beam from the injector which must then pass through the compressors and the insertion devices before being returned to the linac for energy recovery?

Issues of Concern: Wakes, CSR, BBU, Errors, Stability ... 


\section{Electron Beam Requirements for PERL}

1. Transverse Normalized RMS Emittance:

$$
\text { 0.5-1 mm-mrad }
$$

2. Longitudinal Emittance $\left(\mathrm{RMS} \sigma_{\mathrm{L}} \times \sigma_{\mathrm{E}}\right)$ :

$$
\begin{aligned}
& 3 \text { ps } \times 0.09 \% \text { at } 25 \mathrm{MeV} \\
& 100 \mathrm{fs} \times 0.1 \% \text { at } 3 \mathrm{GeV}
\end{aligned}
$$

3. Average Current: $200 \mathrm{~mA}$

$$
\sigma_{R M S}=F W H M / 2.35
$$




\section{Average Current}

$$
\bar{I}=200 \quad m A
$$

\begin{tabular}{|c|c|c|}
\hline Mode & $\begin{array}{c}\text { Charge per bunch } \\
{[\mathrm{nC}]}\end{array}$ & $\begin{array}{c}\text { Spacing } \\
{[\mathrm{cm}]}\end{array}$ \\
\hline Each bucket of $1.3 \mathrm{GHz}$ & 0.15 & 23 \\
\hline Every $3^{\text {rd }}$ bucket or $433 \mathrm{MHz}$ & 0.45 & 70 \\
\hline
\end{tabular}

7 orders magnitude higher repetition rate then for similar beam in LCLS at $120 \mathrm{~Hz}$ 


\section{Electron Bunch Length/Pulse Duration}

\begin{tabular}{|l|c|c|}
\hline Ring & $E[\mathrm{GeV}]$ & $\sigma_{\mathrm{L}}[\mathrm{ps}]$ \\
\hline ALS & 1.5 & 14 \\
\hline SLS & 2.4 & 13 \\
\hline SOLEIL & 2.5 & 12 \\
\hline NSLS XRAY & 2.8 & 158 \\
\hline DIAMOND & 3 & 10 \\
\hline ESRF & 6 & 16 \\
\hline
\end{tabular}

- $3^{\text {rd }}$ generations ring sources have $\sigma_{\mathrm{L}} \sim 12 \mathrm{ps}$.

-Goal of PERL to have $\sigma_{\mathrm{L}} \sim 100 \mathrm{fs}$ ! 


\section{Synchrotron Radiation}

Incoherent Spontaneous Emission $U_{[\mathrm{KeV}]}=88.5 \frac{E_{[\mathrm{GeV}]}^{4}}{\rho_{[\mathrm{m}]}}$ in the $2 \pi$ of dipoles $(\rho=7 \mathrm{~m}$ and $3 \mathrm{GeV})$ :

$$
U=1 \mathrm{MeV}
$$

Coherent enhancement of SR

(J.B. Murphy et al. PA, 1997)

$$
k=\frac{3}{2} \frac{N_{e}}{\gamma^{4}} \cdot\left(\frac{\rho}{2 \sigma_{z}}\right)^{\frac{4}{3}}
$$

\begin{tabular}{|c|c|c|c|}
\hline $\begin{array}{c}\text { Charge } \\
{[\mathrm{nC}]}\end{array}$ & $\begin{array}{c}\text { Bunch } \sigma_{\mathrm{L}} \\
{[\mathrm{ps}]}\end{array}$ & $\begin{array}{c}\text { Enhancement } \\
\text { factor }\end{array}$ & $\begin{array}{c}\text { SR Power } \\
{[\mathrm{MW}]}\end{array}$ \\
\hline 0.45 & 0.1 & 20 & 4.2 \\
\hline 0.45 & 0.5 & 2.4 & 0.68 \\
\hline 0.15 & 0.1 & 7 & 1.6 \\
\hline 0.15 & 0.2 & 2.7 & 0.74 \\
\hline
\end{tabular}




\section{CSR}

Problems: Emittance growth, Energy spread, Radiated power

Energy spread due to CSR (no shielding) Derbenev et. al. TESLA FEL 95-055

$$
\Delta E \approx 0.22 \frac{N r_{0} L_{d} m c^{2}}{\left(R \sigma_{s}^{2}\right)^{2 / 3}}
$$

in the $2 \pi$ of dipoles $(0.15 \mathrm{nC}, 100$ fs bunches, $\rho=7 \mathrm{~m}$ and $3 \mathrm{GeV})$ :

$$
\sigma_{E} \approx 1.3 \cdot 10^{-3}
$$

We are using Elegant and TraFiC4 codes to calculate emittance growth due to CSR. 


\section{CSR shielding}

CSR reduction by parallel plate shielding

(J.B. Murphy et al. PA, 1997)

$g a p \propto\left(\rho \sigma_{z}^{2}\right)^{1 / 3}$
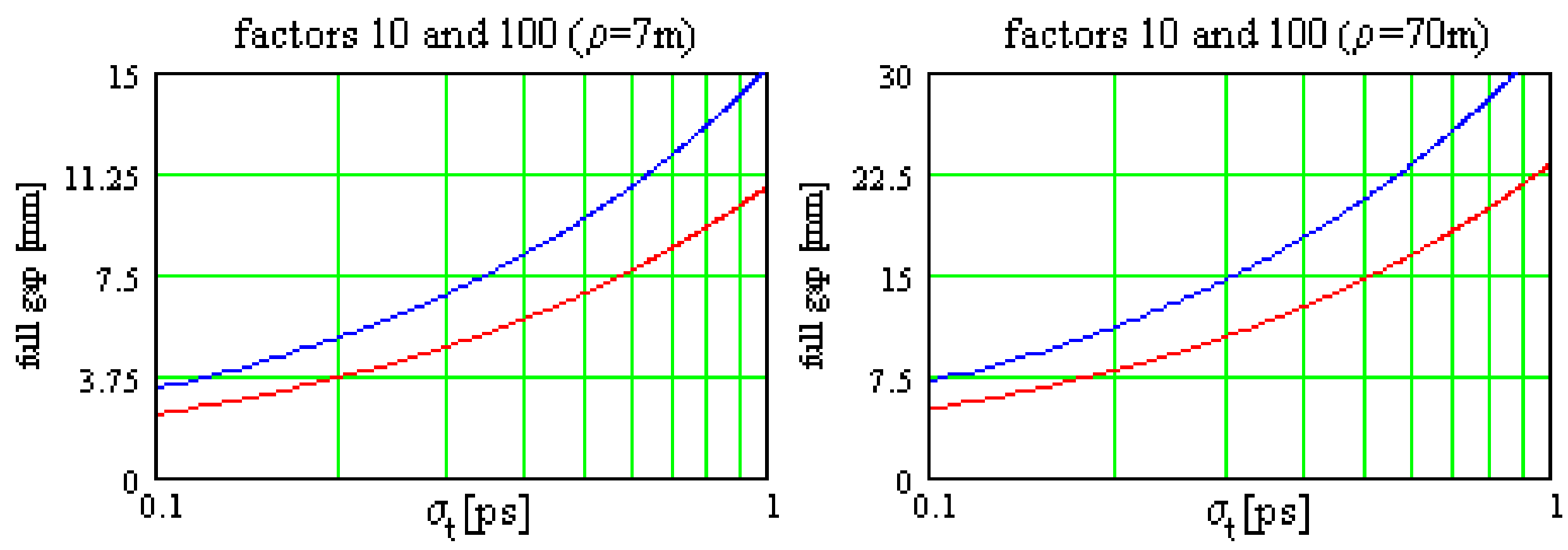

Shielding might be possible and is under study 


\section{Resistive Heating}

Simple heating from resistive wall for small gap chambers

$$
\begin{array}{cl}
P_{[W / m]}=k_{\text {loss }} Q^{2} f_{L}=k_{\text {loss }} I_{\text {ave }}^{2} / f_{L} & \text { High rep rate } \\
k_{\text {loss }}=\frac{c}{\pi a} \frac{\Gamma\left(\frac{3}{4}\right)}{4} \sqrt{\frac{Z_{0}}{2 \sigma \sigma_{z}^{3}}} & \text { is preferable }
\end{array}
$$

\begin{tabular}{|c|c|c|c|c|}
\hline $\begin{array}{c}\text { Charge per } \\
\text { bunch }[\mathrm{nC}]\end{array}$ & $\begin{array}{c}\text { Bunch length } \\
\sigma_{\mathrm{L}}[\mathrm{ps}]\end{array}$ & $\begin{array}{c}\text { Full gap } \\
\text { 2a }[\mathrm{mm}]\end{array}$ & $\begin{array}{c}\mathrm{P}_{[\mathrm{kW} / \mathrm{m}]} \text { for } \\
\mathrm{Cu}\end{array}$ & $\begin{array}{c}\mathrm{P}_{[\mathrm{kW} / \mathrm{m}]} \text { for } \\
\mathrm{Al}\end{array}$ \\
\hline 0.45 & 0.1 & 5 & 3.6 & 5.4 \\
\hline 0.15 & 0.1 & 5 & 1.2 & 1.8 \\
\hline
\end{tabular}

Surface roughness, geometric wakes, etc. are under consideration. 


\section{Scaled Layout}

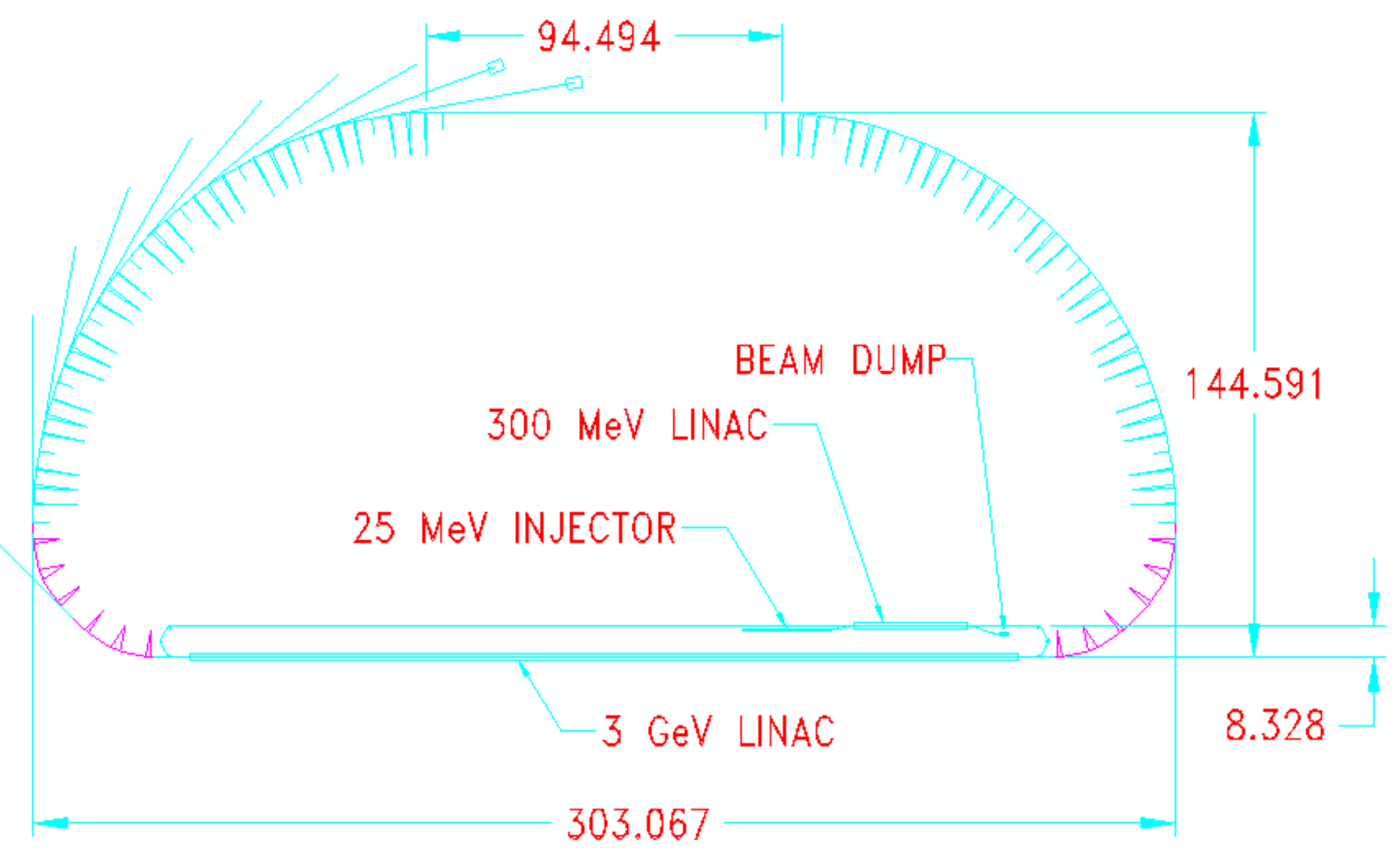

There are 24 straight sections for insertion devices 


\section{Basic Arc Cell Optics for Return Leg and Bunch Compression}

Extended triple bend achromat optics offers ability to tune $\mathrm{R}_{56}$ by changing dispersion in the middle dipole
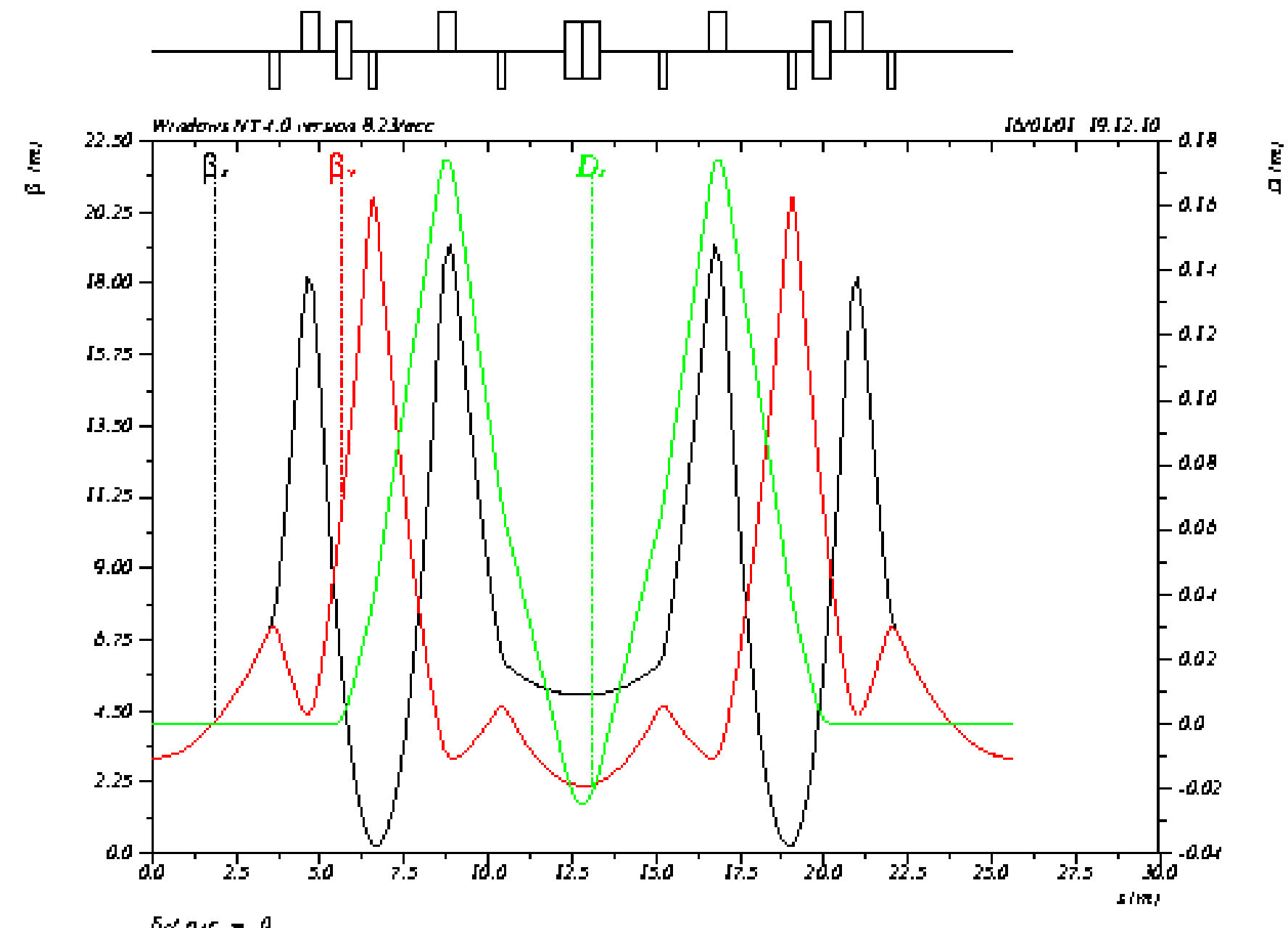

Joble comer $=$ Jwiss 


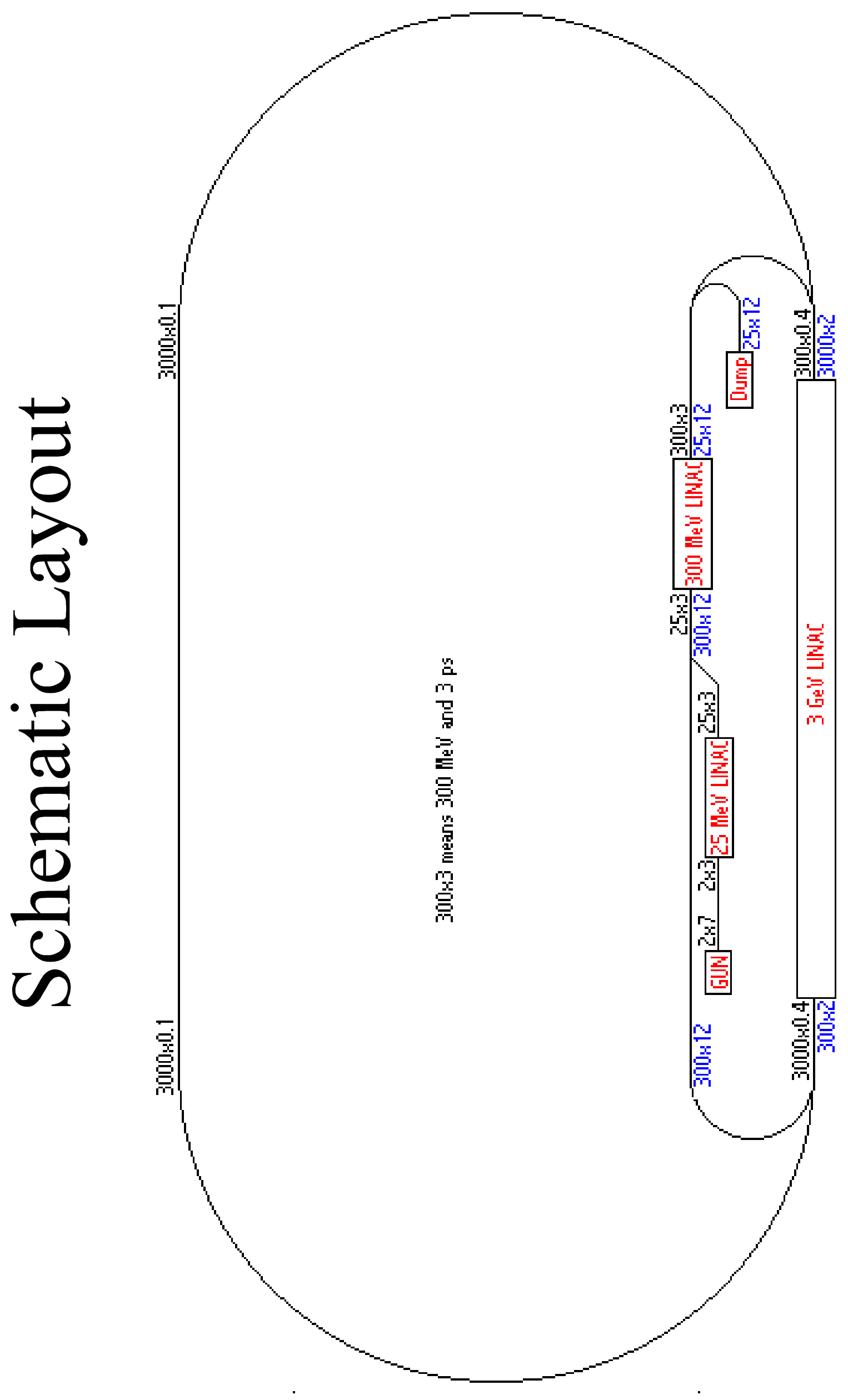




\section{Longitudinal Phase-Space}

ID line width: $\frac{\Delta \lambda}{\lambda}=\frac{1}{n N_{p}} \oplus 2 \frac{\Delta E}{E}$ requires $\frac{\Delta E}{E}<10^{-3}$

$$
F(s, \delta) \propto \exp \left(-\frac{1}{2\left(1-r^{2}\right)}\left[\frac{s^{2}}{\sigma_{L}^{2}}-\frac{2 r s \delta}{\sigma_{L} \sigma_{\delta}}+\frac{\delta^{2}}{\sigma_{\delta}^{2}}\right]\right)
$$

\begin{tabular}{|c|c|c|c|c|c|}
\hline & $\begin{array}{l}\text { Beam } \\
\text { energy } \\
{[\mathrm{MeV}]}\end{array}$ & $\begin{array}{l}\text { Bunch } \\
\text { length } \\
\text { ot[ps] }\end{array}$ & $\begin{array}{c}\text { Uncorrelated } \\
\text { energy spread } \\
\sigma u[\%]\end{array}$ & $\begin{array}{c}\text { Correlated } \\
\text { energy spread } \\
\sigma_{c}[\%]\end{array}$ & $\begin{array}{c}\mathrm{R}[5,6] \\
{[\mathrm{cm}]}\end{array}$ \\
\hline Gun & 2 & 7 & 0.500 & -1.054 & \\
\hline Ballistic compr. (1.6m dri & 2 & 3 & 1.167 & 0.000 & -16.3 \\
\hline Low energy linac & 25 & 3 & 0.093 & 0.000 & \\
\hline Small ER LINAC & 300 & 3 & 0.008 & 0.778 & \\
\hline Small arc compressor & 300 & 0.4 & 0.058 & 0.776 & 12.9 \\
\hline Large ER LINAC & 3000 & 0.4 & 0.006 & 0.078 & \\
\hline Arc-compressor & 3000 & 0.1 & 0.023 & 0.074 & 15.4 \\
\hline X-ray compressor & 3000 & 0.03 & 0.078 & 0.000 & 3.7 \\
\hline
\end{tabular}

We need to budget for some degradation of the longitudinal emittance due to compression and RF curvature effect (no degradation in the table) 


\section{HGHG-based X-ray Device}

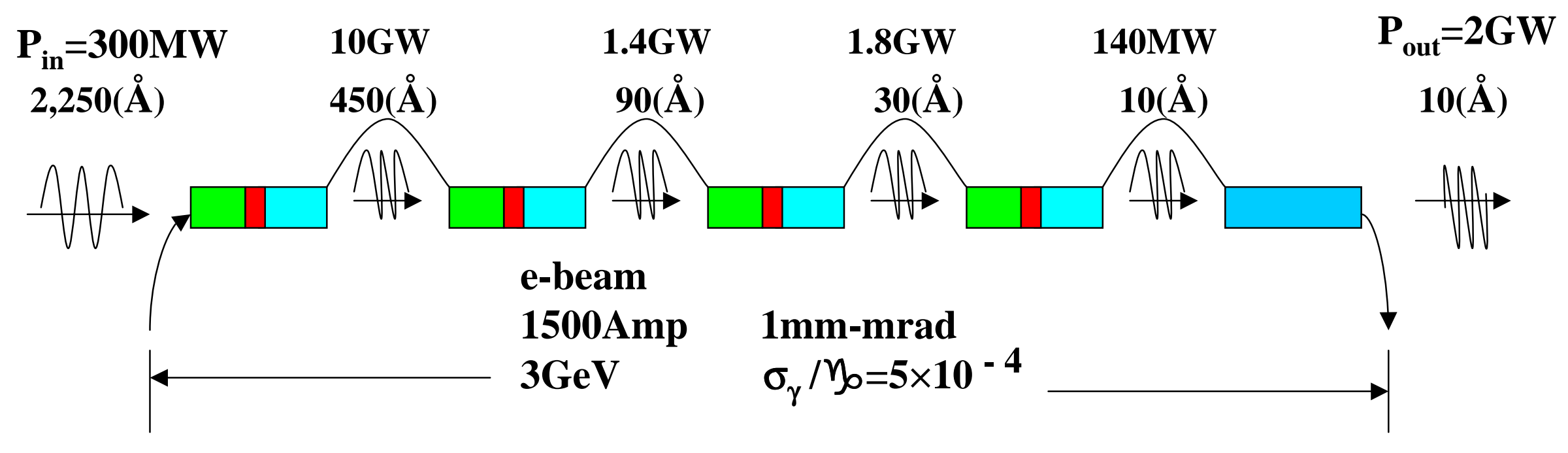

\begin{tabular}{|c|c|c|c|c|}
\hline $\mathbf{I}_{\mathbf{p k}}(\mathrm{Amp})$ & $\boldsymbol{\varepsilon}_{\mathbf{n}}(\mathrm{mm}-\mathrm{mrad})$ & $\mathbf{L}_{\mathbf{W}}(\mathrm{m})$ & $\lambda_{\text {final }}(\AA)$ & $\mathbf{P}_{\text {out }}(\mathrm{GW})$ \\
\hline 1500 & 1 & 46 & 10 & 2 \\
\hline 750 & 1 & 49 & 18 & 1 \\
\hline 2500 & 2 & 35 & 18 & 4 \\
\hline 2500 & 2 & 45 & 10 & 1 \\
\hline
\end{tabular}




\section{FEL mode}

1. Larger charge is preferable for FEL

2. FEL operate at low repletion rate (reduces problem with average heat)

3. Possibly, FEL bunches would be produced by separate gun at low repetition rate. 


\section{Conclusions}

1. The ability to produce and preserve short electron bunches, $\sigma_{\mathrm{L}}<1 \mathrm{ps} \& \sigma_{\mathrm{E}}<10^{-3}$, is a cornerstone of the PERL project.

2. Most of the potential problems are due to coherent effects, as a result lower charge per bunch and a higher repetition rate is preferable.

3. Work is in progress to assess the impact of all the beam dynamic effects on the performance of PERL. 
ISSUES IN LOW EMITTANCE, HIGH AVERAGE

CURRENT DC PHOTOEMISSION GUNS

Charles K. Sinclair Jefferson Lab 


\section{HIGH QUANTUM EFFICIENCY CATHODES}

There are three broad classes of high QE cathodes to consider:

- alkali antimonides - e.g: $\mathrm{Cs}_{3} \mathrm{Sb}, \mathrm{K}_{2} \mathrm{CsSb}$, etc.

- visible light below $\sim 600 \mathrm{~nm}$ required

- alkali tellurides - e.g. $\mathrm{Cs}_{2} \mathrm{Te}, \mathrm{K}-\mathrm{Te}$, $\mathrm{Cs}-\mathrm{K}-\mathrm{Te}$, etc.

- UV light below $\sim 275 \mathrm{~nm}$ required

- NEA semiconductors - e.g GaAs, GaAsP

- visible to near IR light below $\sim 800 \mathrm{~nm}$ required 


\section{High Quantum Efficiency is Essential}

For any linear photoemitter, the current is given by:

$$
i(m A)=\frac{\lambda(n m)}{124} \bullet P_{\text {laser }}(W) \bullet Q E(\%)
$$

So, for example, to provide $200 \mathrm{~mA}$ average current for PERL:

\begin{tabular}{|l|c|c|}
\hline Cathode Type & Wavelength $(\mathrm{nm})$ & $\mathrm{P} \times \mathrm{QE}(\mathrm{W}-\%)$ \\
\hline NEA GaAs & 780 & 31.8 \\
\hline $\mathrm{K}_{2}$ CsSb (PEA) & 532 & 46.6 \\
\hline KCsTe (PEA) & 266 & 93.2 \\
\hline
\end{tabular}

Note that this assumes that every laser photon and every emitted electron are used. Losses of either increase the required $\mathrm{P} \times \mathrm{QE}$. 


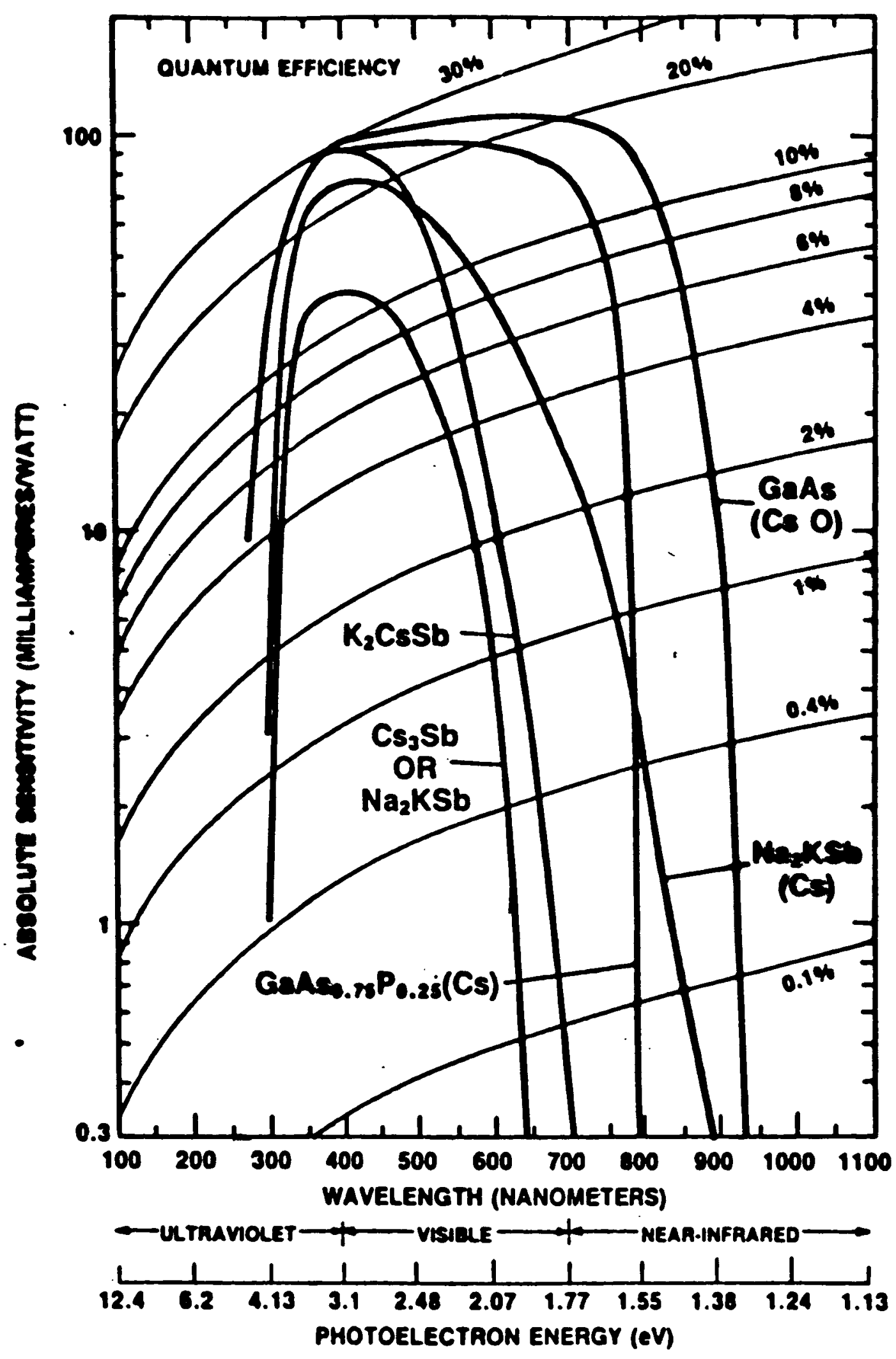




\section{THE THREE-STEP MODEL OF PHOTOEMISSION}

- Optical absorption by electron

- Transport (diffusion) of electron to cathode surface

- Escape of electron through surface potential barrier

For alkali antimonide and alkali telluride photocathodes, the absorption coefficient is $\sim 30 / \mu$, while for negative electron affinity GaAs photocathodes, it is $\sim 1.4 / \mu$. 


\section{ELECTRON AFFINITY}

Electron affinity is defined here as the energy difference between the bottom of the conduction band in a bulk semiconductor, and the vacuum level well outside the semiconductor.

High quantum efficiency photoemitters are of two types - called positive electron affinity (PEA) (e.g. alkali antimonides and alkali tellurides) and negative electron affinity (NEA) (e.g. cesiated GaAs). 
In a positive electron affinity photocathode, electrons MUST escape before they thermalize to the bottom of the conduction band, where they cannot escape, while in negative electron affinity cathodes, the electrons can escape from the bottom of the conduction band, and thus may thermalize before photoemission.

In an NEA photocathode illuminated reasonably close to the bandgap energy, the absorption coefficient is low. The electrons are absorbed over a considerable distance, and thermalize to the conduction band minimum, loosing the history of the wavelength of the illuminating photon. 


\section{EMITTANCE}

$$
\varepsilon_{\text {geom,rms }}=\sqrt{\left\langle x^{2}\right\rangle\left\langle x^{\prime 2}\right\rangle-\left\langle x x^{\prime}\right\rangle^{2}} \text {, and } \varepsilon_{n, r m s}=\beta \gamma \varepsilon_{\text {geom,rms }}
$$

The "full" emittance, defined as four times the rms emittance above, includes $100 \%$ of the beam particles in a uniformly filled phase space, and about $90 \%$ of the particles in a beam with Gaussian profiles for $x$ and $x$ ' (and about $90 \%$ of the particles in a $\mathrm{K}-\mathrm{V}$ beam).

For a beam with a uniformly filled spot of radius $r$, and a MaxwellBoltzmann distribution of velocities characterized by temperature $\mathrm{T}$ :

$$
\varepsilon_{n, r m s}=\frac{r}{2} \sqrt{\frac{k T}{m c^{2}}}, \text { more generally, } \varepsilon_{n, r m s}=\frac{r}{2} \sqrt{\frac{E_{t h}}{m c^{2}}}
$$


There are few high quality measurements of the thermal emittance from photoemission cathodes. The best measurements I know of were made by Bruce Dunham (Ph.D thesis, and PAC '95, p. 1031). He illuminated a NEA GaAs photocathode with a tophat profile laser beam of various wavelengths and spot diameters. His results determine the effective thermal energy, and show that at longer wavelengths, the effective temperature is close to room temperature. 


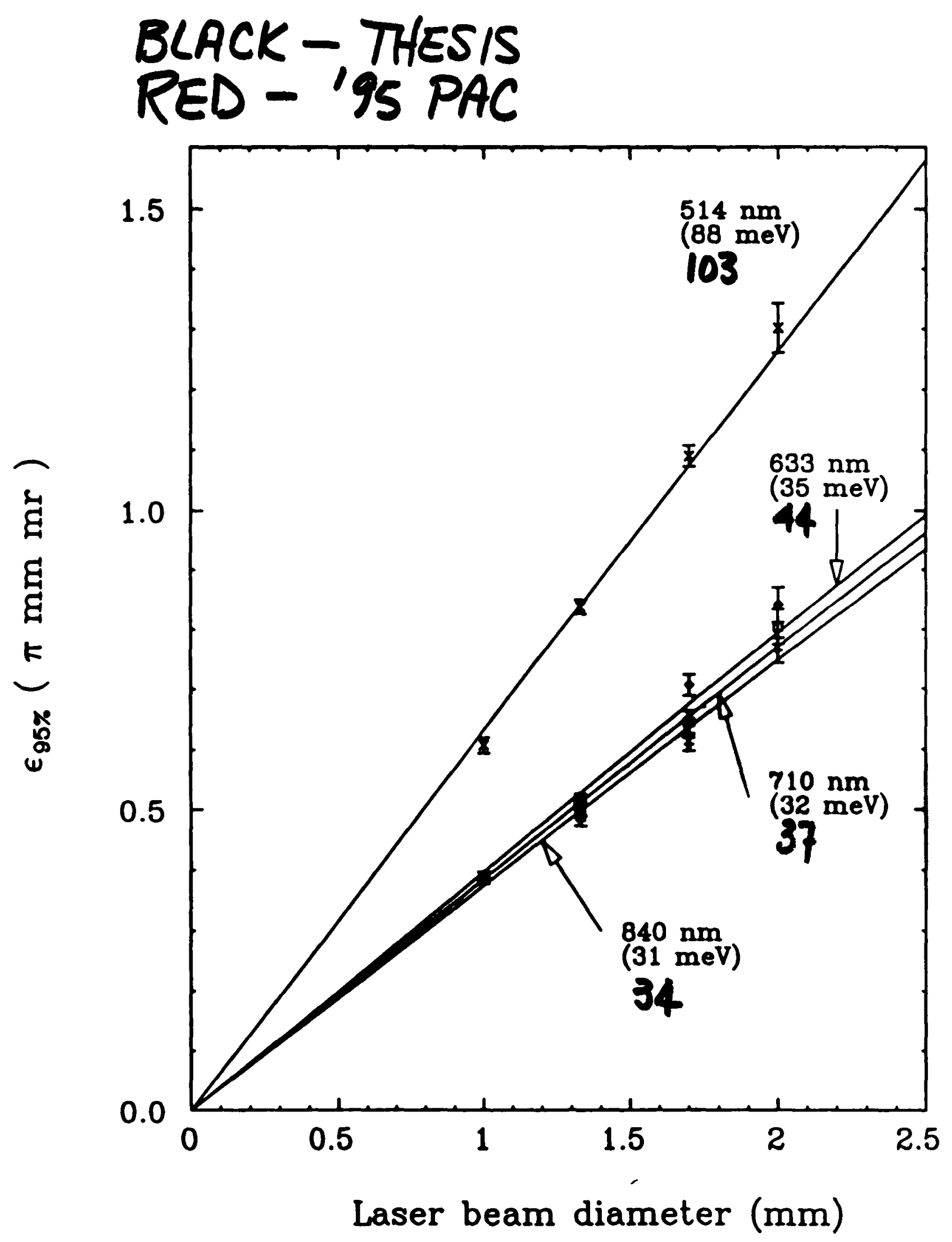

Measured 95\% emittance versus laser beam spot diameter for various optical wavelengths. 


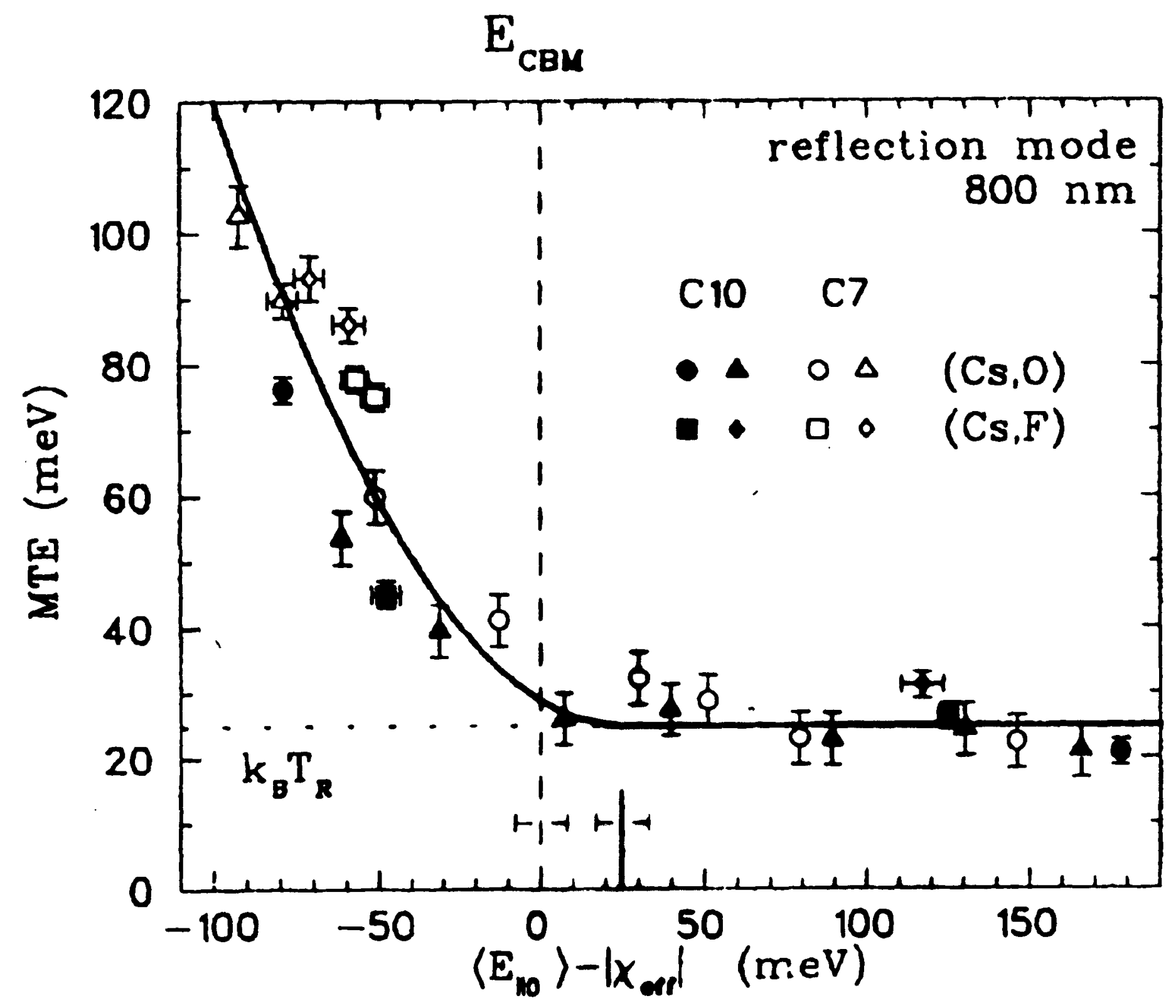




\section{EFFECTIVE TEMPERATURE SUMMARY}

- Dunham obtains wavelength dependent effective cathode temperatures. Within about $0.3 \mathrm{eV}$ of the bandgap, his effective temperature is about 1.5 times the physical temperature.

- Engwall quotes a larger effective temperature than Dunham. His measurements have a very large spread, with the smallest numbers consistent with the Dunham numbers, the largest a factor of two higher.

- In the JLab nuclear physics injector we have measured emittances very close to the Dunham values on a beam with RF structure (499 and $1497 \mathrm{MHz}$ ) and Gaussian laser profiles

- The effective temperature is dependent on the NEA activation

- Cooling the cathode has been demonstrated to reduce the energy spread of a photoemitted beam 


\section{THE IMPACT OF THE THERMAL ENERGY}

- To avoid space charge problems, want:

$$
\begin{aligned}
& \qquad Q_{\text {bunch }} \leq Q_{\text {stored }} / 10, \\
& \text { which leads to: } \\
& Q_{\text {bunch }}(p C) \leq 2.78 E_{\text {cath }}(\mathrm{MV} / \mathrm{m})(r(\mathrm{~mm}))^{2}
\end{aligned}
$$

- If we say $E_{\text {thermal }}=\beta(51 \mathrm{meV})$, then we have:

$$
\begin{gathered}
r(m m)=2 \varepsilon_{n, r m s}(m m-m r a d) \sqrt{\frac{10}{\beta}}, \text { so } \\
Q_{b u n c h}(p C) \leq 111 E_{\text {cath }}(M V / m) \frac{\varepsilon^{2}}{\beta}
\end{gathered}
$$




\section{EXAMPLES}

- With $\beta \sim 6$ and $\varepsilon \sim 1 / 2$, for $154 \mathrm{pC} /$ bunch, need $\mathrm{E}_{\text {cathode }} \sim 33 \mathrm{MV} / \mathrm{m}$. These numbers are about OK for a $\mathrm{Cs}_{2}$ Te cathode, and the cathode field is OK for an RF gun.

- With $\beta \sim 1$ and $\varepsilon \sim 1 / 2$, for 154 pC/bunch, need $E_{\text {cathode }} \sim 5.5$ $\mathrm{MV} / \mathrm{m}$. These numbers are about OK for a GaAs cathode, and the cathode field is OK for a DC gun.

- A $433 \mathrm{MHz}$ RF gun with a $\mathrm{Cs}_{2}$ Te cathode looks like it would require a cathode field too large to be practical.

CONCLUSION: the nature of the cathode will have a lot to do in determining the nature of the gun for these small emittances. 


\section{CATHODE LIFETIME ISSUES}

- At JLab, we operate NEA GaAs cathodes on both the FEL and the nuclear physics accelerators, with total charge delivery from a cathode approaching $1 \mathrm{~A}-\mathrm{hr}$

- PERL will require about $1500 \mathrm{~A}$-hr per operating year.

- JLab GaAs cathode operating lifetimes are limited only by ion back-bombardment. Thus the lifetime is best expressed in terms of delivered coulombs per unit illuminated area - not clock hours. The best results we have give 1/e operating lifetimes of 1.0 to 1.2 times $10^{5}$ coulombs $/ \mathrm{cm}^{2}$.

- The use of multiple illuminated spots from a large area cathode, two readily interchangeable guns, and vacuum improvements may allow the PERL requirement to be met. 


\section{IMPROVING THE GUN VACUUM}

- Massive NEG pump arrays surround the cathode-anode gap

- NEG coated beam tubes act as a pump, a diffusion barrier, and have very low ESD

- Elimination of electrons originating from large radius on the cathode is essential. We anodize a ring at large radius on the cathode wafer to eliminate emission from this region.

- Field emitted electrons degrade the vacuum through ESD, so field emission must be kept at extremely low levels

- Once established, our gun vacuums slowly improve in time, and require no maintenance 


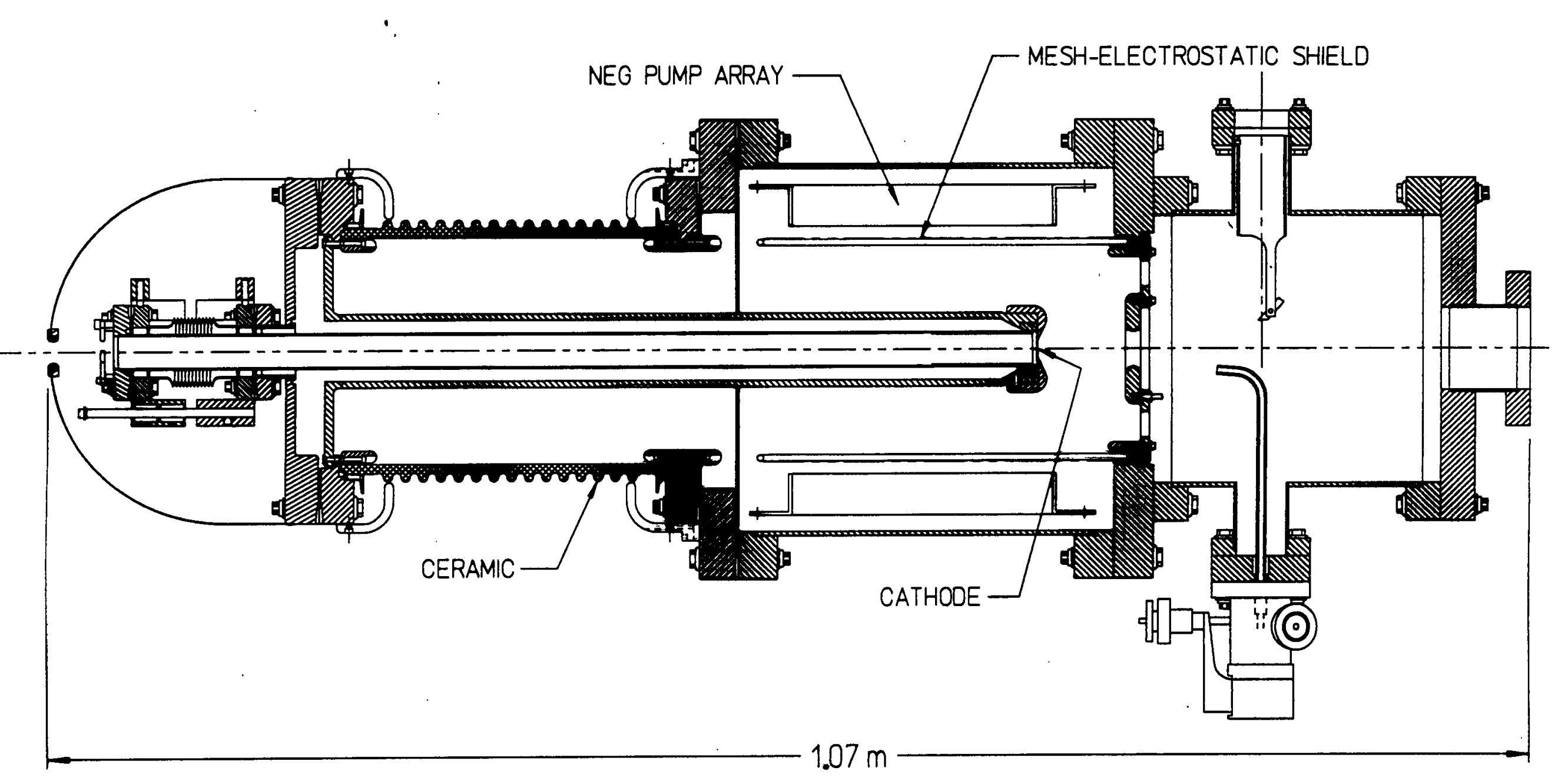




\section{VACUUM - THE NEXT STEPS}

- Develop chambers with reduced outgasing rate

- Develop species-specific pumping

- Investigate the use of diffusion barriers

- Employ impermeable chamber walls

- Develop improved vacuum diagnostics

We believe it should be practical to reach into the $10^{-13}$ torr range with careful work. This should dramatically reduce the ion back bombardment problem. 


\section{LASER ISSUES}

High average power lasers operating at appropriate wavelengths, and with suitable RF time structure are not easy to come by.

We use a Ti:sapphire laser which is very stably mode locked to the accelerator RF (499 or $1497 \mathrm{MHz}$ ) by gain modulation. The gain modulation is provided by an RF gain switched diode laser.

This system has delivered up to $2 \mathrm{~W}$ output, and is in routine use on the accelerator.

The RF output frequency of the laser is readily variable over a huge range - we have operated between $233 \mathrm{MHz}$ and $2.5 \mathrm{GHz}$. The output power is independent of the RF frequency.

We see no reason that this laser cannot be developed to provide at least $10 \mathrm{~W}$ 


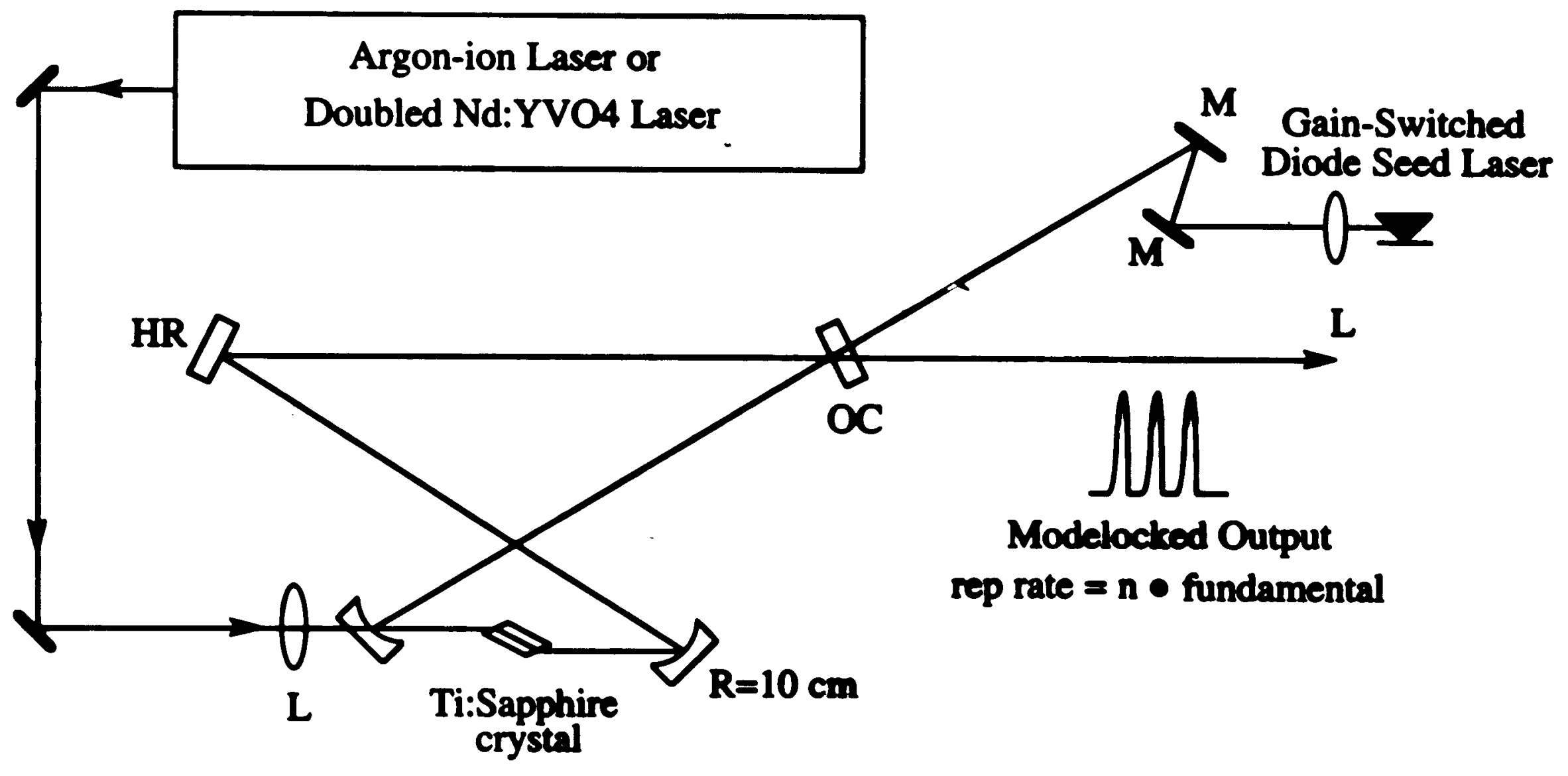

JLAB prototype laser; Alternative to $100 \mathrm{~mW}$ Diode Systems high power, modelocked ti:sapphire 


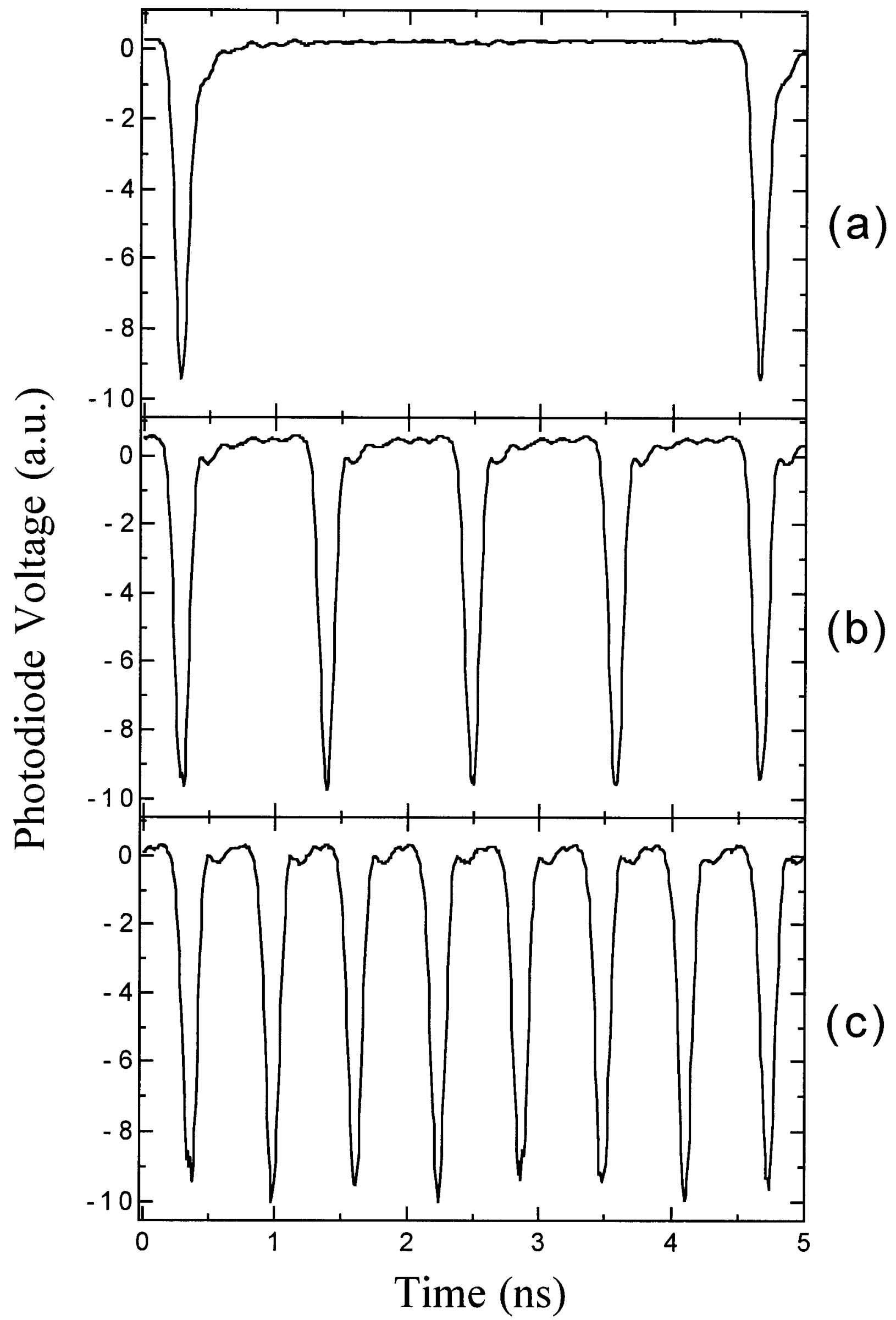




\section{Gun issues}

- High fields are necessary at the cathode (and therefore even higher fields elsewhere on the cathode electrode)

- Field emission from the cathode electrode and its support structure must be very low. Field emitted electrons degrade the vacuum through ESD, and may cause charging of the cathode insulator resulting in catastrophic vacuum failure.

- Ian Brown has developed an ion implantation process to make a uniform, high sheet resistance on a ceramic. We have adapted this to our $500 \mathrm{kV} \mathrm{FEL} \mathrm{gun} \mathrm{with} \mathrm{great} \mathrm{success.}$

- We have treated conventional stainless steel electrodes with plasma-source ion implantation. This reduced the field emission from large area electrodes by over a factor of one thousand. We believe we can operate a DC gun at 20 to $25 \mathrm{MV} / \mathrm{m}$ cathode field. 


\section{CUUM GAP VOLTAGE BREAKDOWN COMPARISON}

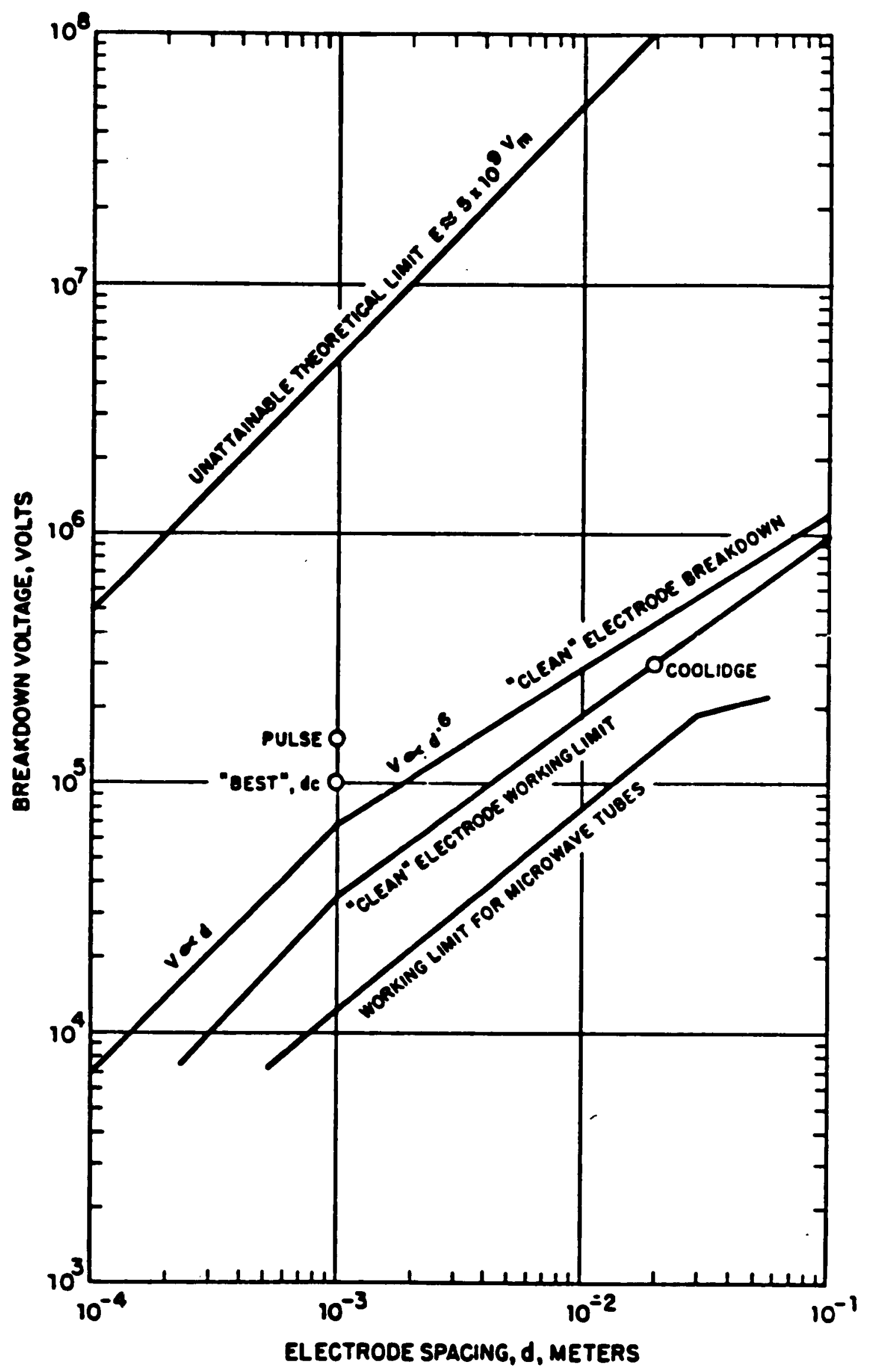




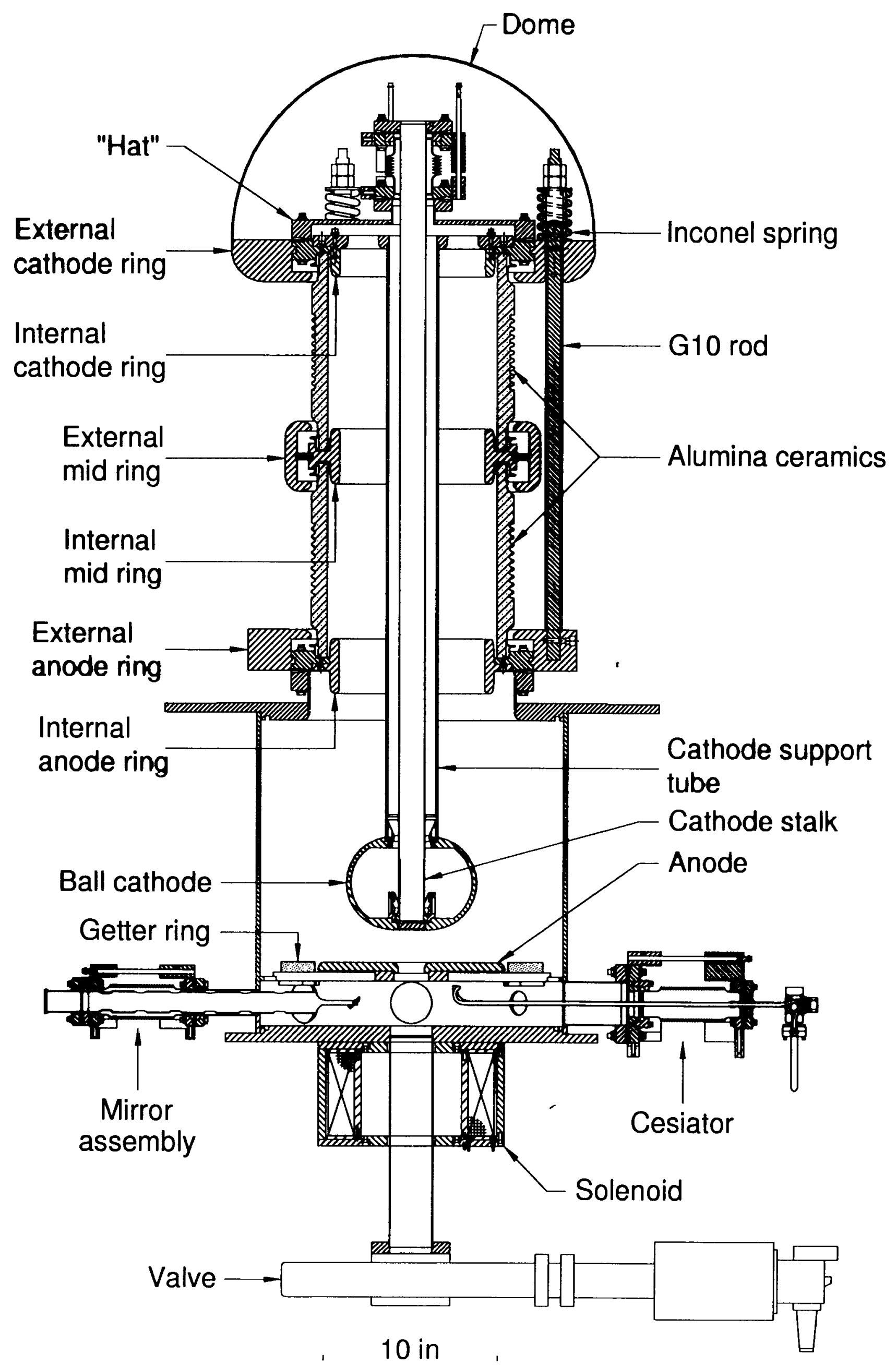




\section{I vs E for 5mm gap 1um SST}

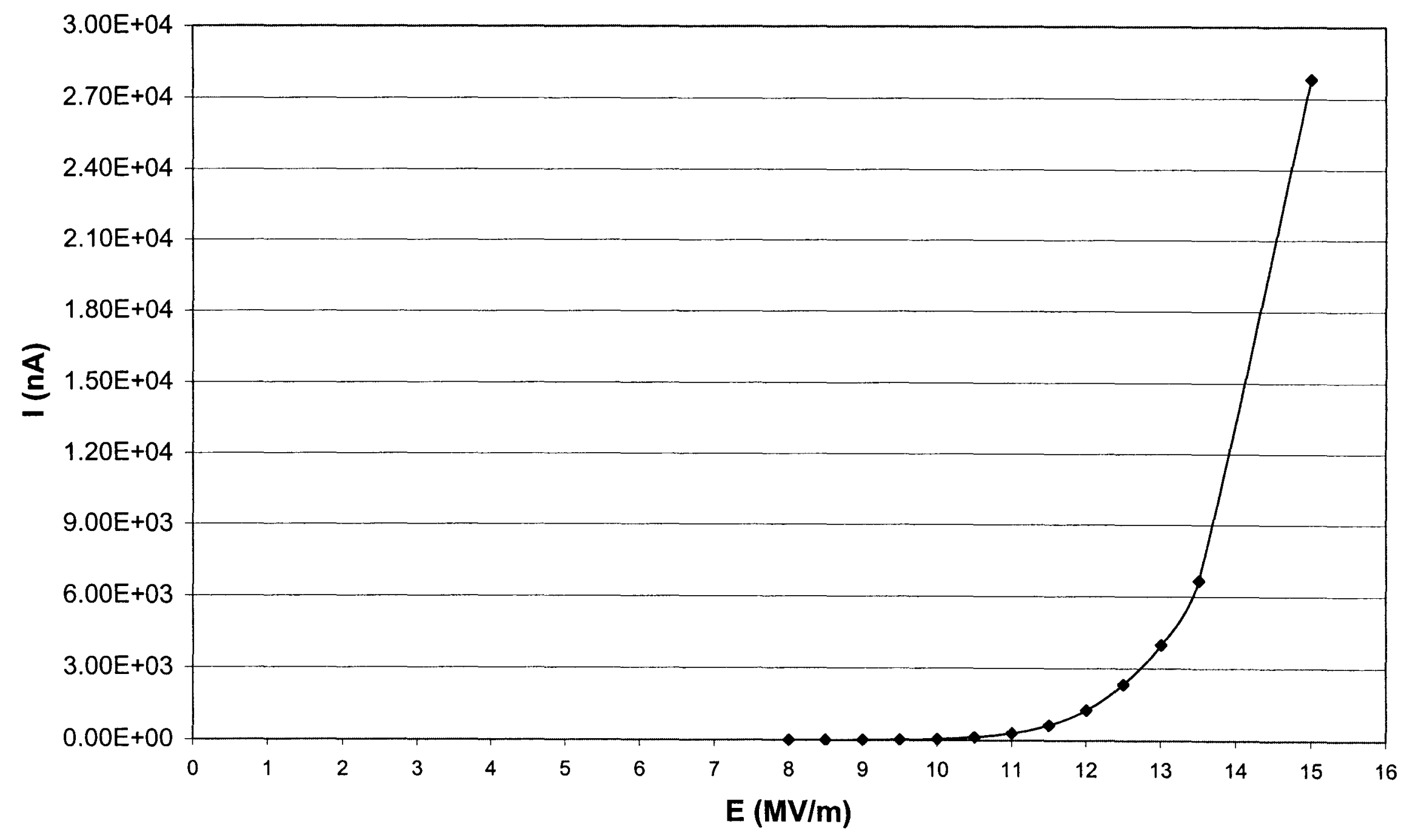


I vs $\mathrm{E}$ for $6 \mathrm{~mm}$ gap 1um Ti

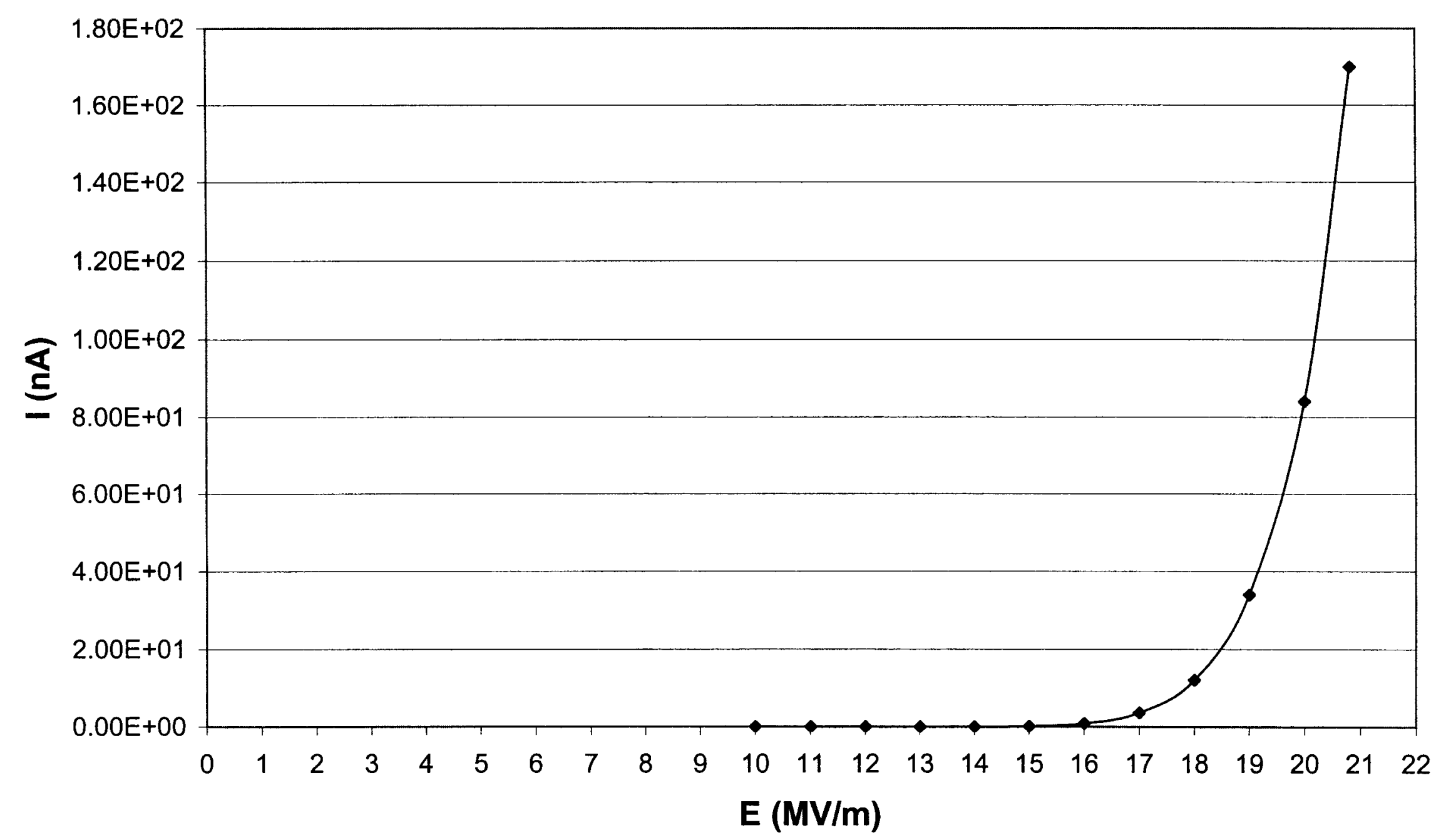


I vs E 4mm gap 9um SST Nitrogen Implanted

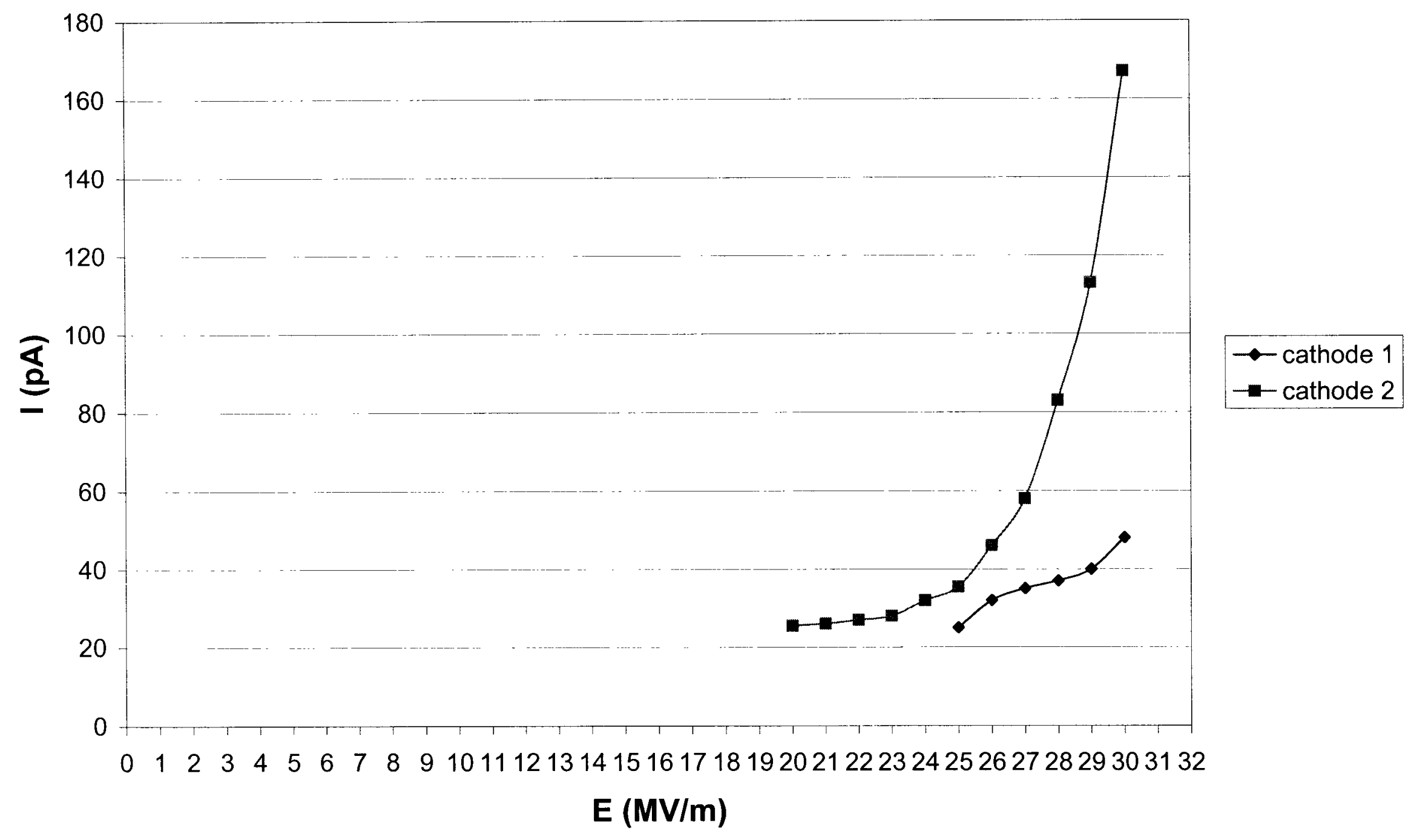




\section{CONCLUSIONS}

- The NEA cathode offers a very small thermal emittance, and the hope that lowering the cathode temperature would reduce this even further.

- Cathode lifetime is presently limited only by ion back bombardment, implying that improved vacuum (which is surely possible) will result in lifetime improvements.

- The primary issues in a DC gun - the cathode insulator and reduction of field emission - appear under control for guns operating up to $\sim 750 \mathrm{kV}$ with $\sim 20 \mathrm{MV} / \mathrm{m}$ cathode field.

- Lasers for any $200 \mathrm{~mA}$ photoemission gun are a challenge. The GaAs cathode offers the advantage of using IR light, avoiding frequency doubling (or more), providing the highest current per watt of light, and using a laser which easily provides the RF structure. 


\section{LASER SYSTEMS FOR DC AND RF GUNS}

\section{BABZIEN, B. SHEEHY, T. SRINIVASAN-RAO, T.TSANG}

Brookhaven National laboratory, Upton, NY 11973

Presented at the workshop on Photoinjector for energy recovery LINAC, Jan. 22-23, 2001 


\section{LASER SYSTEM REQUIREMENTS}

POWER REQUIREMENT:

REQUIRED CURRENT * PHOTON ENERGY/ QE

FOR $200 \mathrm{~mA}$ CURRENT, 2 eV PHOTONS, AND 2\% QE,

LASER POWER $=20 \mathrm{~W}$ ON CATHODE

$\sim 20$ ps FWHM

FLAT TOP TRANSVERSE AND LONGITUDINAL PROFILE

PULSE TRAIN SHAPING

\section{STABILITY}

AMPLITUDE: $\quad 10^{-3}$ pk-pk at $10 \mathrm{MHz}$

TIMING: $\quad 0.2 \mathrm{ps}$ RMS for freq. $>1 \mathrm{MHz}$

TRANSVERSE, LONGITUDINAL PROFILE: 5\% pk-pk

\section{RELIABILITY}

24/7 OPERATION

REDUNDANCY

SCALABILITY 


\section{BASIC LAY OUT OF LASER SYSTEM:}

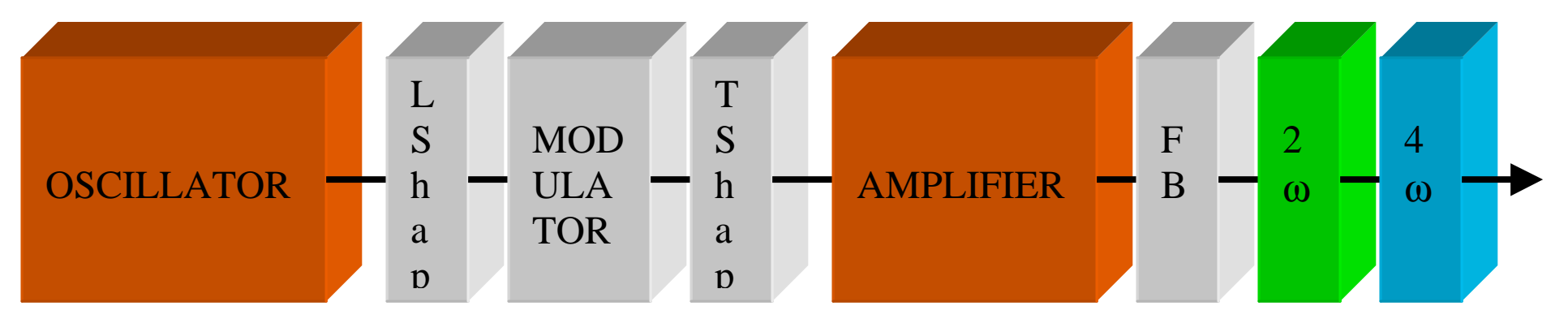




\section{OSCILLATOR:}

a) Single oscillator @ $1.06 \mu \mathrm{m}, 1.3 \mathrm{GHz},<7 \mathrm{ps,} 0.5 \mathrm{~W}$, Custom, commercial unit

Single unit to synchronize

No redundancy

b) Multiple oscillators, each at $1.5 \mu \mathrm{m}, 1.3 \mathrm{GHz}<5 \mathrm{ps}$, tens of $\mathrm{mW}$, Commercial unit

Built in redundancy

Pulse stacking for temporal shaping and Mach-Zender interferometer for slicing Fiber makes alignment relatively easy

Rapid advance due to Communications industry

Suitable for DC/RF gun

Multiple units to synchronize

Requires $3 \omega$ / frequency shifting for multi-alkali cathodes

Scaling to higher powers difficult and requires frequency shifting

May be expensive 


\section{LONGITUDINAL PULSE SHAPING:}

a) Stretching

Conventional

$50 \%$ efficiency

Arbitrary waveform using masks

b) Stacking

Novel technique

No loss

Arbitrary waveform using different path length

Interference effects 


\section{AMPLIFIER:}

\section{Single Amplifier:}

Commercial, diode pumped, high power amplifier modules available

\section{Multiple Amplifiers:}

Fiber Amplifiers with $>10 \mathrm{~W}$ possible

\section{ISSUES:}

\section{Amplitude Stability:}

Diode laser stability driven by current and temperature stability .01\% possible

Saturated or unsaturated operation?

Saturated: Better stability

Gain changes with seed rep. rate

Unsaturated: Gain indep. of rep.rate

Lower stability

Simulations Indicate that $\sim$ *5 -7 degradation of input pulse, depending on the gain 
Spatial and temporal uniformity of micropulses

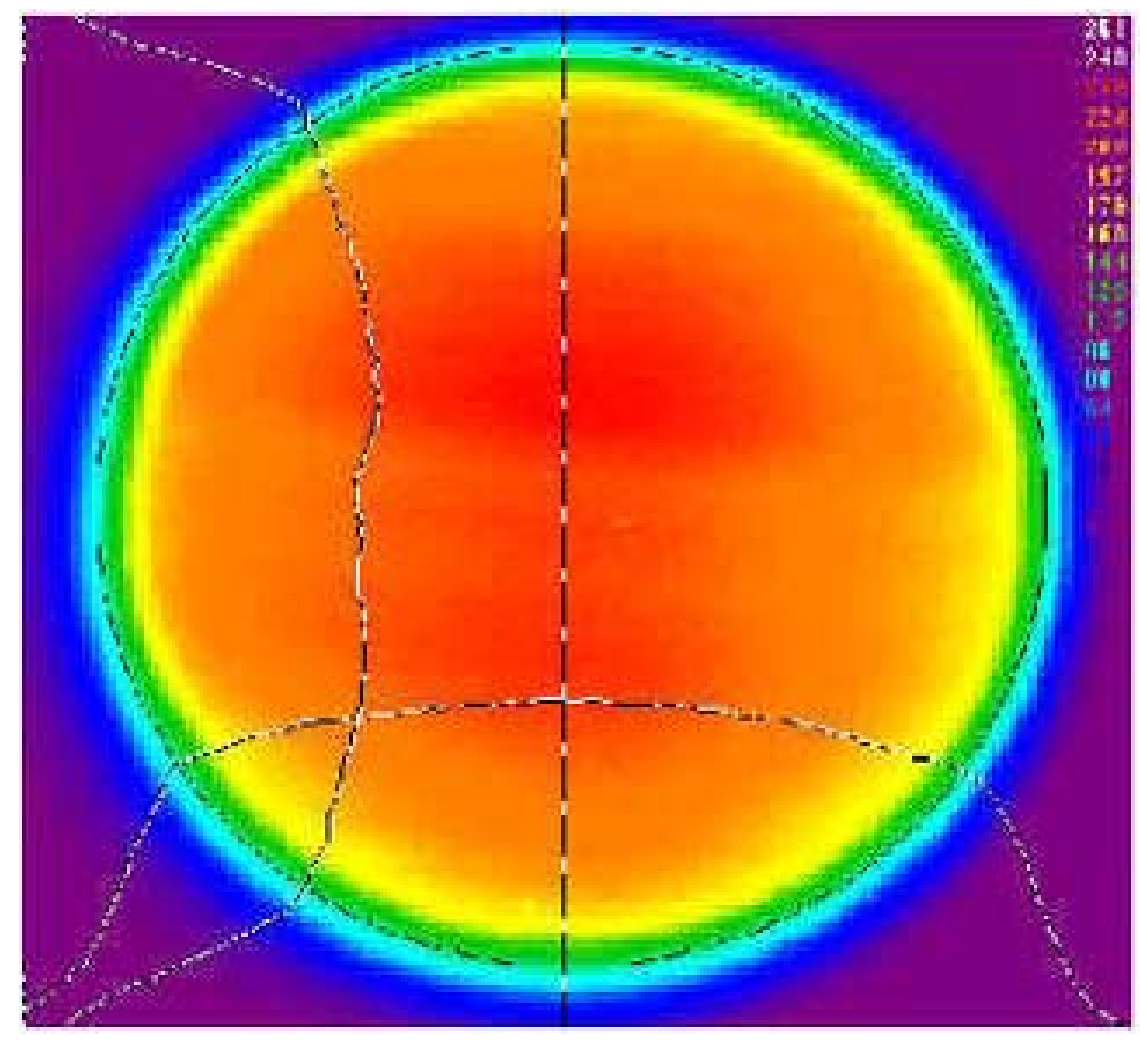

Relay Image of fluorescence of $6.3 \mathrm{~mm}$ diameter, $0.6 \%$ dopant Nd:YAG rod 


\section{FEEDBACK:}

\section{Amplitude}

\section{Timing}

Spatial profile

Temporal profile

\section{DOUBLING CRYSTAL:}

Efficiency:Low peak power and high average power:

$30 \%$ efficiency for the Boeing laser system

$10 \mathrm{~W}$ by intracavity doubling in commercial, diode pumped, CW lasers

Crystal dimensions, Damage threshold to be determined 


\section{VARIOUS OPTIONS AVAILABLE:}

SINGLE OR MULTIPLE HEADS FOR OSCILLATOR AND AMPLIFIERS

LONGITUDINAL SHAPING BY STACKING OR STRETCHING

BULK/MACH-ZENDER MODULATOR

AND MORE

BIGGEST ISSUE: CATHODE LIFETIME AND

MAINTENANCE OF CONTINUOUS OPERATION

CATHODE LIFETIME VARIES FROM HOURS TO WEEK

Ultra high vacuum at cathode is a must

Laser power must be scalable

Quick switch from cathode

LASER SYSTEM MUST BE DESIGNED TO ACCOMMODATE THESE NEEDS 


\title{
Recent Results of the High-Current Advanced FEL Photoinjectors
}

\author{
Dinh Nguyen
}

LANSCE-9

Los Alamos National Laboratory

PERL Photoinjector Workshop

NSLS, Brookhaven National Laboratory

January 22-23, 2001 


\section{Overview of the Advanced Free-Electron Laser (AFEL) Experiment}

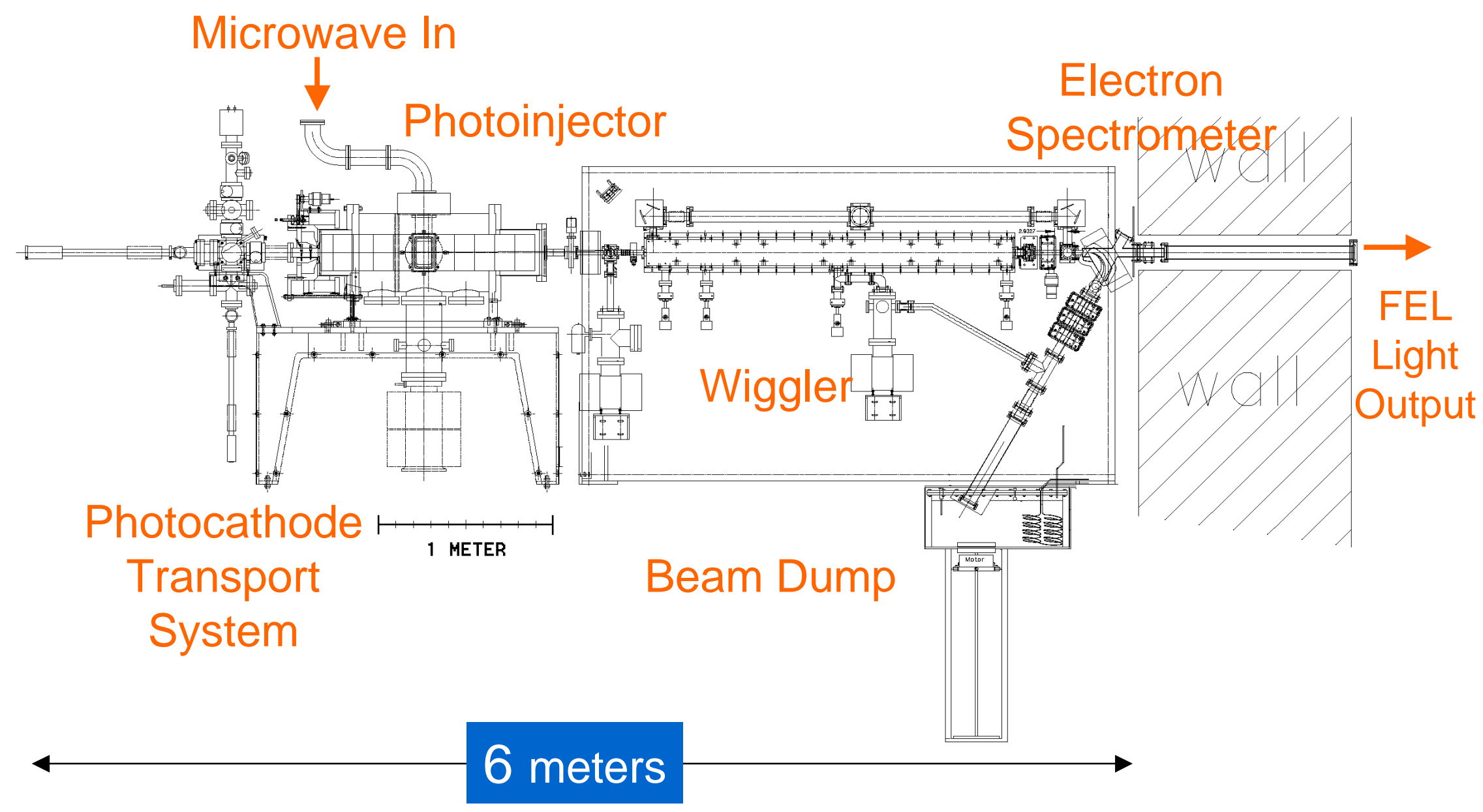




\section{The Advanced FEL Photoinjector Operates at 20 MV/m Gradient and $200 \mathrm{~mA}$ Average Current}

- $1300 \mathrm{MHz}$

- $\mathrm{E}_{\mathrm{b}}=15-20 \mathrm{MeV}$

- $\mathrm{I}_{\text {macro }}=100-400 \mathrm{~mA}$

- $\mathrm{Q}=1-4 \mathrm{nC}$

- $\varepsilon_{\mathrm{rms}}=1.6 \mathrm{~mm}-\mathrm{mrad}$

- $\Delta \gamma / \gamma=0.2 \%$

- Injection $\phi=30^{\circ}$

- Solenoid $=300 \mathrm{~A}$

- Bucking Sol. $=310 \mathrm{~A}$

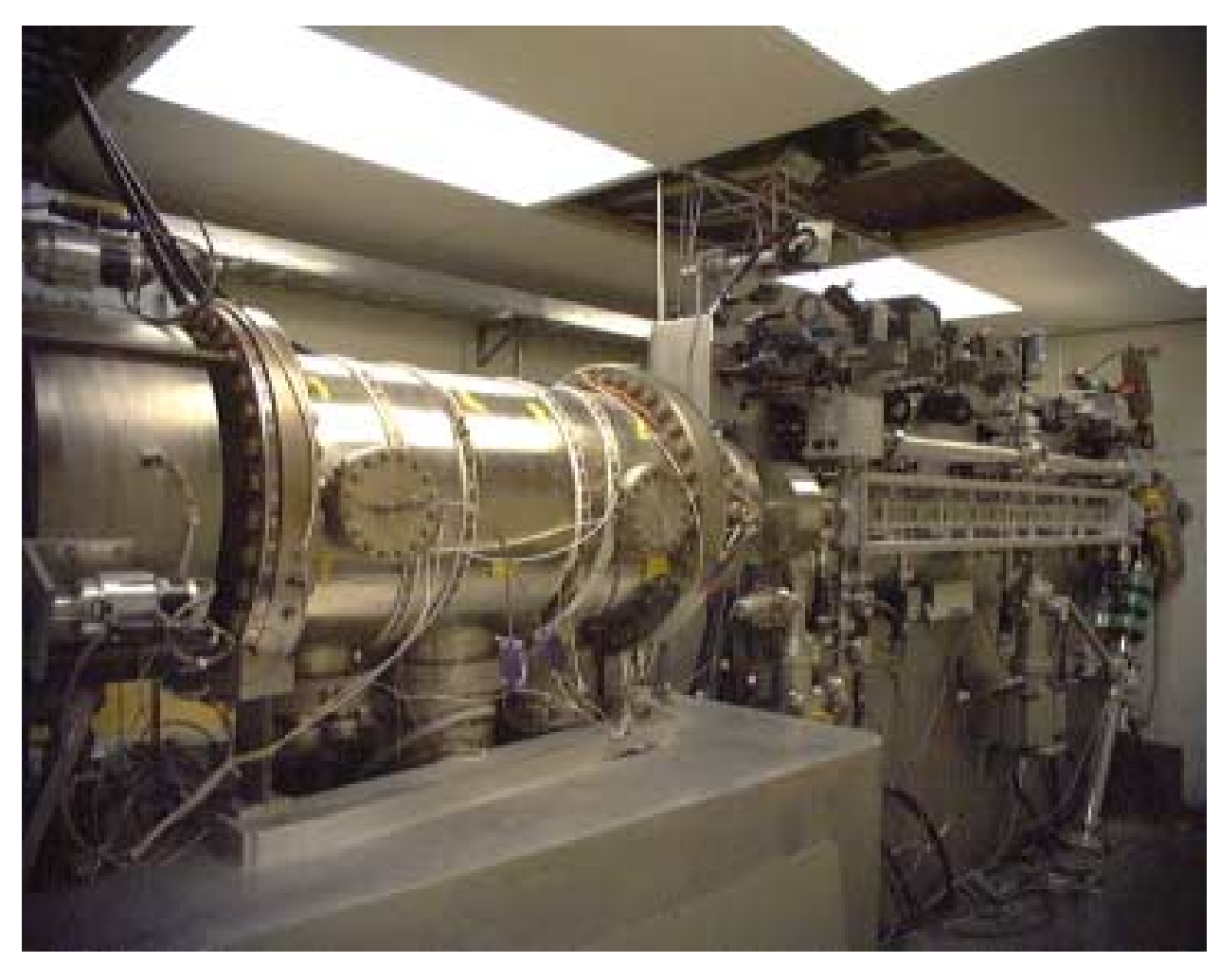




\section{Schematic of the AFEL Photoinjector}

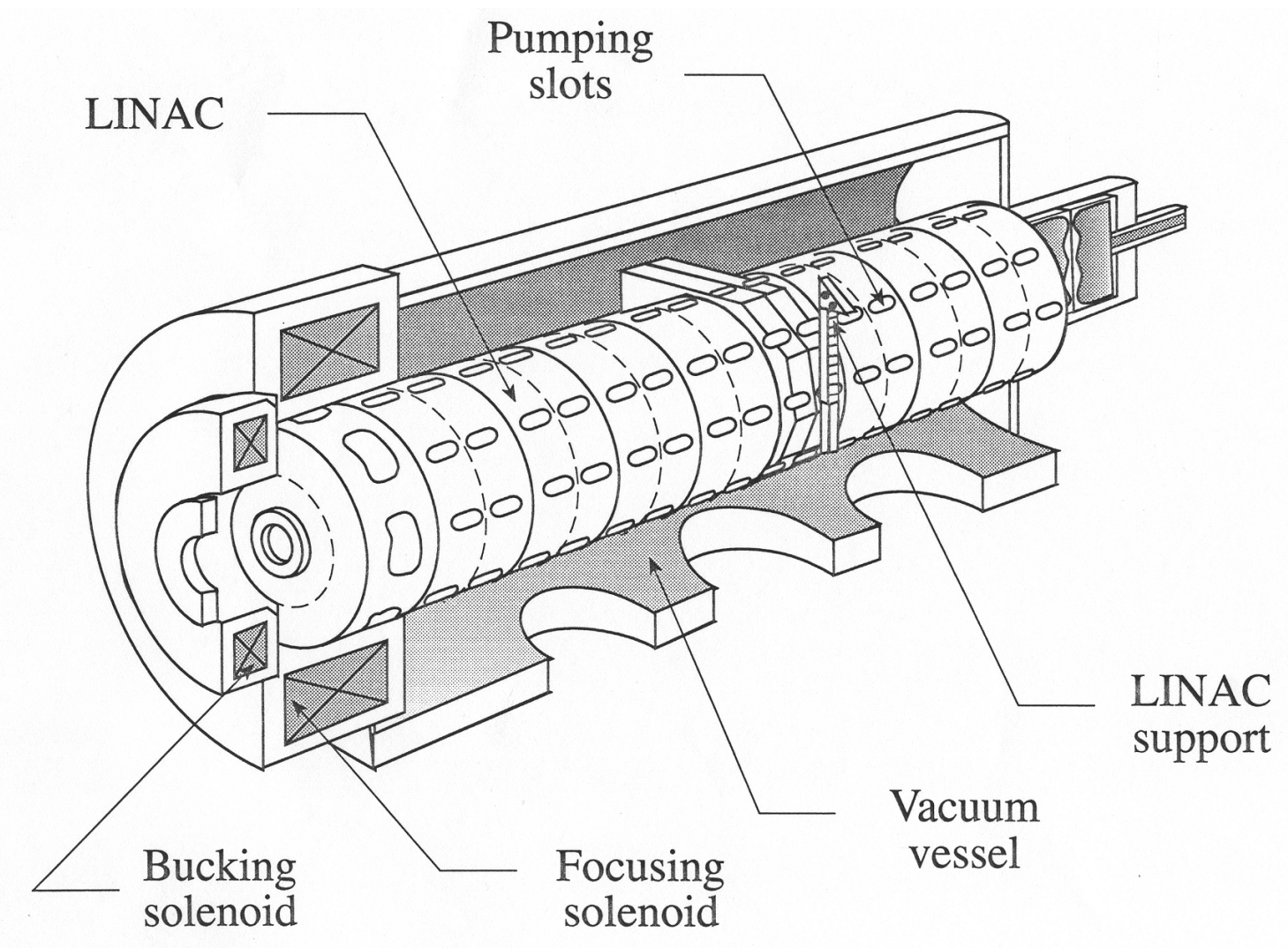

Shunt Impedance $=40 \mathrm{M} \Omega / \mathrm{m}$

Ohmic Loss at $20 \mathrm{MV} / \mathrm{m}=8.8 \mathrm{MW}$ peak

Cryogenic $(77 \mathrm{~K})$ operation reduces ohmic loss by a factor 2 . 


\section{$\mathrm{Cs}_{2}$ Te Energy Levels and Quantum Efficiency}
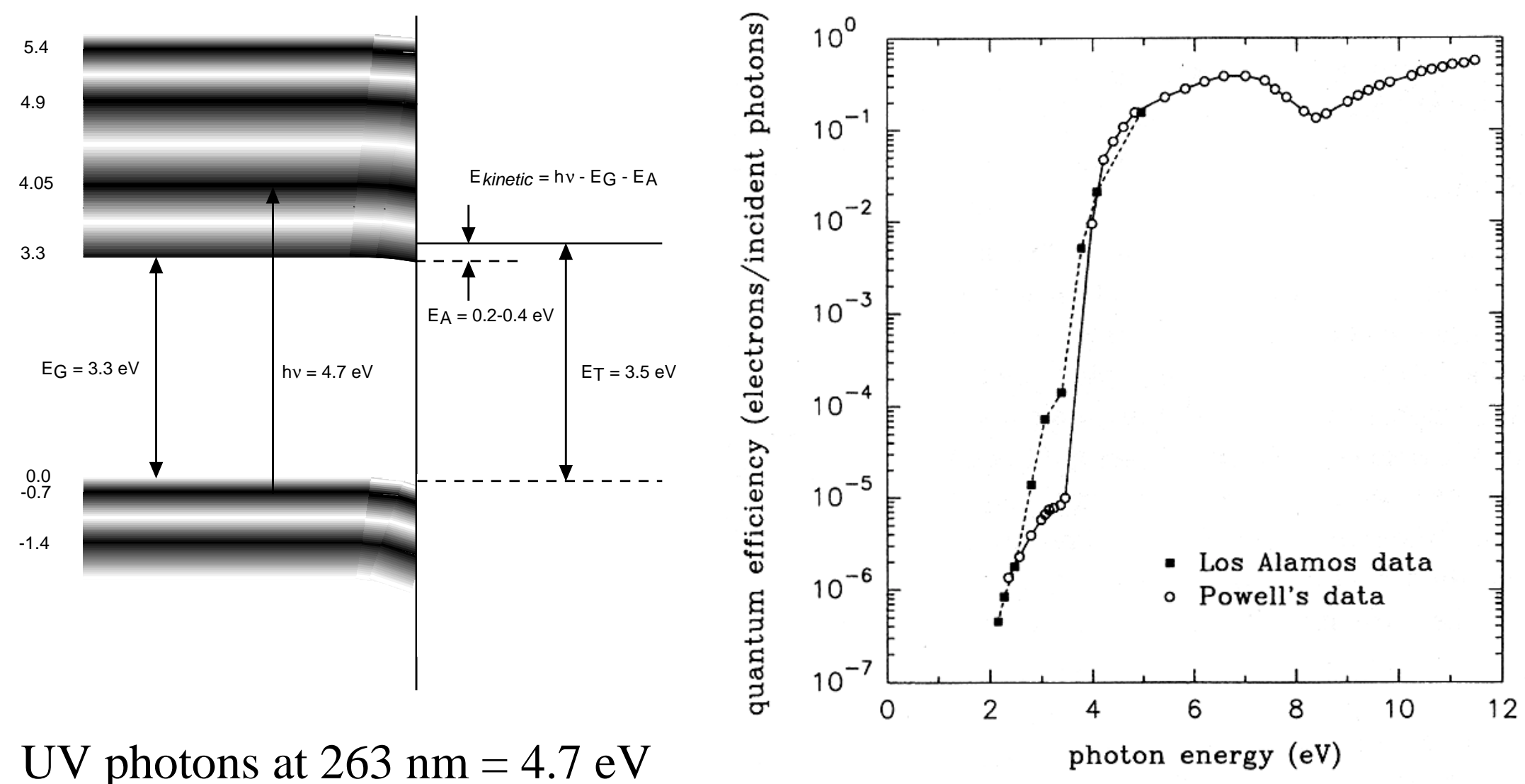

UV photons at $263 \mathrm{~nm}=4.7 \mathrm{eV}$ Electron Affinity $=0.2-0.4 \mathrm{eV}$

Thermal energy of photoelectrons is $\sim 0.2 \mathrm{eV}$

Estimated thermal emittance $=0.3 \mathrm{~mm}-\mathrm{mrad} / \mathrm{mm}$ photoemission radius. 


\section{Beam Measurements with $1 \mathrm{nC}$ Charge and $2.6 \mathrm{~mm}$ Emission Radius}

(Steve Gierman's Thesis)

Emittance measurements with a ps streak camera

Measured slice emittance $1.6 \mathrm{~mm}$-mrad at $1 \mathrm{nC}$

PARMELA slice emittance $0.6 \mathrm{~mm}$-mrad at $1 \mathrm{nC}$ (thermal emittance not included)
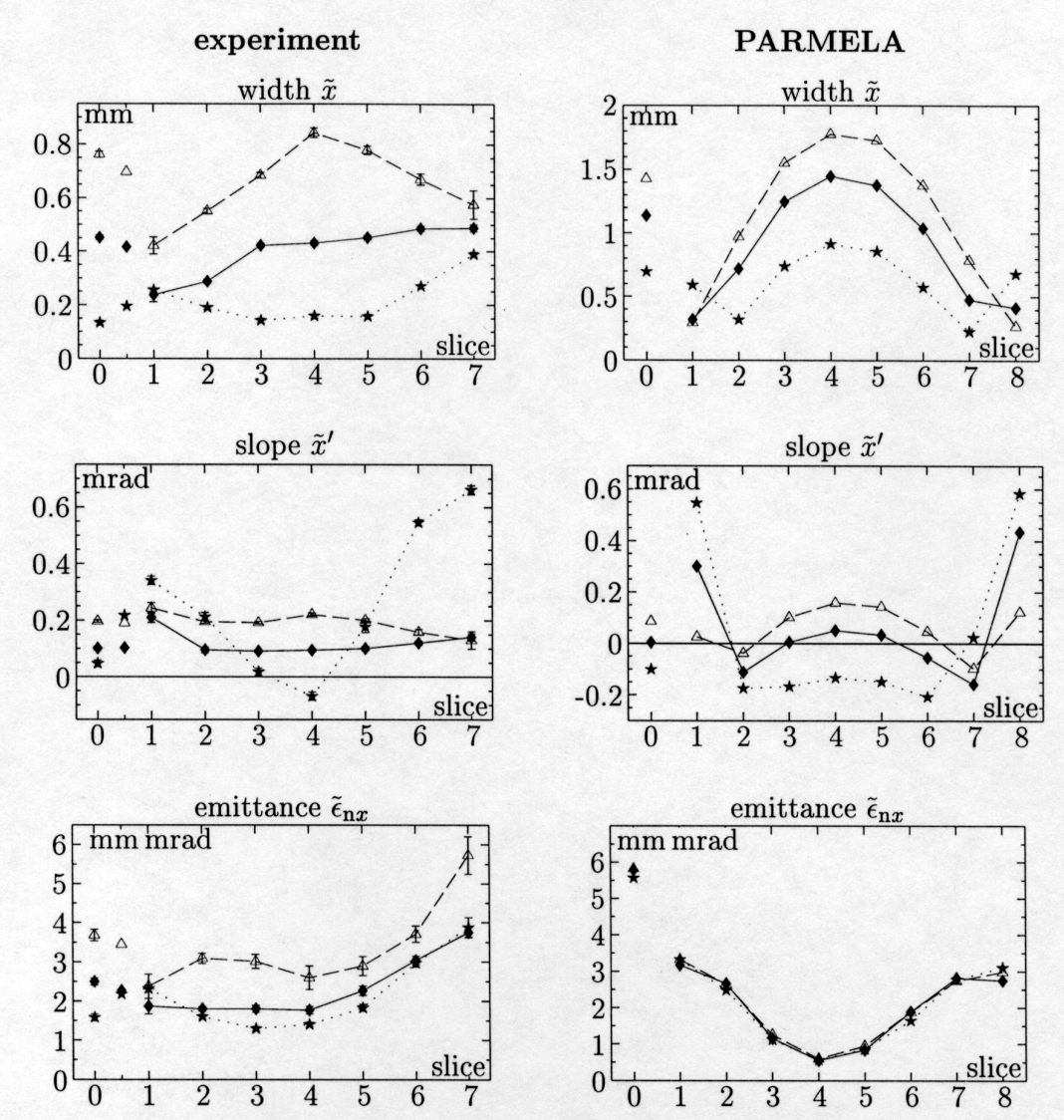

90 A coil current 


\section{Beam Measurements with $<5 \mathrm{nC}$ Bunch Charge and $7 \mathrm{~mm}$ Emission Radius (SASE Experiment)}

Measured rms radius and inferred normalized emittance vs charge
Measured FWHM pulse length and inferred peak current vs charge 


\section{The AFEL Drive Laser Consists of a Diode-pumped Oscillator and two Flashlamp-Pumped Amplifiers}
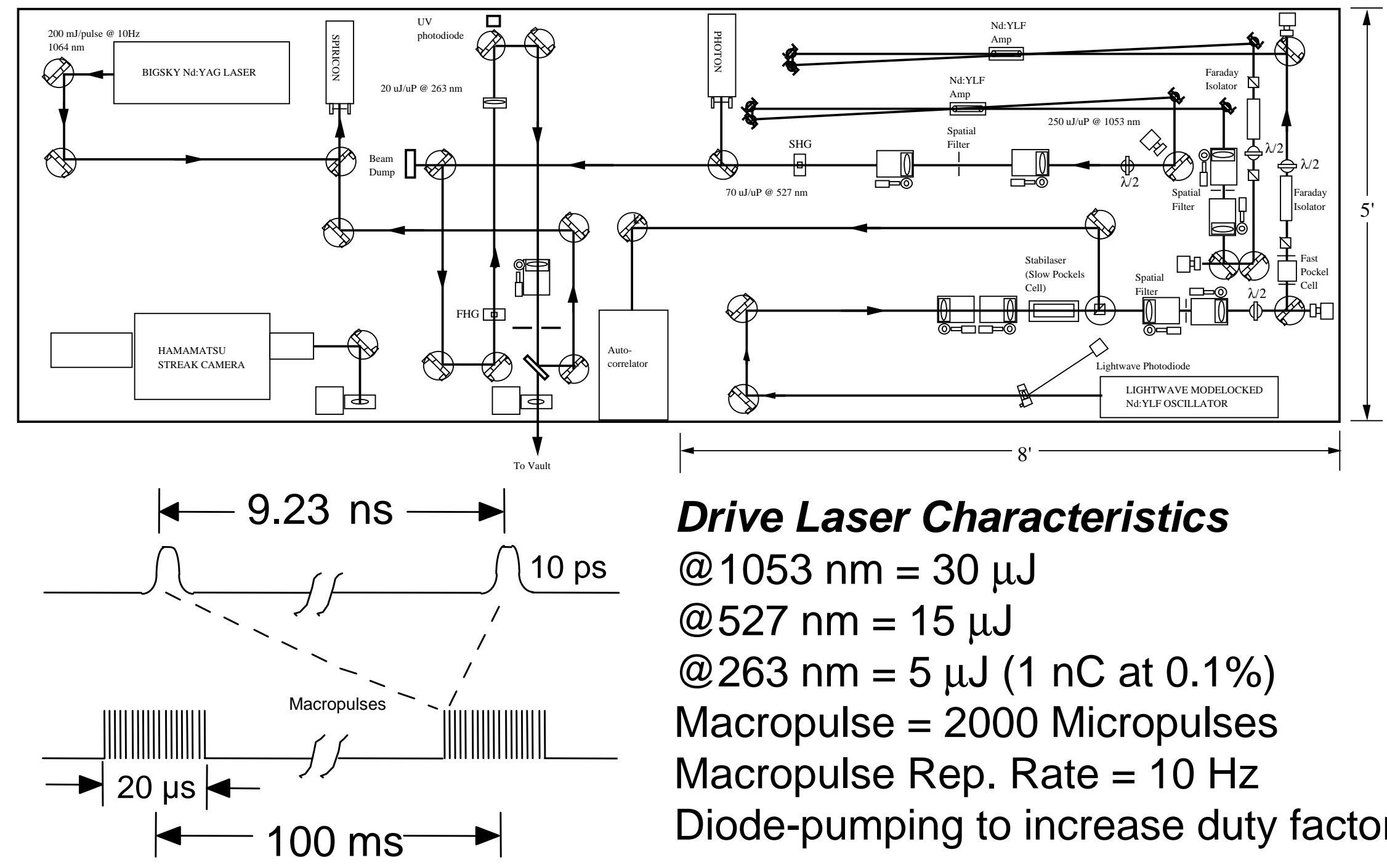

Drive Laser Characteristics

$@ 1053 \mathrm{~nm}=30 \mu \mathrm{J}$

@527 nm = $15 \mu \mathrm{J}$

@263 nm = $5 \mu \mathrm{J}(1 \mathrm{nC}$ at $0.1 \%)$

Macropulse $=2000$ Micropulses

Macropulse Rep. Rate $=10 \mathrm{~Hz}$

Diode-pumping to increase duty factor. 


\section{Stability Tests Show 1/e Lifetime of $\mathrm{Cs}_{2}$ Te Photocathode Is 40 Hours at $J=60 \mathrm{~A} / \mathrm{cm}^{2}$}

Measurements in AFEL Linac $200 \mathrm{~mA}$

Low duty

Measurements* in DC Gun

1/e lifetime $=40$ hours at $\mathrm{J}=60 \mathrm{~A} / \mathrm{cm}^{2}$ $\mathrm{Q}=8 \times 10^{6}$ Coulomb $/ \mathrm{cm}^{2}$

* Courtesy of Etec, Inc. An Applied Materials Company
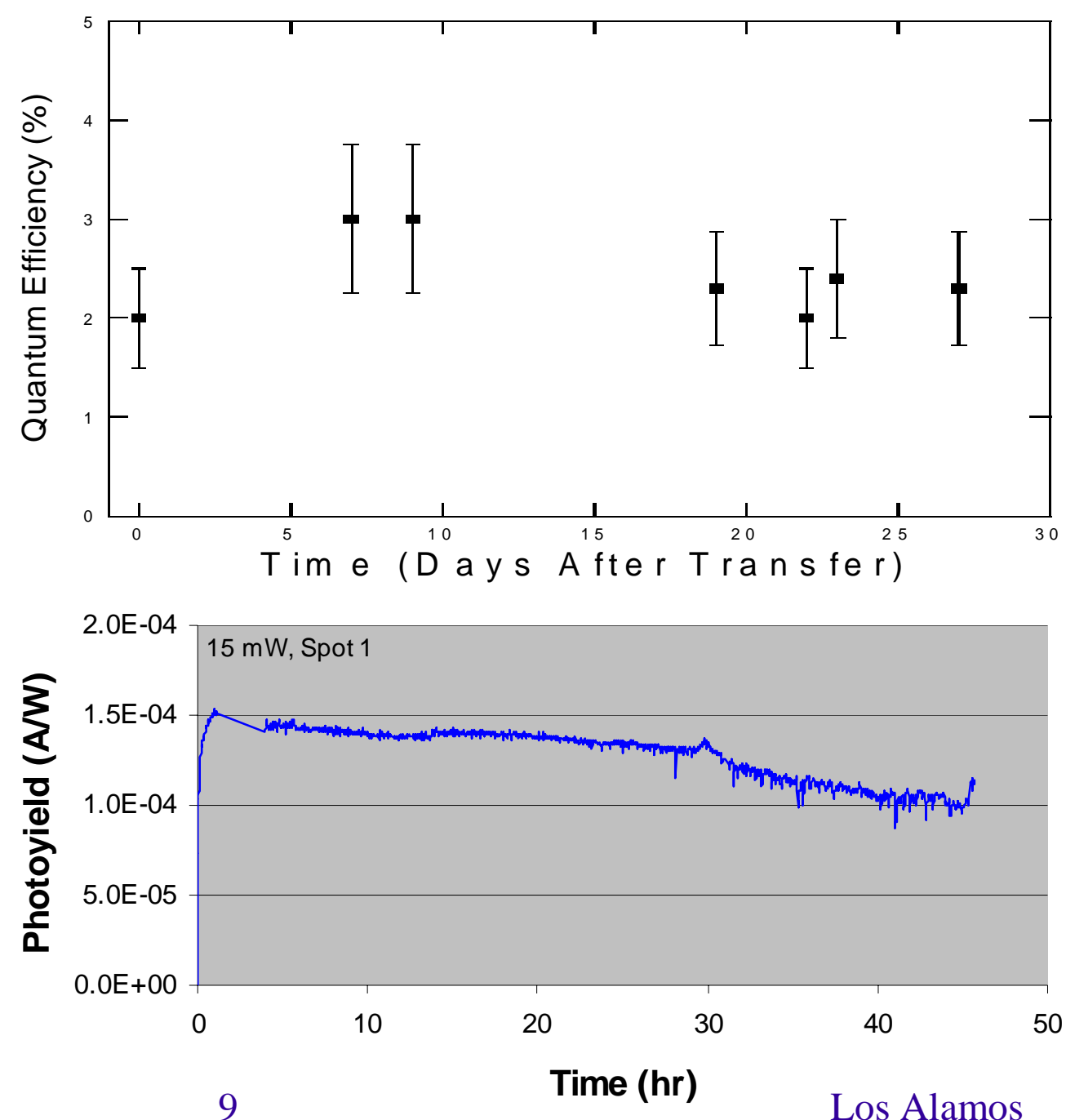


\section{CsBr Coating Passivates $\mathrm{Cs}_{2}$ Te Photocathodes Against Contamination at the Expense of Q.E.}

\begin{tabular}{|c|c|c|}
\hline & Uncoated & Coated \\
\hline & Cs2Te & CSBr:Cs2Te \\
\hline Total thickness of Te (Angstroms) & 150 & 150 \\
\hline Total thickness of Cs (Exposure minutes) & 200 & 170 \\
\hline Reflective QE (@257 nm) before CSBr & $5 \%$ & $10 \%$ \\
\hline Approximate CsBr thickness (Angstroms) & 0 & 90 \\
\hline Reflective QE (@257 nm) after CSBr & NA & $3 \%$ \\
\hline Retlective QE atter N2 exposure for $15 \mathrm{~min}$ & $<0.0001 \%$ & $0.11 \%$ \\
\hline QE atter N2 exposure \& 150C12-day heating & $<0.0001 \%$ & $0.09 \%$ \\
\hline
\end{tabular}

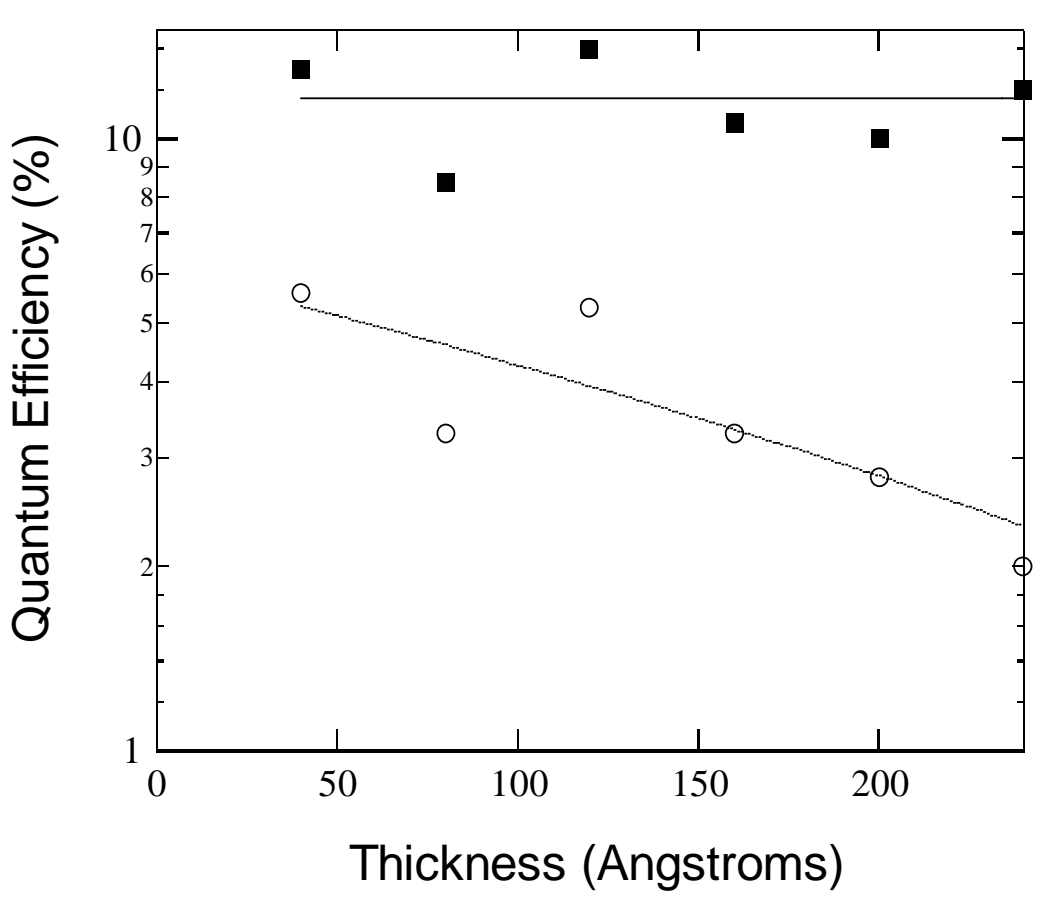




\section{Removable Hexatriacontane Coating Offers Excellent Protection Against Nitrogen Exposure}

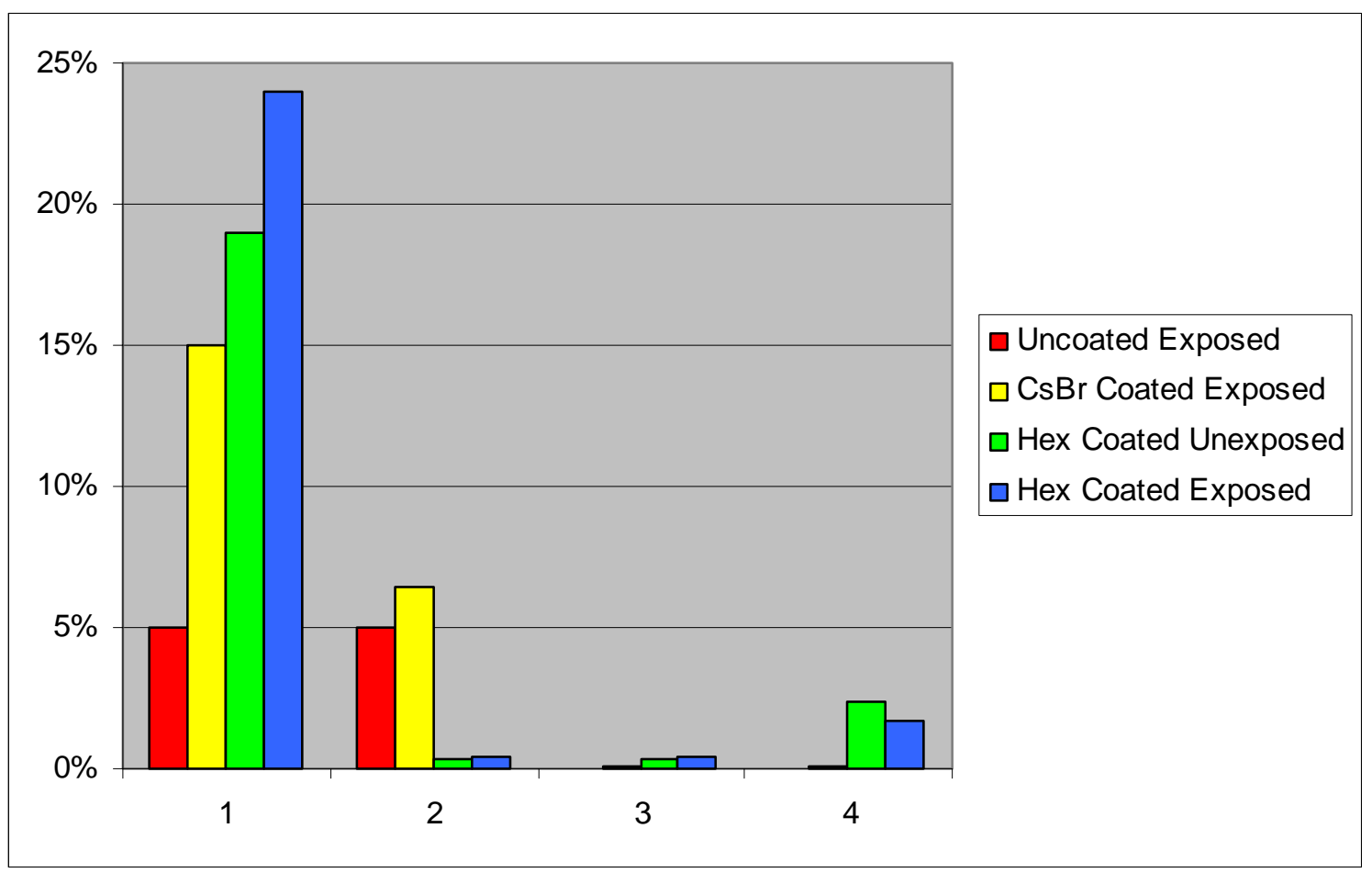

1: Before Coating 3: After Exposed

2: After Coating 4: After Stripping 


\section{Los Alamos Preparation Chamber Provides CVD for Making Semiconductor Photocathodes}

- $\mathrm{Cs}_{2} \mathrm{Te}, \mathrm{CsKTe}$ $\mathrm{CsK}_{2} \mathrm{Sb}, \mathrm{Cs}_{3} \mathrm{Sb}$

- CsBr, NaI, CsI Hexatriacontane

- UHV, RGA

- Thickness Monitor

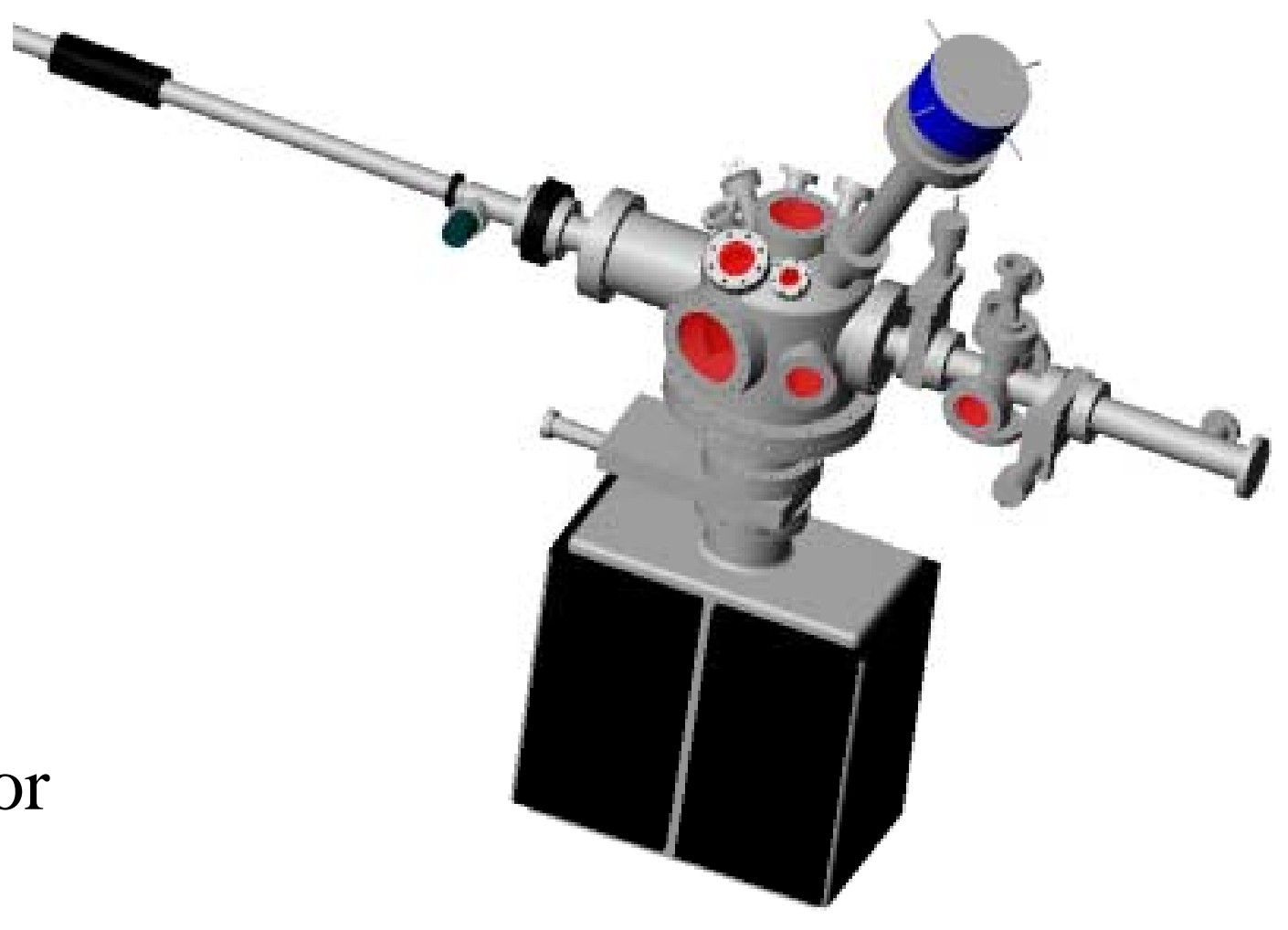

- Cathode transfer mechanism 


\section{Sub-picosecond Accelerator (SPA) for Electron Beam-Pumped Plasma Wakefield}

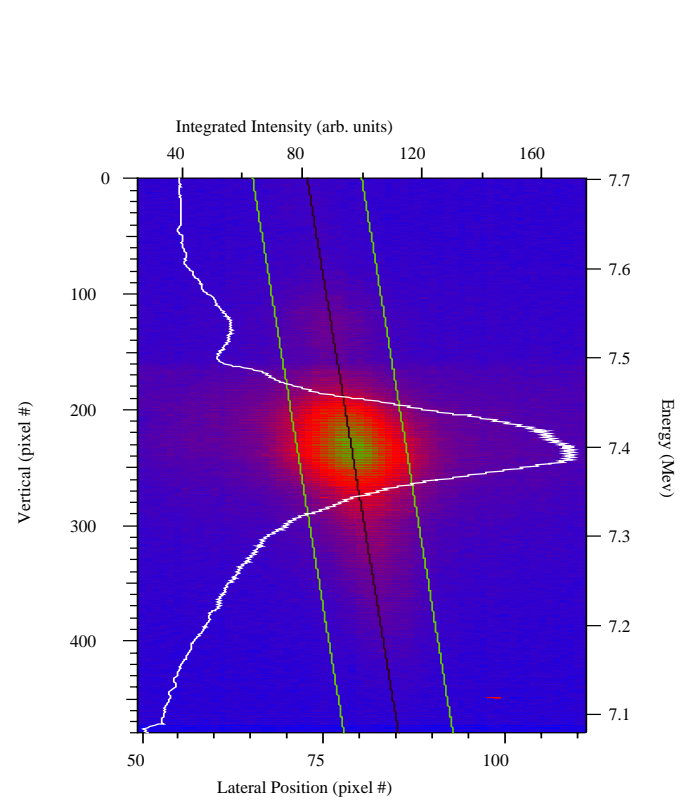

Gas plume off.

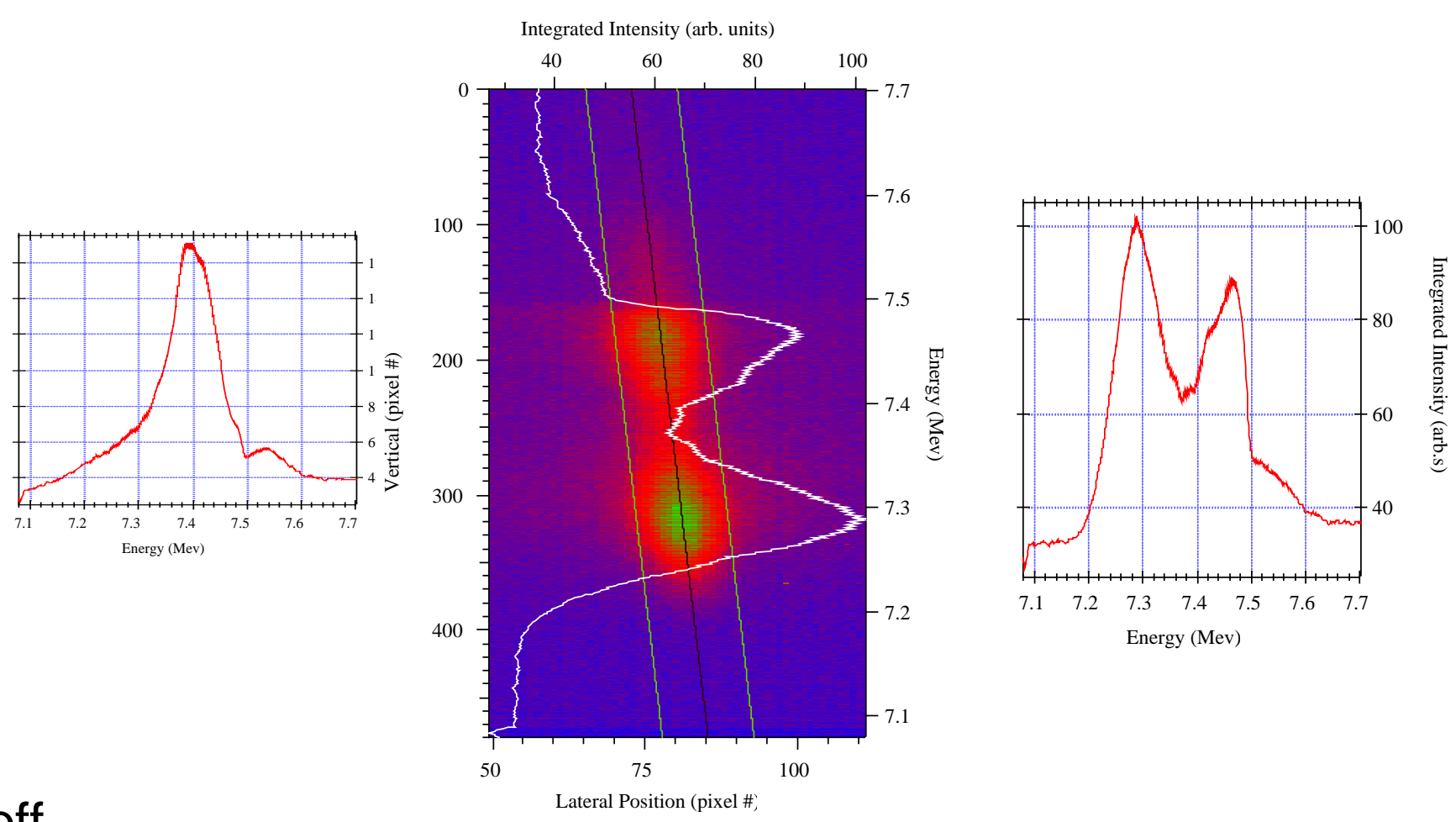

Gas plume on. Demonstrated deceleration gradient is about $60 \mathrm{MV} / \mathrm{m}$. 


\section{Summary}

- The Advanced FEL photoinjector at LANL offers several important capabilities:

$\checkmark$ High average current (up to $400 \mathrm{~mA}$ )

$\checkmark$ Excellent emittance at high charge

$\checkmark$ High repetition rate

$\checkmark$ Cryogenic operation (cut ohmic loss in half)

- Passivating $\mathrm{Cs}_{2} \mathrm{Te}$ photocathode with $\mathrm{CsBr}$ protects against contamination in the linac but not to nitrogen exposure.

- Passivating $\mathrm{Cs}_{2} \mathrm{Te}$ photocathode with removable hexatriacontane demonstrates excellent protection against exposure to 1 atmophere of nitrogen.

- A 1/e lifetime of $40 \mathrm{hrs}$ for continuous operation at 60 $\mathrm{A} / \mathrm{cm}^{2}$ has been achieved.

- Sub-picosecond bunch compression has been demonstrated on the Los Alamos Sub-picosecond Accelerator. 


\title{
$433 \mathrm{MHz}$ High Duty Photocathode RF Gun Experiments
}

\author{
David H. Dowell \\ Boeing Physical Sciences Research Center \\ Seattle, WA
}

Boeing

J. Adamski, FEL Program Manager

T. D. Hayward, Acc. Physics and Design

P. E. Johnson, Acc. HV, RF Systems and Operations

C. A. Lancaster, Controls

H. Mann, Mechanical Engineering

L. Milliman, Controls

C. D. Parazzoli, Theory and Simulation

A. M. Vetter, Acc. and RF Design

D.H. Dowell/PERL Workshop; Jan 22-23, 2001
Collaborators

P.G. O'Shea, University of Maryland

CEA, Bruyeres-le-Chatel, France:

S. Joly

A. Loulergue

G. Haouat

J.P. de Brion

LANL 


\section{Gun Talk Outline}

I. 433 MHz PhotoInjector Design Philosophy.

II. Review of $433 \mathrm{MHz}$ RF Photocathode Gun Technology

A. Gun Design and Demonstrated Performance

B. Cathode QE and Lifetime

C. Drive Laser Performance

III. Electron Beam Quality

A. Transverse Emittance

B. Longitudinal Emittance

IV. Summary and Conclusions 


\section{Historical Perspective}

\section{Motivation:}

Design, build and test an RF photocathode gun capable of operating at high current and high duty factor for GBFEL.

\section{Result:}

A 1992 demonstration of a two-cell, $433 \mathrm{MHz}$ photocathode gun at $32 \mathrm{~mA}$ of average current and $25 \%$ duty factor. 


\section{Photoinjector Design Philosophy}

Use a CW low frequency photocathode gun to generate high charge (1-5 nC) and long (50 ps) micropulses.

Advantages:

Capable of CW operation High charge

Long micropulses Excellent Beam Quality at High Beam Current

Disadvantage:

Cathode field limited to $25-30 \mathrm{MV} / \mathrm{m}$

Accelerate in Low frequency RF cavities.

Advantages:

Minimizes wakefields

CW operation

Disadvantage:

Accelerating gradient limited to $5 \mathrm{MV} / \mathrm{m}$

Linearize and compress to high peak current at $20 \mathrm{MeV}$.

Advantages:

Linearizing improves compression

Reduces space charge emittance growth

Disadvantage:

Emittance growth due to coherence synchrotron radiation

D.H. Dowell/PERL Workshop; Jan 22-23, 2001 


\section{Layout of the $433 \mathrm{MHz}$ PhotoInjector}

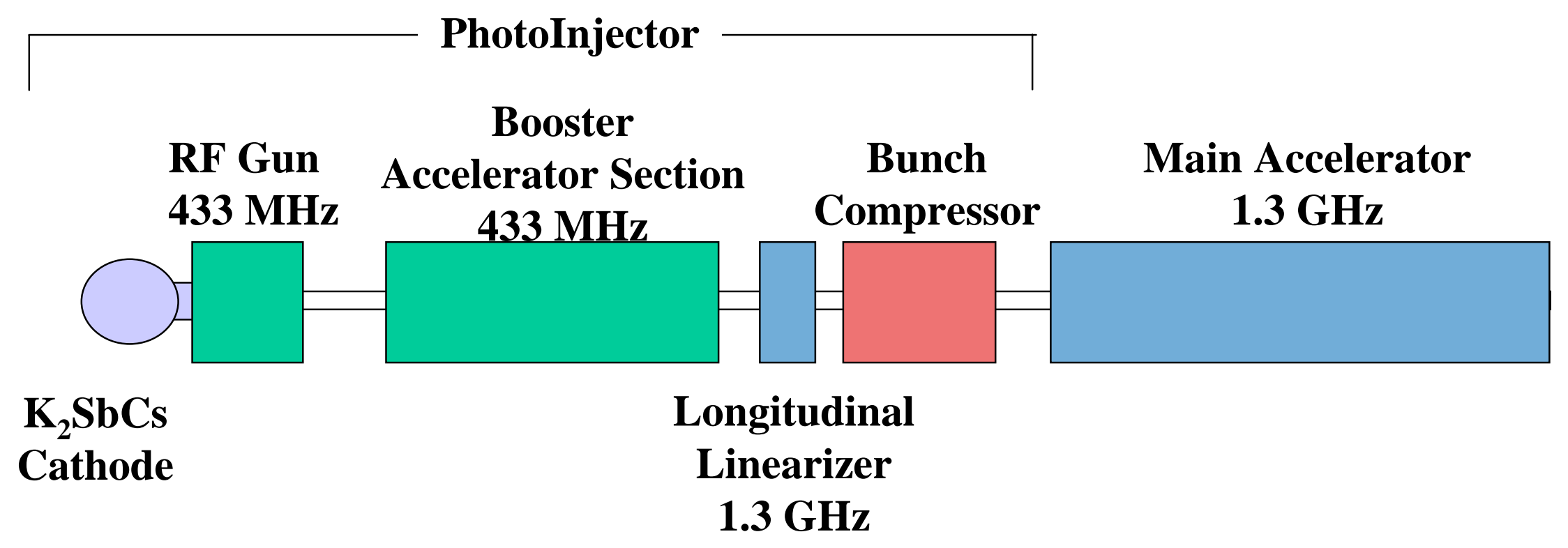




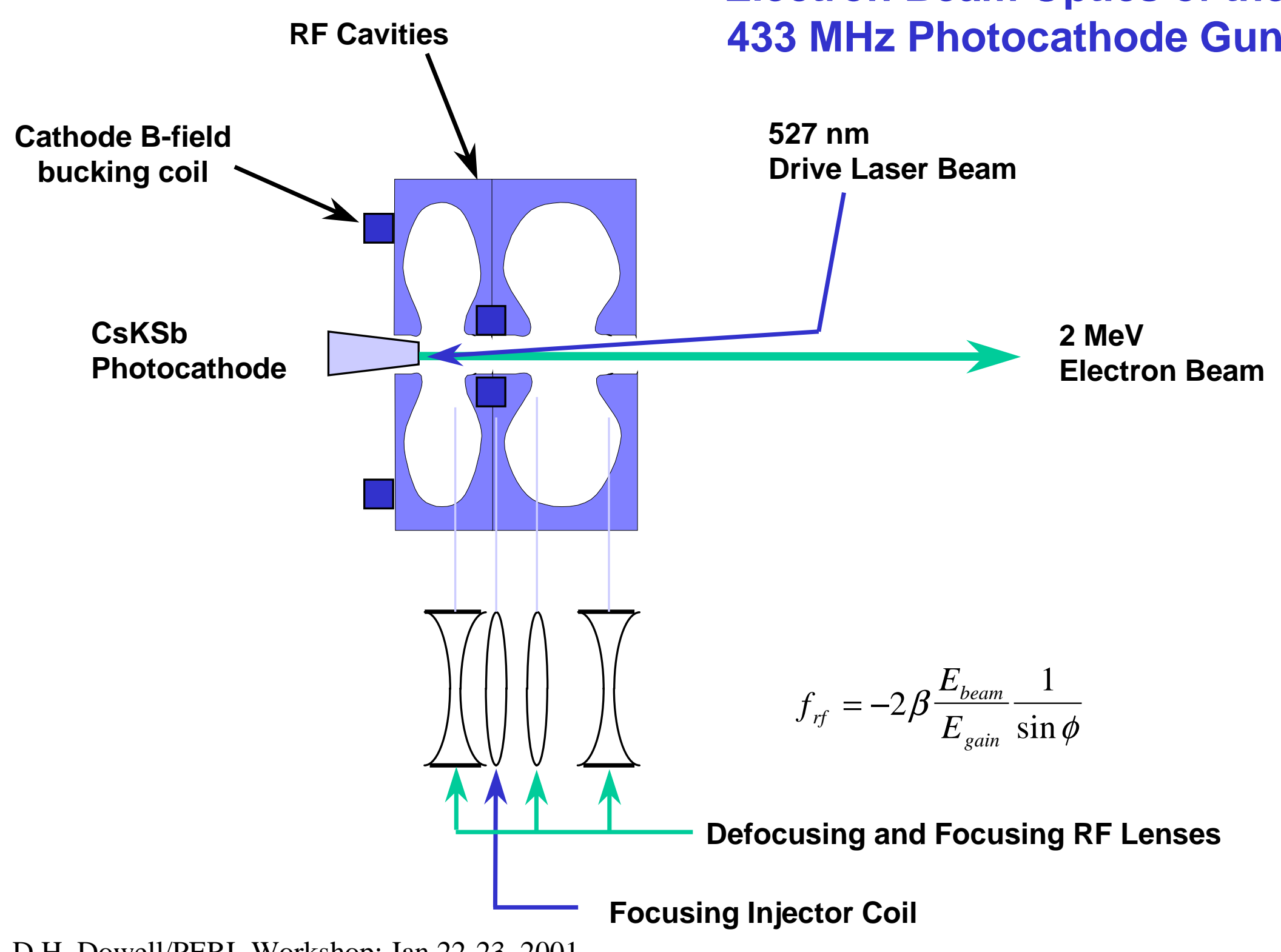

\section{Electron Beam Optics of the $433 \mathrm{MHz}$ Photocathode Gun}

$527 \mathrm{~nm}$

Drive Laser Beam 


\section{The Boeing 433 MHz RF Photocathode Gun}

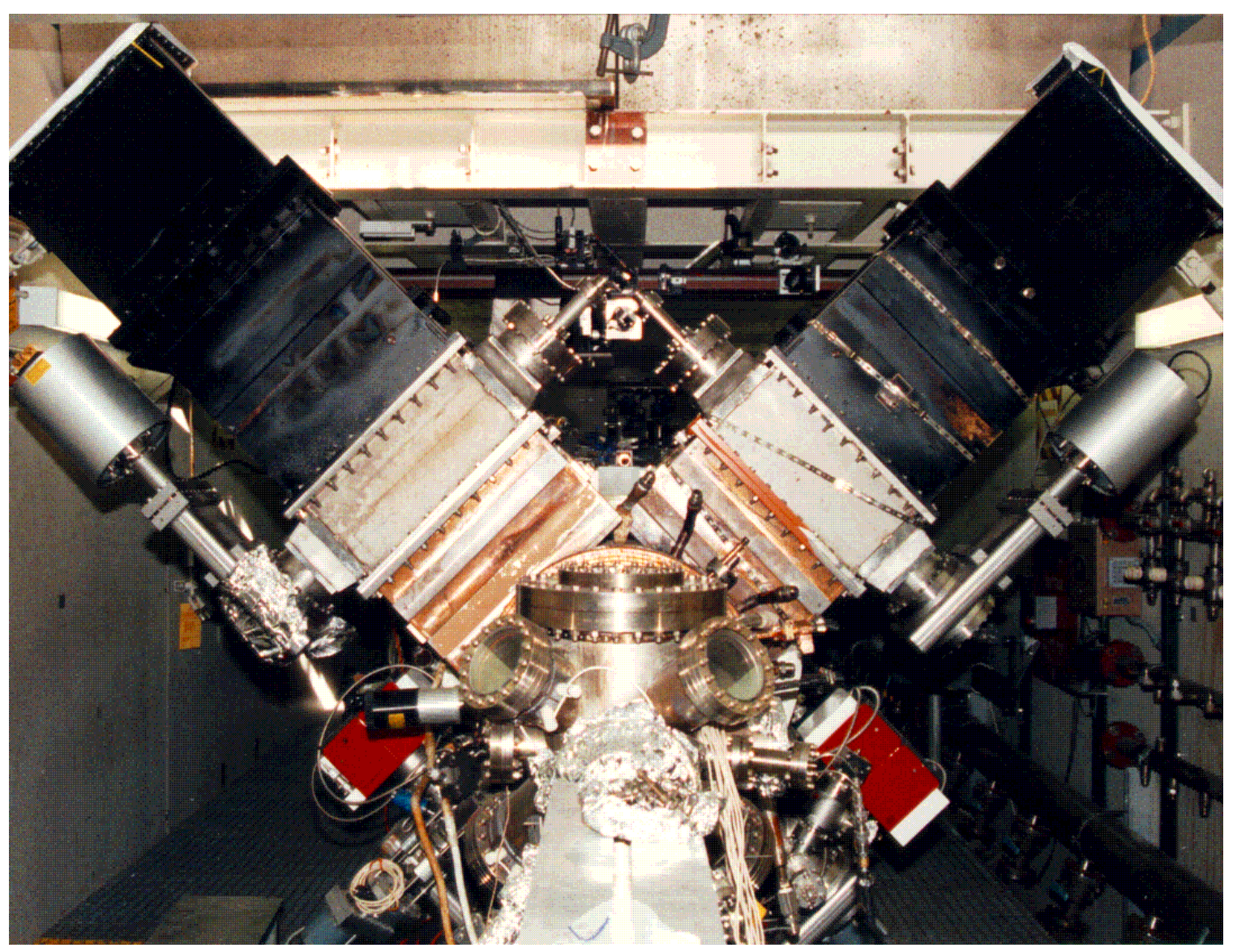




\section{Demonstrated Performance of $433 \mathrm{MHz}$ Photocathode Gun, 1992 H-D Test}

Photocathode Performance:

Photosensitive Material:

Quantum Efficiency:

Peak Current:

Cathode Lifetime:

Angle of Incidence:

Gun Parameters:

Cathode Gradient:

Cavity Type:

Number of cells:

RF Frequency:

Final Energy:

RF Power:

Duty Factor:

Laser Parameters:

Micropulse Length:

Micropulse Frequency:

Macropulse Length:

Macropulse frequency:

Wavelength:

Cathode Spot Size:

Temporal and Transverse Distribution:

Micropulse Energy:

Energy Stability:

Pulse-to-pulse separation:

Micropulse Frequency:

Gun Performance:

Emittance (microns, RMS):

$\mathrm{K}_{2} \mathrm{CsSb}$ Multialkali

$5 \%$ to $12 \%$

45 to 132 amperes

1 to 10 hours

near normal incidence

$26 \mathrm{MV} / \mathrm{meter}$

Water-cooled copper

4

$433 \times 10^{6}$ Hertz

$5 \mathrm{MeV}$ (4-cells)

$600 \times 10^{3}$ Watts

25\%, 30 Hertz and $8.3 \mathrm{~ms}$

$53 \mathrm{ps}$, FWHM

$27 \times 10^{6}$ Hertz

$10 \mathrm{~ms}$

30 Hertz

$527 \mathrm{~nm}$

3-5 mm FWHM

gaussian, gaussian

0.47 microjoule

$1 \%$ to $5 \%$

$37 \mathrm{~ns}$

$27 \times 10^{6}$ Hertz

Charge:

Energy:

Energy Spread:

5 to 10 for 1 to 7 nCoulomb

1 to 7 nCoulomb

$5 \mathrm{MeV}$

100 to $150 \mathrm{keV}$

D.H. Dowell/PERL Workshop; Jan 22-23, 2001 


\section{RF Characteristics of $433 \mathrm{MHz}$ Gun Cavities}

\section{Measured Values}

\begin{tabular}{l|l|l|l|l|}
\multicolumn{5}{|c}{ Measured Gun Cavity RF Characteristics } \\
\hline & \multicolumn{1}{|c|}{ Parameter } & \multicolumn{1}{c|}{ L1 } & \multicolumn{1}{c|}{ L2 } & \multicolumn{1}{c|}{ Units } \\
\hline frequency & $f$ & 433.33 & 433.33 & $\mathrm{MHz}$ \\
\hline shunt impedance & $R=V^{2} / P_{c}$ & 2.86 & 4.28 & $\mathrm{M} \Omega$ \\
\hline coupling coefficient & $\beta$ & 3.1 & 3.1 & \\
\hline
\end{tabular}

Operating Parameters for Existing Gun Cavities External Coupling Coefficient $\beta=3.1$

\begin{tabular}{|ll|c|c|c|}
\hline \multicolumn{1}{|c|}{ Parameter } & L1 & L2 & Units \\
\hline nominal accelerating voltage & $V$ & 0.9 & 1.1 & $\mathrm{MV}$ \\
\hline wall loss power & $P_{c}$ & 285 & 285 & $\mathrm{~kW}$ \\
\hline beam power @ $I_{a v g}=200 \mathrm{~mA}$ & $P_{b}$ & 180 & 220 & $\mathrm{~kW}$ \\
\hline forward power required & $P_{k}$ & 515 & 545 & $\mathrm{~kW}$ \\
\hline reflected power & $P_{r}$ & 50 & 40 & $\mathrm{~kW}$ \\
\hline
\end{tabular}

\section{Optimized for 200 Milliamperes}

Operating Parameters for PERL-Optimized Gun Cavities External Coupling Coefficient $\beta=2.0$

\begin{tabular}{|ll|c|c|c|}
\hline \multicolumn{1}{c}{ Parameter } & & L1 & L2 & Units \\
\hline nominal accelerating voltage & $V$ & 0.9 & 1.1 & MV \\
\hline wall loss power & $P_{c}$ & 285 & 285 & $\mathrm{~kW}$ \\
\hline beam power @ $I_{\text {avg }}=200 \mathrm{~mA}$ & $P_{b}$ & 180 & 220 & $\mathrm{~kW}$ \\
\hline forward power required & $P_{k}$ & 470 & 505 & $\mathrm{~kW}$ \\
\hline reflected power & $P_{r}$ & 5 & 2 & $\mathrm{~kW}$ \\
\hline
\end{tabular}

Data supplied by A.M. Vetter.

See also:

J.L. Warren, T.L. Buller and A.M. Vetter, "Design of MCTD Photoinjector Cavities", Proc. 1989 IEEE PAC, Vol I, pp.420-422. May 20-27, 1989, Chicago, Illinois, 


\section{Types of Photocathodes}

\begin{tabular}{|c|c|c|c|c|c|}
\hline Material & QE Range & Drive Laser Wavelength & $\underset{0}{\stackrel{8}{0}}$ & 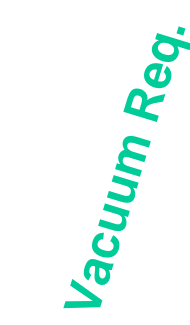 & 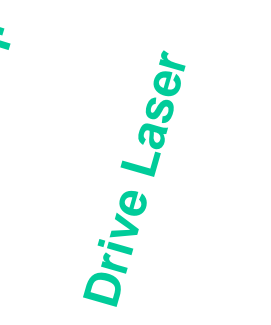 \\
\hline $\begin{array}{l}\text { Metal } \\
\qquad(\mathrm{Cu}, \mathrm{Mo} . . .)\end{array}$ & $\sim 0.02-0.06 \%$ & $260 \mathrm{~nm}, \mathrm{UV}$ & None & $10^{-7} \mathrm{~T}$ & Difficult \\
\hline $\mathrm{CsK}_{2} \mathrm{Sb}$ & $10-14 \%$ & $527 \mathrm{~nm}$ & Difficult & $10^{-10} \mathrm{~T}$ & Moderate \\
\hline CsTe & $10-14 \%$ & $260 \mathrm{~nm}$ & Easy & $10^{-9} \mathrm{~T}$ & $\begin{array}{l}\text { Moderate } \\
\text { to Difficult }\end{array}$ \\
\hline $\mathrm{LaB}_{6}$ & $\sim 0.1 \%$ & $355 \mathrm{~nm}$ & Easy & $10^{-7} \mathrm{~T}$ & Difficult \\
\hline Ga As (Cs) & $1-5 \%$ & $527 \mathrm{~nm}$ & Moderate & $10^{-11} \mathrm{~T}$ & Moderate \\
\hline
\end{tabular}




\section{Photocathode Fabrication Chamber}

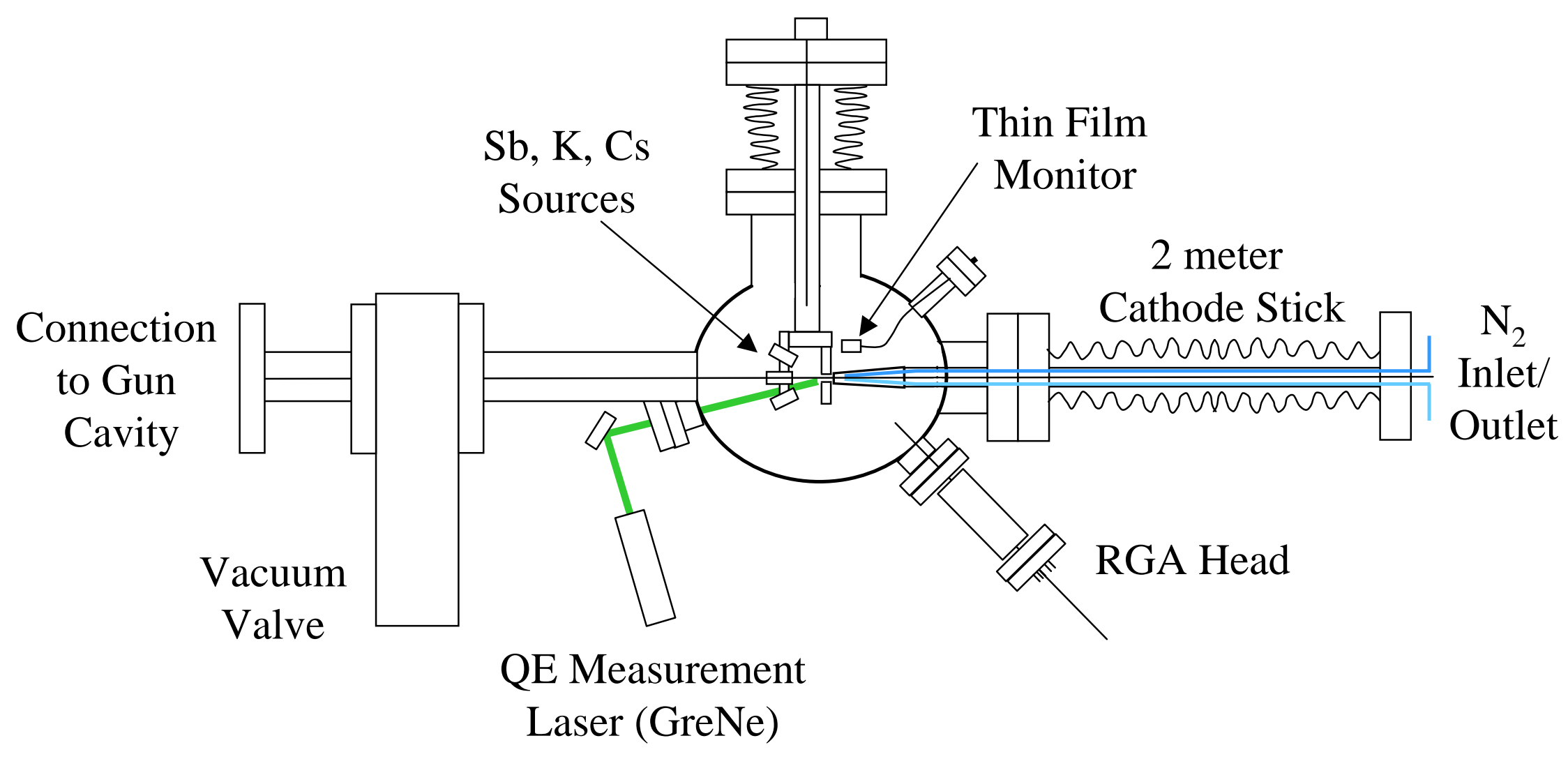


QE Fabrication History and Gun Space Charge Limits

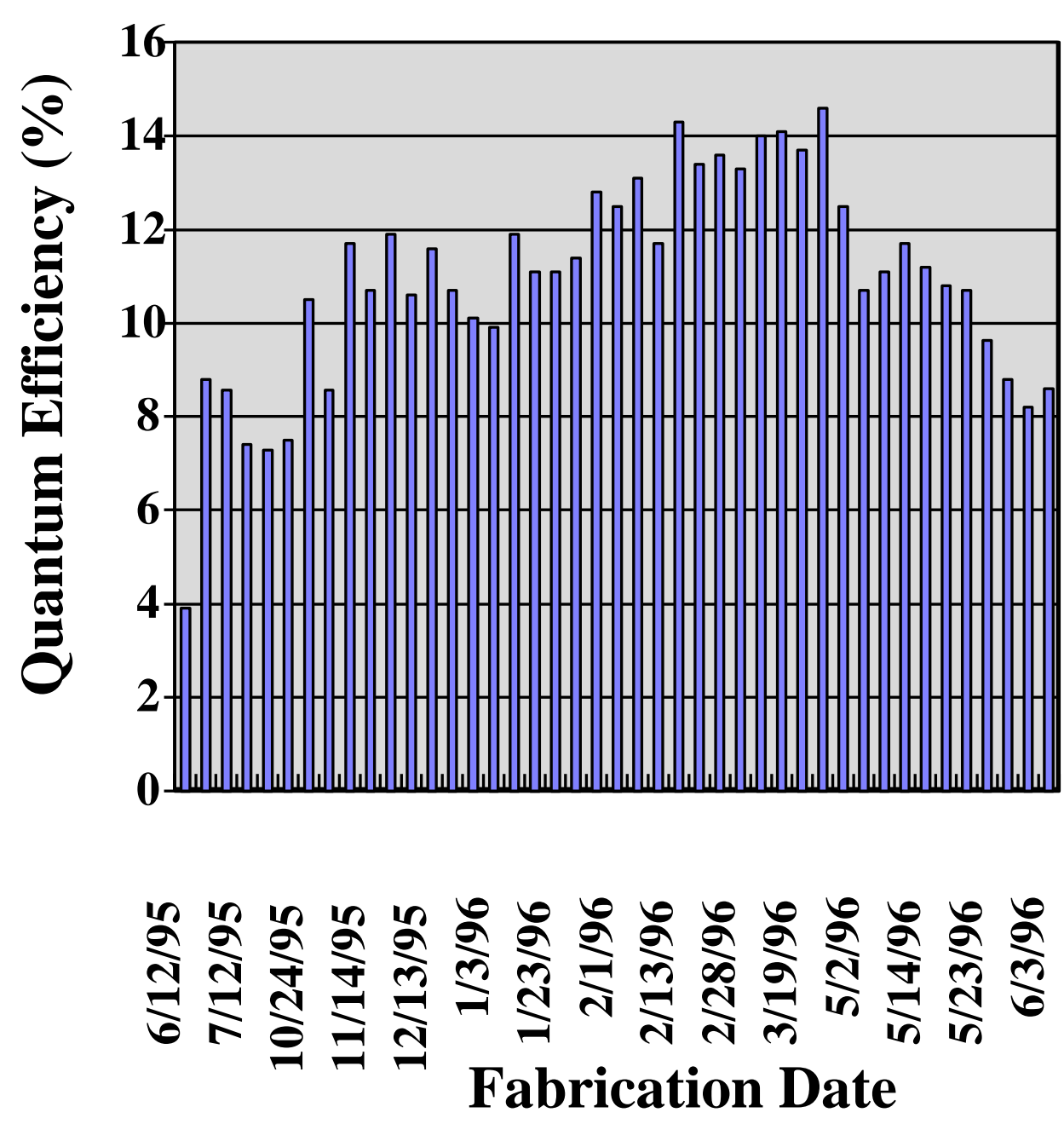

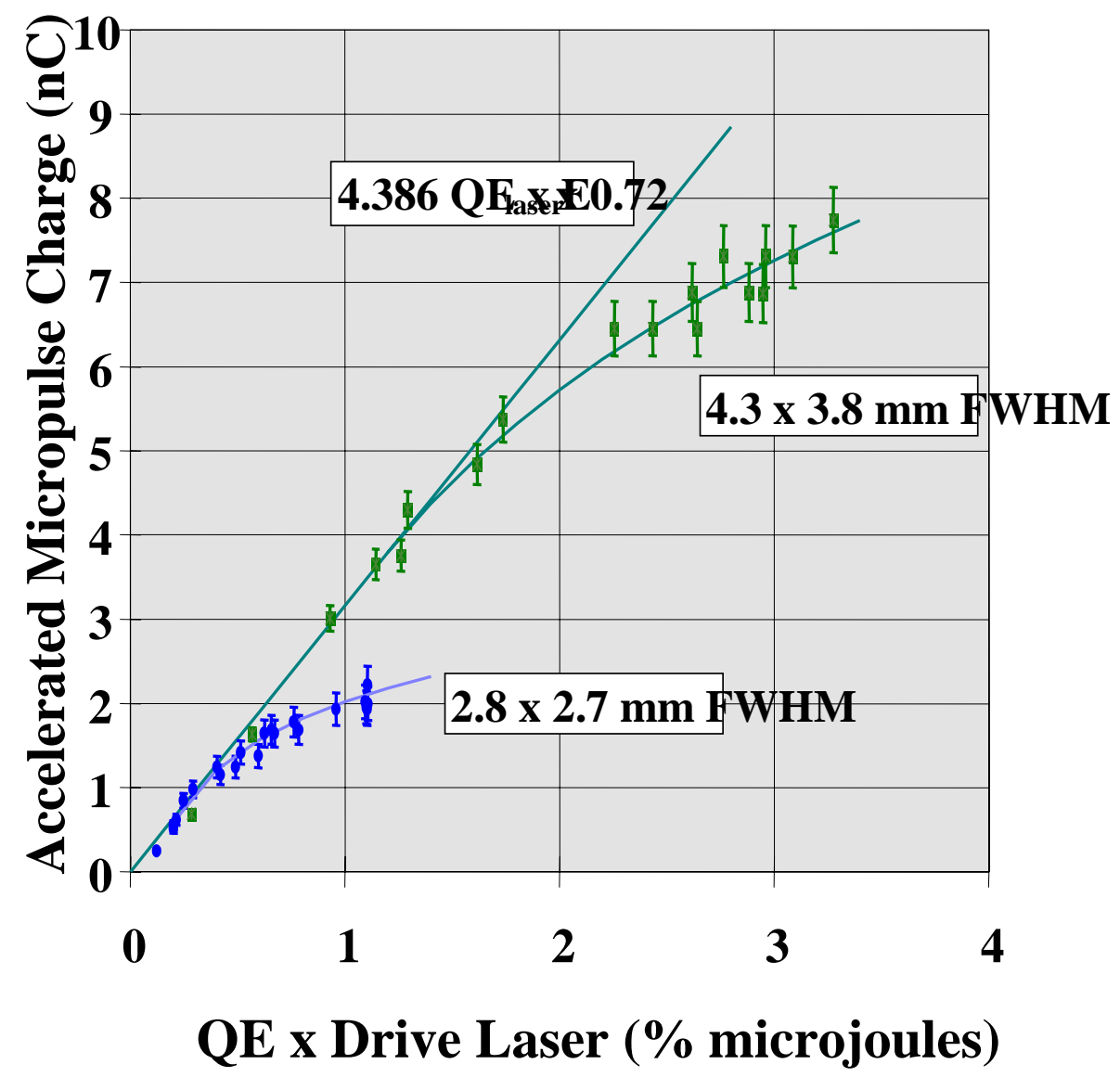

QE x Drive Laser (\% microjoules) 


\section{Cathode Lifetime Vs. $\mathrm{H}_{2} \mathrm{O}$ Partial Pressure}

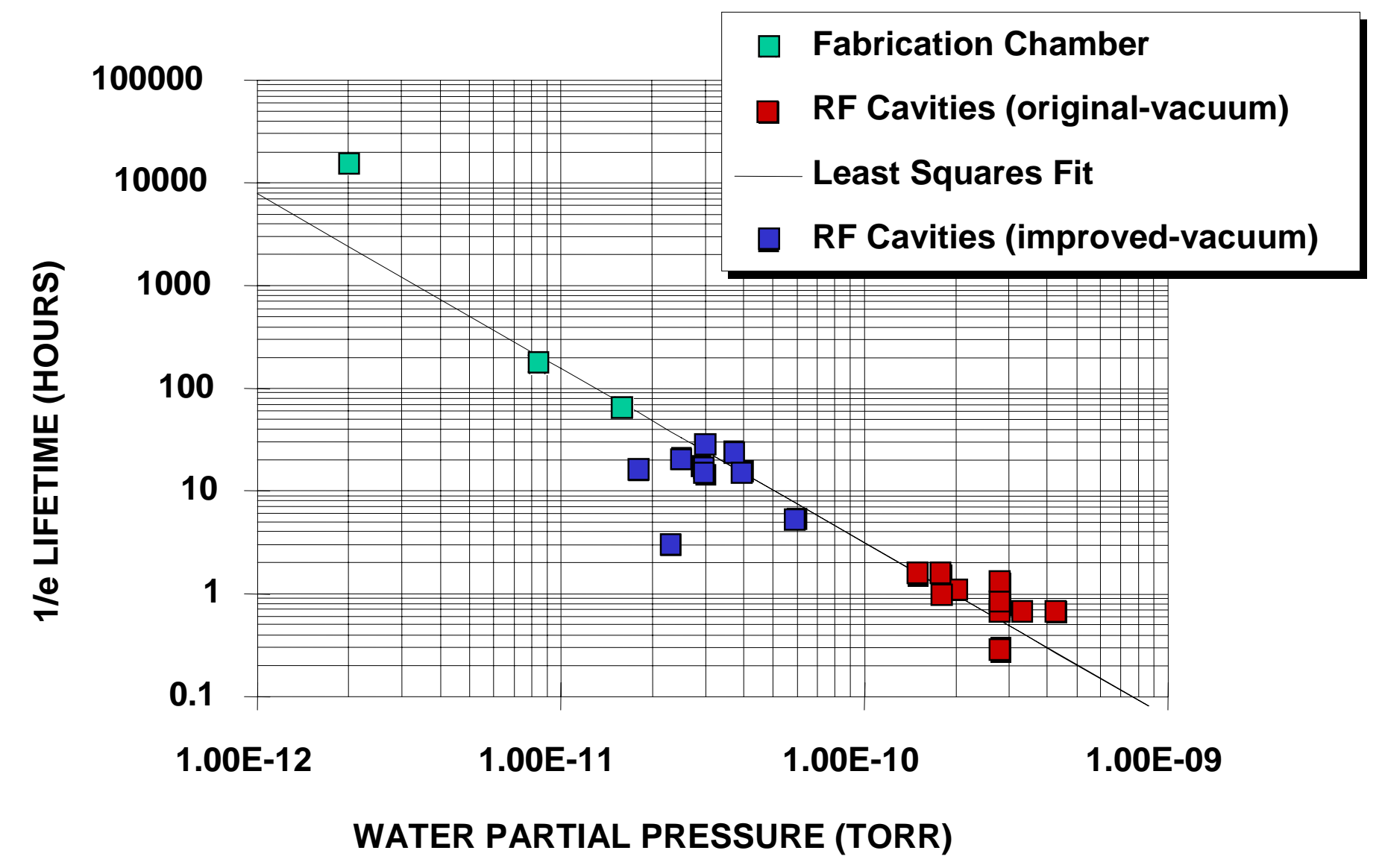




\section{Photocathode 1/e Lifetime Vs. Duty Factor}

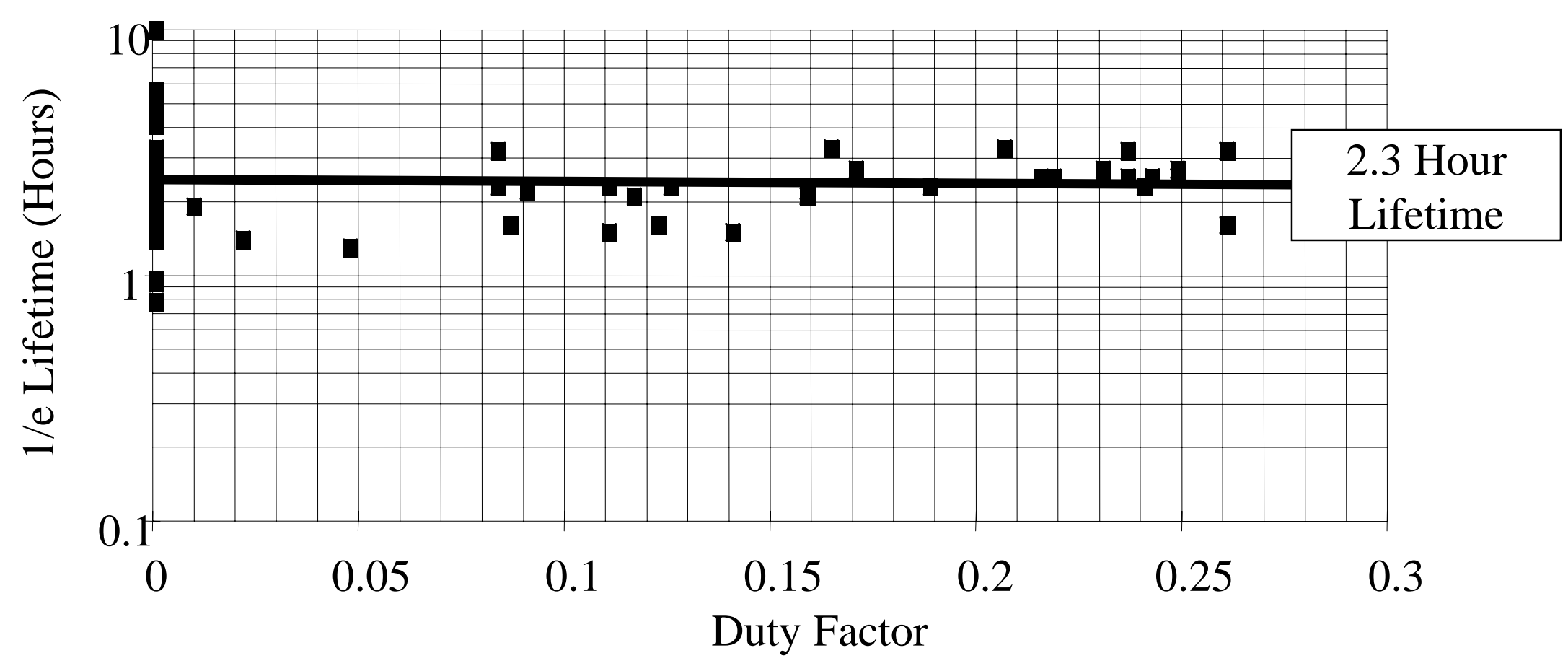




\section{Cathode Rejuvenation and}

\section{Improving Lifetime by Operating with Hot Cathode}

Rejuvenating a used $\mathrm{K}_{2} \mathrm{CsSb}$ cathode by heating it to 120 degrees $\mathrm{C}$.

The quantum efficiency increases at the rate of $2.5 \%$ / hour

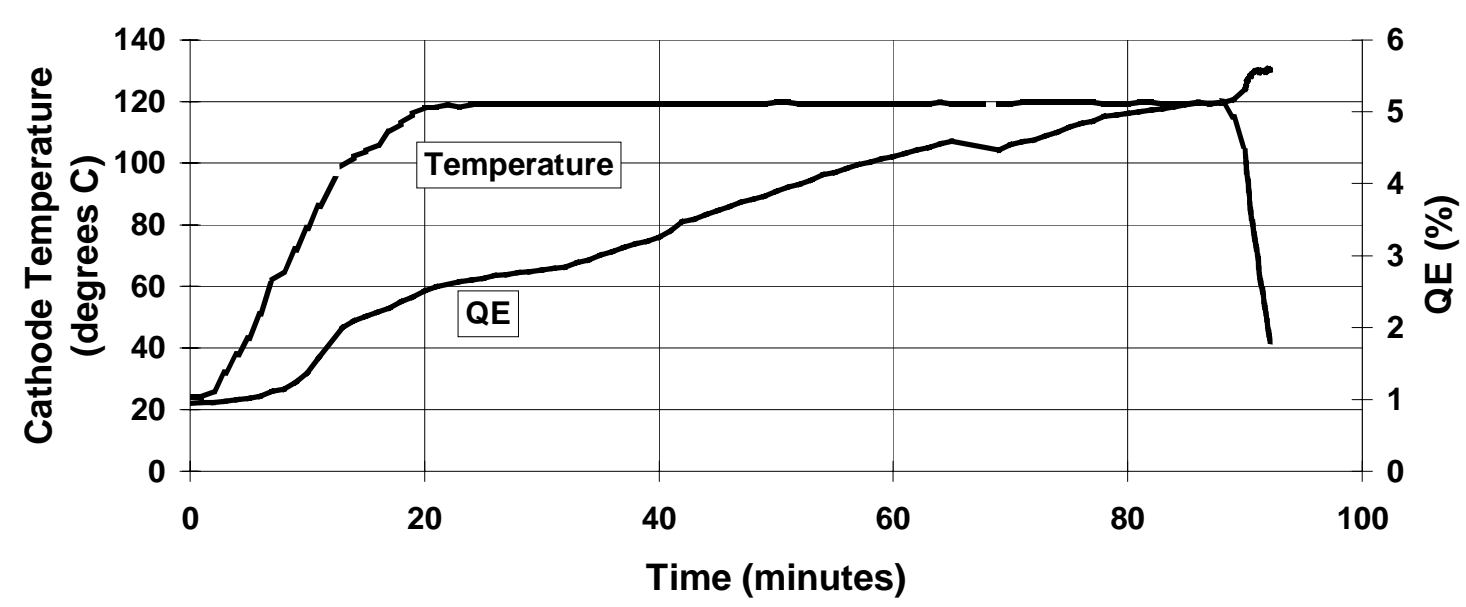

Photocathode quantum efficiency at elevated temperature in the RF cavity vacuum

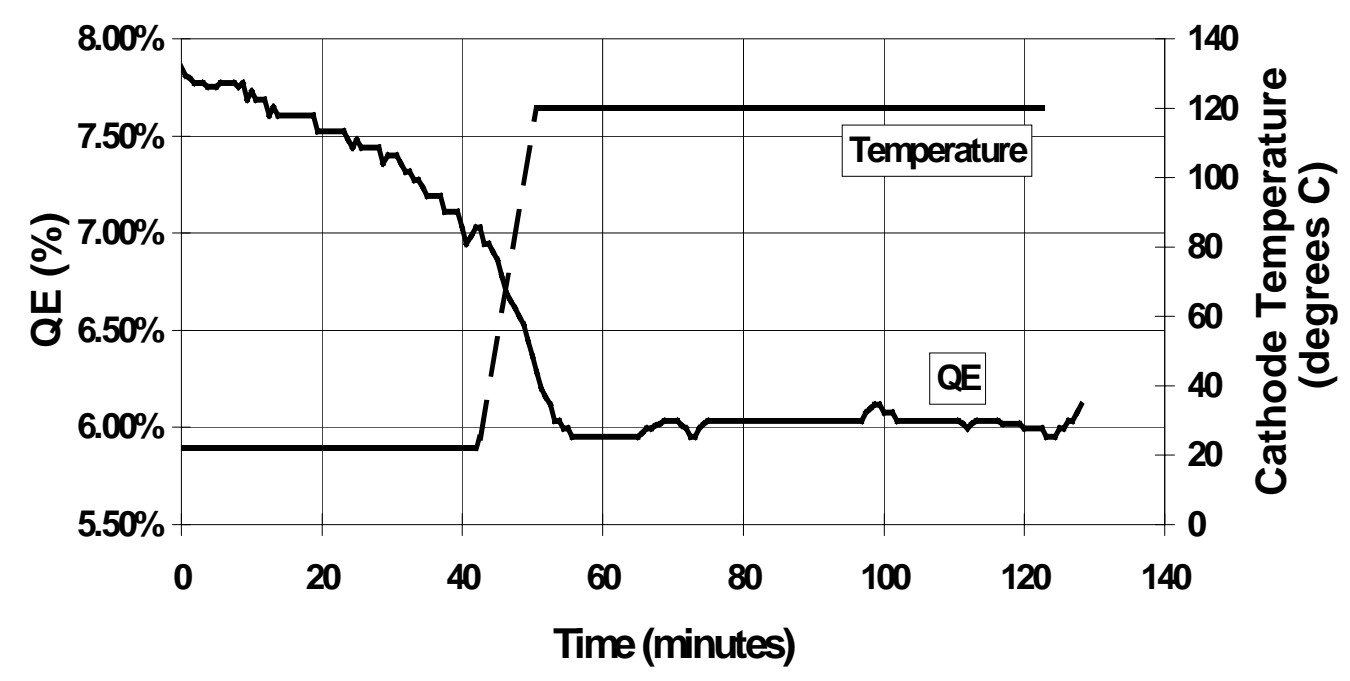




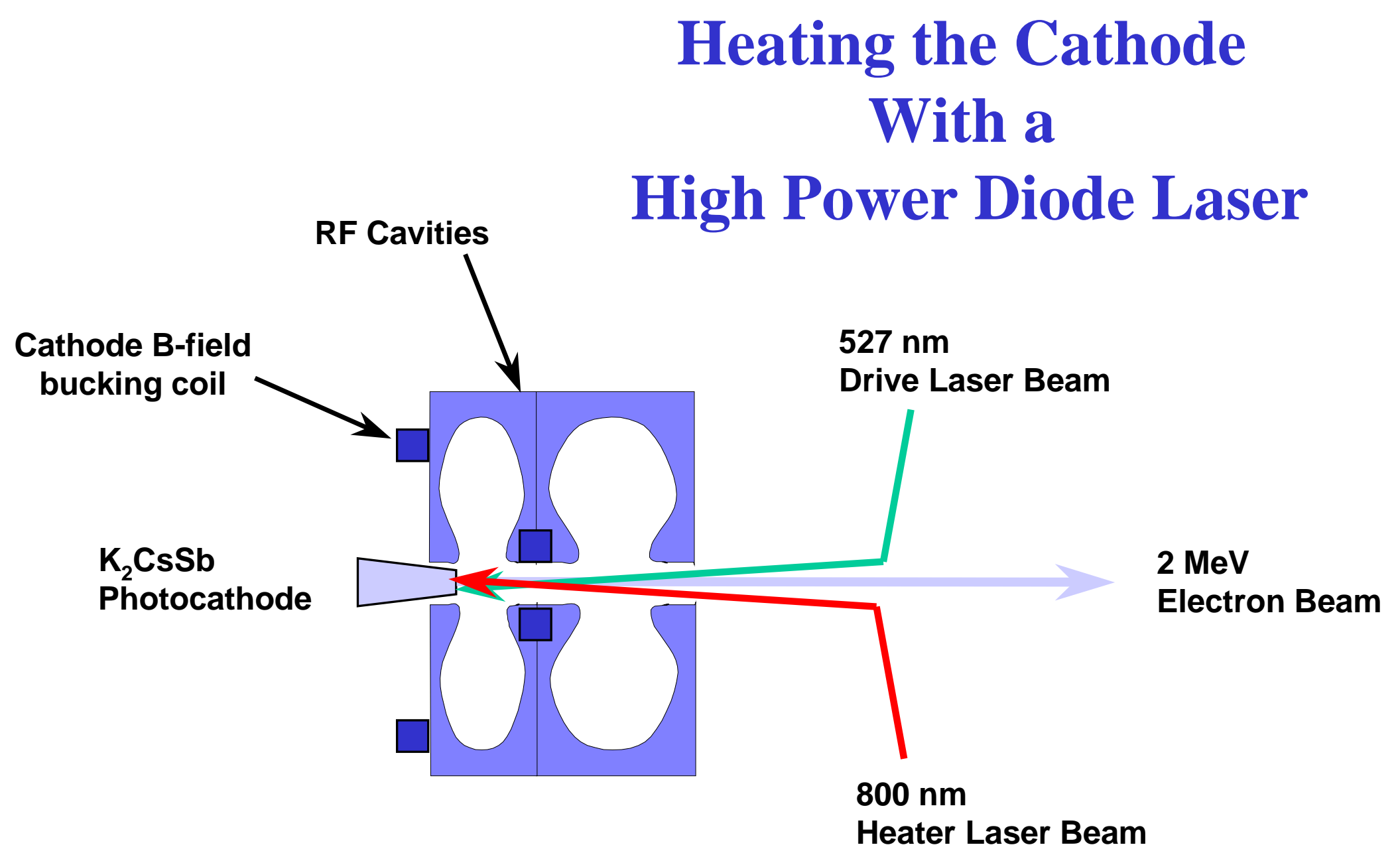

D.H. Dowell/PERL Workshop; Jan 22-23, 2001 


\section{Drive Laser Configuration Used in 1992 High Duty Test}

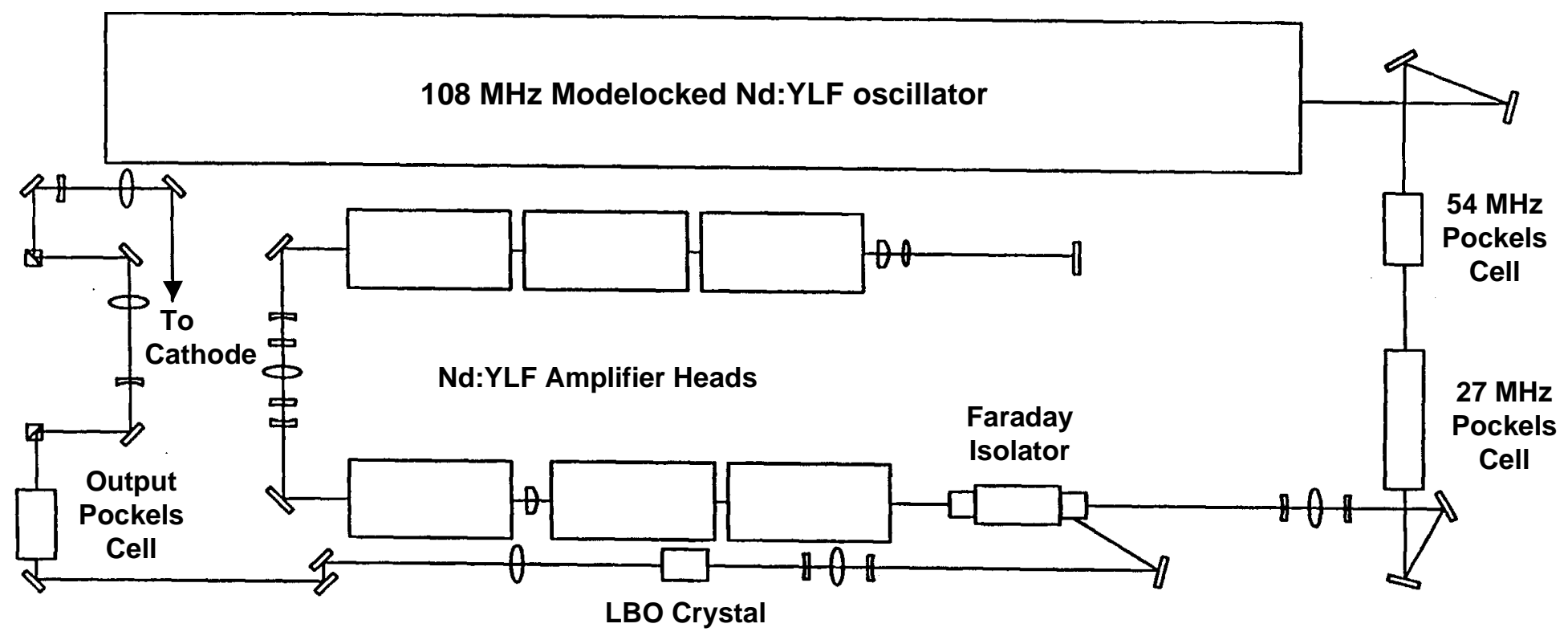




\section{MHz Gun Transverse Beam Quality Measurements 1992 and 1994-1996 Test Results}

\section{Gun Emittance}

Vs.

Microbunch Charge

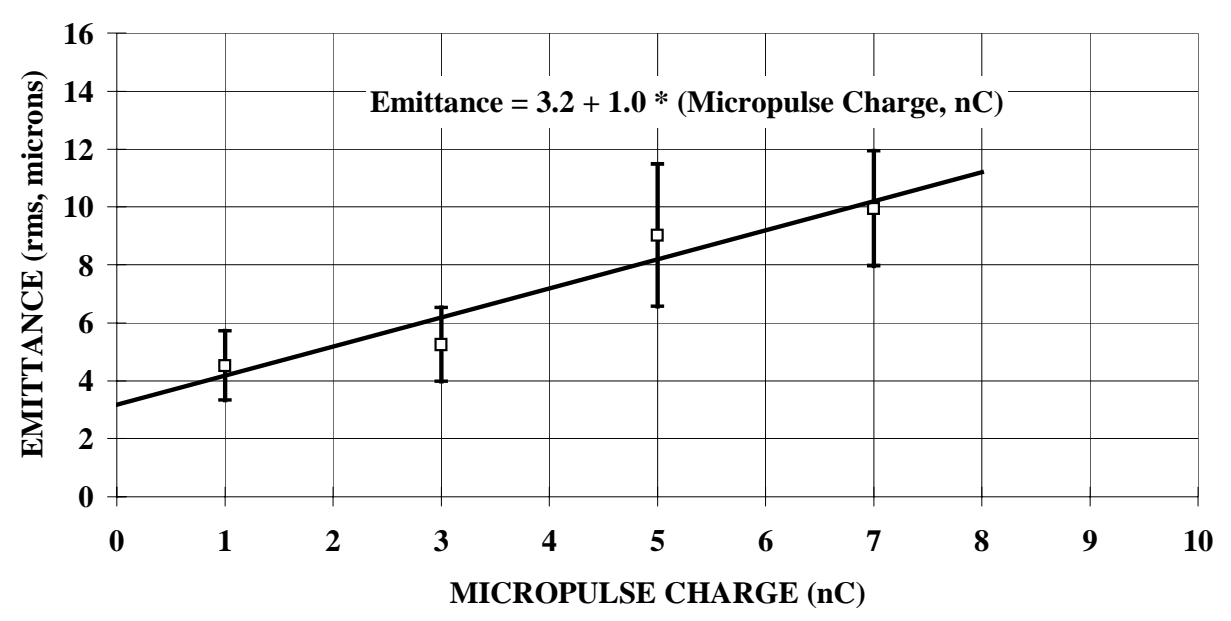

\section{Beam Emittance at 3 nC Gaussian-Gaussian Distributions}

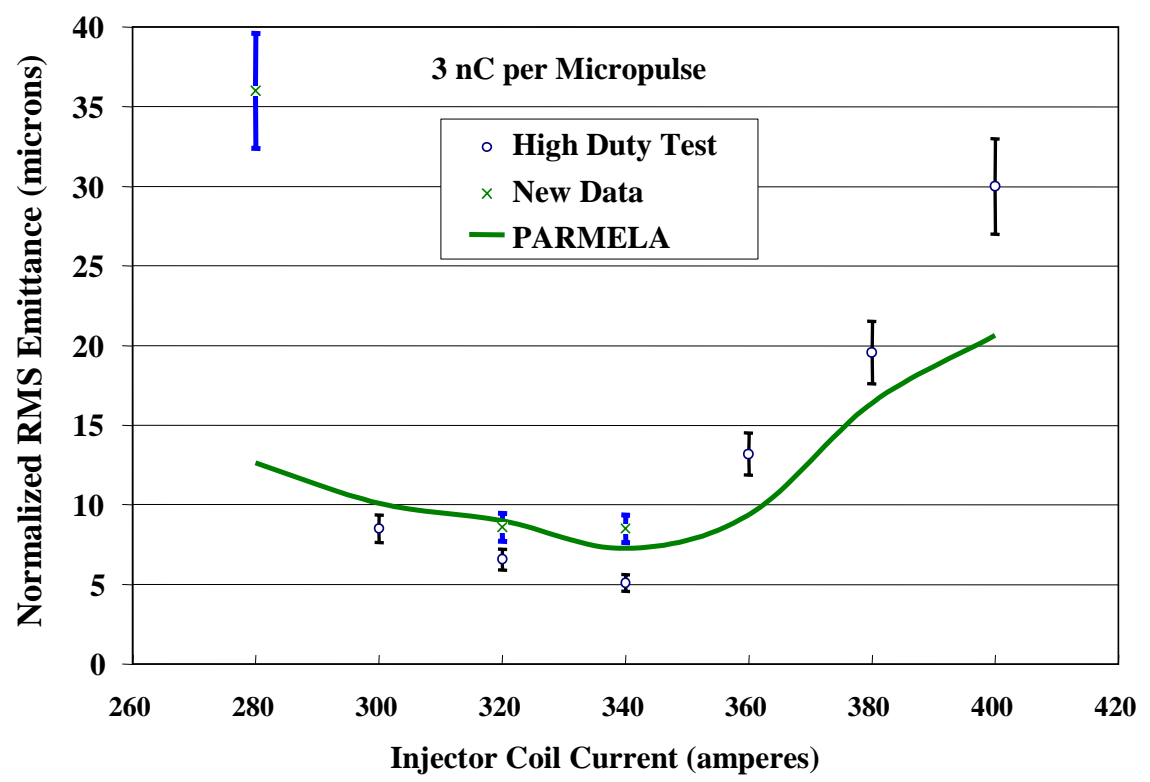




\section{PARMELA_B Simulations at 0.5 nC}

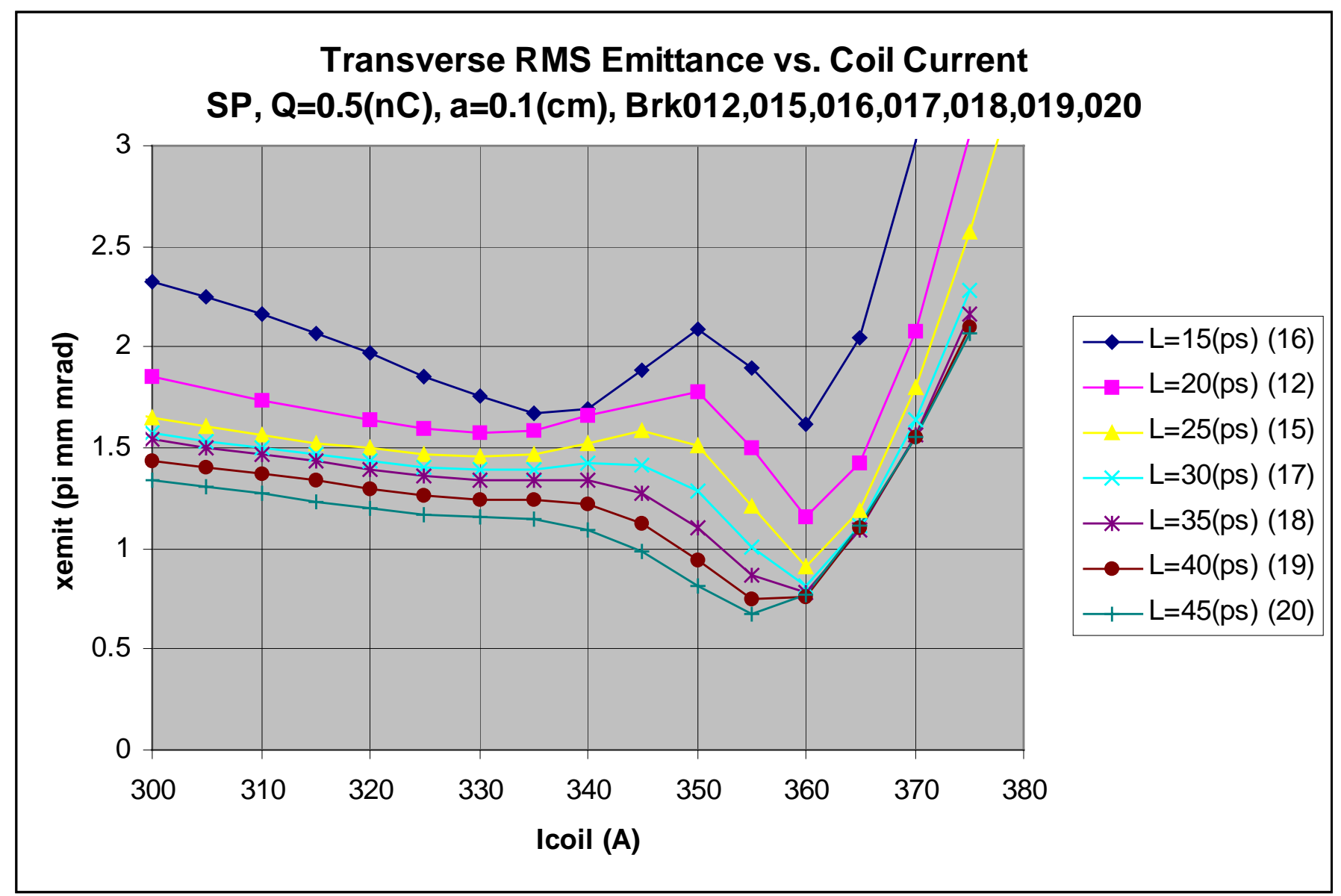

D.H. Dowell/PERL Workshop; Jan 22-23, 2001

PARMELA_B calculations

provided by B. Koltenbah, Boeing 


\section{Longitudinal Emittance}




\section{Components of the ELSA Accelerator Used to Make the Longitudinal Phase Space Measurements}

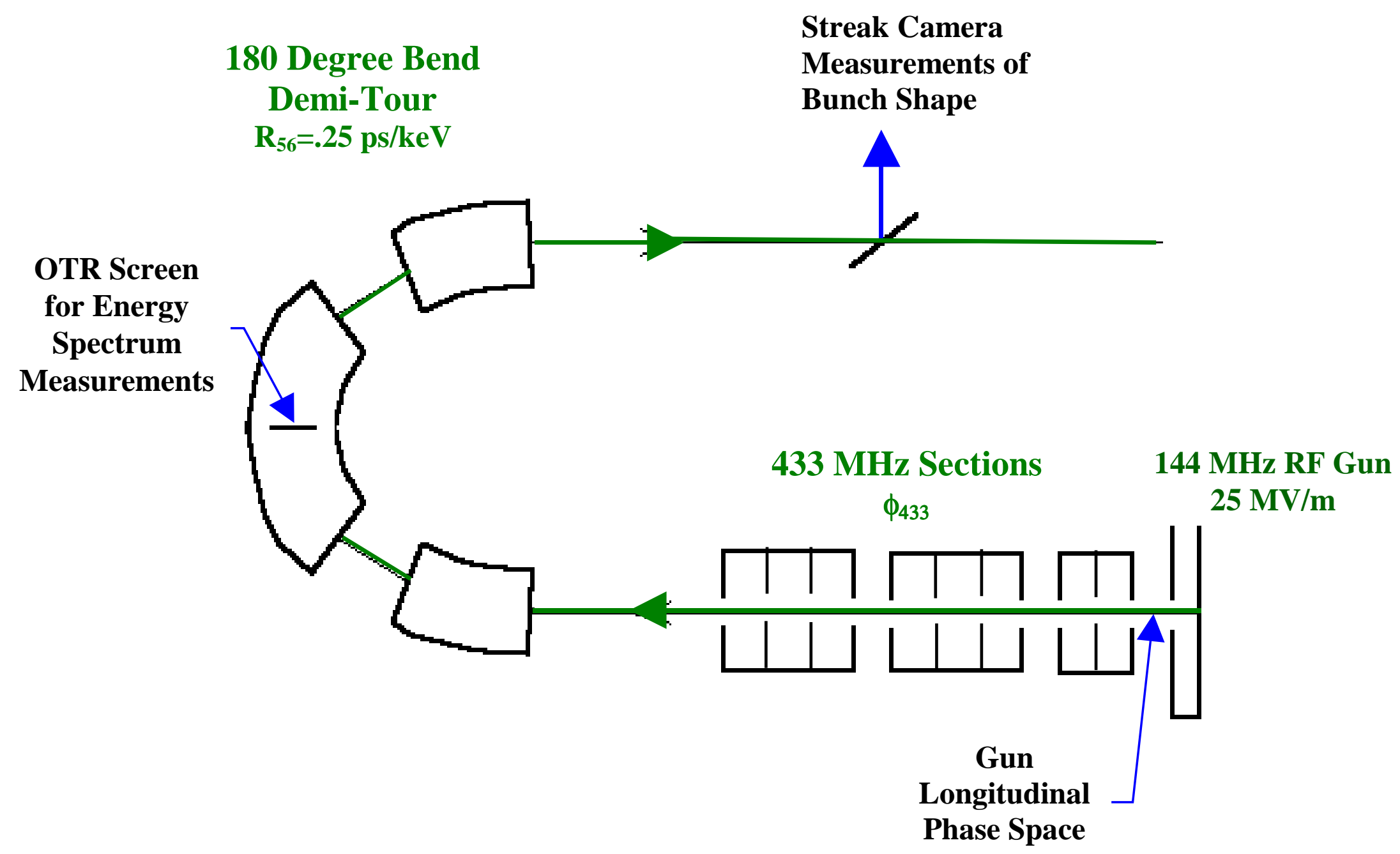




\section{Definition of Phase Space Parameters, Including Correlations}

Longitudinal Beam Ellipse:

$$
\gamma \Delta \mathbf{t}^{2}+2 \alpha \Delta \mathbf{t} \Delta \mathbf{E}+\beta \Delta \mathbf{E}^{2}=\frac{\varepsilon_{\ell}}{\pi}
$$

Longitudinal Beam Matrix:

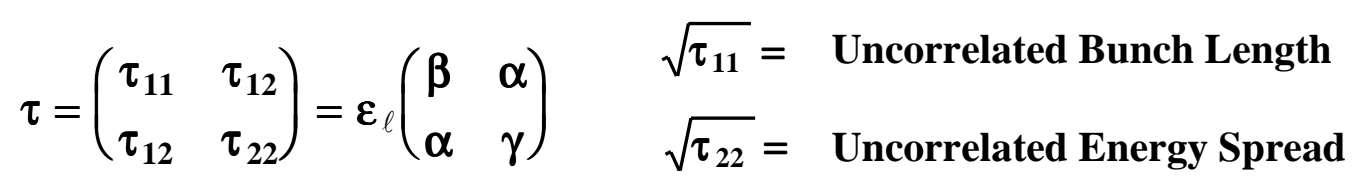

Include correlated emittance by distorting the ellipse boundary using quadratic and cubic terms:

$$
\Delta E=-\frac{\alpha}{\beta} \Delta t \pm \sqrt{\left(\frac{\alpha}{\beta} \Delta t\right)^{2}-\frac{\gamma \Delta t^{2}-\frac{\varepsilon_{\ell}}{\pi}}{\beta}}+\mathbf{a} \Delta \mathbf{t}^{2}+b \Delta t^{3}
$$




\section{Distortions of the Longitudinal Phase Space Ellipse}

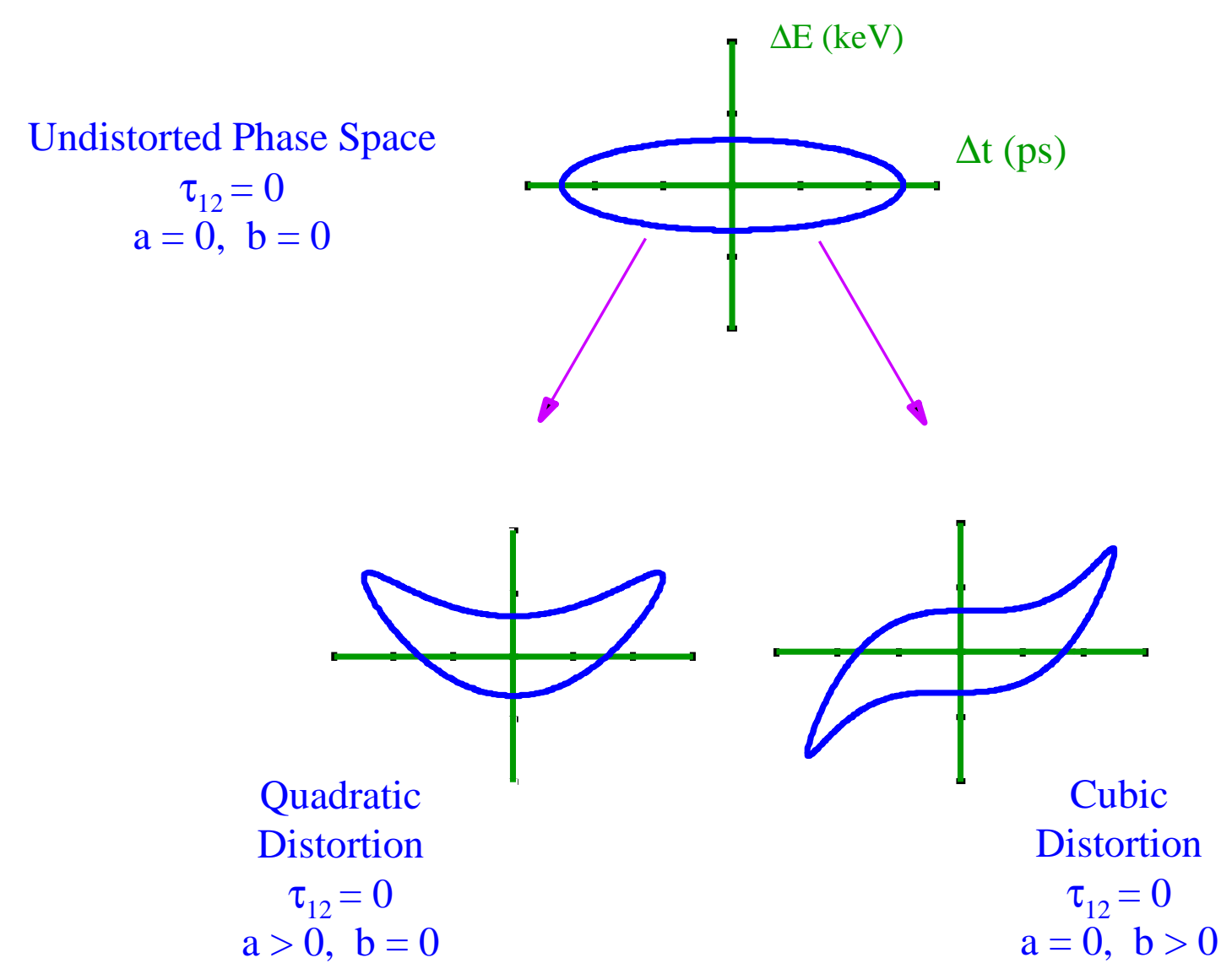




\section{Fits to $1 \mathrm{nC}$ per Microbunch Data}
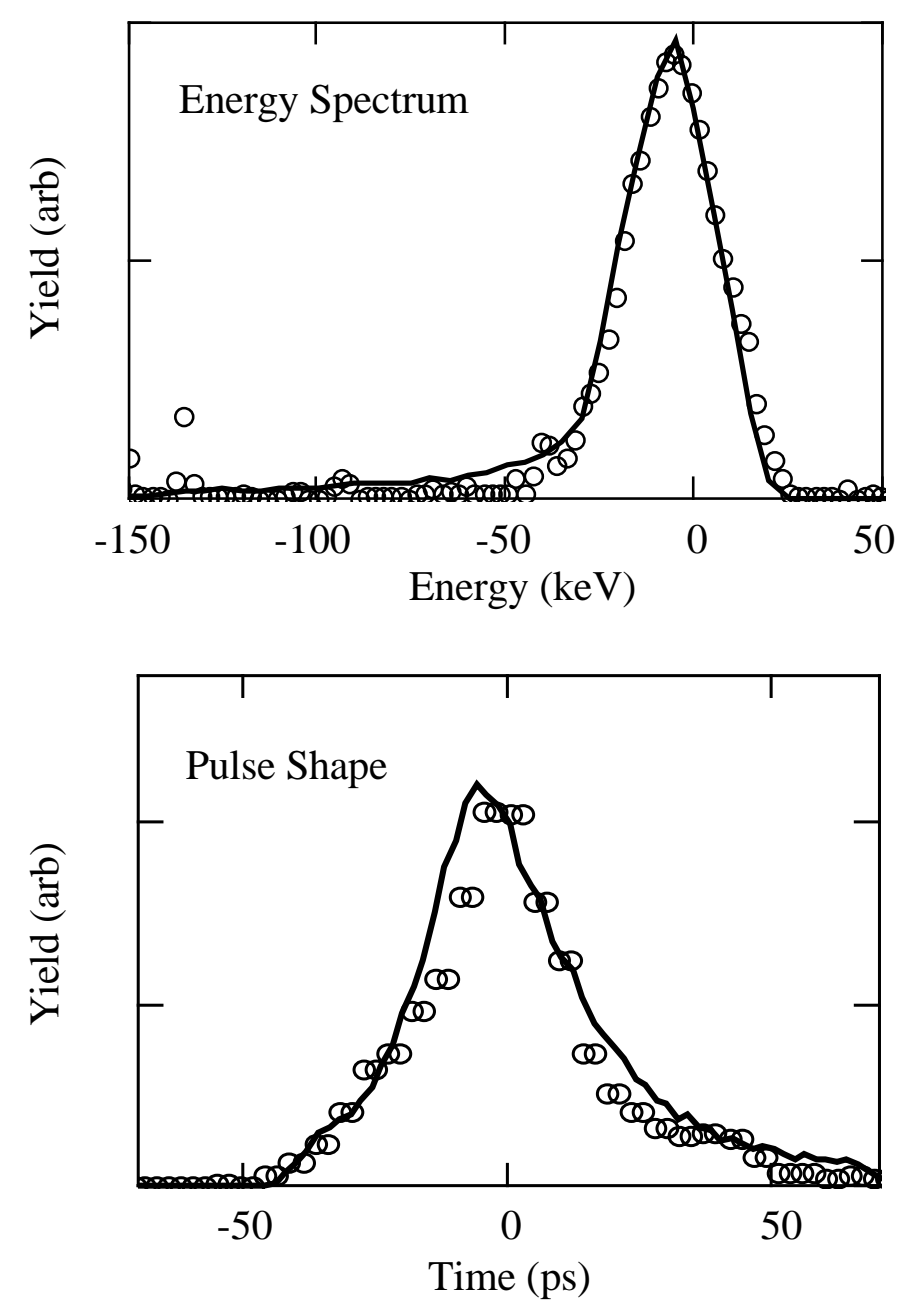

Longitudinal Phase Space Distributions Obtained from the Data Analysis

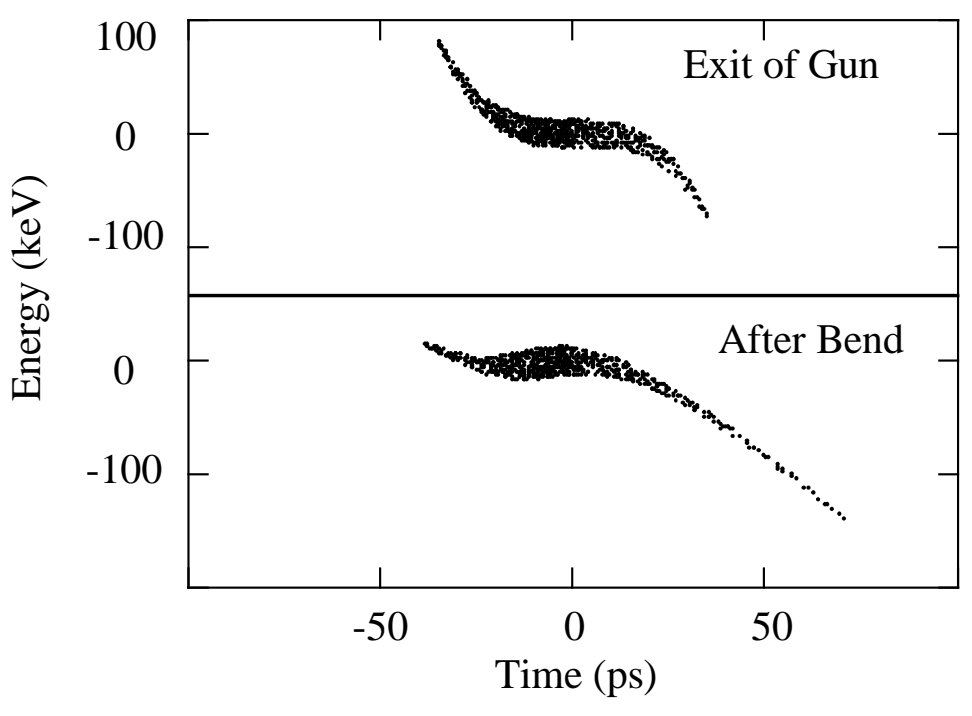

Ref: S. Joly et al.,... 
The Uncorrelated Emittance Grows Linearly with Surface Charge Densities Below the Space Charge Limit

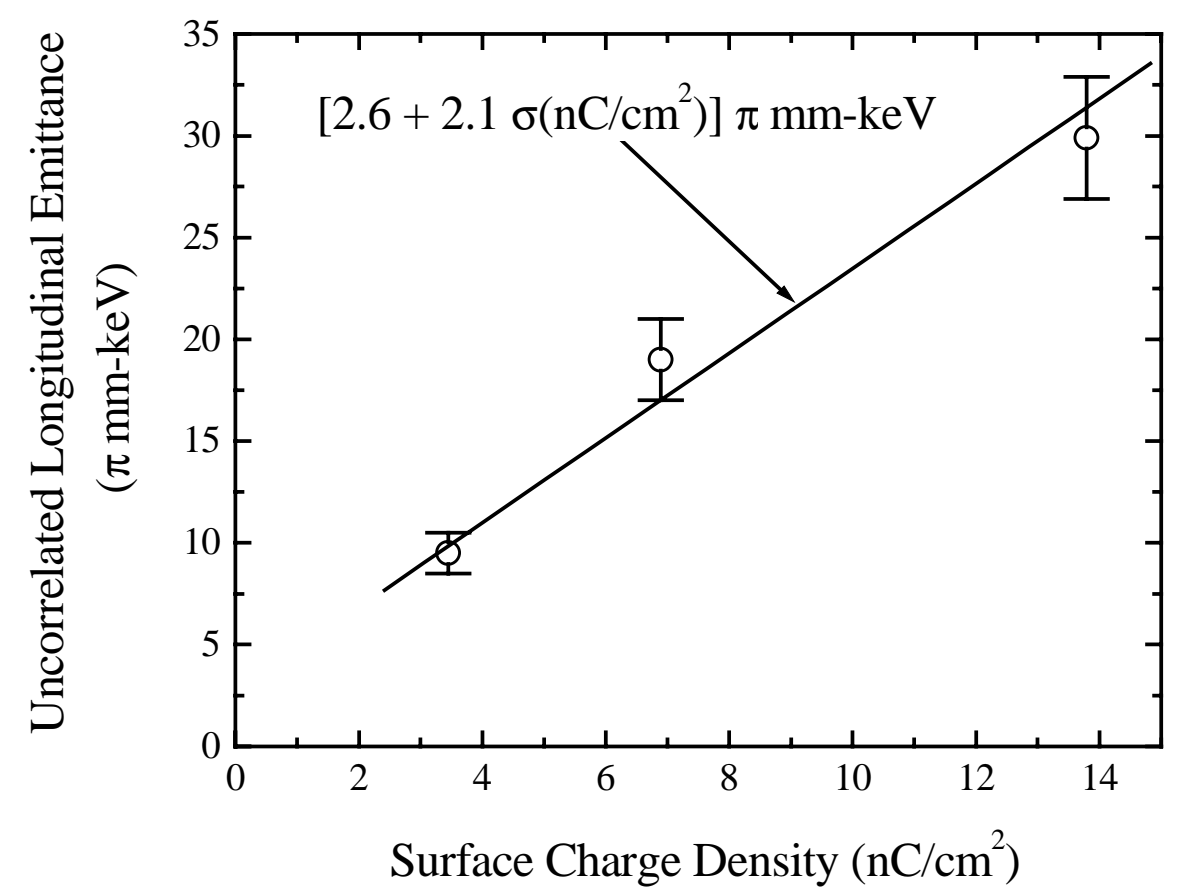

Pulse length a constant 11 ps(rms)

$10 \mathrm{nC} / \mathrm{cm}^{2}$ corresponds to $11 \mathrm{MV} / \mathrm{m}$

D.H. Dowell/PERL Workshop; Jan 22-23, 2001
In These Experiments Most of the Emittance Growth Was due to Increased Energy Spread

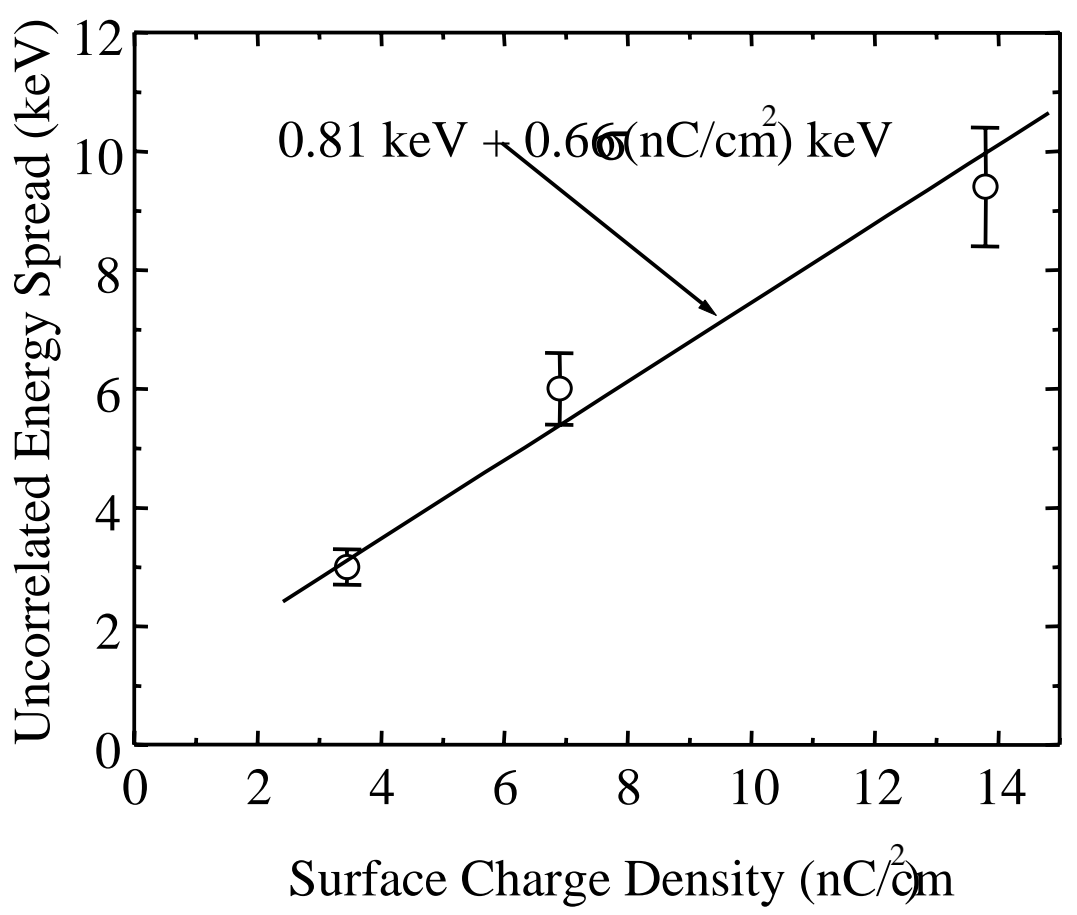

Ref: D.H. Dowell et al., PAC97. 


\section{Summary and Conclusions}

$433 \mathrm{MHz}$ PhotoIjnector Design Philosophy/Approach:

Gun, Booster, Linearizer and Compressor

433 MHz Gun has Demonstrated Most of the PERL Requirements:

Duty Factor

Beam Quality:

Transverse and Longitudinal Emittance

Problems and Unfinished Business:

Cathode Lifetime

Cathode Fabrication

Drive Laser

RF Design and Controls

Experimental Verification of Low Emittance at $0.5 \mathrm{nC}$

Start-to-End Simulations

CSR Experiments 


\title{
The New L-Band 1 1⁄2 Cell Photocathode RF Gun at Argonne
}

\author{
M.E. Conde, W. Gai, R. Konecny, J.G. Power, P. Schoessow, X. Sun, ANL, Argonne, IL 60439, \\ USA
}

\begin{abstract}
We report on the status of the new short bunch, high intensity electron gun at the Argonne Wakefield Accelerator. The 1-1/2 cell L-band photocathode RF gun is expected to produce $10-100 \mathrm{nC}$ bunches with $2-5 \mathrm{ps}$ rms pulse length and normalized emittance less than 100 $\mathrm{mm} \mathrm{mrad}$. The beam energy at the exit of the gun cavity will be in the range $7.5-10 \mathrm{MeV}$. A standing-wave linac structure operating at the same frequency $(1.3 \mathrm{GHz})$ will increase the beam energy to about $15 \mathrm{MeV}$. This beam will be used in wakefield acceleration experiments with dielectric loaded structures. These travelling-wave dielectric loaded structures, operating at 7.8 and 15.6 $\mathrm{GHz}$, will be excited by the propagation of single bunches or by trains of up to 32 electron bunches.
\end{abstract}

\section{INTRODUCTION}

High current short electron beams have been a subject of intensive studies [1]. One of the particular uses for this type of beam is in wakefield acceleration applications. High current $(\mathrm{kA})$ short electron beam generation and acceleration did not materialize until the advent of RF photoinjector technology[2]. Although most photocathode RF gun development has been concentrated on high brightness, low charge applications such as free electron laser injectors, there have been several relatively high charge RF photocathode based electron sources built and operated $[3,4,5]$. In general, there are two approaches to attaining high peak current. One approach is to generate an initially long electron bunch with a linear head-tail energy variation that is subsequently compressed using magnetic pulse compression. The advantage of magnetic compression is that it is a well-known technology and can produce sub-picosecond bunch lengths. However, due to strong longitudinal space charge effects, this technology is limited to relatively low charges $(<10 \mathrm{nC})$.

Another approach is to directly generate short intense electron bunches at the photocathode and then accelerate them to relativistic energies rapidly using high axial electric fields in the gun [3]. The advantage of this approach is that it can deliver very high charges, for example, $100 \mathrm{nC}$ if one uses an L-band gun. This would satisfy the requirements of most electron driven wakefield experiments for both plasma and dielectric structures, if the pulse length is short enough $(<10 \mathrm{ps}$ FWHM). So far, the Argonne Wakefield Accelerator (AWA) has demonstrated the capability of producing $100 \mathrm{nC}, 25-$ 35 ps (FWHM) electron beams at $14 \mathrm{MeV}$. This unprecedented performance was obtained using a half cell photocathode gun cavity and two standing wave irisloaded linac sections [6]. The AWA machine has reached its design goal and has been used for dielectric wakefield [7] and plasma [8] experiments. The initial results are encouraging [9]. Achieving higher gradients in wakefield experiments would require the drive electron pulse to be even shorter and have a lower emittance. In this paper, we discuss the design of a new RF photocathode gun with the capability of producing $10-100 \mathrm{nC}$ with $2-5 \mathrm{ps}$ (rms) pulse lengths.

\section{DESIGN CONSIDERATIONS}

In order to generate high charge and short bunch lengths from a photocathode RF gun, the electric field on the cathode surface has to be very intense. In this way the electrons leaving the cathode surface are quickly accelerated to relativistic velocities, minimizing the bunch lengthening and the emittance growth that the space charge forces produce [10,11]. There is also bunch lengthening and transverse emittance growth at the exit iris of the gun cavity due to the defocusing forces of the RF fields. Thus, this effect also calls for high accelerating gradient and high beam energy at the exit of the gun. It is therefore desirable to have a multicell gun with high accelerating gradient. Practical considerations (mainly a finite amount of RF power) limit the design to $1-1 / 2$ cells. The choice for our new gun design is a Brookhaven type $1-1 / 2$ cell cavity [12] scaled up to $\mathrm{L}$ band operation. This gun will be followed by one of the present linac tanks that exist at the AWA facility.

A detailed numerical study $[13,14]$ of this gun was performed with the codes SUPERFISH and PARMELA [15]. Table 1 summarizes the parameters used in the simulations. These extensive numerical simulations showed a strong dependence of bunch length and emittance with respect to the accelerating gradient in the gun cavity (Fig. 1). Based on these studies, it was decided that an accelerating gradient of $80 \mathrm{MV} / \mathrm{m}$ on the cathode

\footnotetext{
* This work is supported by the Department of Energy, High Energy Physics Division, Advanced Technology Branch under the Contract No. W-31-109-ENG-38.
} 
surface was a good operating point. This requires $10 \mathrm{MW}$ of RF power to be coupled into the gun cavity, which still leaves enough power to run one of the linac tanks. This accelerating gradient yields good values of emittance and bunch length, while still not high enough to make the RF conditioning of the gun a challenging task. (In fact, we recently conditioned a duplicate of the present AWA gun up to a gradient of $125 \mathrm{MV} / \mathrm{m}$ [16].)

Table 1. The gun design parameters as calculated using SUPERFISH.

\begin{tabular}{|l|c|}
\hline \multicolumn{1}{|c|}{ Inner Radius of the Cell, b $(\mathrm{cm})$} & 9.03 \\
\hline Radius of the iris, a $(\mathrm{cm})$ & 2.75 \\
\hline Width of the iris, d $(\mathrm{cm})$ & 1.5 \\
\hline Aperture of the exit $(\mathrm{cm})$ & 2.5 \\
\hline Operating frequency $(\mathrm{GHz})$ & 1.3 \\
\hline Initial beam radius $(\mathrm{cm})$ & 1 \\
\hline Quality factor, Q & 26008
\end{tabular}

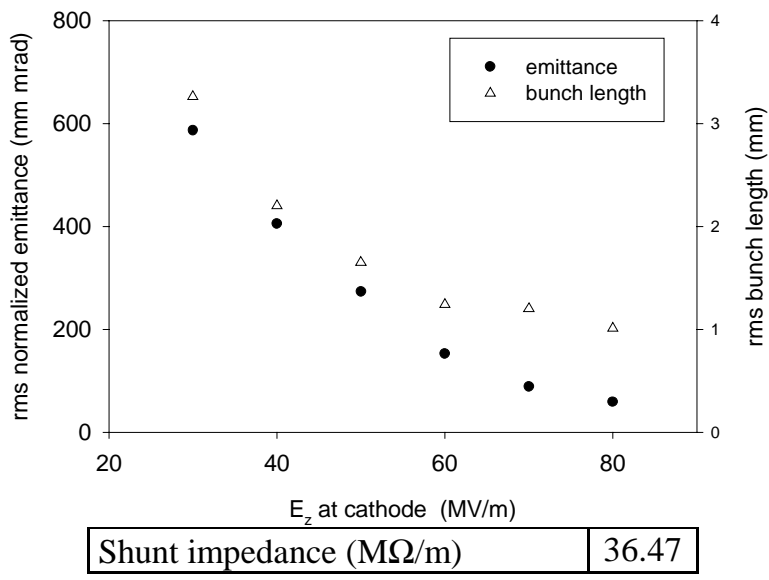

Figure 1: Emittance and bunch length as a function of the accelerating gradient on the cathode surface, for a $40 \mathrm{nC}$ bunch.

\section{CONSTRUCTION AND RF MEASUREMENTS}

The RF gun will be operated with a focusing solenoid and a bucking solenoid to cancel the magnetic field on the plane of the cathode. These two solenoids are exactly next to each other, with the photocathode plane as their plane of symmetry. This maximizes the space available for the RF power coupler over the full cell of the gun. There is a vacuum pumping port in the full cell, located diametrically opposite to the RF coupler, both being at the equator line of the full cell. An RF pickup probe is placed near the vacuum pumping port, relying on the evanescent $\mathrm{RF}$ fields present in that location. An RF tuning plunger is located half way along the circumference of the full cell between the RF coupler and the vacuum pumping port. This breaks the symmetry of the full cell, but it is acceptable in our L-band size cavity. The perturbation of the field lines near the axis of the cavity is negligible. In the half cell, the cathode holder can also function as a tuning plunger, allowing us to adjust the parameters of the two cells, in order to achieve the right resonance frequency for the $\pi$ mode and field balance in the cavity. The cooling channels are drilled along the cylindrical wall of the gun, and also run over part of the back and front plates of the cavity.

Numerical simulations of this final design yield values for the emittance and bunch length that are slightly worse than the ones obtained in reference [13, 14]. This results from the fact that the location and dimensions of the solenoids are not dictated only by the optimization of the beam parameters, but also by other physical constraints. The degradation is however very small, and the gun is still expected to generate very short bunches with low emittance. Results of numerical simulations with PARMELA are shown in Fig. 2. These plots show emittance, bunch length, energy and radial coordinate as a function of the longitudinal coordinate along the accelerator for a bunch charge of $40 \mathrm{nC}$. At the exit of the linac the code predicts a normalized rms emittance of 66 $\mathrm{mm}$ mrad and an rms bunch length of $3.7 \mathrm{ps}$.

Figure 2: Numerical simulations of a $40 \mathrm{nC}$ electron
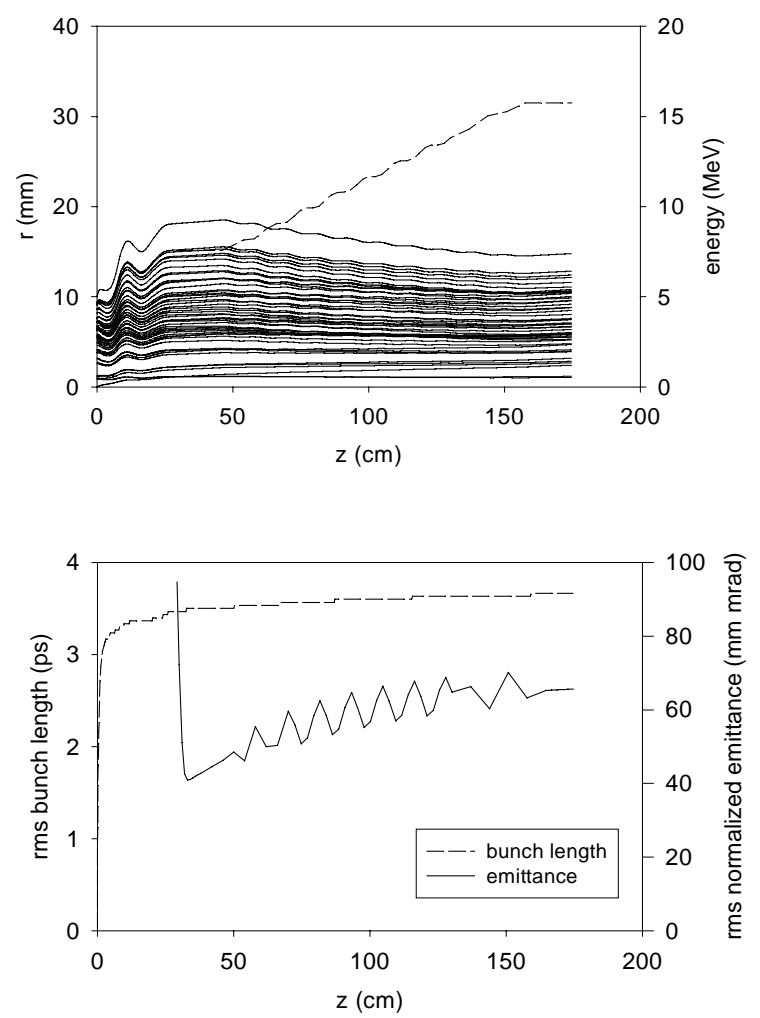

bunch as it propagates along the gun and linac structures: (a) energy and trajectories in the transverse plane; (b) bunch length and emittance.

The gun (Fig. 3) is presently being installed in a test area for RF conditioning and commissioning. The value of the unloaded quality factor $\left(Q_{0}\right)$ of the gun is presently 
21000 , but this number will increase slightly when the final cathode holder (with better electric contact) is installed. Figure 4 shows the profile of the axial electric field along the axis of the cavity measured by the usual bead-pulling technique. After the final brazing cycle the gun cavity became somewhat overcoupled $\left(\mathrm{S}_{11}=-10 \mathrm{~dB}\right)$; a tuning post in the waveguide will improve the coupling.
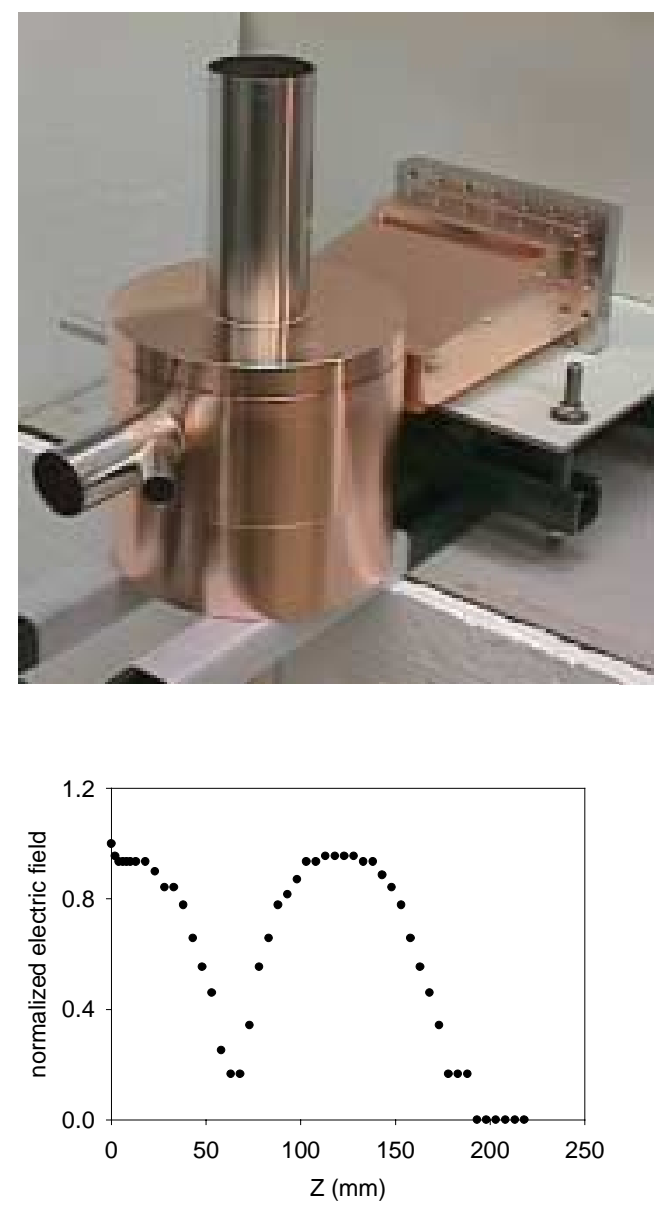

Figure 3: Gun before final brazing cycle.

Figure 4: Profile of axial electric field along the axis of the cavity.

\section{CONCLUSION}

The new AWA photocathode RF gun will dramatically improve the capabilities of our program to study wakefield acceleration in dielectric loaded structures and plasmas. The electron beam produced by this gun is expected to excite wakefields in plasmas with accelerating gradients in excess of $1 \mathrm{GeV} / \mathrm{m}$ with a plasma density of $\sim 10^{14} / \mathrm{cm}^{3}$.

In dielectric loaded structures, this beam will also make a significant improvement over presently attainable gradients. One can use this beam to directly demonstrate collinear wakefield acceleration gradients in excess of 50 $\mathrm{MV} / \mathrm{m}$ corresponding to $200 \mathrm{MW}$ of RF power generated in $30 \mathrm{GHz}$ dielectric structures.

It is worth pointing out that the present AWA photocathode RF gun has achieved unprecedented values of charge per bunch, and has allowed us to advance the understanding of wakefield acceleration in plasmas and in dielectric structures. However, the present gun was designed when only a very limited amount of RF power was available for the experiment ( $2 \mathrm{MW})$. Thus, the beam parameters, namely, bunch length and emittance, suffered serious limitations due to this relatively low level of RF power. The newly designed gun will take advantage of the higher level of RF power now available in the facility, yielding better beam parameters and, consequently, higher accelerating gradients in the wakefield acceleration experiments.

\section{REFERENCES}

[1] C. Travier, Proceedings of Advanced Acceleration Concepts Workshop, Edited by P. Schoessow, AIP Proceedings, No. 335, p.57, 1994.

[2] J. Fraser et al., IEEE Trans. Nucl. Sci., NS-32, p.1719 (1985).

[3] P. Schoessow et al., Proceedings of Particle Accelerator Conference, p.976, 1995.

[4] B. Carlsten, et al., Proceedings of Particle Accelerator Conference, p.985, 1995.

[5] E. Colby et al., Proceedings of Particle Accelerator Conference, p.967, 1995.

[6] M.E. Conde et al., Phys. Rev. ST Accel. Beams 1, 041302 (1998); M.E. Conde et al., Proceedings of Particle Accelerator Conference, p.1996, 1997.

[7] P. Schoessow et al., Proceedings of Particle Accelerator Conference, p.639, 1997.

[8] N. Barov et al., Phys. Rev. ST Accel. Beams 3, 011301 (2000); N. Barov, M.E. Conde, W. Gai, J. Rosenzweig, Phys. Rev. Lett., Vol. 80, No. 1, p.81, 1998.

[9] P. Schoessow et al., Journal of Applied Physics, Vol. 84, No. 2, p.663, 1998.

[10] K.J. Kim, Nuclear Instrumentation and Methods, A275, p.201, 1989.

[11] L.Serafini, J. Rosenzweig, Physical Review E, Vol. 55, No. 6, p.7565, 1997.

[12] K. Batchelor et al., Proceedings of European Particle Accelerator Conference, p.541, 1990.

[13] W. Gai et al., Nucl. Instr. and Meth. A 410, p.431, 1998.

[14] W. Gai et al., Proceedings of Advanced Acceleration Concepts Workshop, Baltimore, 1998.

[15] SUPERFISH and PARMELA, Las Alamos National Lab. Report LA-UR-96-1834, 1997 and LA-UR-961835, 1996.

[16] C.H. Ho et al., Proceedings of Linac98 Conference, Chicago, 1998. 


\title{
Ultra-stable Laser system for Photocathode RF gun Applications
}

\author{
Katsuyuki Kobayashi \\ Sumitomo Heavy Industries (USA), Inc \\ c/o Aculight Corporation \\ 11805 North Creek Parkway S. Bothell, WA 98011 \\ E-mail: kobayashi@aculight.com
}

A part of this work is performed under the management of the Femtosecond Technology Research Association, supported by New Energy and Industrial Technology Development Organization. 
Contents

- Production

Pulrise

- Research \& Development

Amplifier

Square pulse 


\section{Pulrise: Stable UV Laser for Photoinjector}

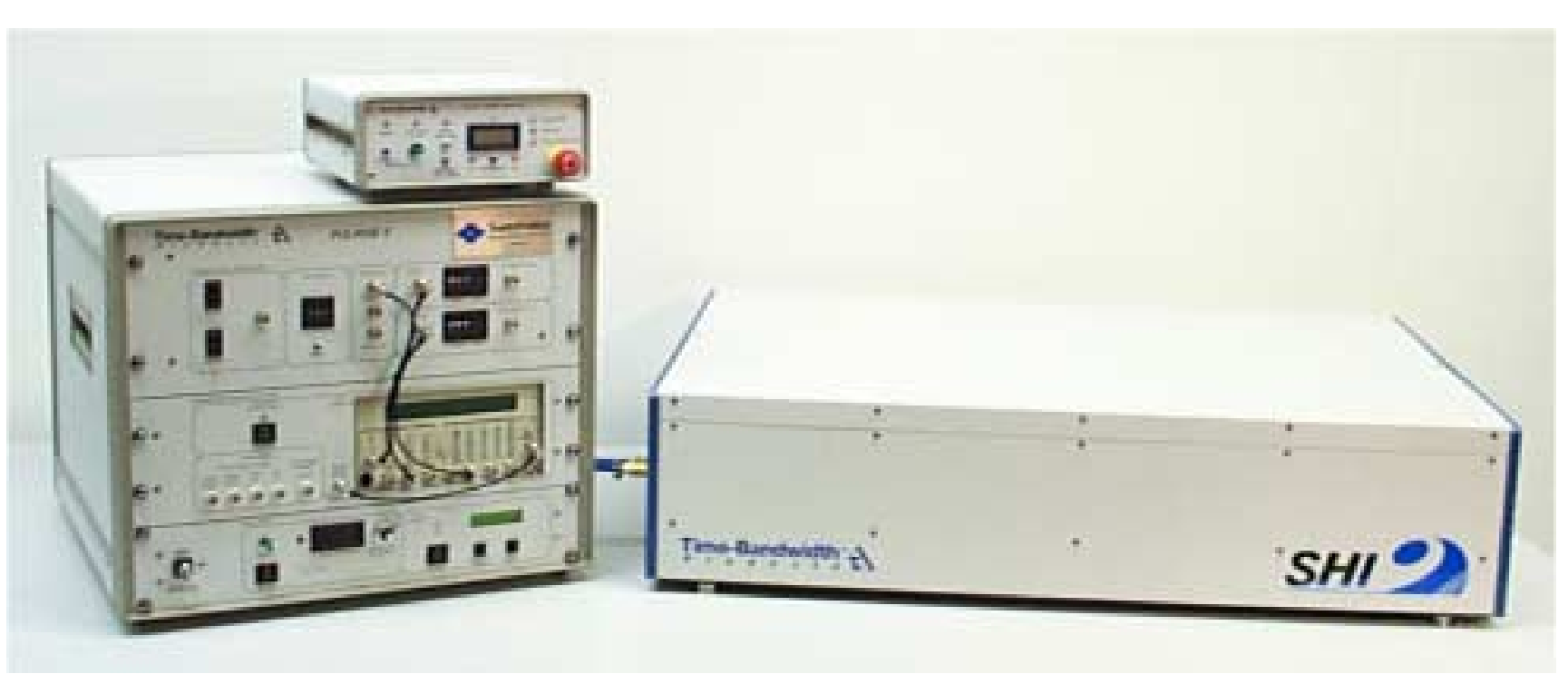

Laser medium Nd:YLF

Pulse width 12 ps @1047 nm, 8 ps @262 nm

Pulse energy 2 mJ @1047 nm, 0.2 mJ @262 nm

Jitter

$<0.5$ ps RMS

Dimension $\quad 600 \mathrm{~mm}(\mathrm{~W}) \mathrm{X} 900 \mathrm{~mm}(\mathrm{D}) \mathrm{X} 300 \mathrm{~mm}(\mathrm{H})$

Repetition rate $25 \mathrm{~Hz}(100 \mathrm{~Hz}$ available) 


\section{Stability of Pulrise}

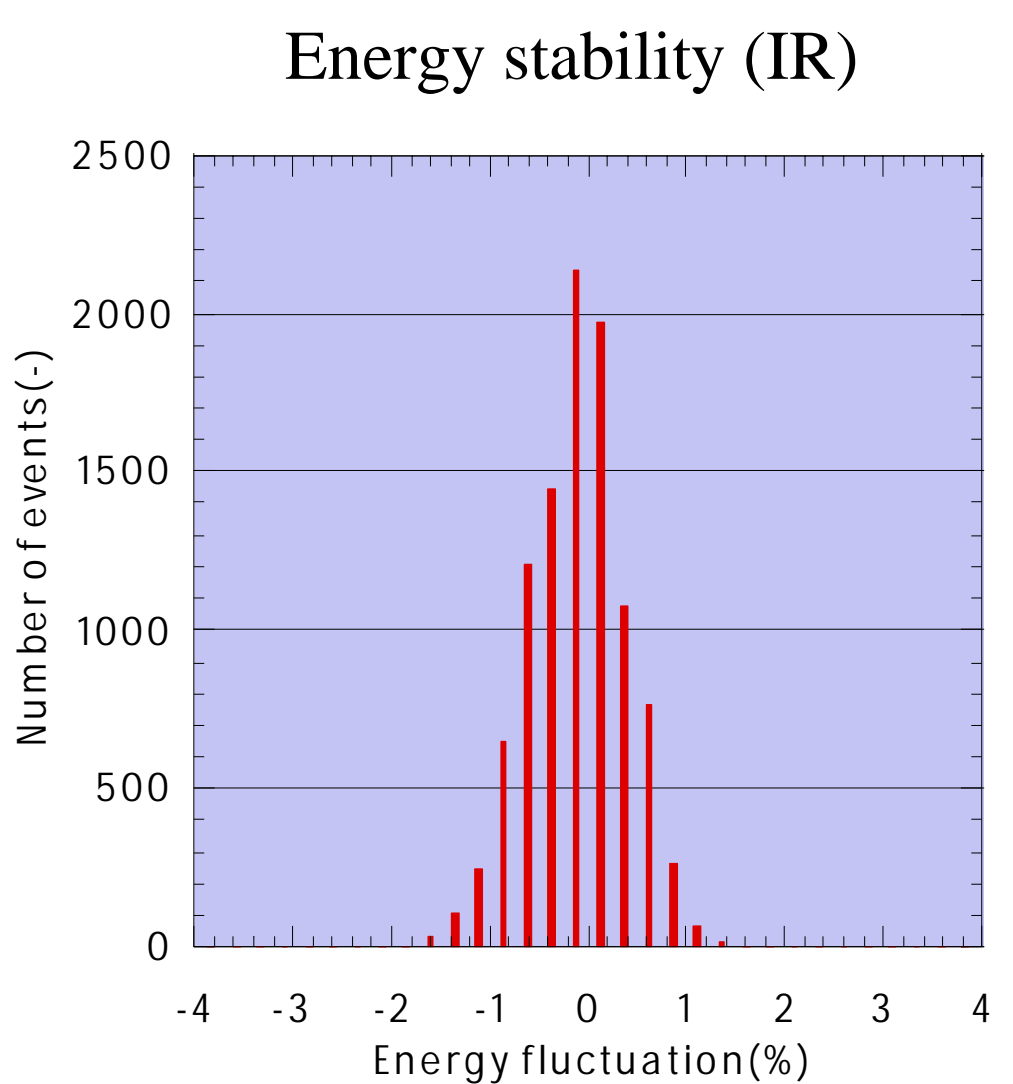

Total shot number

Standard deviation
Beam profile (IR)

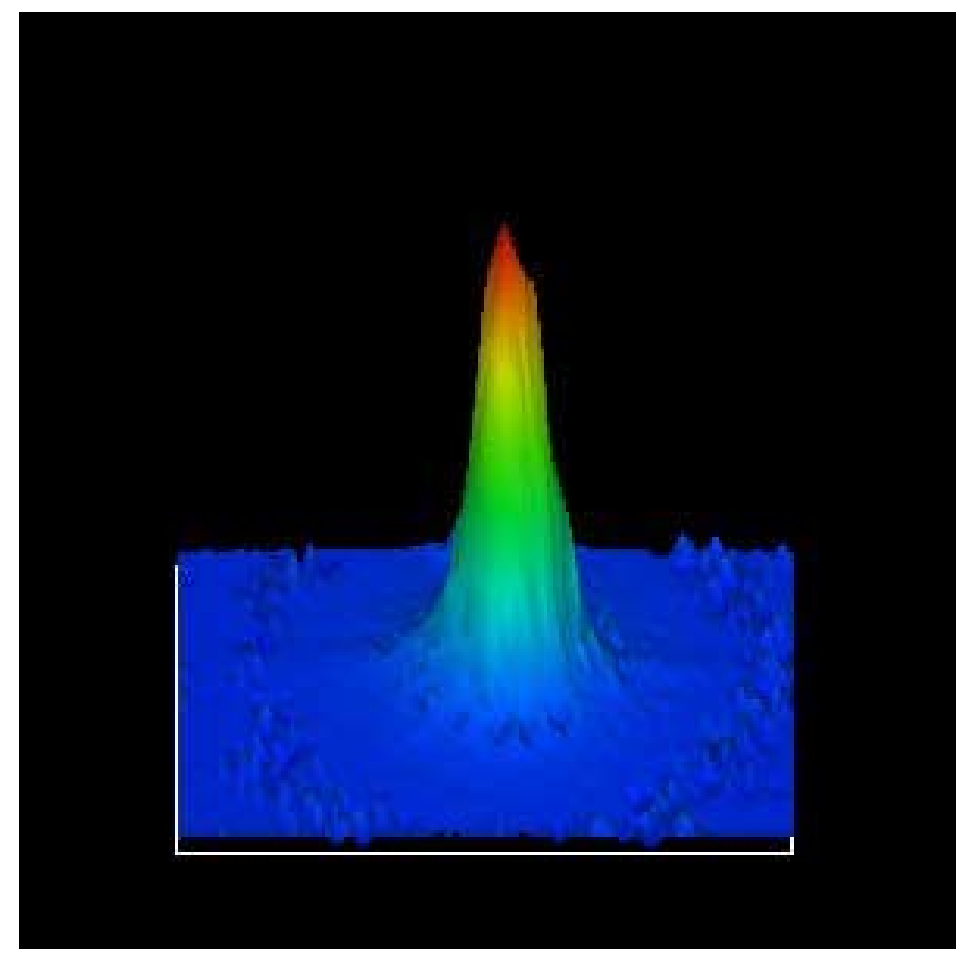

Pointing stability $\sigma=5 \mu \mathrm{rad}$ 


\section{Electron Bunch Characteristics}

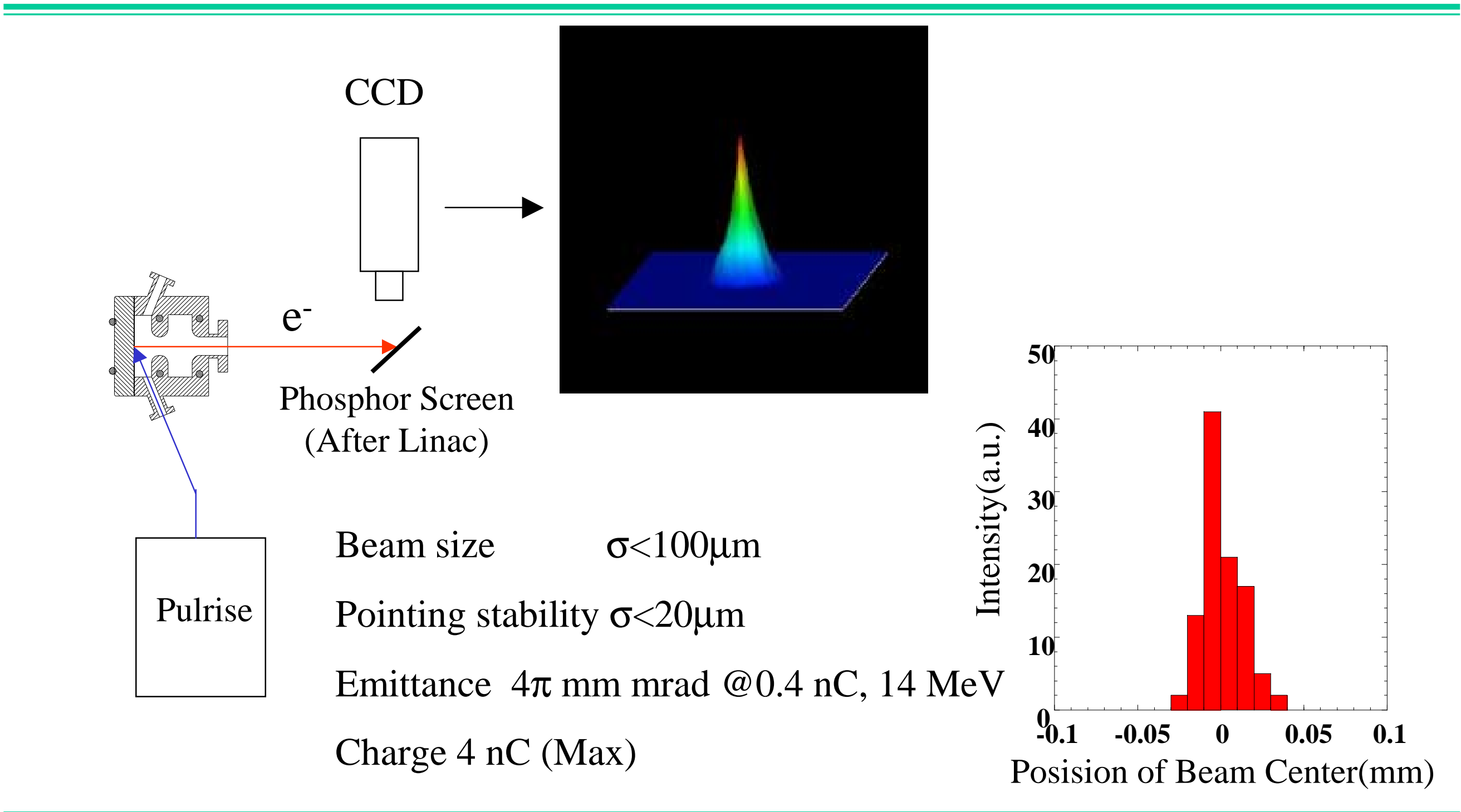




\section{Nd:YLF Amplifier}

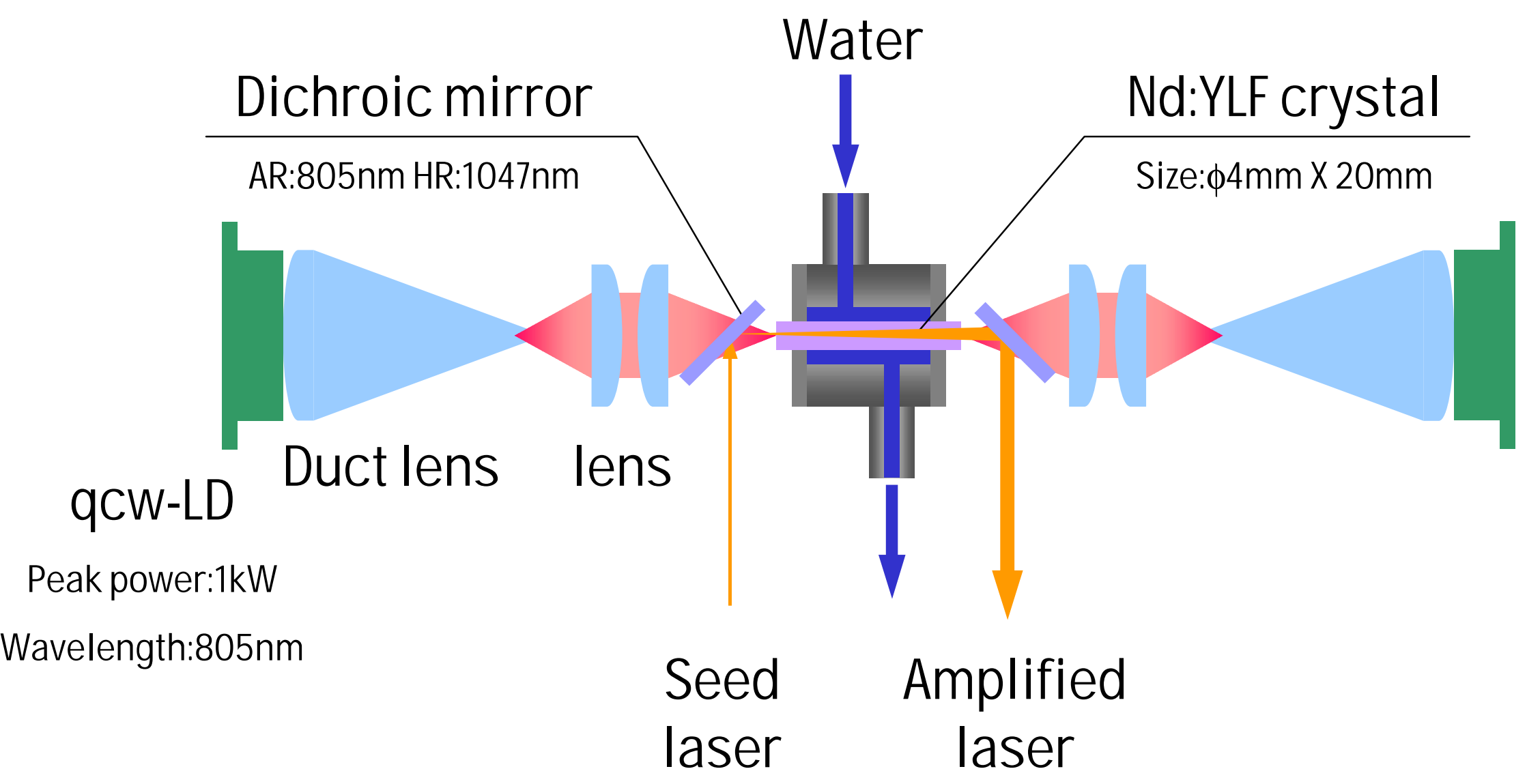


Nd:YLF Amplifier

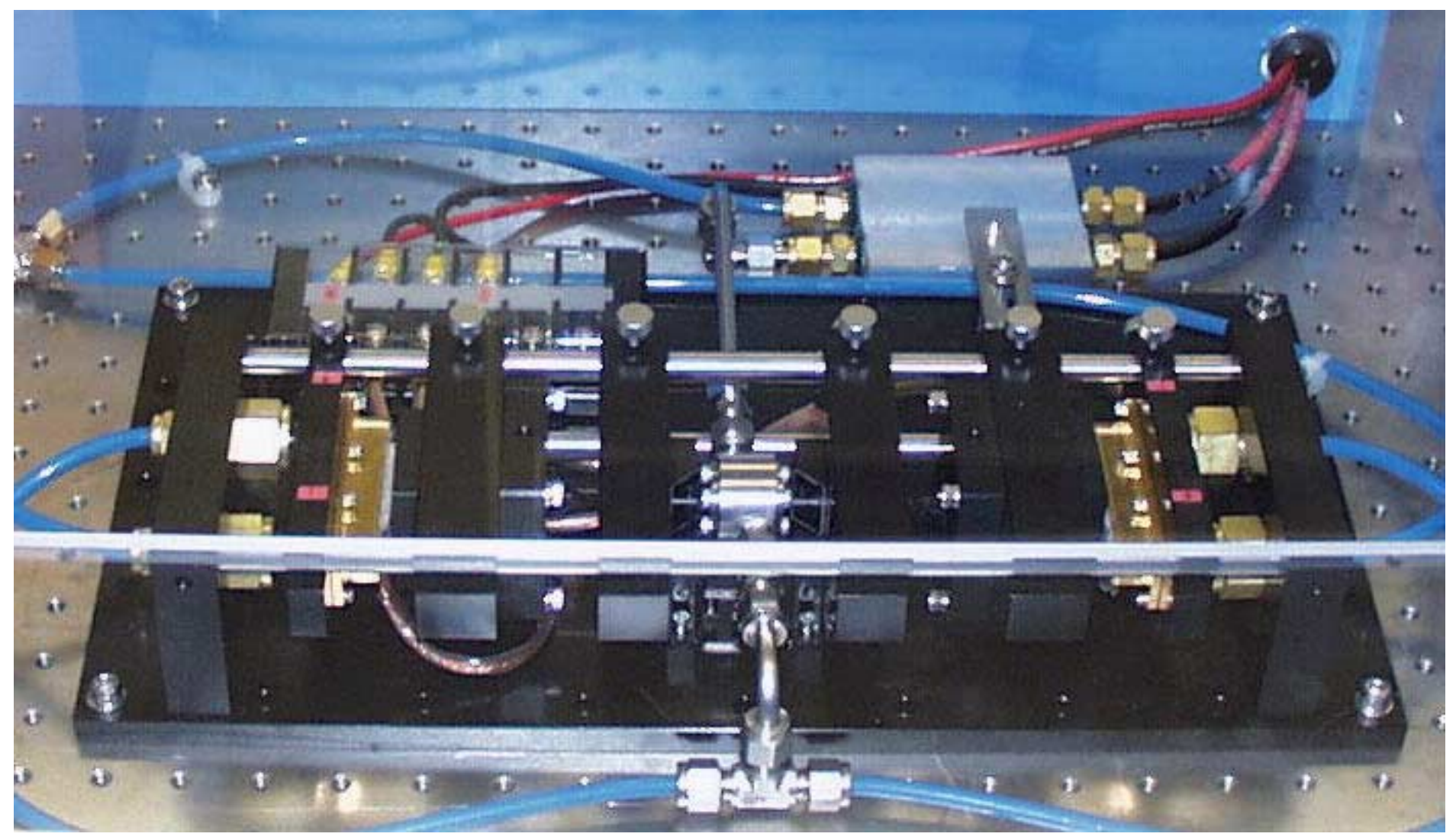




\section{Amplification with 2-pass configuration}

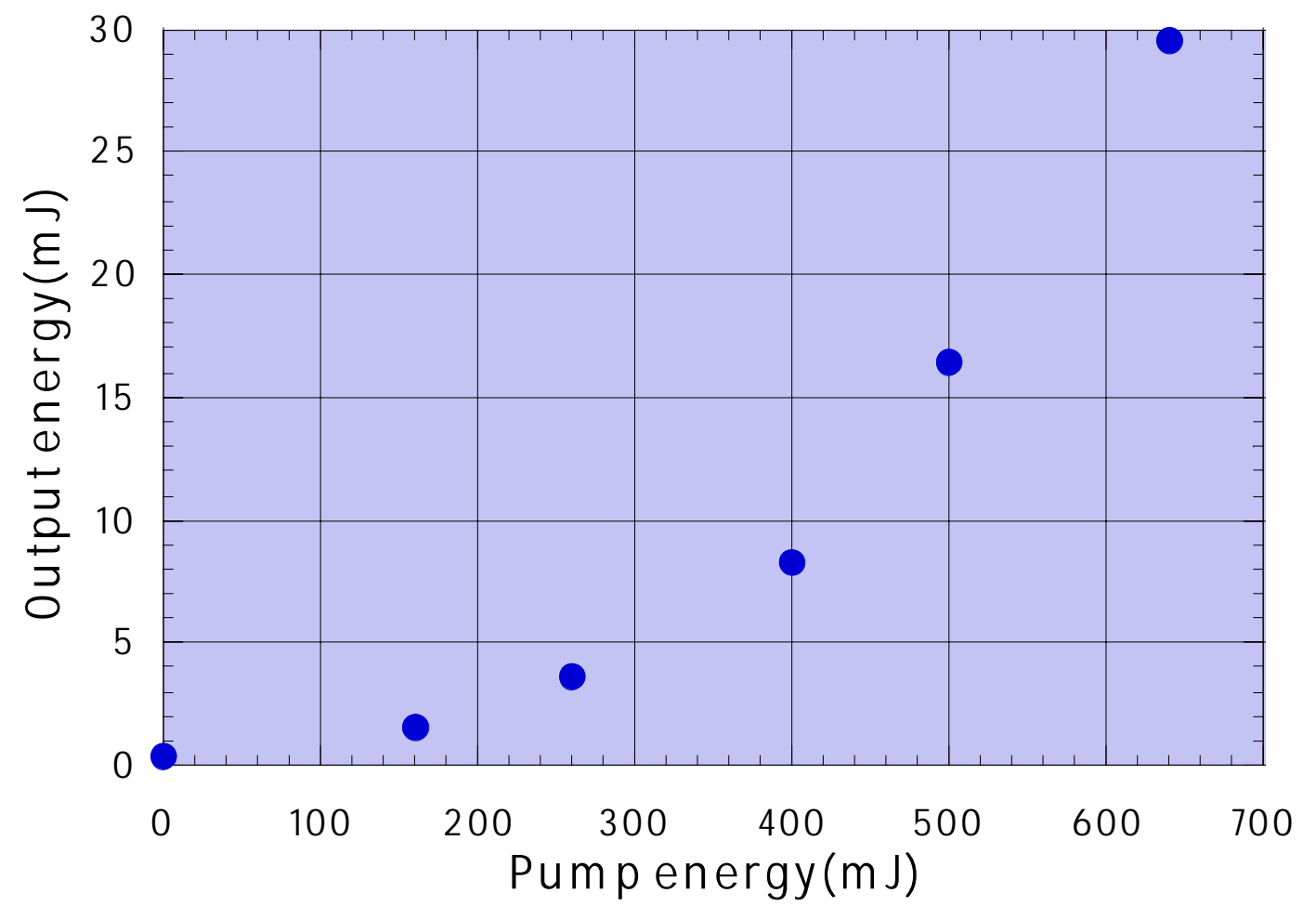

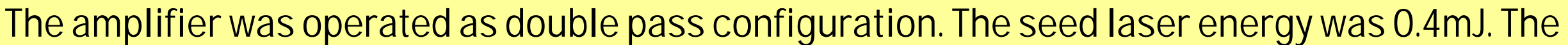

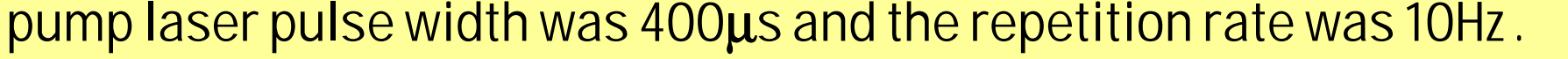




\section{Beam Profile and Focused Diameter}
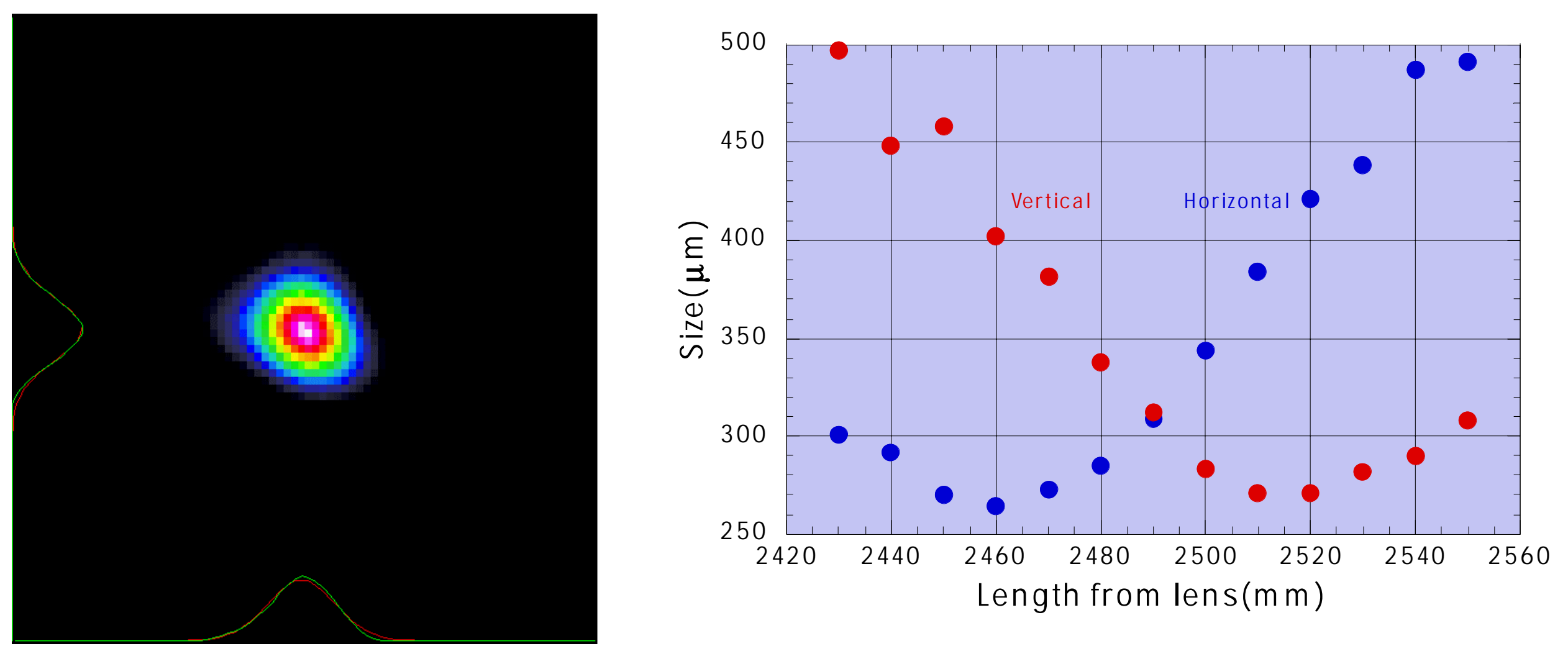

with $2500 \mathrm{~mm}$ lens

The beam diameter (the width of the $1 / \mathrm{e}^{\wedge} 2$ intensity level) was approximately $300 \mu \mathrm{m}$. 


\section{Pulsewidth}

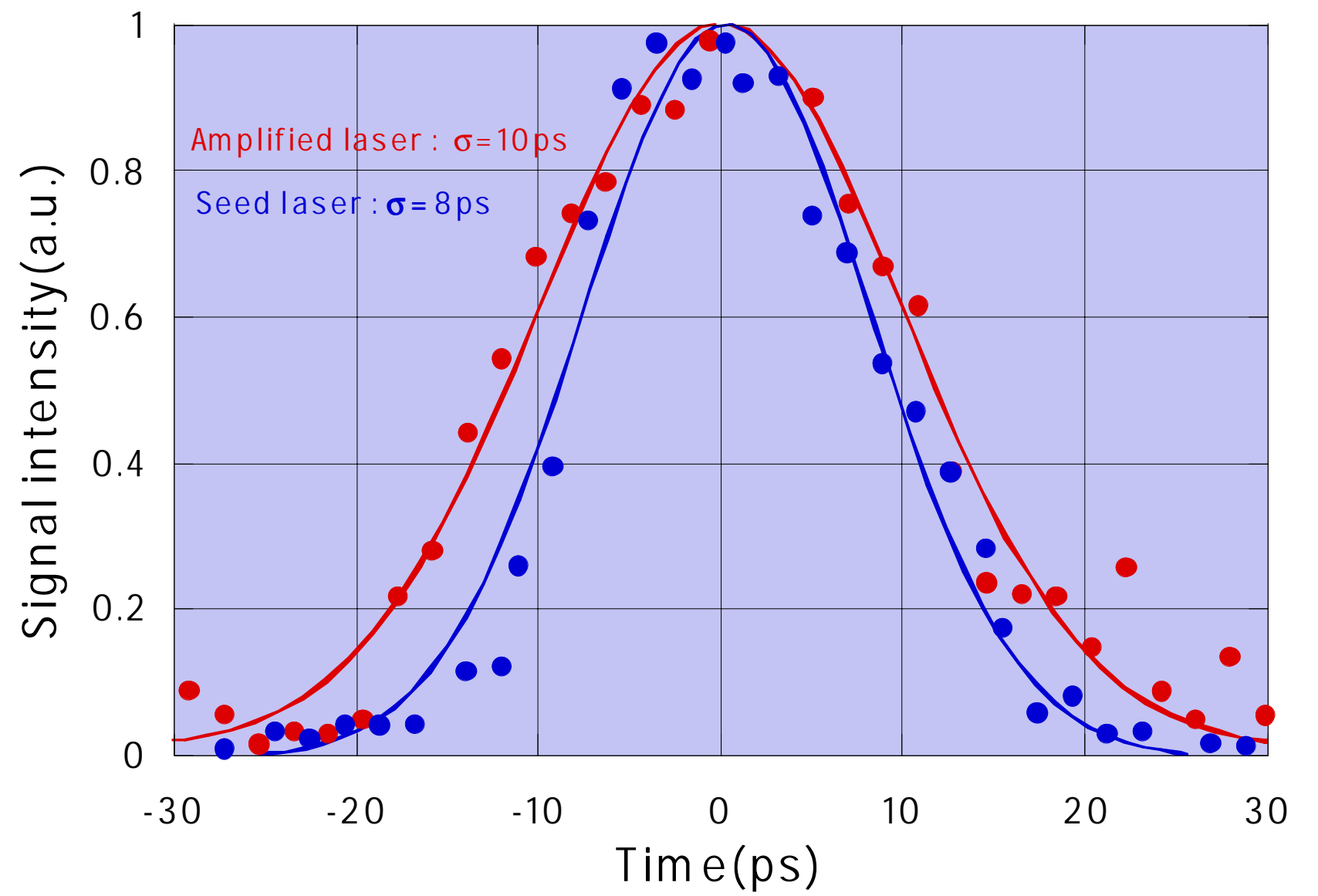




\section{Square Pulse Shaping with a SLM}

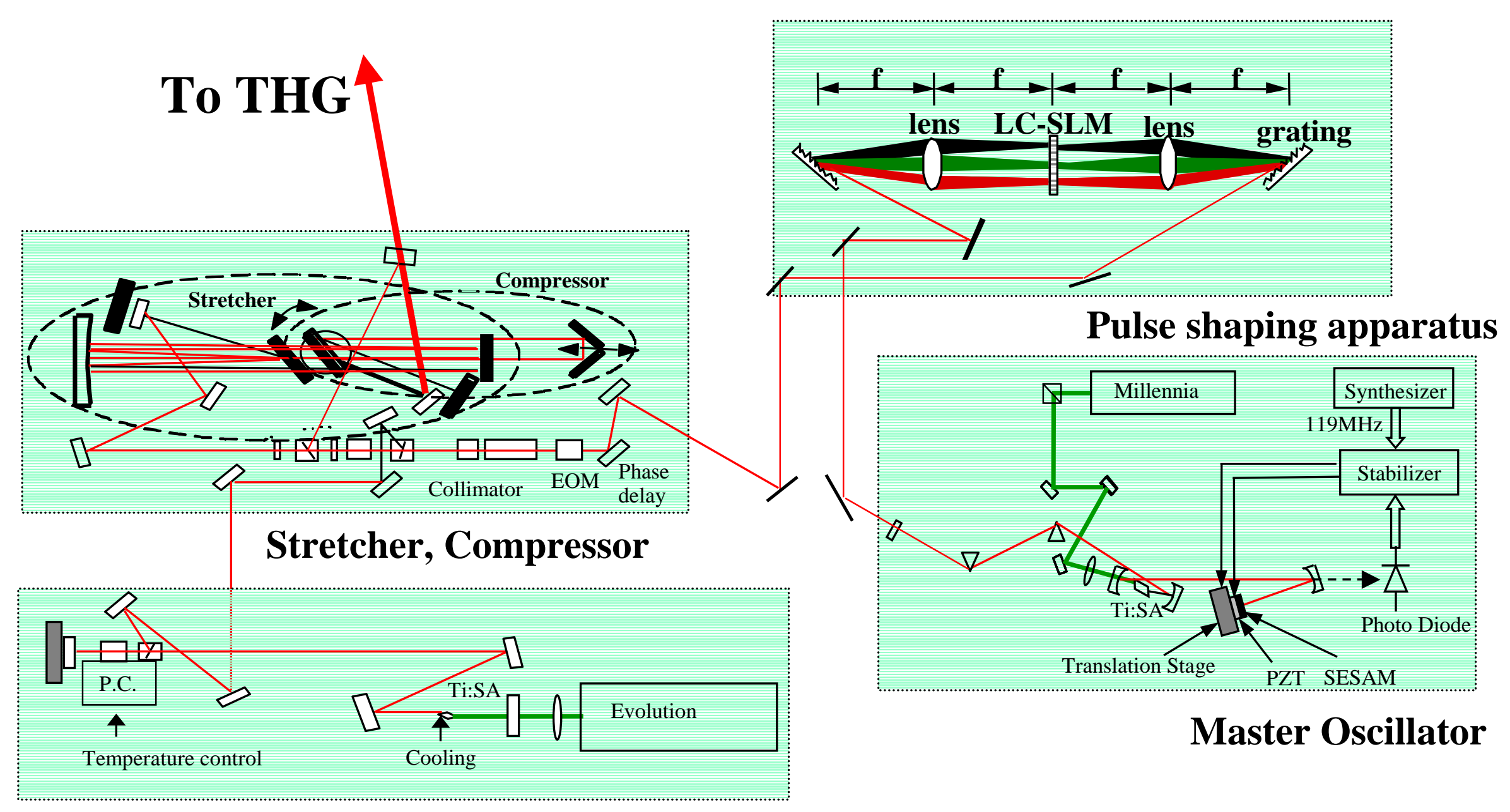

\section{Regenerative amplifier}




\section{Autocorrelation Trace}

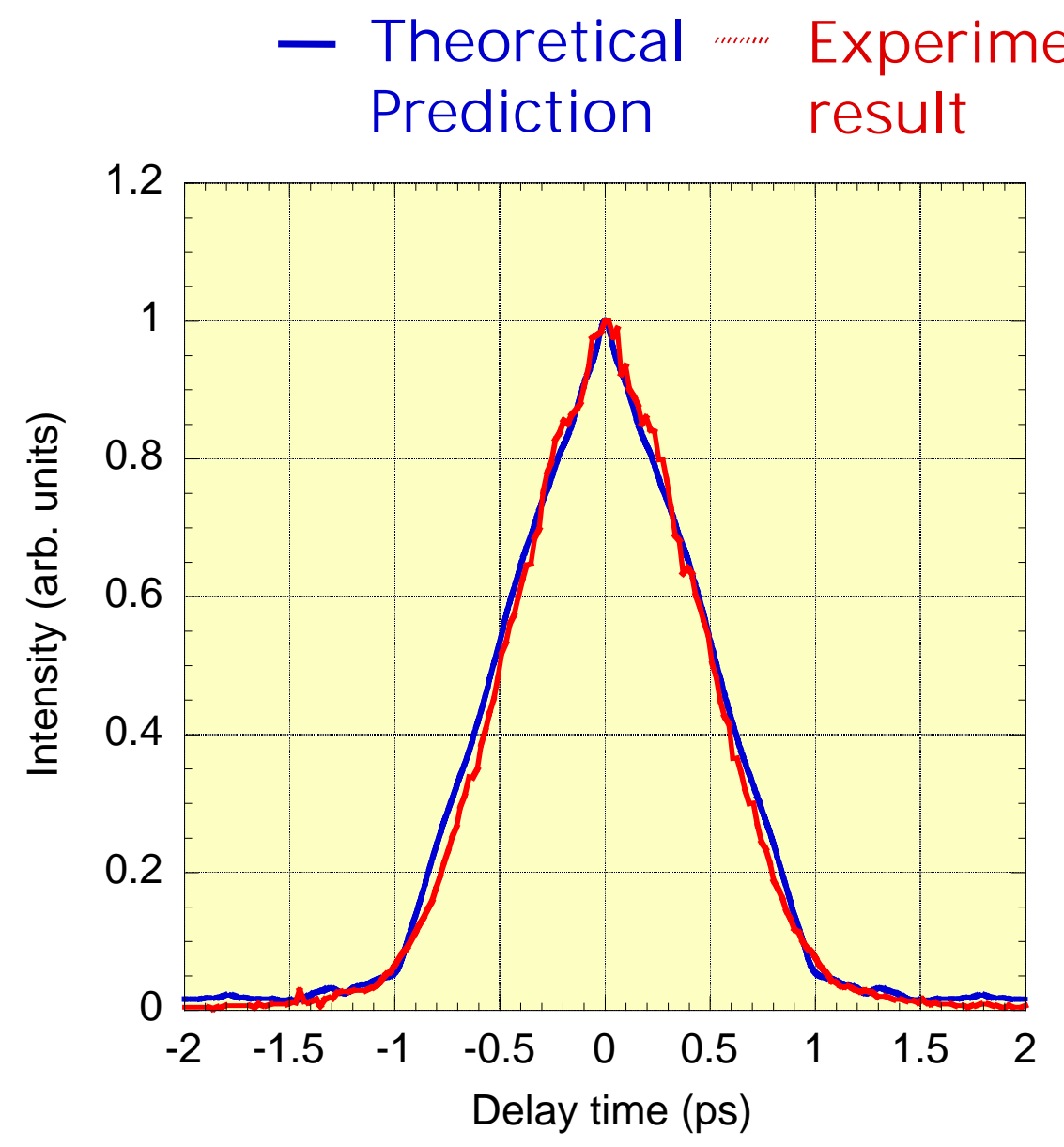

Pulsewidth $1 \mathrm{ps}$

Energy $\quad 0.3 \mathrm{~mJ}$ (IR) repetition $\quad 1 \mathrm{kHz}$

Triangle trace indicates square temporal profile. 


\section{Summery}

Pulrise

Stable UV laser for photo-injector

Already open to the market

Amplifier

Prototype model finished

Continuing for production model

Please visit Photonics West at San Jose

Paper \#4267-30

Poster session 5:30 to 7:00 PM on 1/24

Square Pulse

Trying to generate $\mathrm{THG}$

Katsuyuki Kobayashi: kobayashi@aculight.com 


\title{
A Regenerative Source for a High Average Current Energy Recovery Linacs
}

\author{
Alexander Zholents and Max Zolotorev
}




\section{Scylla and Charibdys of Photocathode guns with a high average current}

\section{Metal cathodes (Cu, Mg):}

excellent lifetime

poor quantum

efficiency;

need ultraviolet

laser

\section{Semiconductor $\left(\mathrm{K}_{2} \mathrm{CsSb}, \mathrm{Cs}_{2} \mathrm{Te}\right)$ :}

\author{
poor lifetime
}

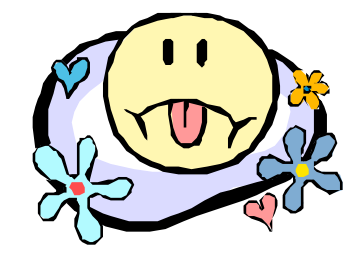

excellent quantum efficiency

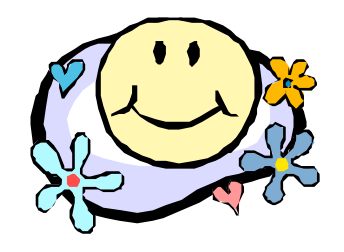




\section{A Recirculating SR Source for Ultrafast X-Ray Science}

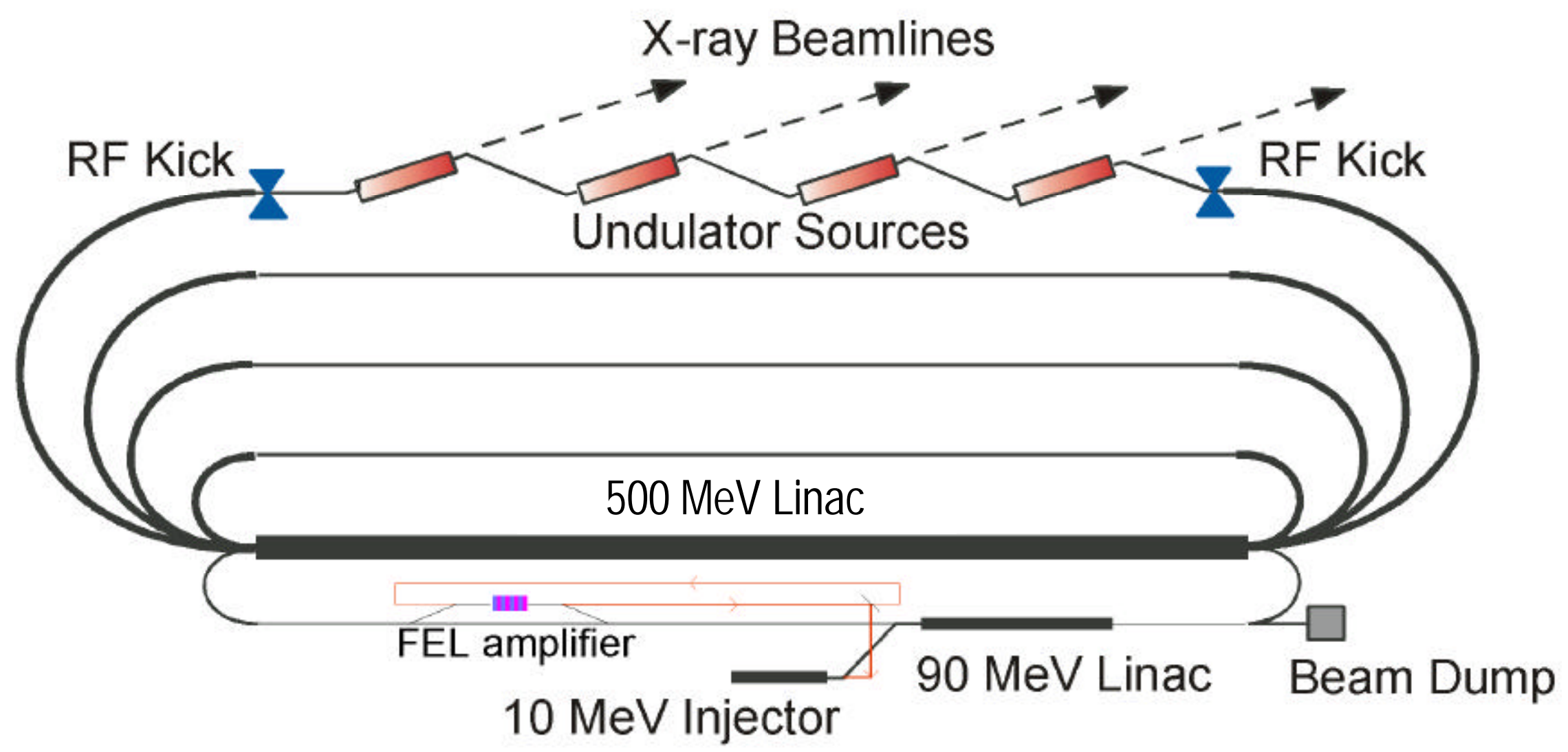

Scale: $100 \mathrm{~m}$

Two stages of pulse compression:

a) electron beam: $10 \mathrm{ps} \longrightarrow 1 \mathrm{ps}$

b) X-rays:

$1 \mathrm{ps} \longrightarrow 100 \mathrm{fs}$ 


\section{Compression of $x$-ray pulses}

is possible due to a correlation between the longitudinal and transverse positions of electrons inside the electron bunch created by the RF orbit deflection in a cavity in the beginning of the final straight section.

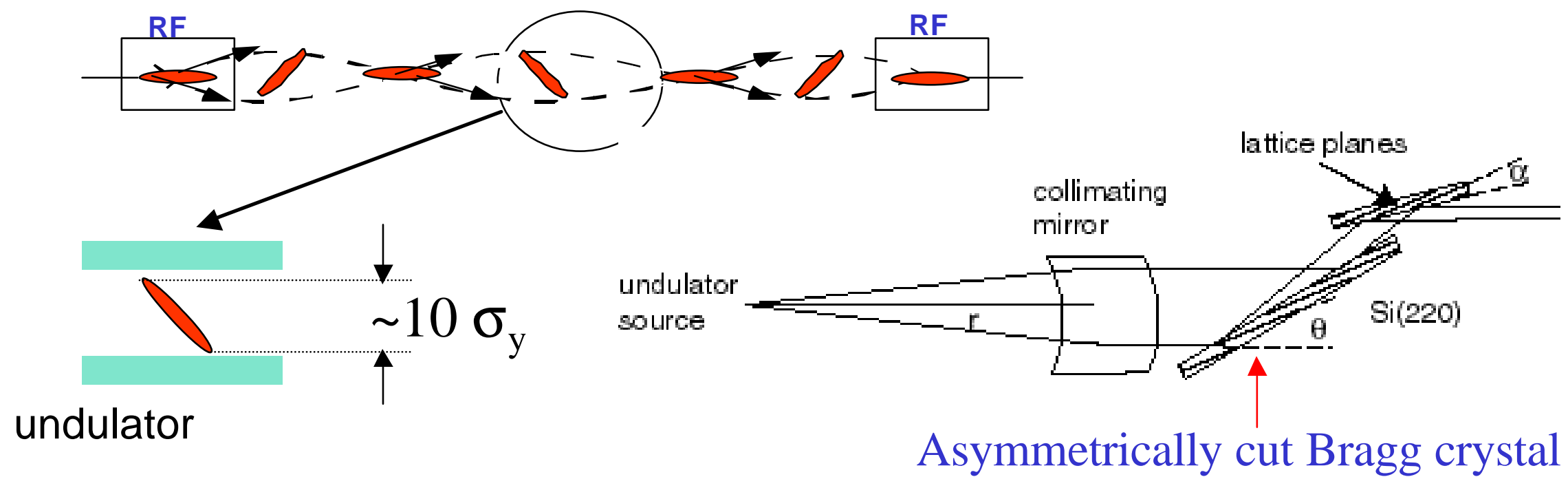

Diffraction limited size of a source at $\lambda 1 \AA$ is $2 \mu \mathrm{m}$

Beam size at $\varepsilon_{\mathrm{n}}=0.4 \mathrm{~mm}-\mathrm{mrad}$ is $\sim 10 \mu \mathrm{m}$ 


\section{Electron beam parameters out of the injector}

Energy

Charge

Normalized rms horizontal emittance

Normalized rms vertical emittance

Energy spread at $20 \mathrm{MeV}$

Pulse length (uniform distribution)

\section{The RF gun parameters:}

RF frequency

Peak electric field on a cathode

Repetition rate of injection pulses

\section{Laser parameters:}

Wavelength (3-rd harmonic of Ti:sapphire laser)

Pulse energy

Pulse length (FWHM)

Repetition rate
$20 \mathrm{MeV}$

$1 \mathrm{nC}$

$20 \mathrm{~mm}-\mathrm{mrad}$

$0.4 \mathrm{~mm}-\mathrm{mrad}$

$15 \mathrm{keV}$

$10 \mathrm{ps}$

$2.6 \mathrm{GHz}$

$100 \mathrm{MV} / \mathrm{m}$

$10-100 \mathrm{kHz}$

$267 \mathrm{~nm}$

$100 \mu \mathrm{J}$

$10 \mathrm{ps}$

$10-100 \mathrm{kHz}$ 


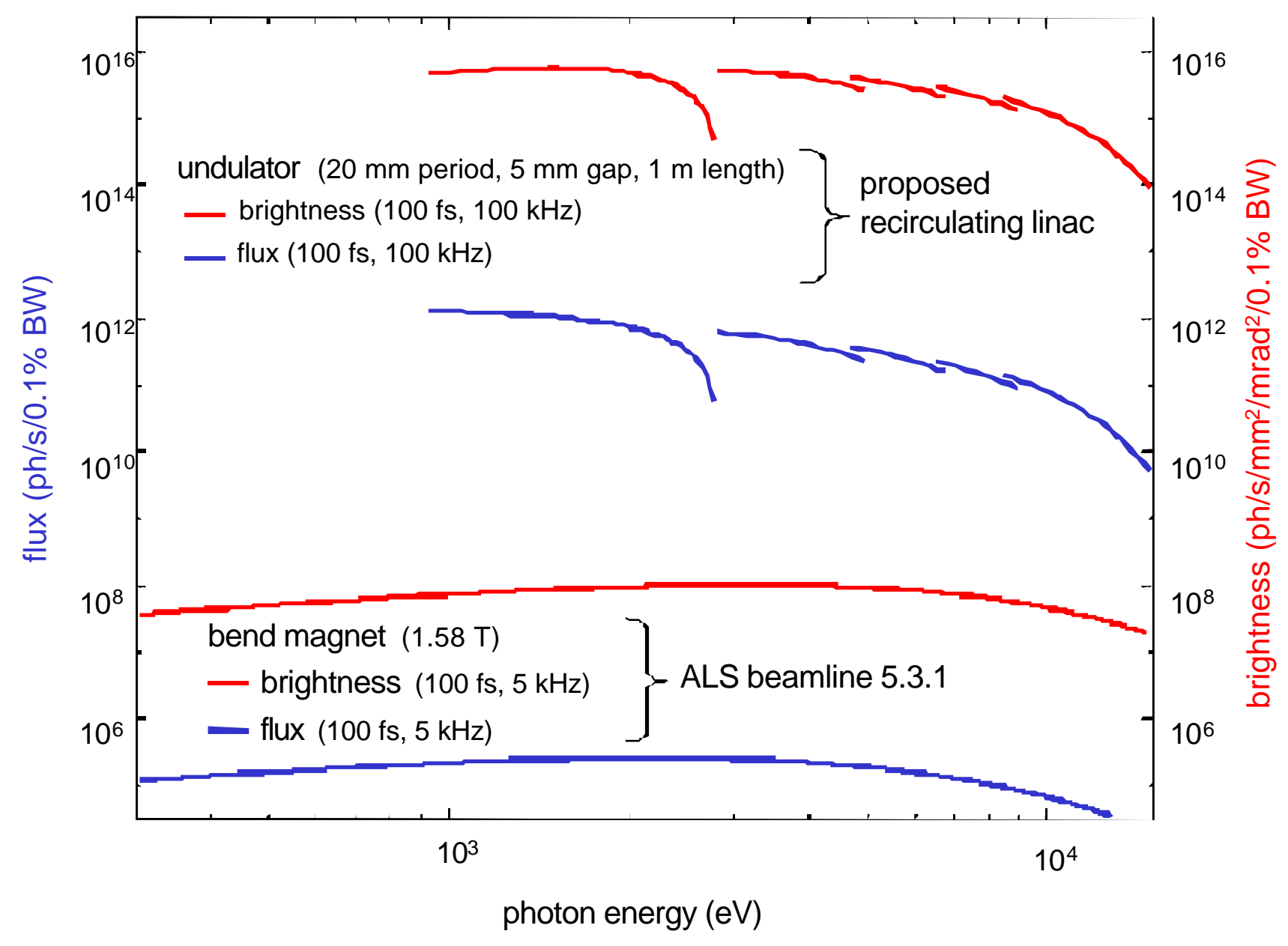

\section{Average femtosecond flux and brightness compared with that of the existing bend-magnet fs beamline.}




\section{Details of a start-up and operation}

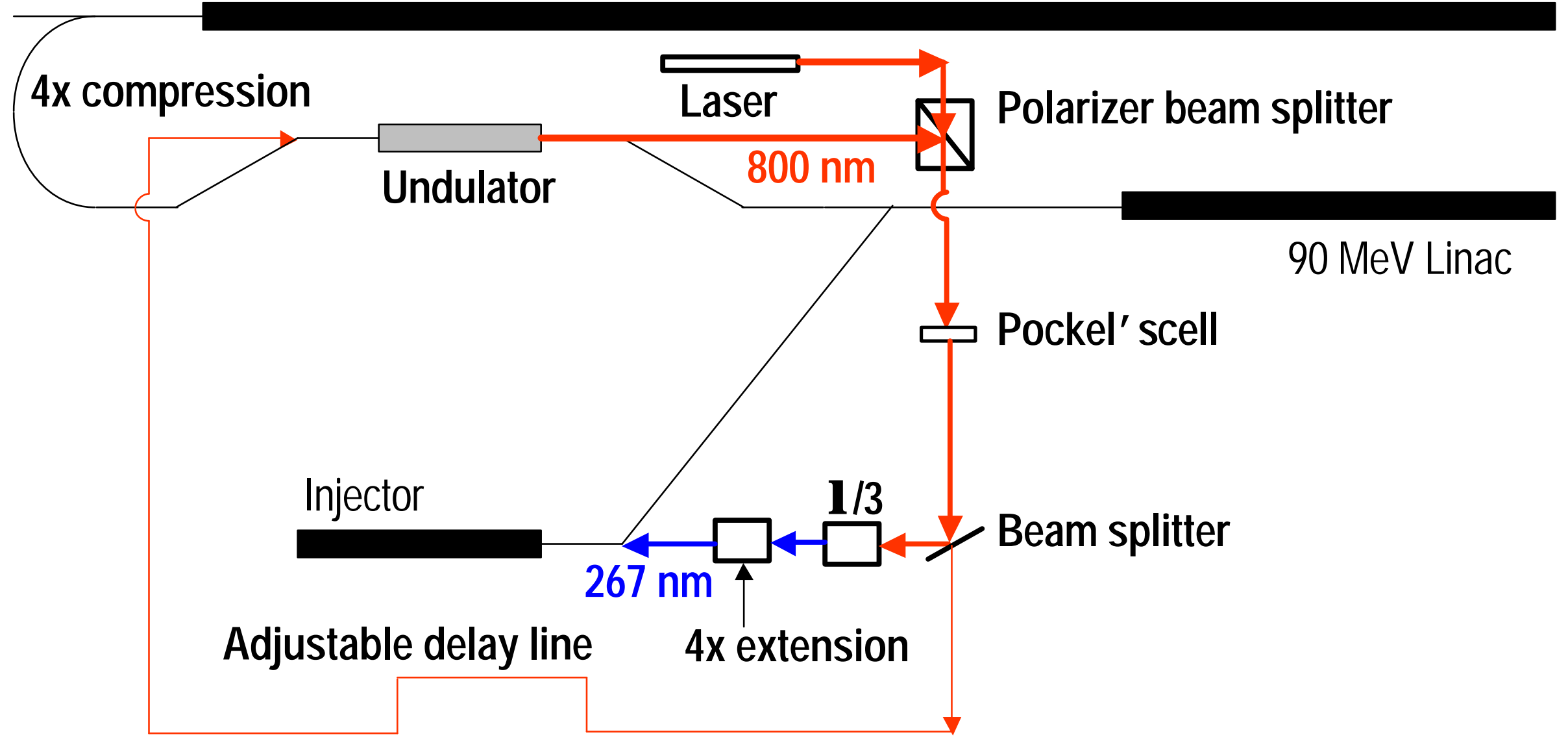

In the regenerative mode (continuous operation):

$1 \mathrm{~mJ} /$ pulse, $20 \mathrm{MHz}$ rep. rate from FEL amplifier at $800 \mathrm{~nm}$ At a start-up ( $1 \mathrm{sec})$ : 


\section{FEL operation}

Undulator: length $=2 \mathrm{~m}$, period $=2 \mathrm{~cm}, \mathrm{~K}=2$

Peak current $=400 \mathrm{~A}$

Light in $=12.5 \mu \mathrm{J}$

Light out $=2500 \mu \mathrm{J}$ 
A_w versus $Z$

19:00:10

20010118

FEMTO

SPARC 10

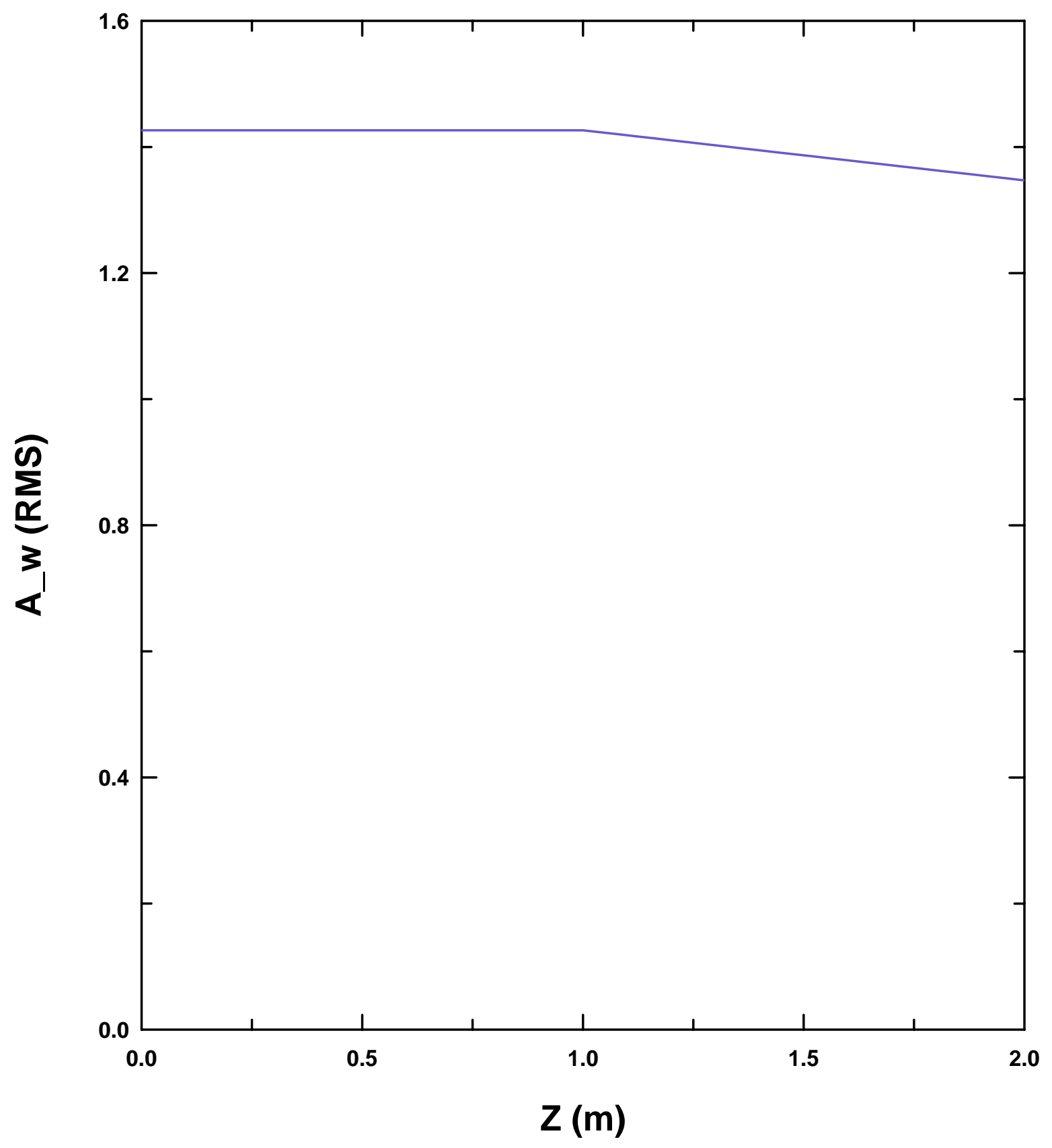


Avg. Field Power vs. Z

19:00:10

20010118

FEMTO

SPARC 10

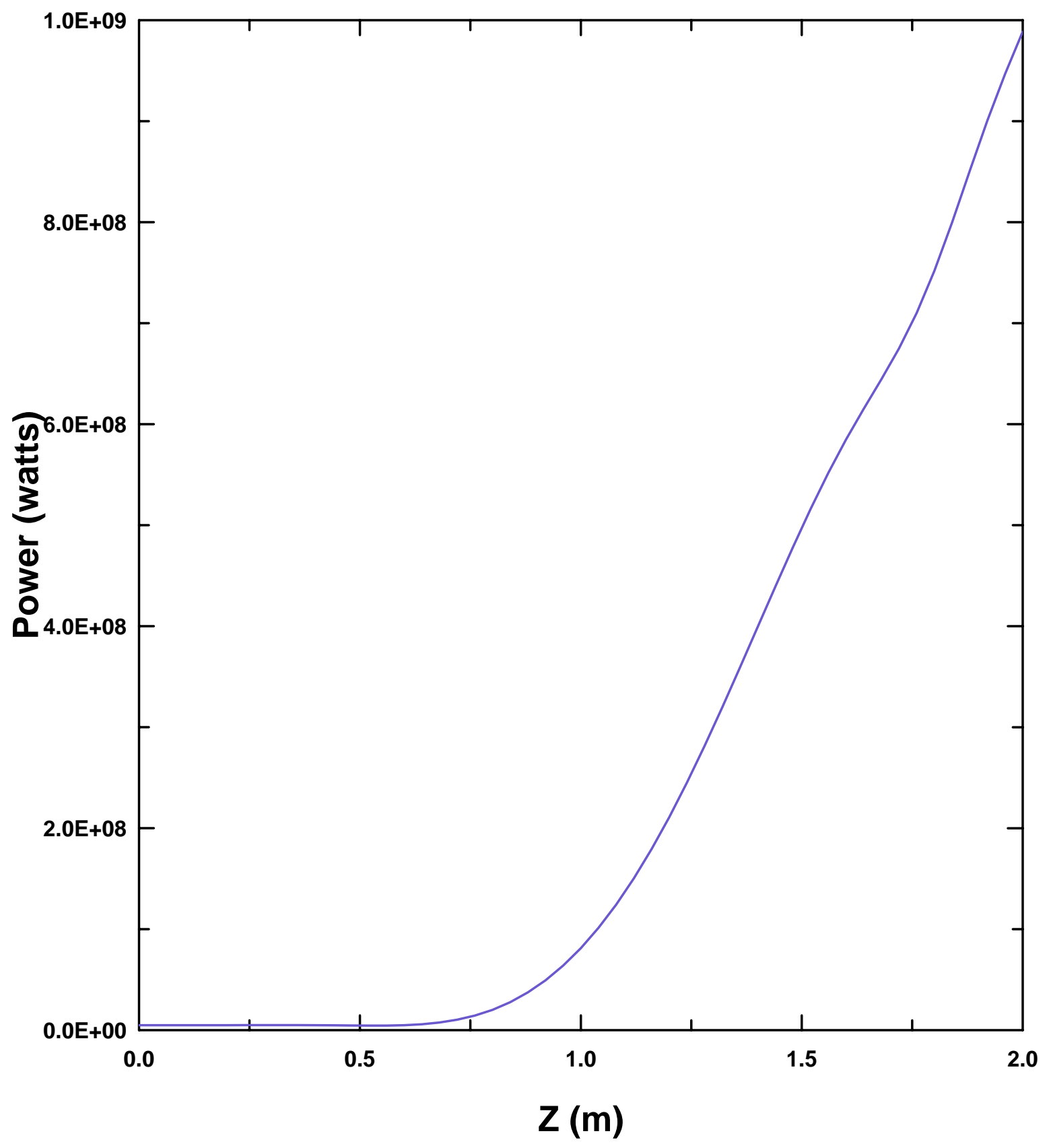


Phase Space at Z $=0.52 \mathrm{M}$

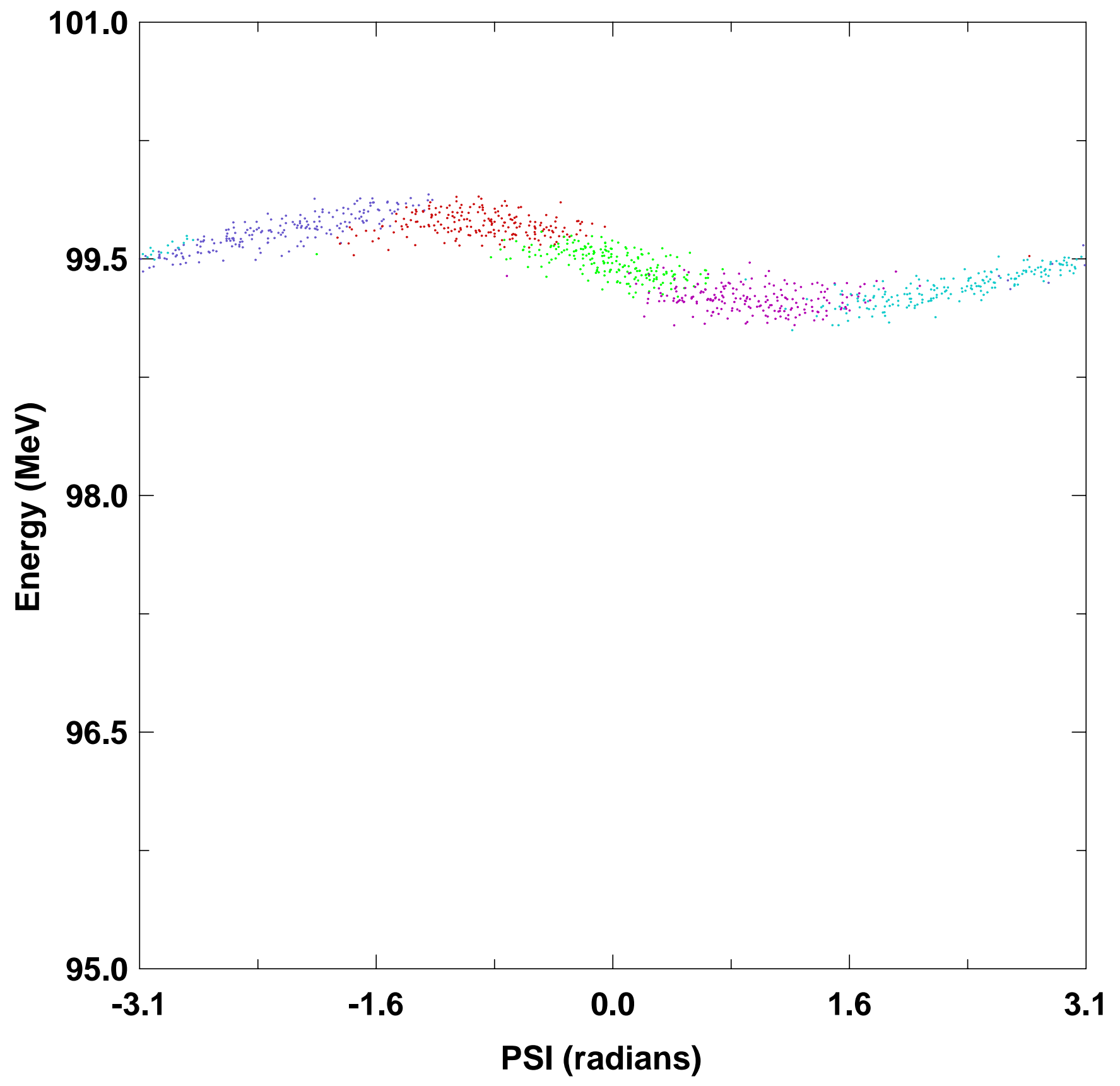


Phase Space at Z $=1.00 \mathrm{M}$

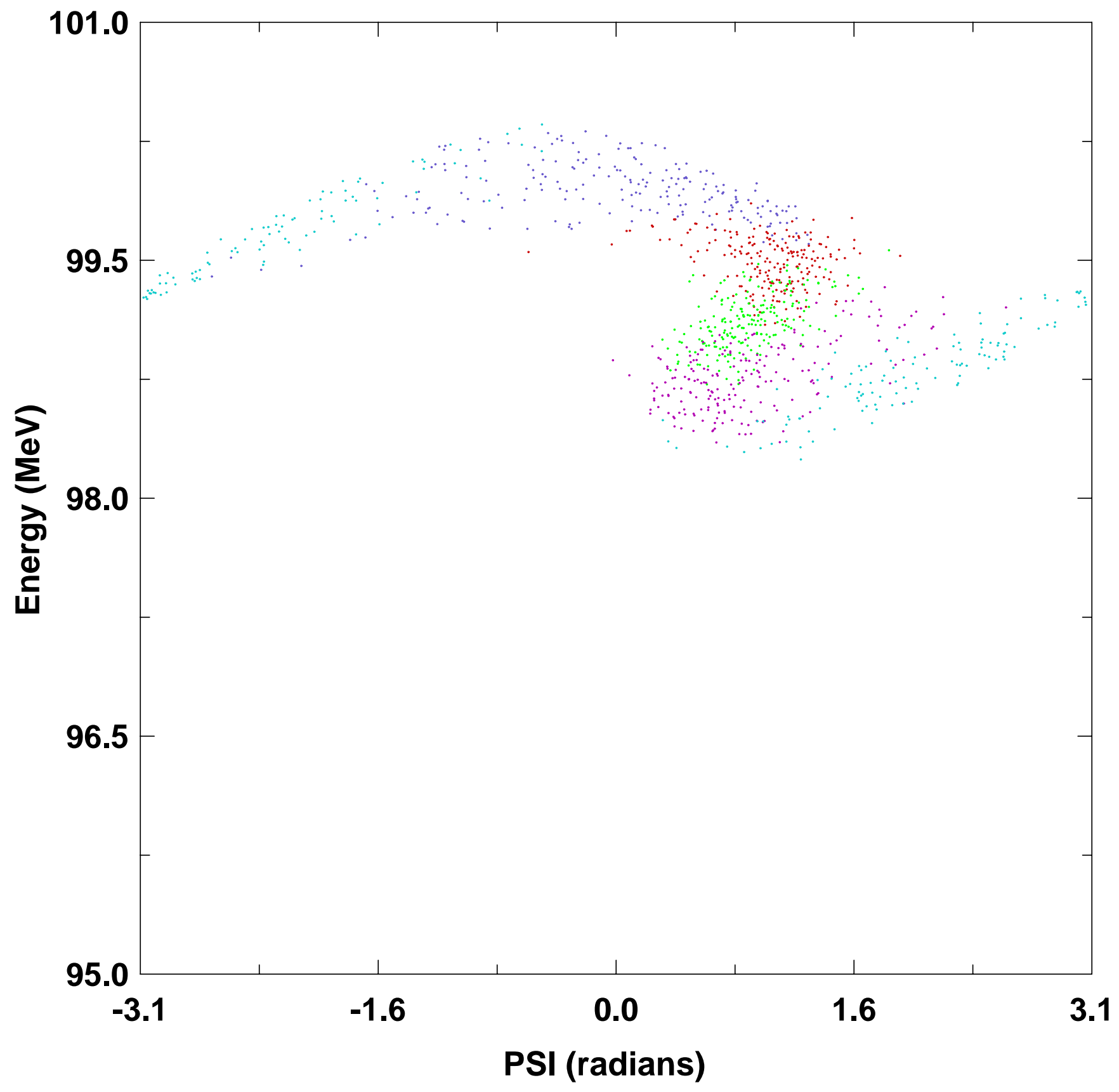


Phase Space at Z $=1.52 \mathrm{M}$

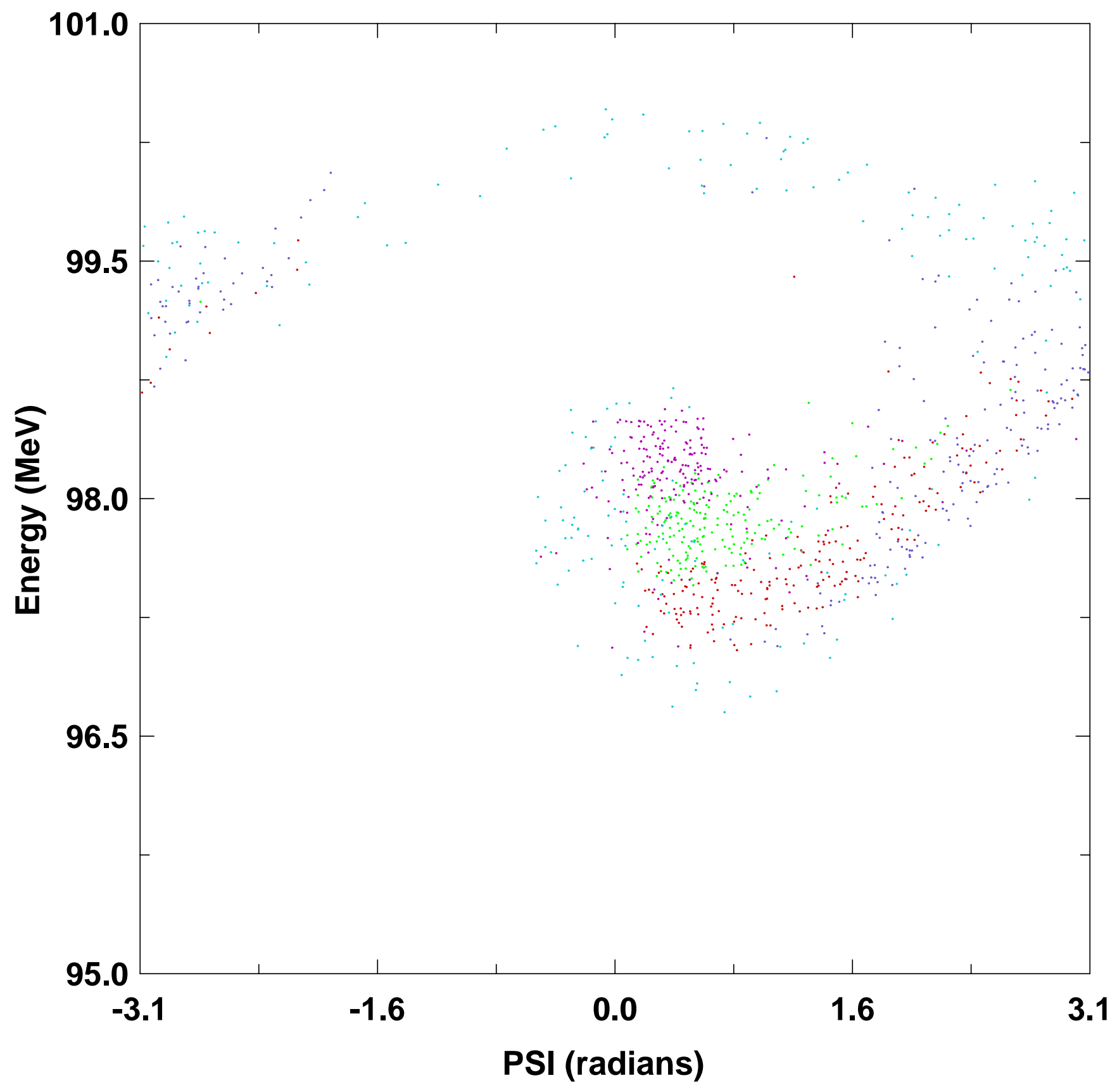


Phase Space at Z $=2.00 \mathrm{M}$

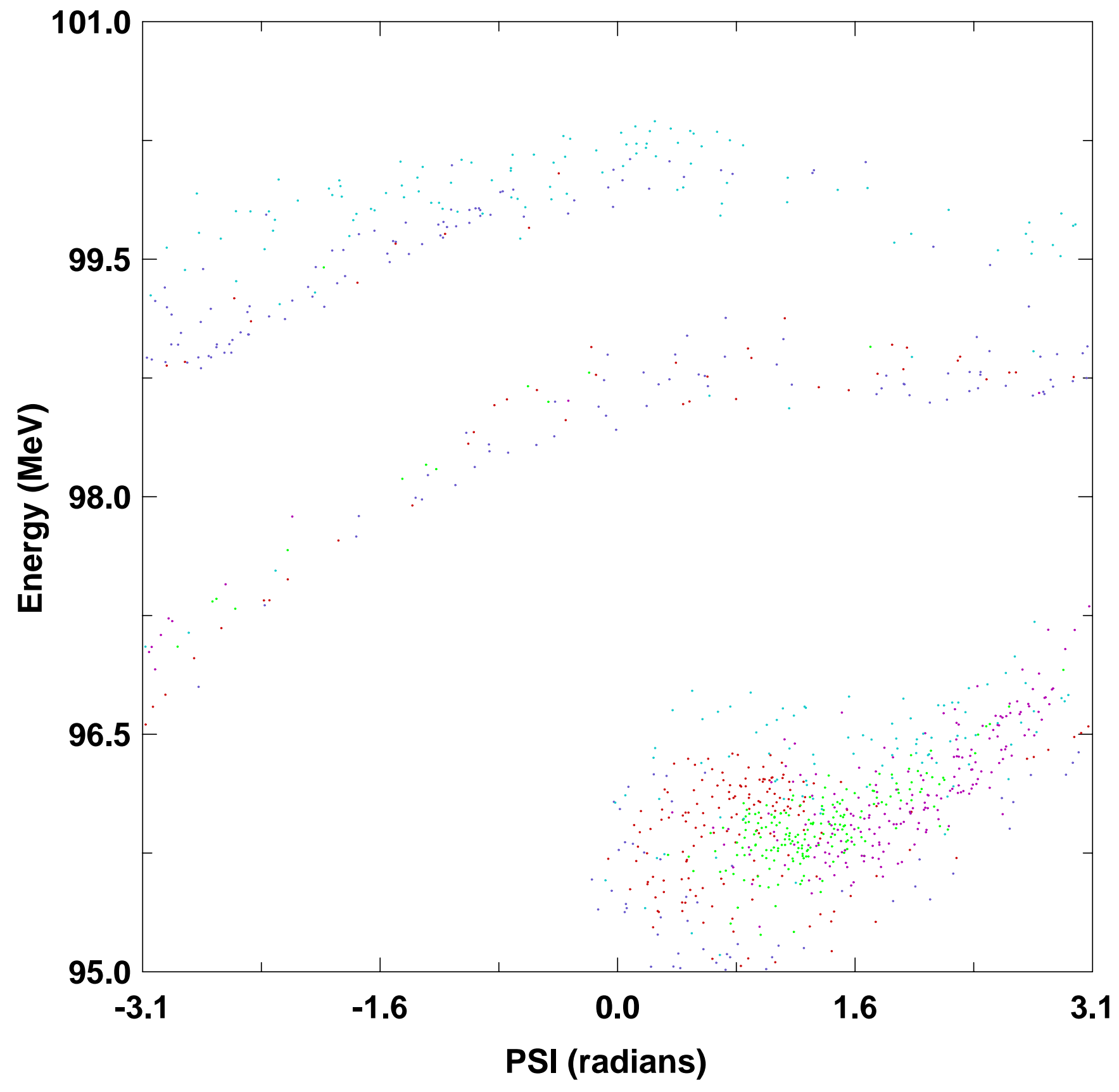




\title{
Femtosecond and Picosecond Lasers for Accelerator Applications
}

\author{
Katsuyuki Kobayashi \\ Sumitomo Heavy Industries (USA), Inc. \\ c/o Aculight Corporation \\ 11805 North Creek Parkway S. Bothell, WA 98011 \\ E-mail: kobayashi@aculight.com
}

A part of this work is performed under the management of the Femtosecond Technology Research Association, supported by New Energy and Industrial Technology Development Organization. 
Feasibility study of PERL photoinjector laser

- Multi-laser combination

Accelerator relating laser technologies of out research work

- Timing stabilization of the amplified pulses

- Synchronization of two mode-locked oscillators 


\section{PERL photoinjector laser}

Requirements for the Laser

Wavelength $\sim 260 \mathrm{~nm}$

Pulsewidth 10 ps

Rep. Rate $1.3 \mathrm{GHz}$

Ave. Power $80 \mathrm{~W}$

$130 \mathrm{MHz}, 8 \mathrm{~W}$ ? Yes !!

Build 10 lasers and combine into one system.

How ? 


\section{Plan 1: Pile up on the surface of the cathode}
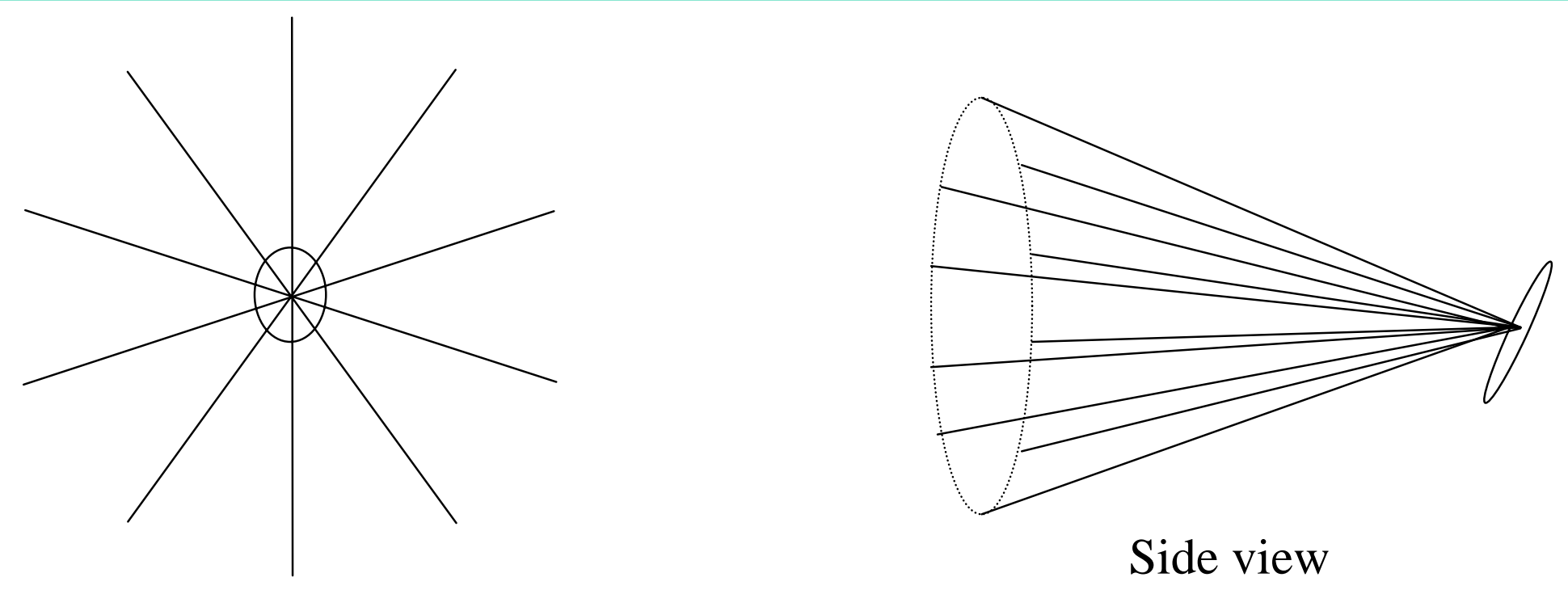

Front view

Advantage

Simple configuration

Disadvantage

Requires 10 view ports

Different incident angles

Different polarization 


\section{Plan 2: Pile up on the surface of the cathode}

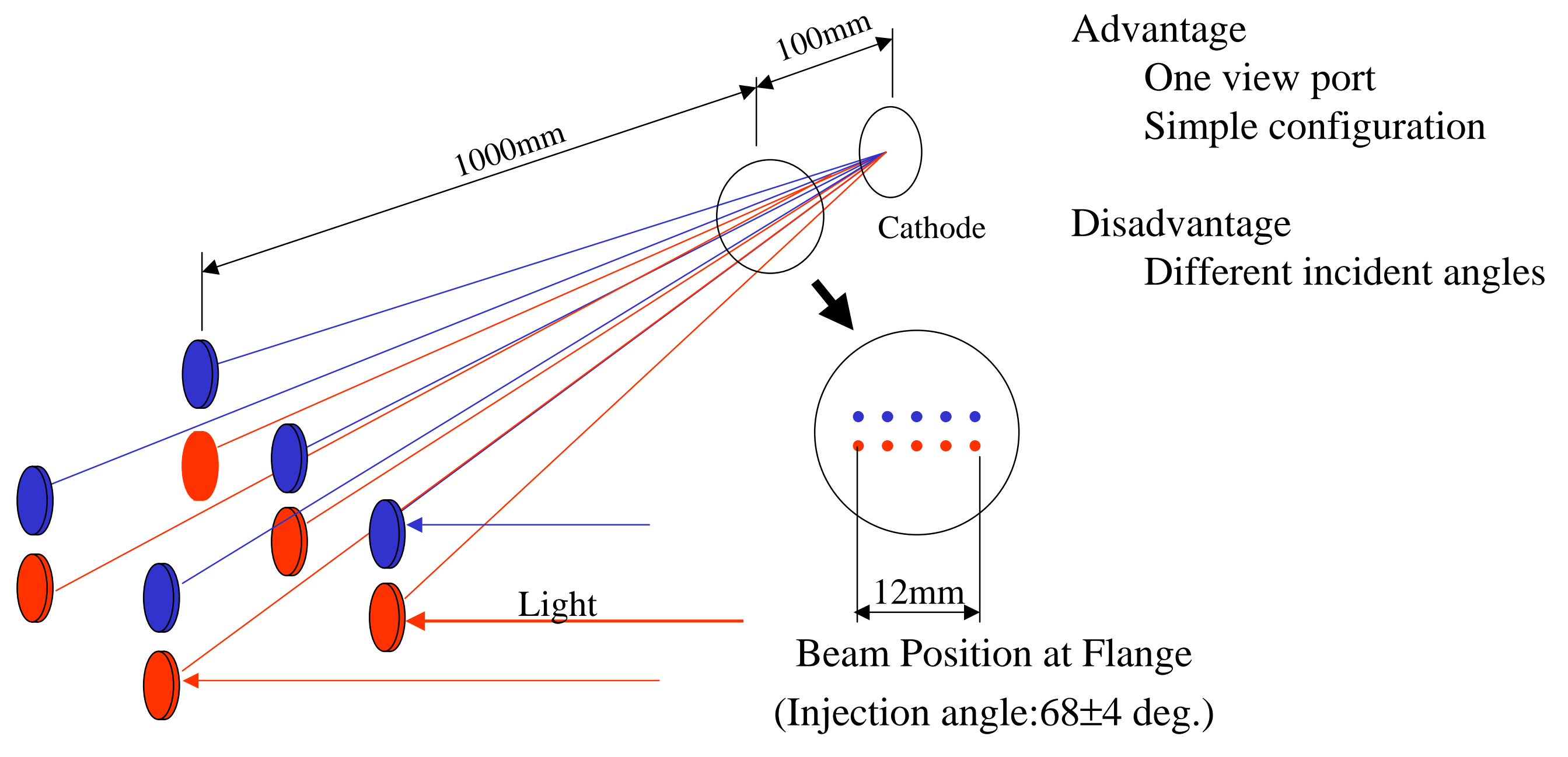




\section{Plan 3: Overlap with a grating (wavelength multiplexing)}

\section{Advantage}

All beams exactly overlap

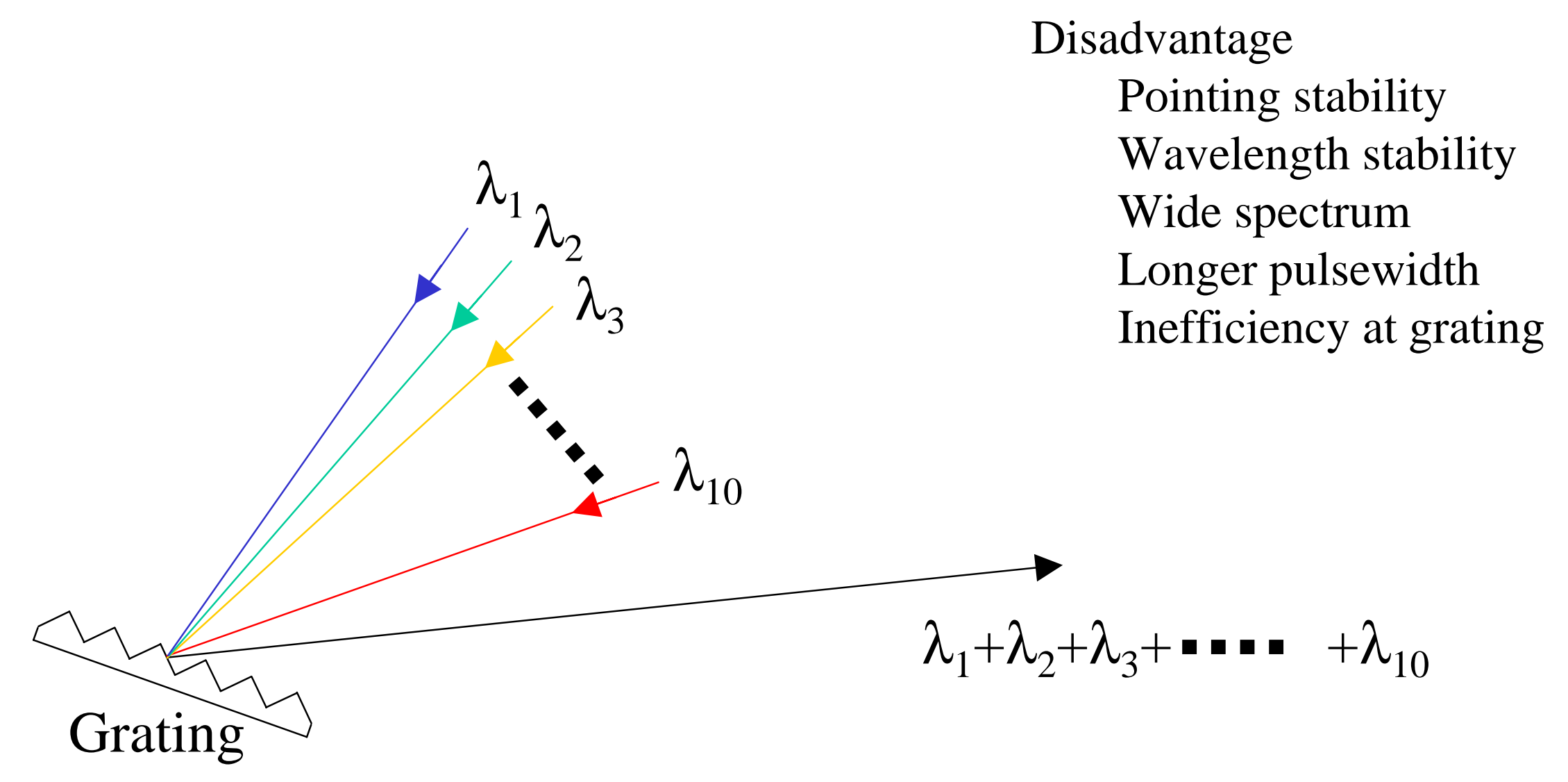




\section{Option: overlapping 2 beams into 1}

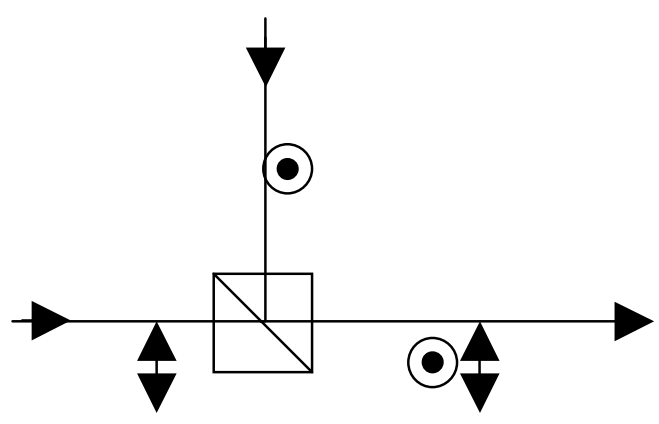

- Two beams are overlapped in a polarized beam splitter.

-Resulting 5 beams incident on the cathode either from 5 directions (plan 1,2) or 1 direction (plan 3). 


\section{Single Pulse jitter measurement}

Statistical method cannot be adopted to low-repetition amplified pulses
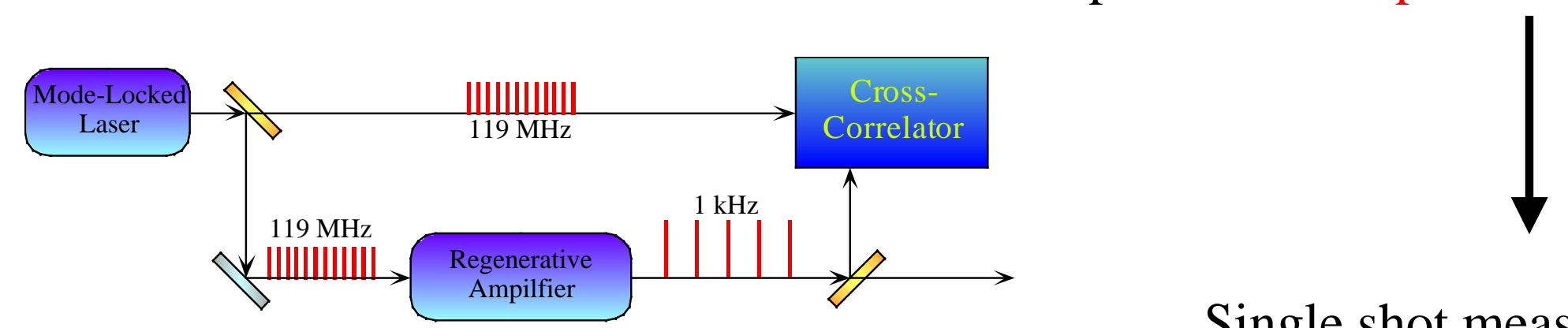

Single shot measurement

Stable oscillator as a reference

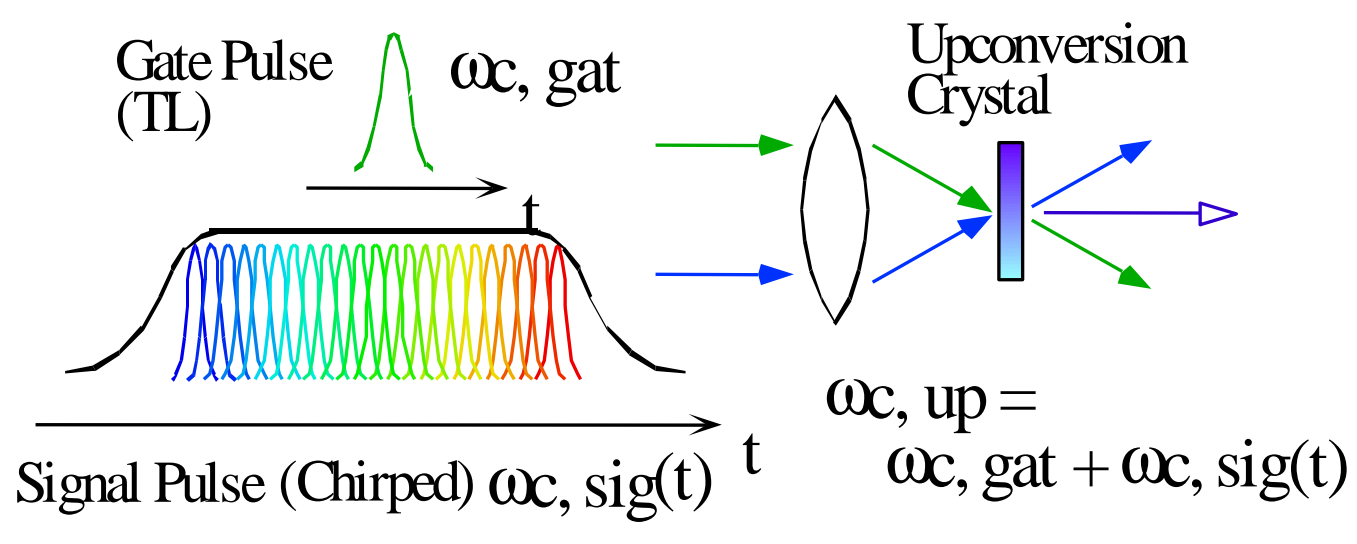

Intentionally stretched pulse 


\section{Measurement of timing fluctuation of the amplified pulses}

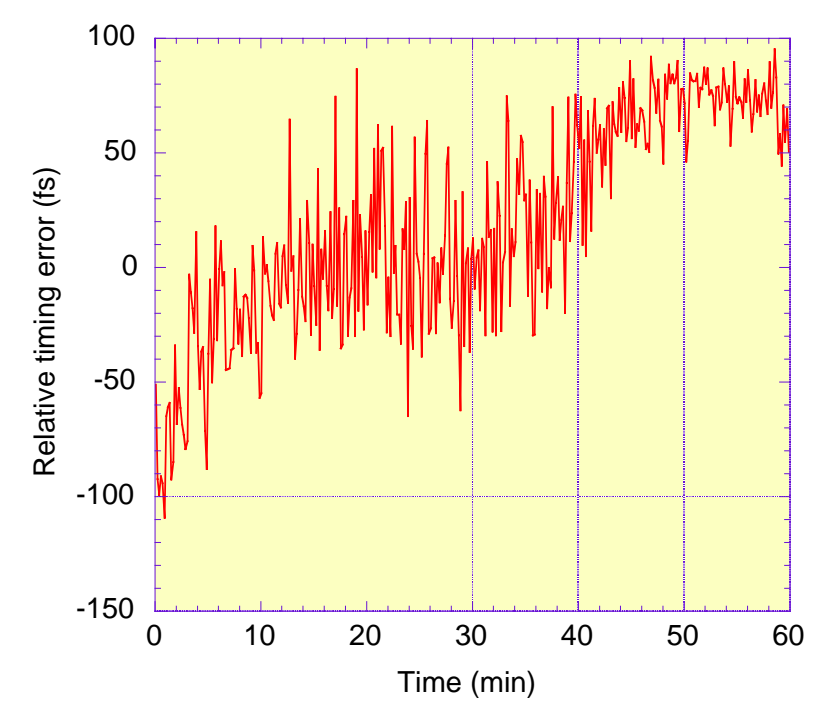

(a) Long term drift

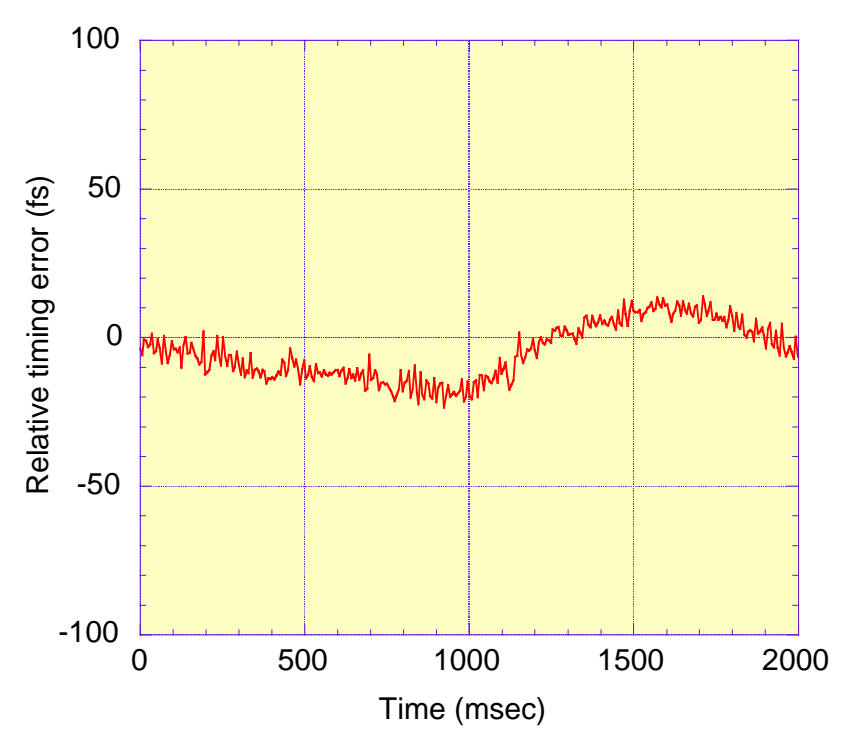

(b) Short term fluctuation

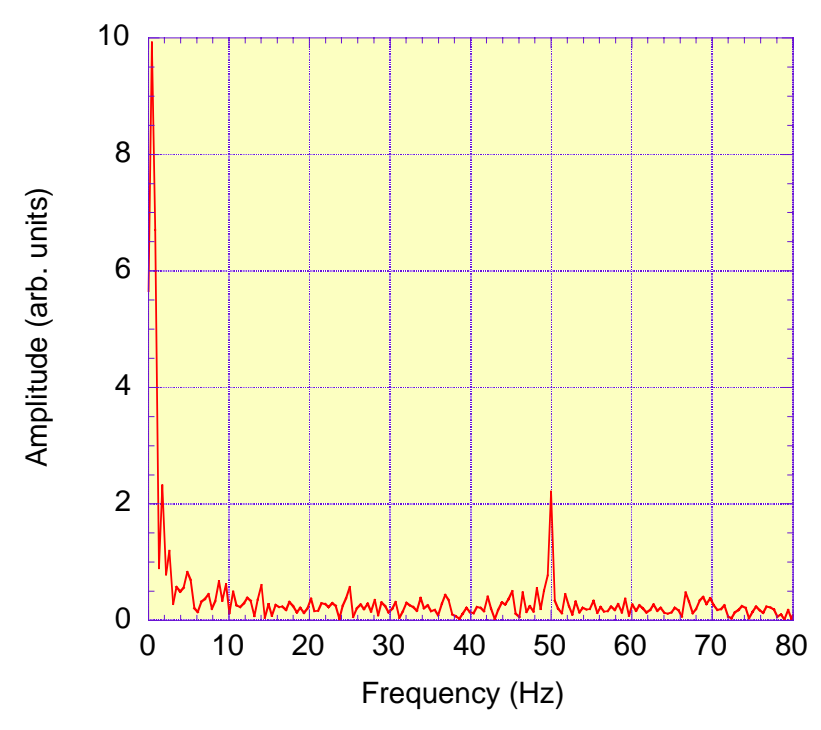

(c) Fourier transform of (b)

Long term (one hour) drift was less than $200 \mathrm{fs.}$ Short term (several seconds) fluctuation was about $10 \mathrm{fs}$. 


\section{Reduction of timing fluctuation of the amplified pulses}
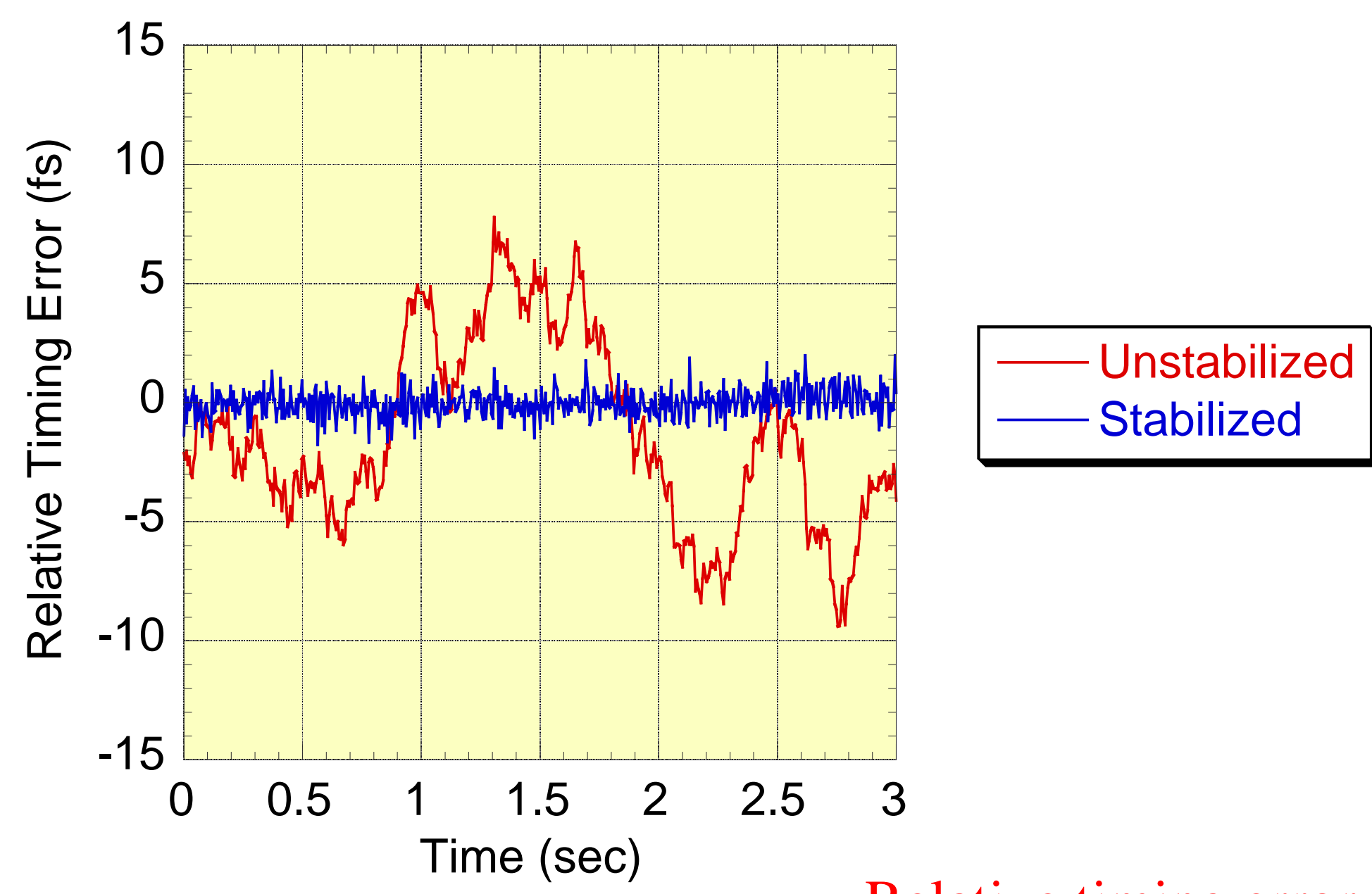

Relative timing error $\sim 0.7 \mathrm{fs}$ (rms) 


\section{Synchronization of two mode-locked oscillators}

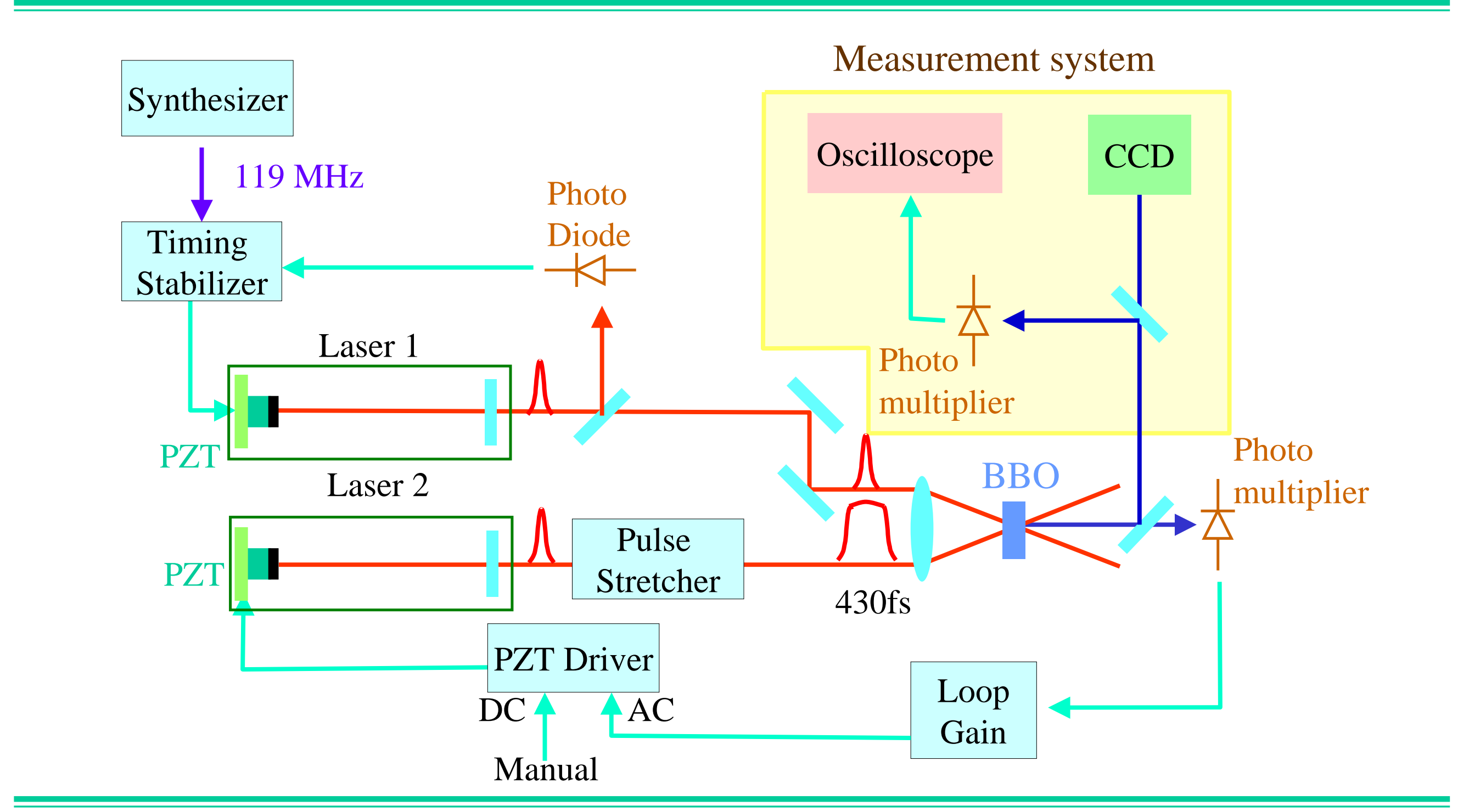




\section{While phase-lock loop is not operating}

- The pulse bunches repeated at the beat frequency.

- Each pulse bunch consists of pulses which had the repetition frequency of $119 \mathrm{MHz}$.
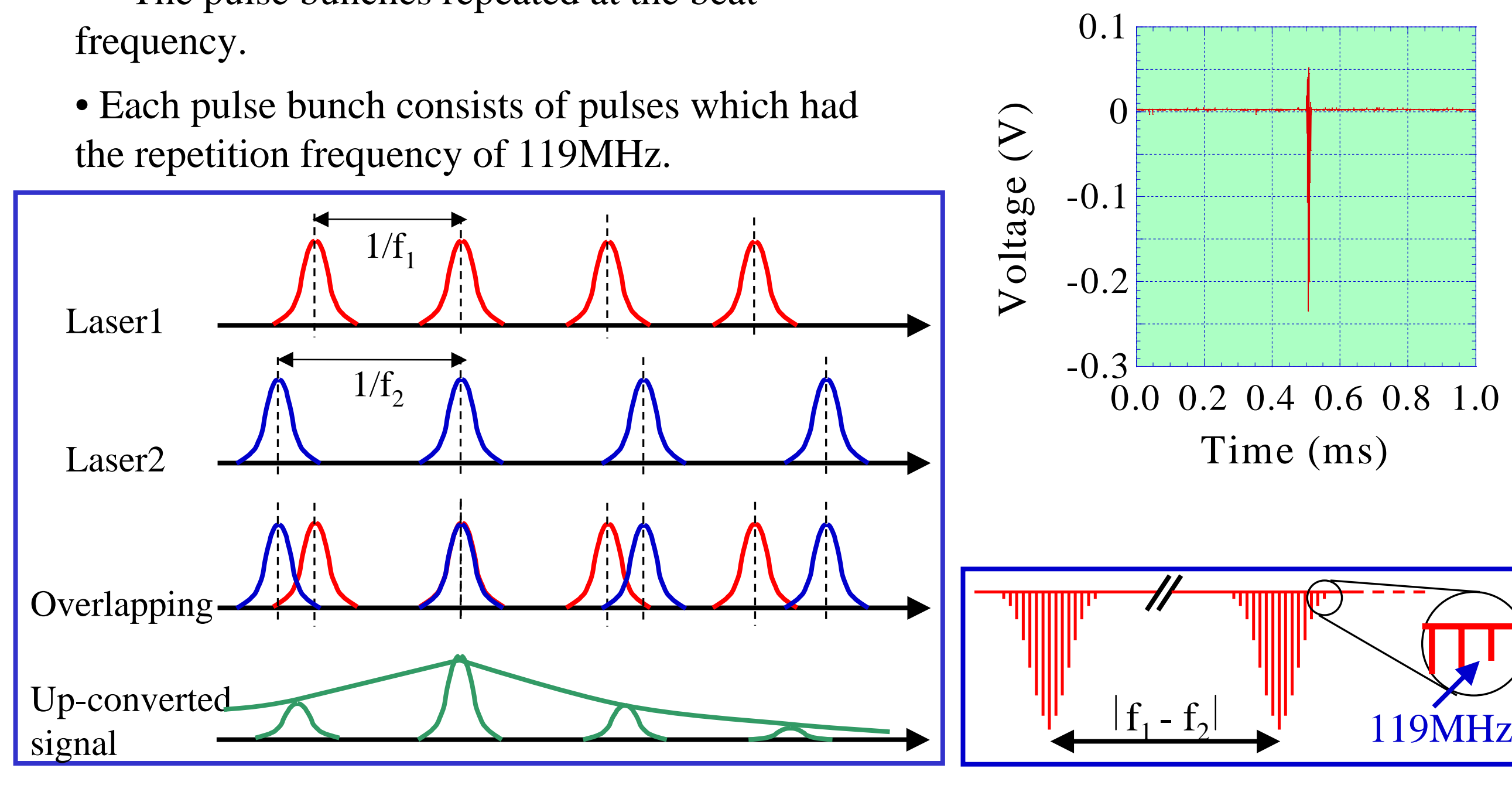


\section{New CSR Experiments: LEUTL SDL}




\section{Experimental Configuration for Measuring CSR Correlations}

Top View:

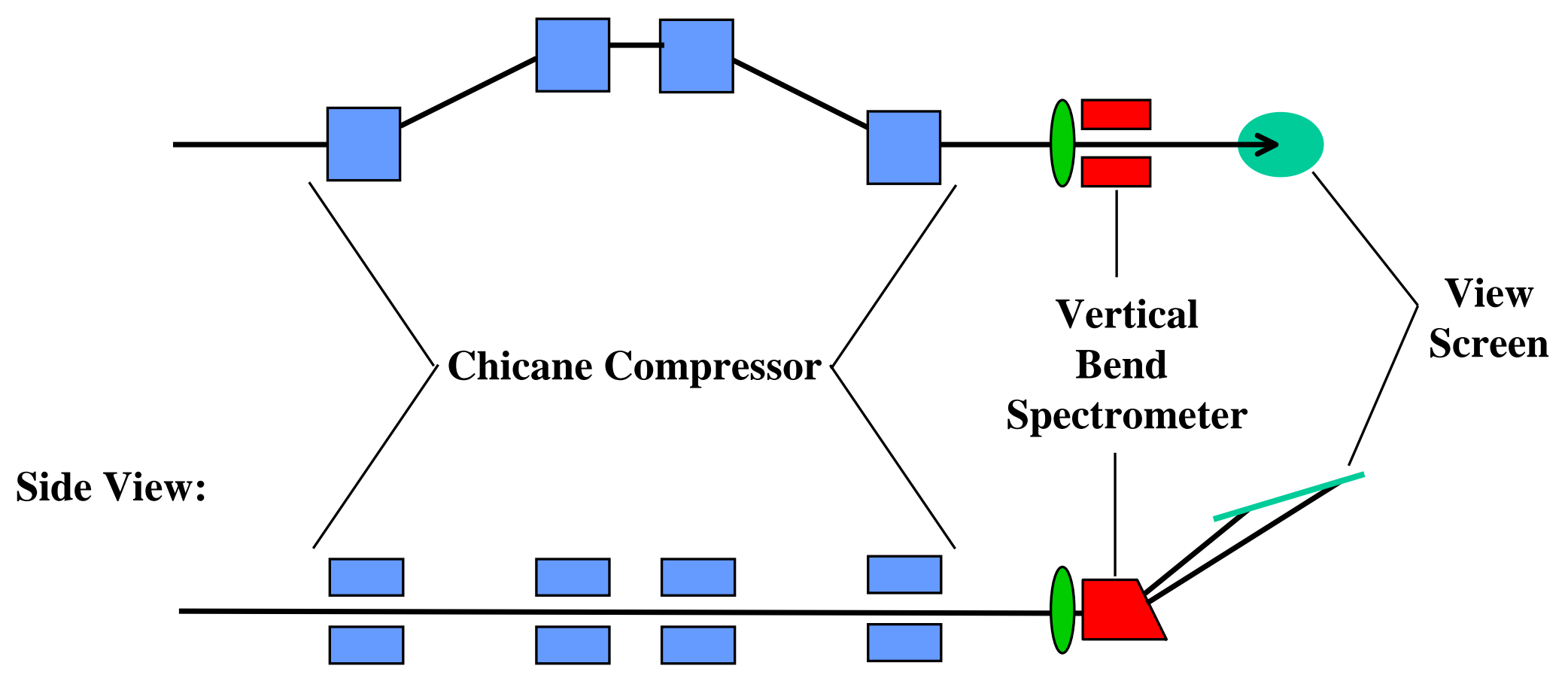




\section{PARMELA Generated Initial Distribution for SDL at Spectrometer Focal Plane}

Initial emittance: $2.2 \pi$, Final emittance: $6.0 \pi$

Compression to 1000 amperes
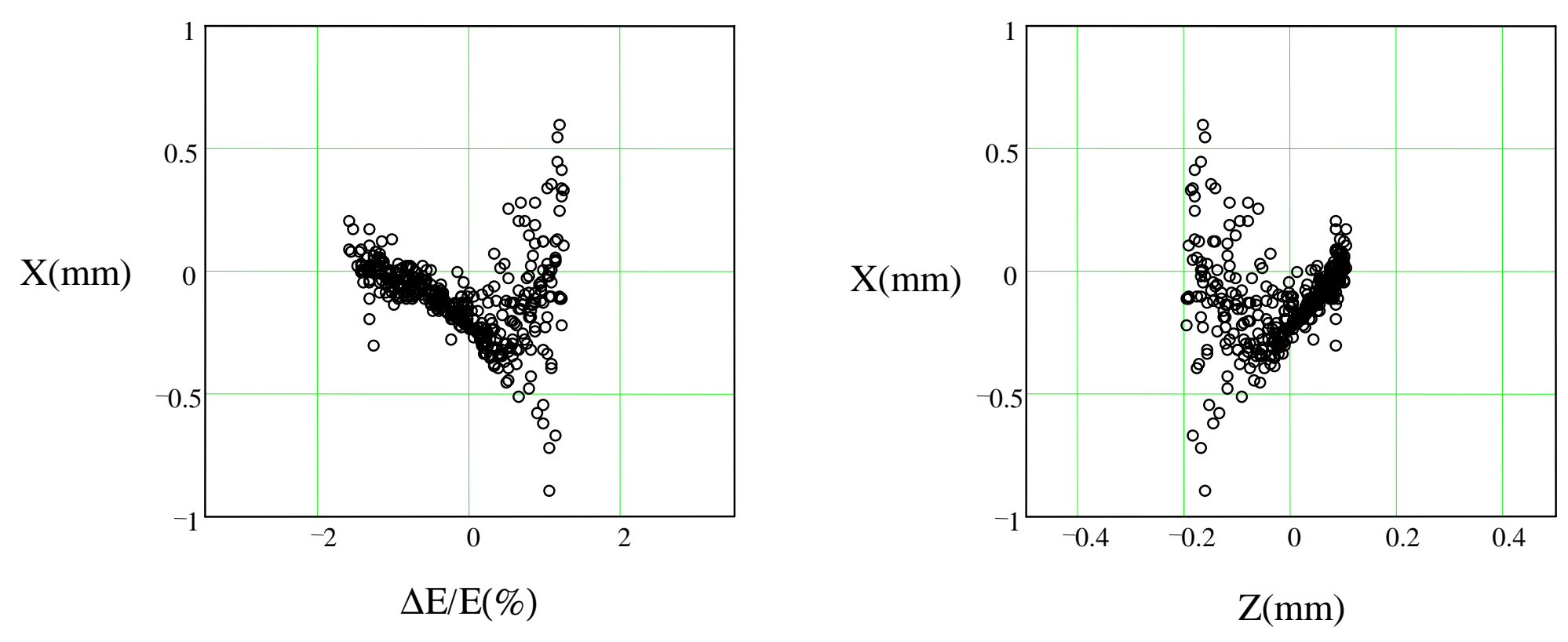

Focal Length $=500 \mathrm{~mm}$ 


\section{Phase lock loop is in operation}

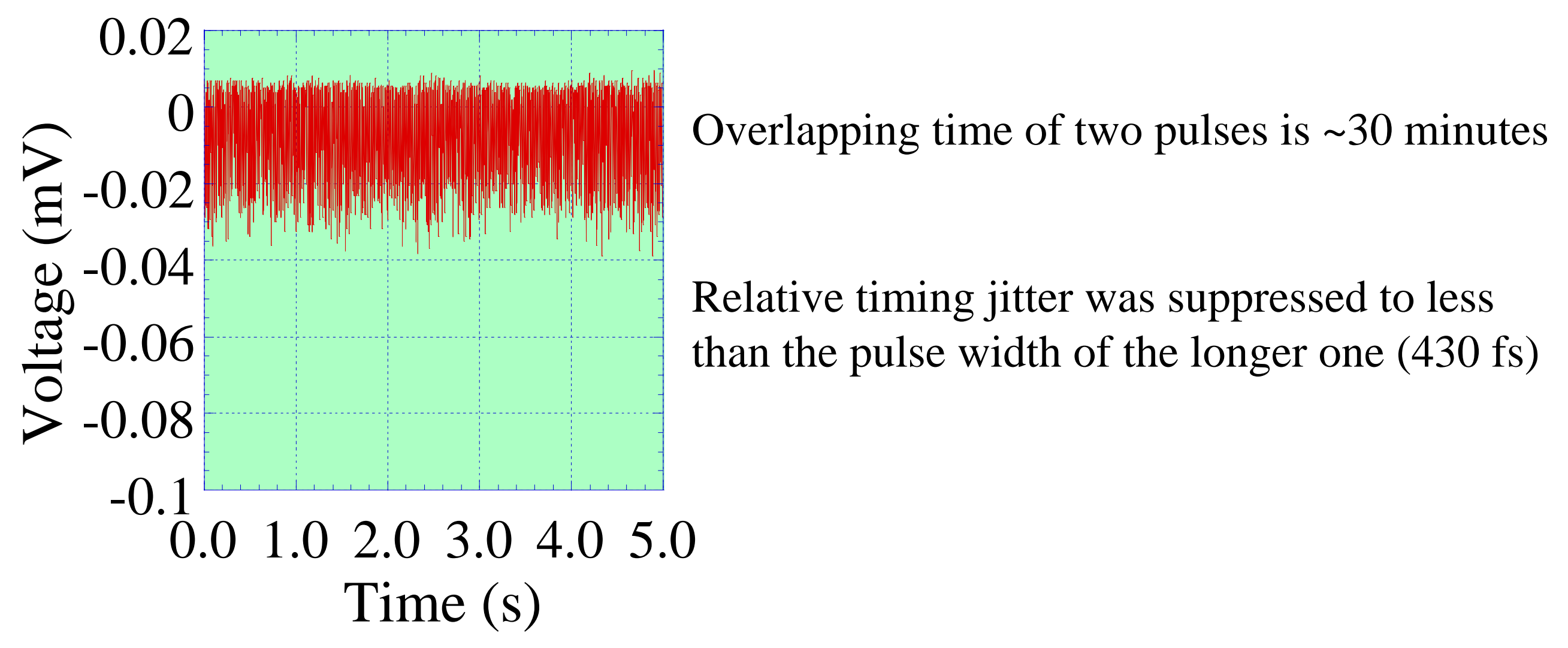




\section{Application sample: $\mathrm{CO}_{2}$ laser seeder}

\section{To $\mathrm{CO}_{2}$ laser}

$1 \mu \mathrm{J}, 10.6 \mu \mathrm{m}, 10 \mathrm{ps}$

$1 \mathrm{~mJ}, 745 \mathrm{~nm}, 10 \mathrm{ps}$

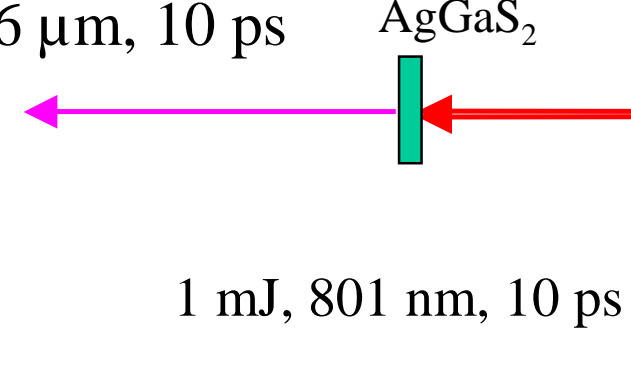

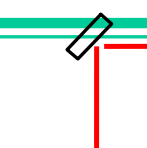


Feasibility study of PERL photoinjector laser

- Proposed to combine 10 lasers into one system

- Discussed on 3 plans to combine

Accelerator relating laser technologies of out research work

- Stabilized the amplified pulse < 1 fs

- Synchronized two mode-locked oscillators

Katsuyuki Kobayashi: kobayashi@aculight.com 


\title{
Preliminary Beam Parameters of PERL Injector with DC Gun
}

\author{
F.Zhou, I.Ben-Zvi, T.Shaftan, X.Wang \\ Brookhaven Accelerator Test Facility \\ Brookhaven National Laboratory \\ Upton, NY 11973, USA \\ January 22, 2001 \\ PERL Photoinjector workshop
}




\section{Outline}

- Beam Required at the exit of PERL Injector

- Schematic Layout of PERL Injector with DC Gun

- Preliminary Simulation Results

- Summary and Outlook 


\section{Beam Requirements at the Injector Exit}

- Beam Current 200 mA,i.e, 0.15 nC/bunch for L-band linac

- Energy about $25 \mathrm{MeV}$ and RMS Energy Spread $25 \mathrm{keV}$

- RMS Bunch Length:3 ps or $0.9 \mathrm{~mm}$

- Transverse Emittance: 1 mm.mrad @ $0.15 \mathrm{nC}$ 


\section{Schematic Layout of the Injector}

DC Gun Buncher $(1.3 \mathrm{GHz}) \quad$ Solenoid
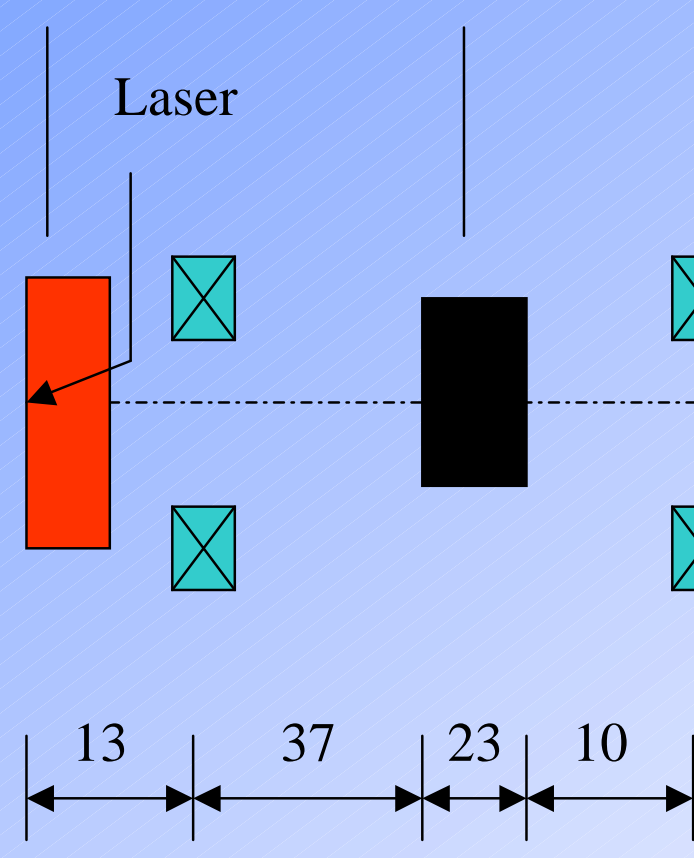

Brookhaven Science Associates U.S. Department of Energy

Q

67
Accel. Structures $(1.3 \mathrm{GHz})$
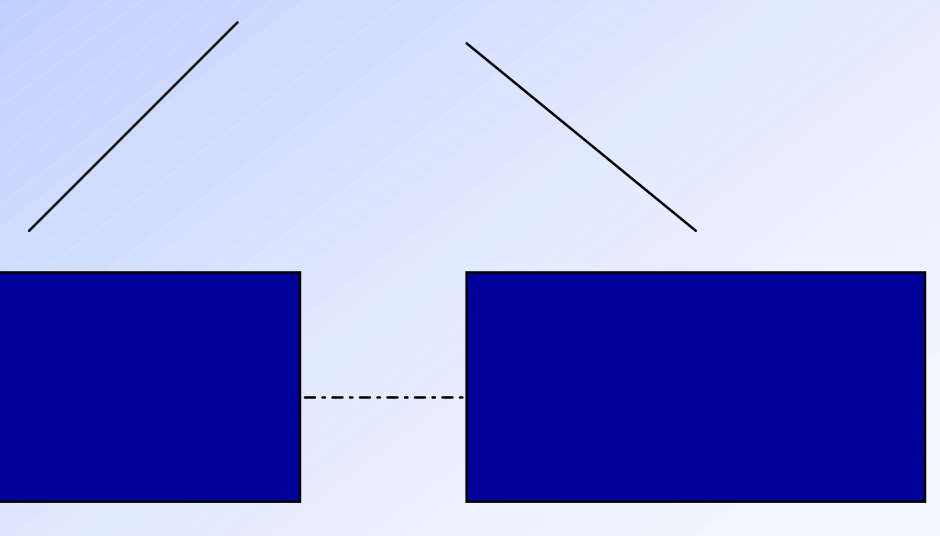

100

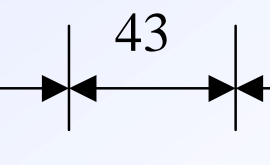

100

NATIONAL LABORATORY 


\section{Beam Simulations}

- Beam simulations starts from the cathode to the injector exit

- A package of widely used computer codes:

POISSON (gun and solenoids),

SUPERFISH (Buncher and accelerating structures)

and beam dynamics simulation code:

ASTRA (A Space Charge Tracking Algoritum ), which is newly developed by K.Floettmann from DESY.

- DC gun

Schematic geometry ( $5 \mathrm{~cm}$ from the cathode to anode) and its electric field $(500 \mathrm{kV})$ 


\section{Gun}
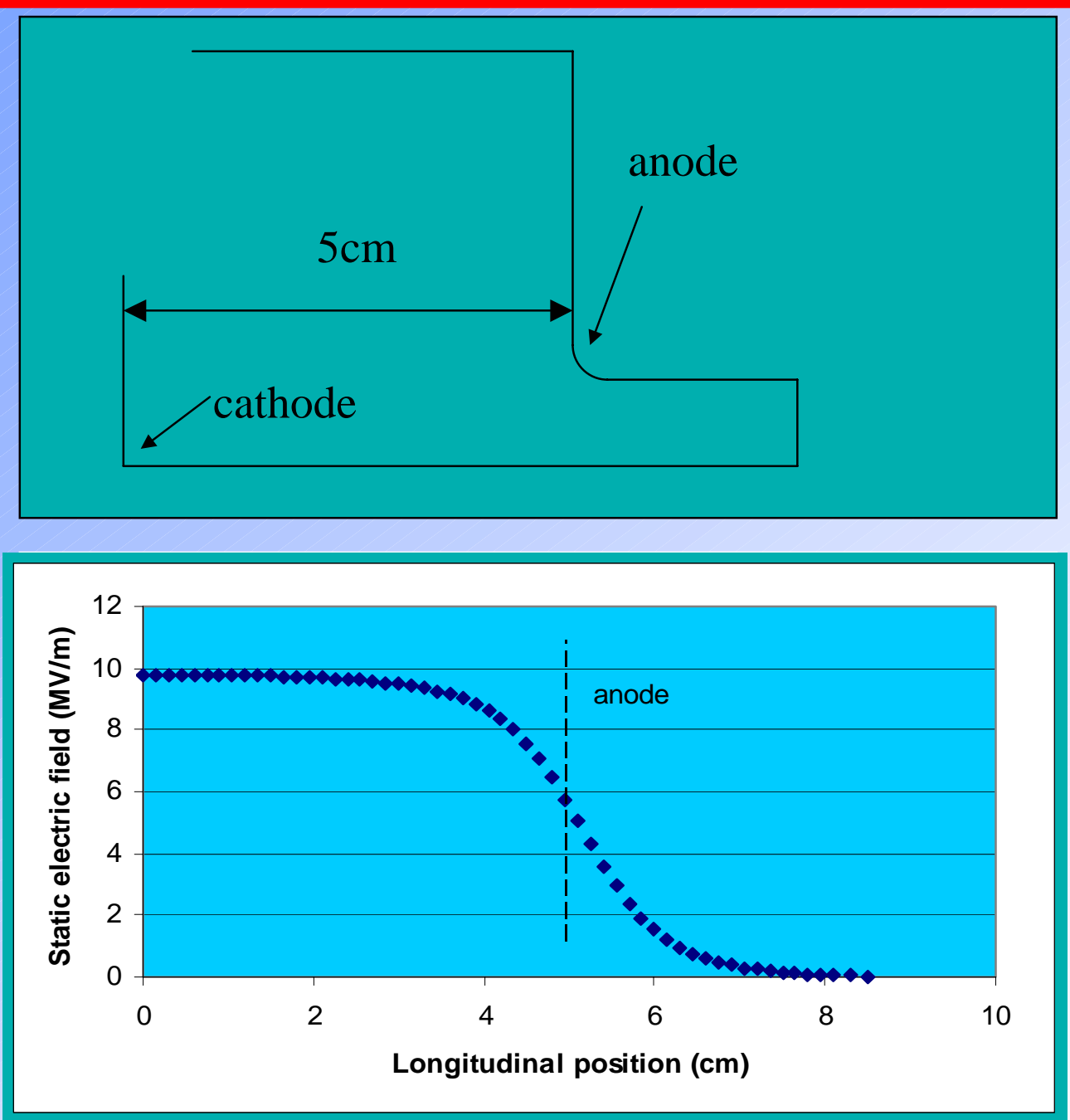

Brookhaven Science Associates

U.S. Department of Energy 


\section{Solenoid Fields}

- $1^{\text {st }}$ solenoid for the emittance compensation is located at $13 \mathrm{~cm}$ from the cathode (just after the gun). Maximum Bz is 520 Gauss.

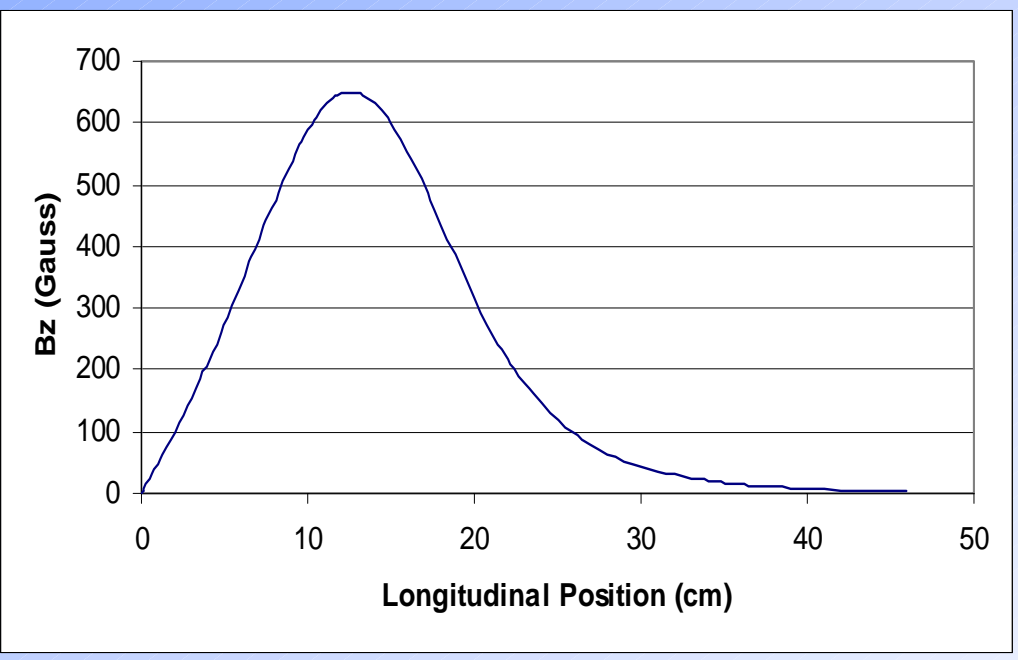

- $2^{\text {nd }}$ solenoid (maximum Bz: 550 Gauss) is located after buncher, which has two functions:

* further emittance compensation, since the energy is still lower, $2.0 \mathrm{MeV}$

${ }^{*}$ optics matching to the accelerating structures 


\section{Buncher}

- Buncher is two-cell standing wave structures $(1.3 \mathrm{GHz}, 7.5 \mathrm{MV} / \mathrm{m})$, which has two functions:

* bunching the bunch from RMS length $10 \mathrm{ps}$ to $3.0 \mathrm{ps}$ ( $3 \mathrm{~mm}$ to $0.9 \mathrm{~mm}$ )

* accelerating the bunch in order to reduce the space charge effect. The RF phase is 30 degrees off-crest and its net energy gain from the buncher is about $1.4 \mathrm{MeV}$.

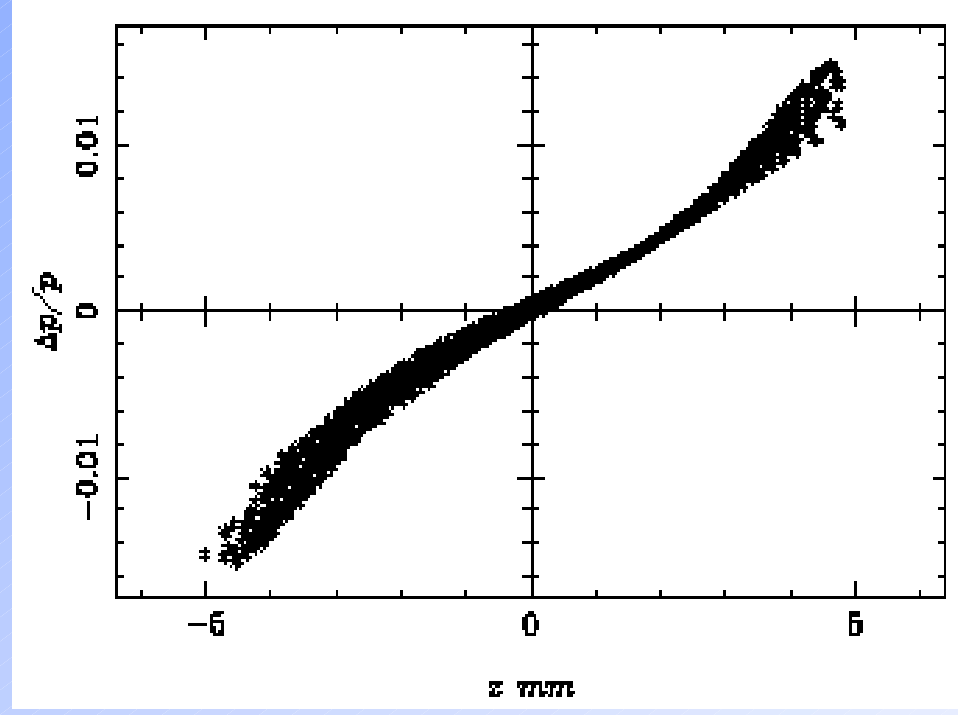

before the buncher

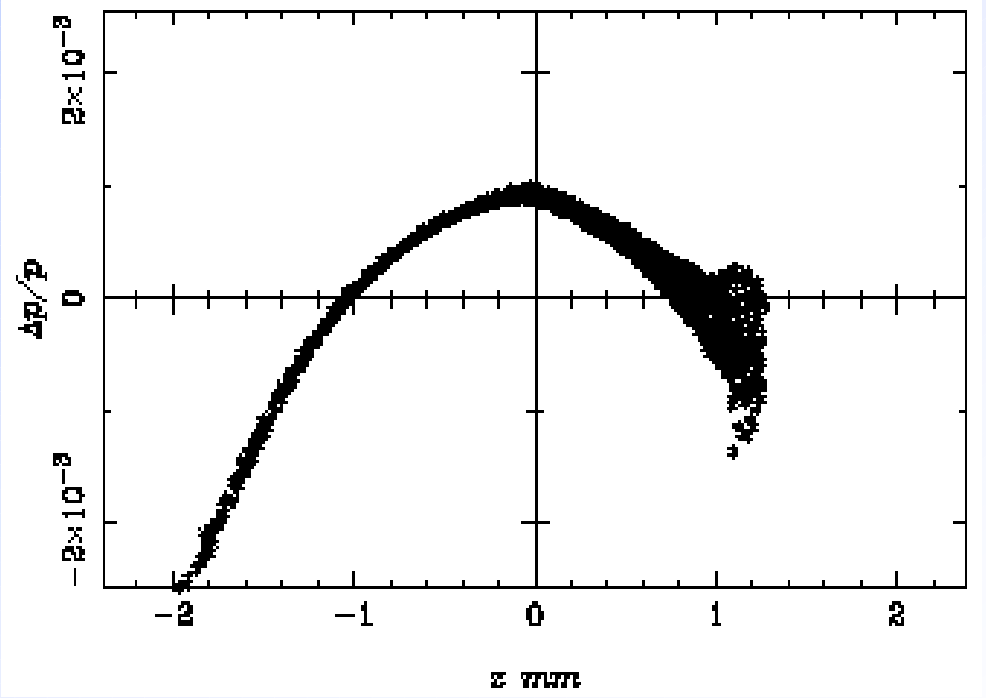

after the buncher 


\section{Buncher}

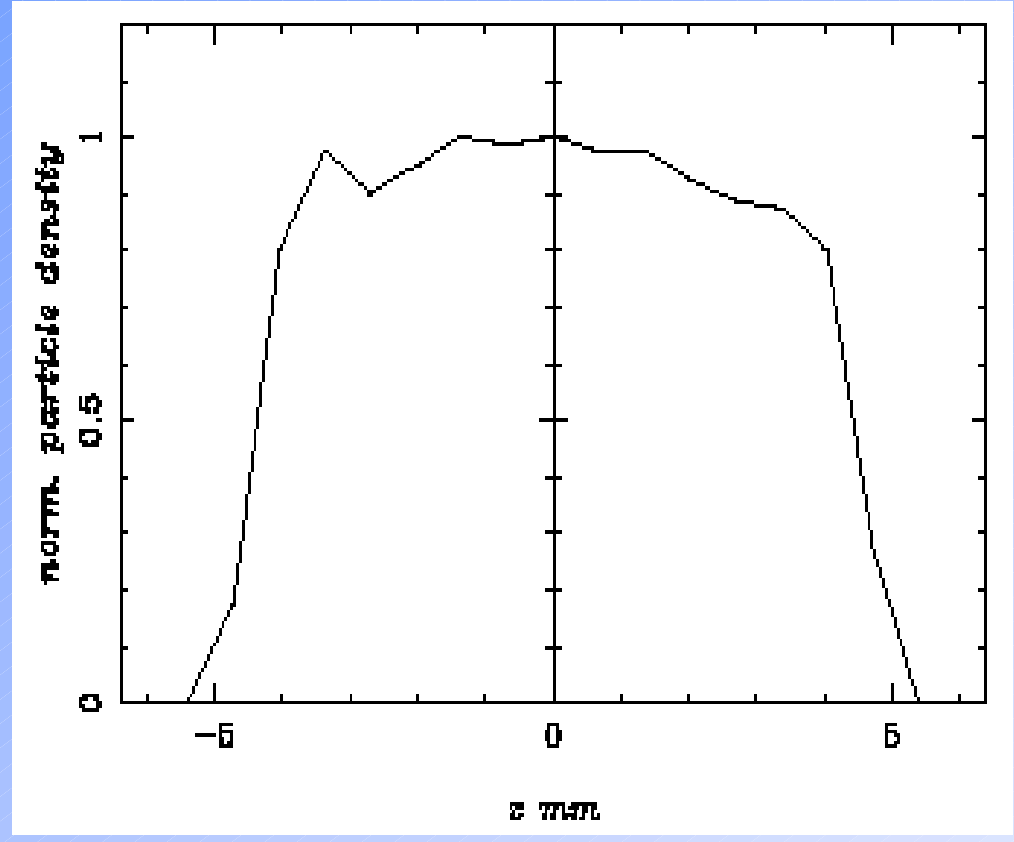

before the buncher

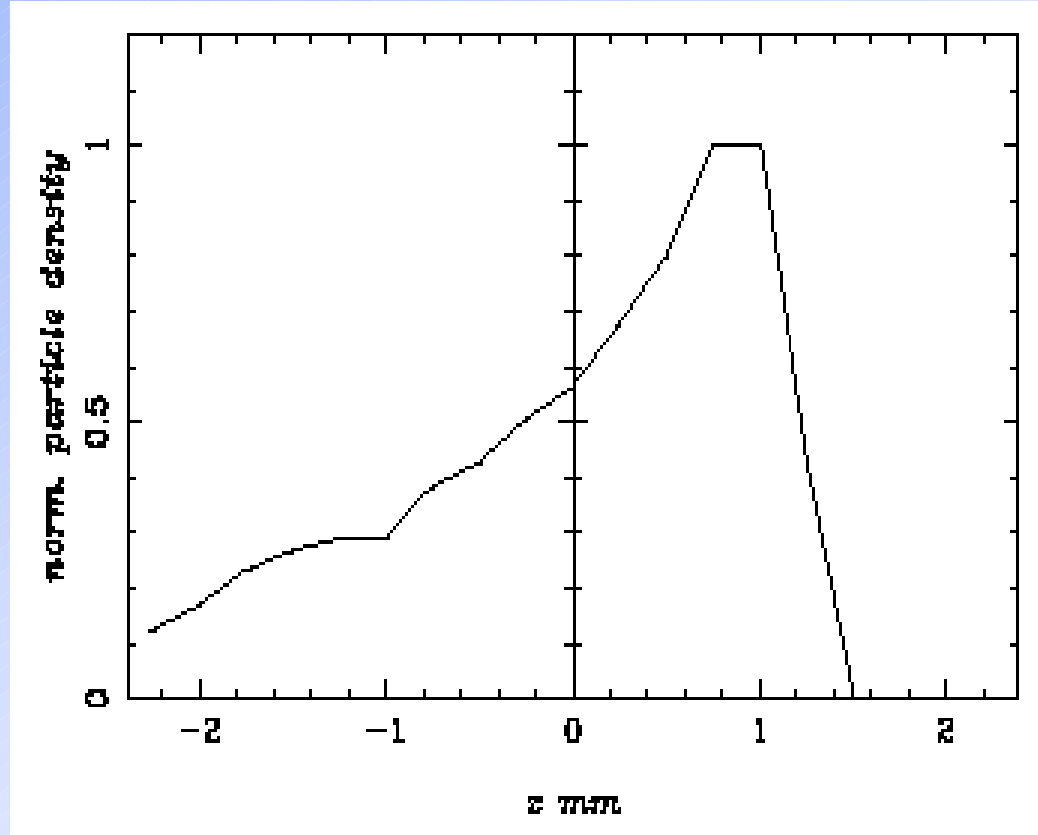

after the buncher 


\section{Accelerating Structures}

- Using the $2^{\text {nd }}$ solenoid to match the beam optics to two accelerating structures. One structure $(1.3 \mathrm{GHz})$ is 9 cell with $1 \mathrm{~m}$, gradient is $10 \mathrm{MV} / \mathrm{m}$. After the acceleration, the beam divergence is reduced greatly and the emittance is slightly decreased and then keeps to be constant.

- The RMS bunch length is slightly modified through two structures and then kept to $3.0 \mathrm{ps}(0.9 \mathrm{~mm})$.

- The energy spread after two structures are $25 \mathrm{keV}$ at the energy of $25 \mathrm{MeV}$. 


\section{Accelerating Structures-Trans. Space}
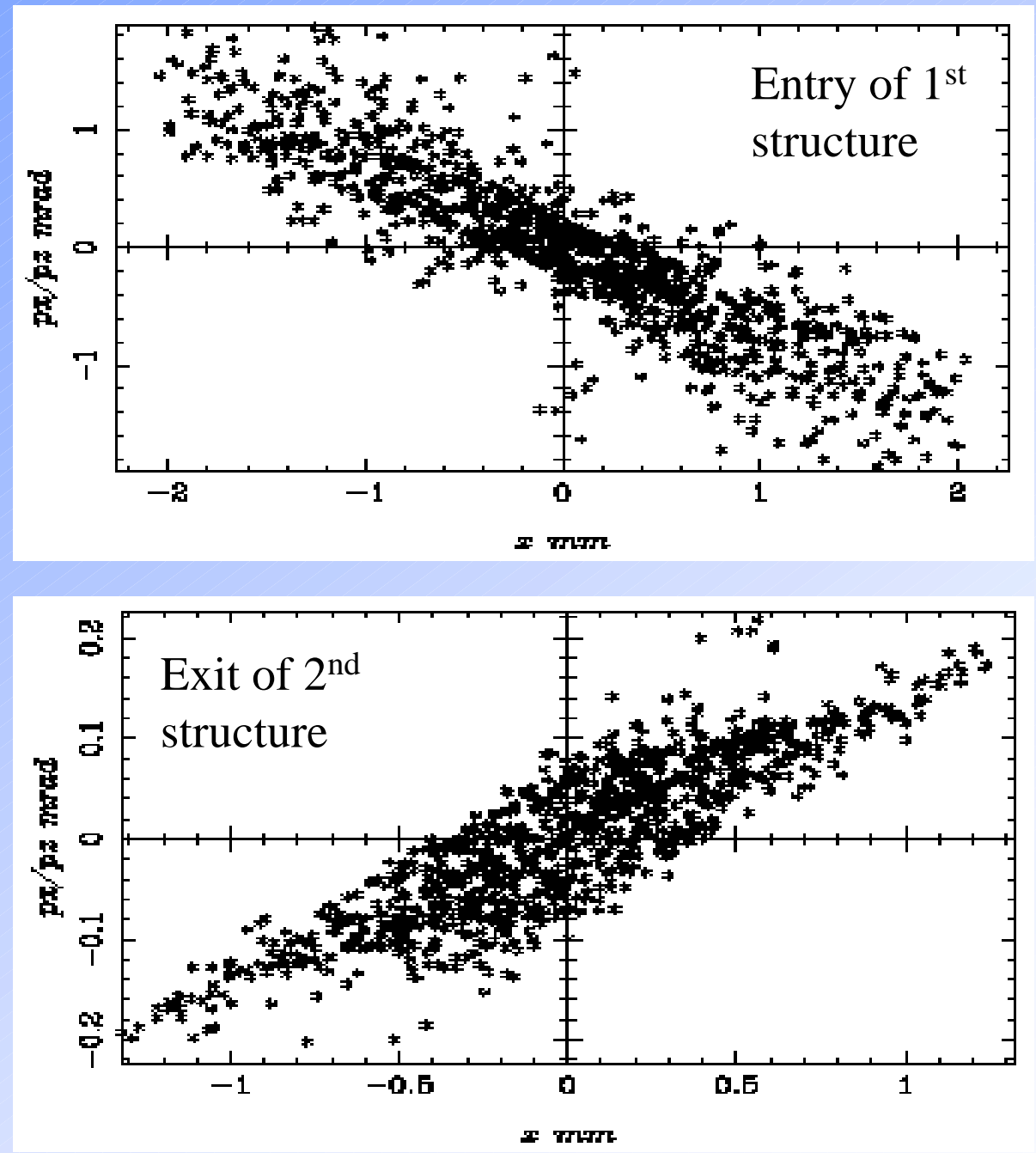

Brookhaven Science Associates U.S. Department of Energy 


\section{Accelerating structures-bunch length}
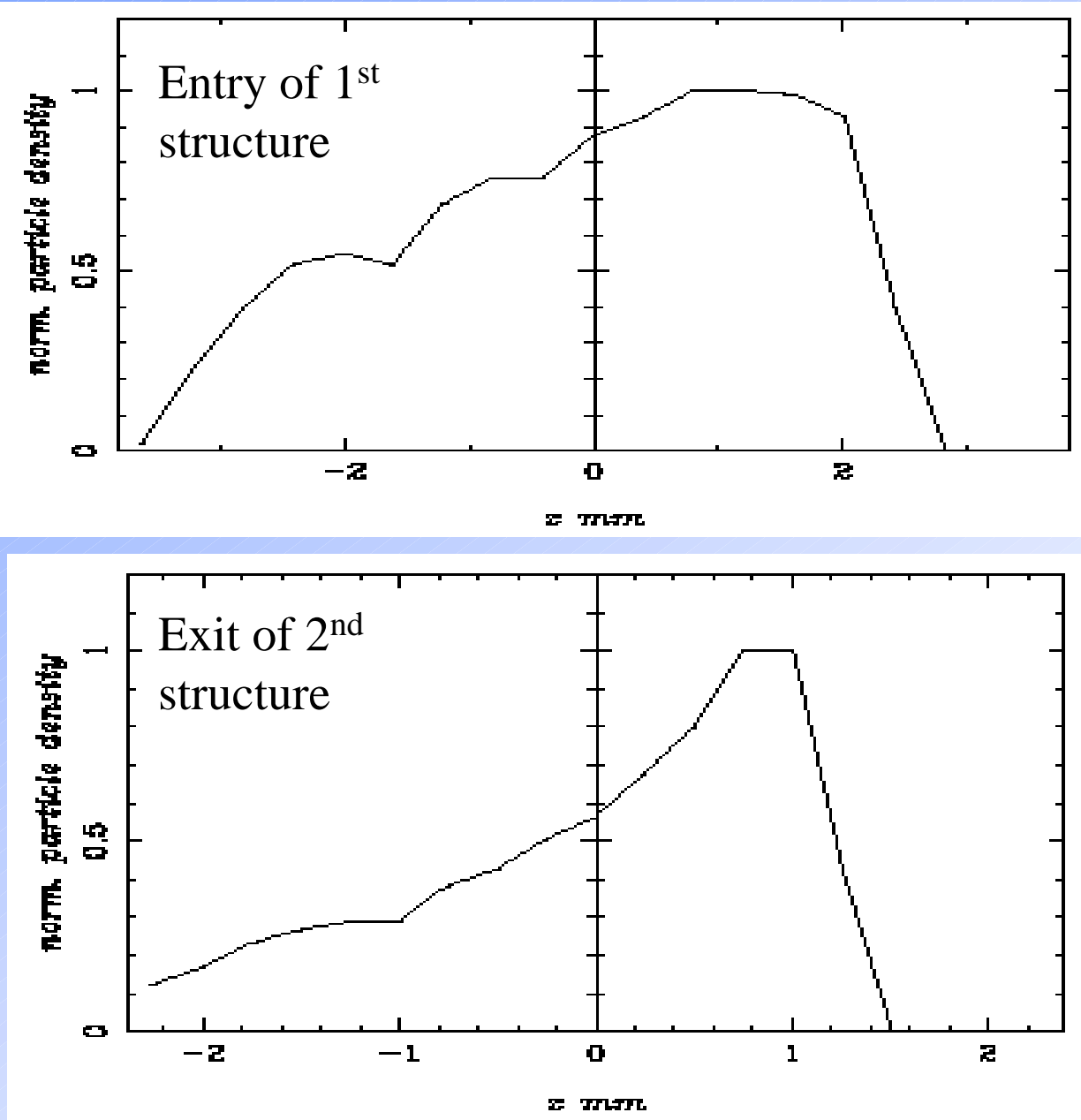

Brookhaven Science Associates U.S. Department of Energy 


\section{Accelerating structures-Energy spread}
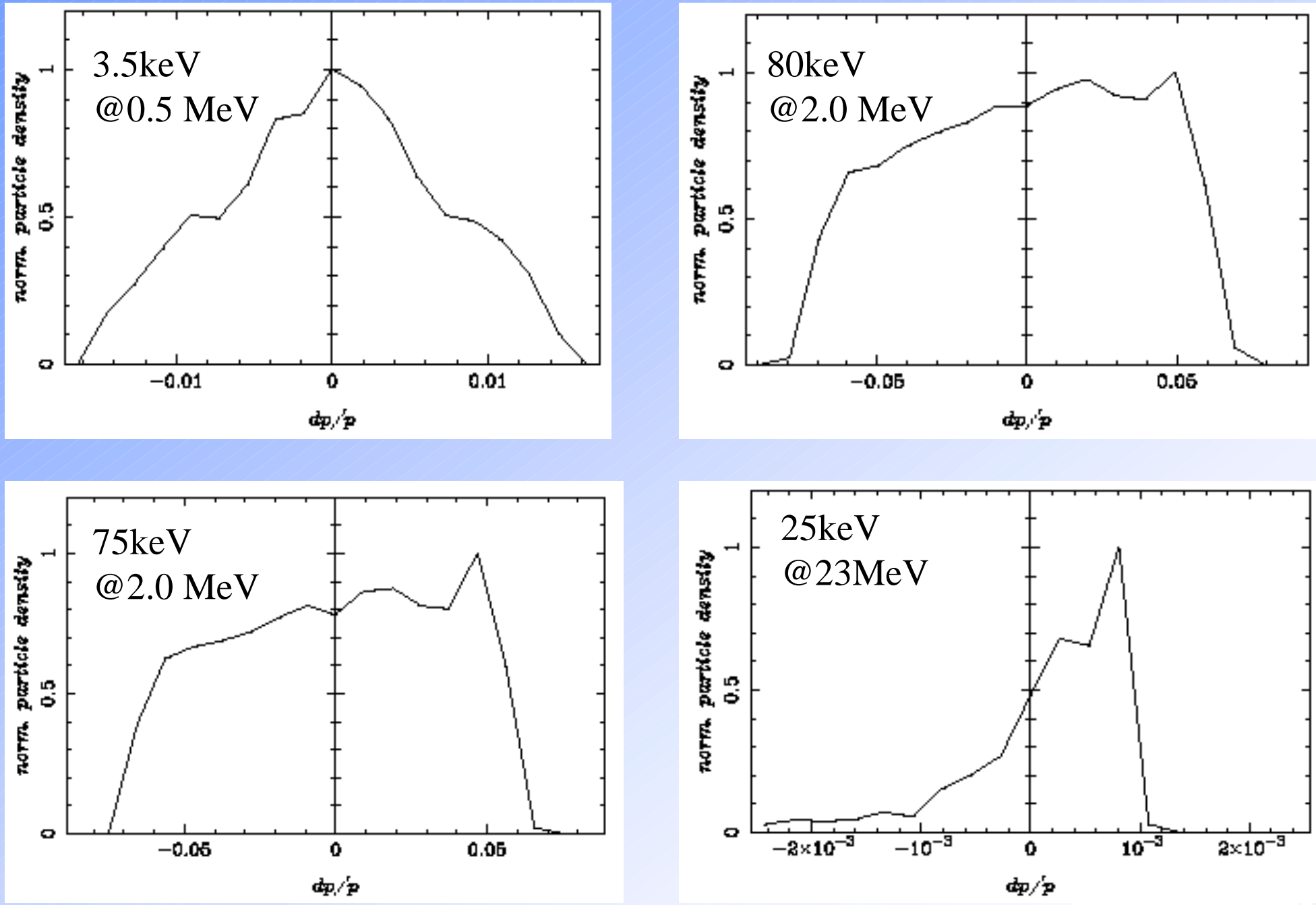

Brookhaven Science Associates U.S. Department of Energy 


\section{Simulation results}

- Simulation starts from cathode. The initial distribution at the cathode :

Longitudinal: Plateau distribution, 25 ps top, 2 ps for rise and fall time, respectively.

Transverse: Uniform, RMS 1mm

Particles: 1000

bunch charge: $0.15 \mathrm{nC}$

- Both longitudinal and transverse emittance,beam size, bunch length, energy spread vs longitudinal position 


\section{Simulation Results: Laser Longi. Distributions on the cathode}
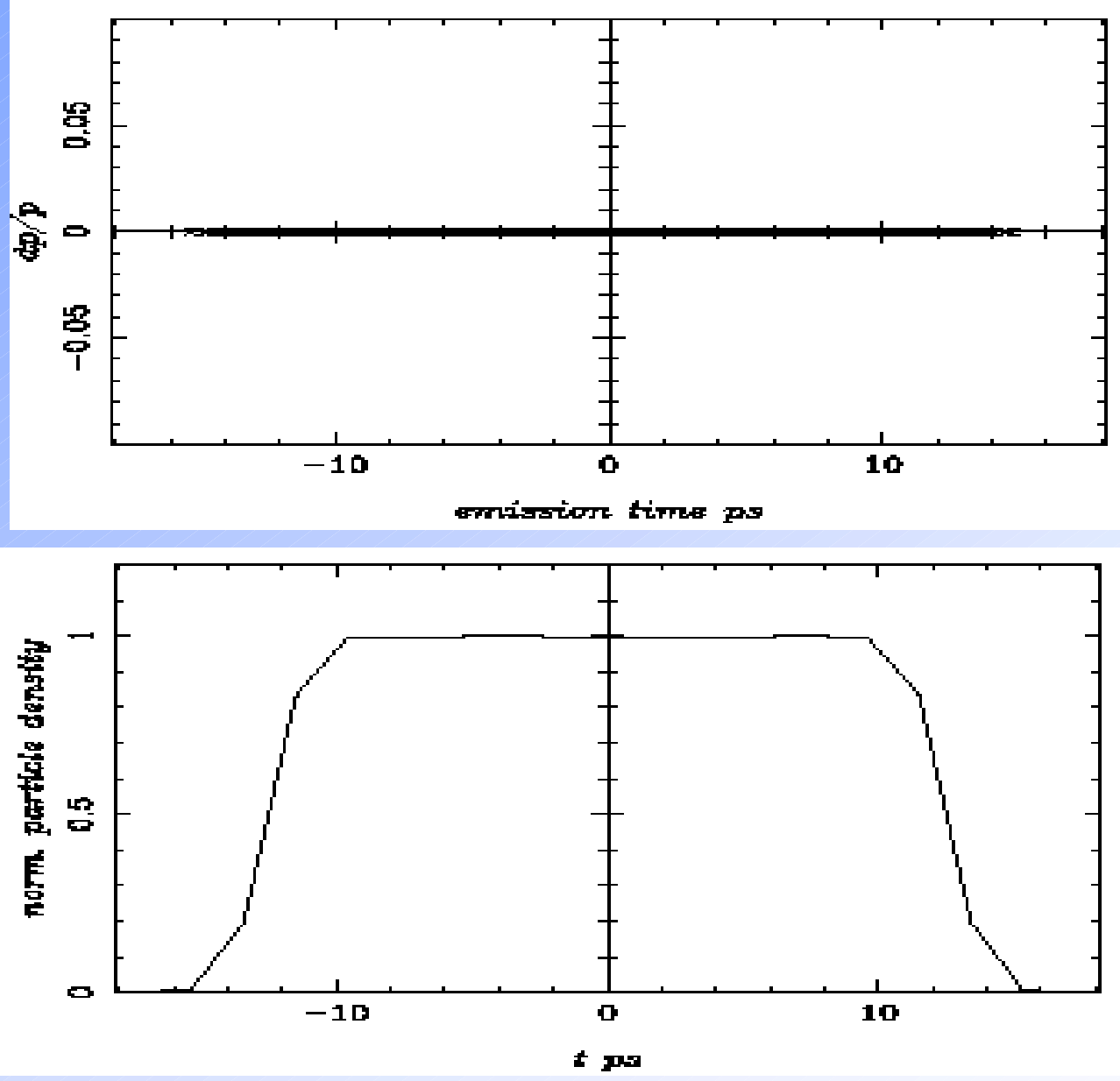

Brookhaven Science Associates U.S. Department of Energy 


\section{Simulation Results: Laser Trans. Distributions on the cathode}
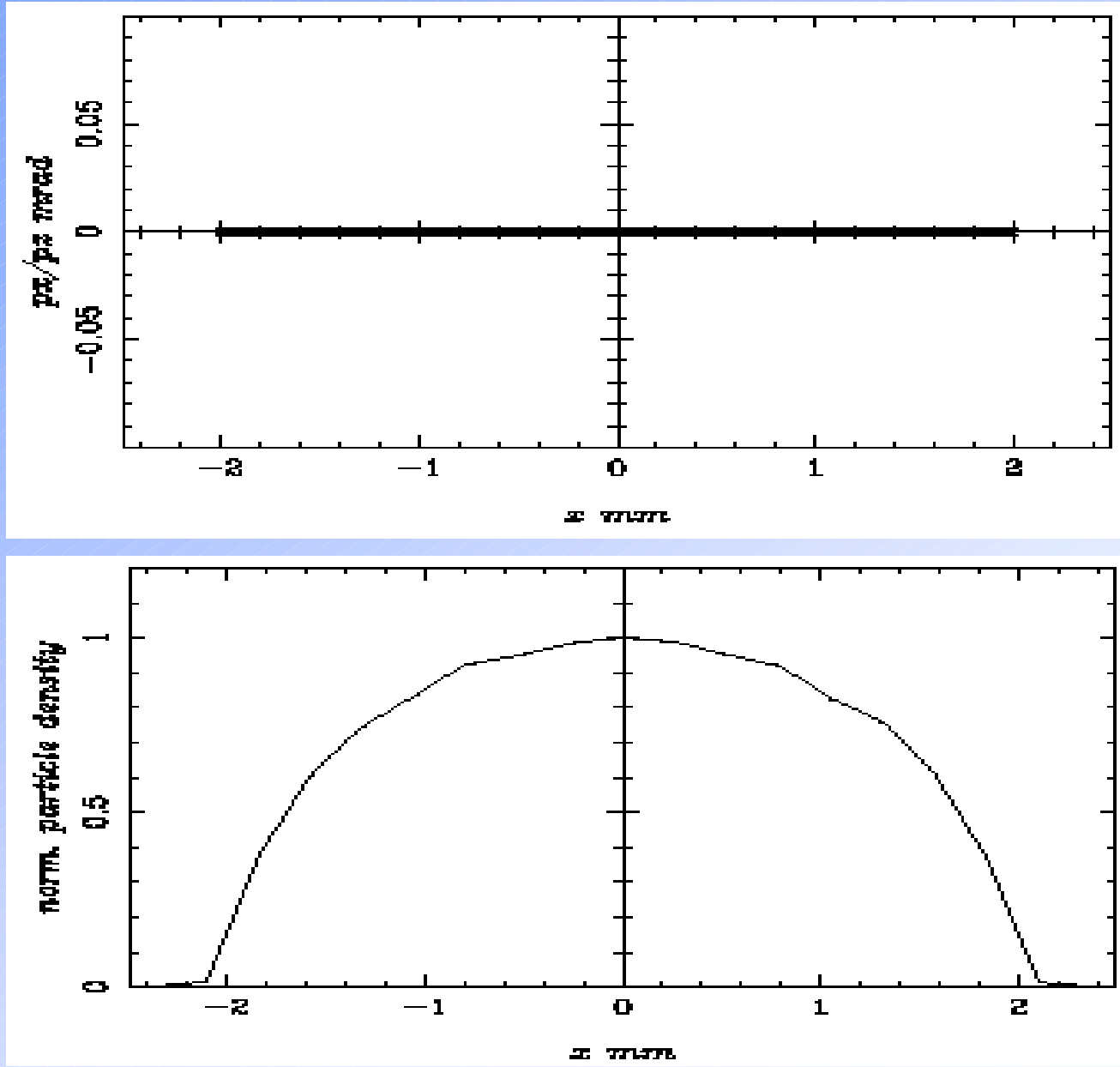

Brookhaven Science Associates U.S. Department of Energy 


\section{Simulation Results: Transverse Emittance and Beam size}
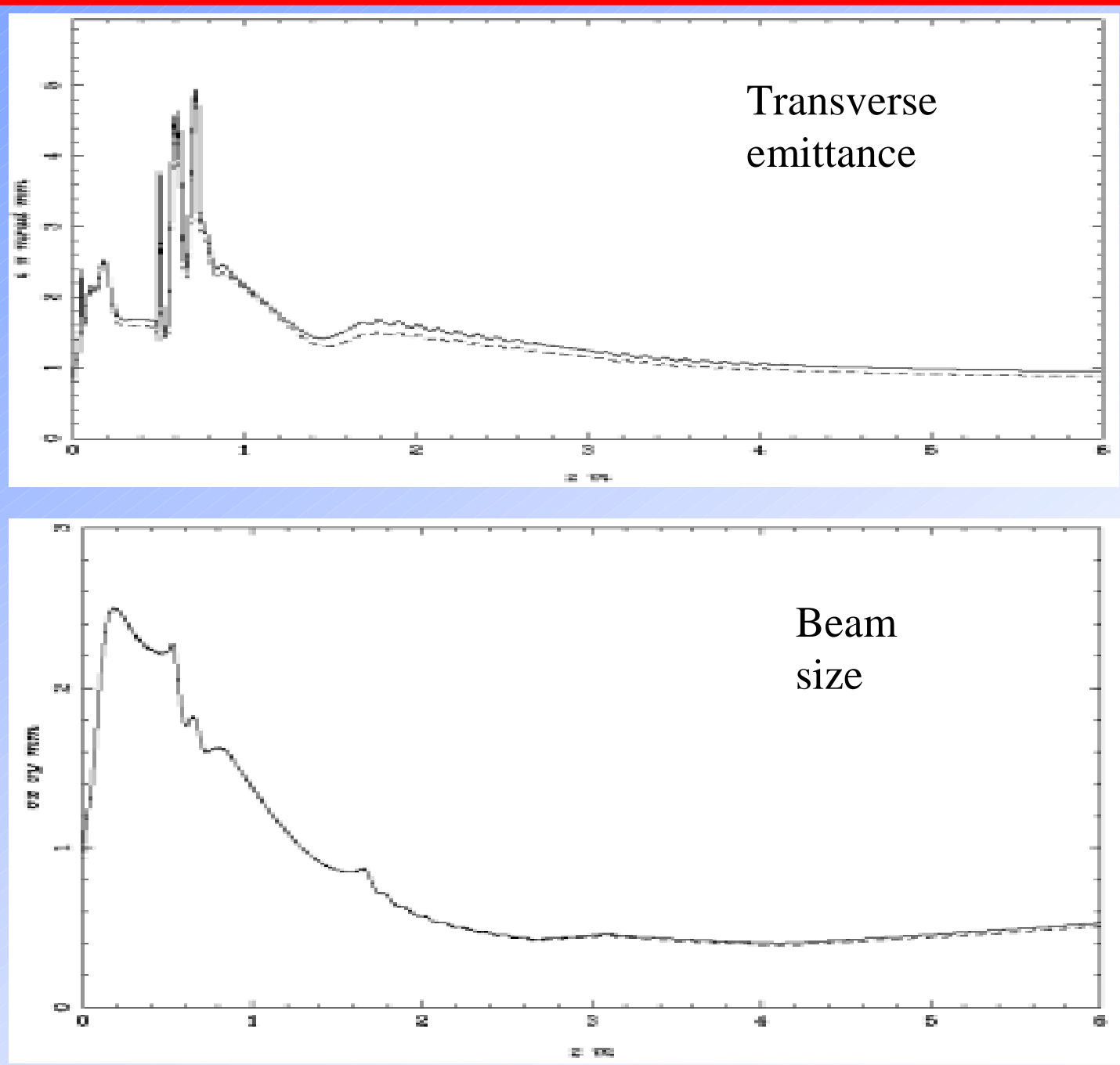

Brookhaven Science Associates U.S. Department of Energy 


\section{Simulation Results:Beam Divergence}

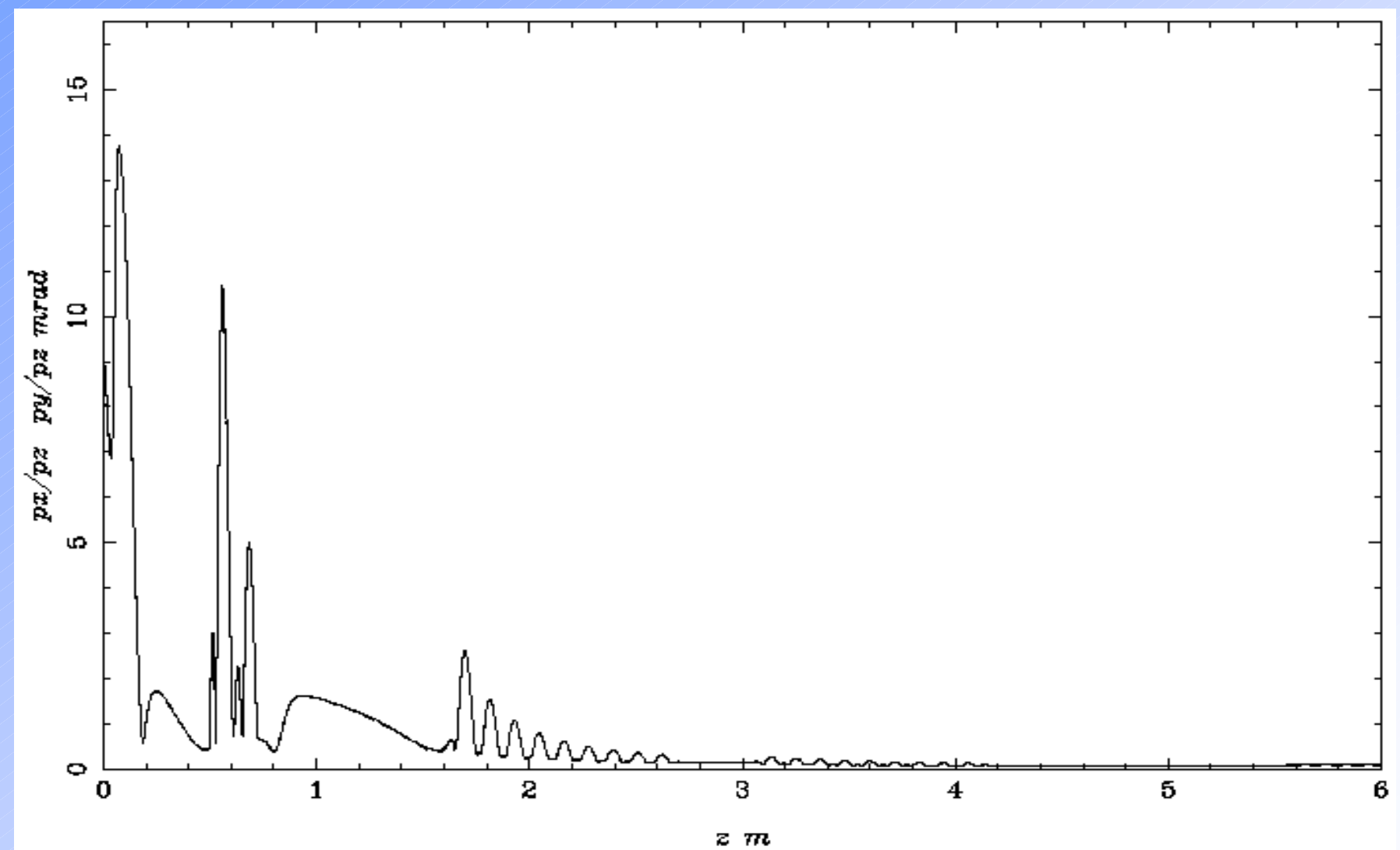

Brookhaven Science Associates U.S. Department of Energy 


\section{Simuation Results:Longitudinal Emittance and Bunch length}
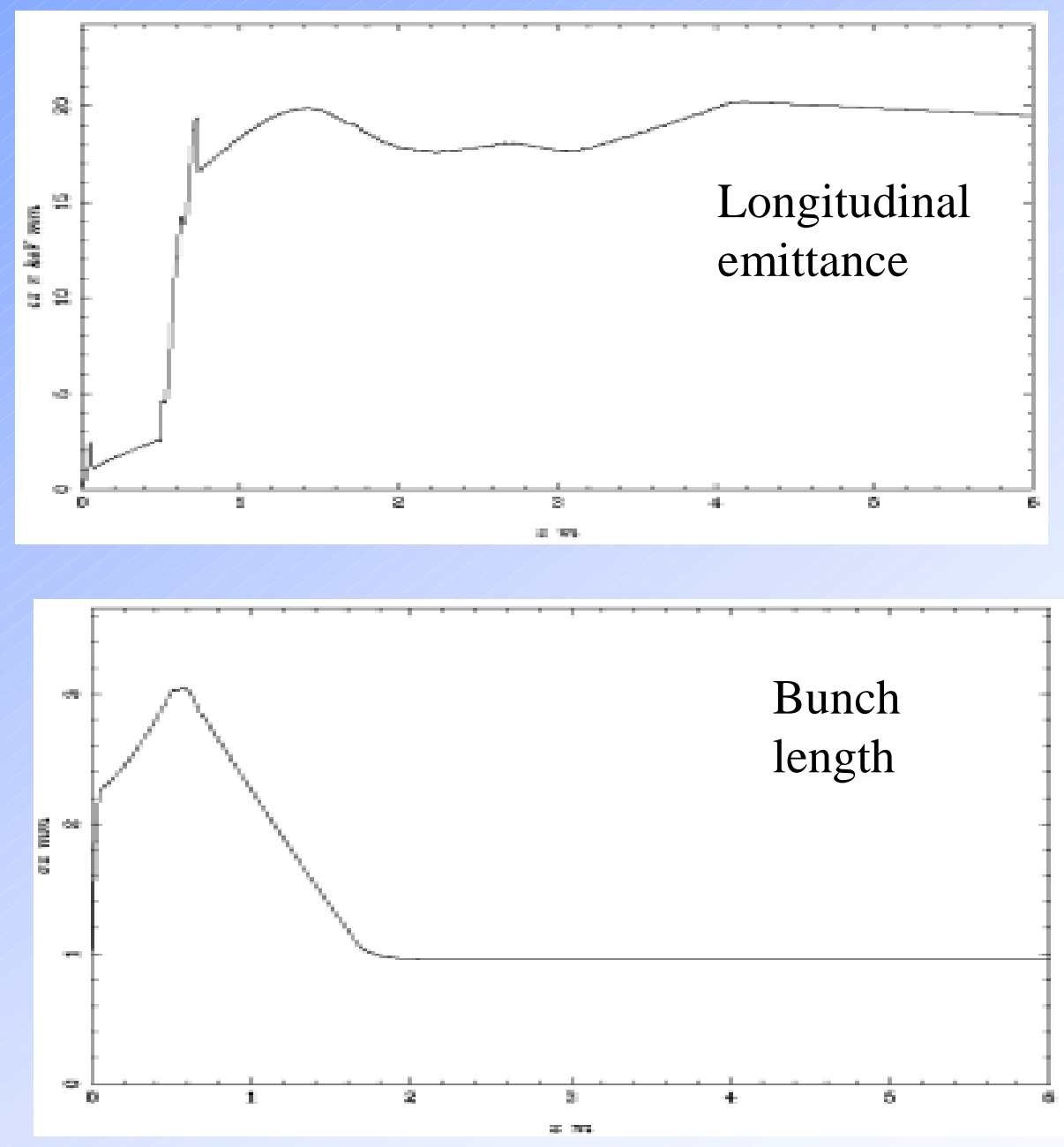

Brookhaven Science Associates U.S. Department of Energy 


\section{Simulation Results:Energy and Energy Spread}
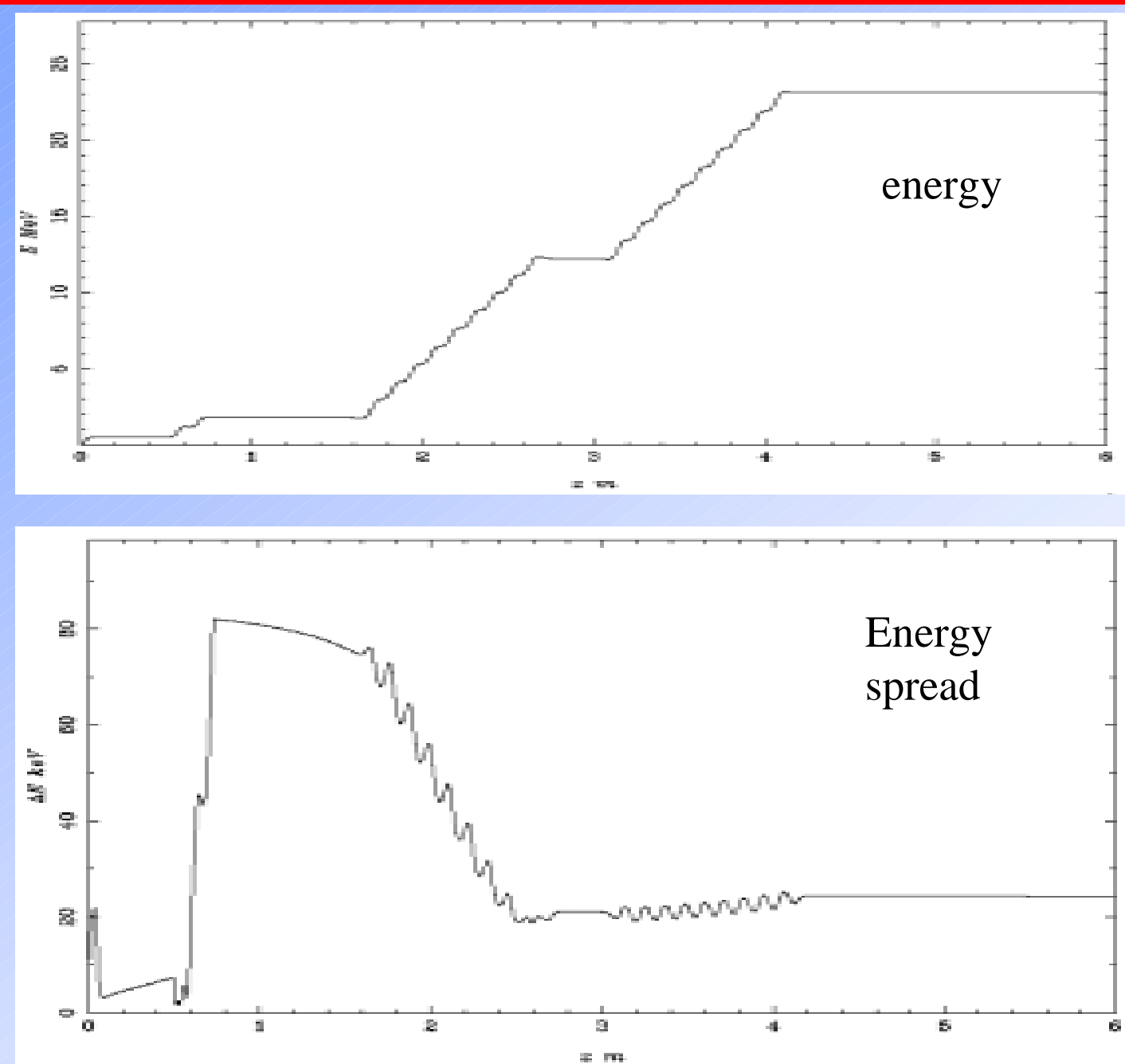

Brookhaven Science Associates U.S. Department of Energy 


\section{Summary and Outlook}

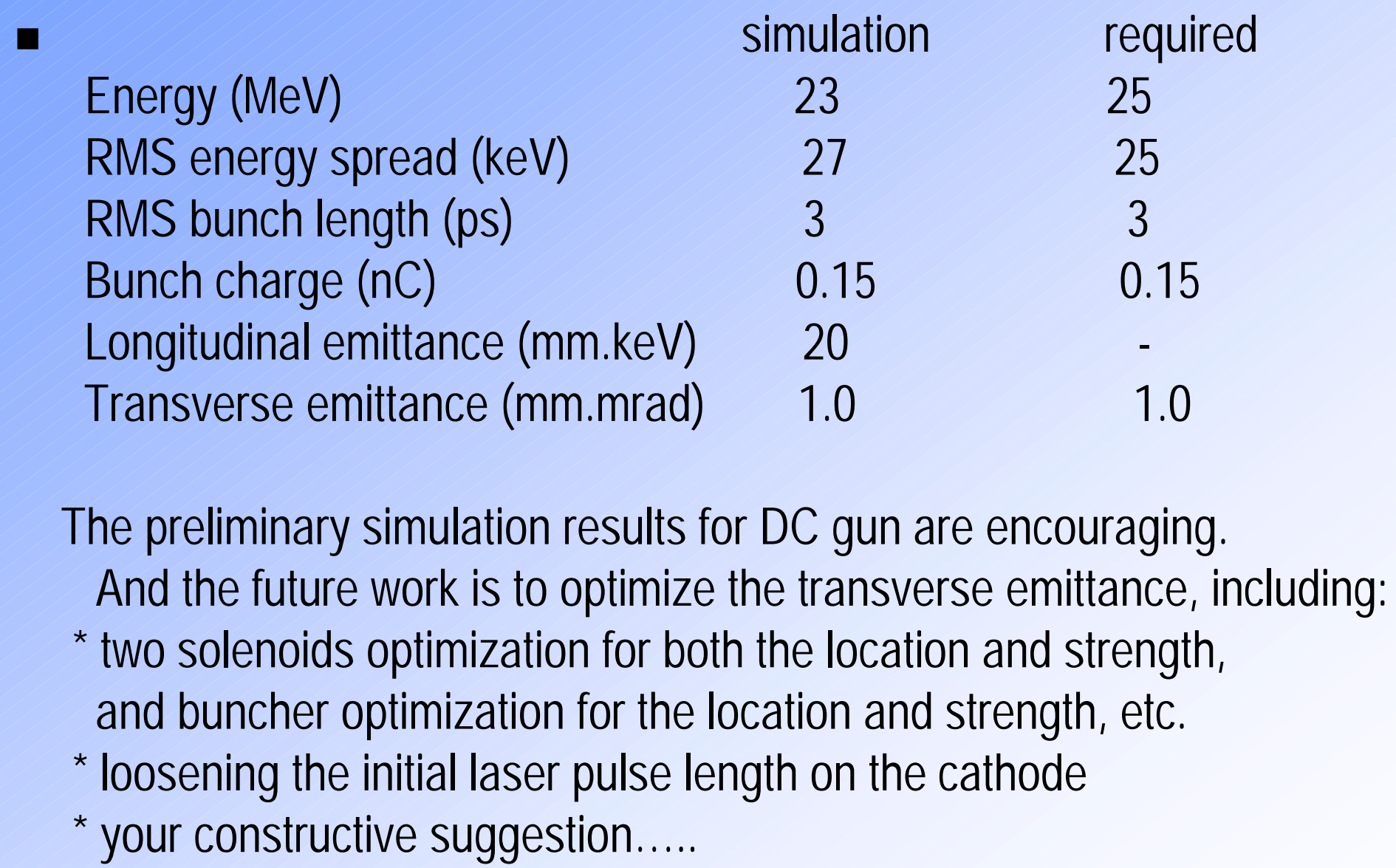


Possible use of PEP-II cavity technology for a photocathode RF gun

\author{
R. Rimmer
}

LBNL 
Intro. to PEP-II cavity

design goals

specifications

fabrication

A photocathode injector study

IFRA study

RF gun cavity

injector cost estimates

frequency scaling

A $714 \mathrm{MHz}$ cavity for NLC damping rings simplified design improved fabrication methods costs

A $1.5 \mathrm{GHz}$ cavity for ALS

simple third harmonic cavity 


\section{Intro. to PEP-II cavity}

$476 \mathrm{MHz}$, single cell with HOM damping High gap voltage and heavy beam loading High HOM load power

High reliability

Thermal stresses high around ports

design: $1 \mathrm{MV}, 150 \mathrm{~kW}, 3 \mathrm{~A}$ beam, 3.5 $\mathrm{M} \Omega$ operation: $850 \mathrm{kV}, 95 \mathrm{~kW}, 2.2 \mathrm{~A}, 3.8 \mathrm{M} \Omega$ highest HOMs $\sim 1.8 \mathrm{k} \Omega, 144 \mathrm{k} \Omega / \mathrm{m}$

Fabrication in-house + industry

Cost $<\$ 150 k$ in quantity of 26 


\section{PEP-II B-Factory}
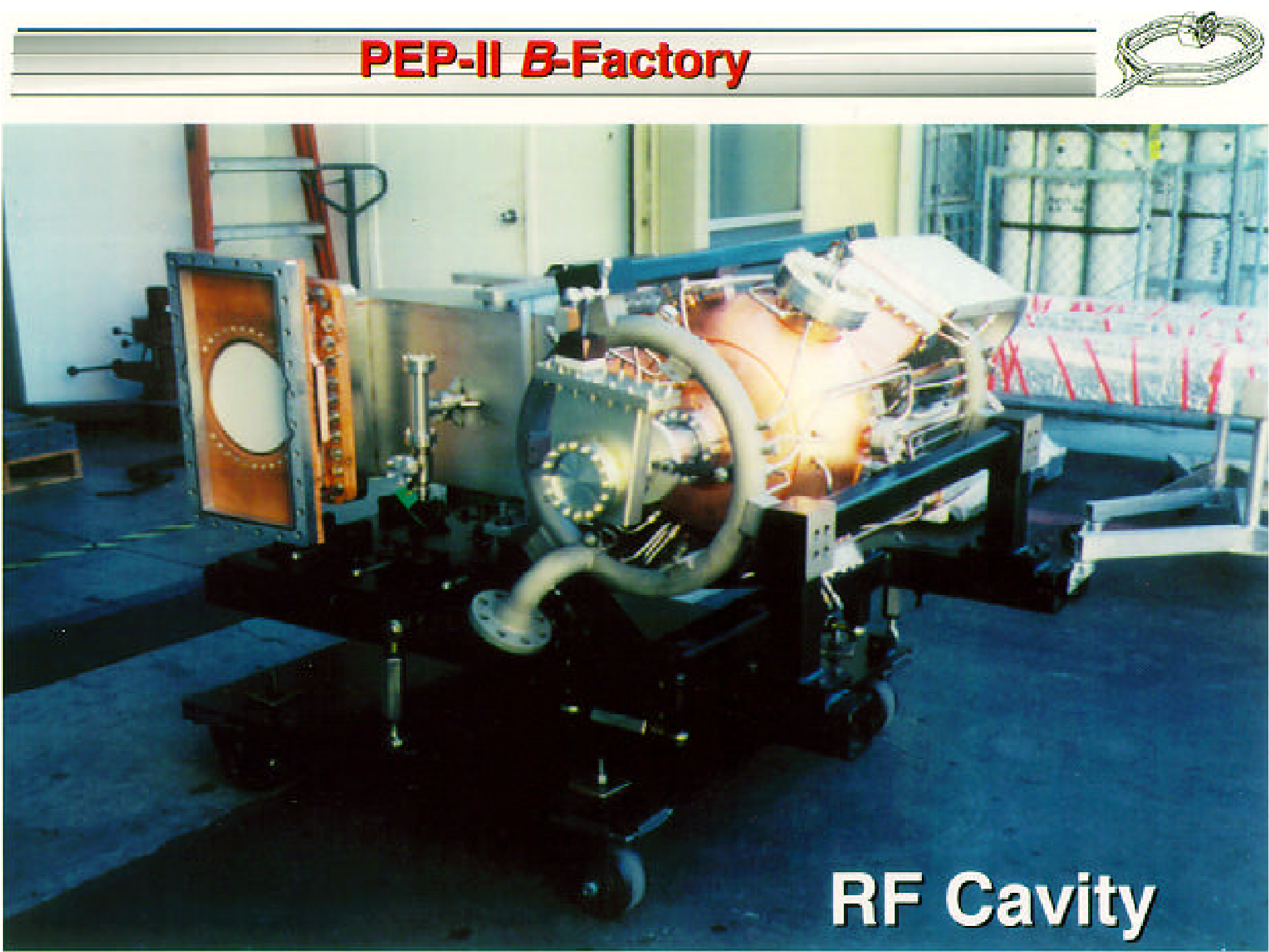


\section{PEP-II B-Factory}

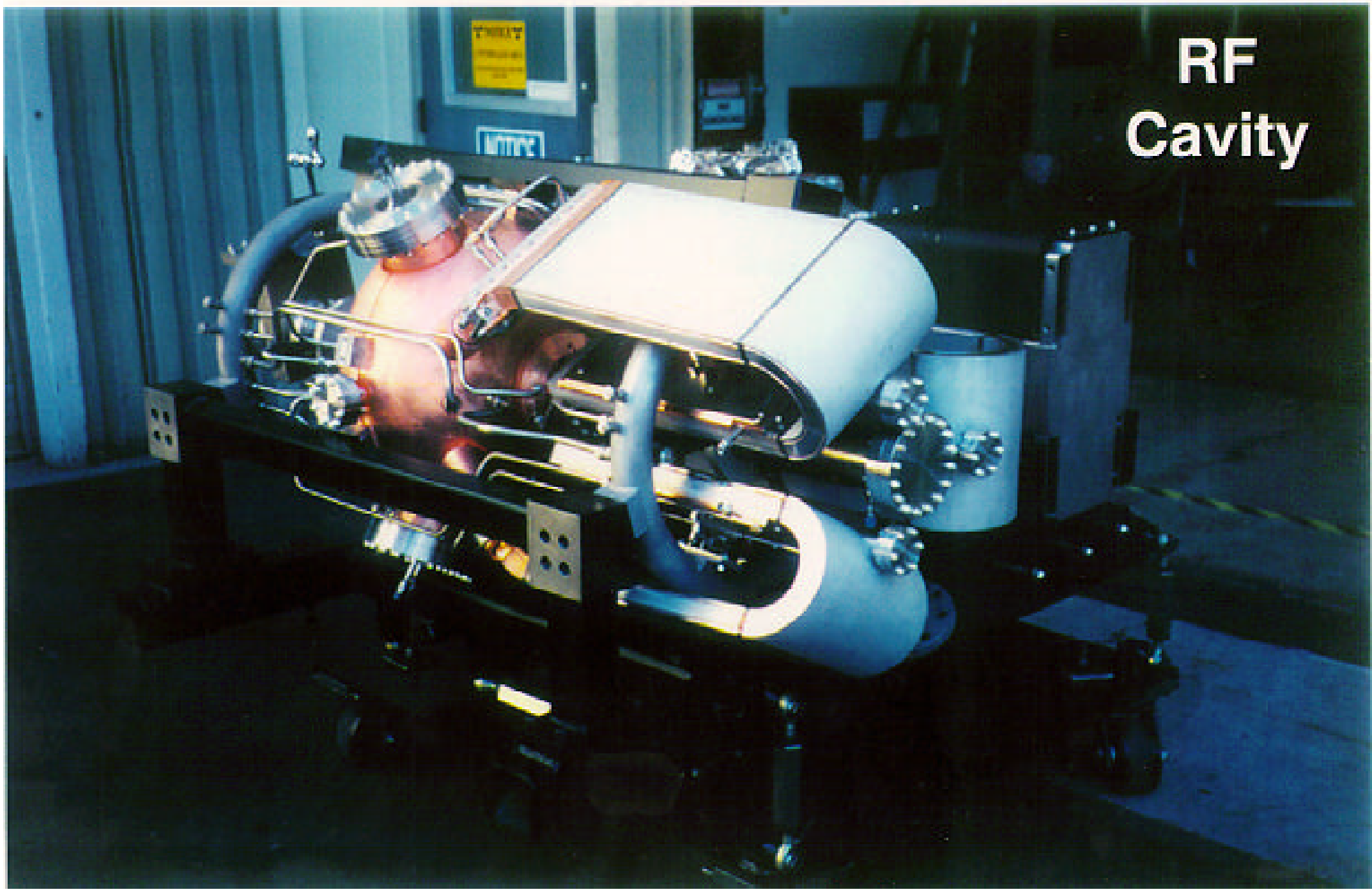




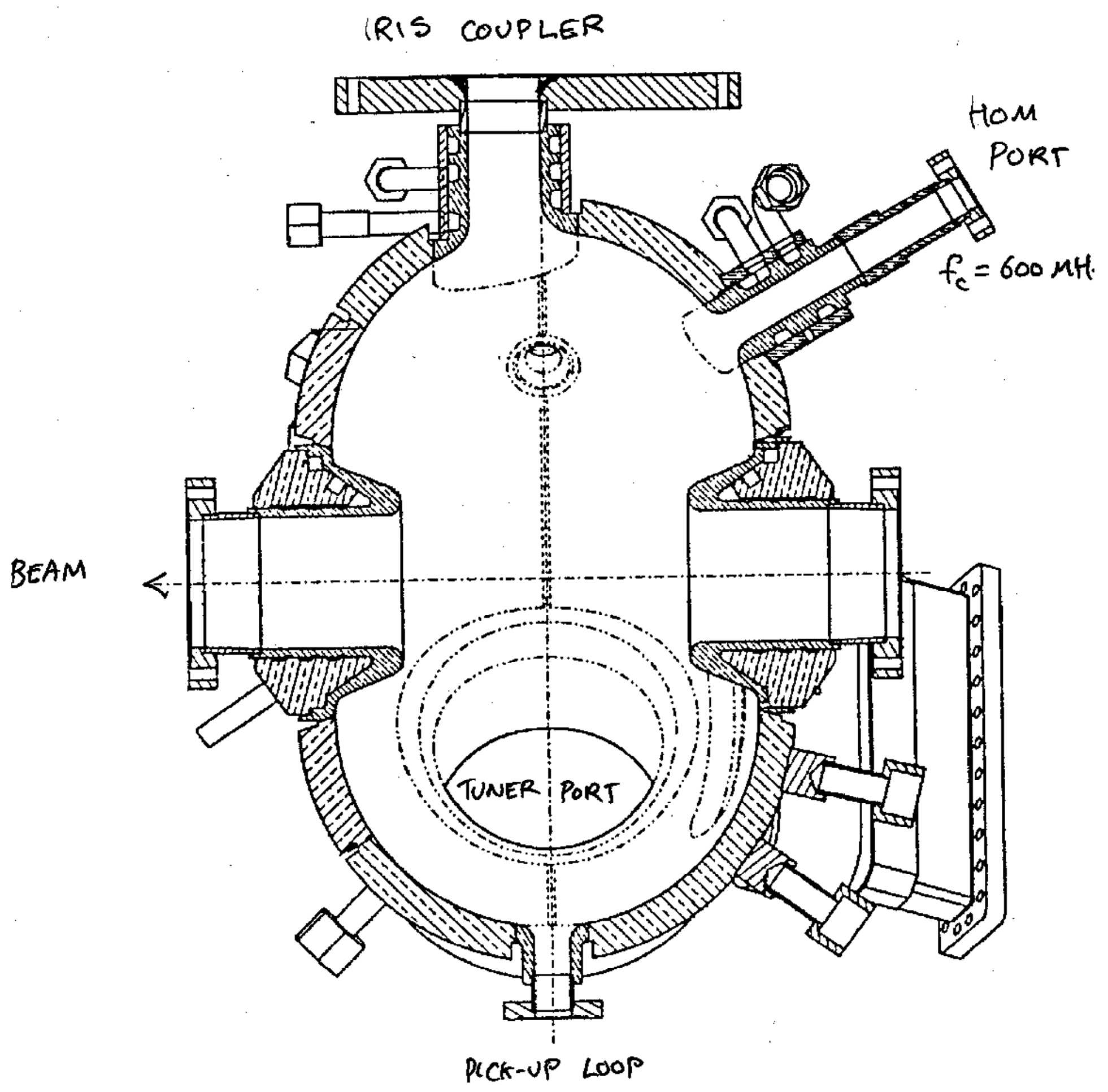

$$
\text { PEP-T RF CAVITY } f_{0}=476 \mathrm{MHz}
$$




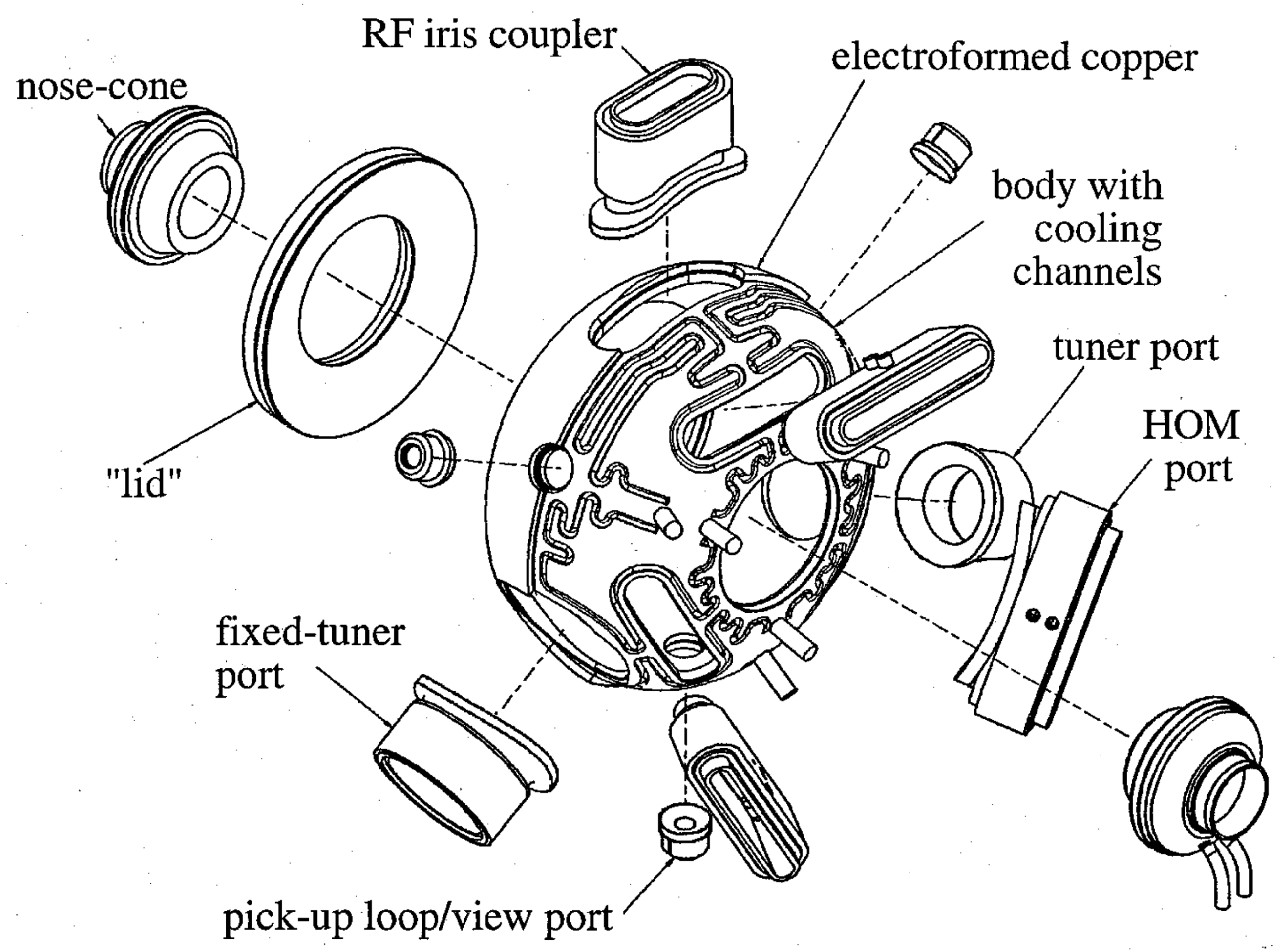




\section{PEP-II RF Cavity produced by \\ Lawrence Livermore National Laboratory}

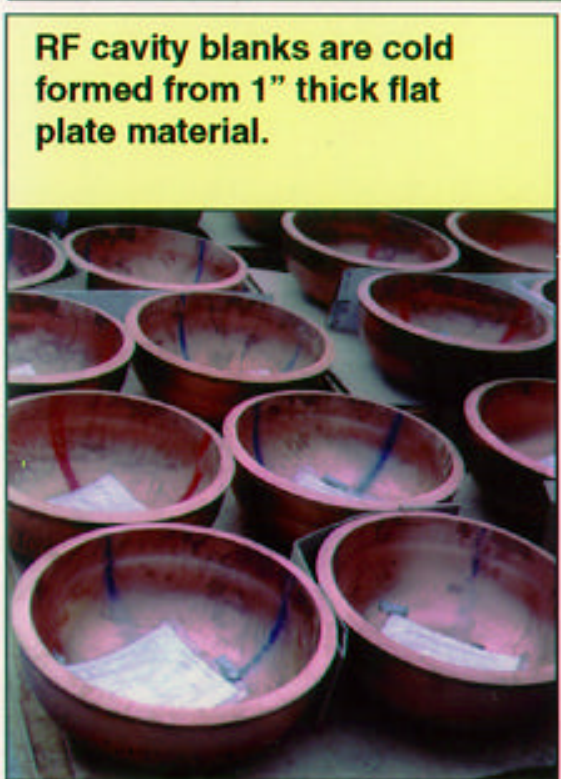

Full penetration EB vacuum welds attach tuner, Iris and HOM ports.

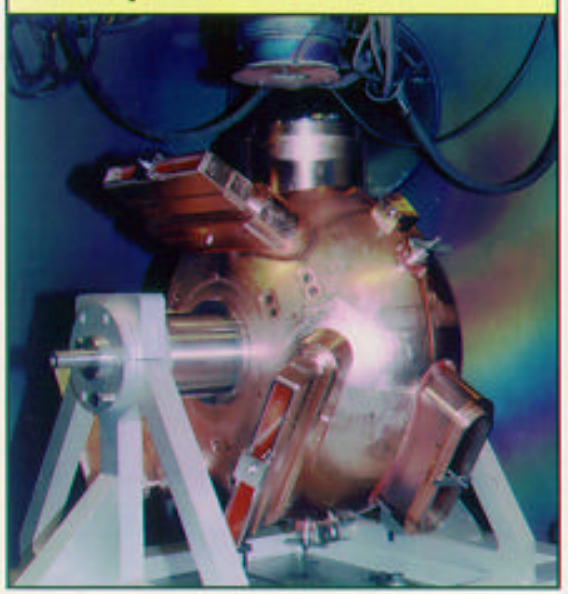

37937-mf-u-001

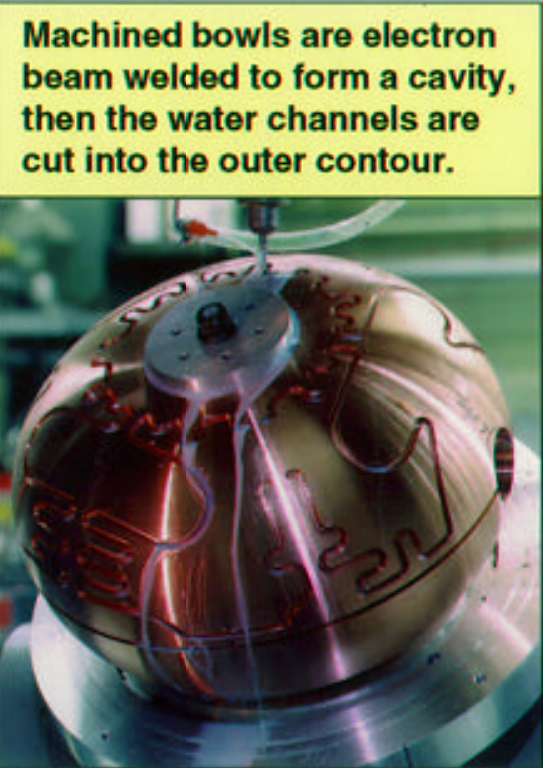

The RF surface is finish turned to a 16 Ra finish using LLNL's Diamond Turning Machine \#3.

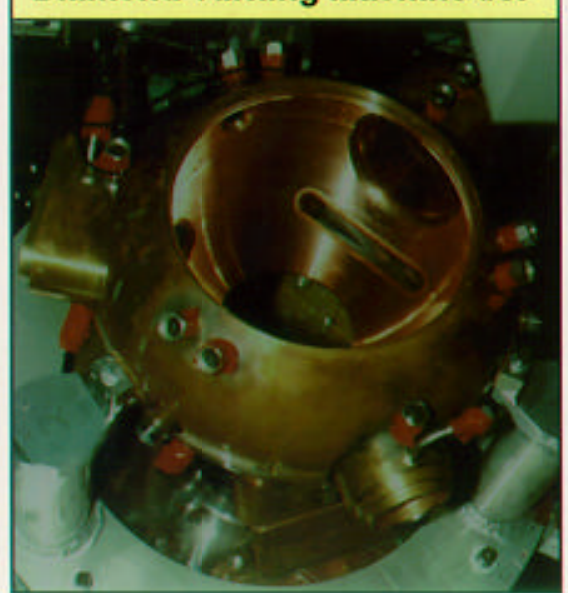

Wax is placed into the water channels prior to plating $3 / 8$ " thickness of OFE copper. Note future port location.

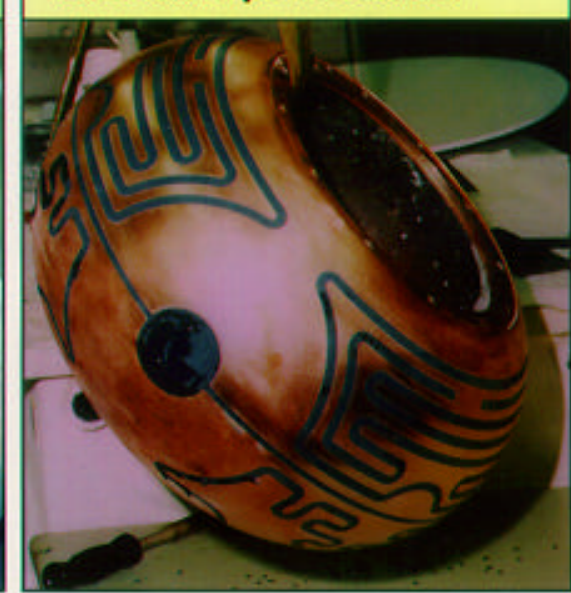

The beams noses are EB welded into the cavity following a precise tuning process.

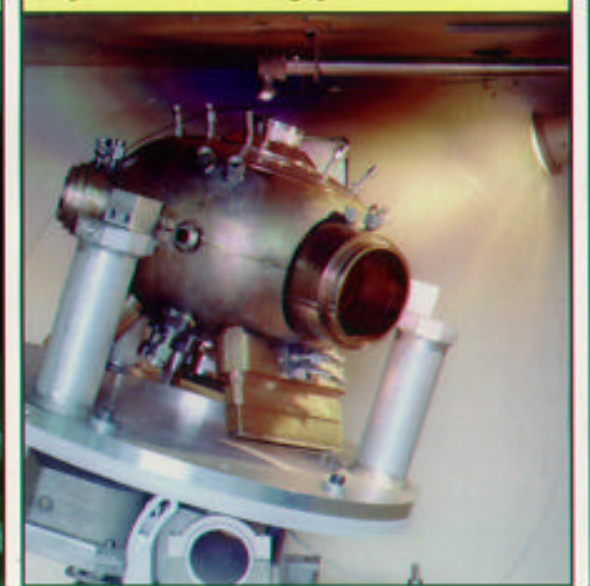

Penetrations for various ports are cut through the electroplated cavity body. Note the blue wax in $\mathbf{4}$ holes.

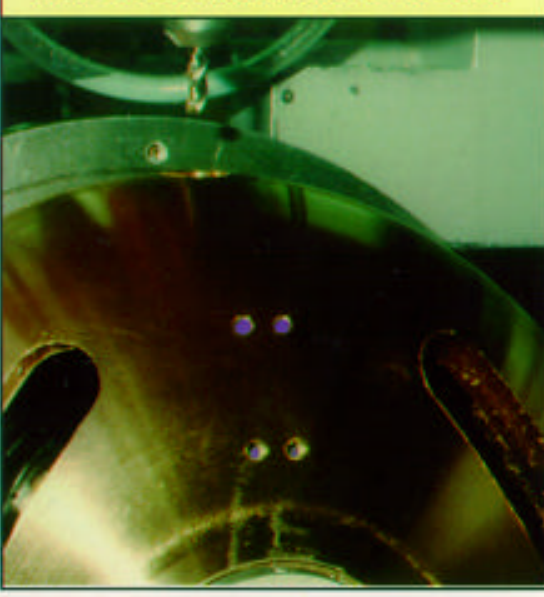

The final product is cleaned for UHV and ready for shipment.

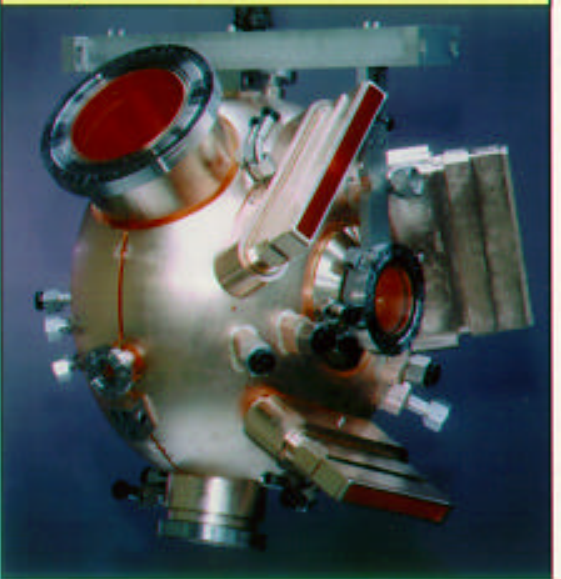




\section{Lessons learned from PEP-II cavity fabrication}

End product works very well

Fabrication processes worked well

- cold-forming of bowls

- vacuum/ $/ \mathrm{H}_{2}$ brazing

- multi-axis machining

- e-beam welding

- electro-forming

thermal stress problem dominated by current concentration around HOM ports

cost \& complexity

- complex cooling channel geometry

- large number of ports, many different types

- most needing multi-axis machining

- tight tolerances for brazed-on water jackets

- rectangular knife-edge flanges 


\section{A photocathode injector study}

IFRA study for power beaming

based on "off the shelf" technology

CW RF gun cavity

shape optimized for low beta, max. Rs

gradient, emittance limited by Pwall

fabrication would be based on PEP-II

injector cost estimates

based on PEP-II costs, \$-mid-1990's

frequency scaling 


\section{Ignition Feedback Regenerative Amplifier}

$200 \mathrm{~kW}$ at $0.84 \mu \mathrm{m}$

- 200 A peak current

- 0.15 A average current

- $10 \mathrm{~mm}$ mrad normalized emittance

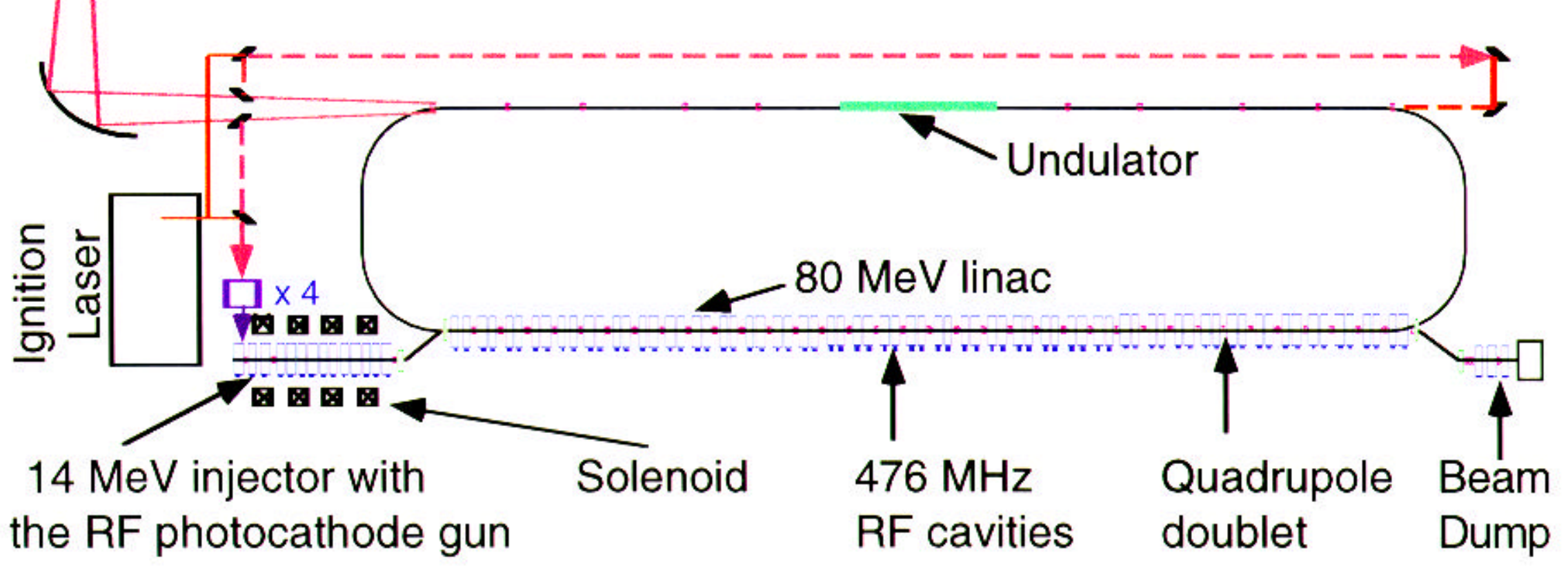




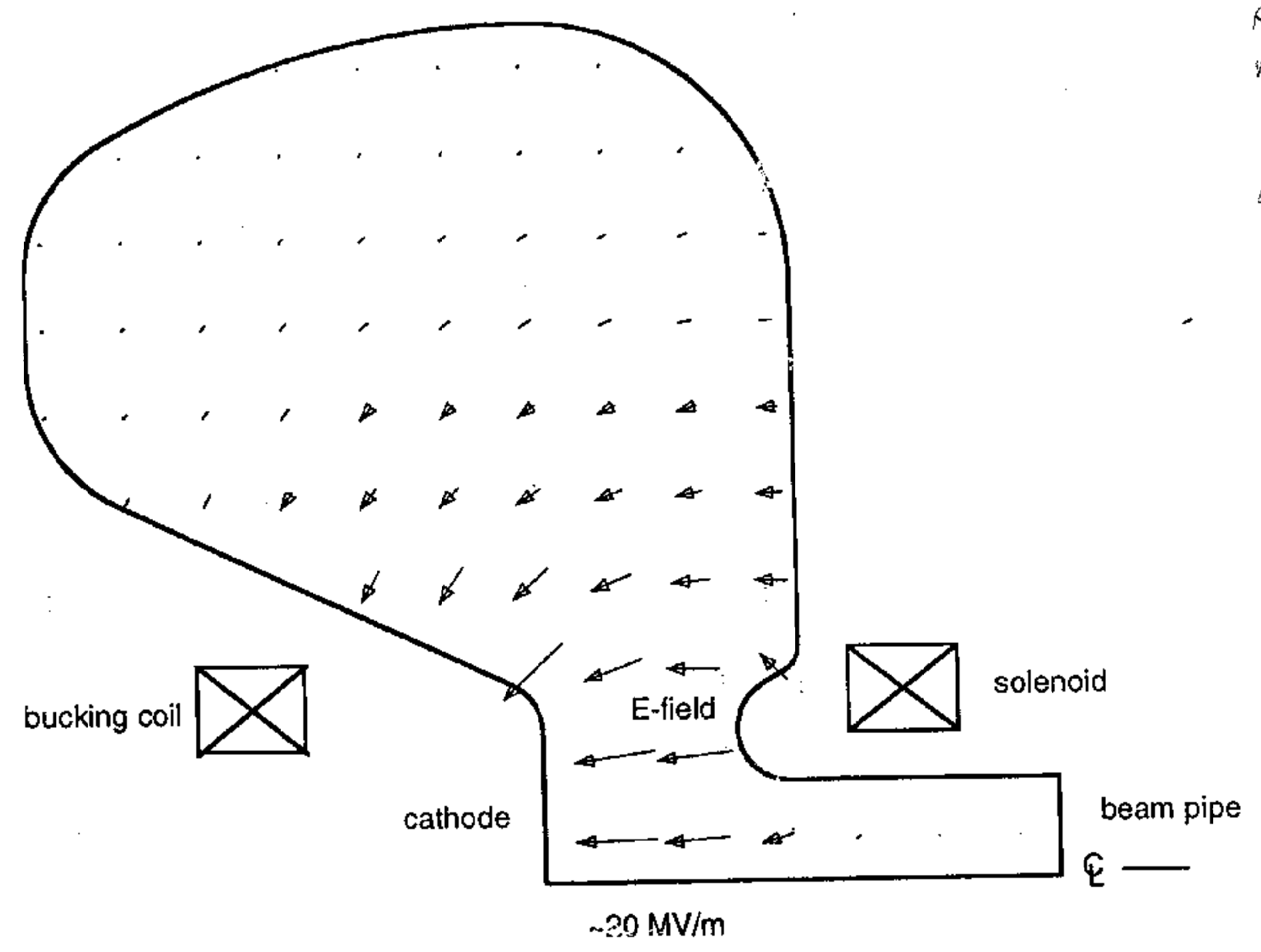

$R=2.17 \mathrm{M.2}$ (no tranait-fime $R_{Q}=95.7 \Omega$ correction)

$c_{i}=22687$

$E_{20} 3183 \mathrm{nu} / \mathrm{ma} 100$ 她.

Possible photocathode cavity geometry 


\section{$\therefore$}

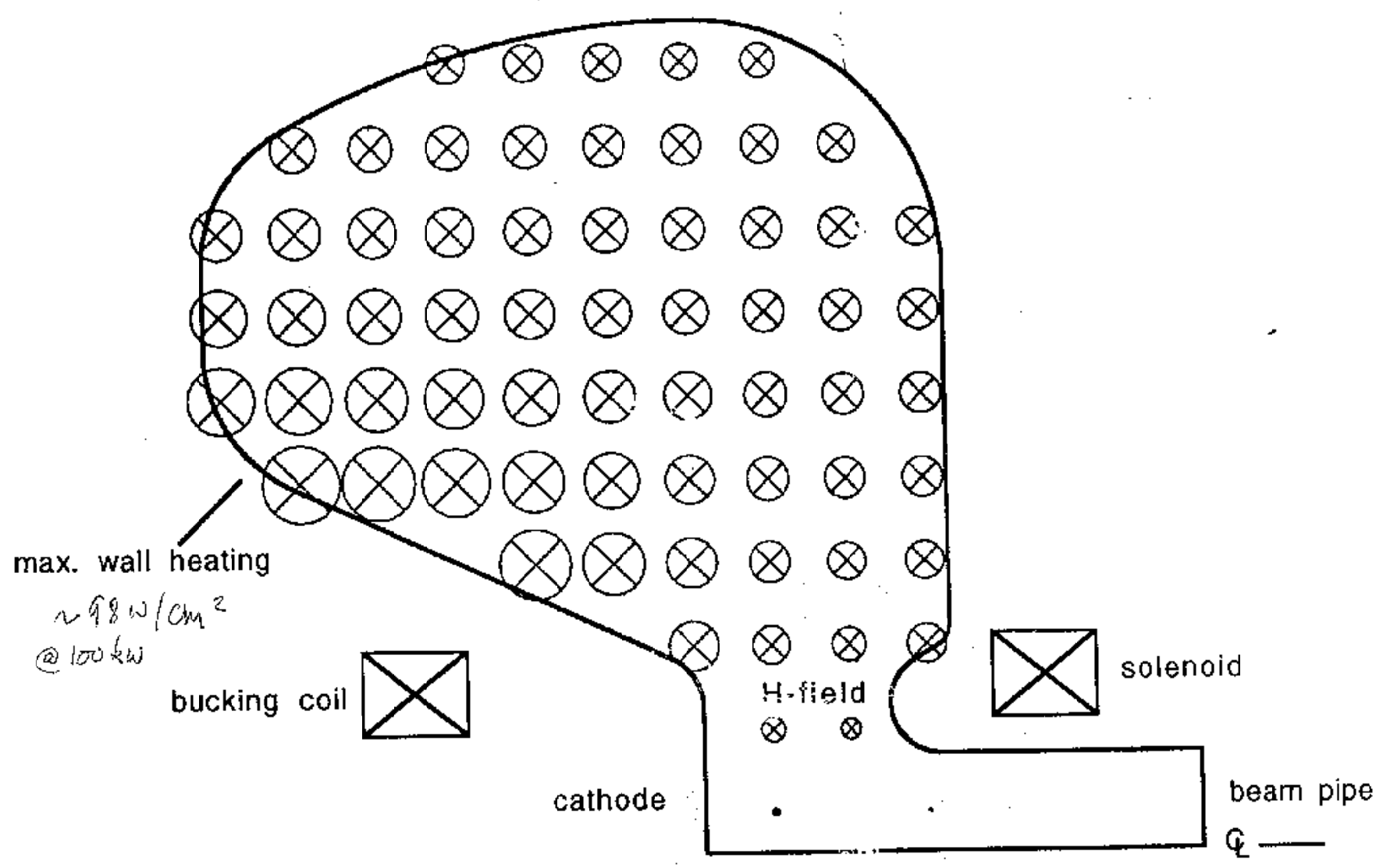

Possible photocathode cavity geometry 


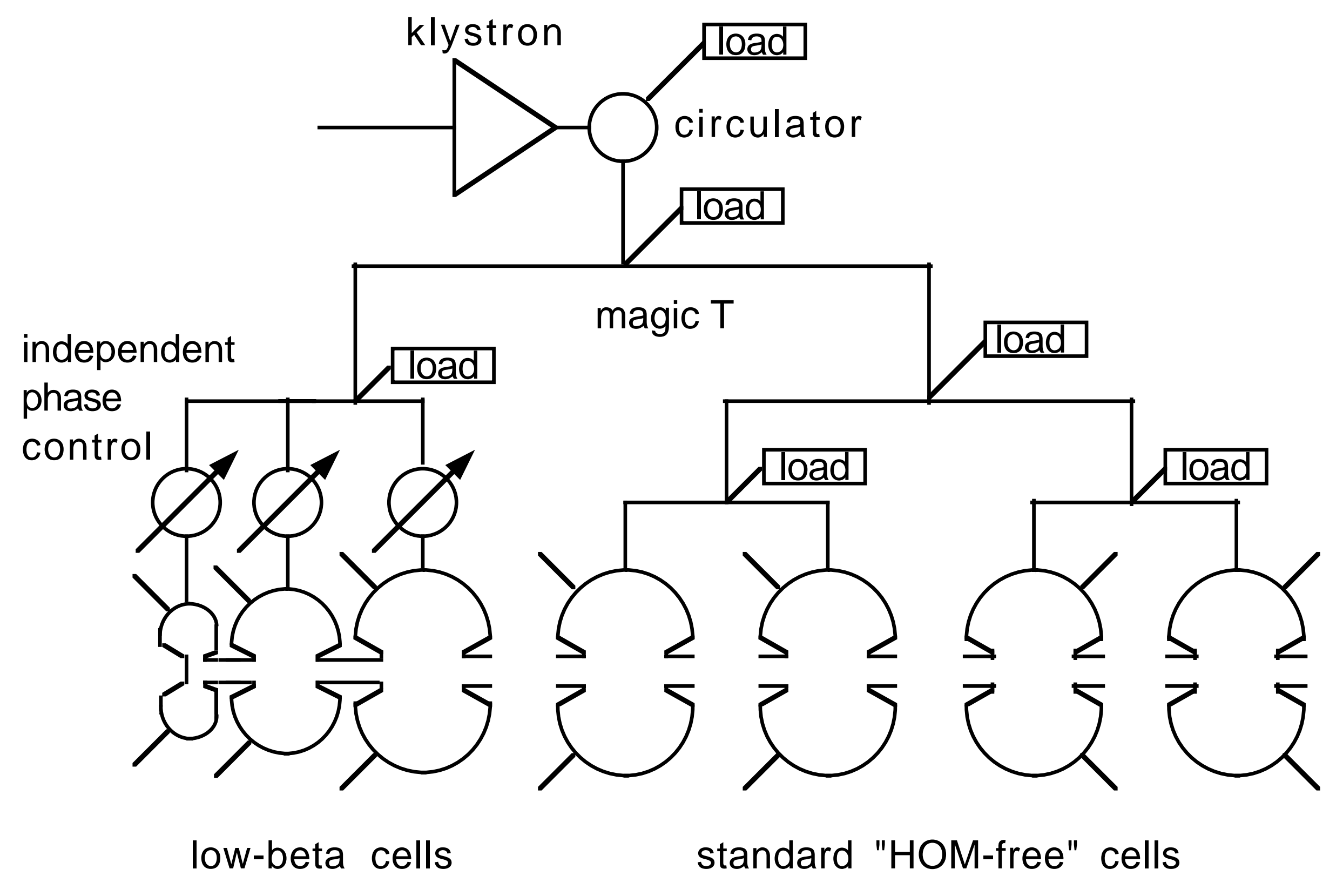


PB RF Stn cost

\begin{tabular}{|c|c|c|c|c|c|c|c|}
\hline \multirow[t]{2}{*}{ Item } & \multirow{2}{*}{$\begin{array}{c}\text { cost per item } \\
\mathrm{k} \$\end{array}$} & \multicolumn{2}{|c|}{$1 E$} & \multicolumn{2}{|r|}{$\mathrm{IER}$} & \multicolumn{2}{|c|}{$\not B$} \\
\hline & & \# required & $k \$$ & \# required & $\mathrm{k} \$$ & $\#$ required & $k \$$ \\
\hline Klystron (Phillips, $1.2 \mathrm{MW}$ ) & 335 & 1 & 335 & 1 & 335 & 1 & 335 \\
\hline Cavity (1 MV max) & 150 & 4 & 600 & 2 & 300 & 8 & 1200 \\
\hline HOM loads ( 3 per cavity) & 17 & 12 & 204 & 6 & 102 & 24 & 408 \\
\hline Coupler box (1 per cavity) & 19 & 4 & 76 & 2 & 38 & 8 & 152 \\
\hline $500 \mathrm{~kW}$ Window (1 per cavity) & 21 & 4 & 84 & 2 & 42 & 8 & 168 \\
\hline Fixed tuner (1 per cavity) & 2 & 4 & 8 & 2 & 4 & 8 & 16 \\
\hline Variable tuner (1 per cavity) & 20 & 4 & 80 & 2 & 40 & 8 & 160 \\
\hline Raft (1 per cavity) & 12 & 4 & 48 & 2 & 24 & 8 & 96 \\
\hline Circulator (AFT) & 155 & 1 & 155. & 1 & 155 & 1 & 155 \\
\hline Waveguide network & 68 & 1 & 68 & 1 & 68 & 2 & 136 \\
\hline Low-level RF & 85 & 1 & 85 & 1 & 85 & 2 & 170 \\
\hline HV power supply & 375 & 1 & 375 & 1 & 375 & 1 & 375 \\
\hline Cooling system & 40 & 1 & 40 & 1 & 40 & 1 & 40 \\
\hline Cables & 35 & 1 & 35 & 1 & 35 & 1 & 35 \\
\hline Misc, installation etc. & 60 & 1 & 60 & 1 & 60 & 1 & 60 \\
\hline total station cost $(\mathrm{k} \$)$ : & & & 2253 & & 1703 & & 3506 \\
\hline total station voltage $(\mathrm{kV})$ : & & & 3200 & & 1700 & & 6800 \\
\hline beam power available $(\mathrm{kW})$ : & & & 800 & & 990 & & 360 \\
\hline voltage cost performance $(\$ N)$ : & & & 0.70 & & 1.00 & & 0.52 \\
\hline power cost performance $(\$ / W)$ : & & & 2.82 & & 1.72 & & 9.74 \\
\hline
\end{tabular}


PB RF Stn cost

\begin{tabular}{|c|c|c|c|c|c|}
\hline \multirow[t]{2}{*}{ Item } & cost per item & \multicolumn{2}{|c|}{$4 \times 5$ cell } & \multicolumn{2}{|c|}{$2 \times 5$ cell } \\
\hline & $\mathrm{k} \$$ & \# required & $\mathbf{k} \$$ & \# required & $\mathbf{k} \$$ \\
\hline Klystron (Phillips, $1.2 \mathrm{MW}$ ) & 335 & 1 & 335 & 1 & 335 \\
\hline Cavity (f MV max) & 500 & 4 & $2000(\$ 500 \mathrm{k} \mathrm{ea})$ & 2 & $1000(\$ 500 \mathrm{k} \mathrm{ea})$ \\
\hline HOM loads ( 3 per cavity) & 17 & 24 & 408 & 12 & 204 \\
\hline Coupler box ( 1 per cavity) & 19 & 4 & 76 & 2 & 38 \\
\hline $500 \mathrm{~kW}$ Window (1 per cavity) & 21 & 4 & 84 & 2 & 42 \\
\hline Fixed tuner (1 per cavity) & 2 & 20 & 40 & 10 & 20 \\
\hline Variable tuner ( 1 per cavity) & 20 & 20 & 400 & 10 & 200 \\
\hline Raft (1 per cavity) & 12 & 4 & 48 & 2 & 24 \\
\hline Circulator (AFT) & 155 & 1 & 155 & 1. & 155 \\
\hline Waveguide network & 68 & 1 & 68 & 1 & 68 \\
\hline Low-level RF & 85 & 1 & 85 & 1 & 85 \\
\hline HV power supply & 375 & 1 & 375 & 1 & 375 \\
\hline Cooling system & 40 & 1 & 40 & 1 & 40 \\
\hline Cables & 35 & 1 & 35 & 1 & 35 \\
\hline Misc., installation etc. & 60 & 1 & 60 & 1 & 60 \\
\hline total station cost $(\mathrm{k} \$)$ : & & . & 4209 & & 2681 \\
\hline total station voltage $(\mathrm{kV})$ : & & & 12650 & & 8500 \\
\hline beam power available $(\mathrm{kW})$ : & & & $300 ?$ & & $150 ?$ \\
\hline voltage cost performance $(\$ / V)$ : & & & 0.33 & & 0.32 \\
\hline power cost performance $(\$ / W)$ : & & & 14.03 & & 17.87 \\
\hline
\end{tabular}


Gun/injector options

A: Photocathode gun plus 2.5 PEP-II based accelerating modules (length $\sim 21 \mathrm{~m}$, cost $\sim 10.5 \mathrm{M}$ )

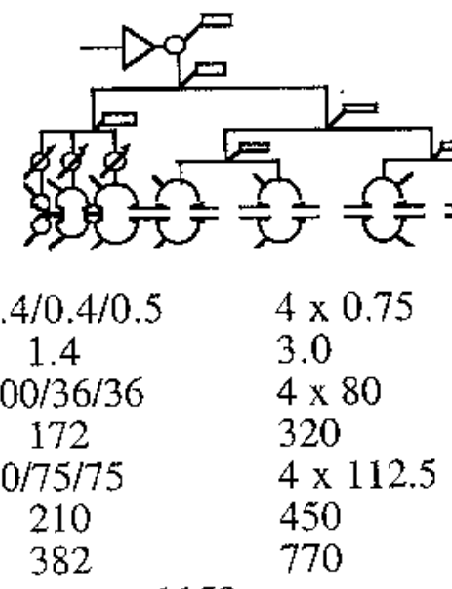

1152

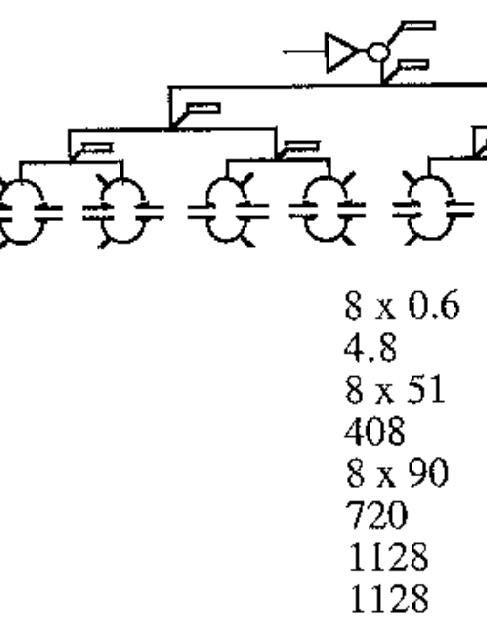

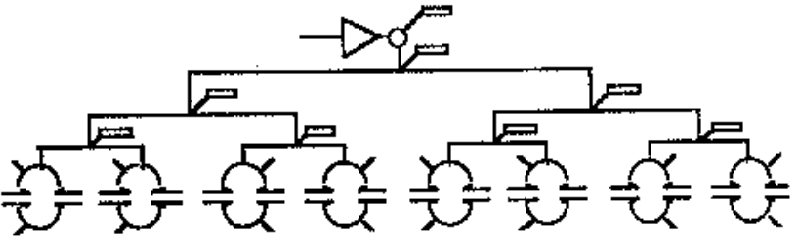

$8 \times 0.6$

4.8

$8 \times 51$

408

$8 \times 90$

720

1128

1128
$14 \mathrm{MV}$

$1308 \mathrm{~kW}$

$3408 \mathrm{~kW}$

B: Photocathode gun plus five five-cell cavities (length $\sim 12 \mathrm{~m}$, cost $\sim \$ 8.8 \mathrm{M}$ )

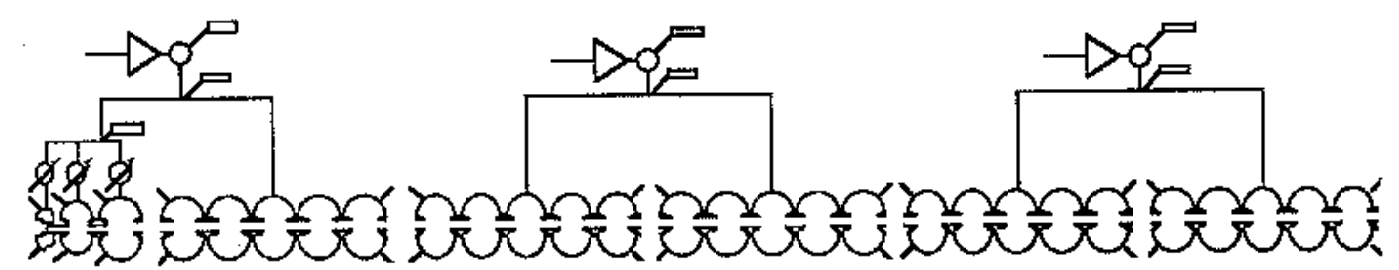

$\begin{array}{lcl}\text { Veff (MV) } & 0.4 / 0.4 / 0.5 & 5 \times 0.6 \\ \text { total (MV) } & 1.4 & 3.0 \\ \text { Pcell (kW) } & 100 / 36 / 36 & 5 \times 45 \\ \text { total (kW) } & 172 & 225 \\ \mathrm{~Pb}(\mathrm{~kW}) & 60 / 75 / 75 & 5 \times 90 \\ \text { total }(\mathrm{kW}) & 210 & 450 \\ & 382 & 675 \\ \text { Pkly }(\mathrm{kW}) & & 1057\end{array}$

$10 \times 0.48$
4.8
$10 \times 28.8$
288
$10 \times 72$
720
1008
1008

$10 \times 0.48$
4.8
$10 \times 28.8$
288
$10 \times 72$
720
1008
1008

$14 \mathrm{MV}$

$973 \mathrm{~kW}$

$3073 \mathrm{~kW}$ 


\section{IFRA accelerator options ( $14-80 \mathrm{MV})$}

R.R. $8 / 98$

A: Module based on eight PEP-II cavities

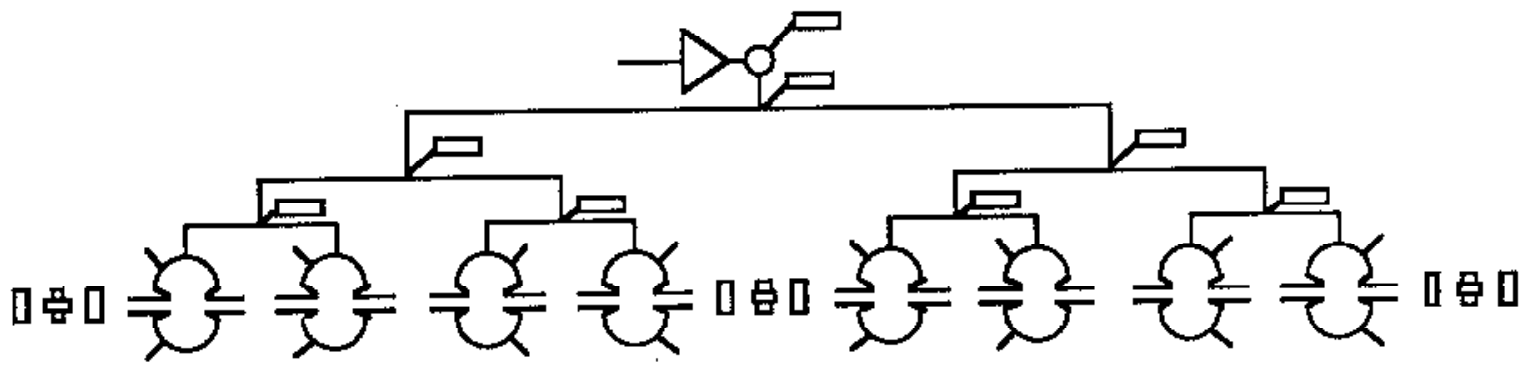

$$
\begin{array}{llll}
\mathrm{Rs}=8 \times 3.5 \mathrm{M} \Omega & (70 \% \text { of MAFIA Q }) & =28 \mathrm{M} \Omega & \\
\mathrm{V}=8 \times 0.85 \mathrm{MV} & (\text { length } \sim 6.927 \mathrm{~m}) & =6.8 \mathrm{MV} & (-1 \mathrm{MV} / \mathrm{m}) \\
\mathrm{P}=8 \times 103 \mathrm{~kW} & & & \\
\text { cost }=\$ 3.5 \mathrm{M} \text { ea., } & & & \\
\text { total cost }=\$ 45.5 \mathrm{MV} \text { requires } 12 \text { stations }+1 \text { length }=90 \mathrm{~m} \text {, operating power } & =9.9 \mathrm{MW} & \text { tinjectar } & \$ 56 \mathrm{M}
\end{array}
$$

B: Module based on four five-cell cavities

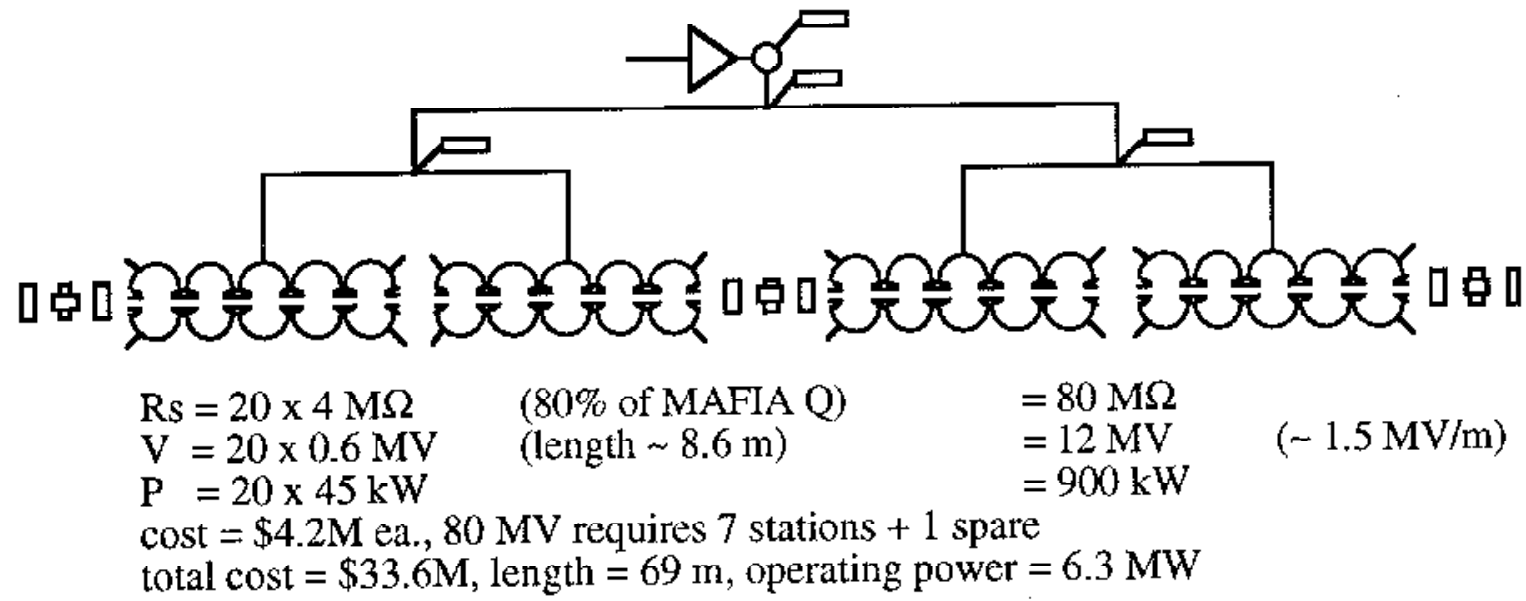

C: Module based on two high-power five-cell cavities

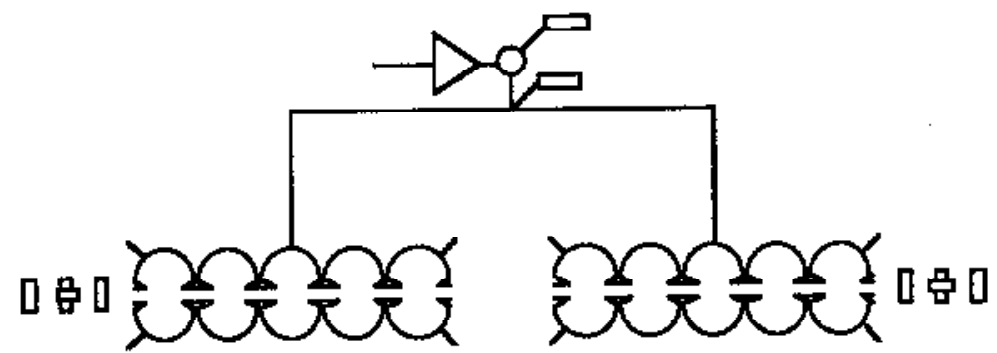

$$
\begin{array}{lll}
\mathrm{Rs}=10 \times 3.5 \mathrm{M} \Omega & (70 \% \text { of MAFIA Q }) & =35 \mathrm{M} \Omega \\
\mathrm{V}=10 \times 0.85 \mathrm{MV} & (\text { length } \sim 4.281 \mathrm{~m}) & =8.5 \mathrm{MV} \\
\mathrm{P}=1=\mathrm{x} 103 \mathrm{~kW} & & =1030 \mathrm{~kW}
\end{array}
$$

cost $=\$ 2.7 \mathrm{M}$ ea., $80 \mathrm{MV}$ requires 10 stations +1 spare

total cost $=\$ 29.7 \mathrm{M}$, length $=47 \mathrm{~m}$, opcrating power $=10.3 \mathrm{MW}+$ injector $=\$ 38.5 \mathrm{M}$ 


\section{Scaling}

for a set shape $R / Q$ is a constant. Scaling the size to different frequencies for a given voltage the parameters vary as follows:

Quality factor $Q$, and shunt impedance $R_{S} \alpha 1 /$ sqrt(f)

power $\alpha$ sqrt $(f)$

surface area $\alpha 1 / f^{2}$

wall-power density $\alpha$ f2.5

Electric field strength increase $\alpha f$

Kilpatrick criterion increases with frequency

Cavity with $500 \mathrm{kV}$ at $714 \mathrm{MHz}$ similar to PEP-II at $800 \mathrm{kV}$ 


$$
\mathrm{f}=1.64 \mathrm{E}_{\mathrm{k}} \mathrm{e}^{\left(-8.5 / \mathrm{E}_{\mathrm{k}}\right)}
$$

\section{Klipatrick criterion}

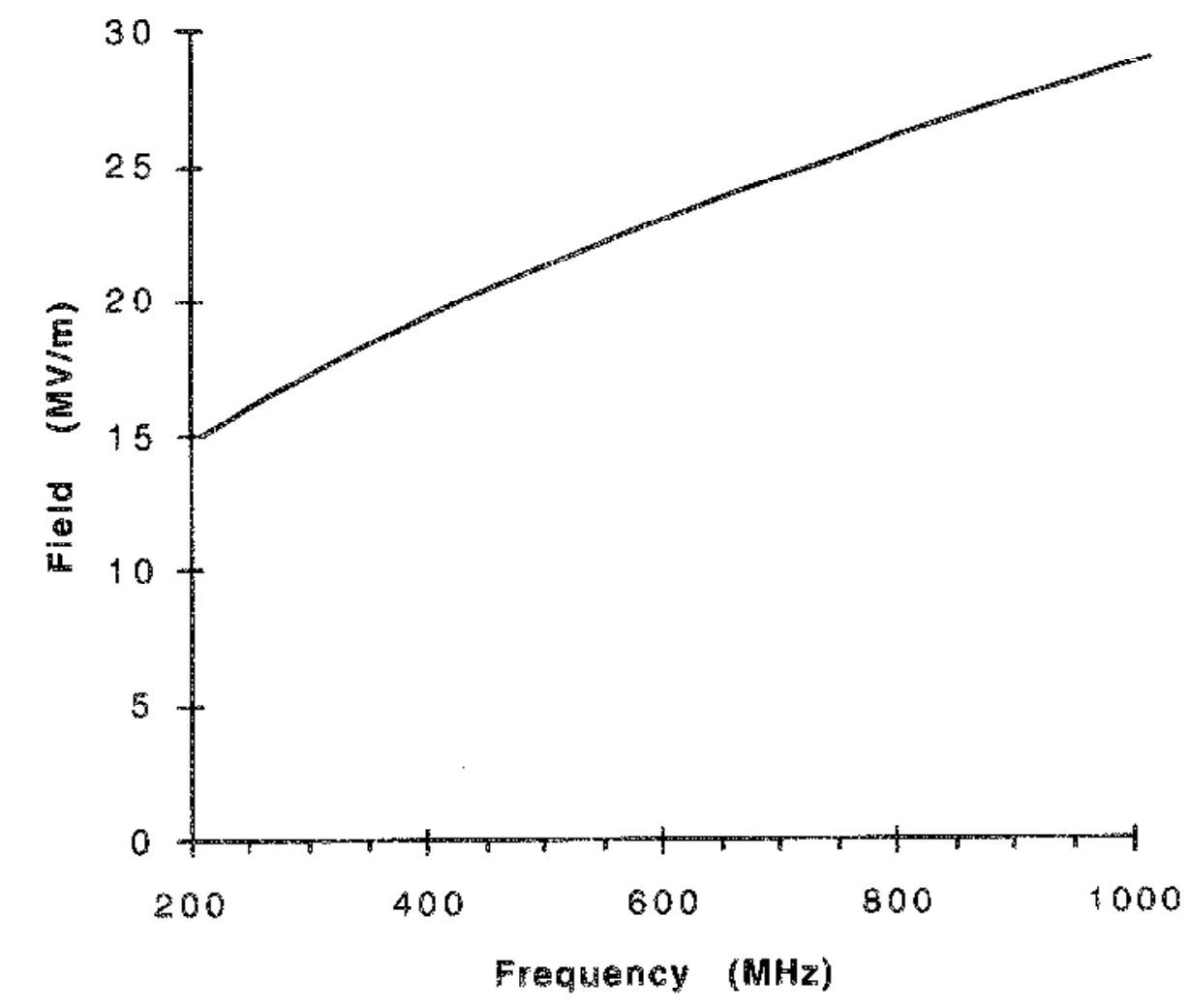




\section{A $714 \mathrm{MHz}$ cavity for NLC damping rings}

simplified design

rounded body, "dumbbell" HOM ports

improved fabrication methods

fewer, simpler parts and operations

costs

$\$ 100-150 \mathrm{k}$ ea. depending on labor rates

$(\$ 200 k+13,600$ hours for 10 cavities)

Cheaper in China! 


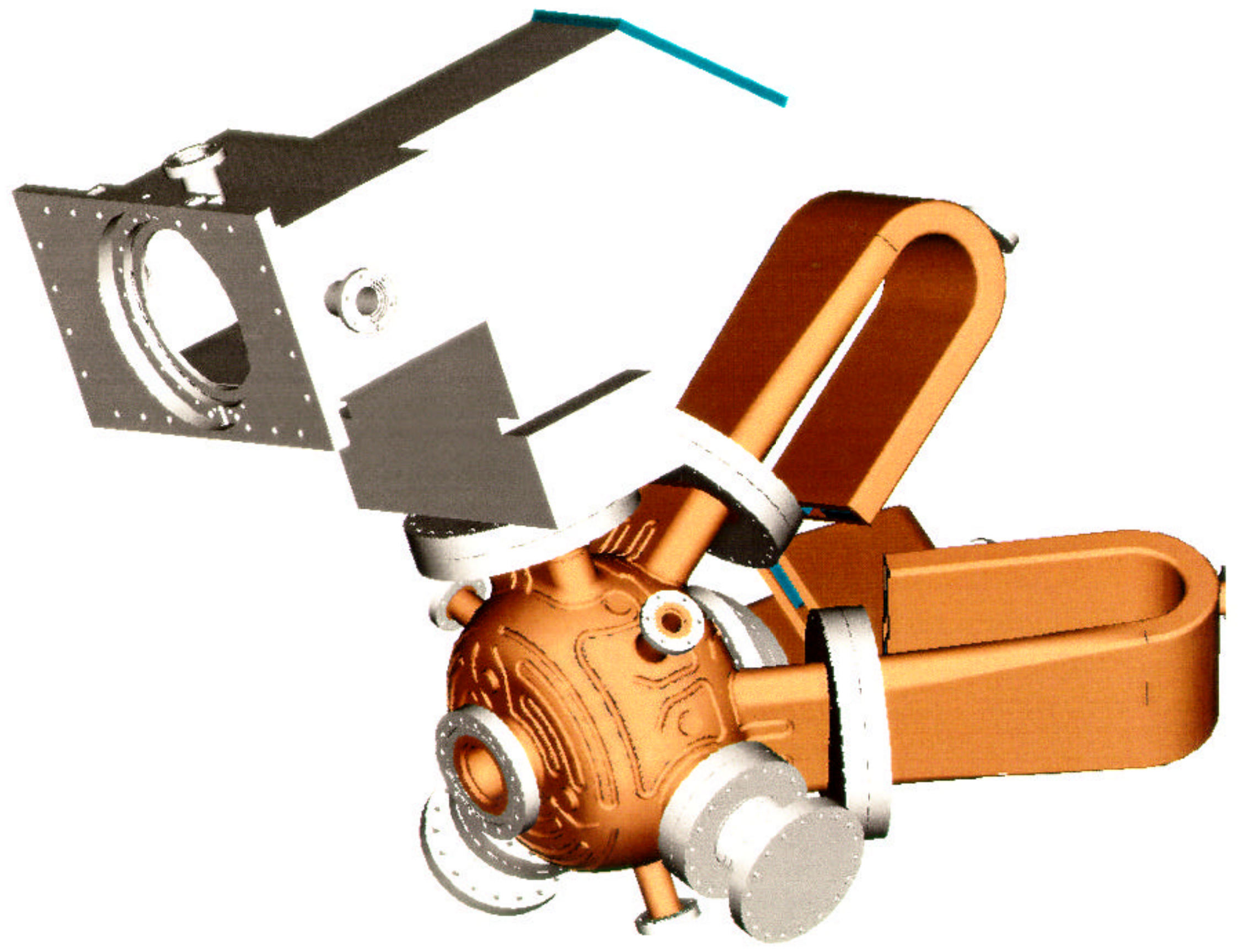




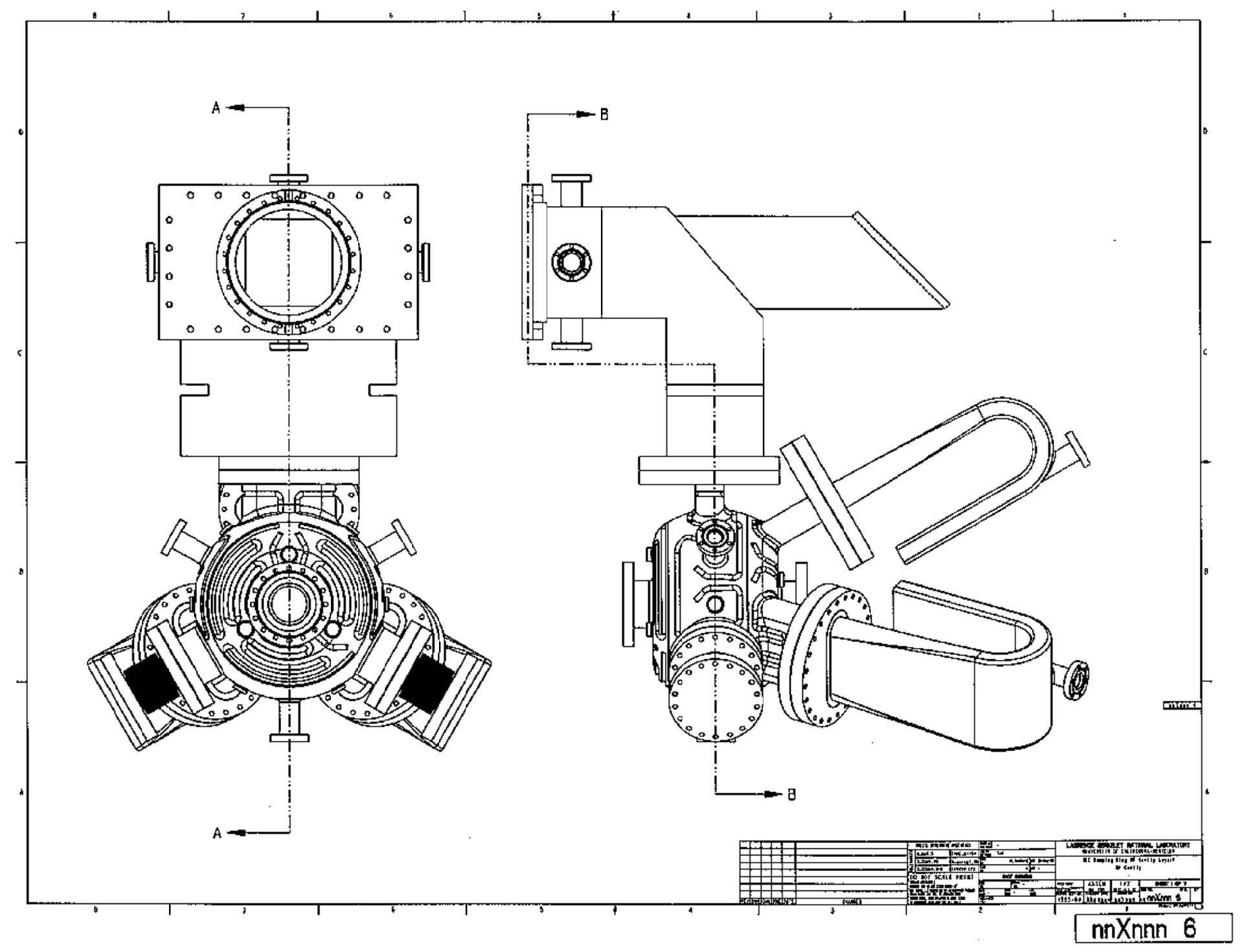




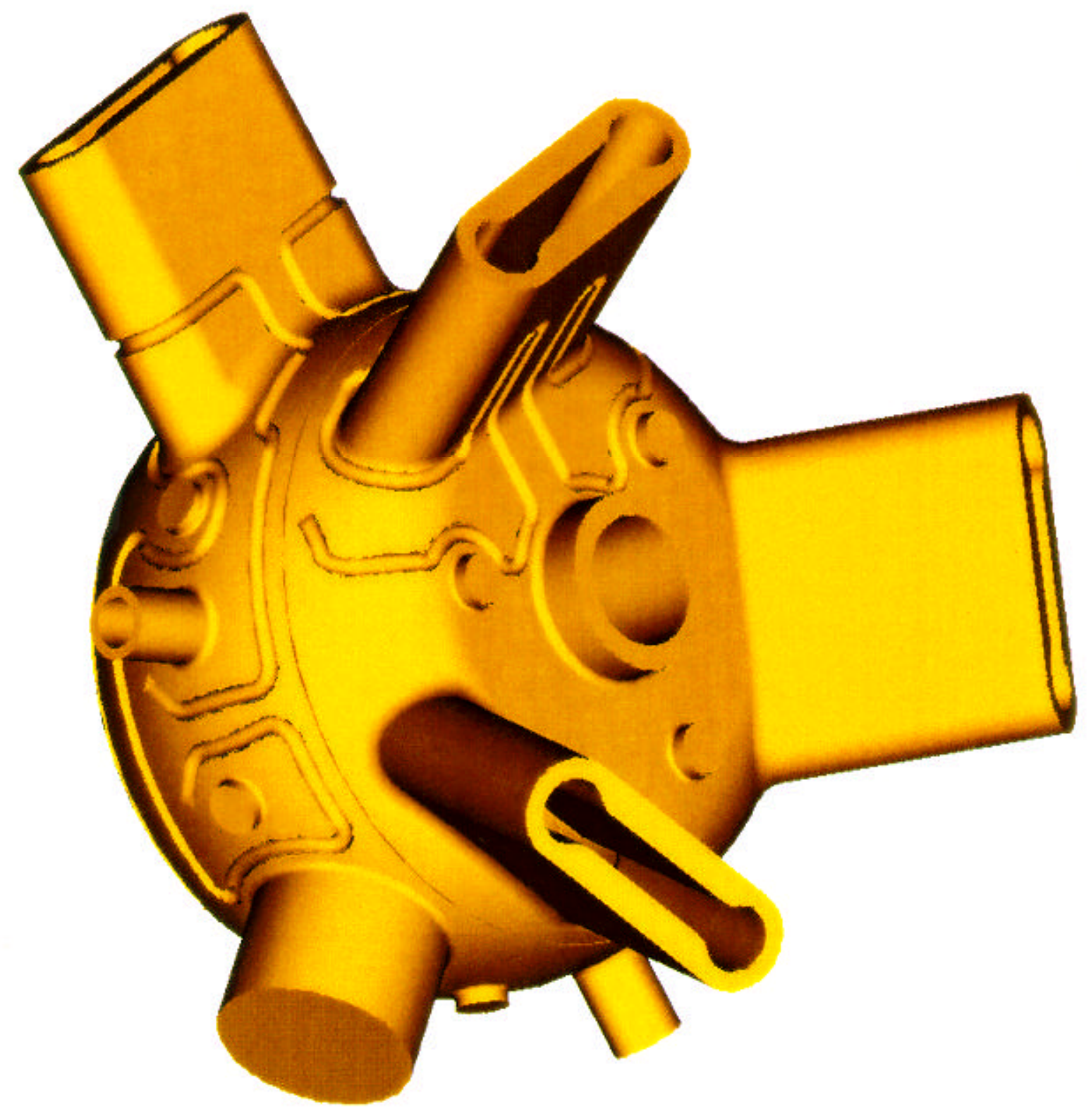




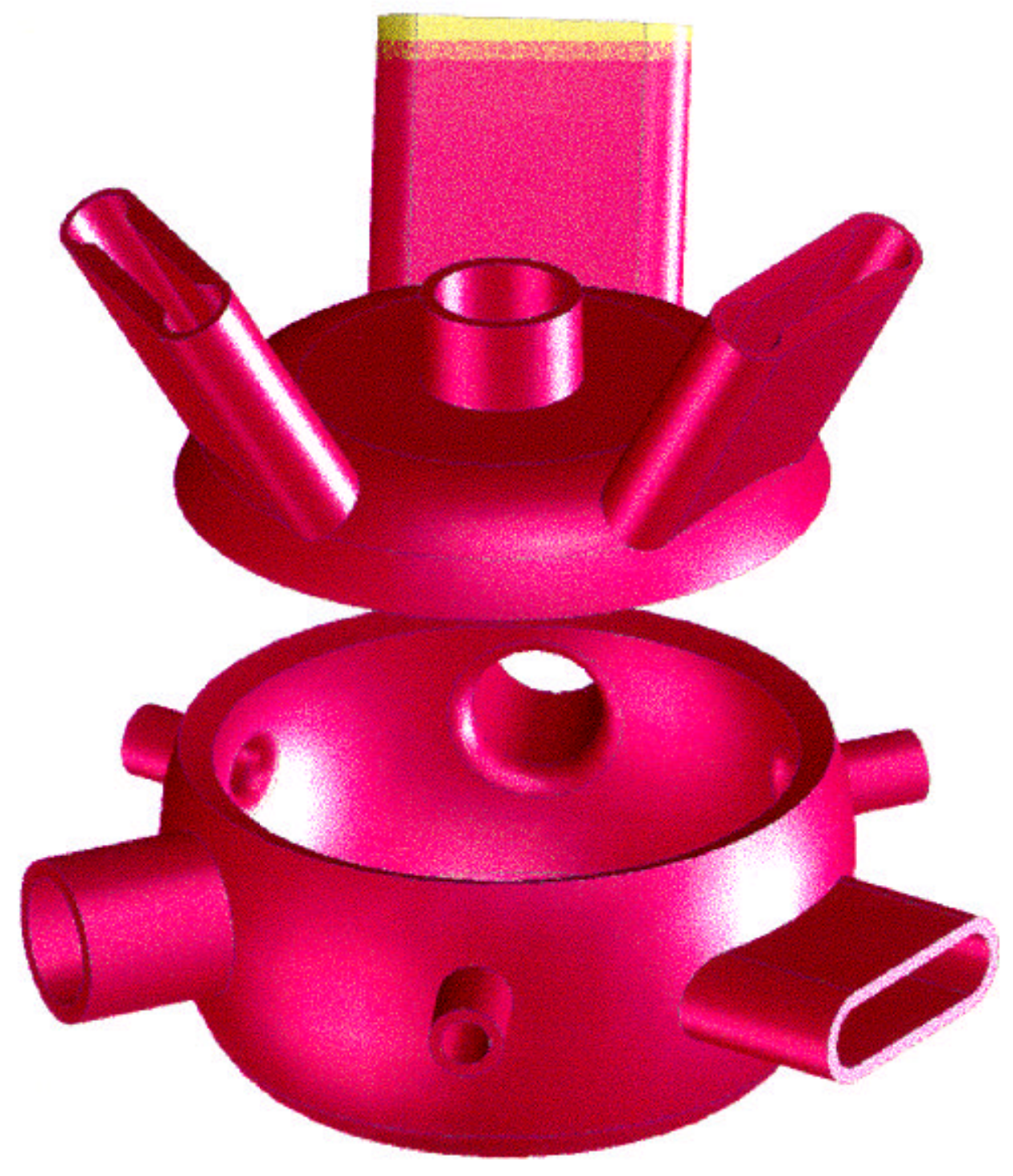




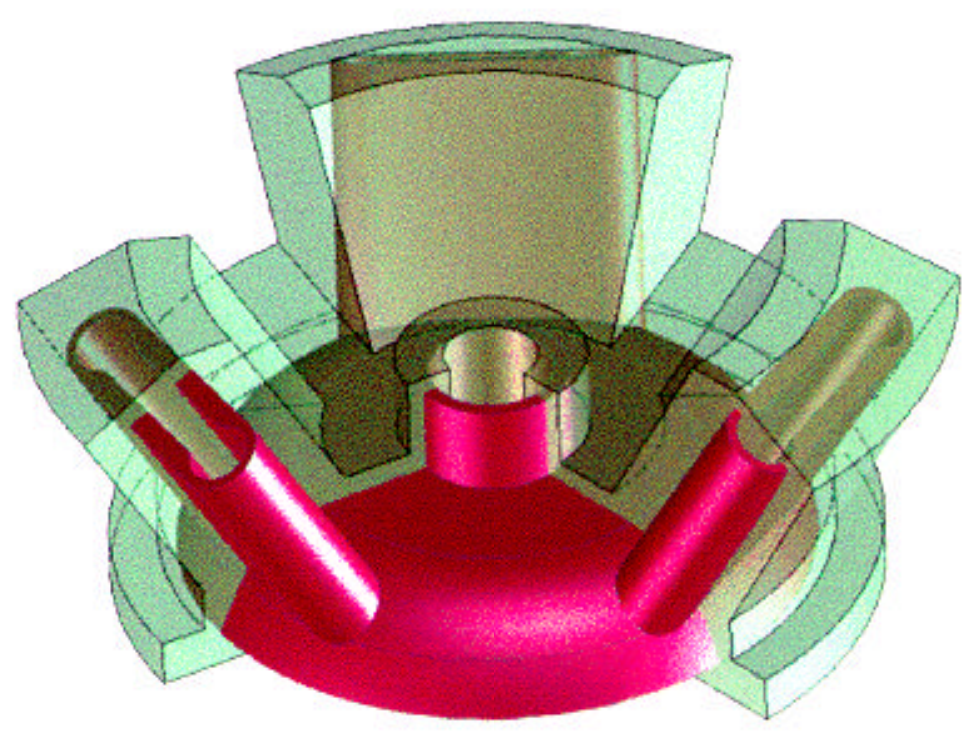

Figure 46. "Lid" forging.

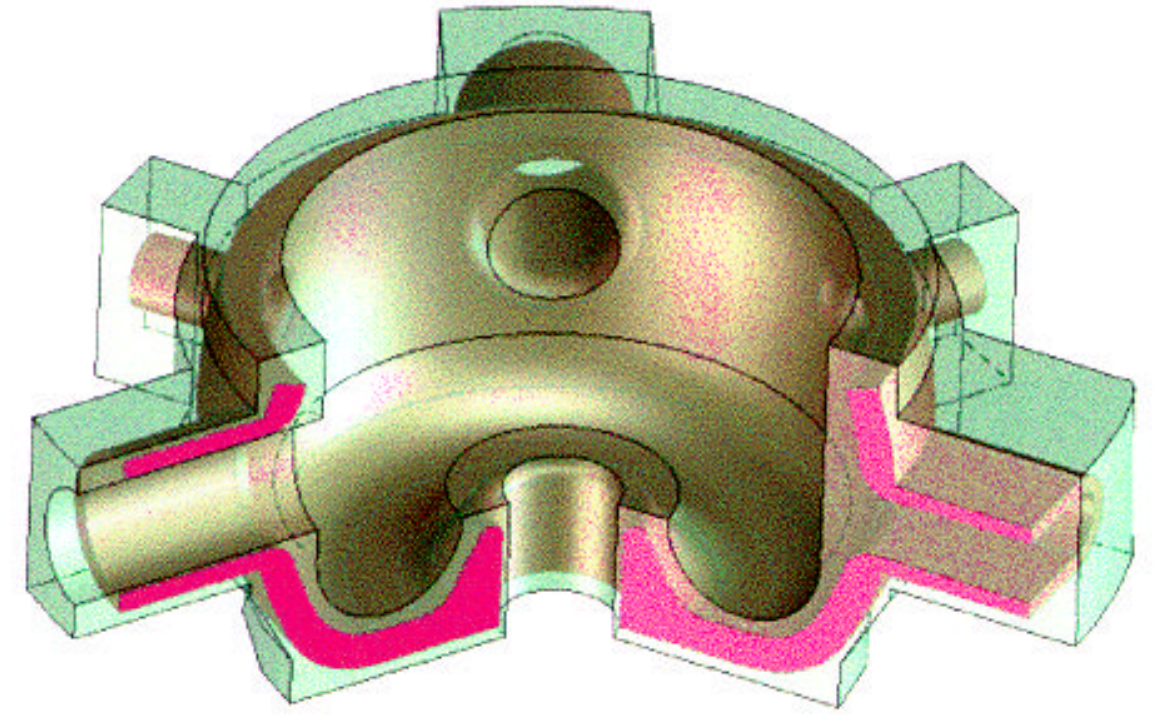

Figure 47. "Body" forging. 


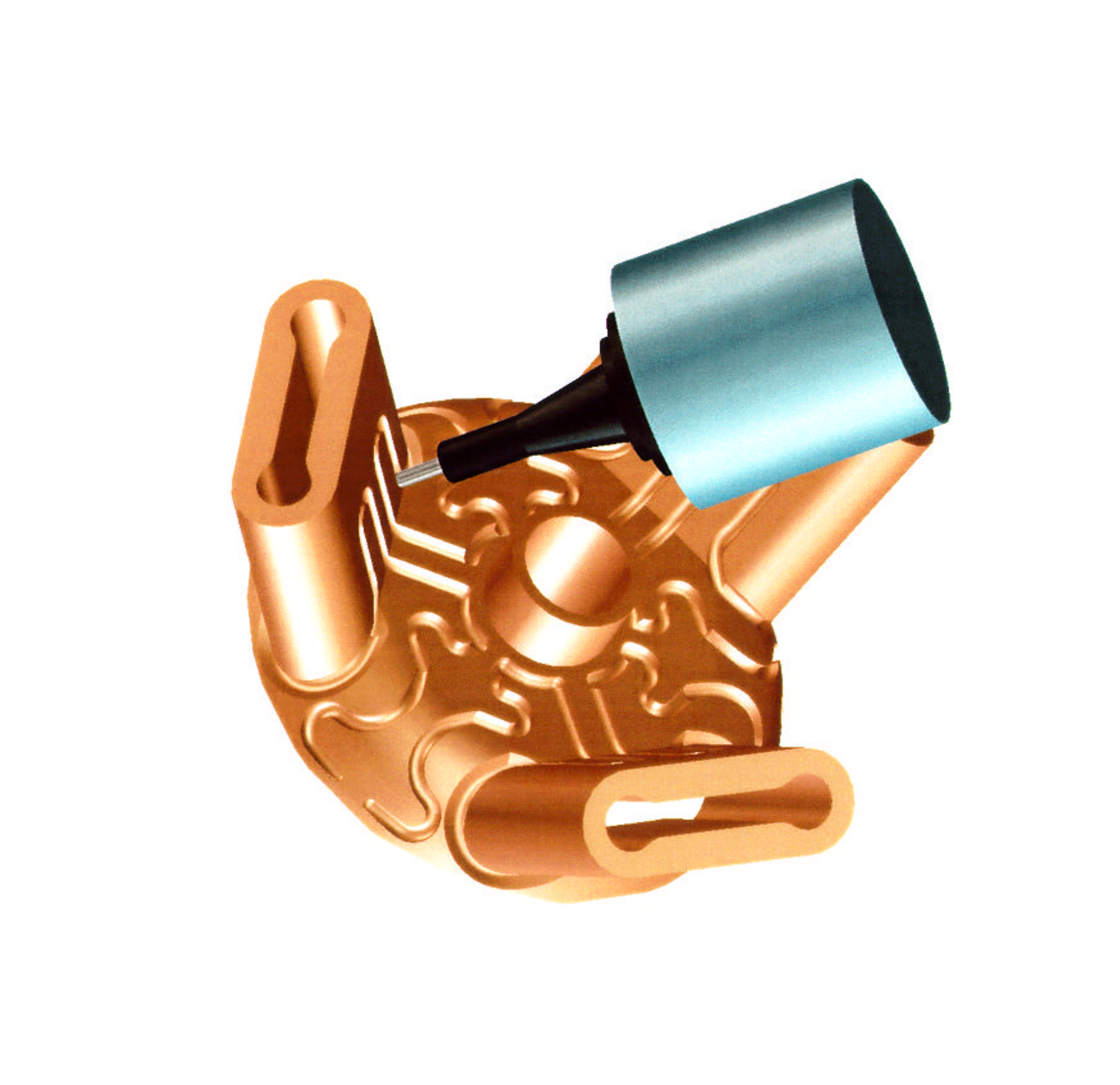




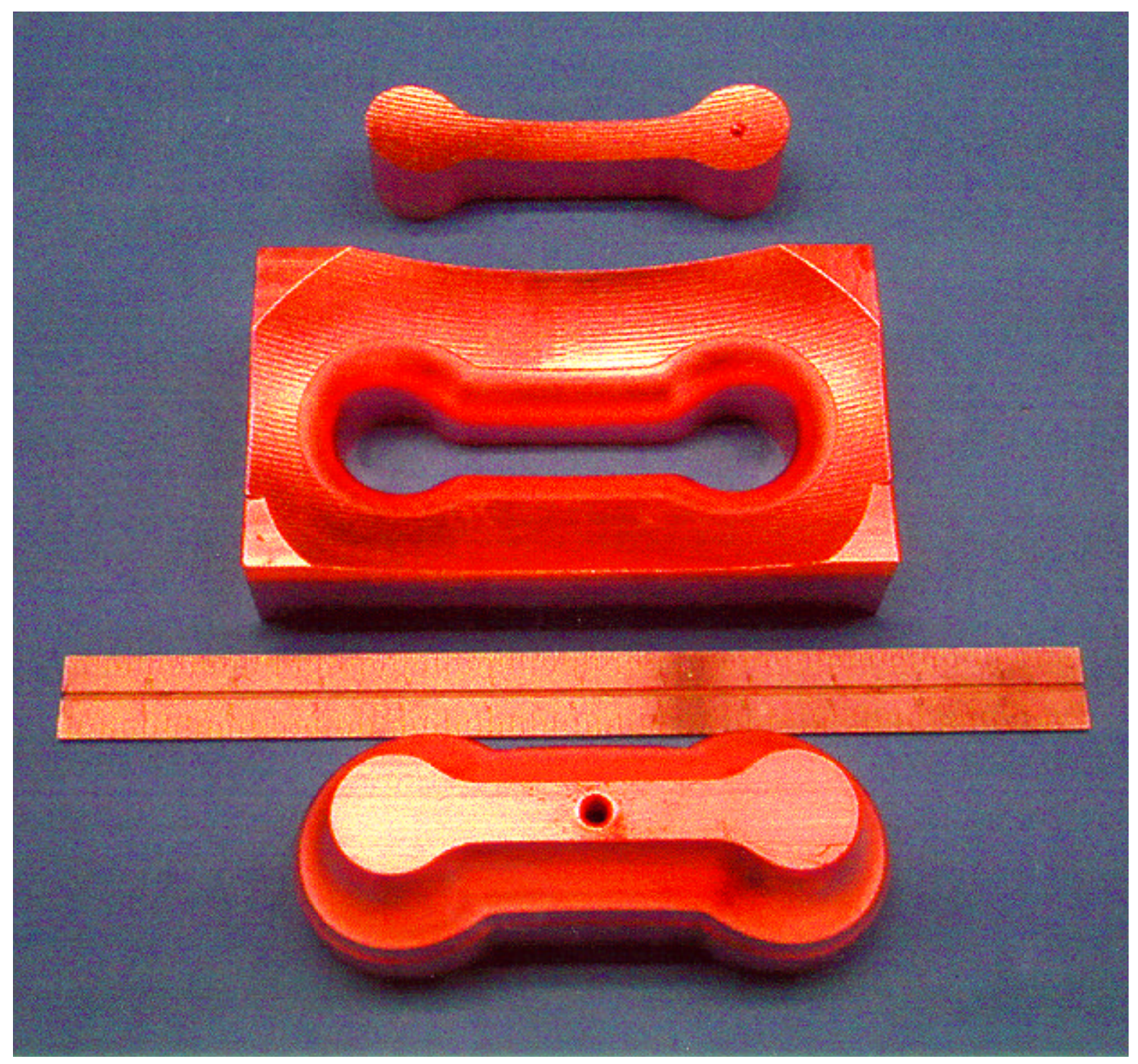


A 1.5 GHz cavity for ALS

6 simple third harmonic cavities

$5 \mathrm{~kW} \mathrm{CW}, 125 \mathrm{kV}$ (conservative)

no HOM ports

PEP-II type body channels

made from OFE Cu plate stock

e-beam welded and plated

made in house and quickly so $\$ \$ \$$ ! (z00k ea.) 


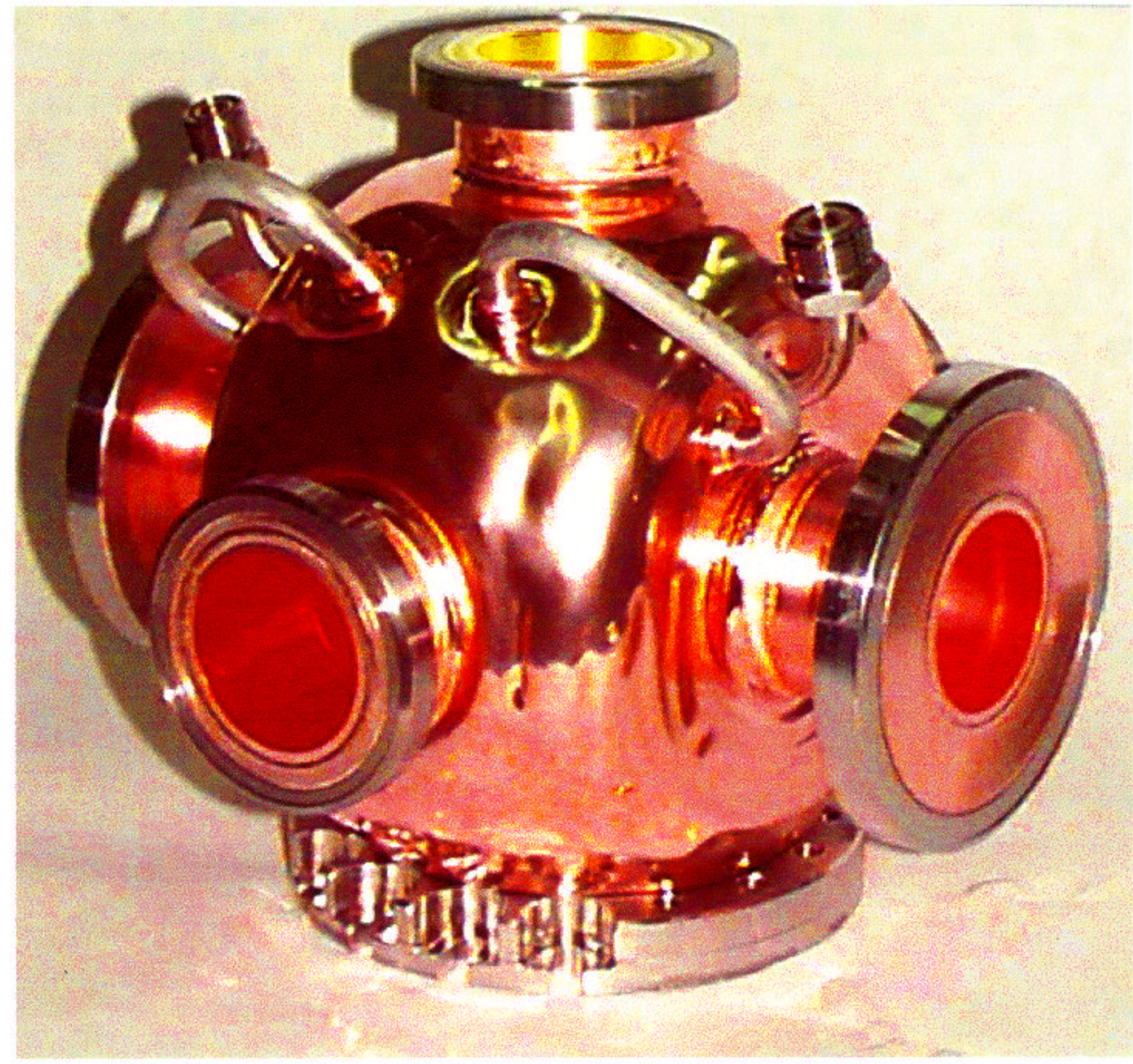




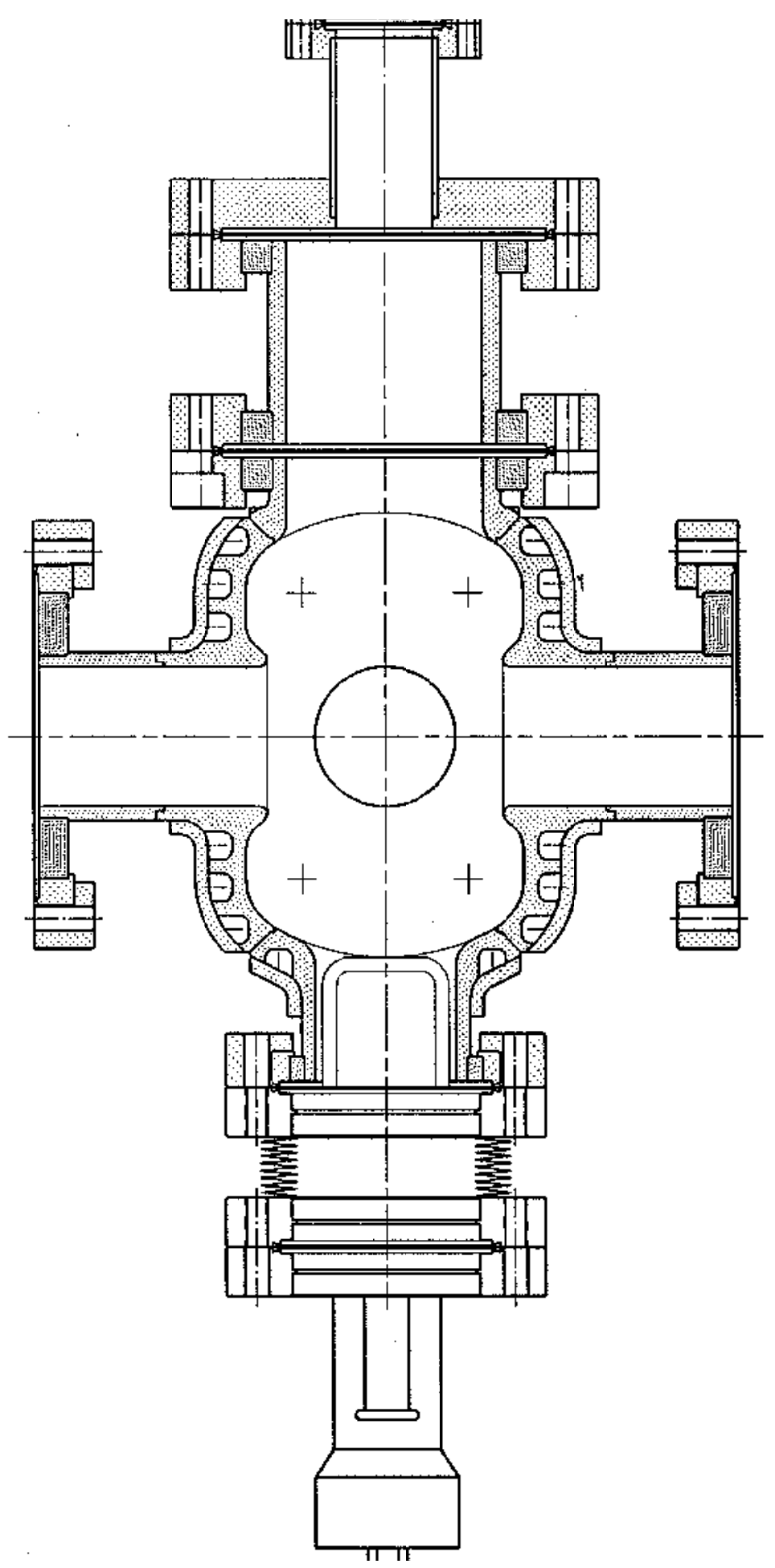




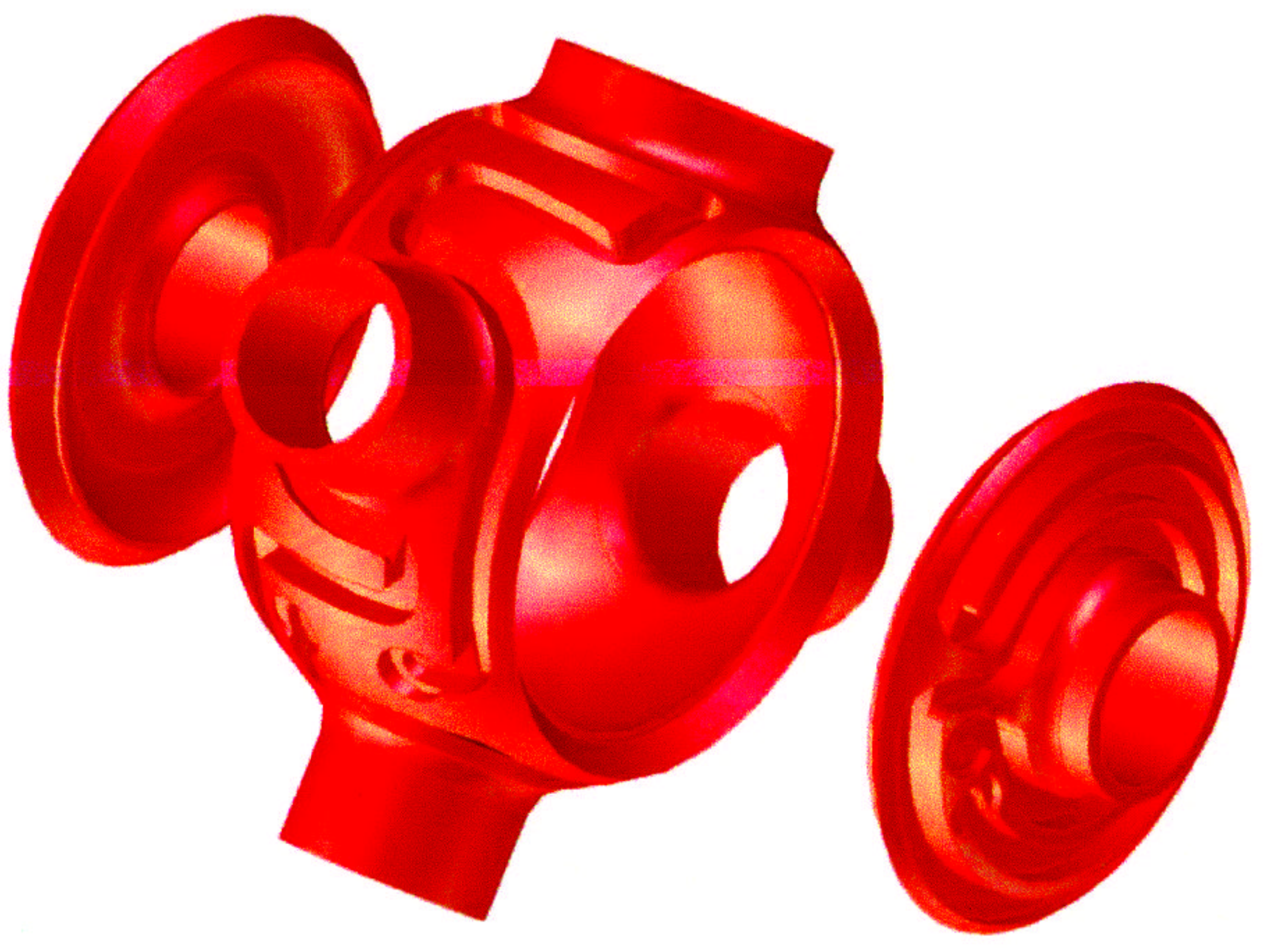




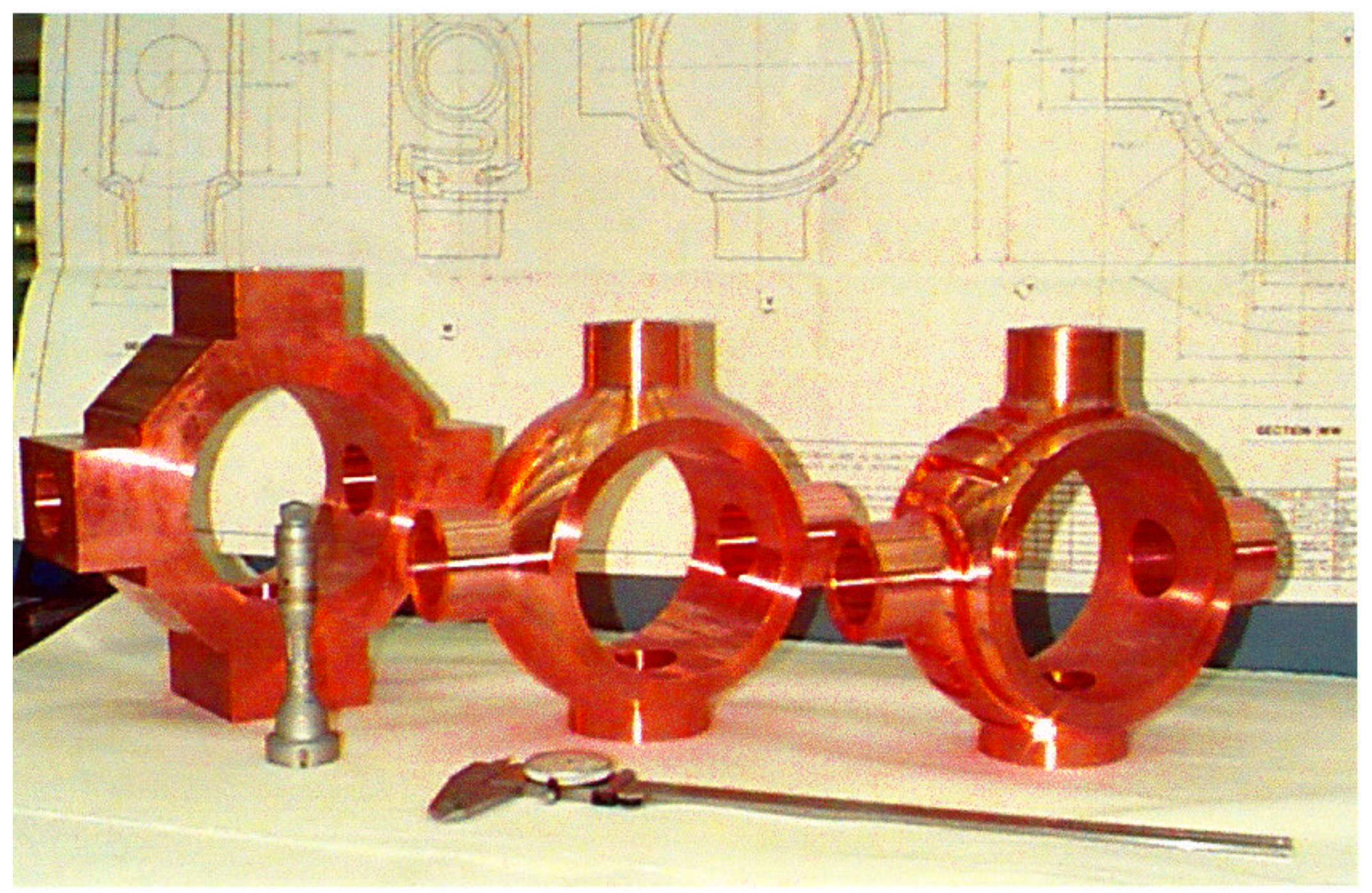




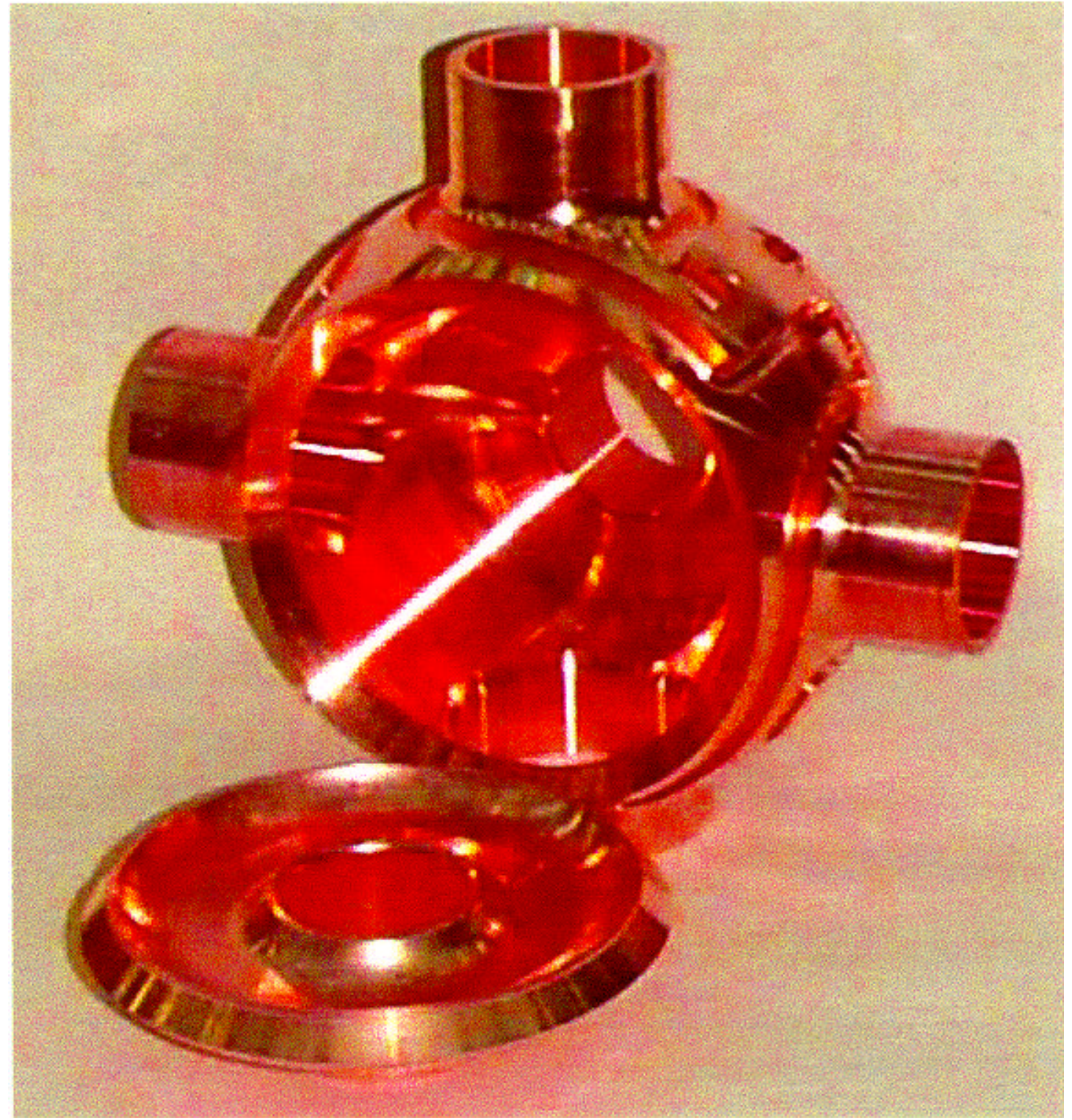




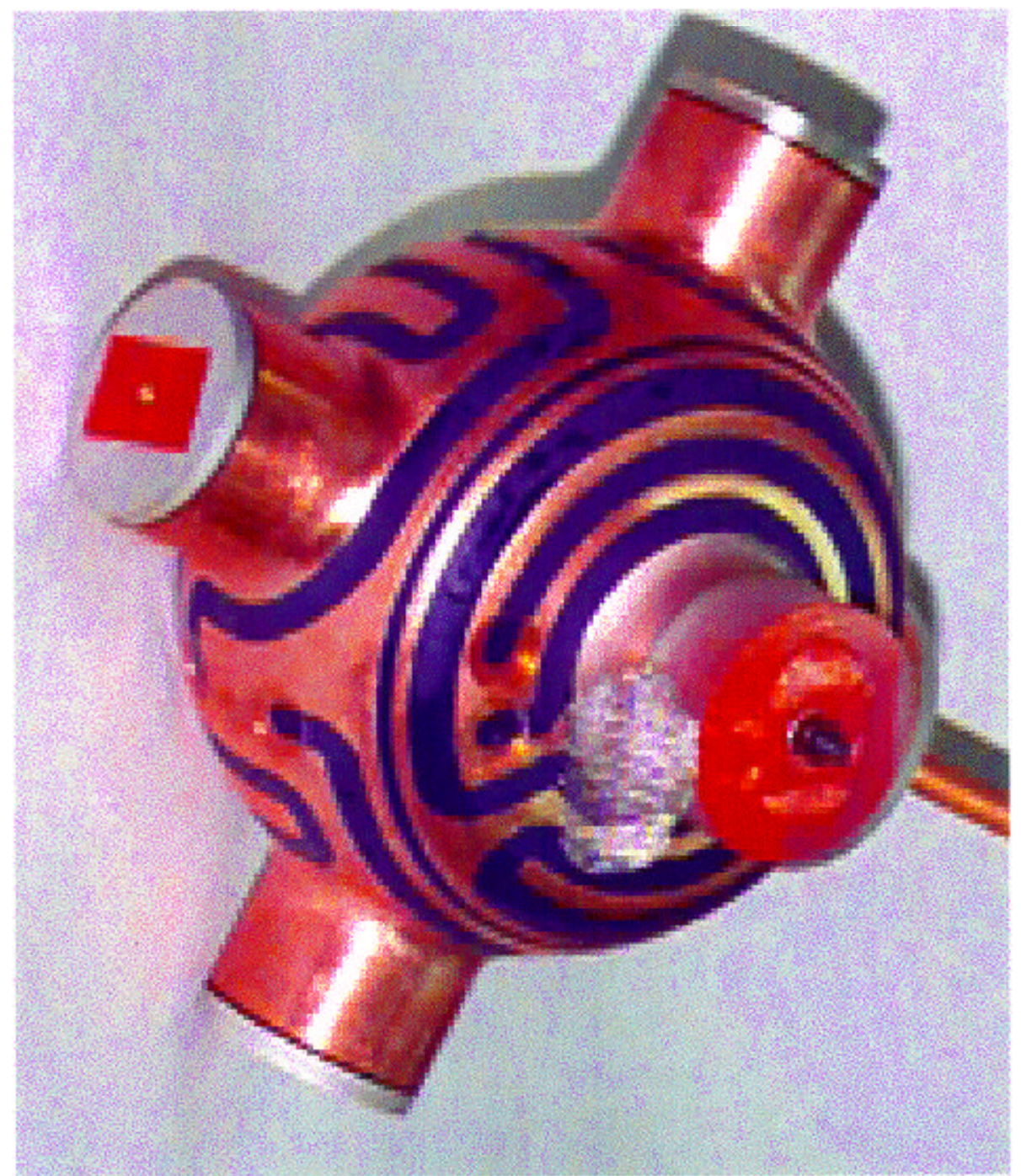




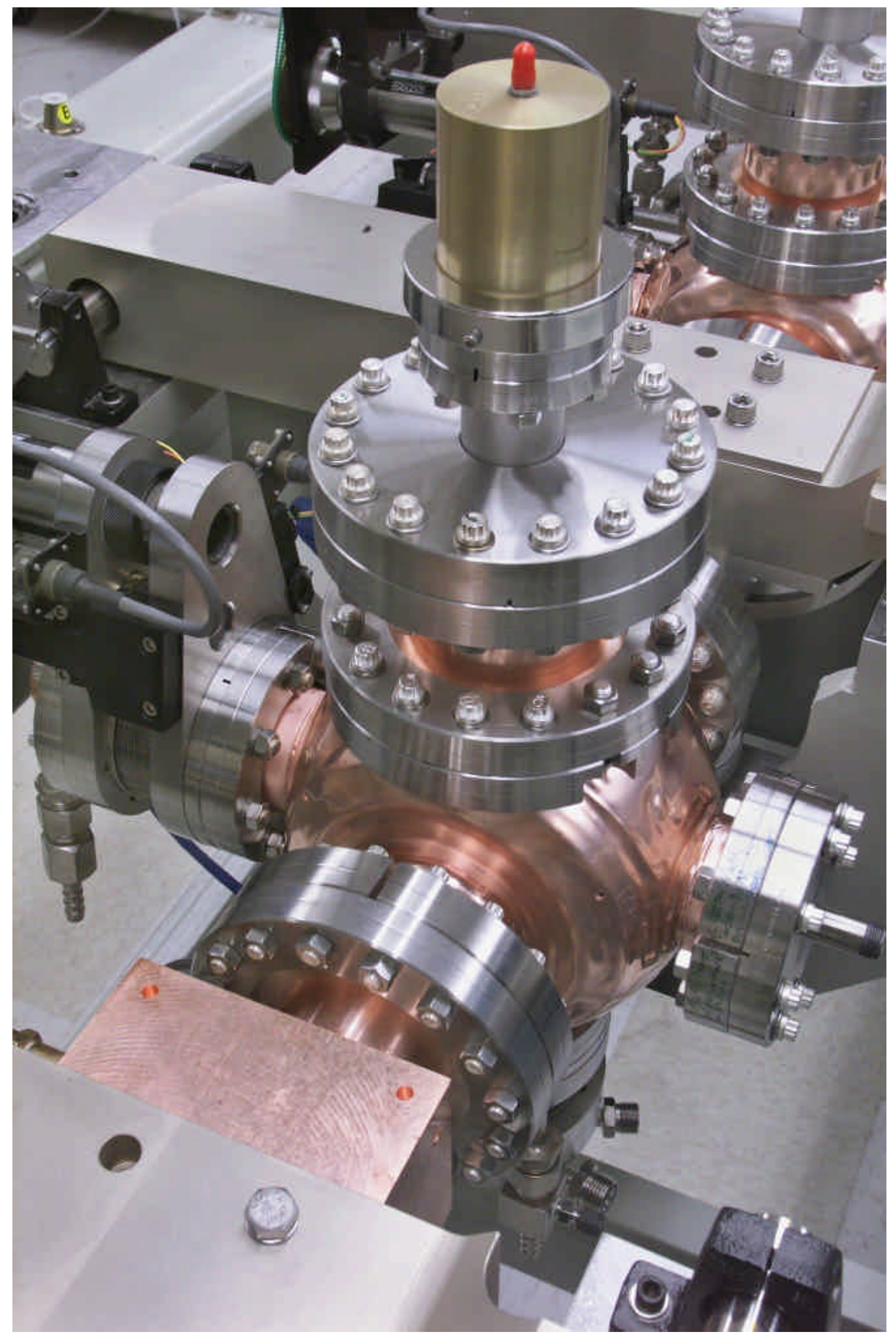




\title{
A $433 \mathrm{MHz}$ Cavity Based Photocathode RF Gun Injector For PERL
}

\author{
David H. Dowell \\ Boeing Physical Sciences Research Center \\ Seattle, WA
}




\section{PhotoInjector Talk Outline}

I. PhotoInjector Design Philosophy.

II. Non-Linearities in Bunch Compression

III. Bunch Compression Using a RF Linearizer

IV. Experimental Results

CSR and Space Charge Effects

VI. Strawman Design for PERL

VII. Conclusions 


\section{Photoinjector Design Philosophy}

Use a CW low frequency photocathode gun to generate high charge (1-5 nC) and long (50 ps) micropulses.

Advantages:

Capable of CW operation High charge

Long micropulses Excellent Beam Quality at High Beam Current

Disadvantage:

Cathode field limited to $25-30 \mathrm{MV} / \mathrm{m}$

Accelerate in Low frequency RF cavities.

Advantages:

Minimizes wakefields

CW operation

Disadvantage:

Accelerating gradient limited to $5 \mathrm{MV} / \mathrm{m}$

Linearize and compress to high peak current at $20 \mathrm{MeV}$.

Advantages:

Linearizing improves compression

Reduces space charge emittance growth

Disadvantage:

Emittance growth due to coherence synchrotron radiation

D.H. Dowell/PERL Workshop; Jan 22-23, 2001 


\section{Layout of the $433 \mathrm{MHz}$ PhotoInjector}

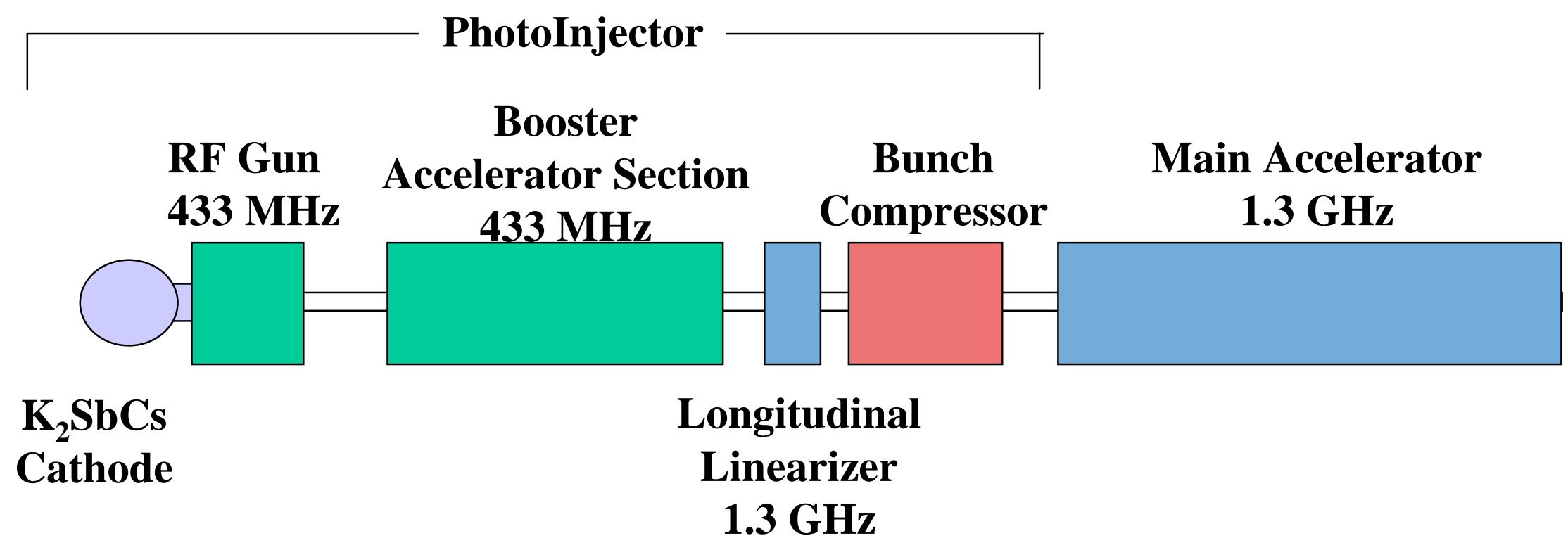




\section{Non-Linearities in Bunch Compression}

Long microbunches are distorted in longitudinal phase space due to wakefields and RF curvature.

$433 \mathrm{MHz}$ cavities introduce minimal wakes, but still cause significant curvature.

Introduce a RF section at third harmonic (1300 MHz) to cancel curvature of $433 \mathrm{MHz}$ booster. 


\section{Bunch Compression Using a RF Linearizer}

Transform initial longitudinal paraxial coordinates, $\Delta \mathrm{E}$ and $\Delta \mathrm{t}$, through the booster:

$$
\begin{aligned}
& \Delta \mathrm{E}_{1}=\mathrm{E}_{433}\left[\cos \left(\omega_{433} \Delta \mathrm{t}_{0}+\phi_{433}\right)-\cos \left(\phi_{433}\right)\right]+\Delta \mathrm{E}_{0} \\
& \Delta \mathrm{t}_{1}=\Delta \mathrm{t}_{0}
\end{aligned}
$$

Perform similar transformation through third harmonic section:

$$
\begin{aligned}
& \Delta \mathrm{E}_{2}=\mathrm{E}_{1300}\left[\cos \left(\omega_{1300} \Delta \mathrm{t}_{1}+\phi_{1300}\right)-\cos \left(\phi_{1300}\right)\right]+\Delta \mathrm{E}_{1} \\
& \Delta \mathrm{E}_{2}=\mathrm{E}_{1300}\left[\cos \left(3 \omega_{433} \Delta \mathrm{t}_{0}+\phi_{1300}\right)-\cos \left(\phi_{1300}\right)\right]+\mathrm{E}_{433}\left[\cos \left(\omega_{433} \Delta \mathrm{t}_{0}+\phi_{433}\right)-\cos \left(\phi_{433}\right)\right]+\Delta \mathrm{E}_{0} \\
& \Delta \mathrm{t}_{2}=\Delta \mathrm{t}_{1}=\Delta \mathrm{t}_{0}
\end{aligned}
$$

The third harmonic RF cancels the $433 \mathrm{MHz}$ curvature when second derivative wrt $\Delta \mathrm{t}_{2}$ is zero:

$$
\left.\frac{\mathrm{d}^{2} \Delta \mathrm{E}_{2}}{\mathrm{~d} \Delta \mathrm{t}_{2}^{2}}\right|_{\Delta \mathrm{t}_{2}=0}=0 \longrightarrow \mathrm{E}_{1300} \cos \left(\phi_{1300}\right)=-\frac{\mathrm{E}_{433} \cos \left(\phi_{433}\right)}{9}
$$

Generally, $\phi_{433}=0$, and $\phi_{1300}$ is chosen to generate energy slew:

$$
\begin{aligned}
& \Delta \mathrm{E}_{3}=\Delta \mathrm{E}_{2} \\
& \Delta \mathrm{t}_{3}=\mathrm{R}_{56} \Delta \mathrm{E}_{2}+\Delta \mathrm{t}_{2}
\end{aligned} \longrightarrow \begin{gathered}
\text { For } \mathrm{E}_{433}=20 \mathrm{MeV}, \mathrm{R}_{56}=50 \mathrm{ps} / \mathrm{MeV}: \\
\mathrm{E}_{1300}=3.2 \mathrm{MeV}, \phi_{1300}=50^{\circ}
\end{gathered}
$$




\section{The 20 MeV RF Photoinjector}

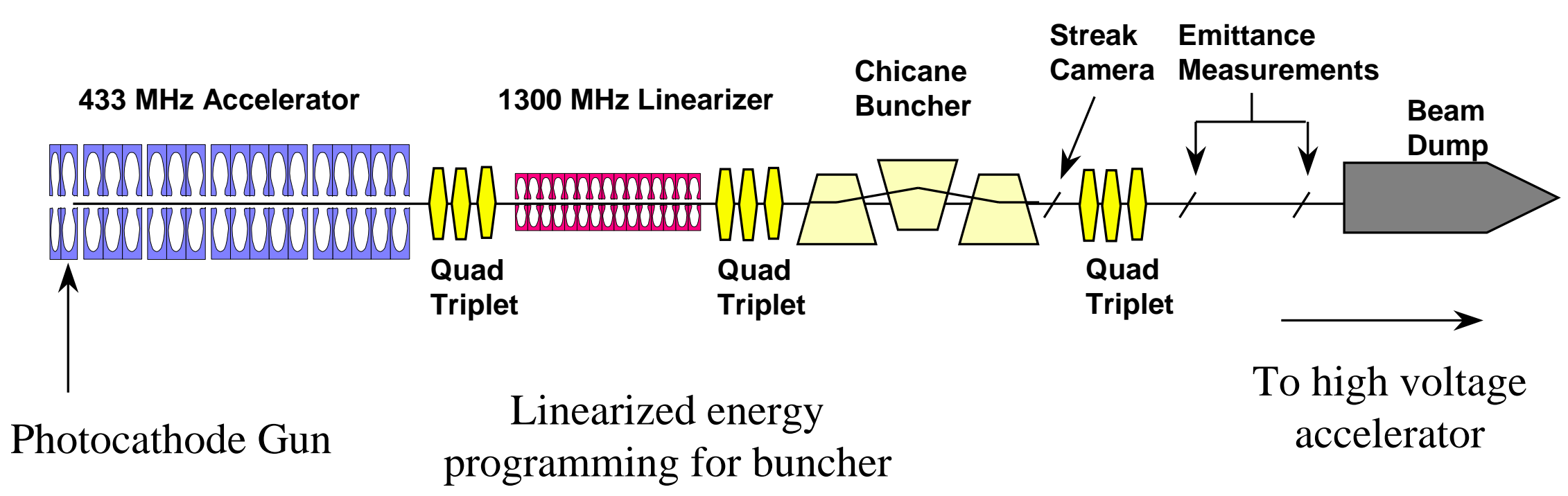




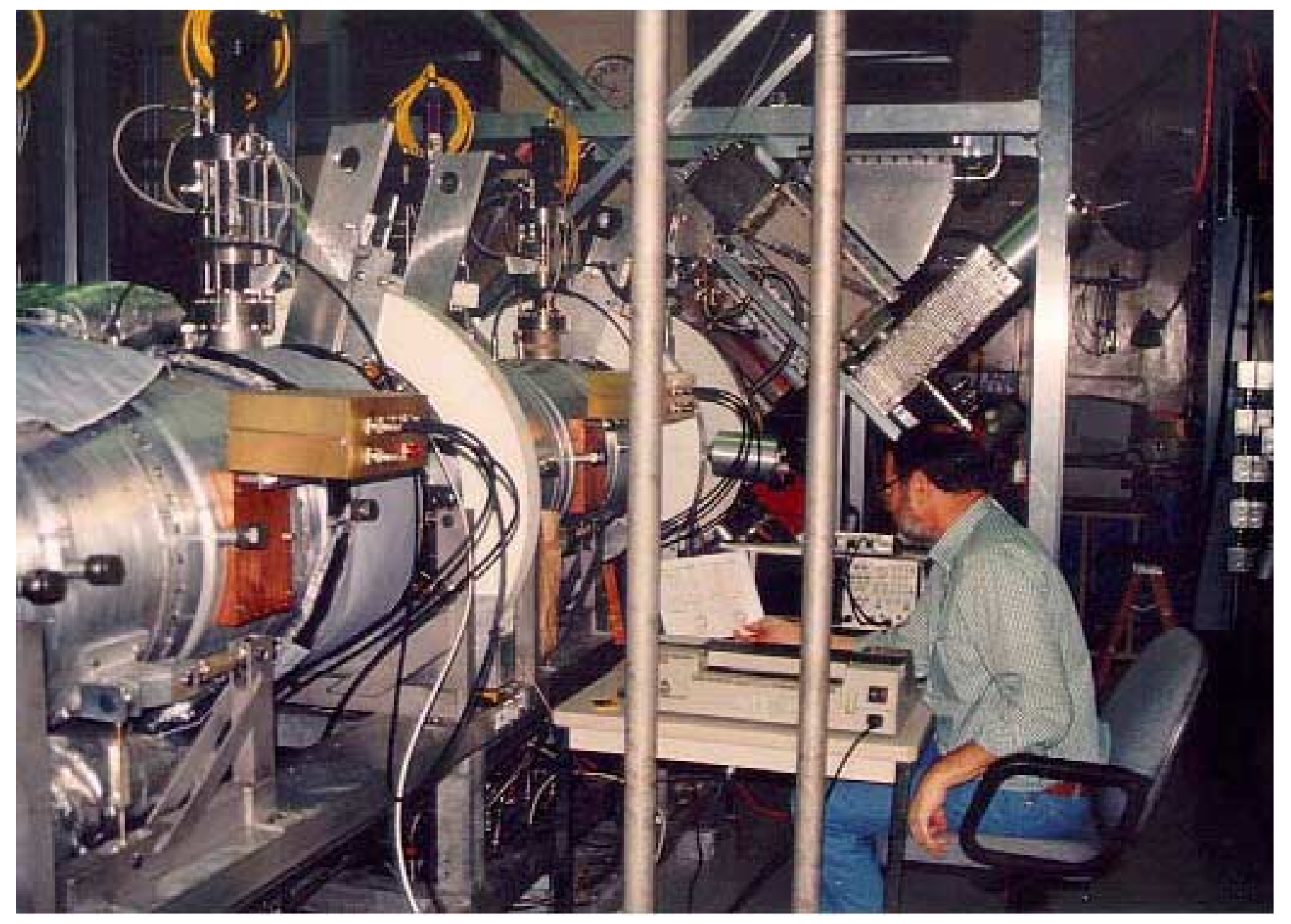

D.H. Dowell/PERL Workshop; Jan 22-23, 2001 
Cooling and RF Feed for $433 \mathrm{MHz}$ 5-Cell Section

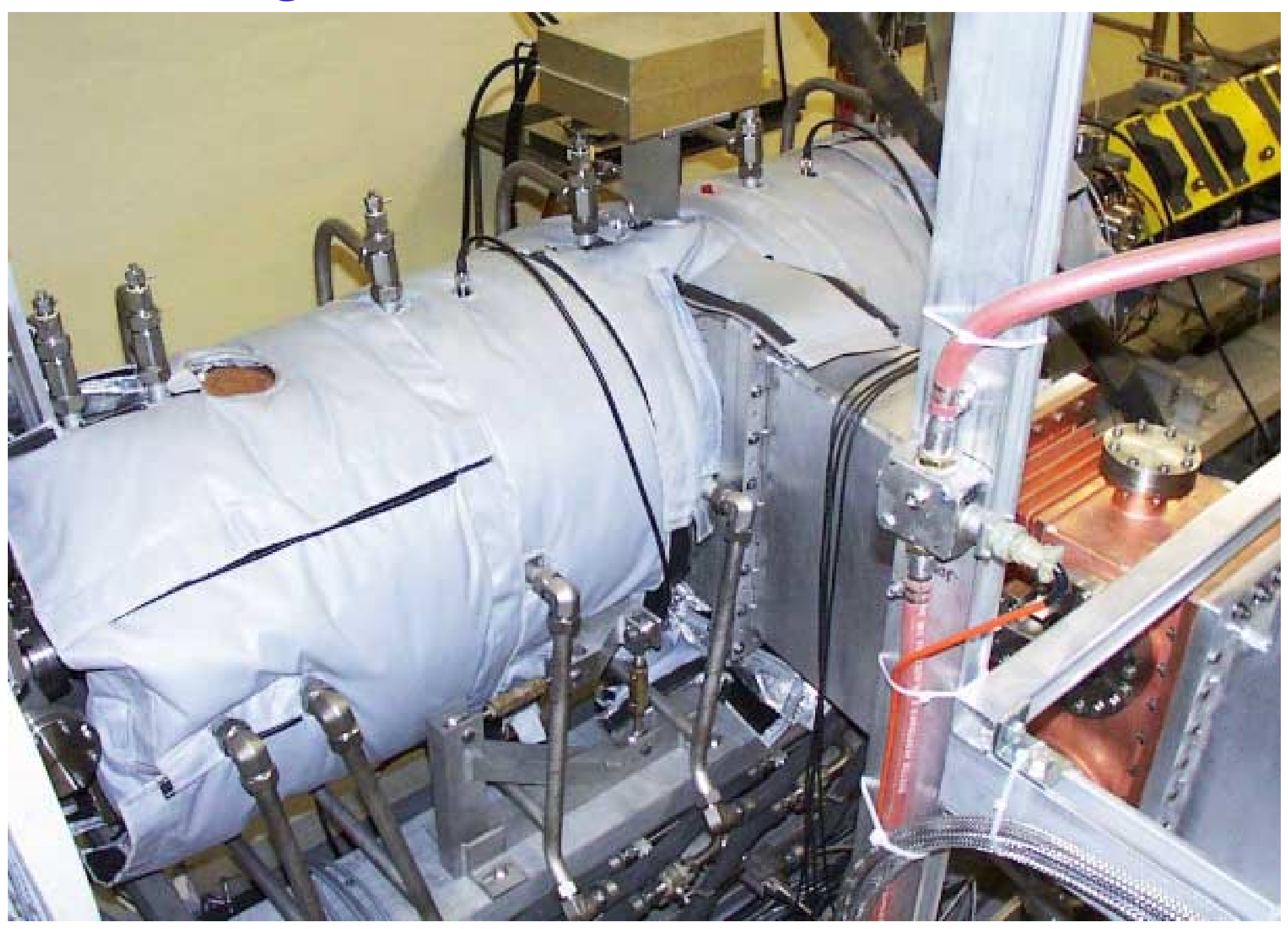

D.H. Dowell/PERL Workshop; Jan 22-23, 2001 


\section{3-Cell and 5-Cell APLE Cavity Booster}

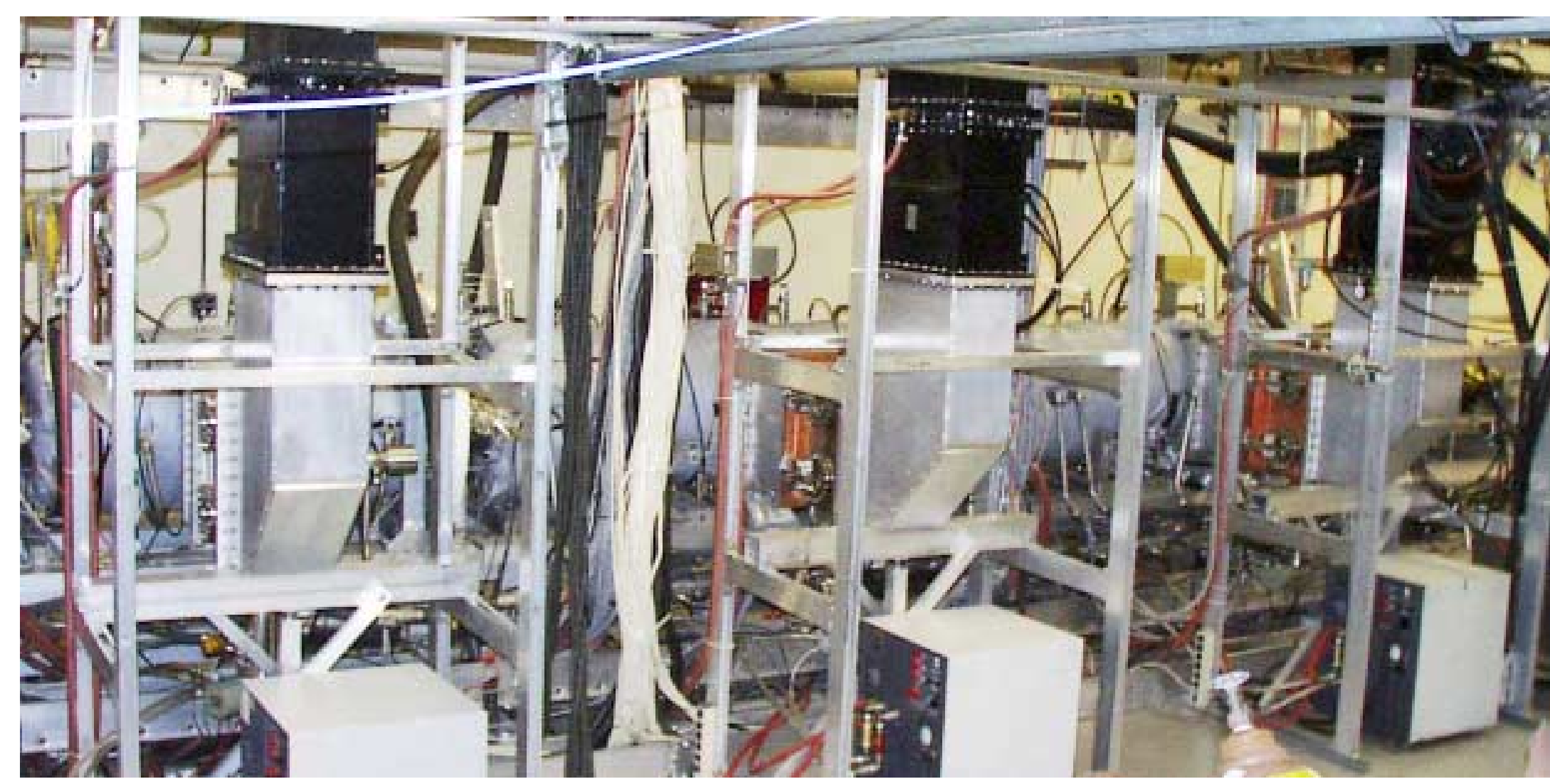

3-Cell Accelerator Cavity
5-Cell Accelerator Cavities 


\section{RF Characteristics of $433 \mathrm{MHz}$ Booster Cavities}

RF characteristics of APLE 5-cell cavity \# 2

Derived from measurements

\begin{tabular}{|ll|cl|}
\hline \multicolumn{2}{|c|}{ Parameter } & \multicolumn{2}{c|}{ Value } \\
\hline \hline frequency & $f$ & 433.33 & $\mathrm{MHz}$ \\
\hline shunt impedance & $R=V^{2} / P_{c}$ & 41.5 & $\mathrm{M} \Omega$ \\
\hline coupling coefficient & $\beta$ & 2.56 & \\
\hline
\end{tabular}

Operating parameters of APLE 5-cell cavity \# 2

Optimized for PERL conditions.

\begin{tabular}{|ll|rl|}
\hline \multicolumn{2}{|c|}{ Parameter } & \multicolumn{2}{c|}{ Value } \\
\hline nominal accelerating voltage & $V$ & 3.5 & $\mathrm{MV}$ \\
\hline wall loss power & $P_{c}$ & 295 & $\mathrm{~kW}$ \\
\hline beam power @ $I_{a v g}=200 \mathrm{~mA}$ & $P_{b}$ & 700 & $\mathrm{~kW}$ \\
\hline forward power required & $P_{k}$ & 1015 & $\mathrm{~kW}$ \\
\hline reflected power & $P_{r}$ & 20 & $\mathrm{~kW}$ \\
\hline
\end{tabular}

Tables provided by A.M. Vetter. 


\section{5-Cell APLE Cavity Power Allocation Peak Energy Gain $=3.5 \mathrm{MV}$}

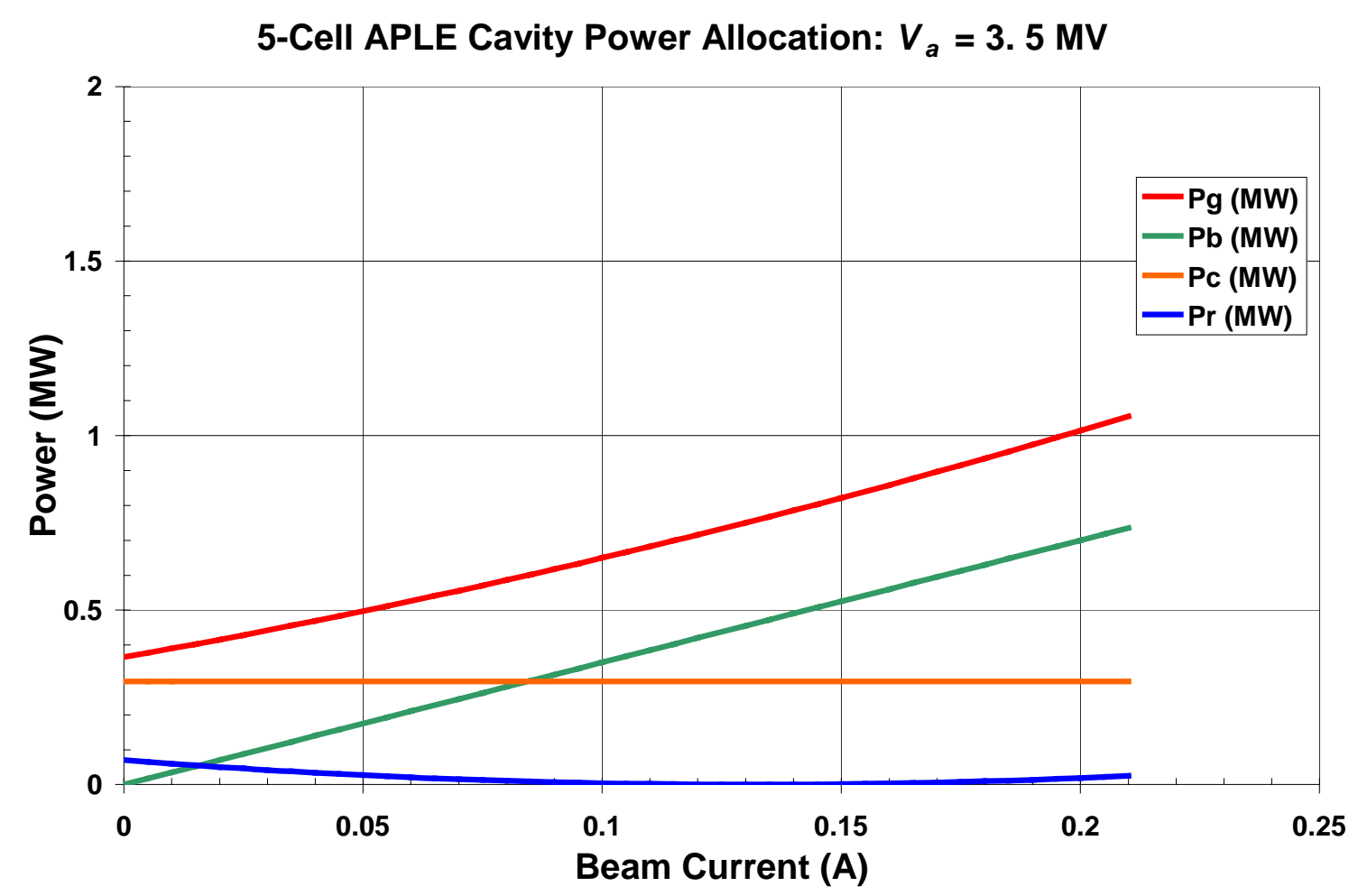

Generator, beam, cavity loss, and reflected power as functions of beam current for 5-cell APLE cavity operation at 3.5 MV. Optimized for PERL operation.

Figure courtesy of A.M. Vetter. 


\section{$1300 \mathrm{MHz}$ Linearizer and Three-Dipole Chicane Compressor}

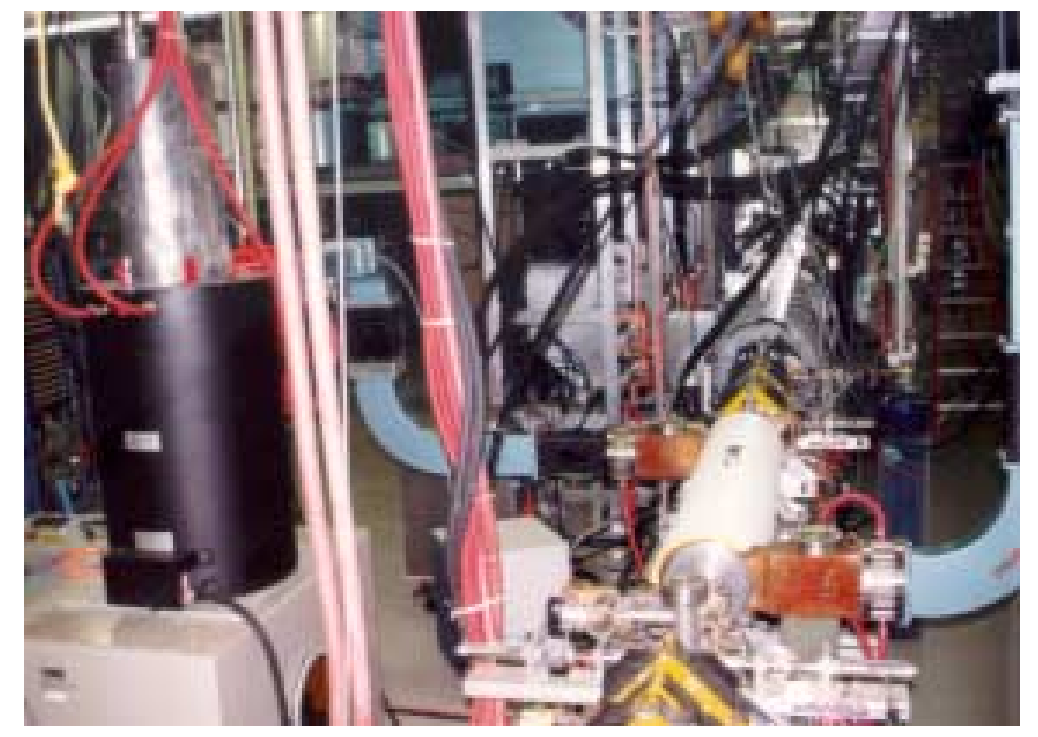

$1300 \mathrm{MHz}$ (third harmonic) energy spectrum programming

for bunch compression

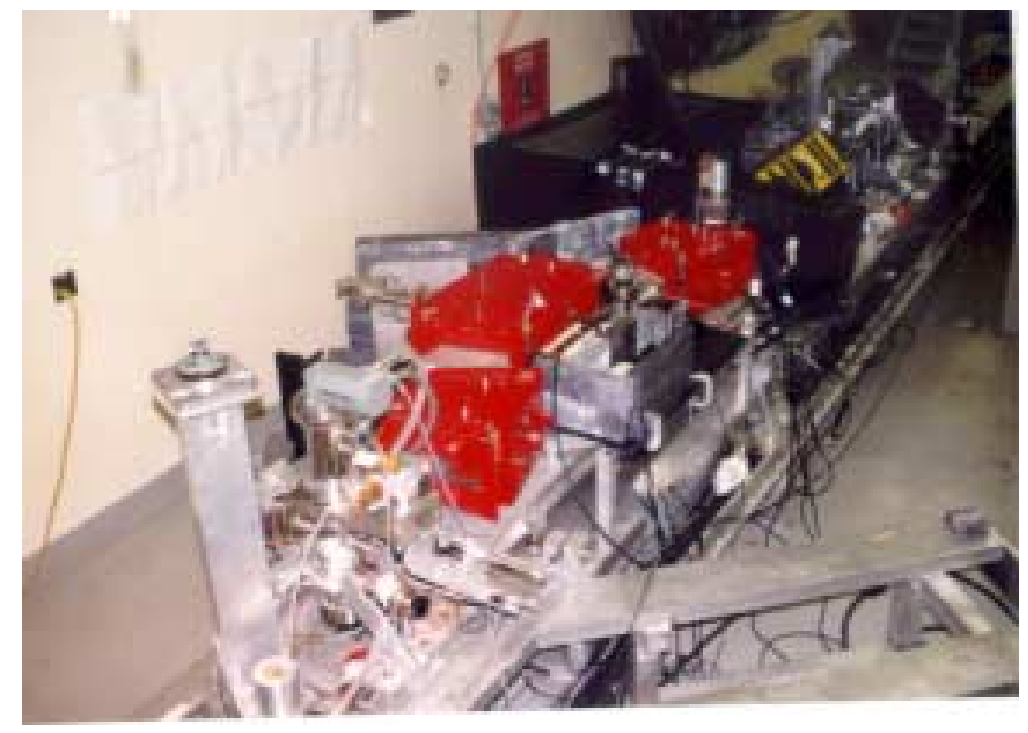

Three dipole magnetic buncher and diagnostics 


\section{Boeing Chicane Buncher}

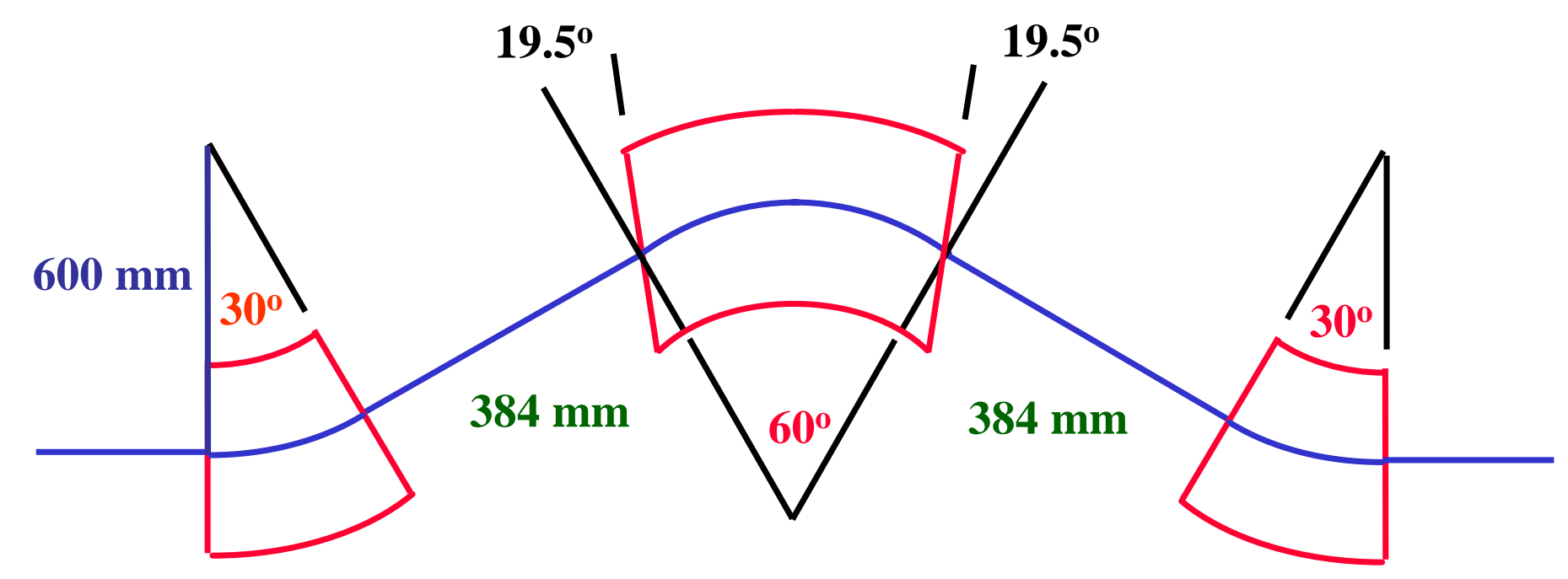

Achromatic chicane composed of three $n=1 / 2$ dipoles. 
Pulse compression occurs at two linearizer phases, but the pulse is linearized only at the decelerating phase and at 1/9 the $433 \mathrm{MHz}$ RF field.
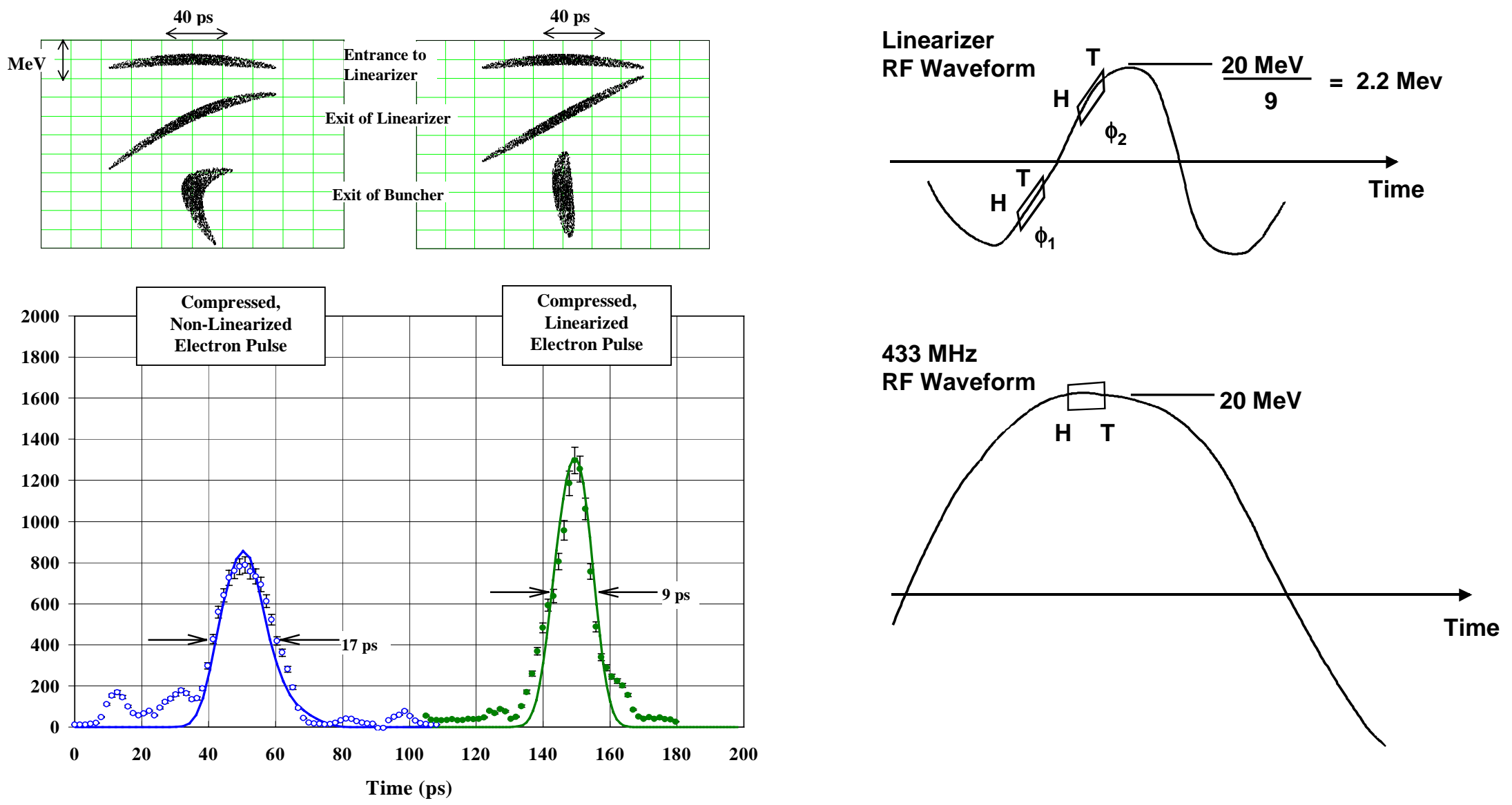

D.H. Dowell/PERL Workshop; Jan 22-23, 2001 


\section{Bend Plane Emittance Growth During Pulse Compression}

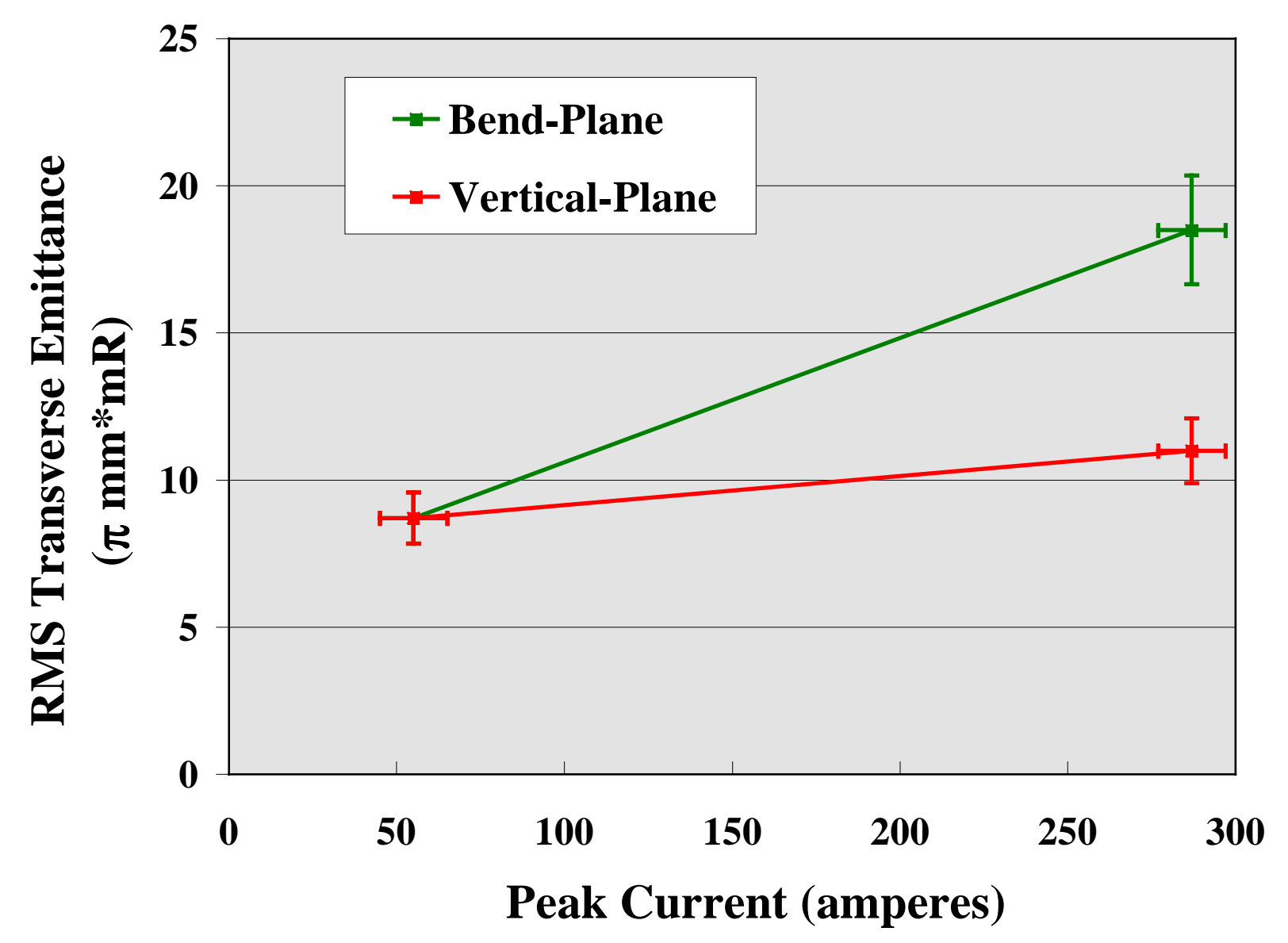




\title{
Coherent Synchrotron Radiation Induced Emittance Growth
}

\author{
Electron Microbunch Traveling in an Arc
}

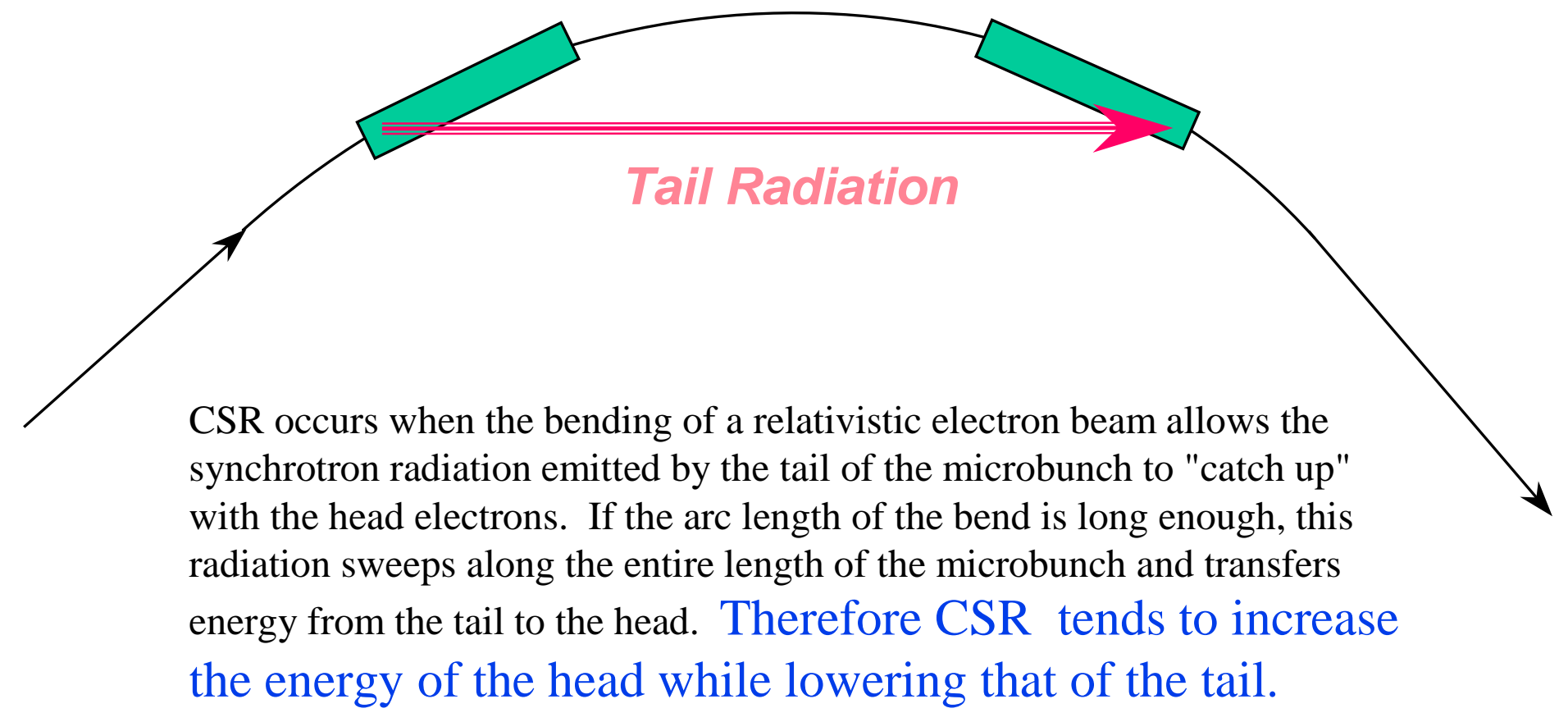

Ref: Y.S. Derbenev et al., DESY TESLA-FEL Technical Note 95-05(1995) 


\section{Transient CSR Radiation at the Magnetic Field Boundary}

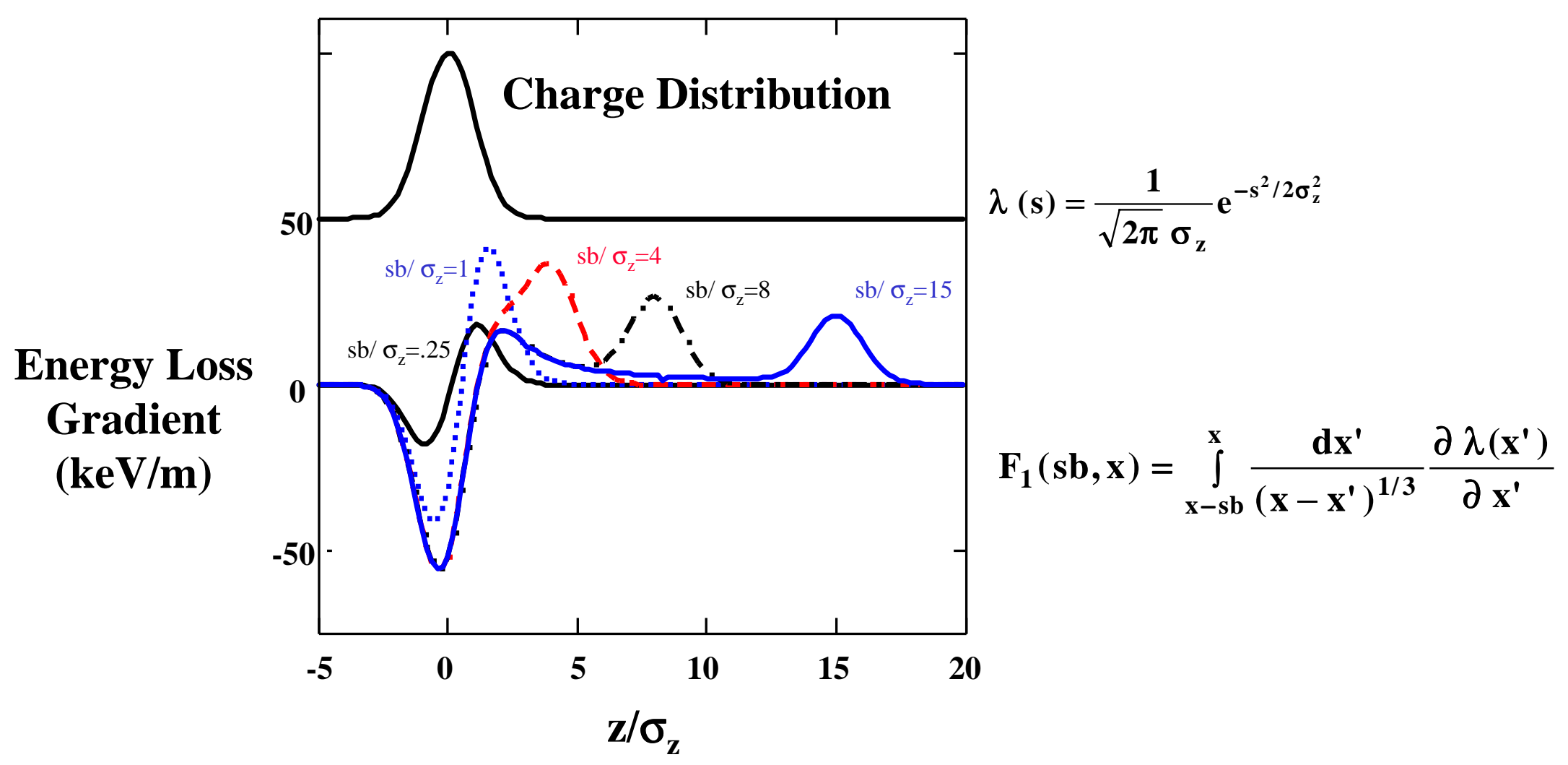

Ref: D.H. Dowell and P.G. O'Shea, “Coherent Synchrotron Radiation Induced Emittance Growth in a Chicane Buncher", contribution to PAC'97. 


\section{Coherent Synchrotron Radiation Induces Correlated Emittance Growth}

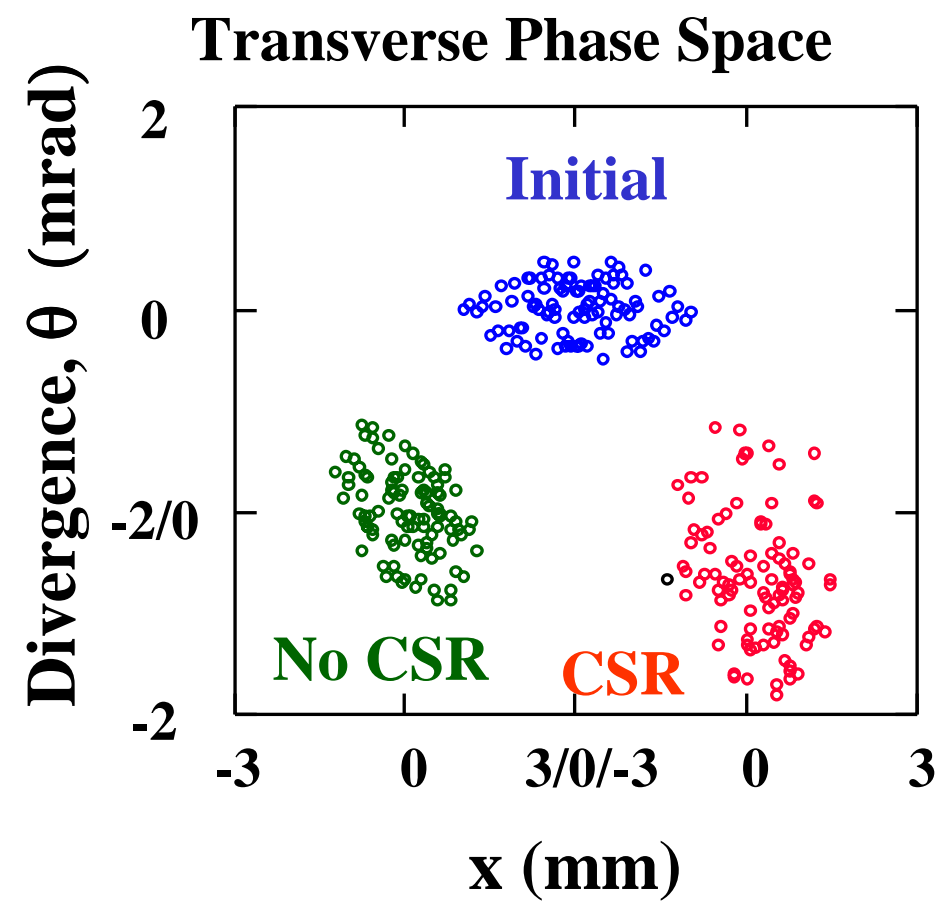

The initial and final transverse phase space distributions, with and without CSR, when the microbunch is compressed. The distributions are offset for display only.

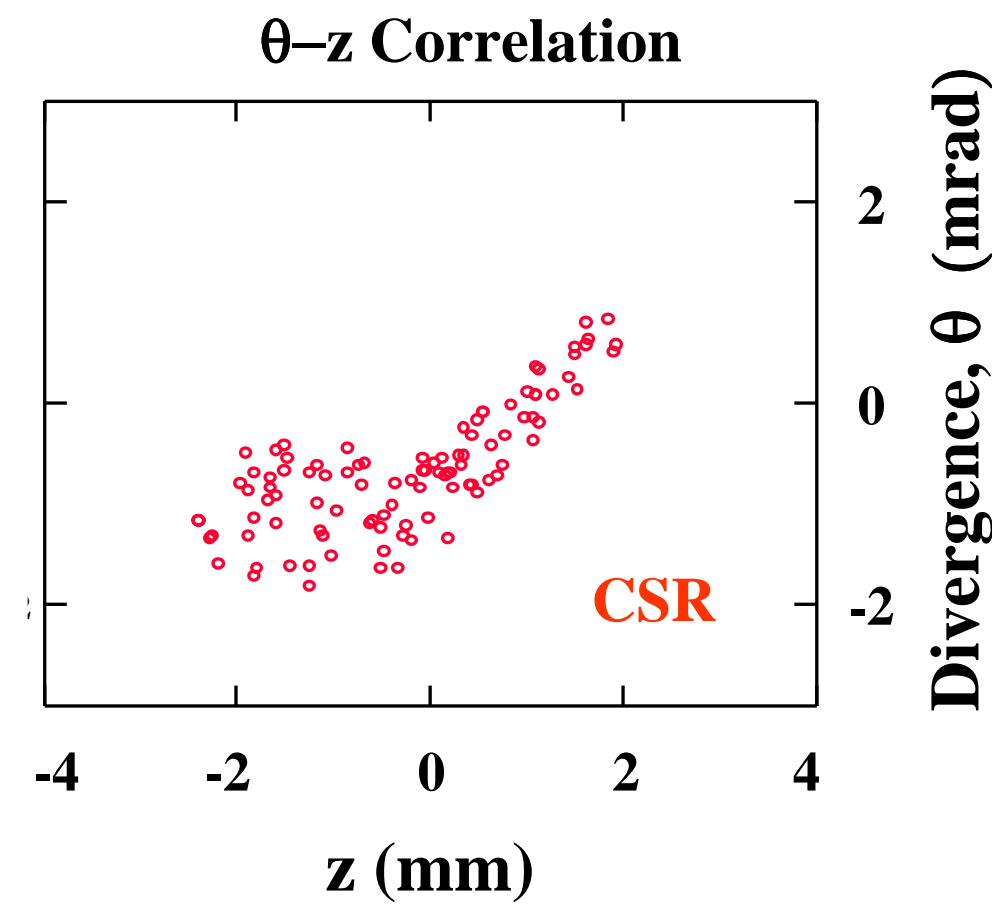

The correlation of divergence with longitudinal position at the exit of the chicane due to CSR. 


\section{Comparison of Experiment with PARMELA and CSR Emittance Calculations.}

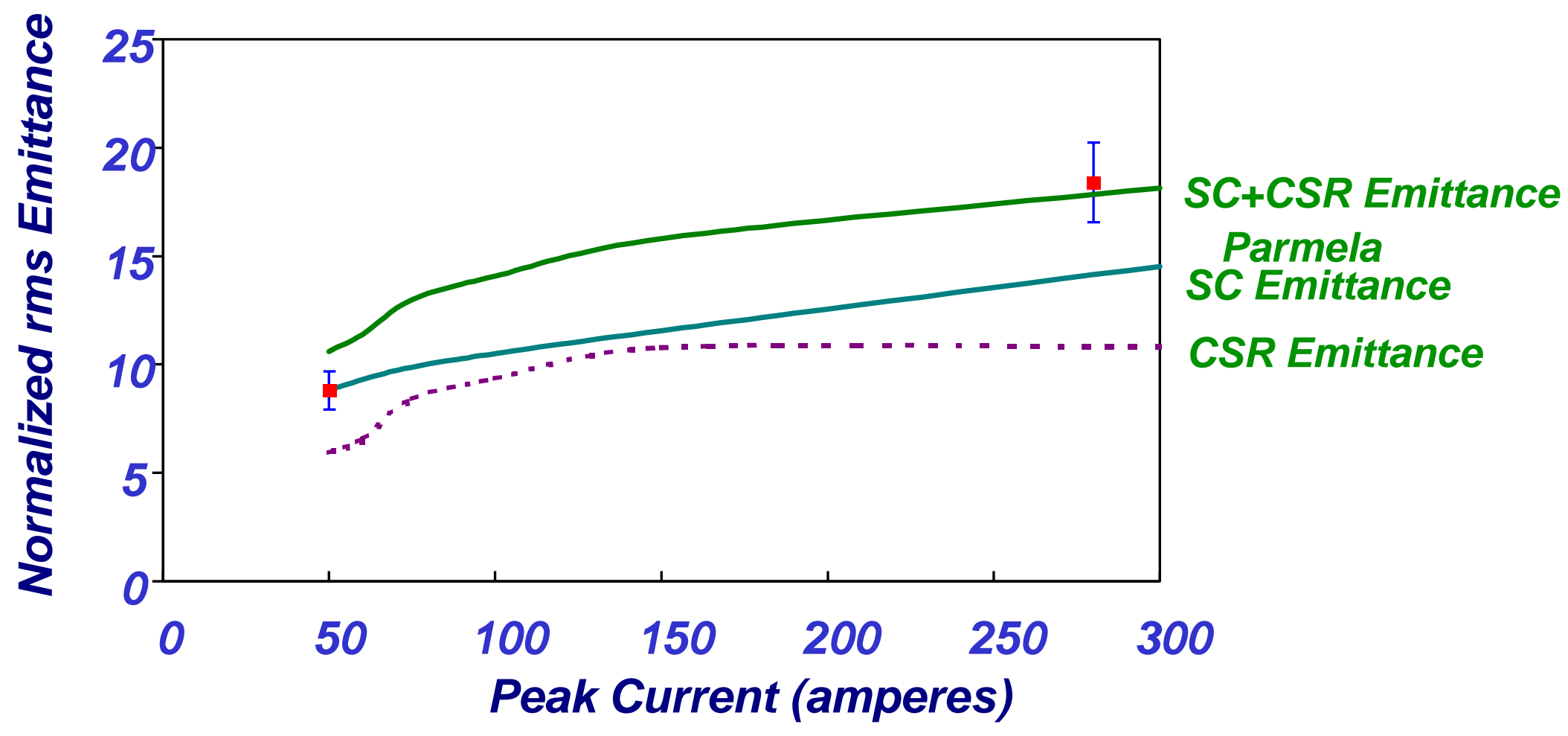




\section{Compressor and CRS Diagnostic at LEUTL (APS-ANL)}

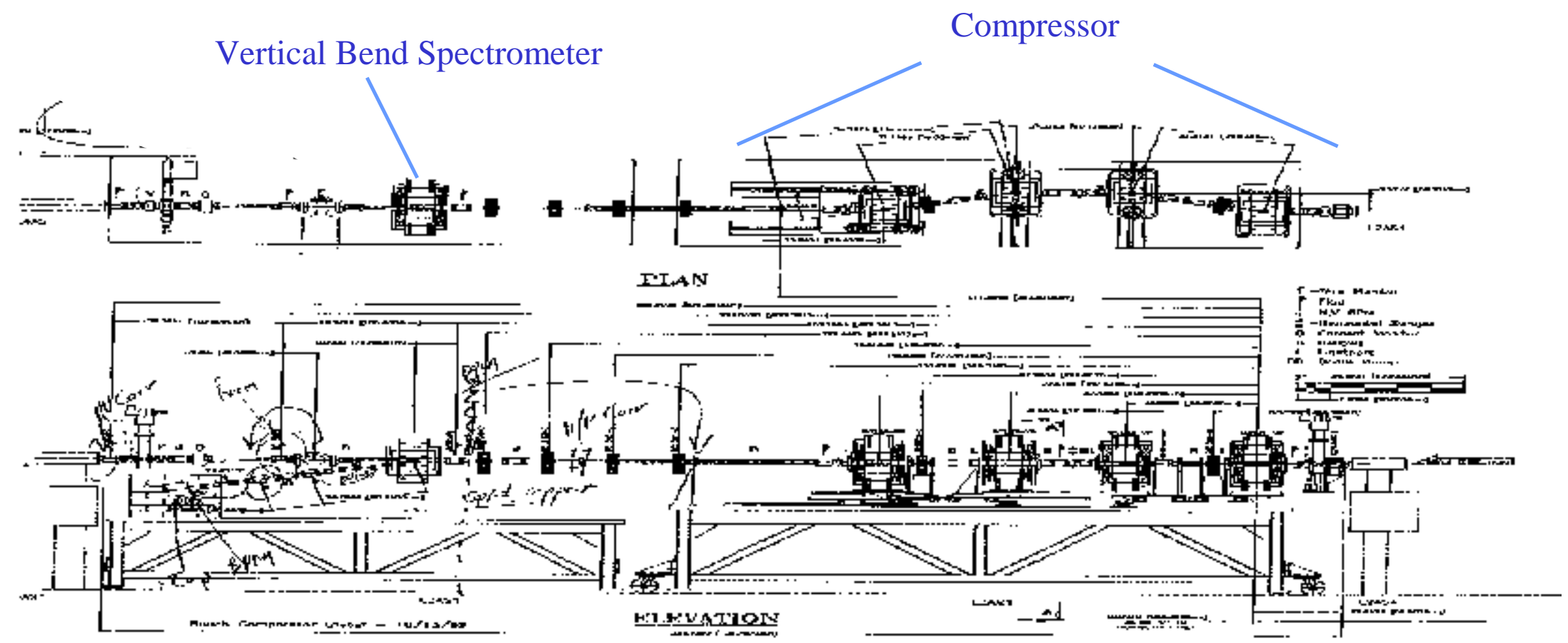




\section{Dowell's CSR diognostic simulation}

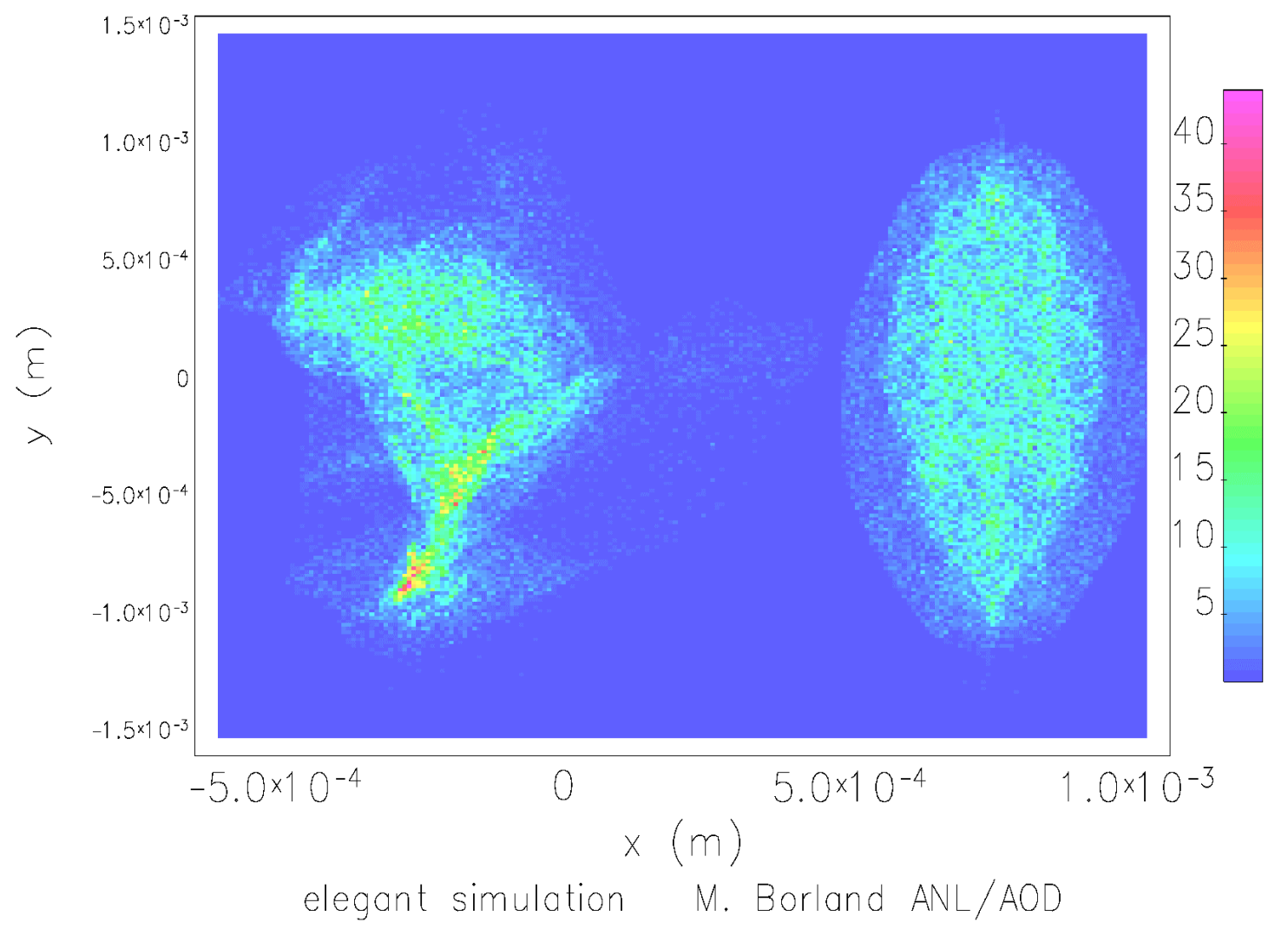

D.H. Dowell/PERL Workshop; Jan 22-23, 2001 


\section{Strawman Design for PERL: Compression Re-circulation}




\section{Compression With PERL Parameters, Linearized Phase Space}

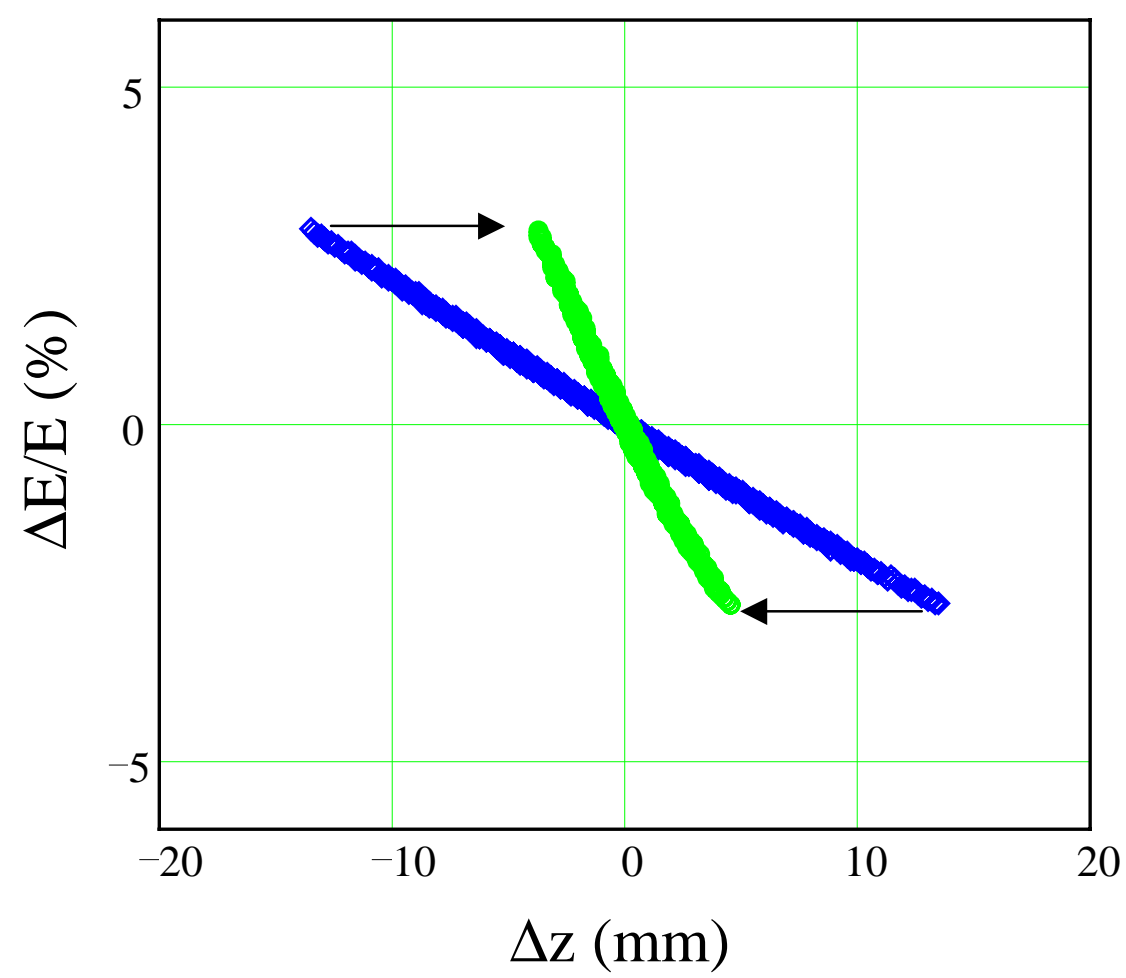

Boeing Style Compressor $10 \mathrm{MeV}$ Compression

Bunch Length: 49=>15 ps

Emittance (CSR only): 1=>1.3 microns

Peak Current: 10=>34 amps

D.H. Dowell/PERL Workshop; Jan 22-23, 2001
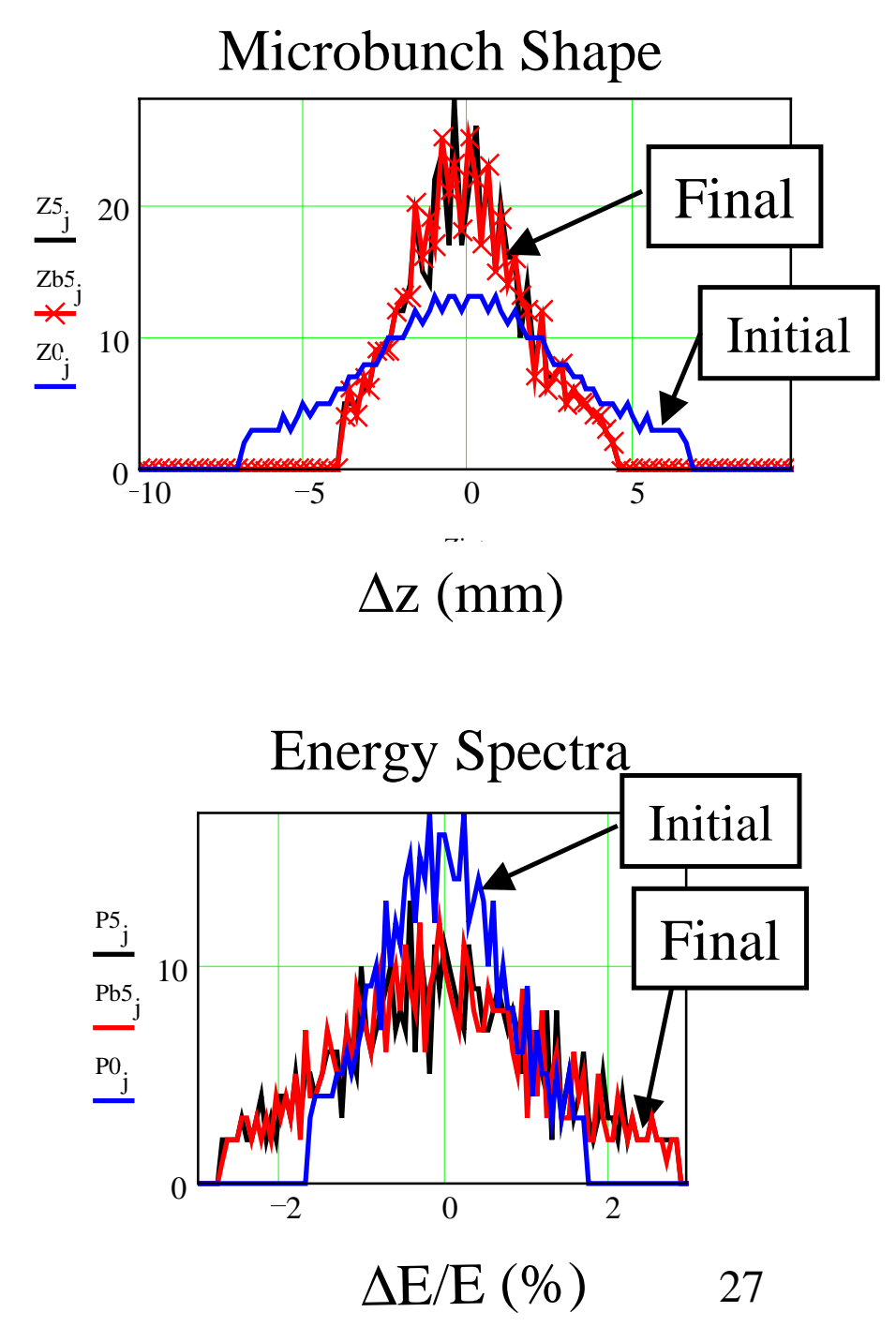


\section{Compression With PERL Parameters, Non-Linearized Phase Space}

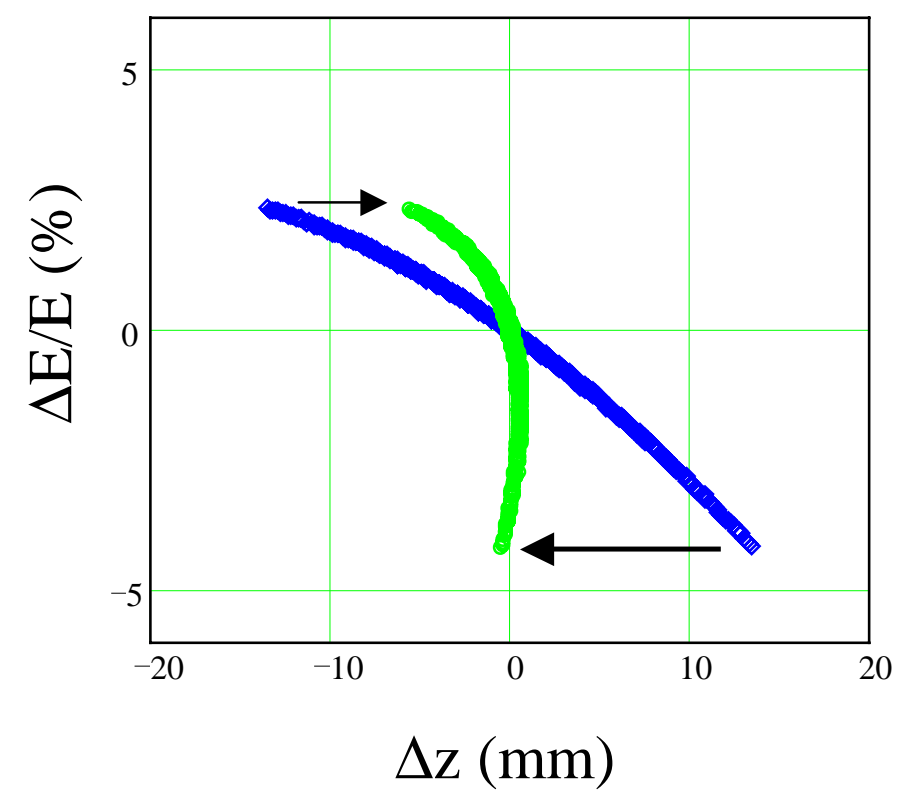

Boeing Style Compressor $10 \mathrm{MeV}$ Compression Bunch Length: $47=>10$ ps

Emittance (CSR only): 1=>1.05 microns (?)

Peak Current: $10=>48$ amps

D.H. Dowell/PERL Workshop; Jan 22-23, 2001
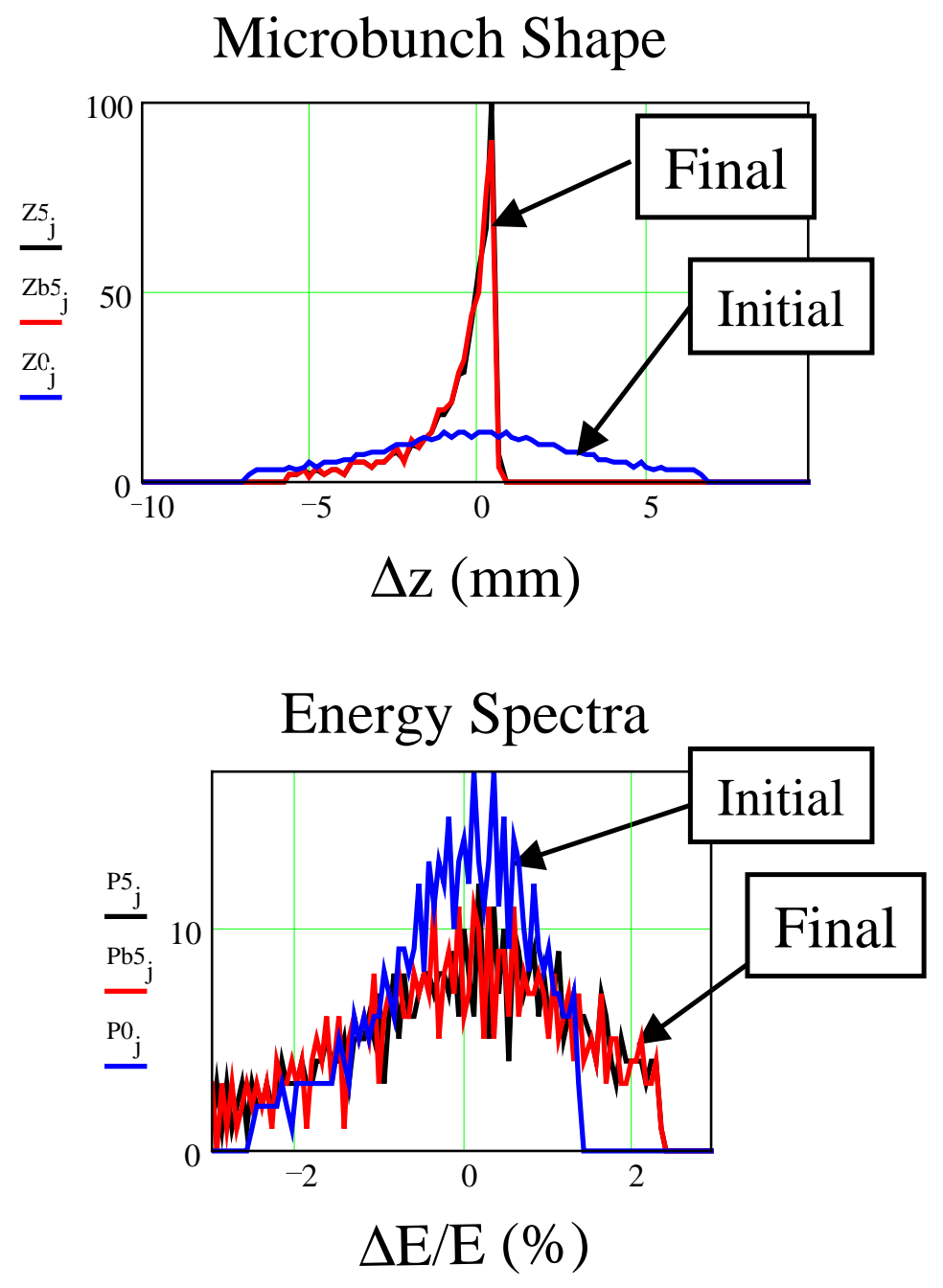


\section{Possible PERL Configuration}

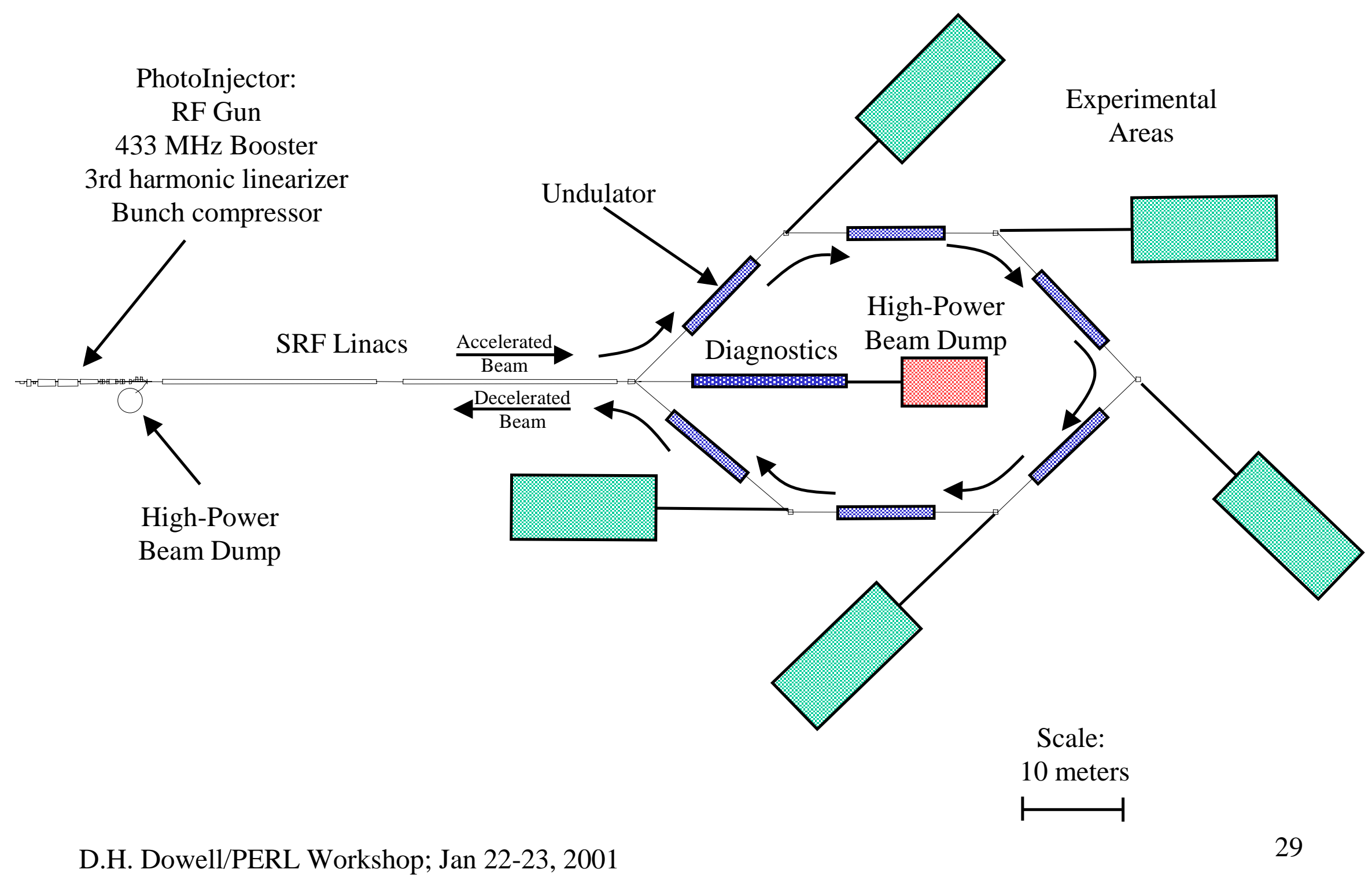




\section{Summary and Conclusions}

433 MHz-Based PhotoInjector Configuration:

Gun, Booster, Linearizer, Compressor

Non-linearity Due to RF Waveform

Requires Third-Harmonic Linearizer

$433 \mathrm{MHz}$ APLE Cavities Satisfy PERL Requirements

Short 1300 MHz SRF Section Needed for Linearizer

Preliminary CSR Calculations Show Some Emittance Growth Full Start-to-End SC+Wakes Calculation Still Needed

Counter-Propagating Beams in E-Recovery Proposed 


\section{Results of the Fermilab High-Brightness RF Photo-injector}

Jean-Paul Carneiro (Fermilab and Université d'Orsay), For the A0 Group (N. Barov, D. Carrigan, M. Champion, D. Edwards, H. Edwards, M. Fitch, J. Fuesrt, W. Hartung, A. Melissinos and J. Santucci )

Presented at the Workshop on Photo-injector for Energy Recovery Linac, BNL, 01-22-01. 


\section{BEAM PARAMETERS}

- Total Energy $\approx 17 \mathrm{MeV}$ (typical run)

- Gun peak accelerating field $=35$ to $40 \mathrm{MV} / \mathrm{m}(4.5$ to $5 \mathrm{MeV})$

- Superconducting on axis cavity accelerating field $=12 \mathrm{MV} / \mathrm{m}$

• Energy spread @ $1 \mathrm{nC}=0.25 \%$

- Bunches per macropulse $=800($ design $), 10-100($ typical run $)$

- Bunch spacing $=1 \mu \mathrm{s}$

- Repetition Rate $=1-10 \mathrm{~Hz}$ (design), $1 \mathrm{~Hz}$ (typical run)

- Bunch charge $=1$ to $8 \mathrm{nC}$

- Bunch length @ $8 \mathrm{nC}$ uncompressed $\approx 10$ ps sigma (measured)

- Bunch length @ $8 \mathrm{nC}$ compressed $\approx 2$ ps sigma (measured)

- Peak current $@ 8 \mathrm{nC}$ for a compressed beam $\approx 1.6 \mathrm{kA}$ (measured)

- Beam radius at cathode $=1.5 \mathrm{~mm} @ 1 \mathrm{nC}, 3 \mathrm{~mm} @ 8 \mathrm{nC}$

- Transverse rms emittance, normalized @ 1nC $\approx 3-4$ mm-mrad (measured, uncompressed)

- Transverse rms emittance, normalized @ 8nC $\approx 13 \mathrm{~mm}-\mathrm{mrad}$ (measured, uncompressed) 


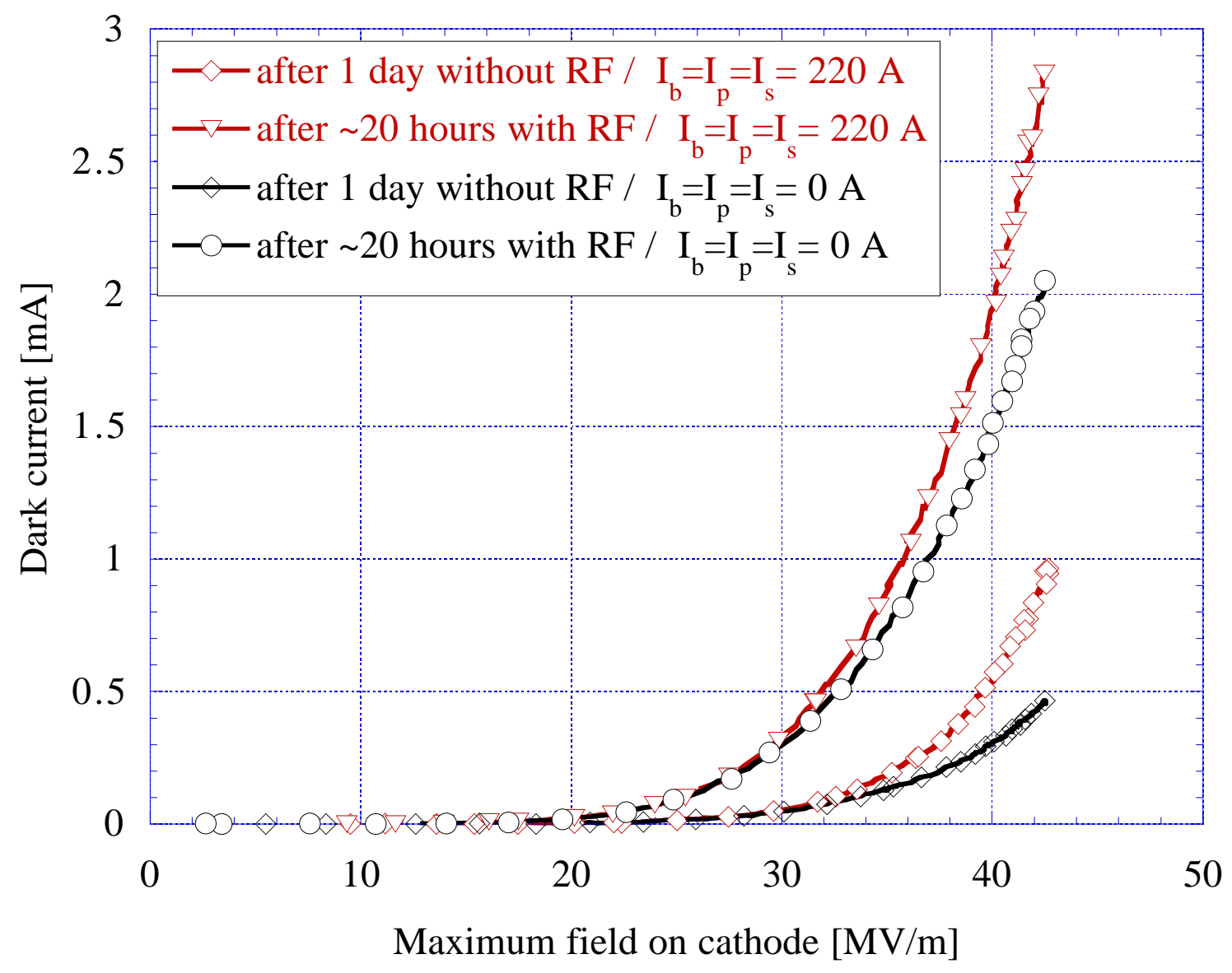



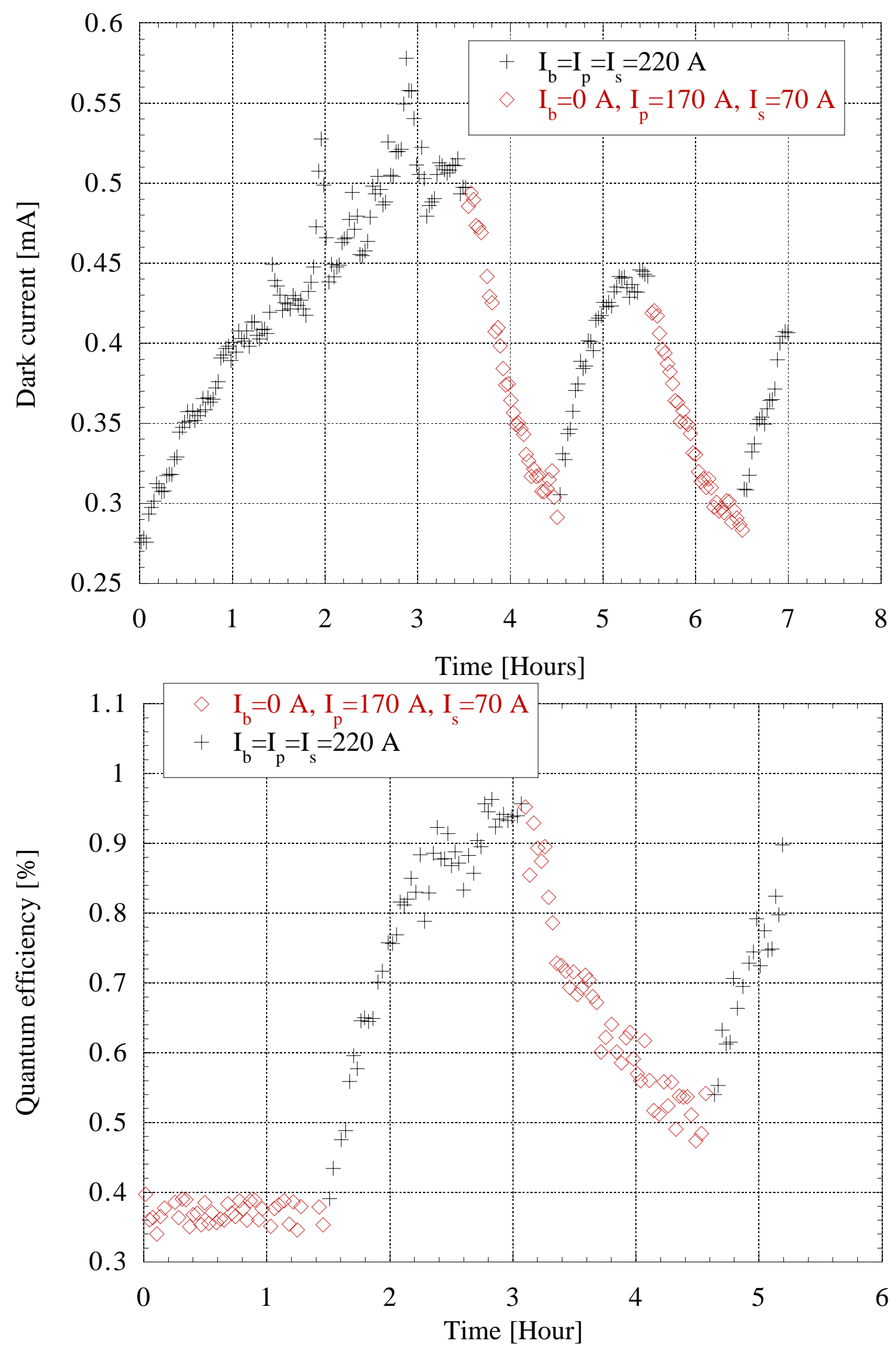


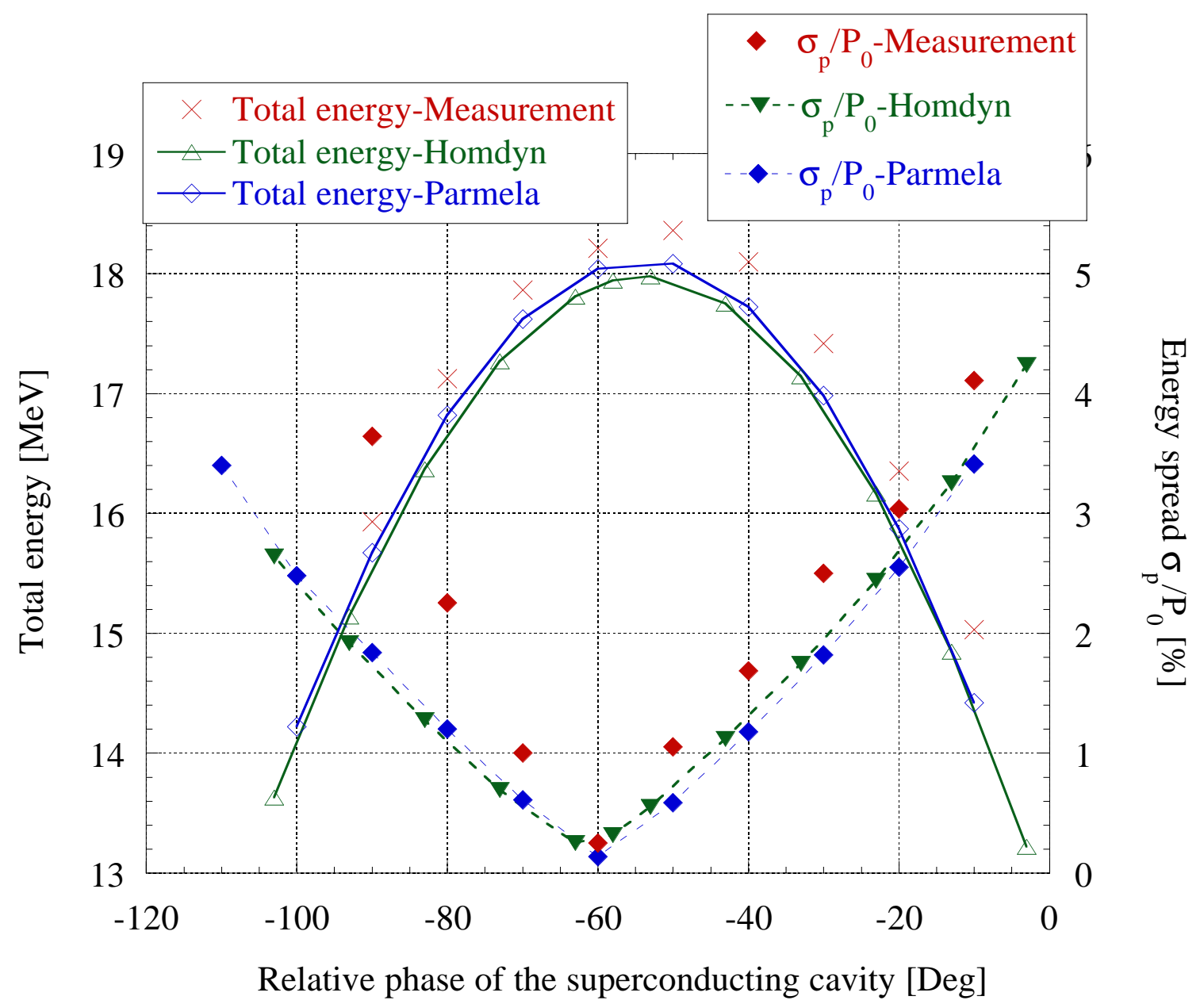




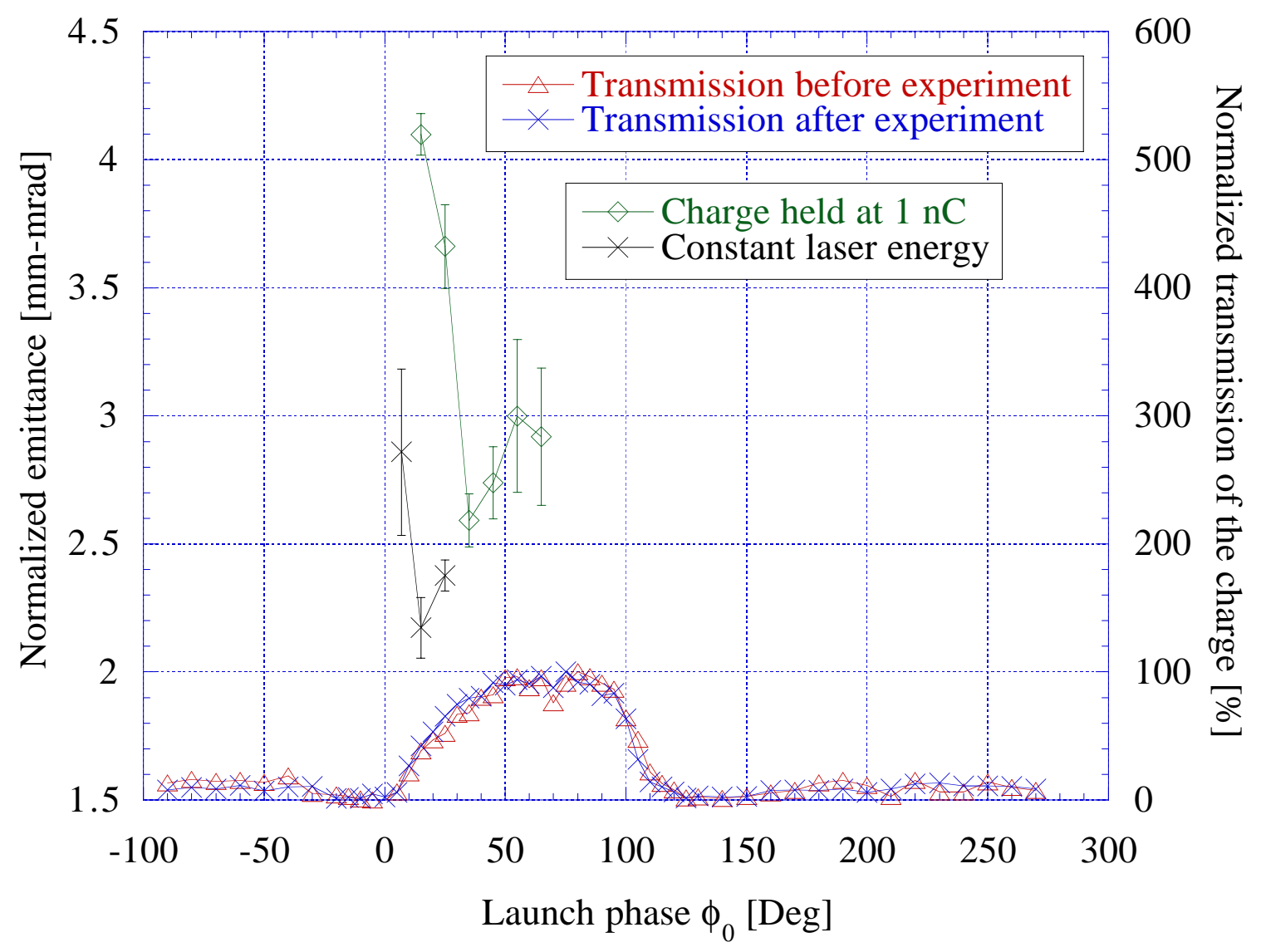



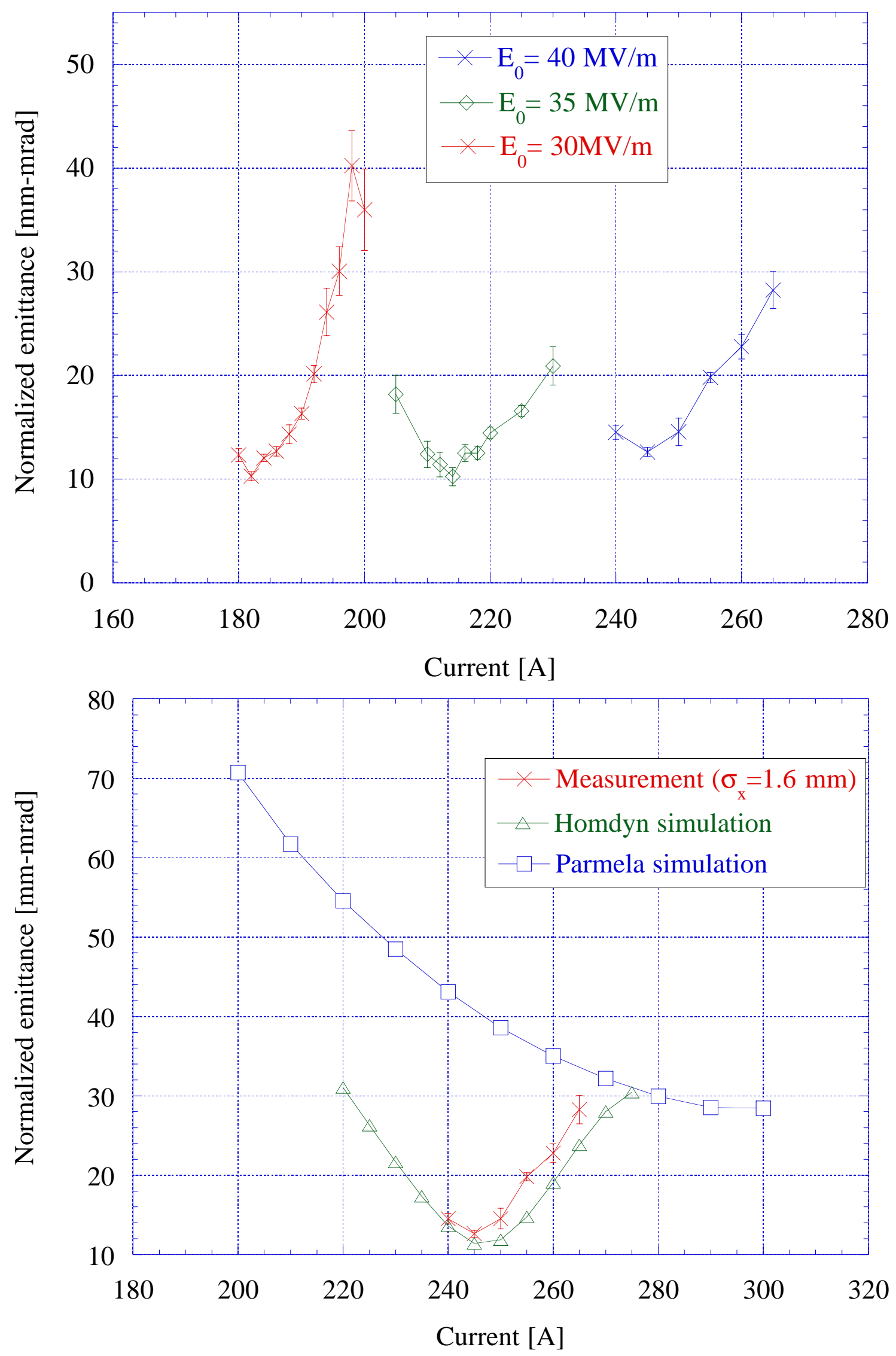

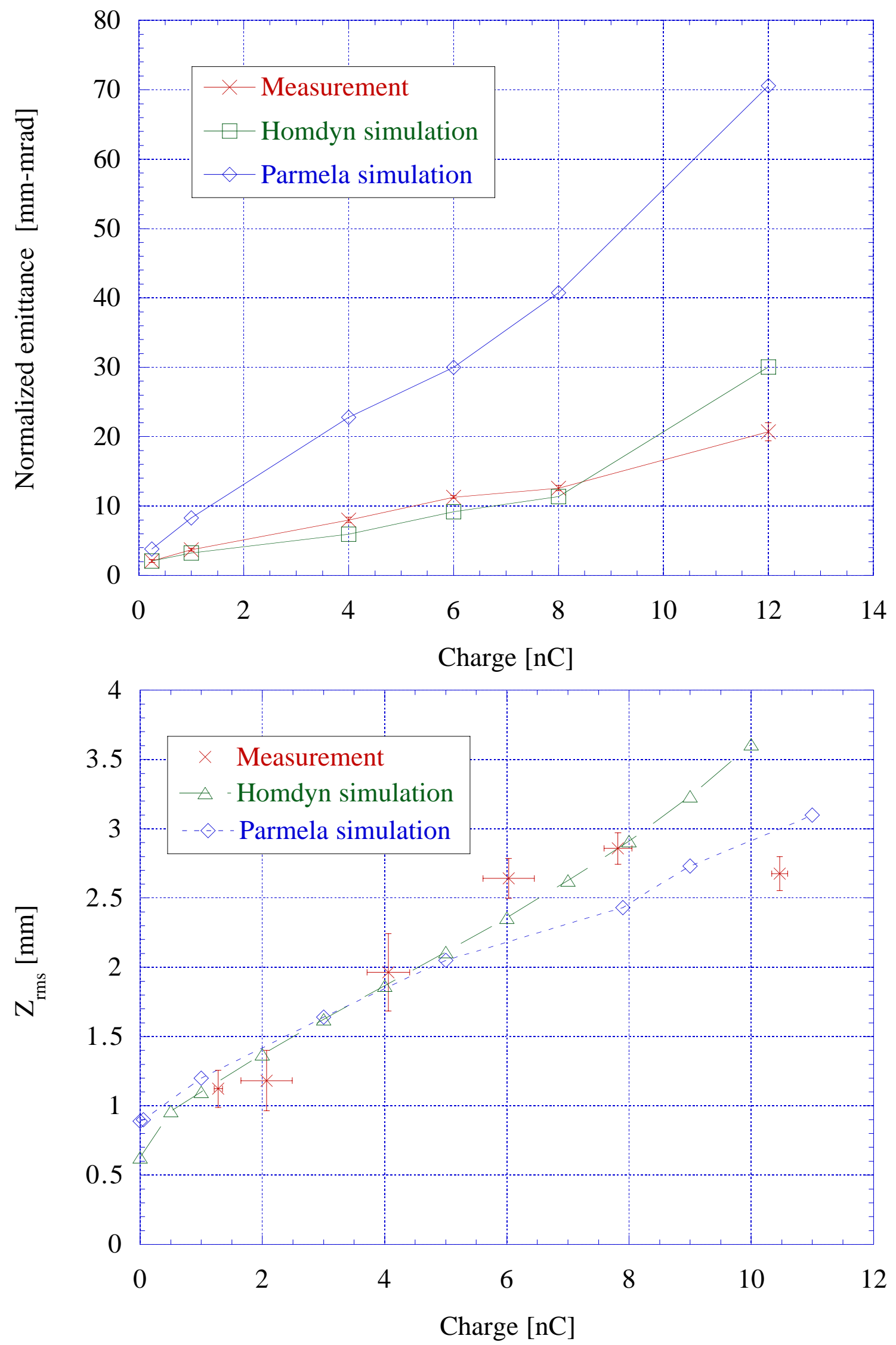


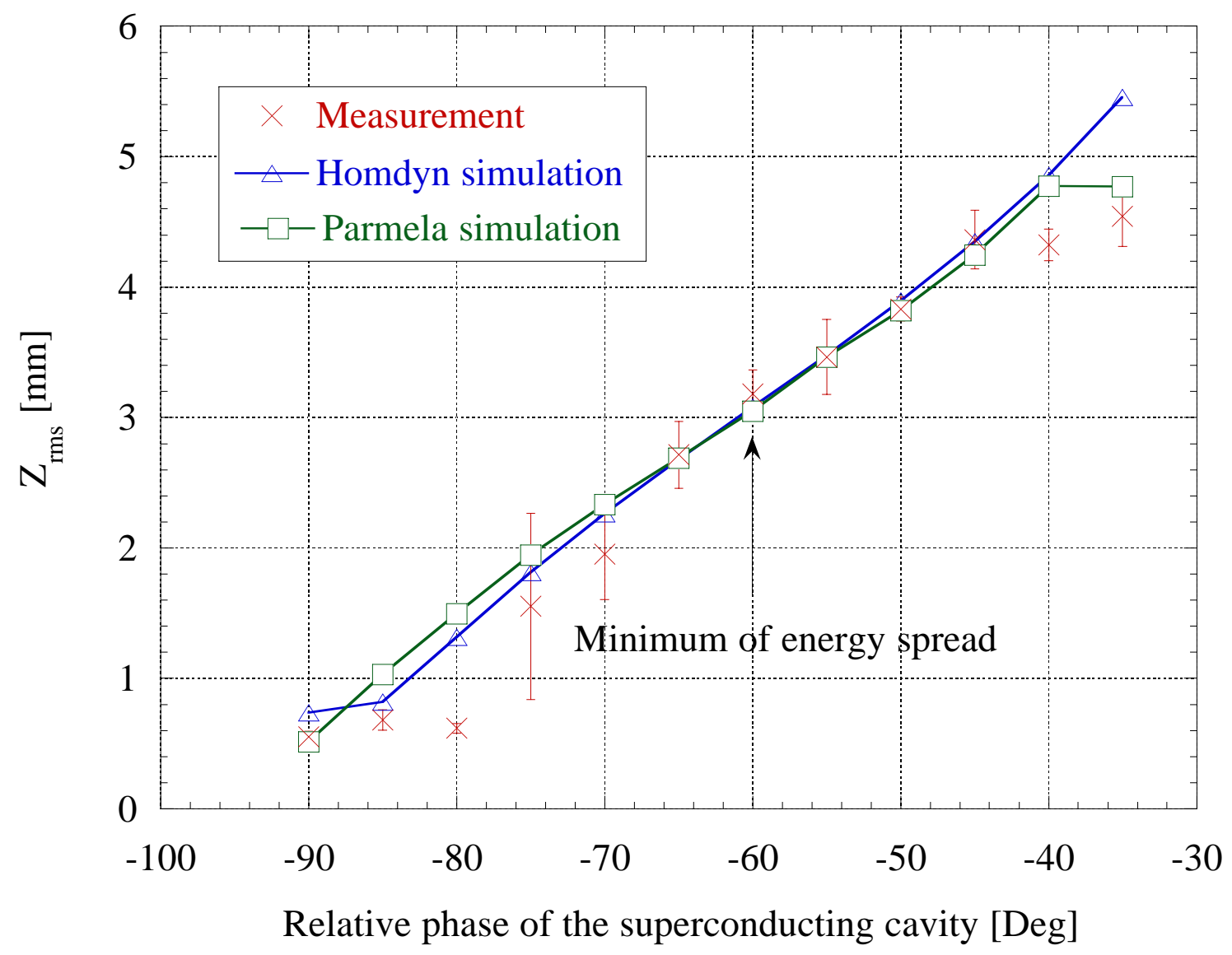




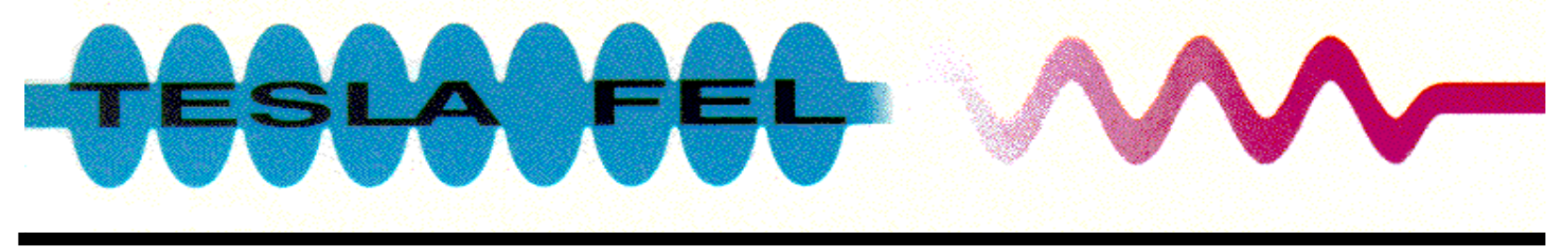

\title{
High Brightness Photo-Injectors for the TESLA X-FEL, TTF II User Facility and...
}

\author{
Ph. Piot \\ injector (beam dynamics) working group: \\ M. Ferrario (INFN-LNF), K. Flöttmann (DESY), B. Grigoryan
}

(YerPhi), T. Limberg (DESY) and Ph. Piot (DESY)

\section{Abstract}

The TESLA linear collider will integrate a X-ray (1 Angstr.) free-electron laser which requires electron bunches with $5000 \mathrm{kA}$ peak current and slice emittance (over the cooperation length) of $1.6 \mathrm{~mm}-\mathrm{mrd}$. On an other end the Tesla Test Facility will be upgraded to drive a User Facililty (TTF II) capables of lasing around $6 \mathrm{~nm}$. Both projects require high performance electron sources. We discuss the concept adopted for beam generation up to the emittance-dominated regime along with the first stage compression. 


\section{-Contents-}

Introduction: some learnings from Tesla Test Facility I

- overview

- injector

- compression

"Ingredients" for a new Injector Design:

- new gun design

- new simulation tools

Proposed TESLA X-FEL Injector [report TESLA-FEL 01-03]:

- overview and requirements for the TESLA X-FEL

- beam dynamics issues

Modification for TTF II User Facility:

- overview and requirement for the TTF User Facility

- practical issue

- discussion of a low charge option

Conclusions and Future Directions

- the Test Facility in DESY-Zeuthen (Berlin)

- ... 
TTF phase I - Injector Overview-

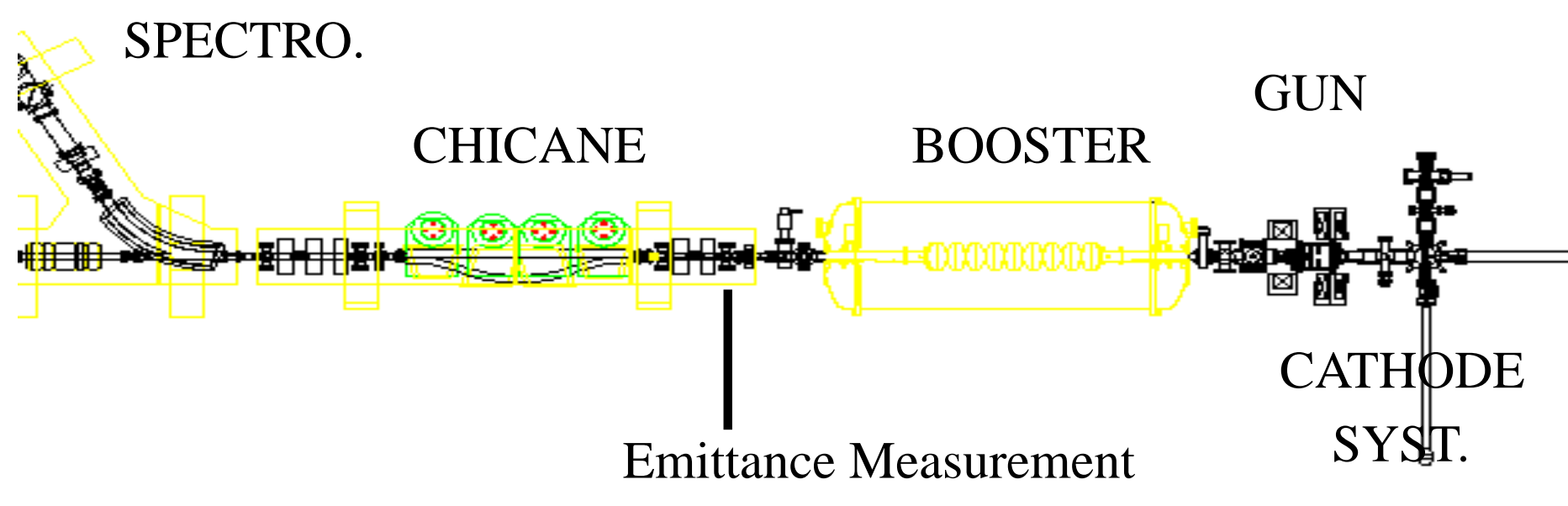

TYPICAL OPERATION: $1 \mathrm{nC}$ (FEL mode), 4 nC (LC mode)

LASER: Gaussian pulse ( 5 ps rms $->12$ ps)

$\lambda=260 \mathrm{~nm}$

for $1 \mathrm{nC}, \mathrm{r}=1.5 \mathrm{~mm}$

GUN: 1+1/2 L-Band (f=1.3 GHz) Gun "FERMI GUN"; peak field $=35 \mathrm{MV} / \mathrm{m}$

RF "flat top" up to $800 \mathrm{msec}$

"two solenoids splitted scheme"

BOOSTER: single 9-cell TESLA cavity

average gradient $11.5 \mathrm{MV} / \mathrm{m}$

phase chosen to compensate correlated energy spread

from gun

CHICANE: not commissioned yet!

DIAGNOSTICS: spectrometer line for energy + energy spread four OTR/ YaG / alumina screens

one multislit device for emittance meas.

several locations where emittance can be measured with the "quad scan" technique 


\section{EMITTANCE MEASUREMENTS at 1nC}

Most of the measurement performed with the multislit mask, but also with the quadrupole scan technique. Measurements have been compared with simulations with ASTRA.

Sensitivity to gun phase

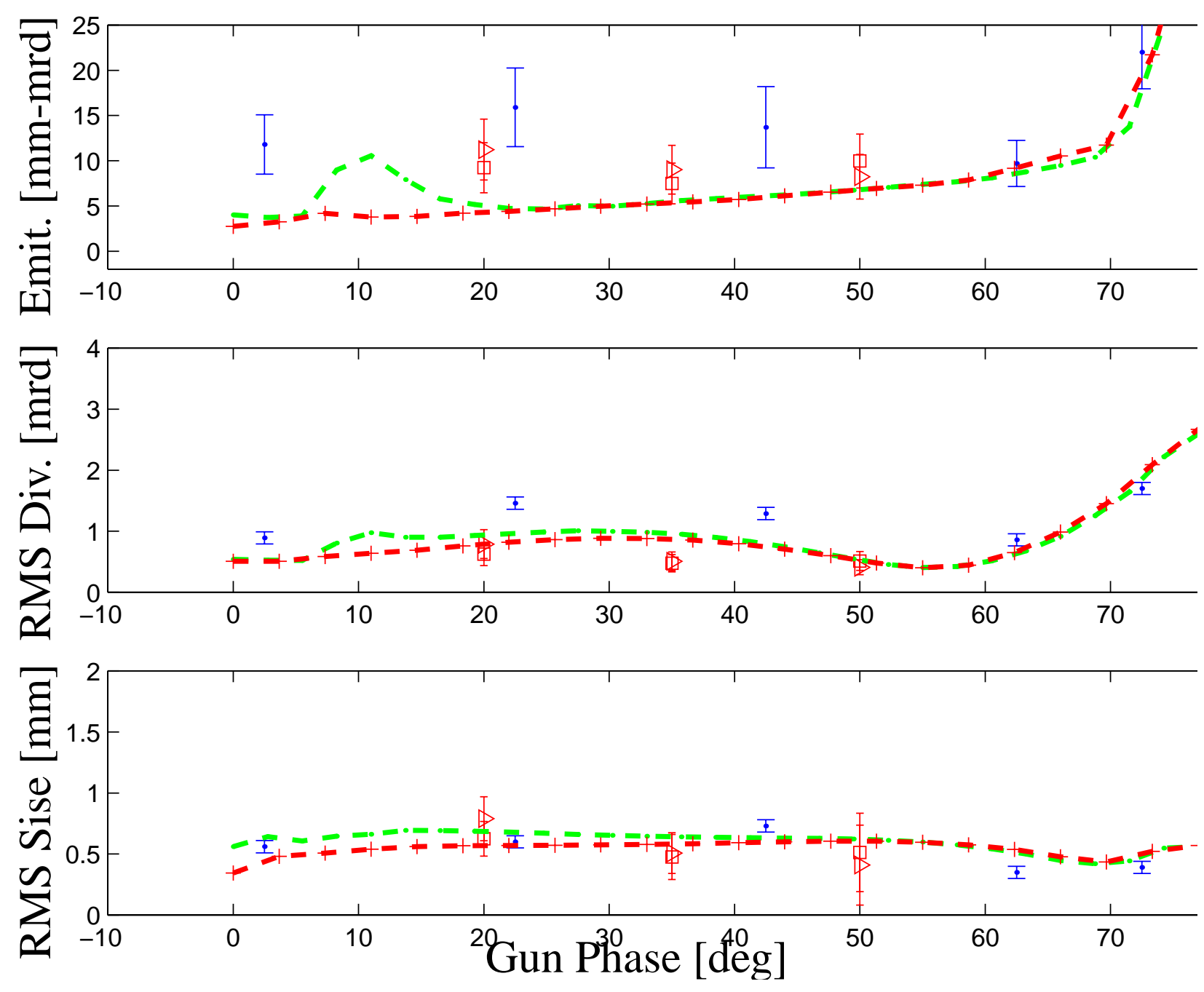

dots: multislits; square/ diamond: quad scan (x/y) green: simulation with Astra

red: simulation with Astra including Schottky effect 


\section{sensitivity versus solenoids settings}
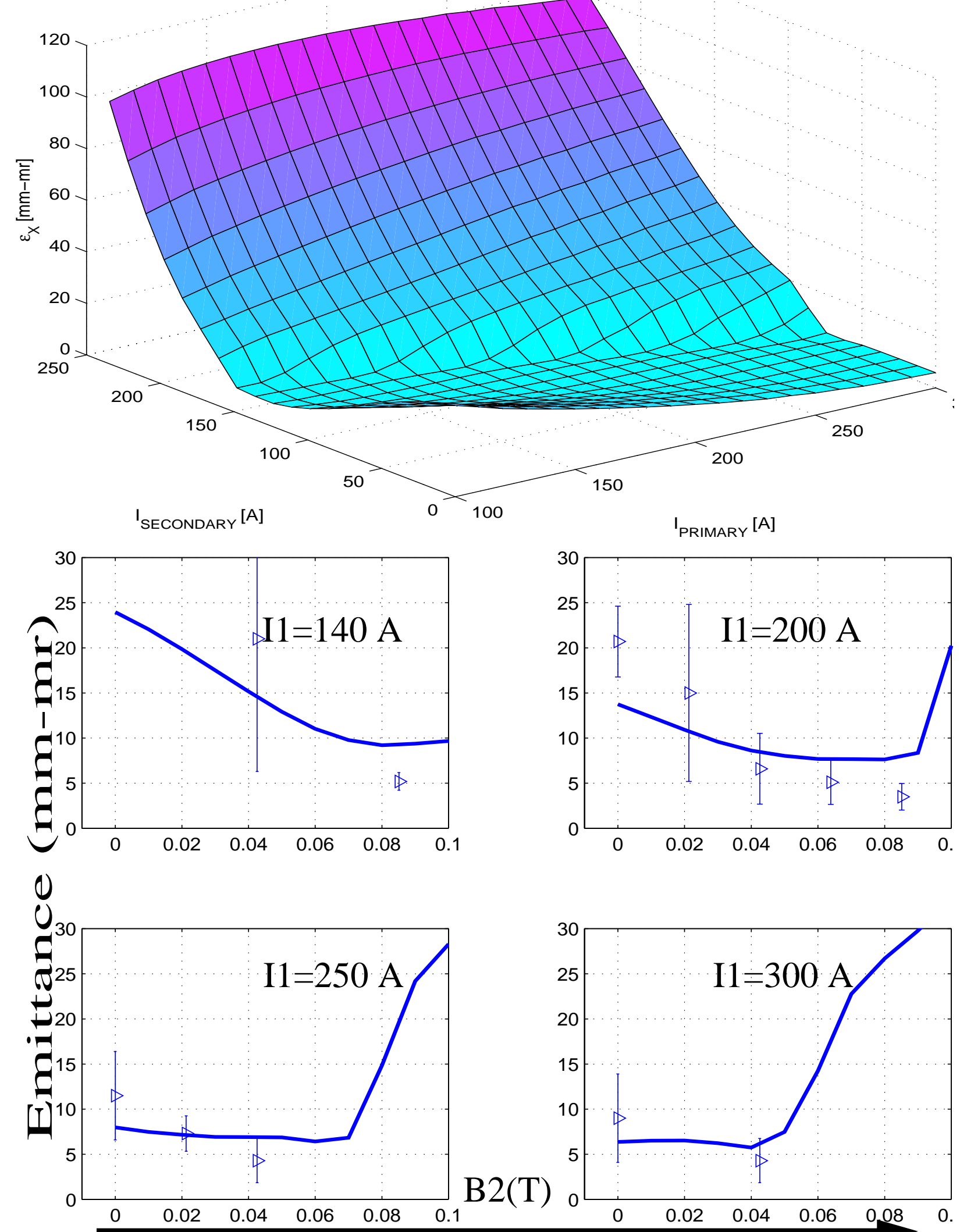

$\mathrm{B} 2(\mathrm{~T})$

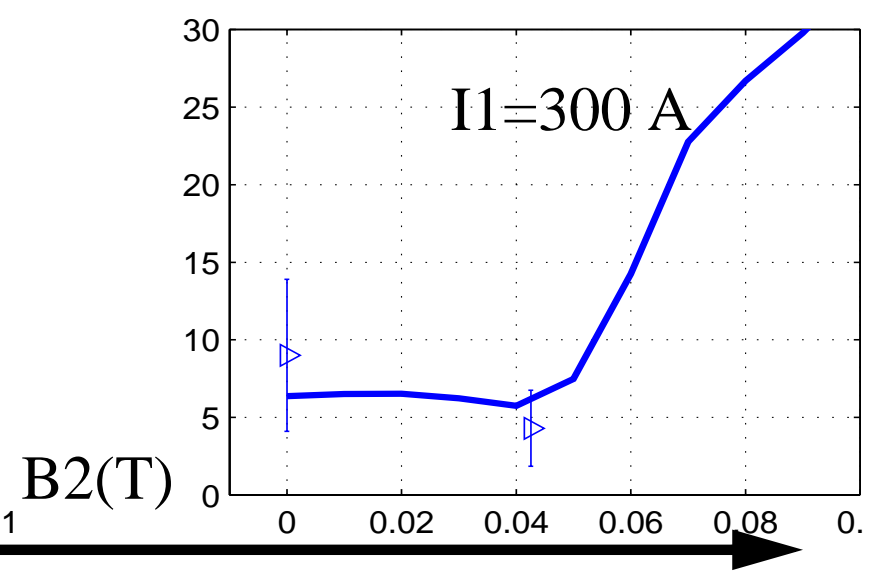

Ph. Piot, Deutsches Elektronen Synchrotron HH / JAN-2001 


\section{TTF phase I - Compression Issues-}

* bunch compression to 500 microns (RMS) with magn. chicane
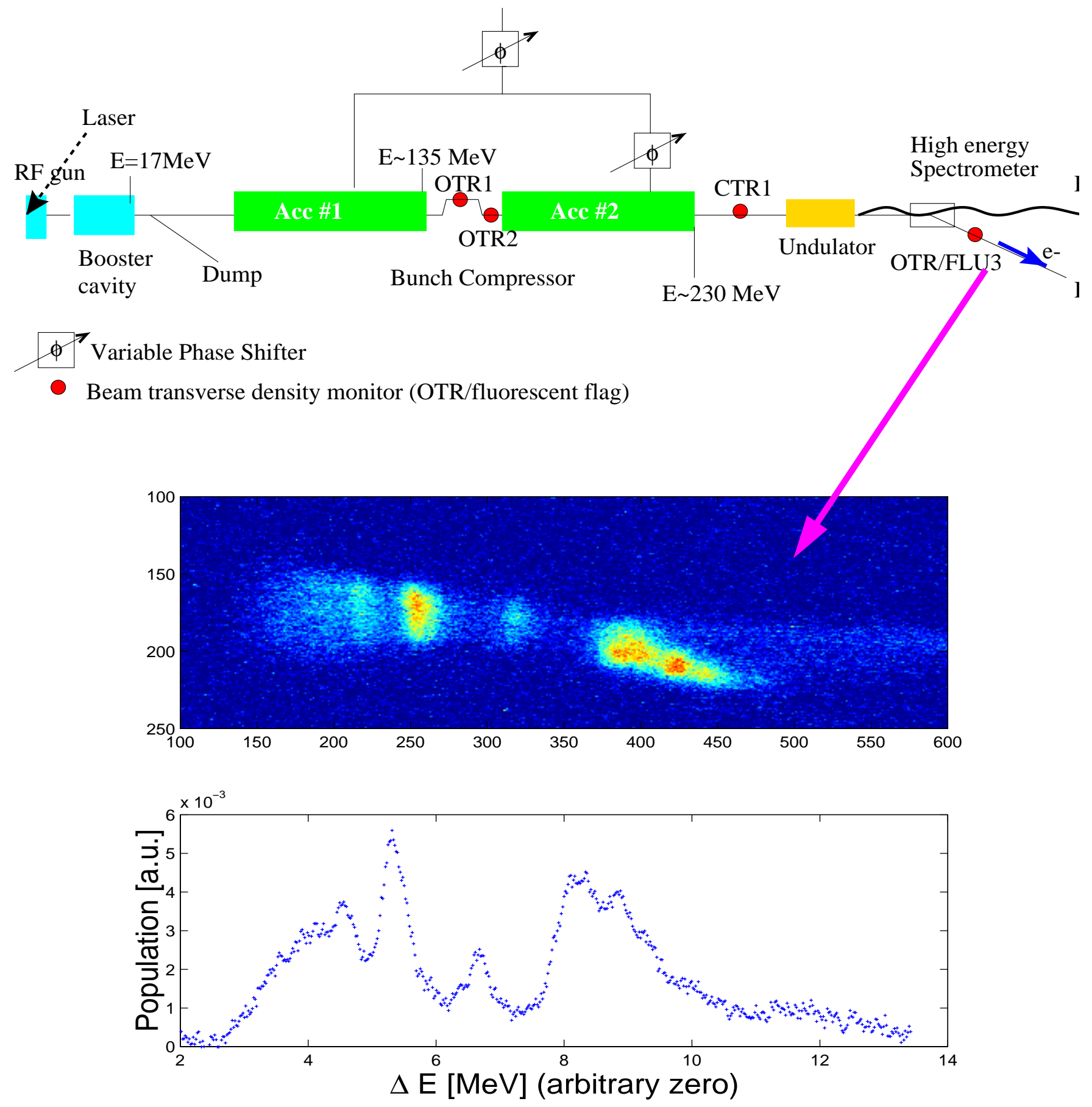

-> anytime the bunch compressor was operated for max. compression we observed a "fragmentation" of the energy profile: wakefield in ACC \#1 (NO!), CSR (MAYBE...) 


\section{TTF Phase I - Compression issue -}

\section{Dependency of the energy profile vs $\Delta \phi$ of ACC \#1}

(max comp for $\phi=-8 \mathrm{deg}$ )
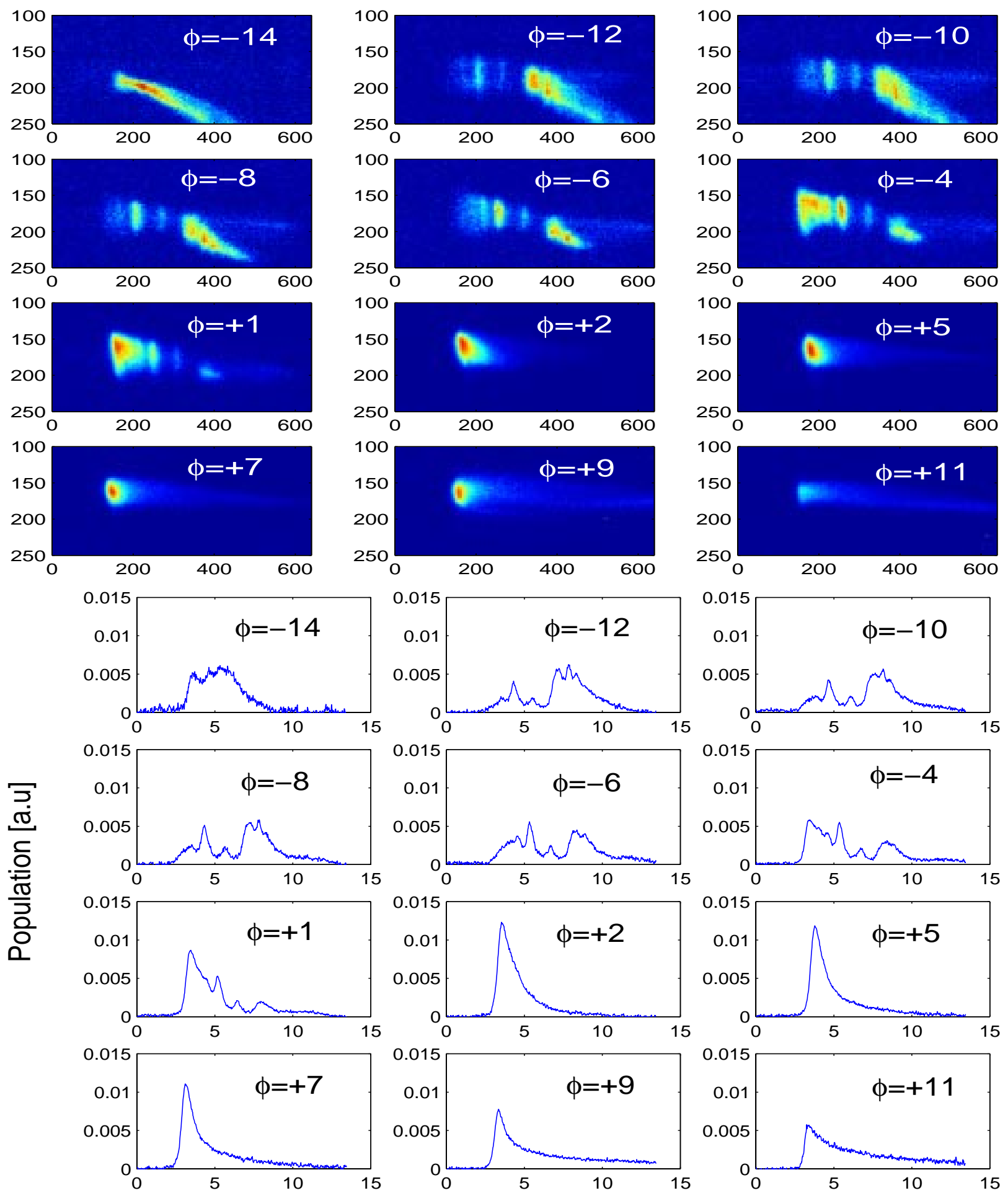

Energy $[\mathrm{MeV}]$ 
TTF 1 - modeling of the compression -

Magnetic Bunch Compressor

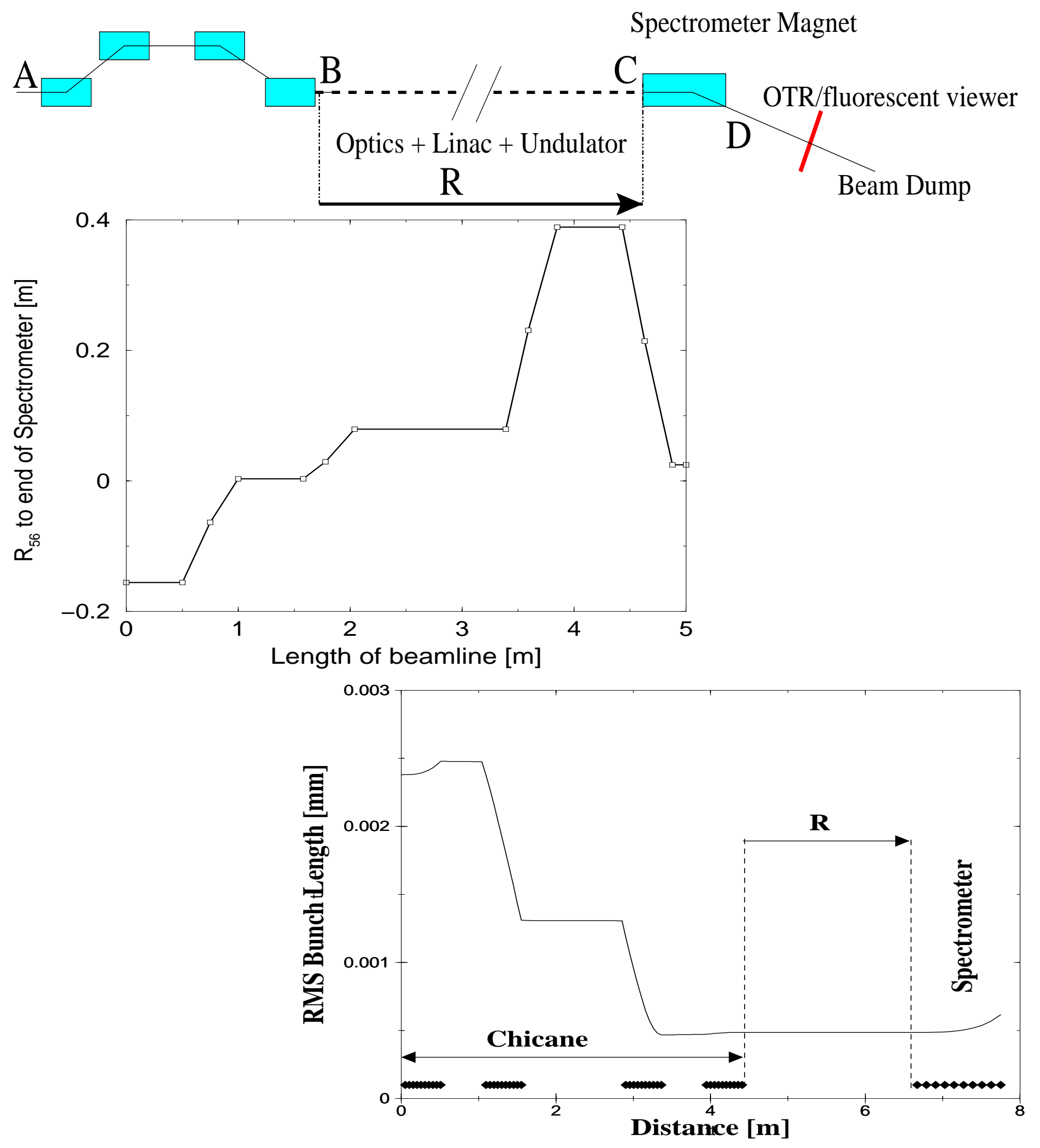


TTF I -compression modeling -

* Initial phase space obtained from ASTRA macroparticle code simulations of the realistic injector.
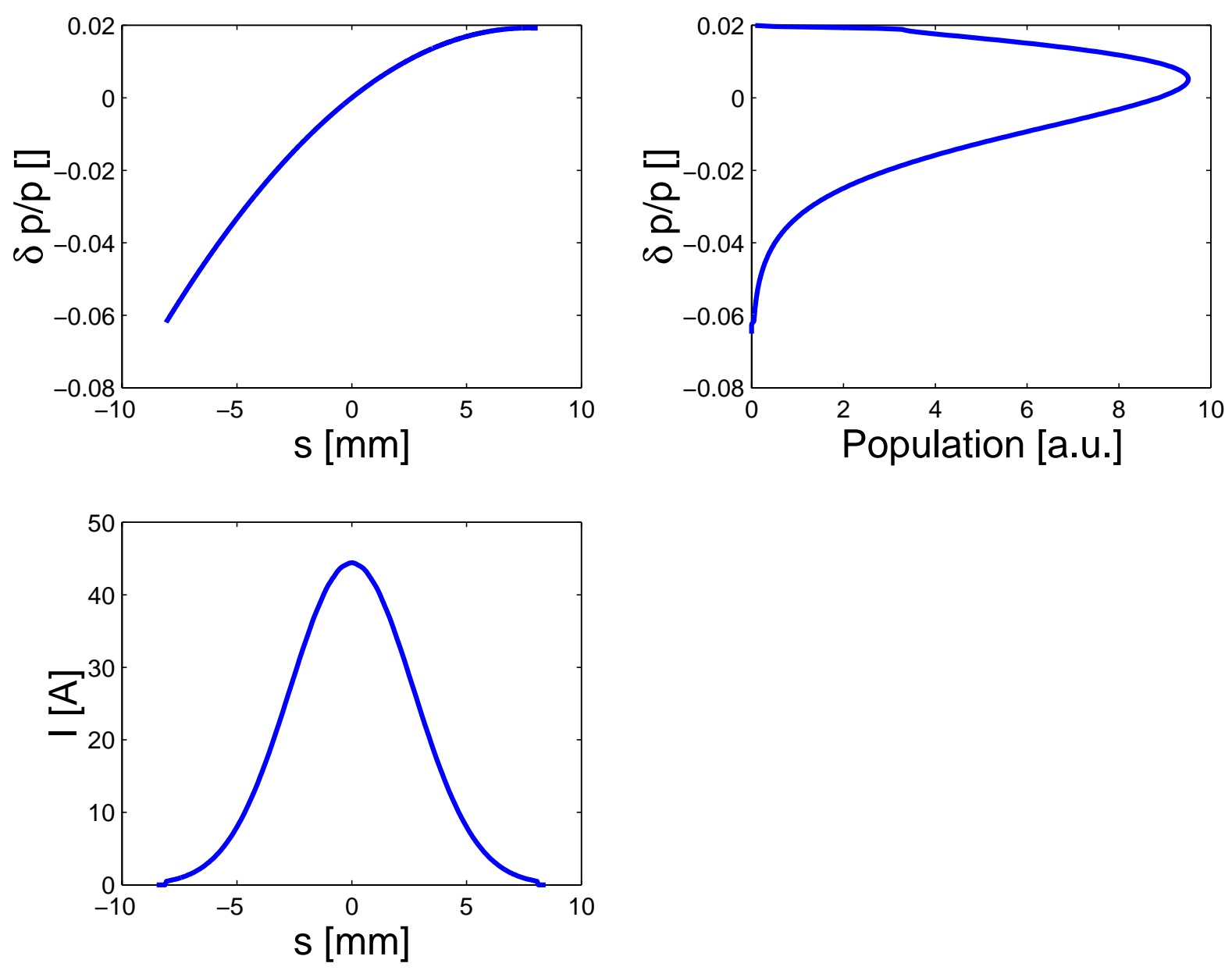

* because of rather long bunch (estimated to $2.5 \mathrm{~mm}$ ) at the injector front end $->$ the bunch accumulates curvature during acceleration in accelerator module ACC \#1

* this "curvature" can strongly enhanced the CSR wake (see Rui Li's paper at EPAC 2000 Wien Austria) 
Modeling of the compressor [R56=180 mm]

Use of TraFiC4 with 1200 macroparticles, using a self consistent algorithm.

Longitudinal phase space at compressor exit
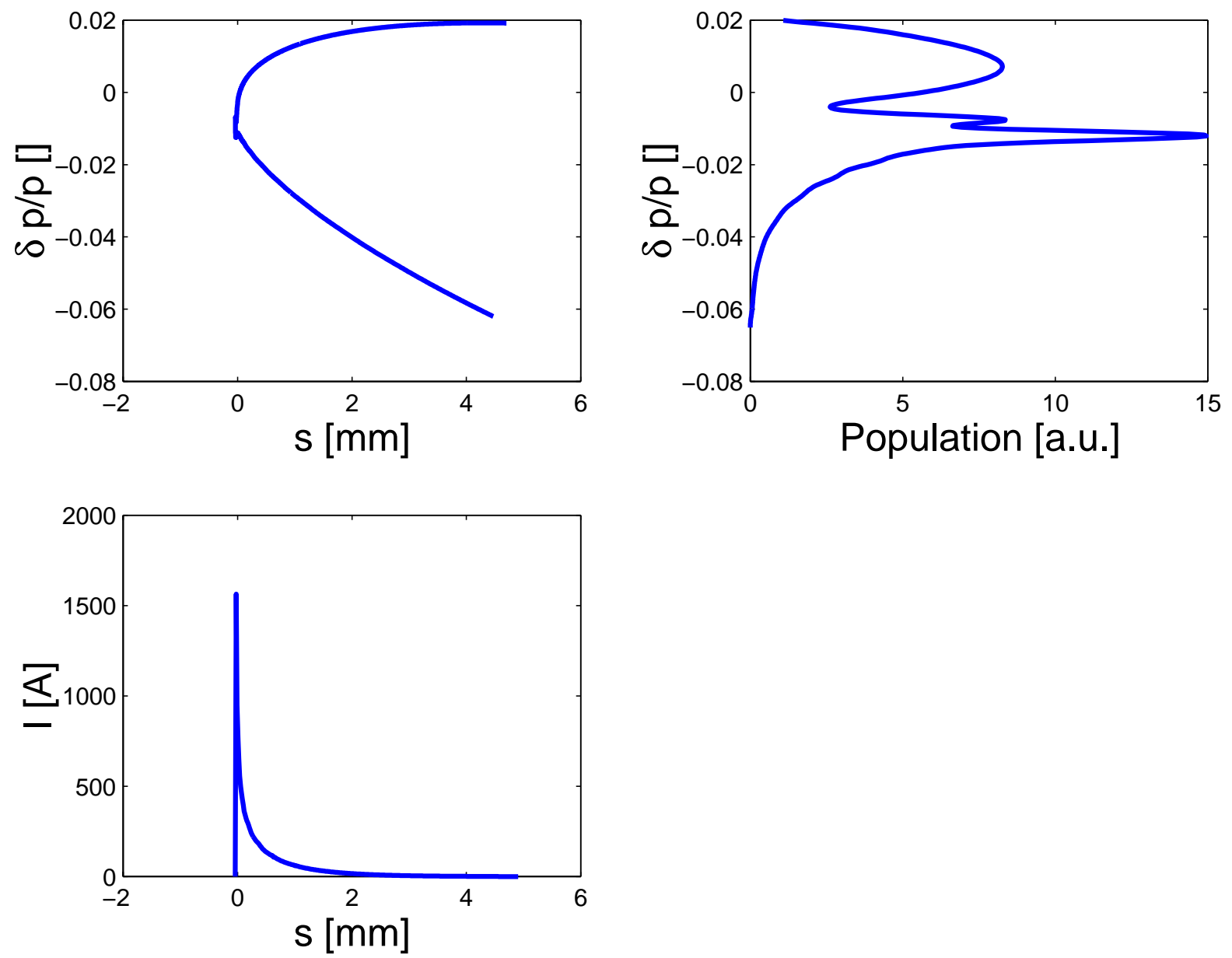

=> energy profile fragments 
Longitudinal phase space at spectrometer exit
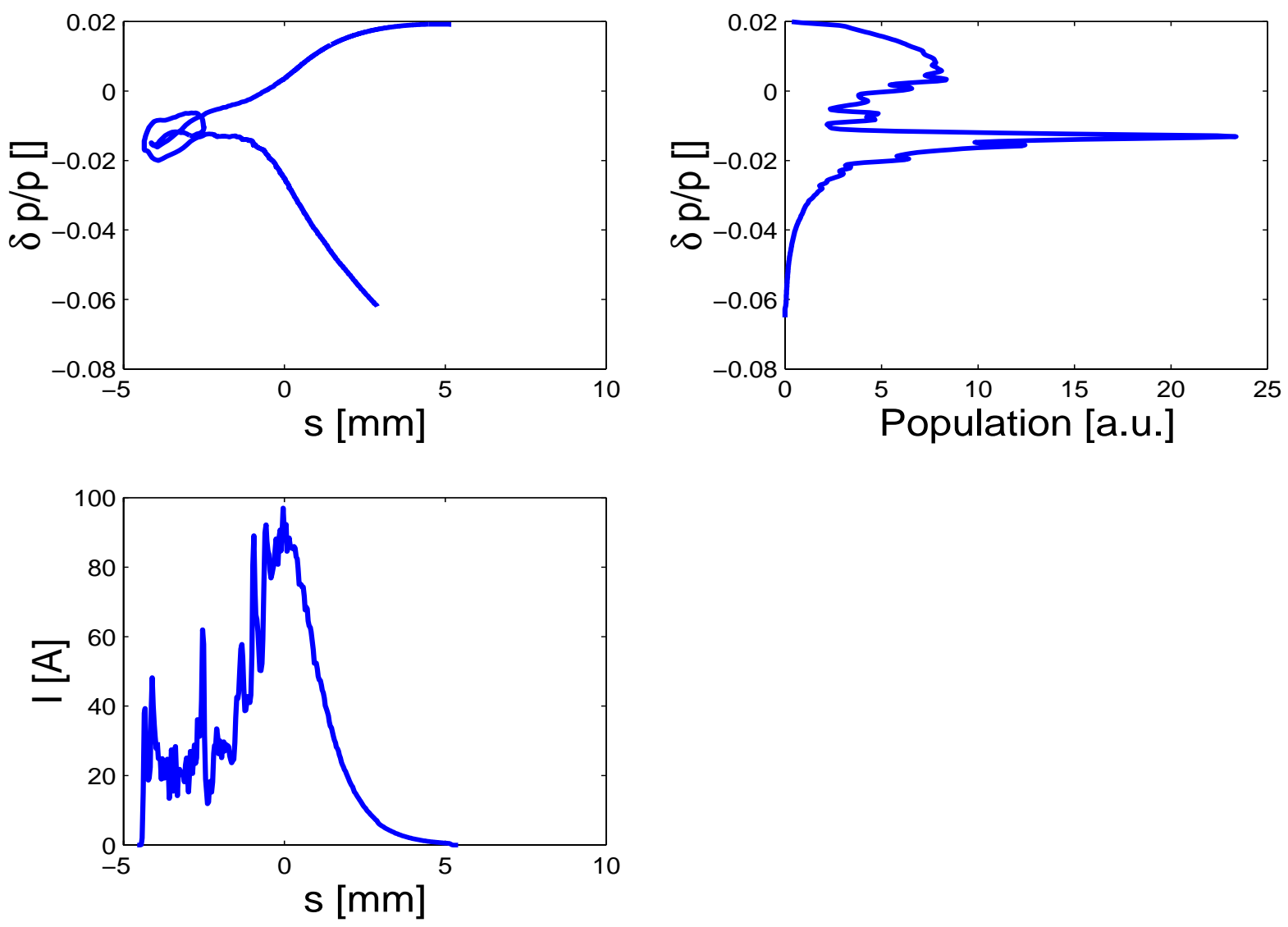

Comparison with the observed energy profile
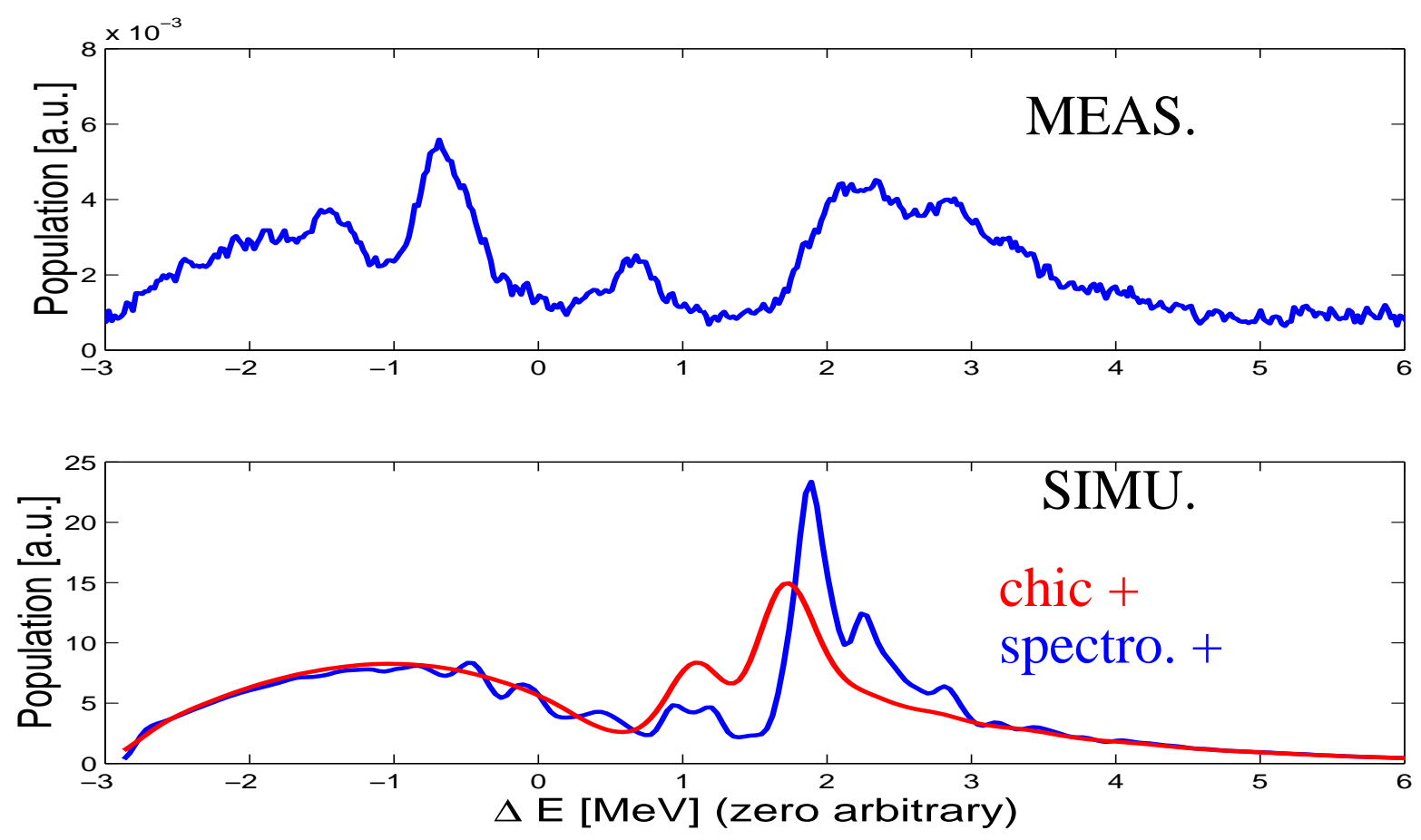
New "Ingredients" for the design of the TESLA XFEL injector new tools: ASTRA [K. Floettmann], HOMDYN [M. Ferrario]

- ASTRA: a macroparticle code with radial mesh grid, that includes space charge + .

- HOMDYN: a multislice envelope code based on a semi-analytical approach. Slices are uniform cylinder.

HOMDYN very fast --> allows a large scan of the parameters ASTRA slower but include nonlinear space charge --> used for fine optimization + generation of "realistic distributions" for integrated modeling [e.g. using ELEGFANT, GENESIS]

new gun (fully symmetric)

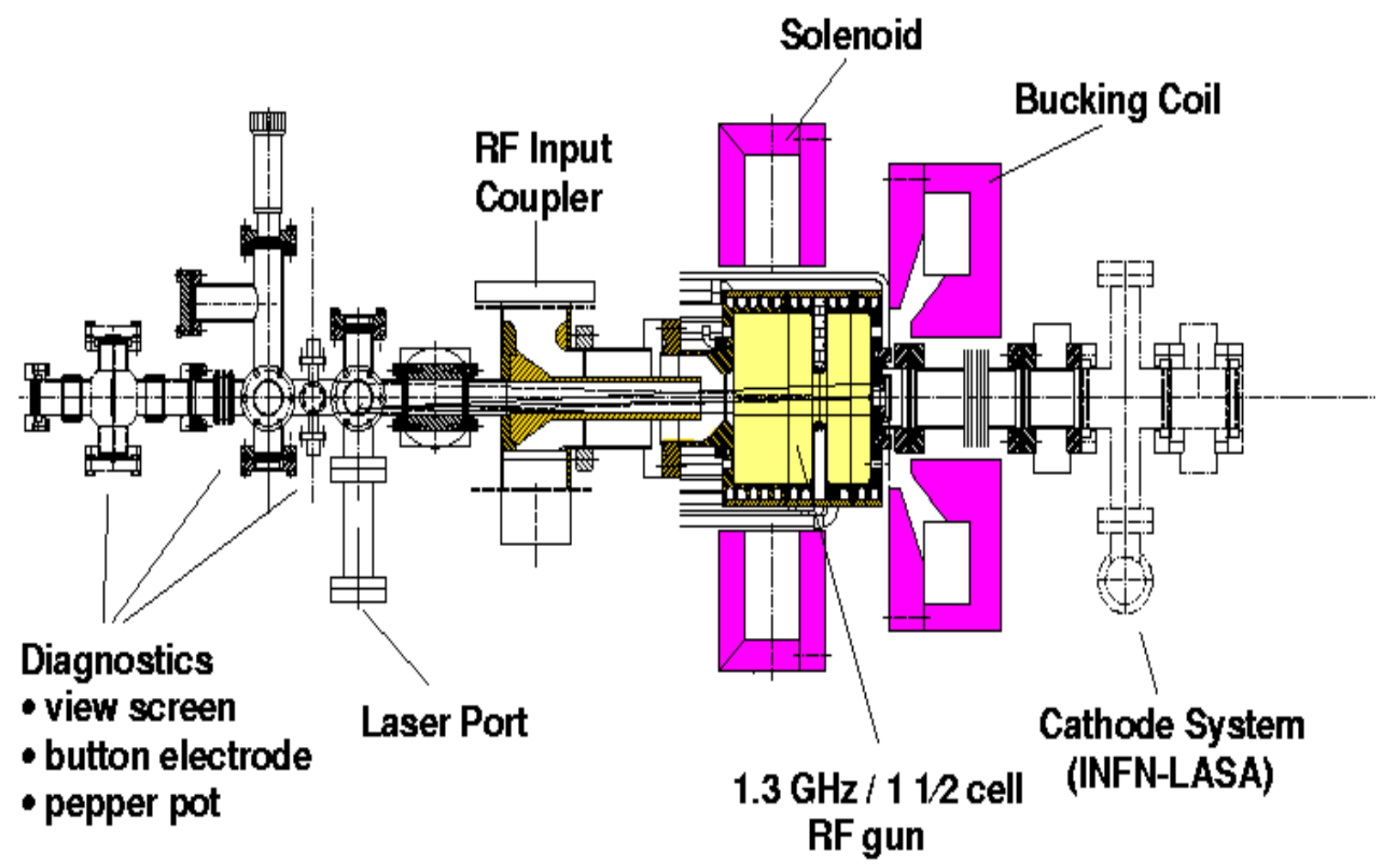




\section{DESIGN PHILOSOPHY:}

* Starting point: optimum operation point of LCLS [M. Ferrario]

- confirmed with Astra

- scaled to $\mathrm{f}=1.3 \mathrm{GHz}$

* then use the field profiles of the DESY gun + solenoid configuration and optimized. Gun peak field taken: $60 \mathrm{MV} / \mathrm{m}$

\section{OPTIMIZATION OF THE LASER PARAMETERS}
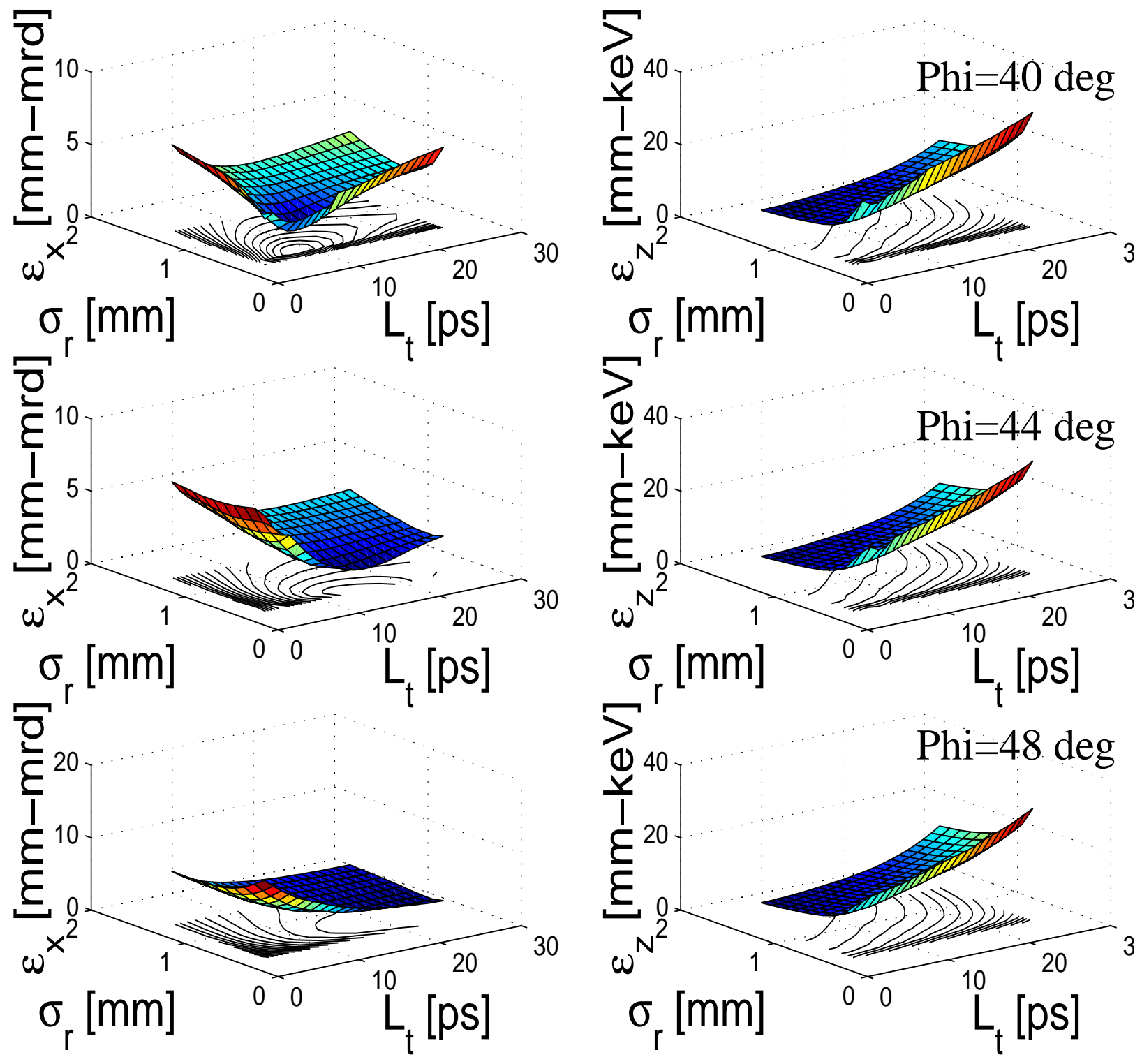


\section{LASER + GUN PARAMETERS}

spot size $=1.5 \mathrm{~mm}$ radius (unif.) pulse length $=20 \mathrm{ps}$, launch phi=44 deg, Gun peak field=60 MV/m

\section{COMPENSATION DRIFT and ACCELERATION}

One possible matching is the invariant envelope match, the beamspot, $\sigma_{r}$, at a $S W$ booster entrance should obey:

$$
\begin{gathered}
\sigma_{r}^{\prime}=0 \\
\sigma_{r}=\frac{2}{\bar{\gamma}^{\prime}} \sqrt{\frac{I}{3 I_{A} \gamma}}
\end{gathered}
$$

I: peak current, $\gamma$ : incoming beam energy, IA Alvfen current, and $\gamma$ ': average accelerating gradient of the booster.

$\square$ The match is achieved in a way that it corresponds to a point where emittance is naturally decreasing

$\square$ The effects of the booster is to: (1) shift the minimum emittance to higher energy and (2) provide some emittance damping $\Rightarrow$ solenoid peak field $=0.2 \mathrm{~T}$

\section{CHOICE OF BOOSTER:}

A TESLA standard cryomodule (i.e. 8 9-cell TESLA cavity). After optimization: the first 4 cavities are operated at $12 \mathrm{MV} / \mathrm{m}$, and the next 4 to the maximum expected gradient $25 \mathrm{MV} / \mathrm{m}$ $\square$ reached energy is $142 \mathrm{MeV}$

$\square$ Injection of long bunch in the accelerating cavity yields curved longitudinal phase space $=>$ set a limit on bunch compression!!! 


\section{ACHIEVED PARAMETERS:}

transverse emit $<0.5 \mathrm{~mm}$-mrd (without thermal emittance) longitudinal emit $\sim 350 \mathrm{keV}-\mathrm{mm}$ !!!

\section{CORRECTION OF LONGITUDINAL PHASE SPACE}

[report TESLA-FEL-01-04]

Most of the longitudinal emittance is coming from curvature (i.e. an electron energy offset is correlated to the longitudinal position): $\delta(s)=\alpha_{1} s+\alpha_{2} s^{2}+O\left(s^{3}\right)$ => can use some higher RF field harmonic to correct this nonlinear dependence [well known trick in storage ring (flat topping) also applied to linear accelerator at BOEING FEL]
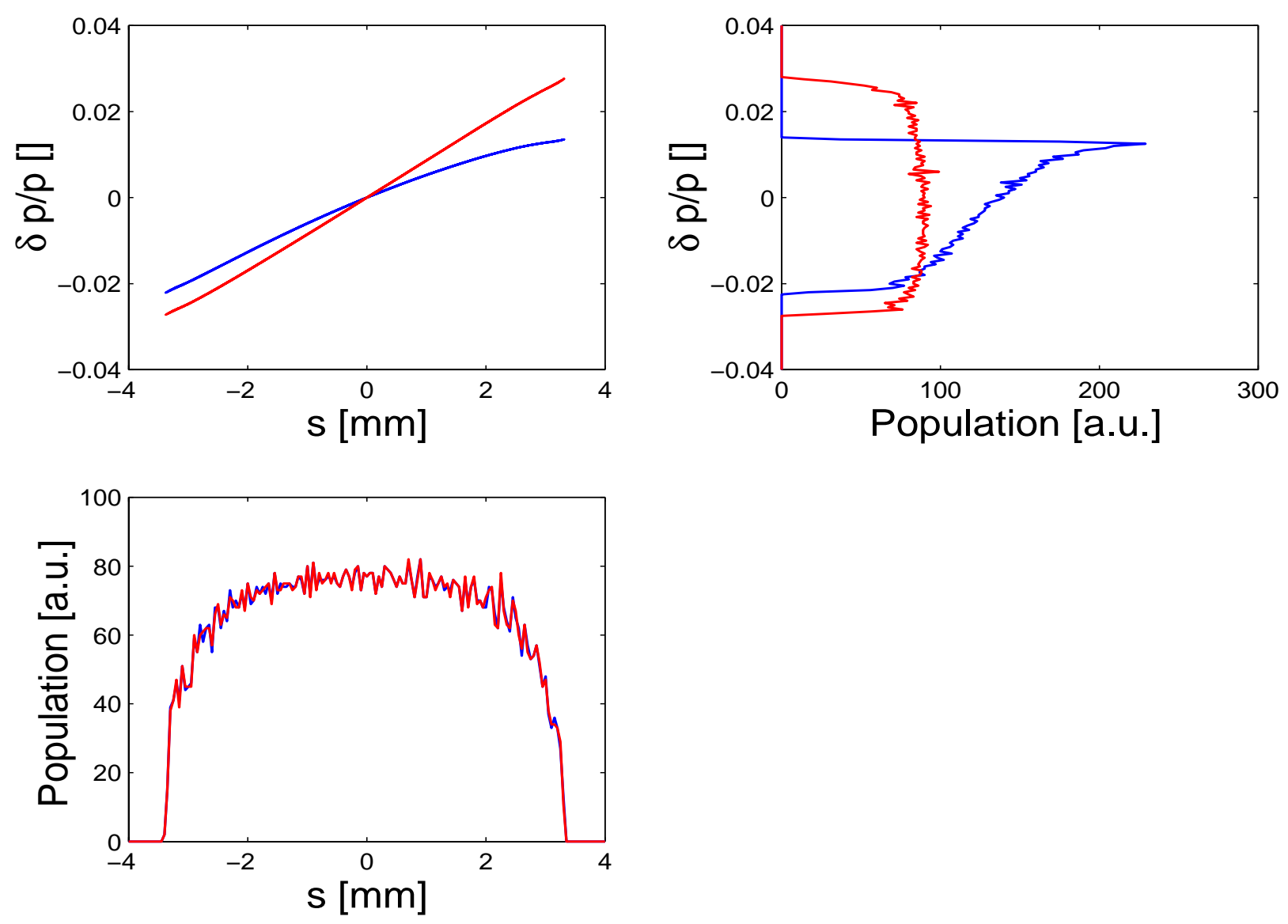

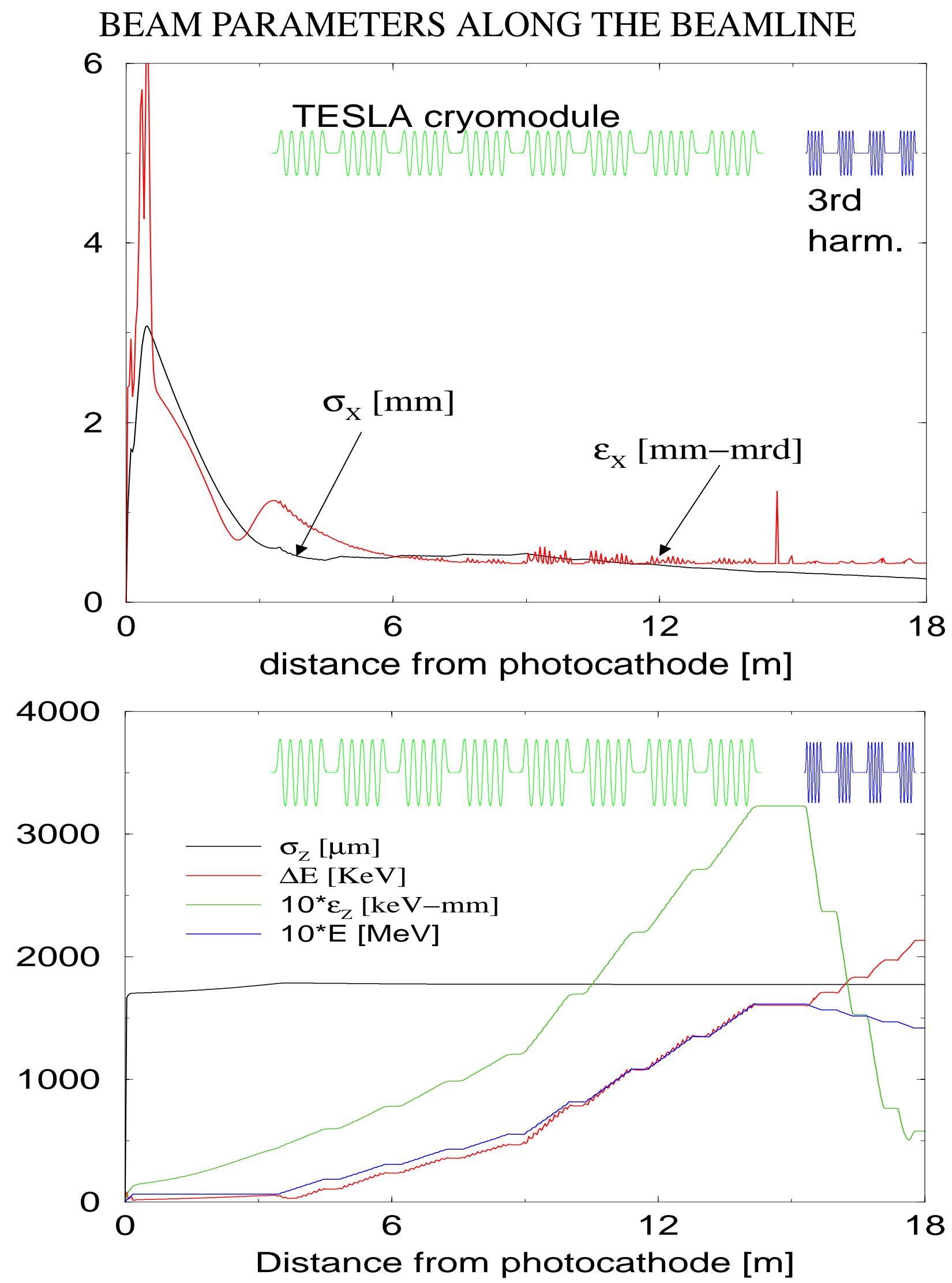


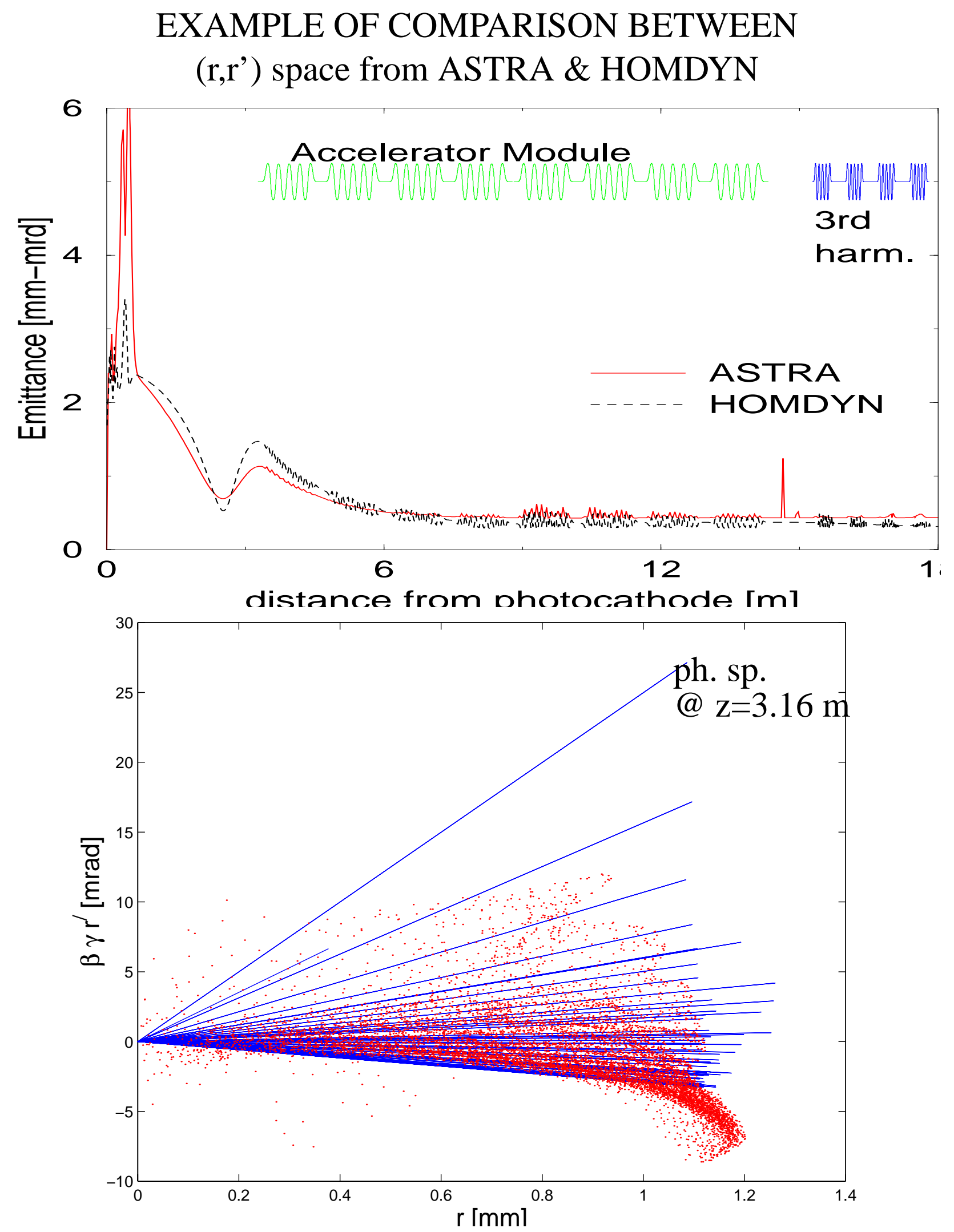

(for the long. phase space the codes show better agreement) 

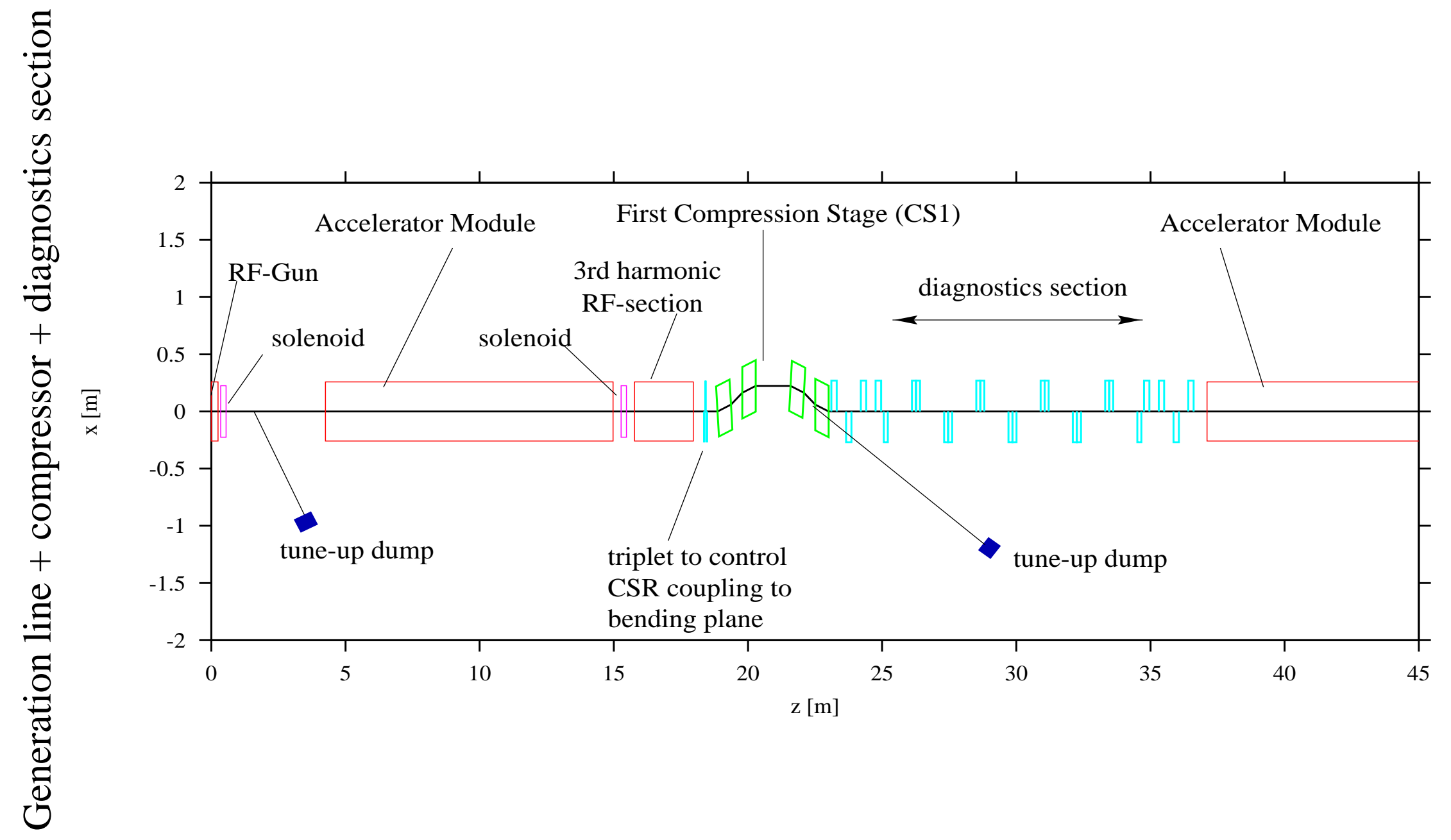
Parameters obtained downstream of the generation line $(\mathrm{z}=18 \mathrm{~m})$
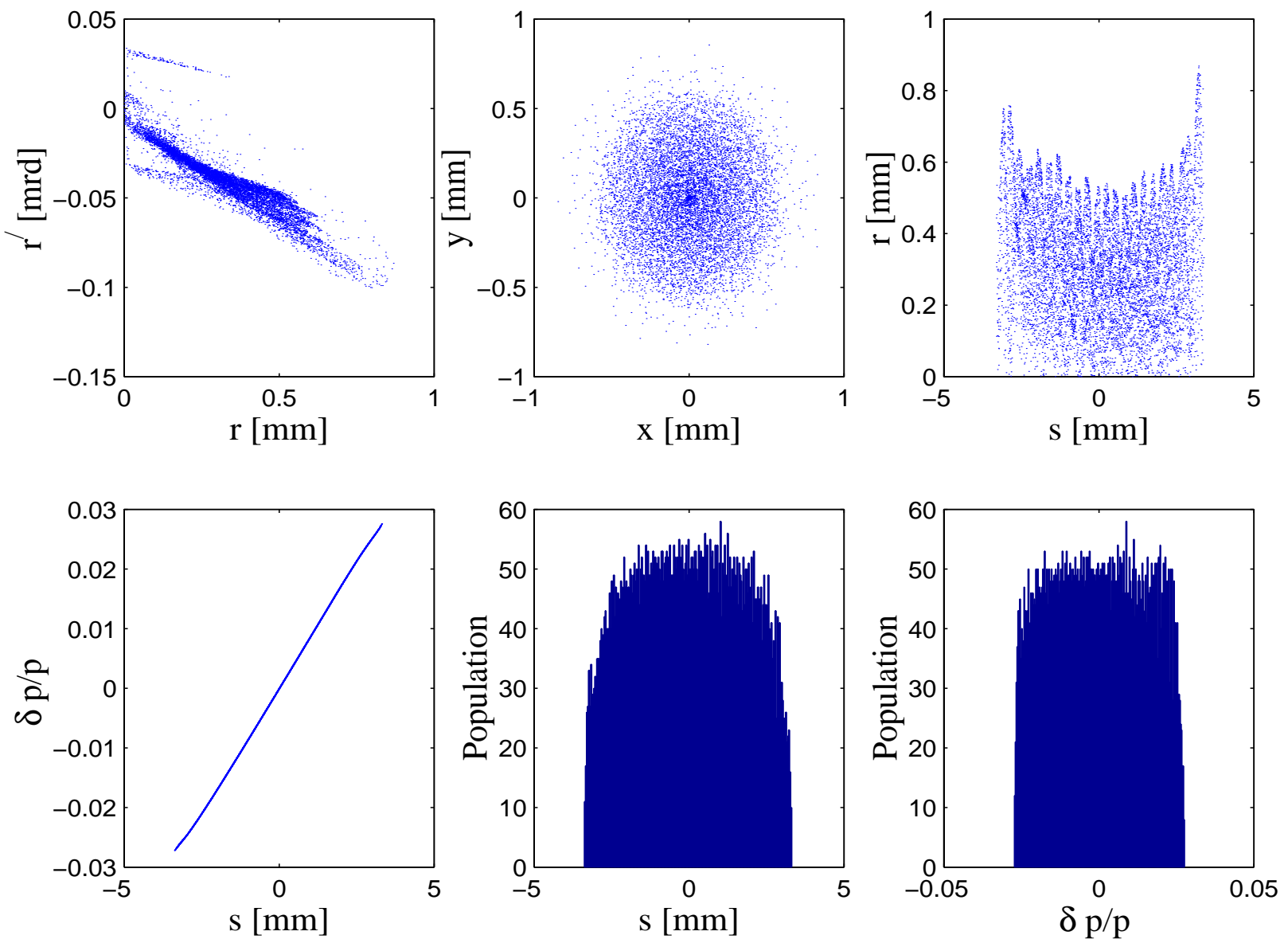

Table 1: beam param @ gener +

\begin{tabular}{|l|l|l|}
\hline \multicolumn{1}{|c|}{ parameters } & \multicolumn{1}{c|}{ values } & \multicolumn{1}{c|}{ units } \\
\hline \hline rms bunch length & 1.77 & $\mathrm{~mm}$ \\
\hline kinetic energy & 142.4 & $\mathrm{MeV}$ \\
\hline rms energy spread & 2132.3 & $\mathrm{keV}$ \\
\hline transverse emittance & 0.43 & $\mathrm{~mm}-\mathrm{mrd}$ \\
\hline trans. emit. (w. 0.73 th. emit.) & 0.90 & $\mathrm{~mm}-\mathrm{mrd}$ \\
\hline longitud. emittance & 57.79 & $\mathrm{keV}-\mathrm{mm}$ \\
\hline betaX/ betaY & $43.35 / 43.74$ & $\mathrm{~m}$ \\
\hline AlphaX/AlphaY & $5.15 / 5.20$ & --- \\
\hline
\end{tabular}




\section{Simulation of the compressor using TraFiC4 code}

* Minimize emittance growth by proper matching

* Many iterative runs --> Optimum requires shielding with $8 \mathrm{~mm}$ height beam pipe

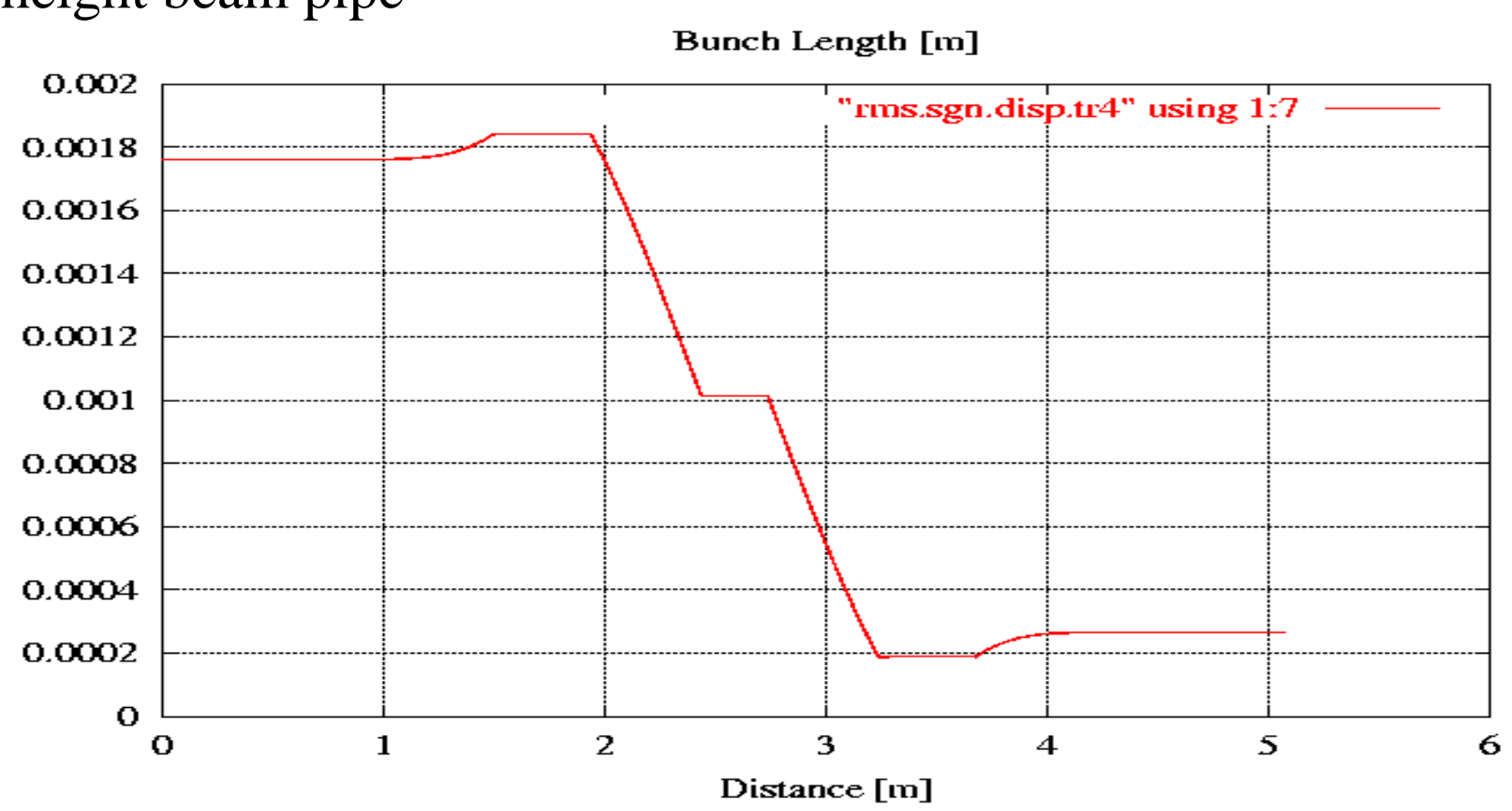

Emittances [m-rd]

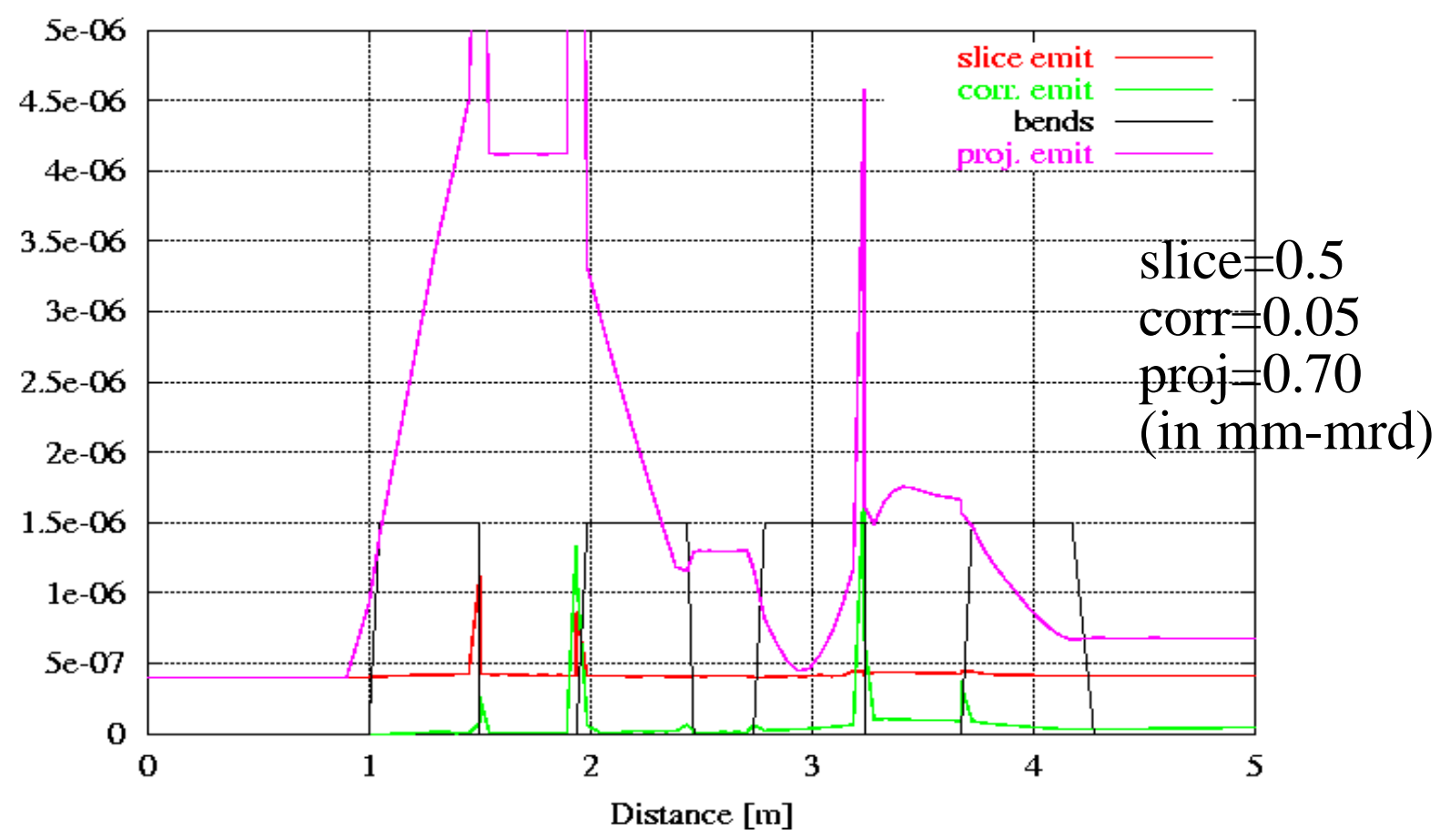




\section{Effects of beam compression in CS1 on the beam density}
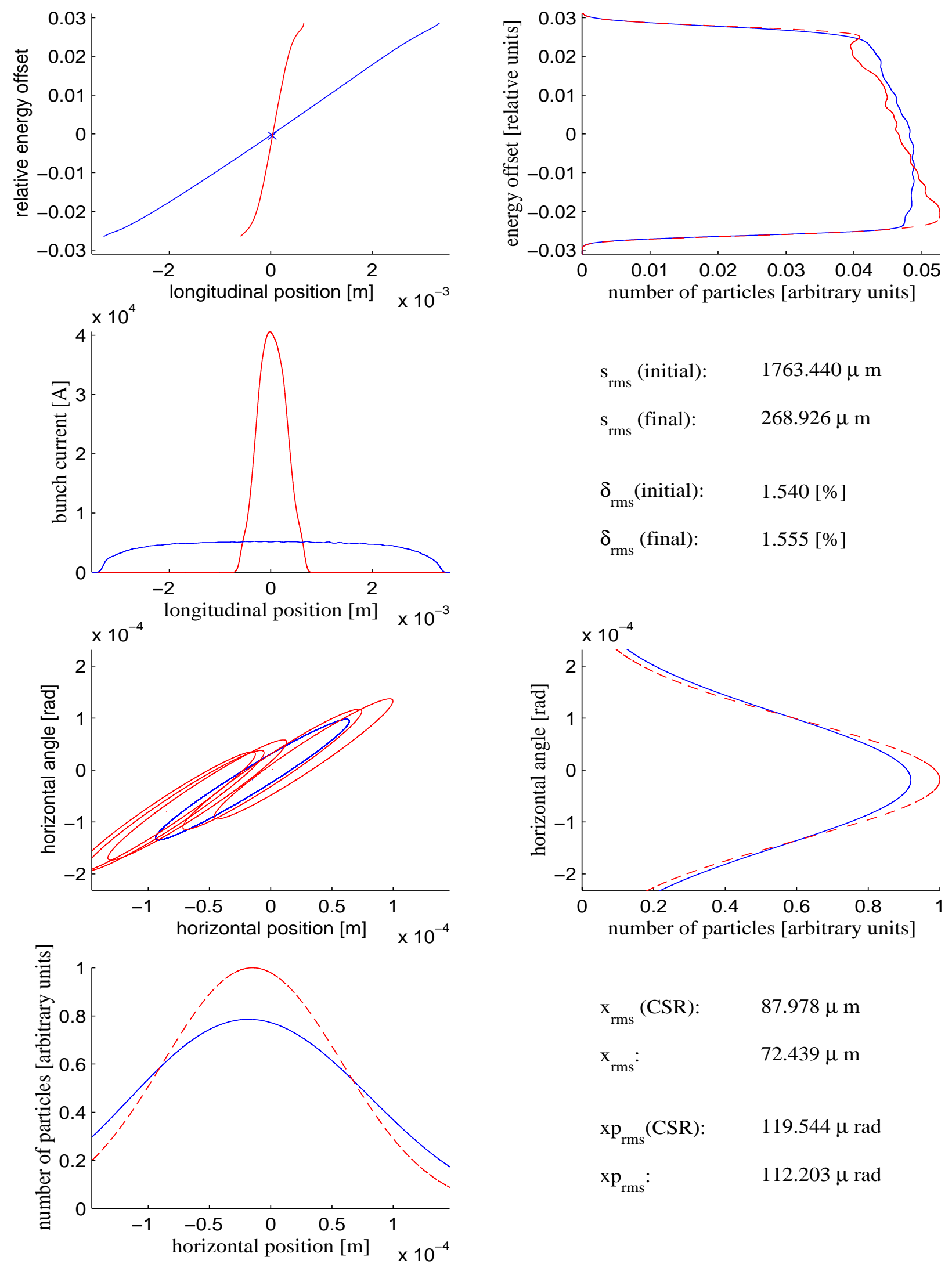

$\delta_{\text {rms }}$ (initial): $\quad 1.540[\%]$

$\delta_{\text {rms }}$ (final): $\quad 1.555[\%]$

number of particles [arbitrary units]

$$
\begin{array}{ll}
\mathrm{x}_{\mathrm{rms}}(\mathrm{CSR}): & 87.978 \mu \mathrm{m} \\
\mathrm{x}_{\mathrm{rms}}: & 72.439 \mu \mathrm{m} \\
\mathrm{xp}_{\mathrm{rms}}(\mathrm{CSR}): & 119.544 \mu \mathrm{rad} \\
\mathrm{xp}_{\mathrm{rms}}: & 112.203 \mu \mathrm{rad}
\end{array}
$$


TTF User Facility (TTF Phase II): Overview + Requirement [see report TESLA-FEL 95-

mode of operation: train of 800 bunches @ 1MHz; train rep. rate: $10 \mathrm{~Hz}$

Table 2: $1 \mathrm{nC}$ beam requirements, $\lambda=6 \mathrm{~nm}$

\begin{tabular}{|l|l|l|}
\hline \multicolumn{1}{|c|}{ parameters } & \multicolumn{1}{c|}{ values } & \multicolumn{1}{c|}{ units } \\
\hline \hline Bunch charge & 1 & nC \\
\hline Peak Current & 2.5 & kA \\
\hline Trans. Emit. & 2.0 & mm-mrd \\
\hline Long. Emit. & 30 & KeV-mm \\
\hline dp/p & 0.001 & -- \\
\hline
\end{tabular}

Table 3: $100 \mathrm{pC}$ beam requirements, $\lambda=6 \mathrm{~nm}$

\begin{tabular}{|l|l|l|}
\hline \multicolumn{1}{|c|}{ parameters } & \multicolumn{1}{c|}{ values } & \multicolumn{1}{c|}{ units } \\
\hline \hline Bunch charge & 0.1 & $\mathrm{nC}$ \\
\hline Peak Current & 600 & $\mathrm{~A}$ \\
\hline Trans. Emit. & 1.0 & $\mathrm{~mm}-\mathrm{mrd}$ \\
\hline Long. Emit. & 10 & $\mathrm{keV}-\mathrm{mm}$ \\
\hline $\mathrm{dp} / \mathrm{p}$ & 0.001 & -- \\
\hline
\end{tabular}

First lasing light for users $20 \mathrm{~nm}==>$ requirement are relaxed e.g. at $1 \mathrm{nC}$, Transv. emit. $4 \mathrm{~mm}-\mathrm{mrd}$ 
TTF2 - INJ. UPGRADE (STILL UNDER DISCUSSION)

\section{A - SHORT TERM (for commissioning + 1st light 20 nm)} current gun: $40 \mathrm{MV} / \mathrm{m}$ laser: five stacked 2 ps (rms) Gaussian pulse gun moved close to the cryomodule + capture cavity removed

\section{B - LONGER TERM (toward 6 nm light)}

symmetric gun: $40 \mathrm{MV} / \mathrm{m}$

realistic laser five stacked 2 ps (rms) Gaussian pulse $==>$ compare to $(\mathrm{A})$ better emittance

\section{C - LONGER++ TERM (6 nm light)}

symmetric gun: $40 \mathrm{MV} / \mathrm{m}$

realistic laser five stacked 2 ps (rms) Gaussian pulse install $3.9 \mathrm{GHz} \mathrm{RF}$-section

$==>6 \mathrm{~nm}$ User Facility with specs.

\section{D - CONTINUOUS UPGRADE}

Design is flexible enough to quickly modify the low energy region depending on what we learn from our Gun Test Facility in DESY-Zeuthen [BERLIN].

$==>$ step-by-step the injector will evolve toward the TESLA XFEL injector

BUT (C) relies on the availability of a $3.9 \mathrm{GHz}$ RF-system. An $\mathrm{R} \& \mathrm{D}$ effort just started within the TESLA coll. to scale the 1.3 GHz TESLA cavity to $3.9 \mathrm{GHz}$.

\section{$==>$ everybody is welcome to join this effort!}




\section{TRANS. BEAM PARAMETERS IN TTF2 INJECTOR}

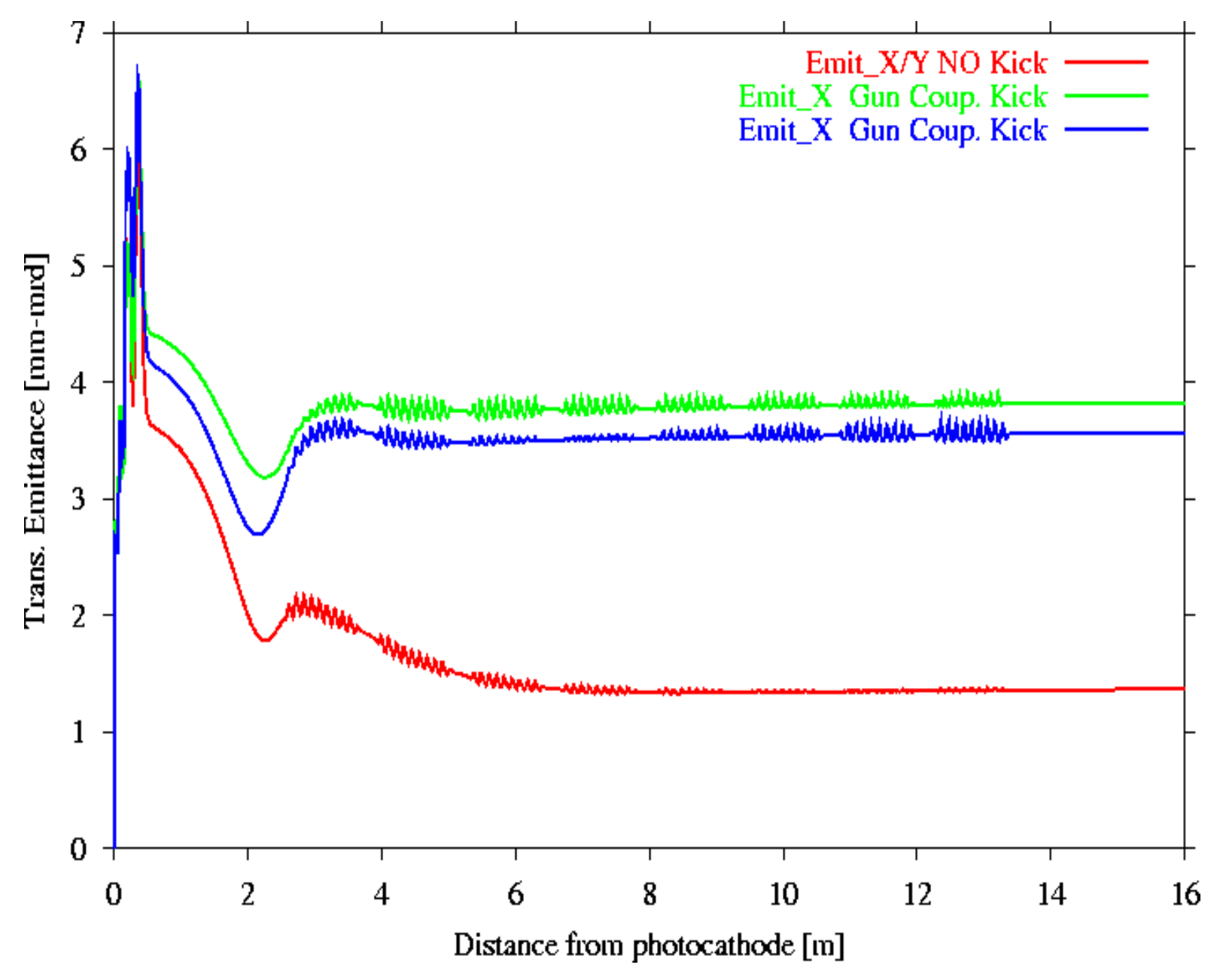

* coupler kick simulated in ASTRA using meas./HFSS simulations from E. Colby (A0, FERMILAB

* long. beam parameters: not a problem to achieve what we want 


\section{COMMENTS ON LOW CHARGE OPERATION $(100 \mathrm{pC} / \mathrm{BUNCH})$}

- reduce all the charge dependent effects

- parameter can be achieved

$==>$ BUT average power of the FEL decrease....

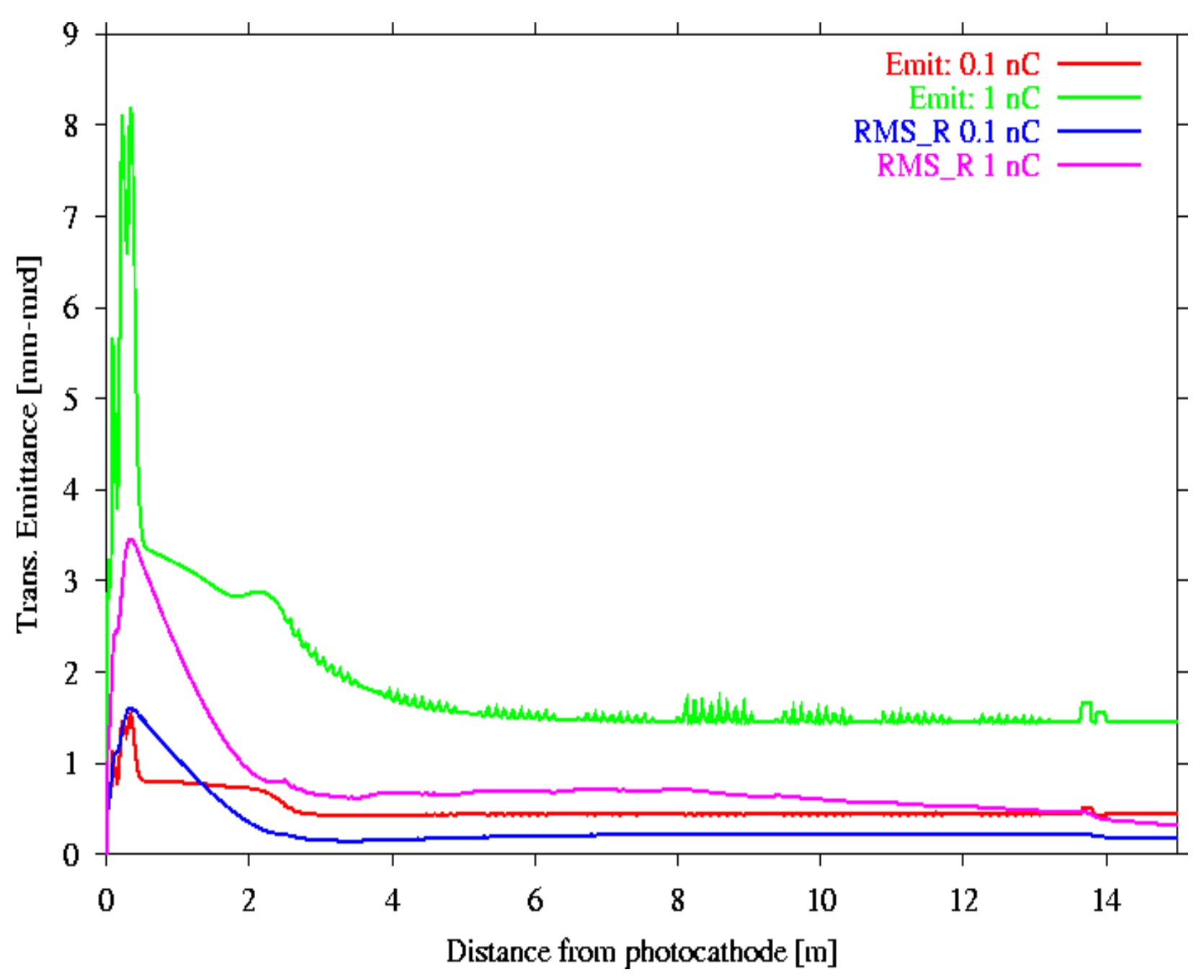




\section{THE PHOTOINJECTOR TEST FACILITY AT DESY ZEUTHEN}

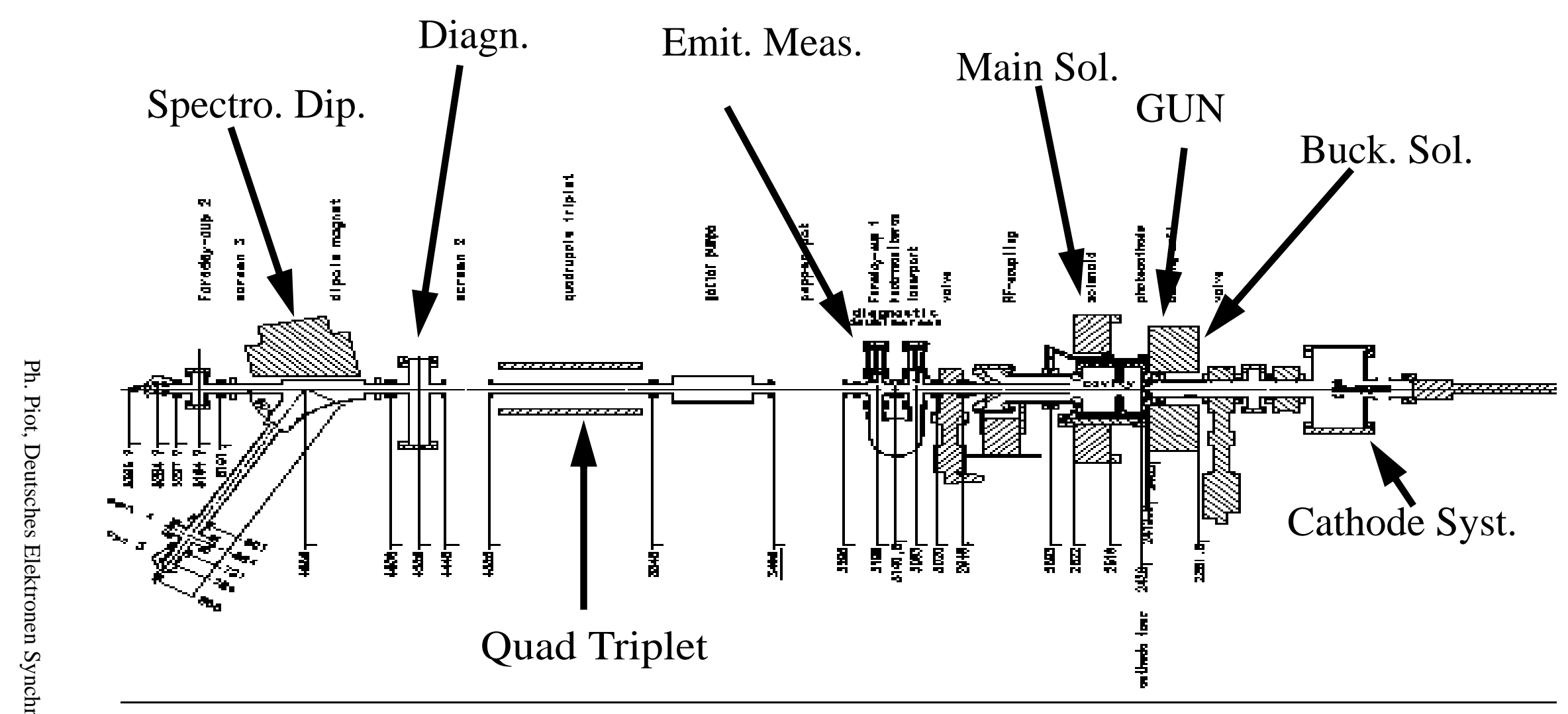




\section{EXPERIMENTAL PROGRAM AT THE PHOTOINJECTOR TEST FACILITY IN ZEUTHEN (NON-EXHAUSTIVE LIST!):}

\section{PHASE I: concentrate on generation line (gun + drift)}

- test a new laser system from Max Born Institute

- gun characterization (test of new design)

- charge scaling

- benchmark of space charge algorithm

$-\ldots$

\section{PHASE II: acceleration}

- test the new Cut Disk Structure (CDS) [V. Paramonov] design in the context of TESLA positron injector

- demonstrate the emittance damping + matching concept

- generation of flat beam

- und viele mehr!

\section{PHASE III: ???}




\section{CONCLUSION}

* Full simulation of injectors systems for TESLA X-FEL, for the TTF II User Facility have been performed. Emittance within specification can be achieved

* In the case of the TESLA X-FEL emittance in the sub-mm-mrd can be achieved downstream of the first stage compression at 142 $\mathrm{MeV}$

* for TESLA X-FEL, full tracking of the generated phase space density using TraFiC4, Elegant thereby including collective effects (CSR, resistive + geometric wake field) results in a transverse emittance of $0.9 \mathrm{~mm}$-mrd (slice) and $2.6 \mathrm{~mm}$-mrd (projected) upstream of the undulator $==>$ phase space density passed to GENESIS (for simulation of the FEL properties)

* TTF II proposed new injector (by multi-stage upgrade) will be a step toward the TESLA X-FEL injector.

* EXPERIMENTAL SIDE:

--> This summer our Injector Test Facility should produce first ebeam

--> dedicated beam time at Tesla Test Facility:

to perform study of CSR bunch self interaction,

to study the impact of surface roughness on the beam 


\section{First order simulations of L-band RF gun for PERL}

X.Y. Chang,X. J. Wang and I. Ben-Zvi

Brookhaven Accelerator Test Facility

National Synchrotron Light Source

Brookhaven National Laboratory

Upton, NY 11973, USA 


\section{Outline}

- Introduction

- First order simulations of L-band RF gun for PERL

- Conclusion and Summary 


\section{Introduction}

\section{- Requirements for beam parameters:}

PERL requires $200 \mathrm{~mA}$, and $0.5 \mathrm{~mm}$-mrad normalized RMS Emittance. 25Mev at linac exit. For 1300Mhz RF gun it needs 150 pc per bunch.

\section{- Why use L-band injector?}

A. Same as main linac frequency, simplify operation, such as cost, synchronization.

B. Potential for higher field operation $(\propto \sqrt{f})$ which can reduce space charge effect. 


\section{- Major issues in L-Band RF Gun injector.}

A. Field on cathode for a 1.6 cell RF gun:

\begin{tabular}{|l|l|l|}
\hline Field gradient & $45 \mathrm{C}^{\circ}$ & $\mathrm{LN}_{2}$ \\
\hline $50 \mathrm{Mv} / \mathrm{m}$ & $4.5 \mathrm{MW}$ & $1.1 \mathrm{MW}$ \\
\hline $25 \mathrm{Mv} / \mathrm{m}$ & $1.1 \mathrm{MW}$ & $350 \mathrm{KW}$ \\
\hline $15 \mathrm{Mv} / \mathrm{m}$ & $350 \mathrm{KW}$ & $110 \mathrm{KW}$ \\
\hline
\end{tabular}

A $L N_{2}$ cooling method is proposed to improve $Q$ and vacuum, therefore, reduce required power and extend cathode lifetime.

B. Heat dissipation problems:

We can relieve this problem by trying using a bigger size cavity works at higher order mode. 


\section{- Longitudinal phase space $\left(\propto \varphi^{3}\right)$ :}

Our major promise is to make the volume of 6-D phase space minimum at linac exit, Not only the transverse or longitudinal emittance. $\left(\varepsilon_{\mathrm{z}} \times \varepsilon_{\mathrm{x}}^{2}\right)$

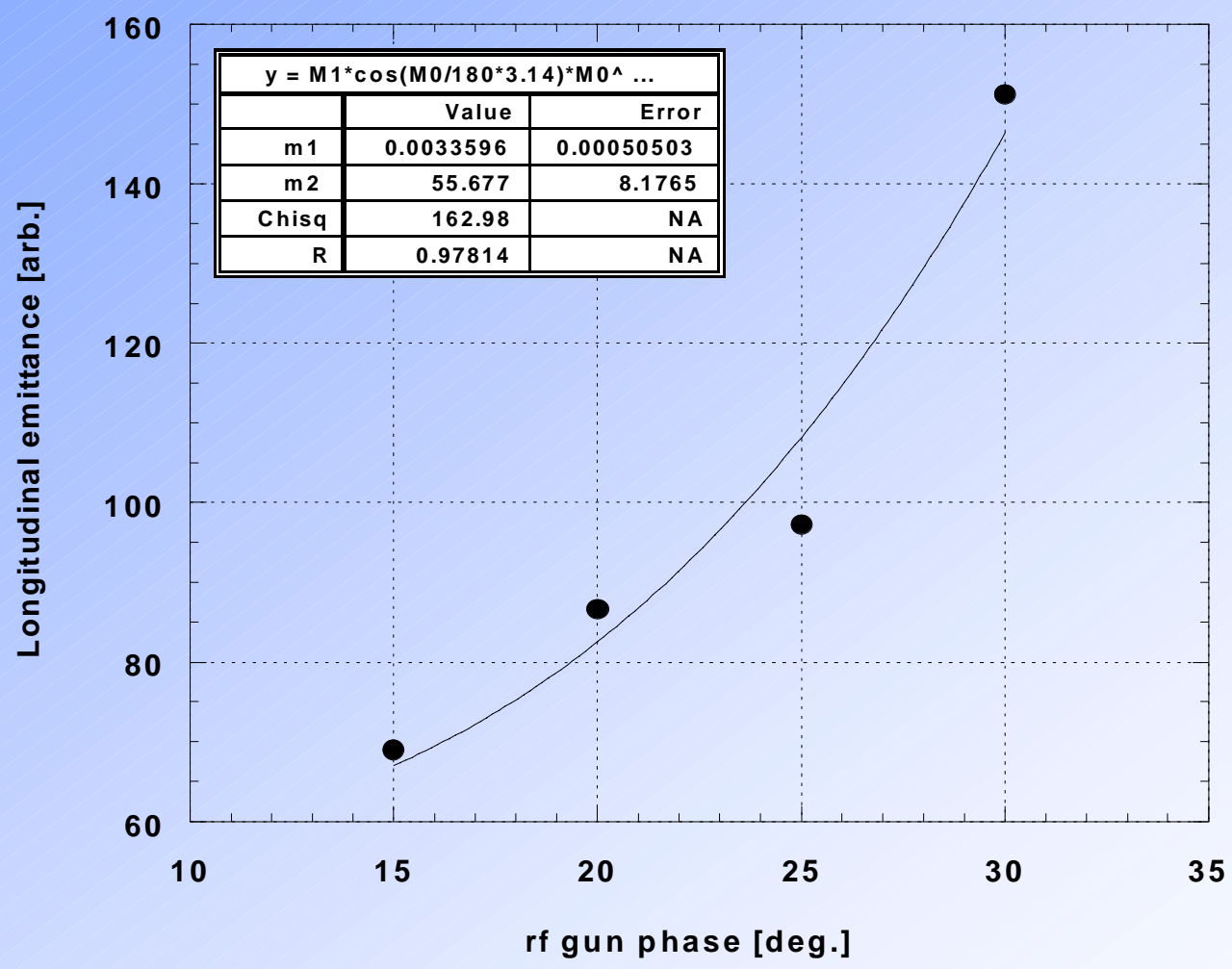




\section{- Introduction of ANL gun:}

The starting point for our studies is based on ANL gun, which is originally used for high intensity short bunch injector. This saves our time of quickly getting start.

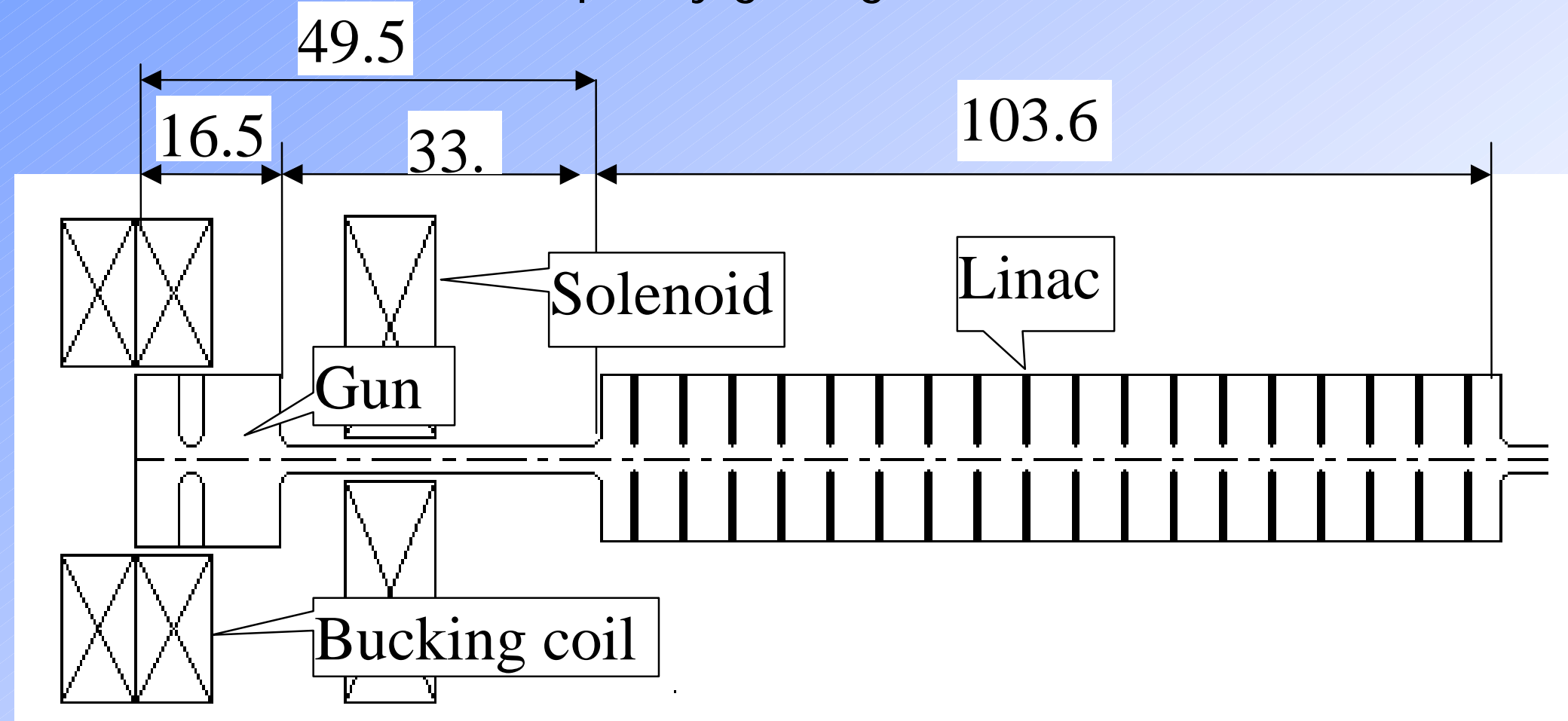

Schematic of L-band RF injector system at ANL(cm) Brookhaven Science Associates U.S. Department of Energy 

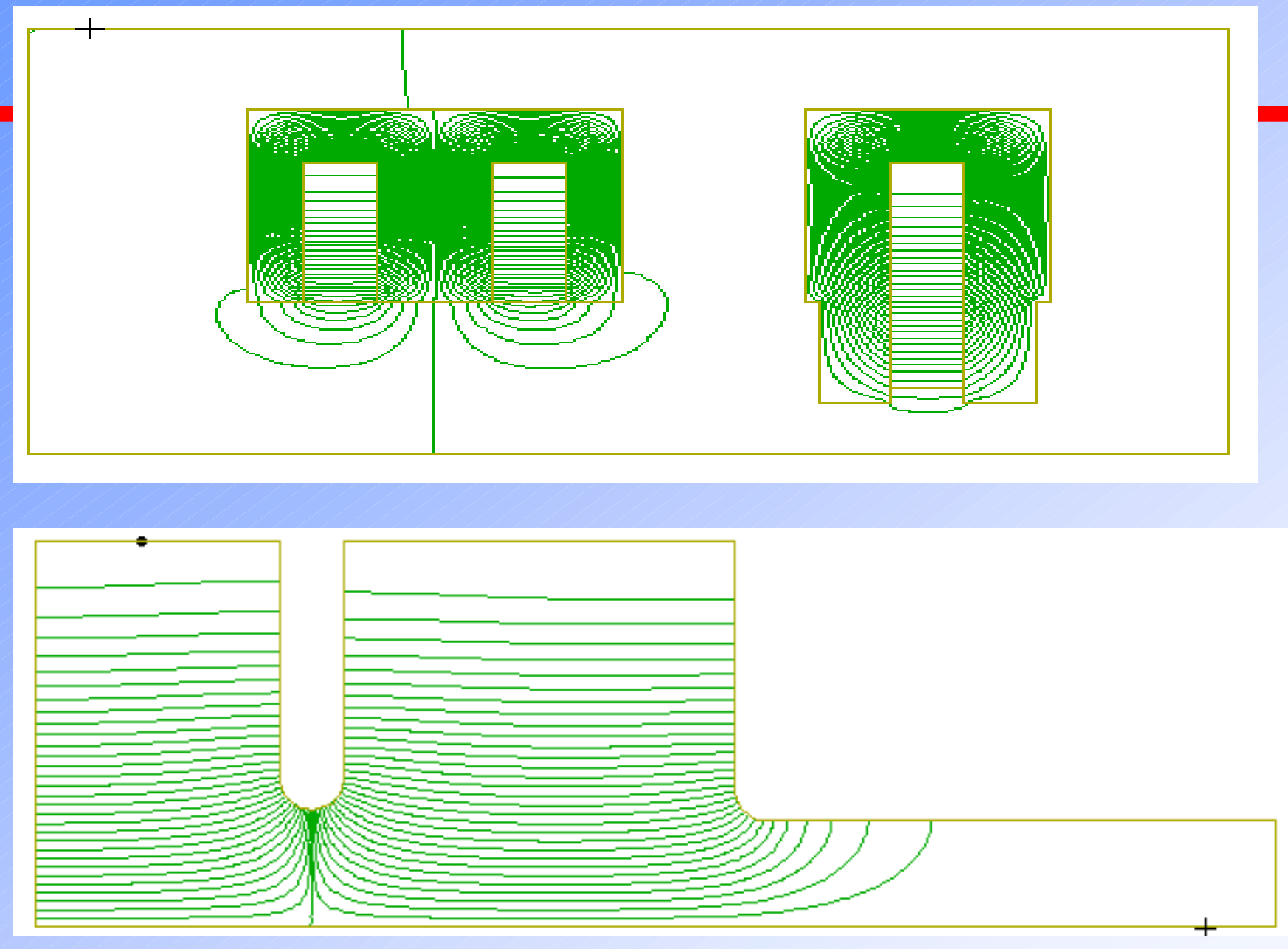

Field distributions of ANL solenoid and 1.5 cell gun 


\section{Simulation}

- Programs be used: POISSON ( for solenoids).

SUPERFISH (Gun, Buncher and accelerating structures) PARMELA (for beam dynamics).

- Layout of L-band RF gun injector for PERL 


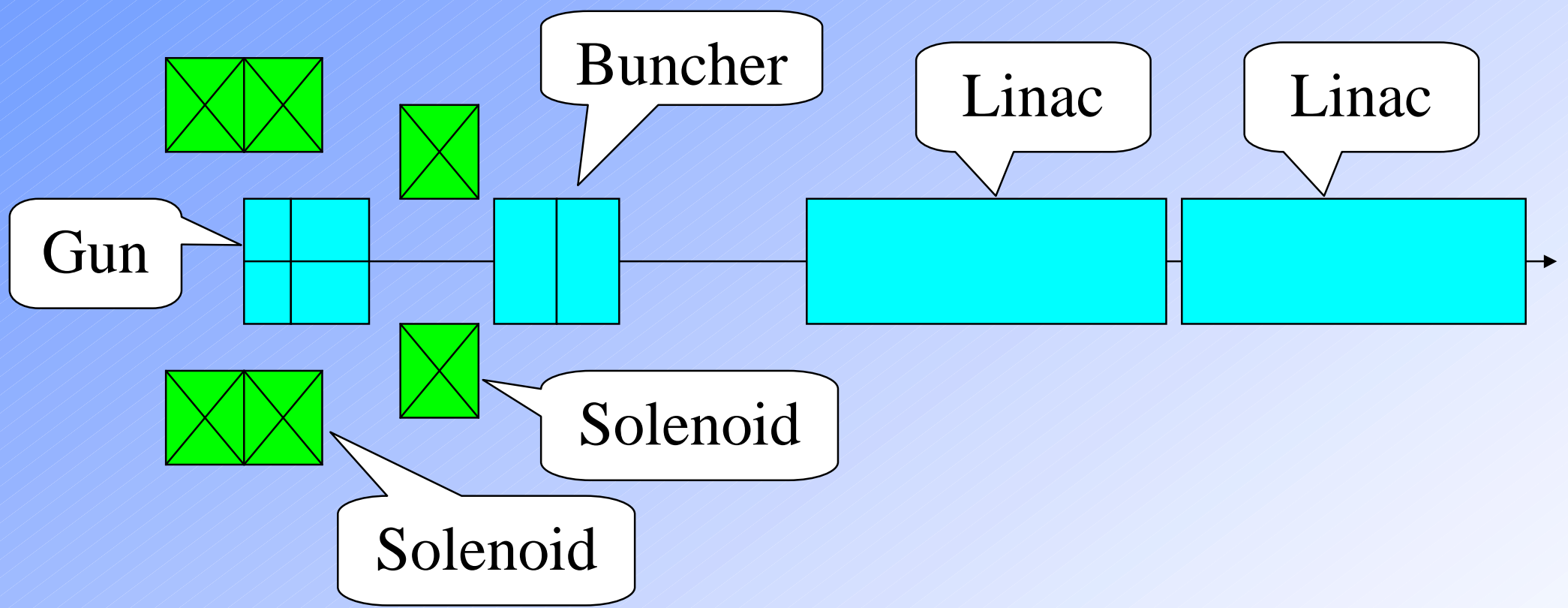

Layout of L-band RF gun injector for PERL 


\section{- Comparison of $25 \mathrm{Mv} / \mathrm{m}$ and $15 \mathrm{Mv} / \mathrm{m}$ fields on cathode.}

To minimize the heat problem, we decided to concentrate on $15 \mathrm{MV} / \mathrm{m}$ case.
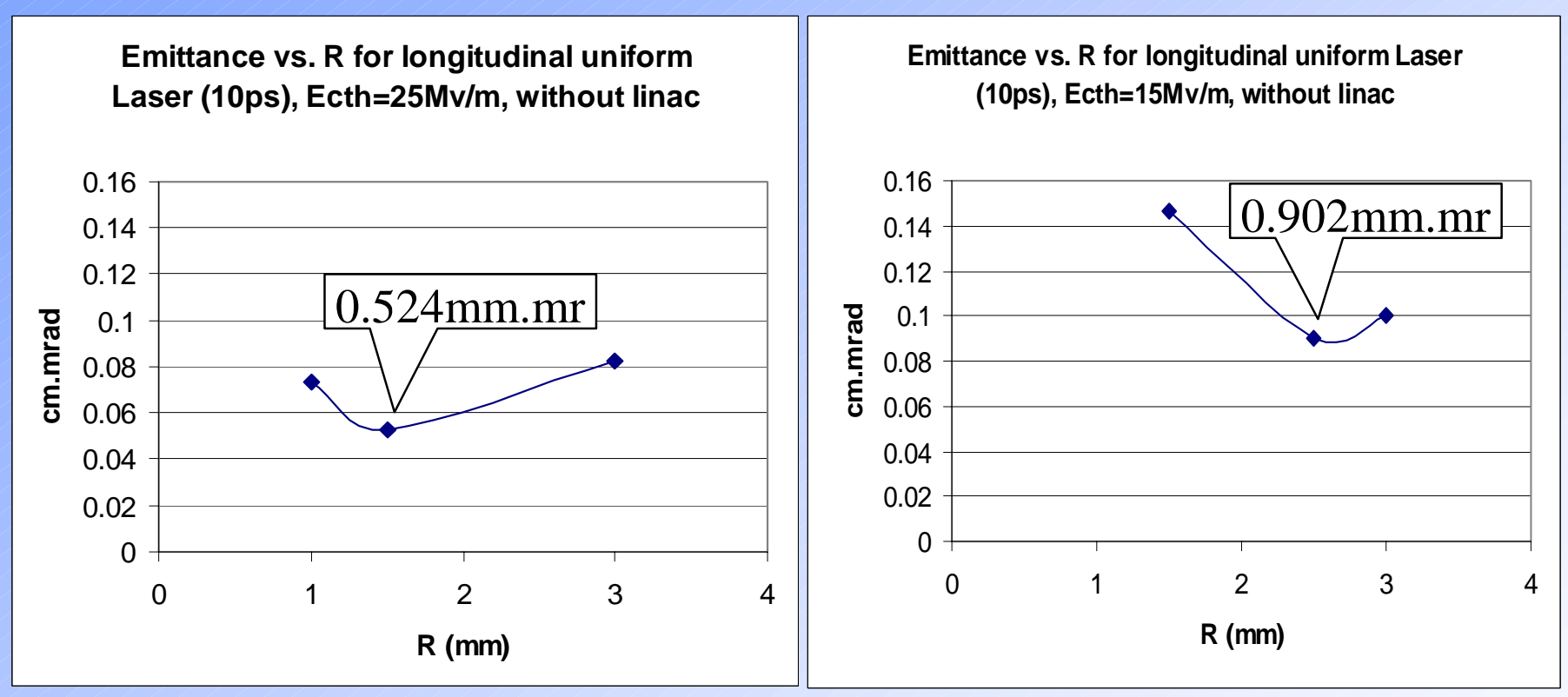

Comparison of $25 \mathrm{Mv} / \mathrm{m}$ and $15 \mathrm{Mv} / \mathrm{m}$ fields on cathode 


\section{- Performance of $15 \mathrm{Mv} / \mathrm{m}$ field on cathode, no linac. 1.5 gun}

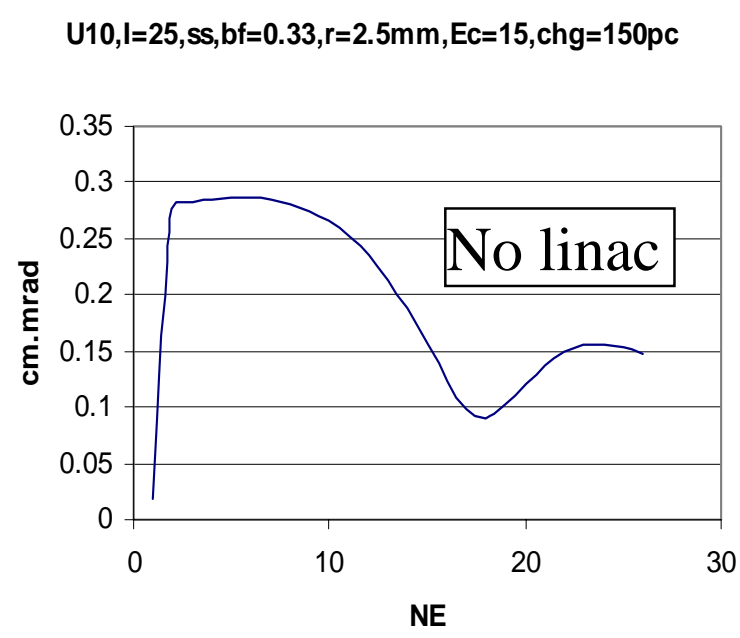

Emittance vs. initial phase for uniform $10 \mathrm{ps}, \mathrm{Ec}=15 \mathrm{Mv} / \mathrm{m}$

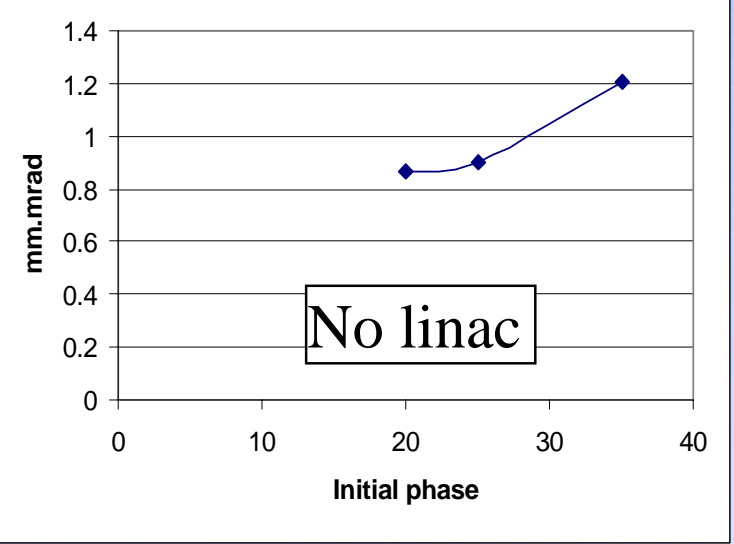

Emittance vs. bunch length for $E c=15 \mathrm{Mv} / \mathrm{m}$

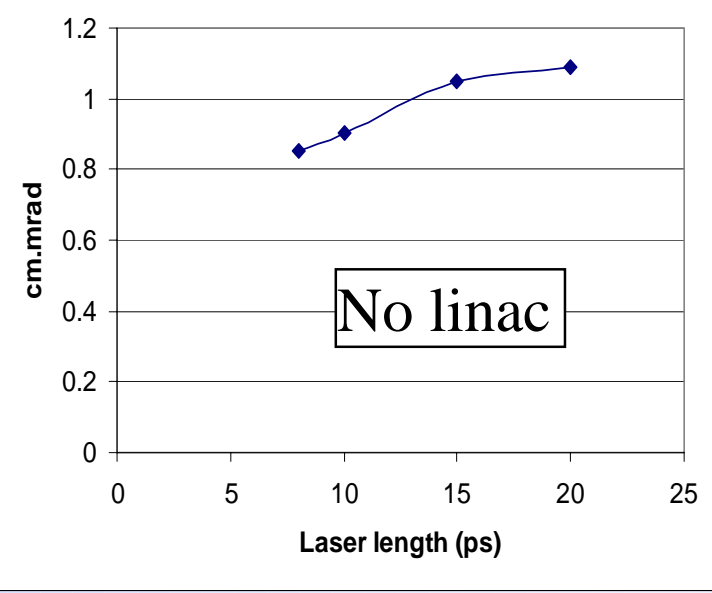

Emittance evolution for 2 linacs, Elinac $=9.5$,energy $=17.69$

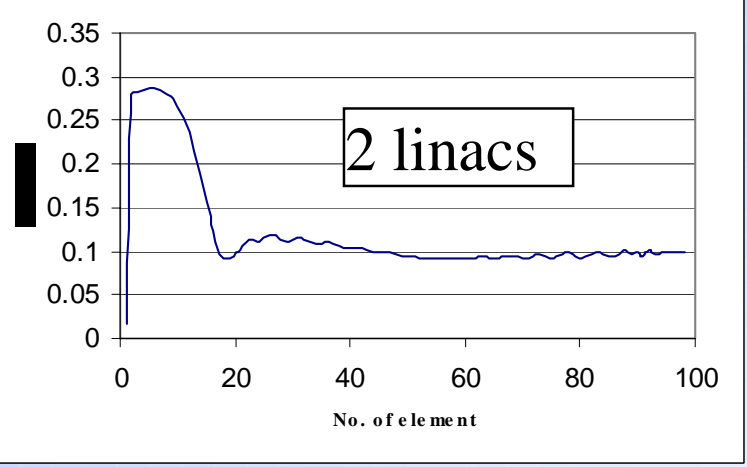

Emit. Vs. drift length

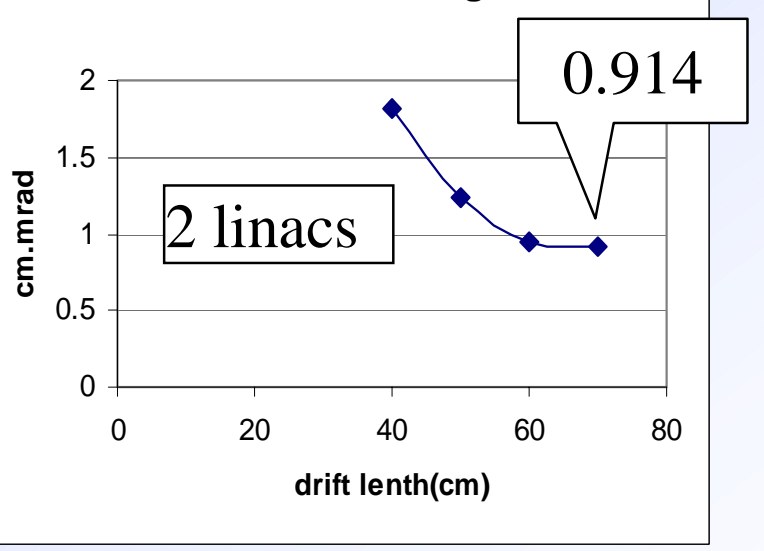


- 2.5 cell gun.

\section{A. Why use 2.5 cell gun ?}

For $30 \%$ increase power, to achieve higher energy at gun exit (1.4---2.35 MeV), which leads to significant reduce in space charge effect.

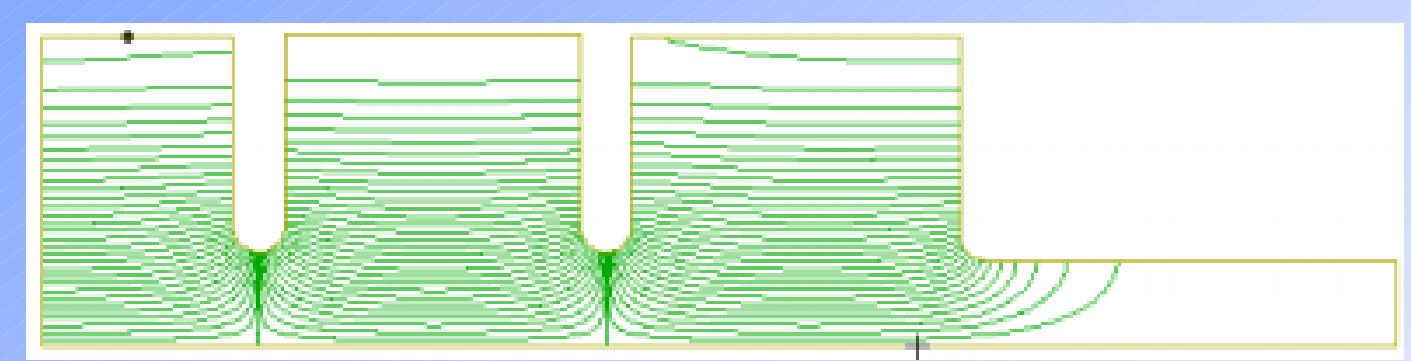

Field distribution for 2.5 cell gun
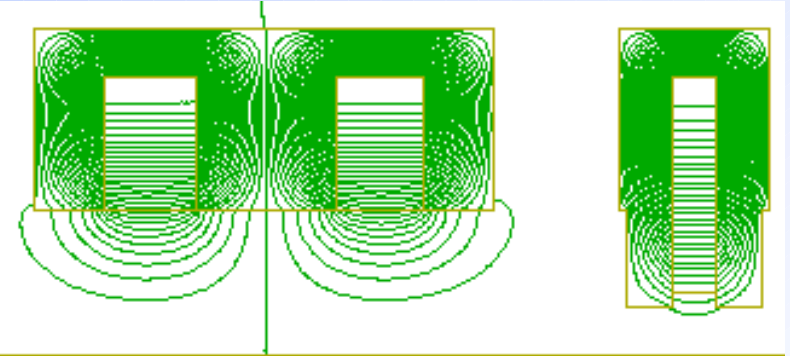

Solenoid for 2.5 gun 


\section{- Performance of a 2.5 cell gun, no bunching cavity}

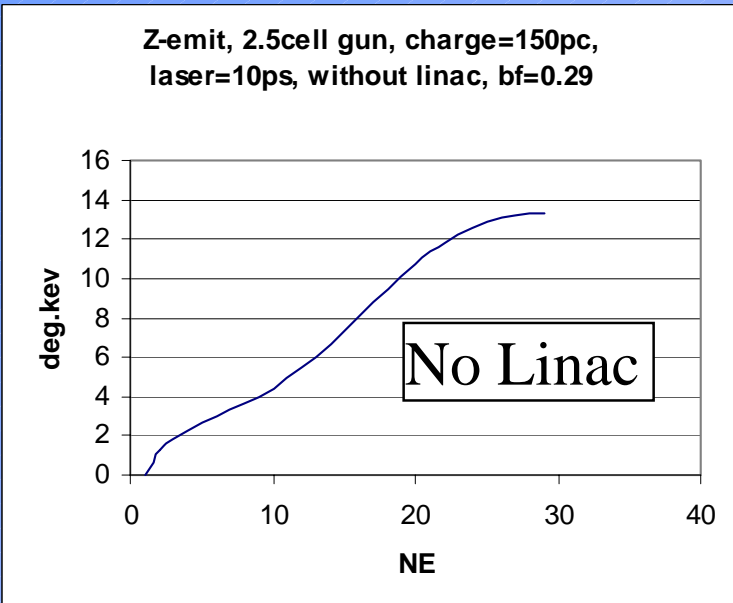

Z-emittance vs. B field for 2.5 cell gun

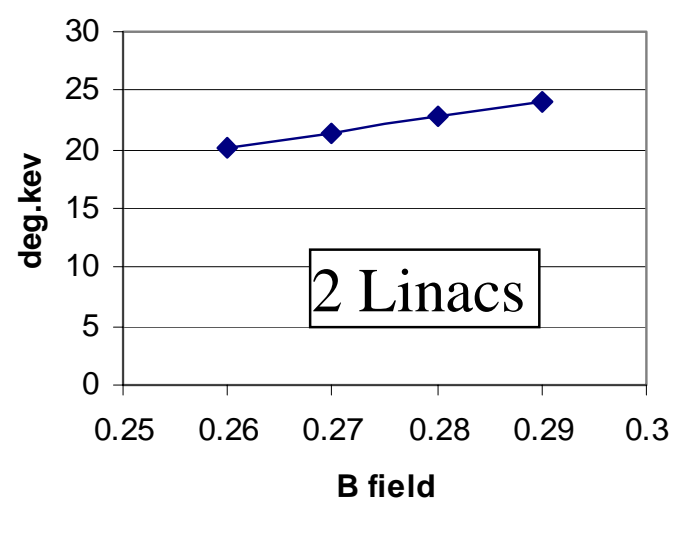

Brookhaven Science Associates U.S. Department of Energy

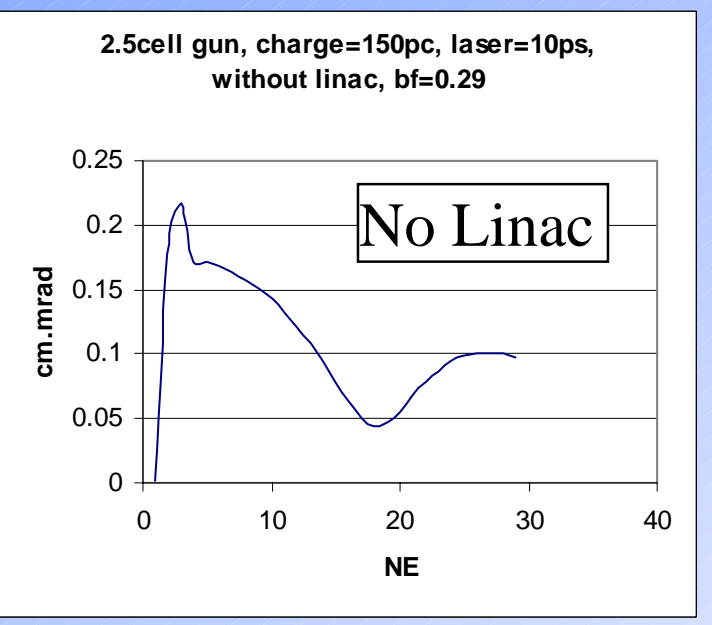

X-emittance vs. B field for 2.5 cell gun

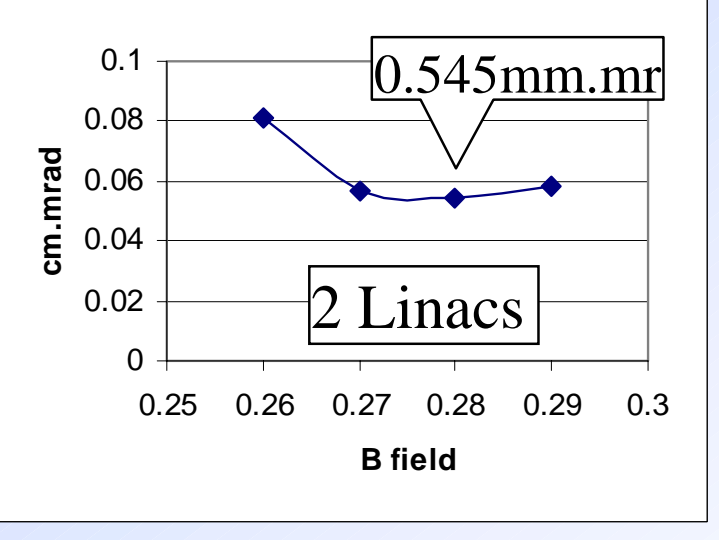

13
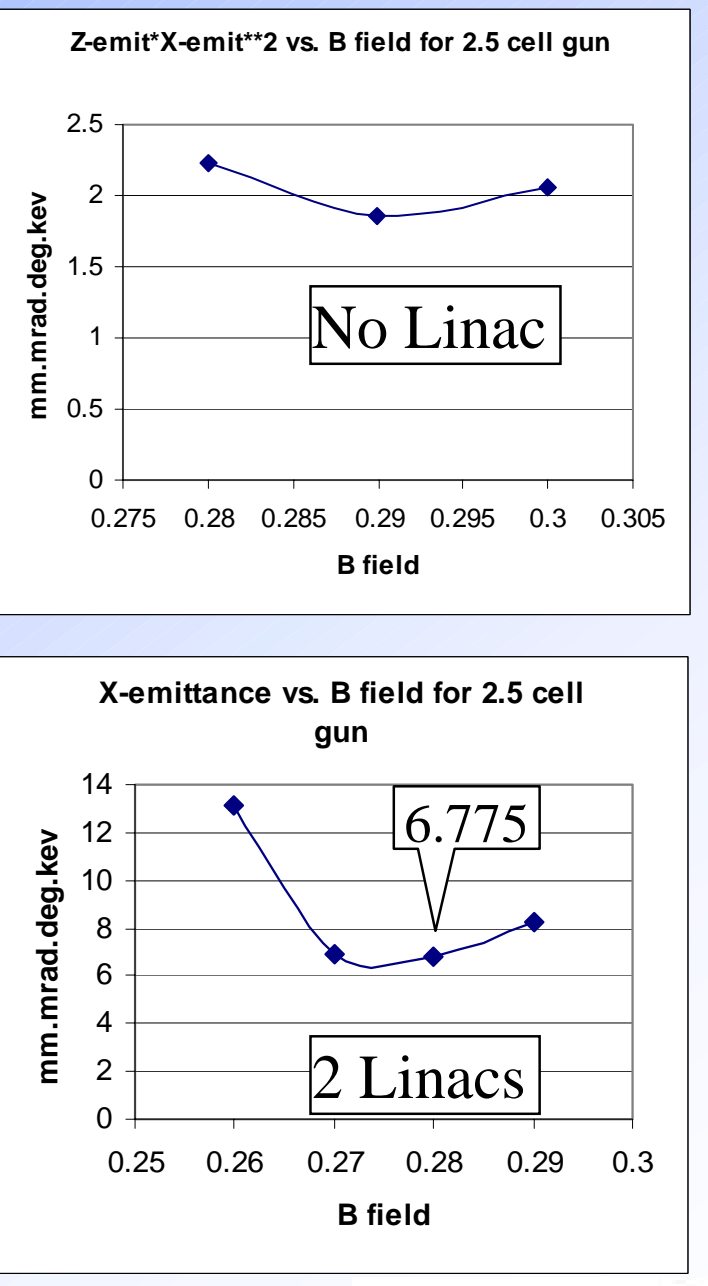

BROOKHAUEN 

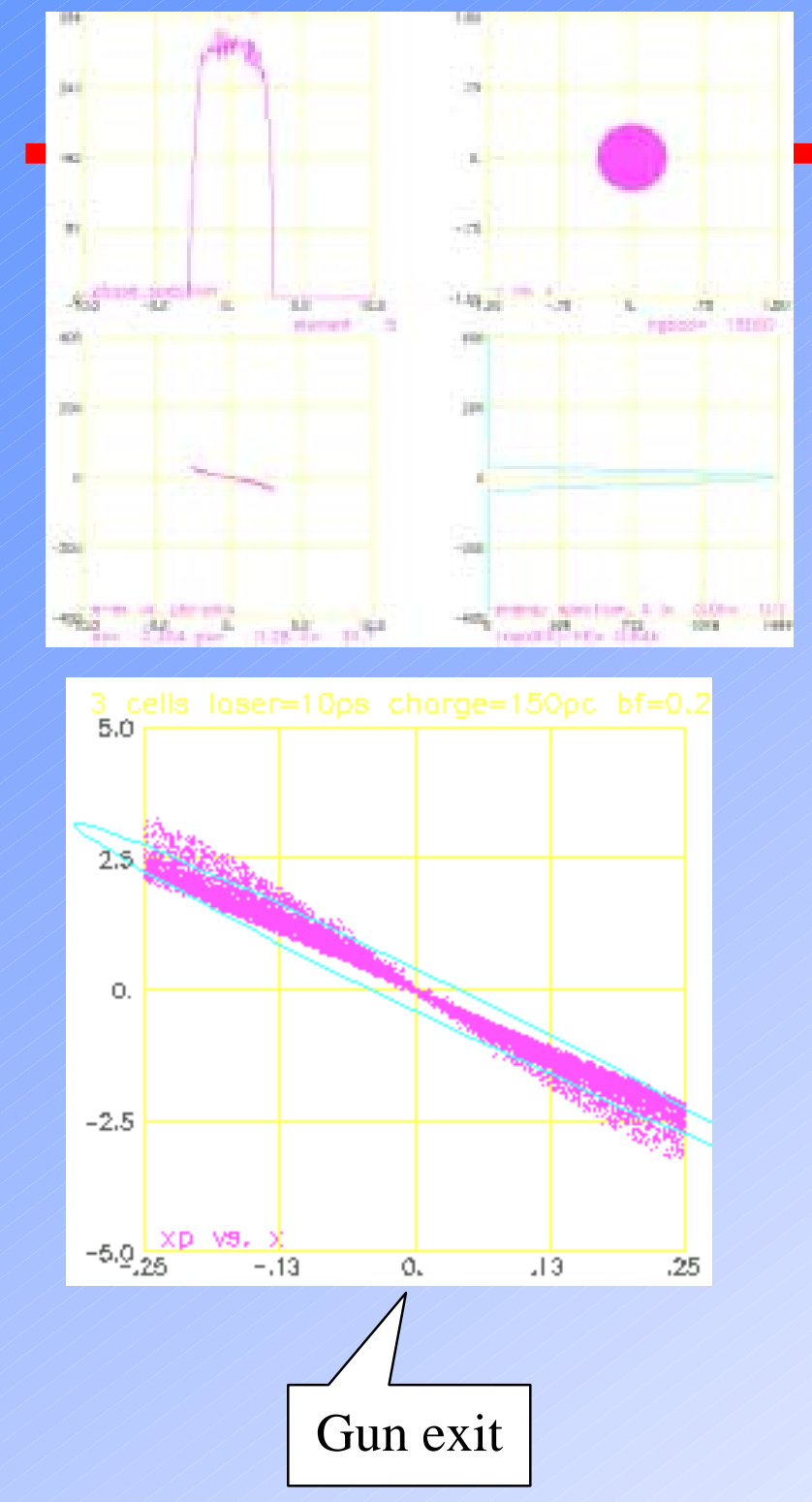

Brookhaven Science Associates U.S. Department of Energy
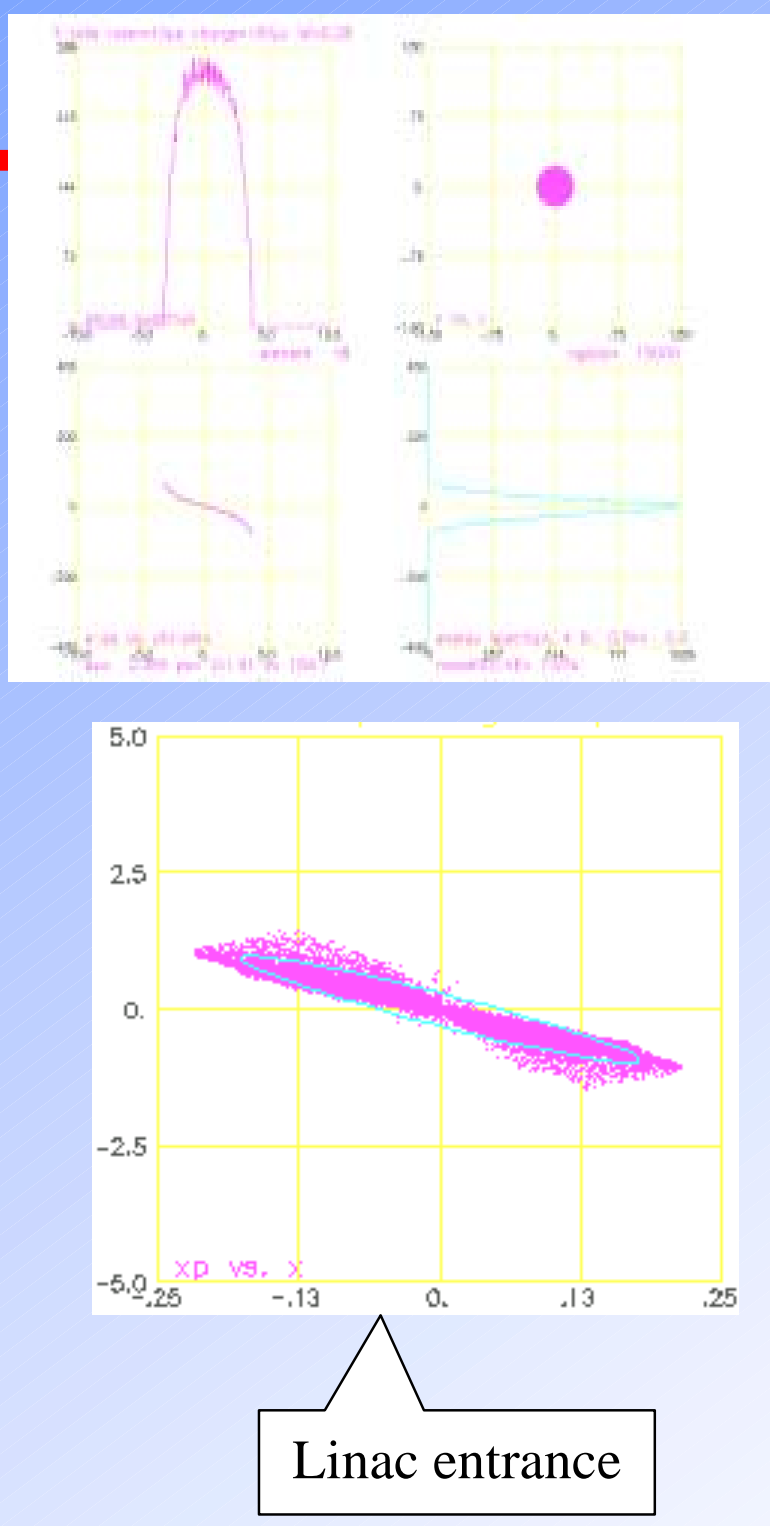

14

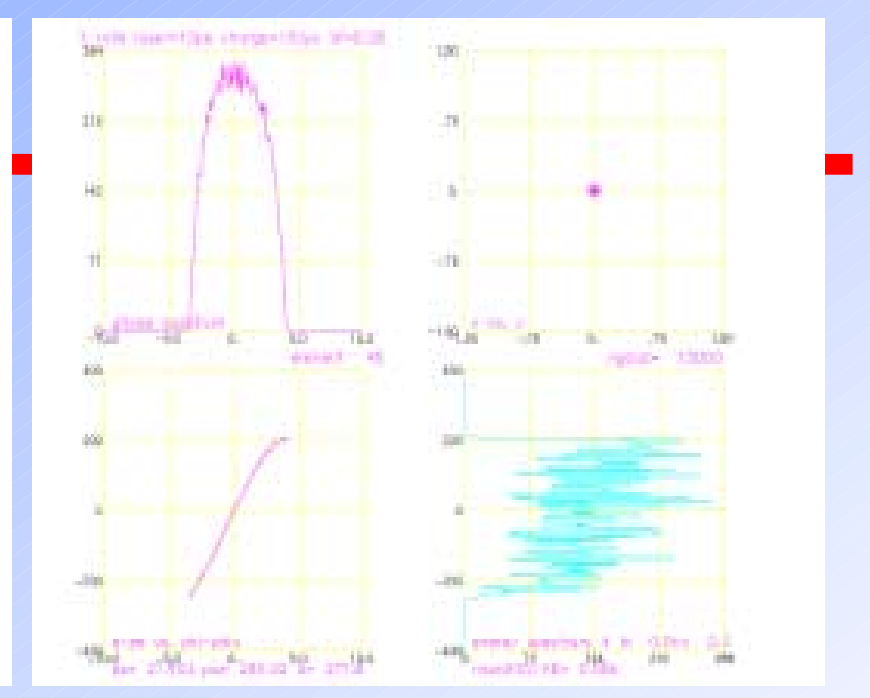

Energy $=21.9, \mathrm{RMS}(\mathrm{dkE}) / \mathrm{kE}=0.58 \%$ $\sigma_{\mathrm{z}}=1.24 \mathrm{~mm}$

2.5

o.

$-2.5$

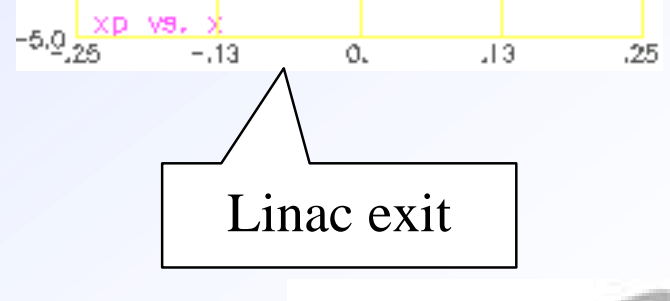

BROOKHAUEN

NATIONAL LABORATORY 


\section{- 2.5 cell with bunching cavities.}

The bunching cavity is used for the purpose of bunching beam before entering linac. But by using 2.5 cell gun, beam energy is a little bit too high for bunching. In case $\mathrm{E}=2.35 \mathrm{Mev}$, it needs $3 \%$ energy spread and $1 \mathrm{~m}$ drift space to compress $1 \mathrm{~mm}$. So, this way may not be efficient. But it does work.

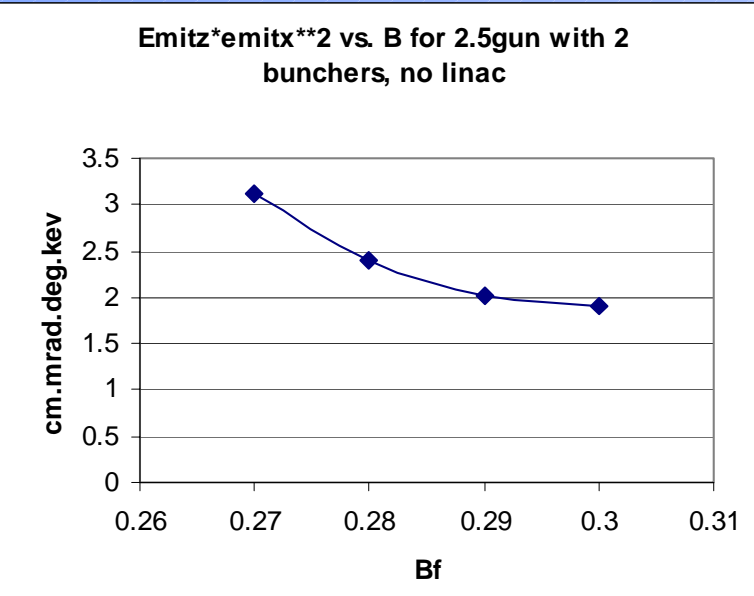

No linac

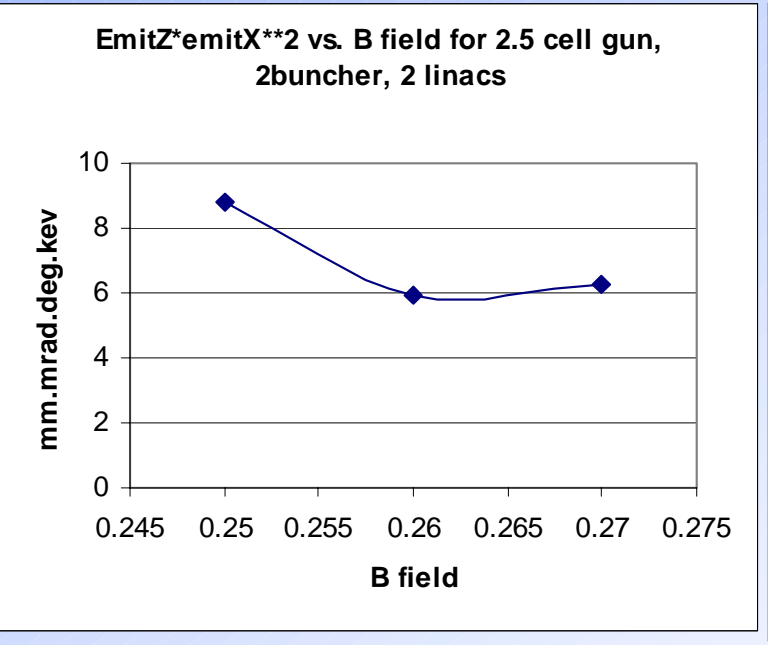

2 linacs

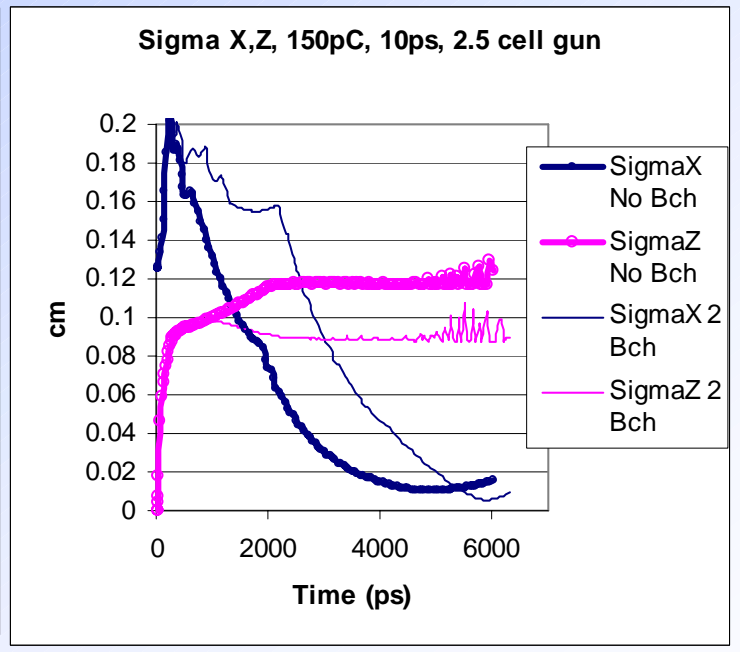

$\sigma_{\mathrm{z}}$ and $\sigma_{\mathrm{x}}$ 


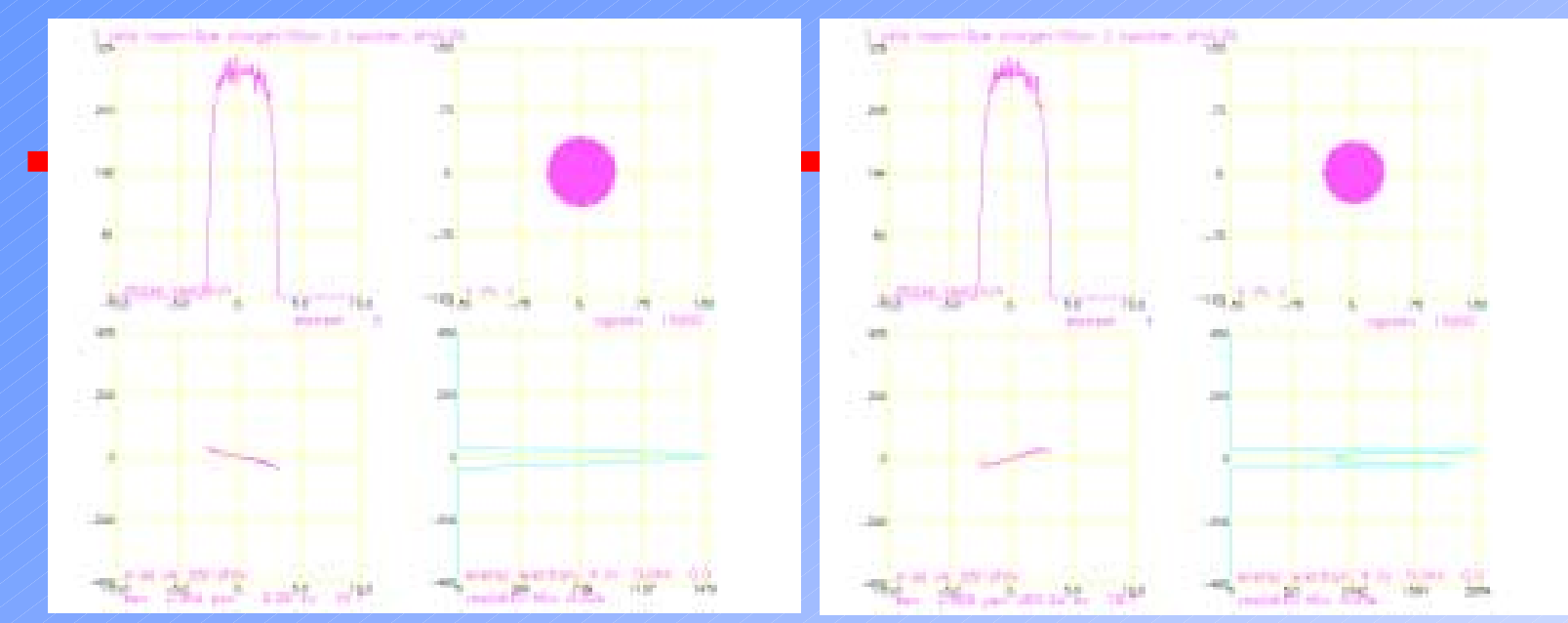

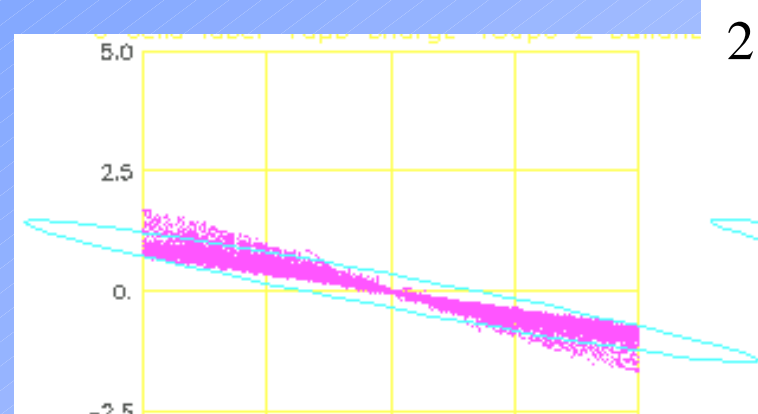

$-2.5$

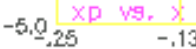

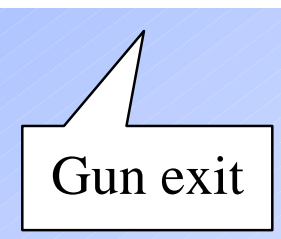

2.5 cell gun, 2 bunchers, 2 linacs

2.5

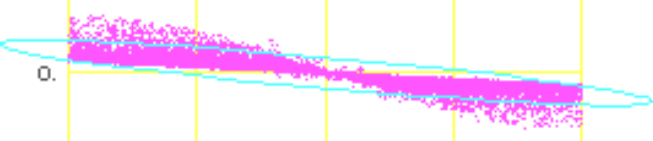

$-2.5$

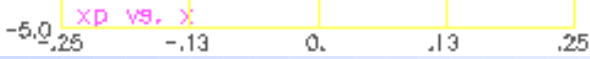

Buncher exit

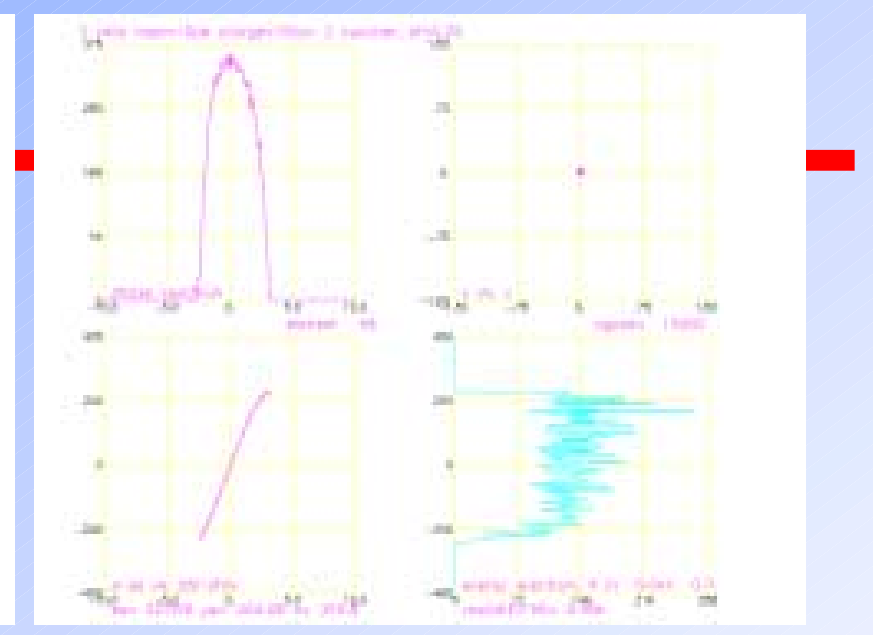

Energy $=22.1, \mathrm{RMS}(\mathrm{dkE}) / \mathrm{kE}=0.58 \%$ $\sigma_{\mathrm{z}}=0.891 \mathrm{~mm}$

2.5

o.

$-2.5$

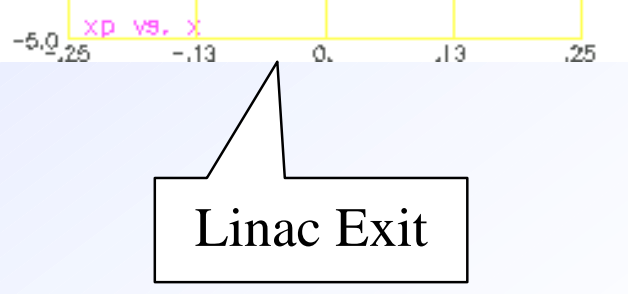

Brookhaven Science Associates U.S. Department of Energy 


\section{- 75 pC, $7 \mathrm{ps}, 2.5$ cell gun, no bunching cavity}

As space charge becomes lower, performance is much better, $\varepsilon_{\mathrm{z}} \times \varepsilon_{\mathrm{x}}{ }^{2}$ is $1 / 7$ that of $150 \mathrm{pc} . \sigma_{\mathrm{z}}=0.828 \mathrm{~mm}$,

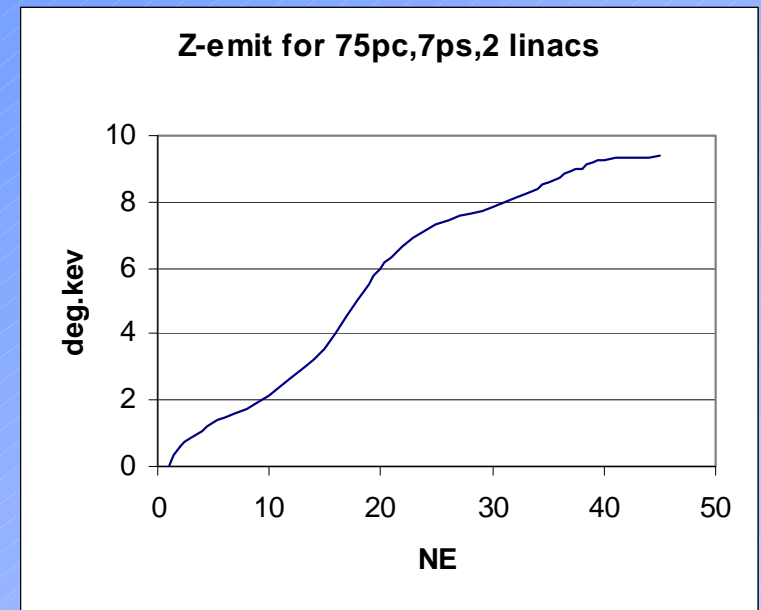

Brookhaven Science Associates U.S. Department of Energy
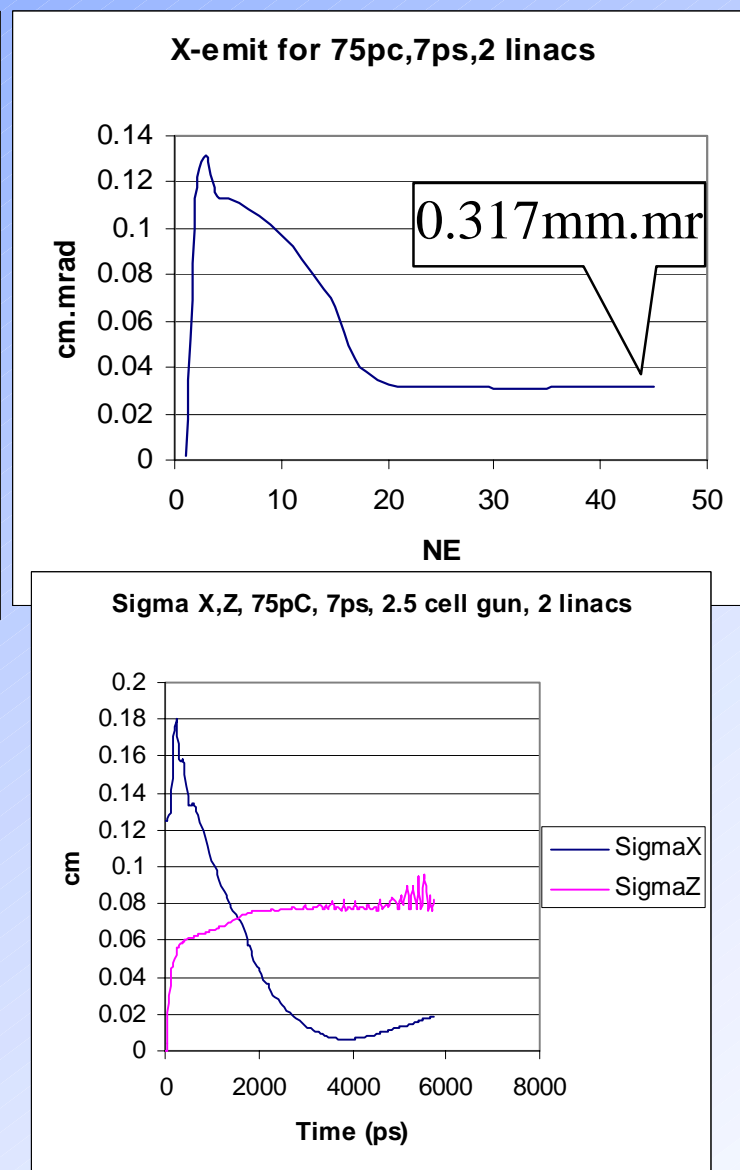

17

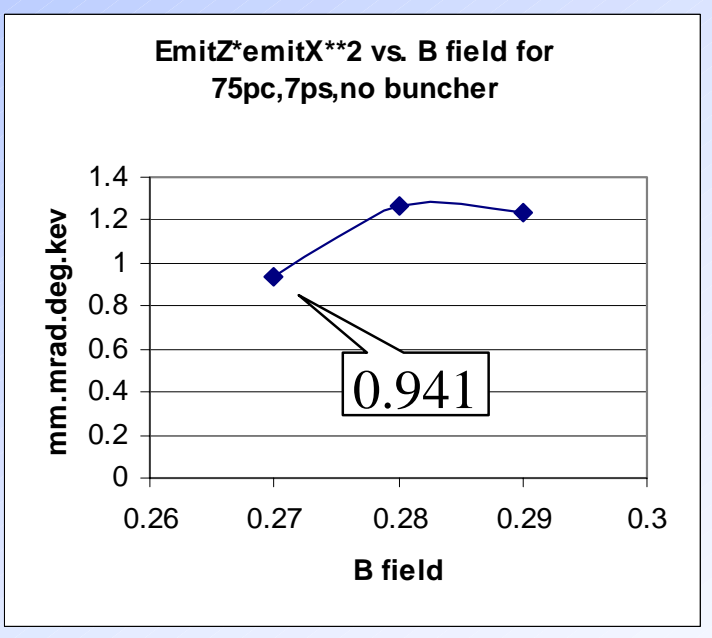

BROOKHAUEN

NATIONAL LABORATORY 

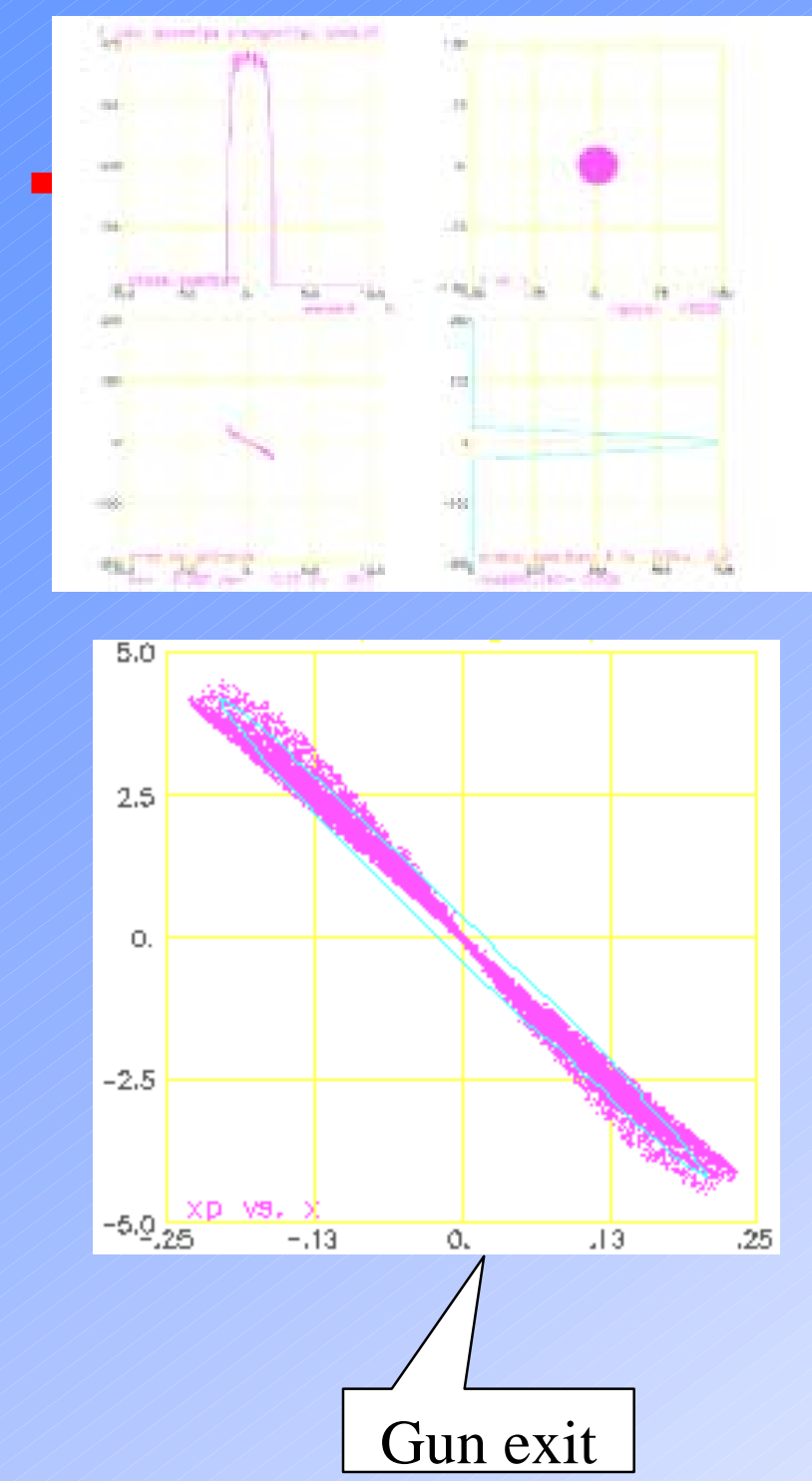

Brookhaven Science Associates U.S. Department of Energy

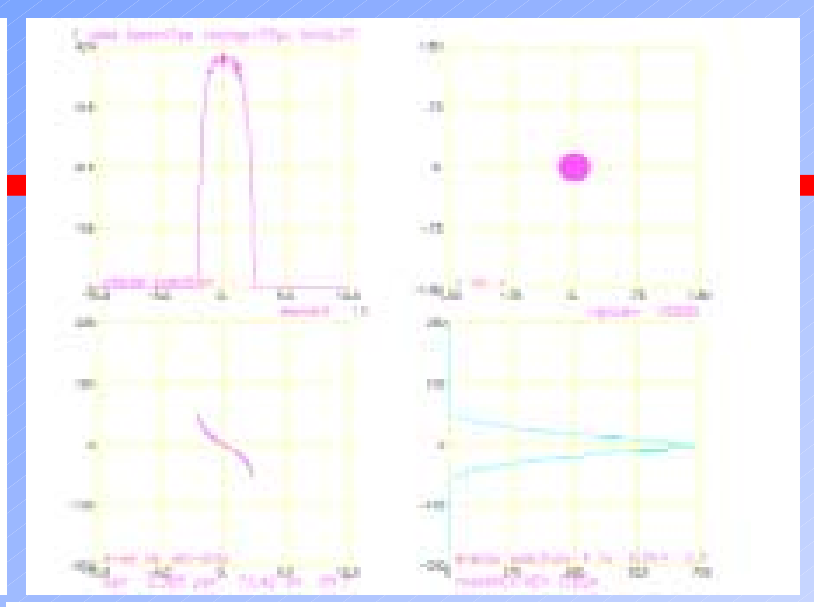

$75 \mathrm{pc}, 7 \mathrm{ps}, 2.5$ cell gun, 2 linacs

2.5

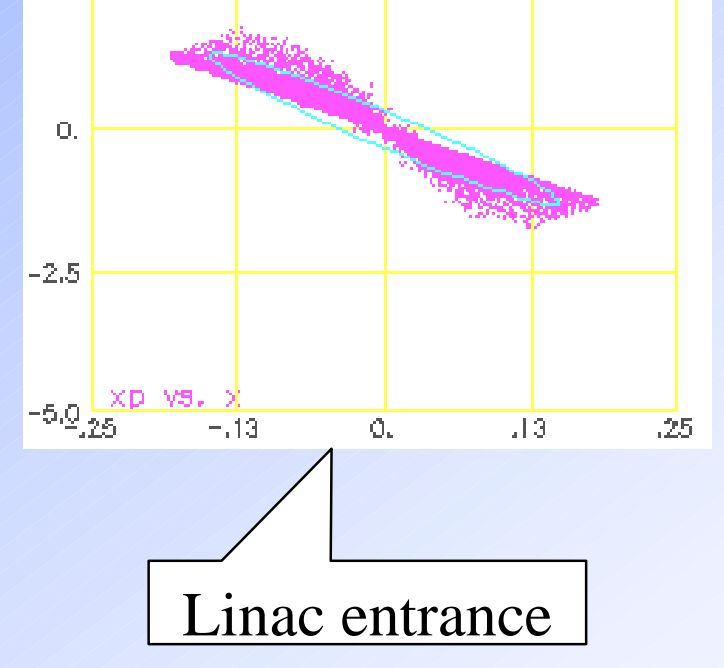

Energy $=22.1, \mathrm{RMS}(\mathrm{dkE}) / \mathrm{kE}=0.28 \%$ $\sigma_{\mathrm{z}}=0.828 \mathrm{~mm}$

$2, \frac{1}{2}$

o.

$-2,5$

$-5 . x_{.25}$ y. $x$

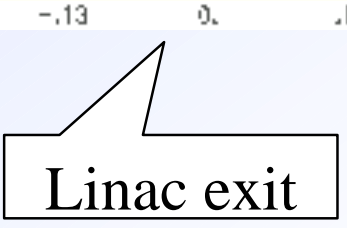


- 75 pC, $7 p s, 2.5$ cell gun, no bunching cavity, optimization
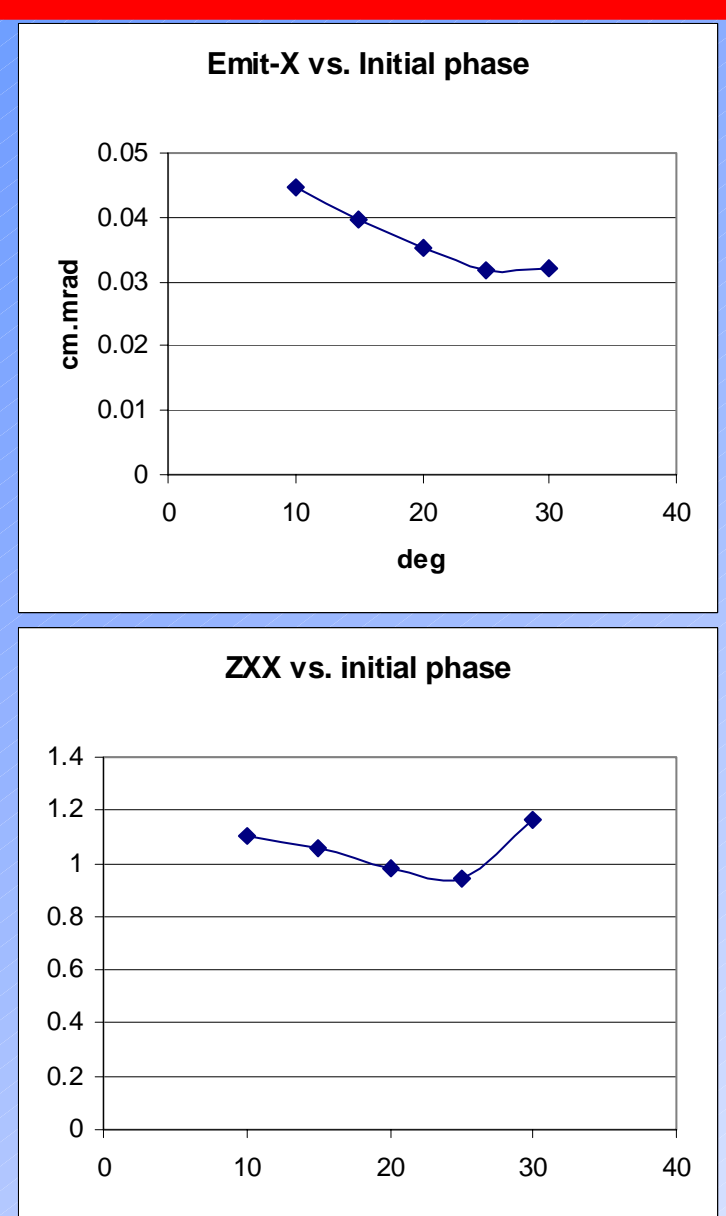

Initial Phase

\section{Brookhaven Science Associates}

U.S. Department of Energy
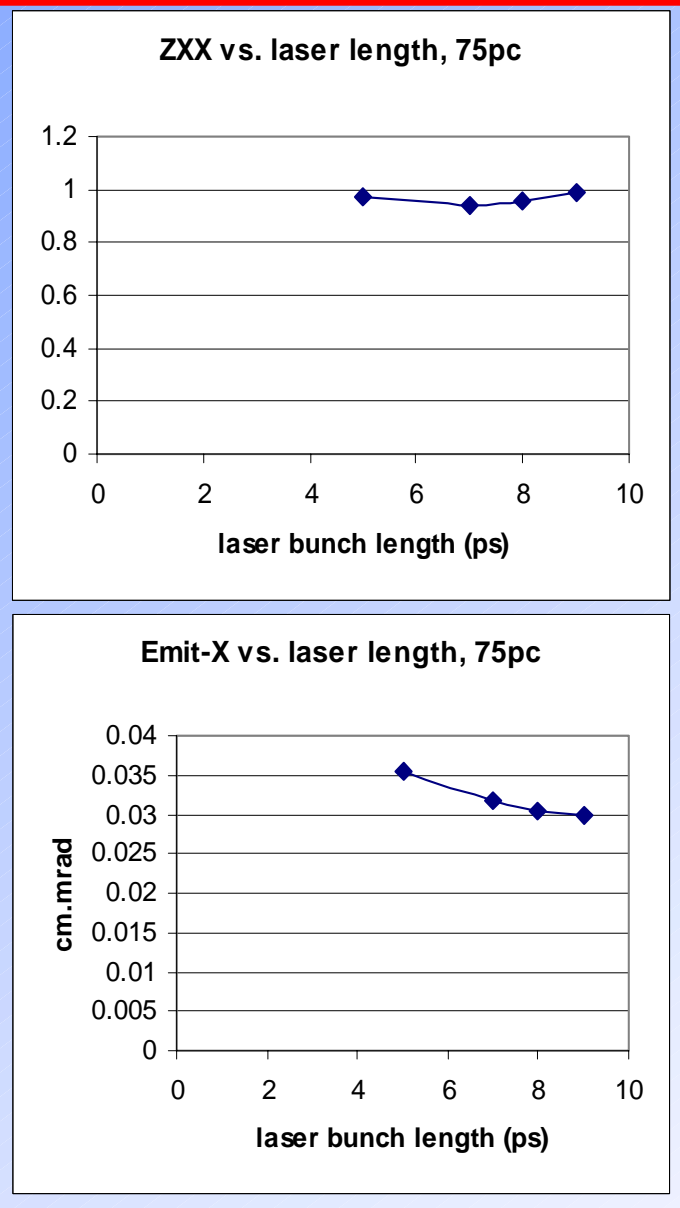

\section{Bunch Phase}

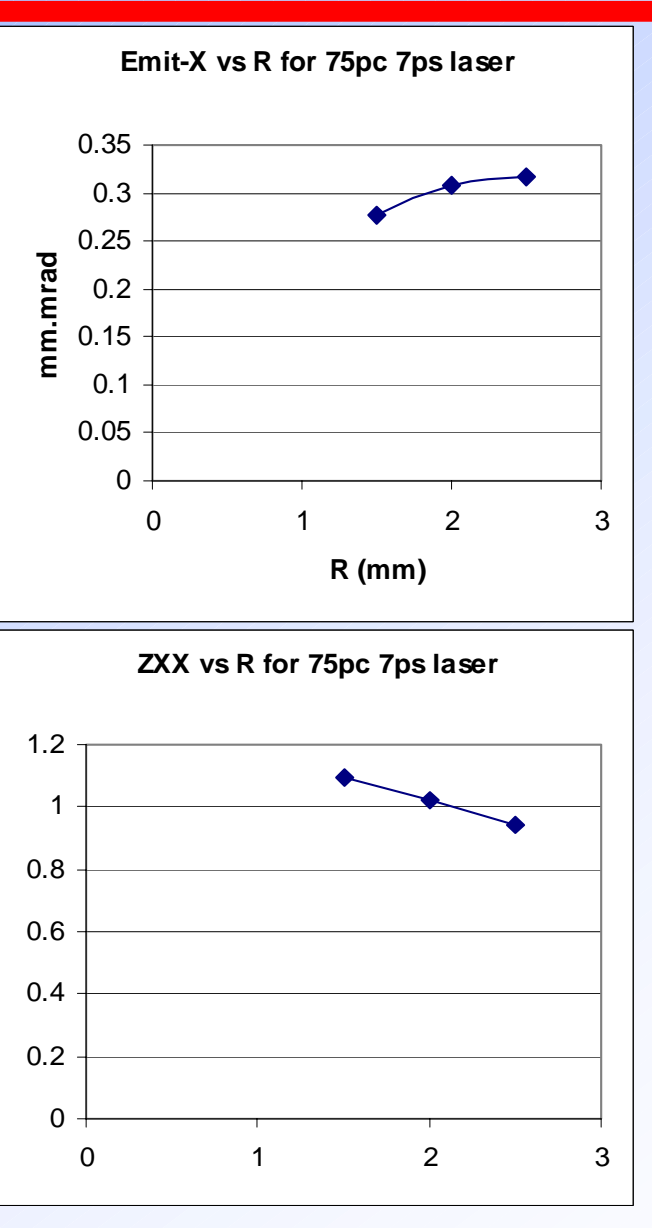

$\mathrm{R}$ 


\section{- 75pc, Gaussian, FWHM 7ps, 2.5 cell gun}

As the technique of shaping longitudinal laser distribution is difficult, using longitudinal Gaussian distribution can simplify the system and then improve system stability.
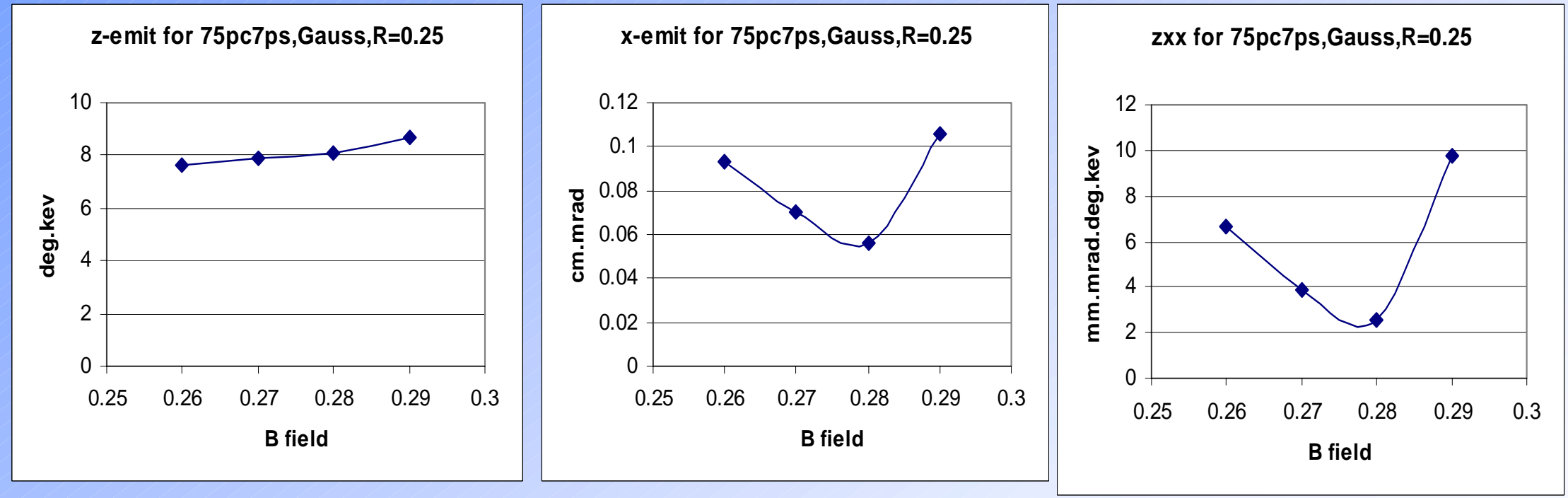

A typical case for 75pc Gaussian distribution 


\section{- Optimization for 75pc, 7ps, Gaussian.}
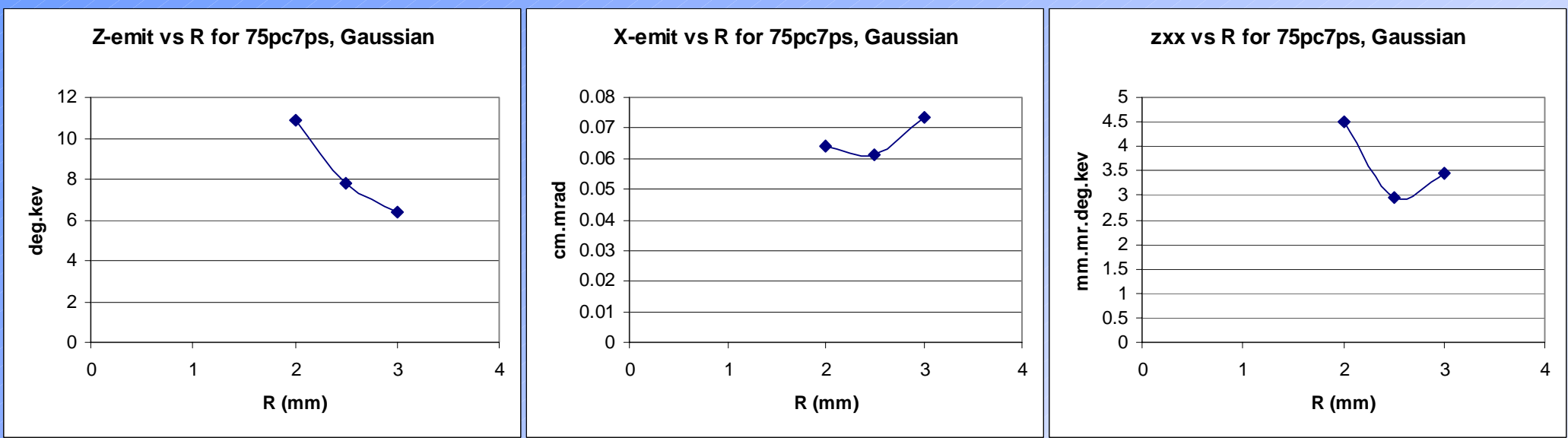

\section{Z-emit,X-emit and ZXX vs. R}
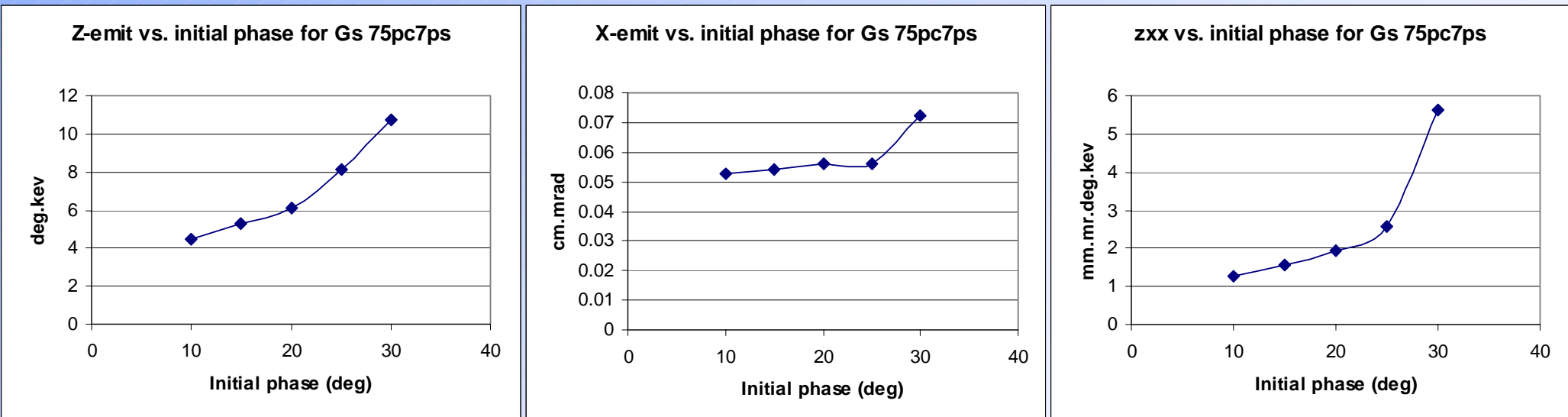

\section{Z-emit,X-emit and ZXX vs. initial phase}

Brookhaven Science Associates

U.S. Department of Energy 


\section{- Higher order mode cavity.}

By using a gun working at high mode can increase its size. The total power loss on wall increases $60 \%$, but the power dissipation density on wall decreases $60 \%$, which can relieve the heat handling problem. As the field distribution is almost the same as original gun, beam dynamics do not change much. The problem is it will be difficult to apply enough solenoid field in gun because the diameter becomes larger.

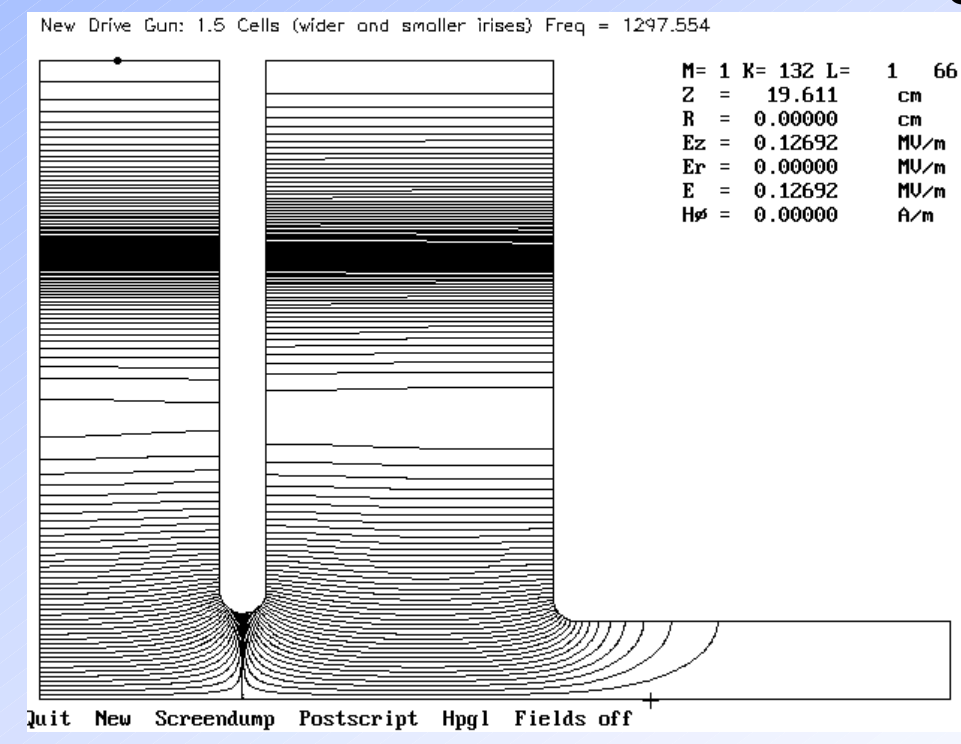




\section{Conclusion and Summary}

- L-band photocathode RF gun is capable of producing PERL quality beam.

- A 1.5 cell L-band with $25 \mathrm{Mv} / \mathrm{m}$ can produce beam required by PERL.

- 2.5 cell gun is better than 1.5 cell gun. Assume the electric field on cathode we can achieved is $15 \mathrm{Mv} / \mathrm{m}$, by using the 2.5 cell gun, for charge $=150 \mathrm{pC}$, we can reach at least the following beam performance at linac exit:

Energy $=21.9 \mathrm{Mev}, R M S(\mathrm{dkE}) / \mathrm{kE}=0.58 \%, \sigma_{z}=1.24 \mathrm{~mm}$,

$\varepsilon_{z}=22.8$ deg.kev, $\varepsilon_{x}=0.545 \mathrm{~mm} . \mathrm{mrad}$, $\varepsilon_{z} \times \varepsilon_{x}^{2}=6.775 \mathrm{~mm}^{2} . \mathrm{mrad}^{2}$. deg.kev.

- For a $75 p C$ charge, the performance is improved greatly. Energy $=22.1 \mathrm{Mev}, R M S(\mathrm{dkE}) / \mathrm{kE}=0.28 \%, \sigma_{z}=0.828 \mathrm{~mm}$, $\varepsilon_{z}=9.364$ deg. kev, $\varepsilon_{x}=0.317 \mathrm{~mm} . \mathrm{mrad}$, $\varepsilon_{z} \times \varepsilon_{x}^{2}=0.941 \mathrm{~mm}^{2} . \mathrm{mrad}^{2}$.deg.kev.

- To simplify the system and improve its stability performance, longitudinal Gaussian distribution laser is considered. We found that the lower the initial phase, the better the performance. When $\phi_{1}=10 \mathrm{deg}, \sigma_{z}=0.673 \mathrm{~mm}$ $\varepsilon_{z}=4.464 \mathrm{deg} . \mathrm{kev}, \varepsilon_{x}=0.529 \mathrm{~mm} . \mathrm{mrad}$, $\varepsilon_{z} \times \varepsilon_{x}^{2}=1.249 \mathrm{~mm}^{2} . \mathrm{mrad}^{2}$.deg.kev. 


\section{Conclusion and Summary}

- Using higher mode cavity RF gun can reduce the power density on the gun cavity wall. More geometric optimization could lead to power reduction $10 \%$.

- More studies need to be done, such as:

A. Performance of higher order mode gun.

B. Study the possibility of shaping the cathode to increase RF focusing near cathode.

C. Parameter optimization.

D. Thermal stress and heat flow calculation are needed

We would like to express our gratitude to Dr W. Gai and $\mathrm{M}$. Conde for providing their gun input files. 


\title{
Beam Dynamics Issues at PERL Injector X.J. Wang \\ National Synchrotron Light Source
}

\author{
1. Introduction. \\ 2. Beam dynamics Major issues at PERL injector \\ A. Space charge effect. \\ B. Longitudinal phase space. \\ C. Transverse phase space bifurcation. \\ D. Nonlinear longitudinal phase compensation. \\ E. Linear space charge compensation. \\ 3. Summary
}




\section{Introduction}

The advantage of PERL over traditional light source is that, small transverse emittance couple with small longitudinal emittance. All beam dynamics issues addressed by Vitaly also exist in PERL injector, such as Wake field, BBU, but other issues even more important at injector:

1. Space charge effect.

2. Transverse phase space bifuracation caused by space charge effects.

3. Longitudinal phase.

4. Non-linearity in compensation in compressor by wake field or space charge effect. 


\section{Space charge effect}

$$
\begin{aligned}
& x^{\prime \prime}+k(s) x-\frac{2 r_{e} N / \ell}{a^{2} \gamma^{3}} x=0 \\
& z^{\prime}=\frac{\delta}{\gamma^{2}} \\
& \delta^{\prime}=\frac{3 r_{0} N}{\gamma^{3} \beta^{2} \ell_{b}^{3}} f(a, b) z
\end{aligned}
$$

Energy is the most effective reduce space charge effect, asymmetry in transverse and longitudinal space charge effect must be kept in mind all time for PERL.

Z-emittance(rms) vs. Z(Bf=0.30,0.25,0.20)

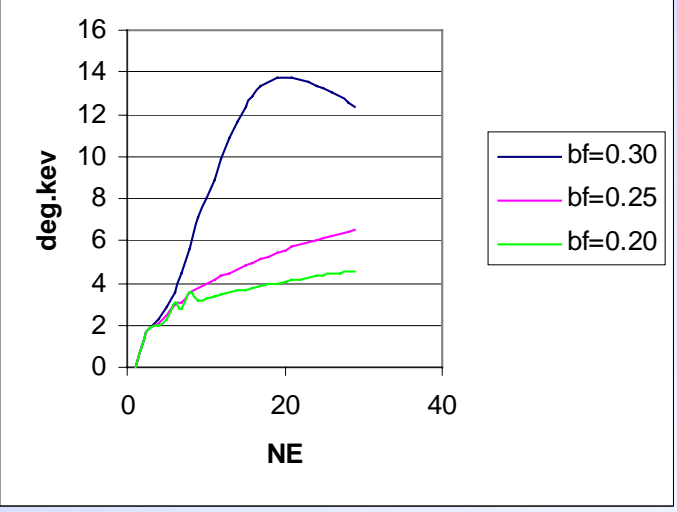

X-emittance $(\mathrm{rms})$ vs. $Z(B f=0.30,0.25,0.20)$

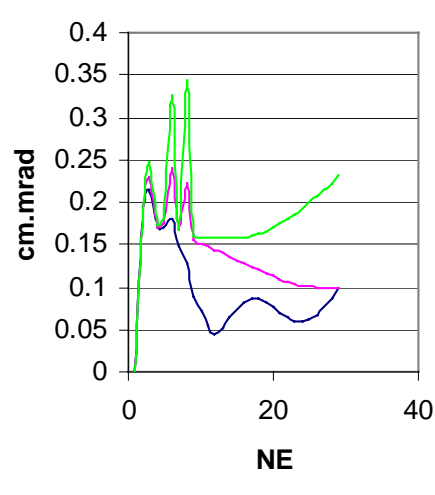




\section{Transverse phase bifurcation}

Studies were done at Jlab to understand transverse Phase Bifurcation due to space charge. We now observed at L-band injector studies. This could be important if beam will lost in later stage. We need to look into several solution on this problem:

1. Beam scrapper.

2. Reduce charge.

3. Modify the longitudinal beam distribution.

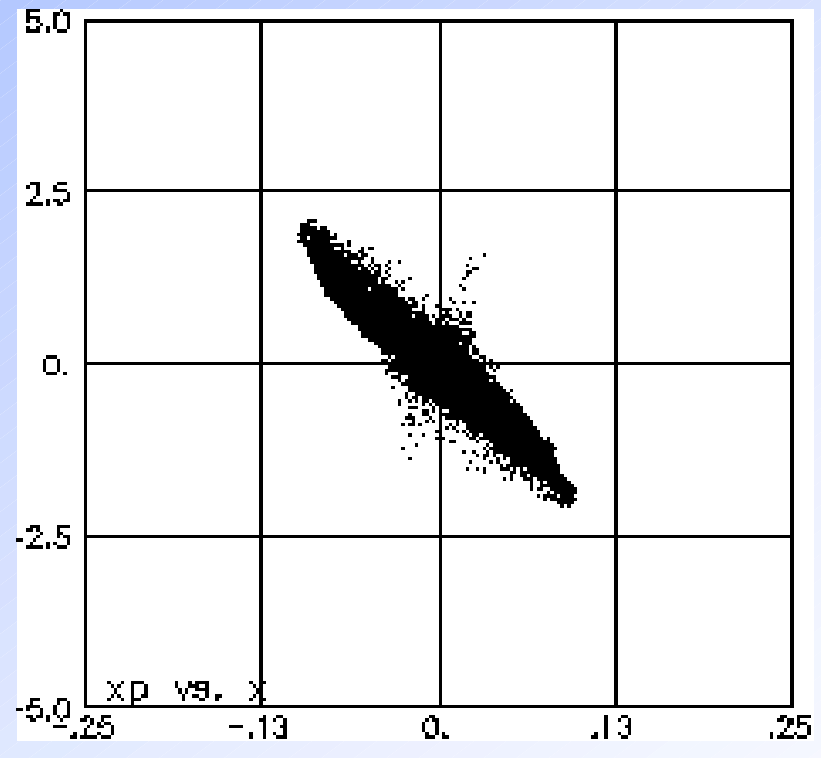

BROOKHRUEN

NATIONAL LABORATORY 


\section{Longitudinal phase space compensation}

\section{H. Liu, NIM A}
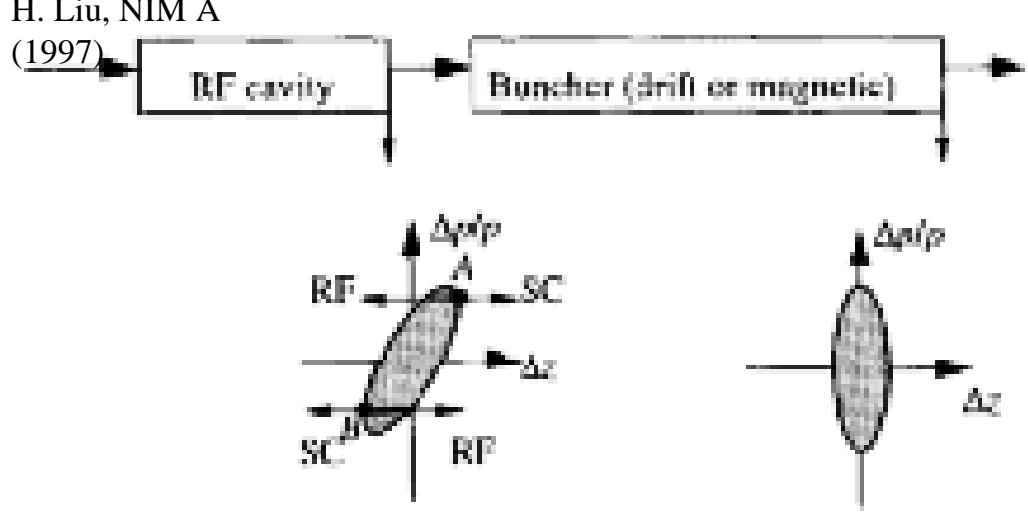
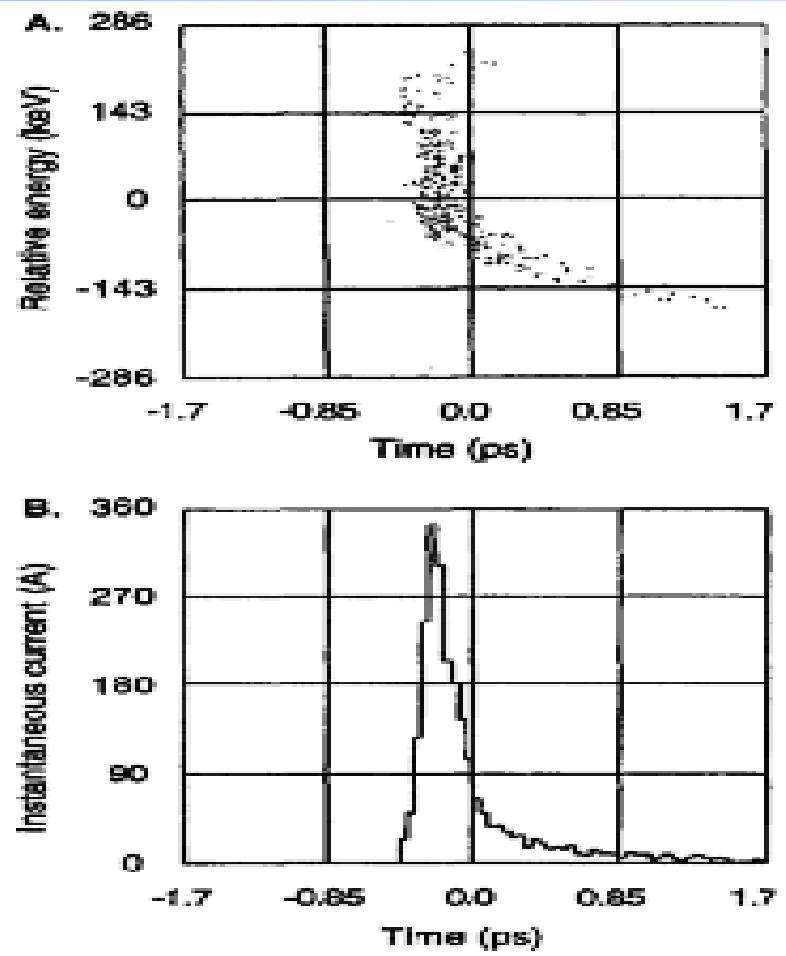

Fiz. 5. PAFMELA simulation resulis for a 0.1 we bunch after compresion to a FWHM of D.22 pa. da Langitucinal phase space,

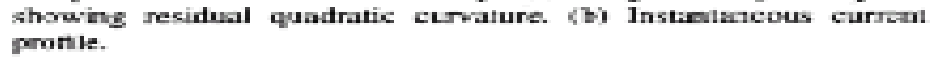

B. Carlsten, NIM A (1996) 


\section{Longitudinal Emittance Compensation}
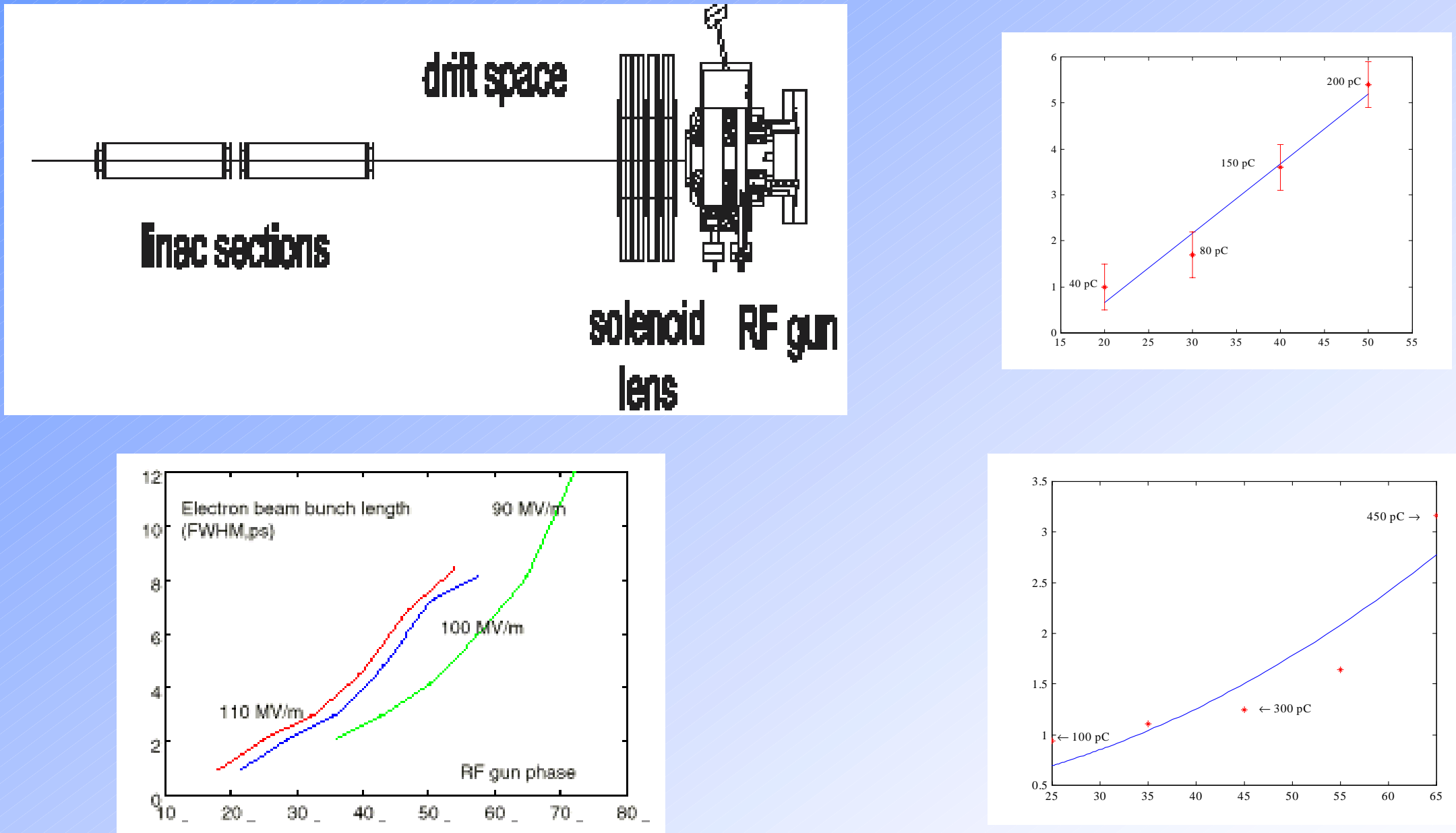

Brookhaven Science Associates U.S. Department of Energy

X.J. Wang et al, Phys Rev. E and PAC 97

6
BROOKHAUEN

NATIONAL LABORATORY 


\section{RF Longitudinal Emittance}
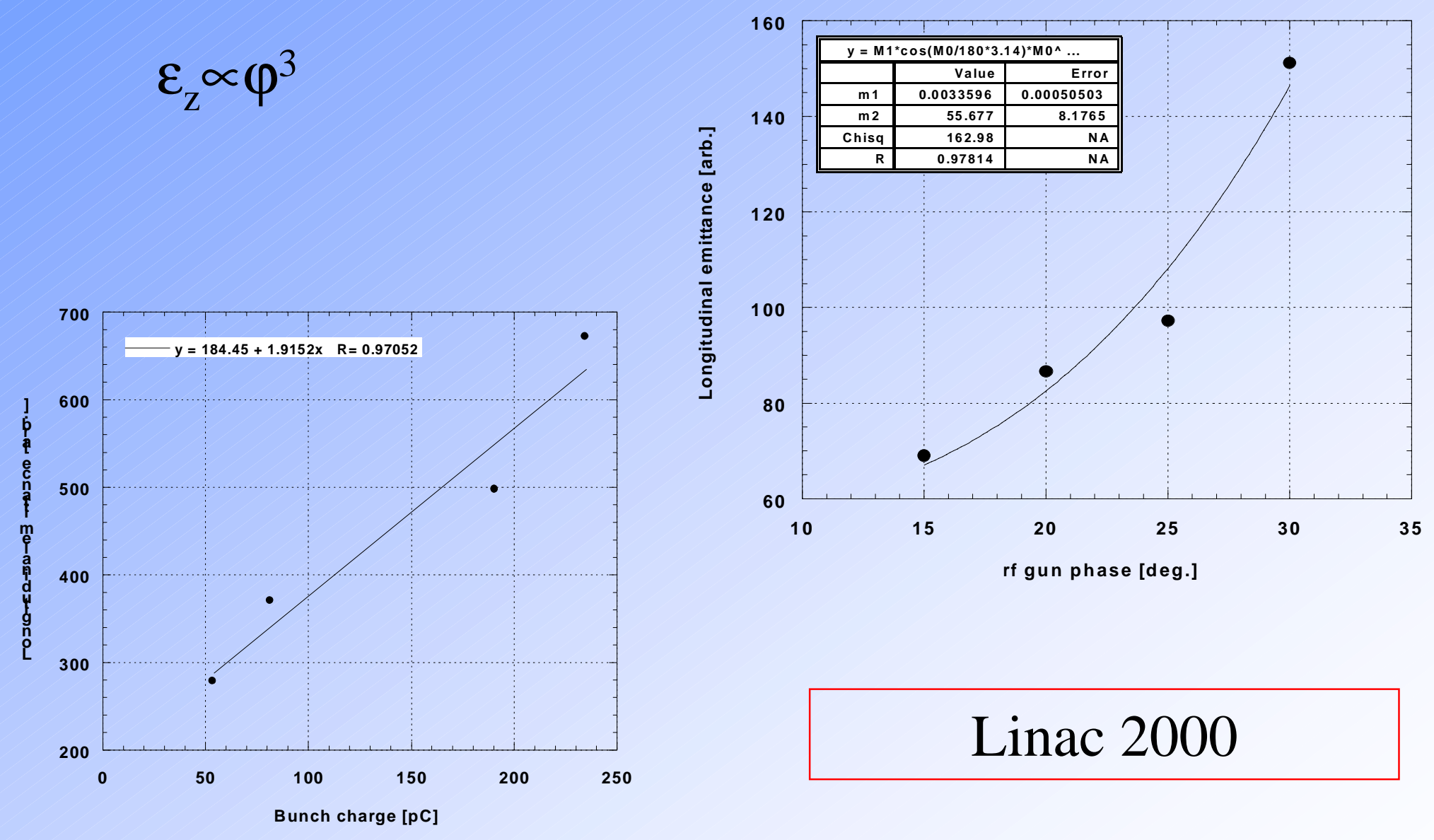

\section{Linac 2000}




\section{Beam Instrumentation Consideration for PERL Injector X.J. Wang}

-Introduction.

-Instrumentation consideration:

1. Laser and optical system.

2. Gun diagnostics.

3. Boost linac.

4. Beam characterization at $25 \mathrm{MeV}$.

- Issues and Summary 


\section{Introduction}

- Charge per bunch: $0.15 \mathrm{nC}$ or $0.45 \mathrm{nC}$

- High rep rate: 1300 or $433 \mathrm{MHz}(200 \mathrm{~mA})$

- Normalized RMS emittance: $1 \mathrm{~mm}-\mathrm{mrad}$

- Longitudinal RMS emittance: 3 ps * $23.2 \mathrm{KeV} @ 25 \mathrm{MeV}$

- Uptime: 24 hrs/day, 25 days/month, 11 months/yea (Stability and reliability)

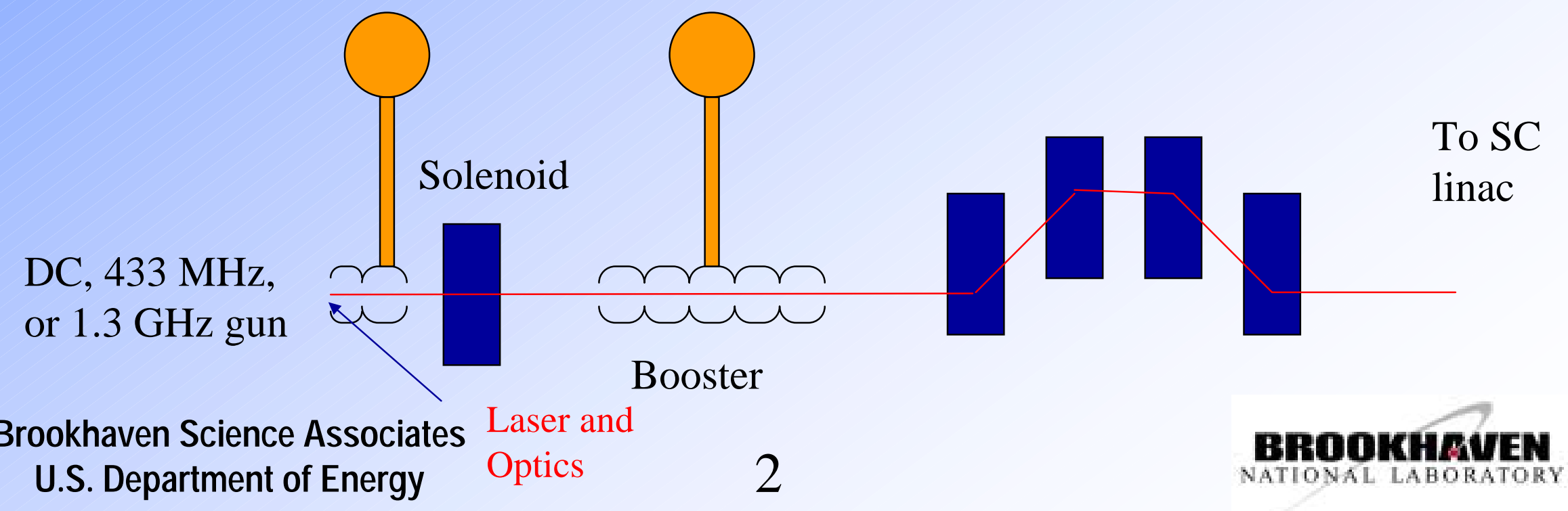




\section{Introduction - continue}

For high average current beams, identify methods to measure:

- Vertical and horizontal emittance.

- Energy and energy spread.

- Bunch length.

- Charge.

- Peak and average current.

- Bunch-to-bunch timing jitter.

Identify feedback systems required for laser and RF.

Special beam instrumentation issues for PERL injector:

- Laser and its transport optics.

-High average power.

-Safety interlock - loss monitoring.

-Beam characterization in 6-D.

-Space charge and relative low energy.

- Jitter - timing and position 


\section{PERL Injector instrumentation - Laser}

Laser energy.

Profile and position.

Timing jitter

Cathode-QE and

Uniformity

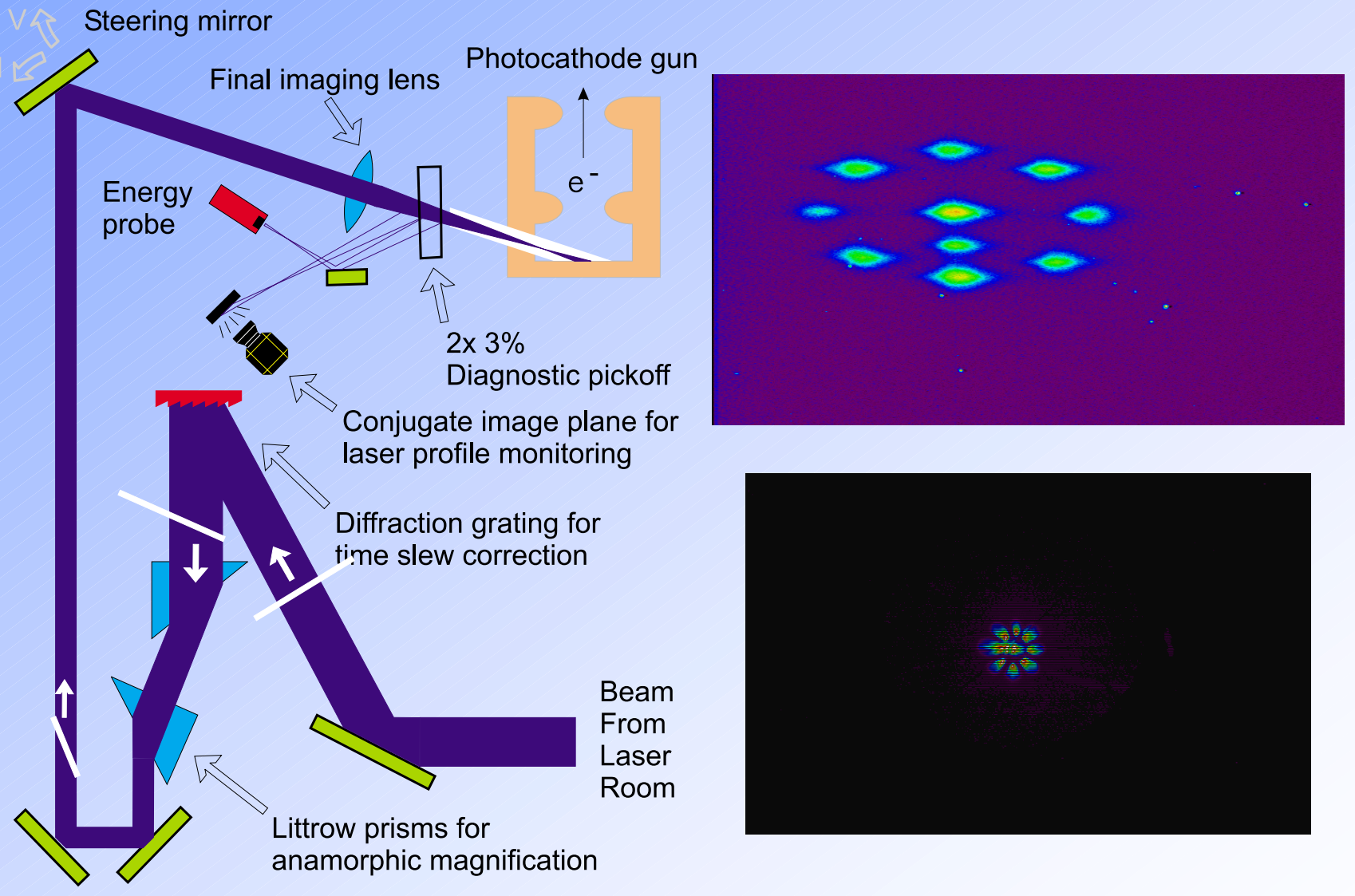

Special equipment: Streak Camera or

Brookhaven Science Associates U.S. Department of Energy gated CCD Camera.

4
BROOKHAVEN

NATIONAL LABORATORY 


\section{PERL Injector instrumentation - ATF Laser}

BNL ATF is the only multi-user facility based on the photocathode RF gun injector, it has demonstrated:

1. Good beam quality for users.

2. Delivery more 1000 year each year in the last two years.

Brookhaven Science Associates U.S. Department of Energy
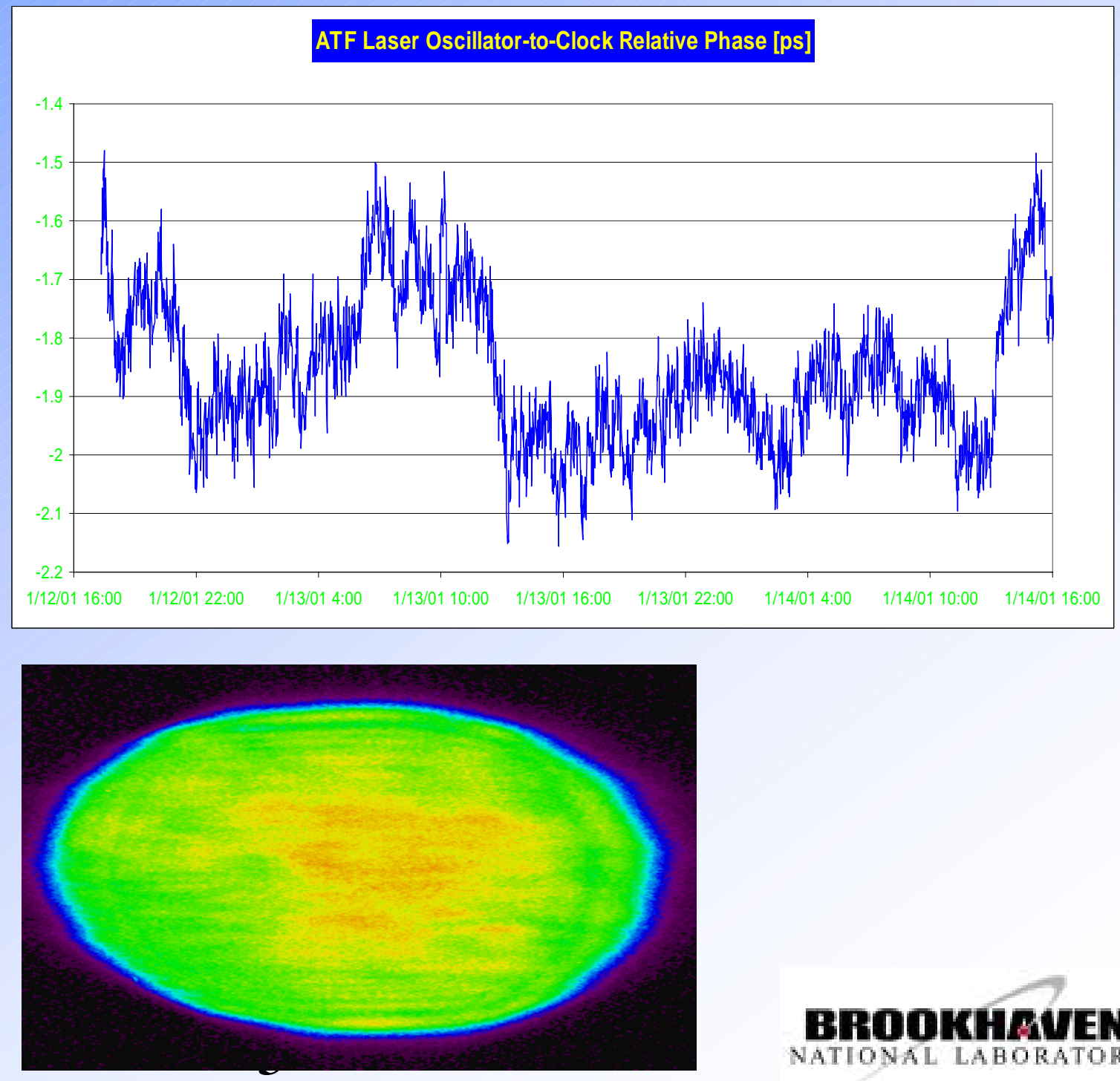

BROOKHAUEN

NATIONAL LABORATORY 


\section{PERL Injector instrumentation - Gun}

\section{Charge. \\ Energy \\ RF gun phase}

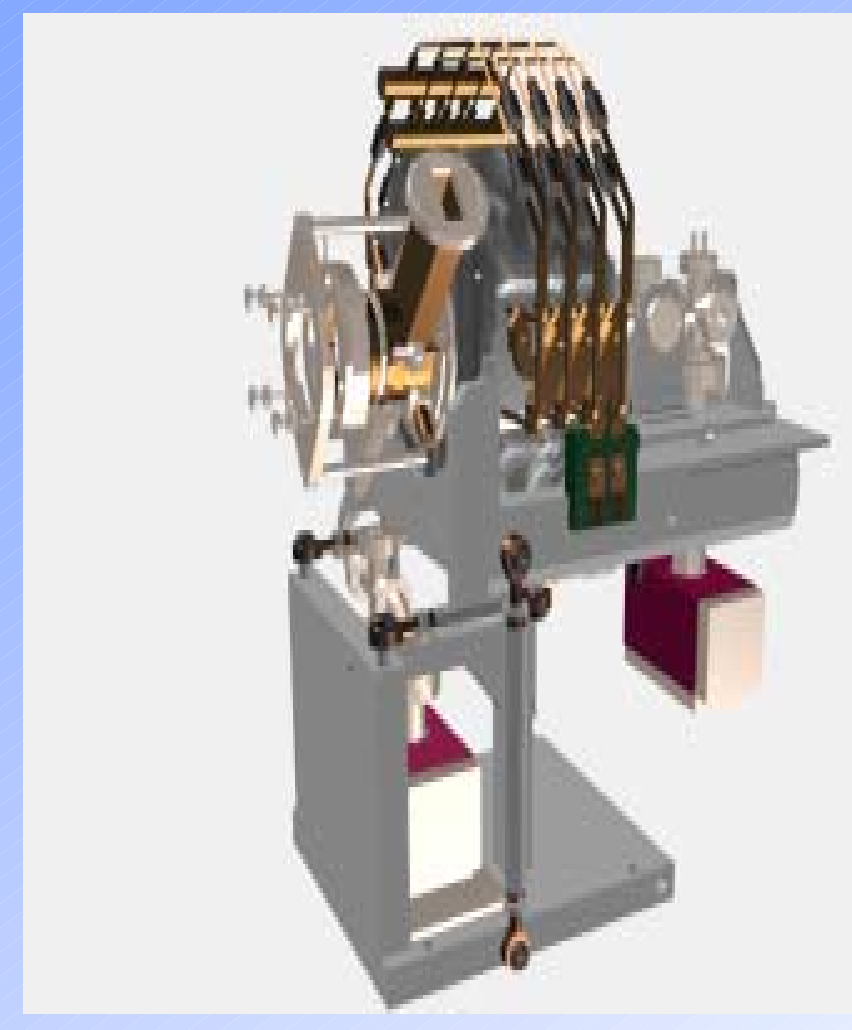

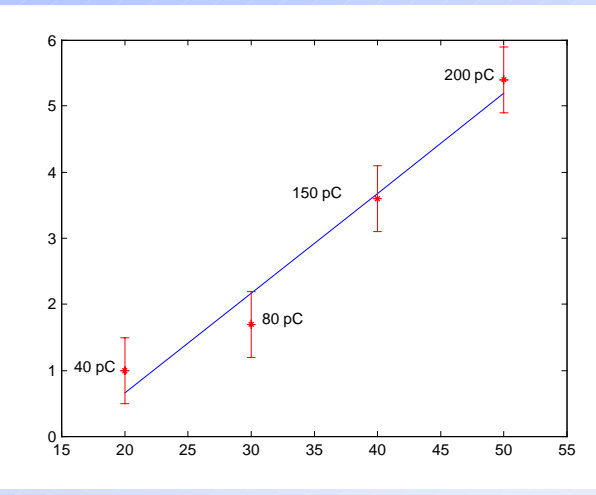

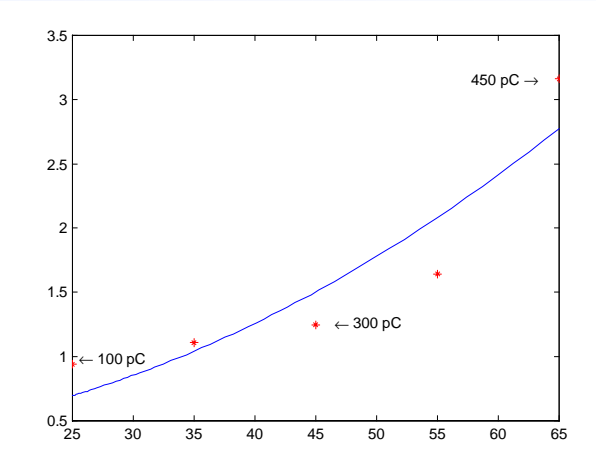

Brookhaven Science Associates U.S. Department of Energy 


\section{PERL Injector instrumentation - Gun cont.}
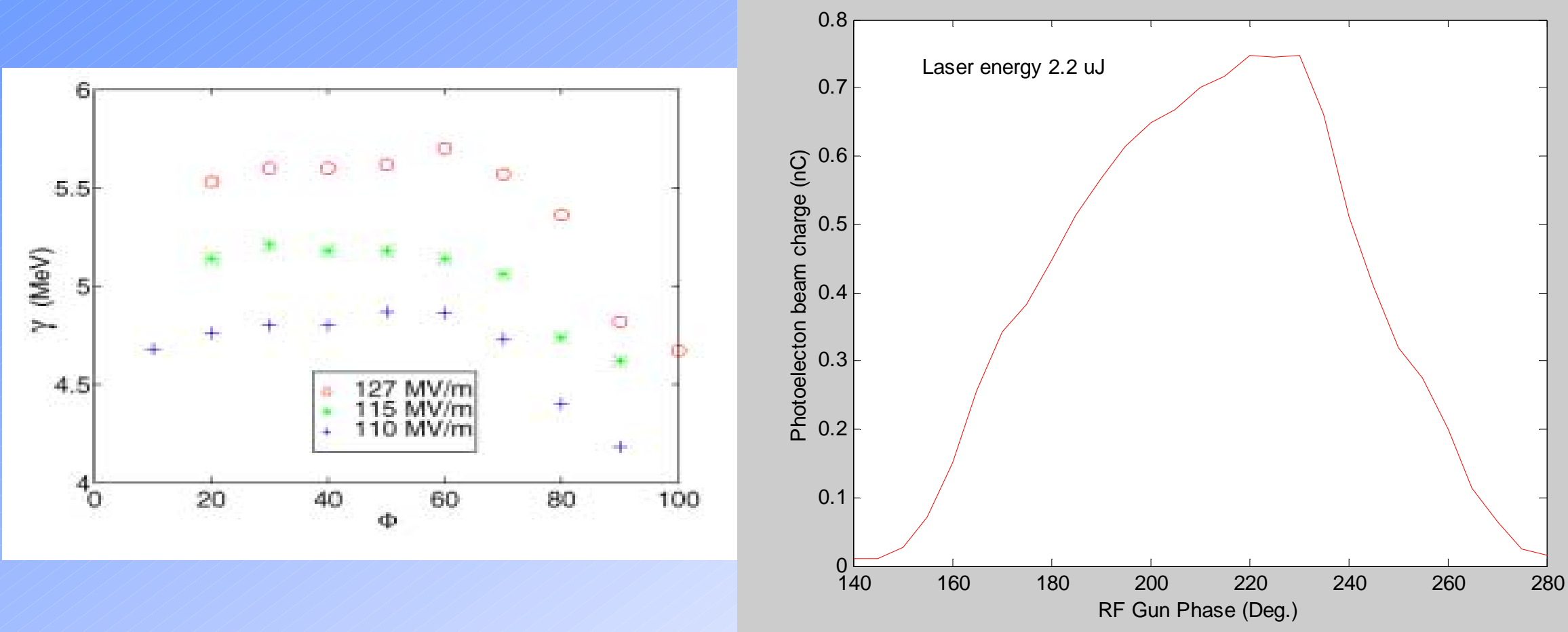

Brookhaven Science Associates U.S. Department of Energy 


\section{PERL Injector instrumentation - Tìming jitter}

$V_{\text {video }}=V_{\text {beam }} x V_{\text {local }} \sin \varphi$

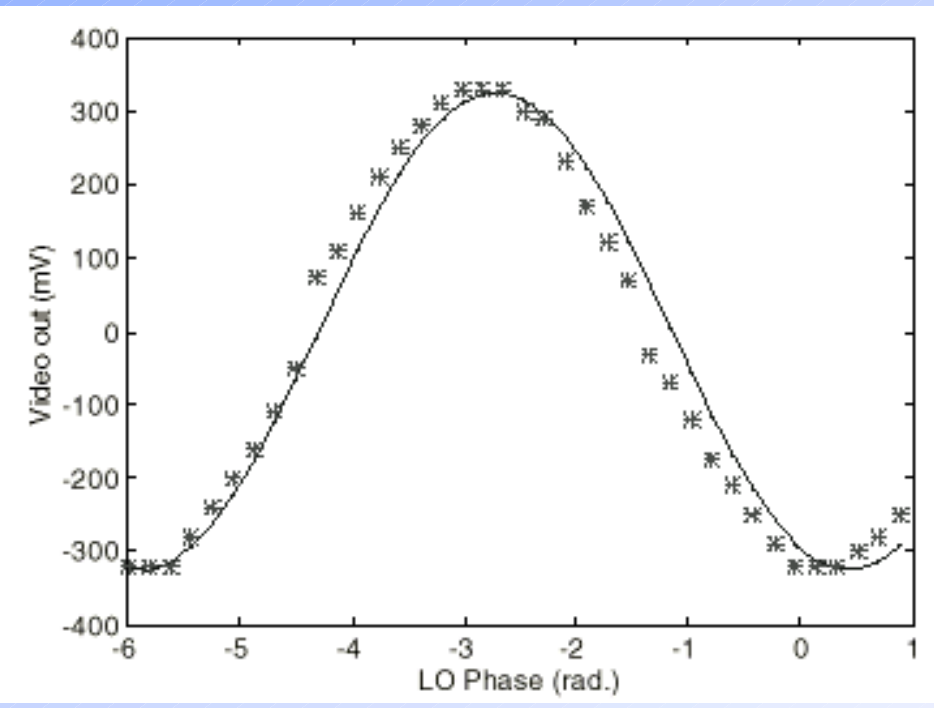

Brookhaven Science Associates U.S. Department of Energy

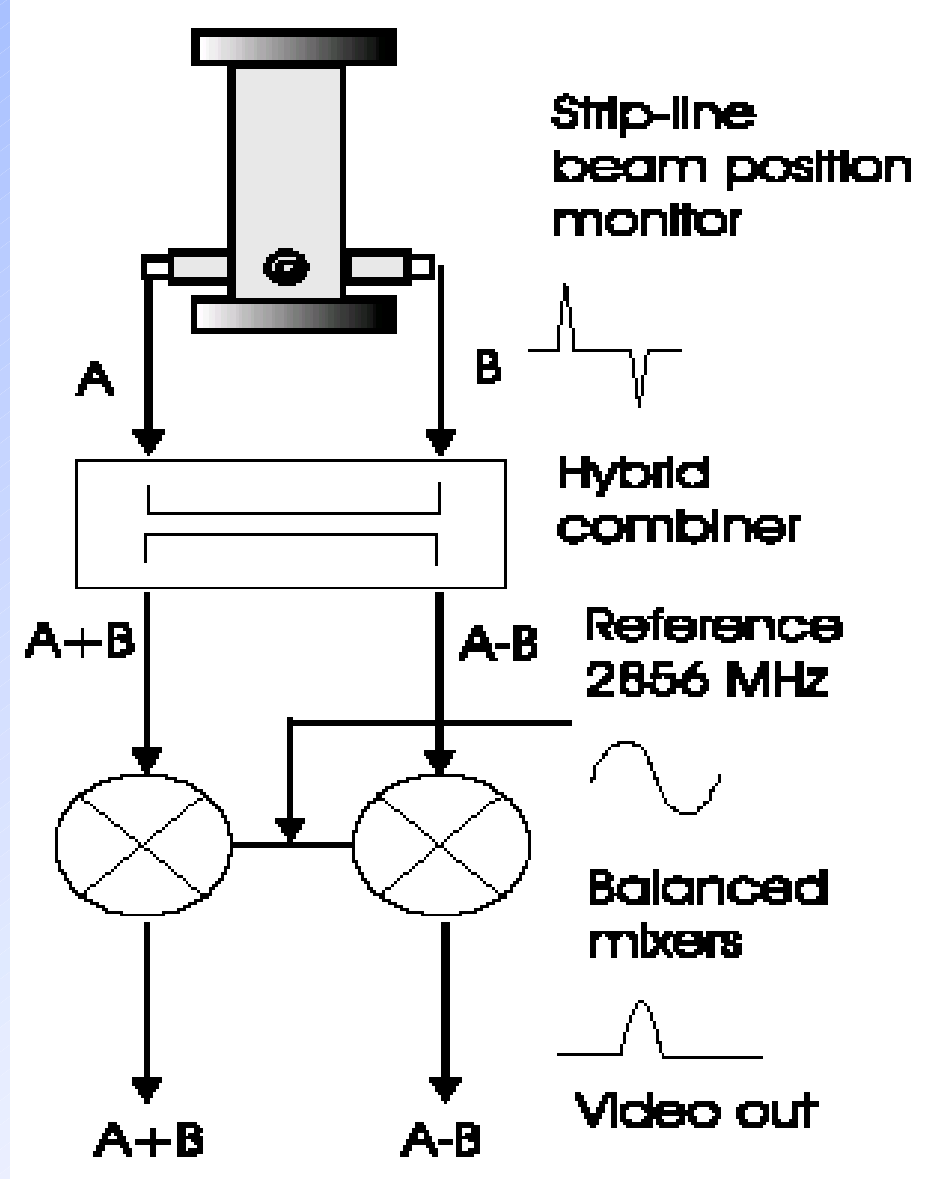

8 


\section{PERL Injector instrumentation - Boost Linac}

Energy.
Positions before and
after the linac.
Beam position inside
the linac.
Beam profile after
linac.

$$
f=\frac{b \bullet g}{b+g}
$$

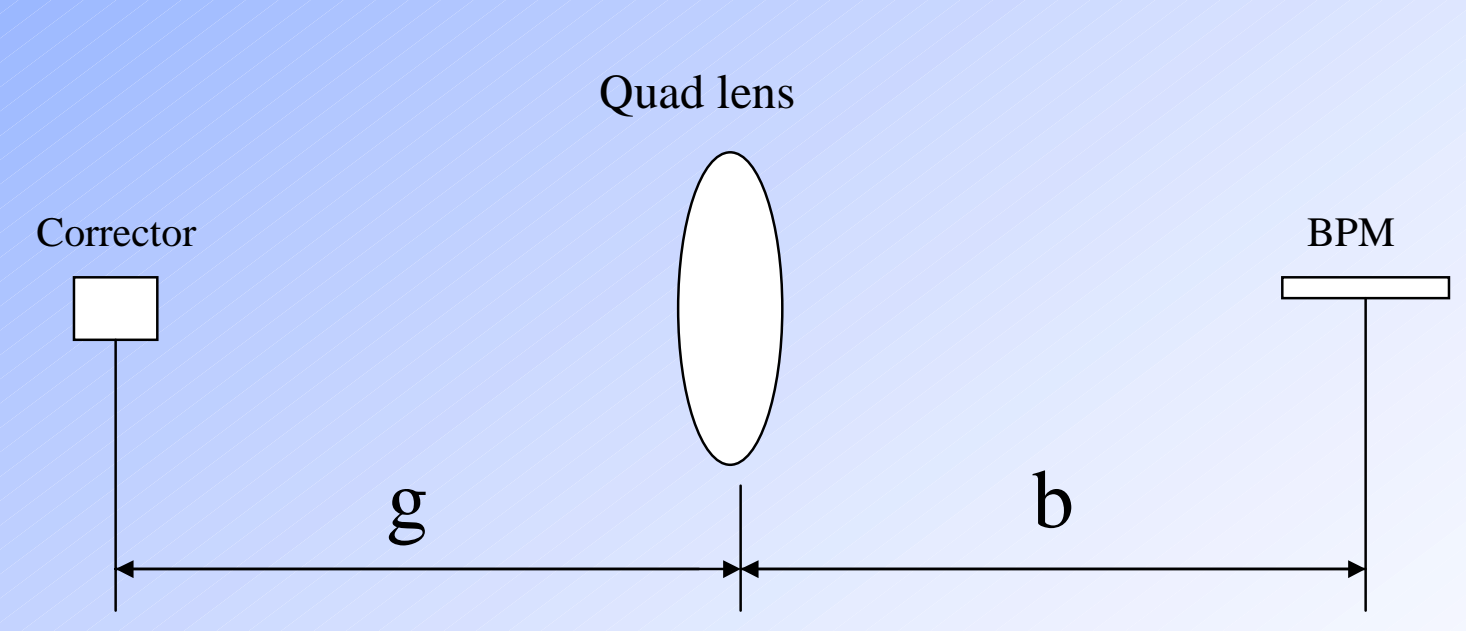




\section{PERL Injector instrumentation - $25 \mathrm{MeV}$}

Transverse emittance - pepper port, Q-scan, multimonitors.

Bunch length, Energy spread - pick up cavity

Beam jitter - BPM for position and timing jitter

$$
\begin{aligned}
& \Delta x=\sqrt{2} \frac{x}{a}\left[1-\left(3 \frac{\sigma_{x}-\sigma_{y}}{a^{2}}+\frac{x^{2}-3 y^{2}}{a^{2}}\right)\right] \\
& \Delta y=\sqrt{2} \frac{y}{a}\left[1-\left(3 \frac{\sigma_{y}-\sigma_{x}}{a^{2}}+\frac{y^{2}-3 x^{2}}{a^{2}}\right)\right]
\end{aligned}
$$

$$
x^{\prime \prime}+k(s) x-\frac{2 r_{e} N / \ell}{a^{2} \gamma^{3}} x=0
$$$$
\begin{aligned}
& z^{\prime}=\frac{\delta}{\gamma^{2}} \\
& \delta^{\prime}=\frac{3 r_{0} N}{\gamma^{3} \beta^{2} \ell_{b}^{3}} f(a, b) z
\end{aligned}
$$

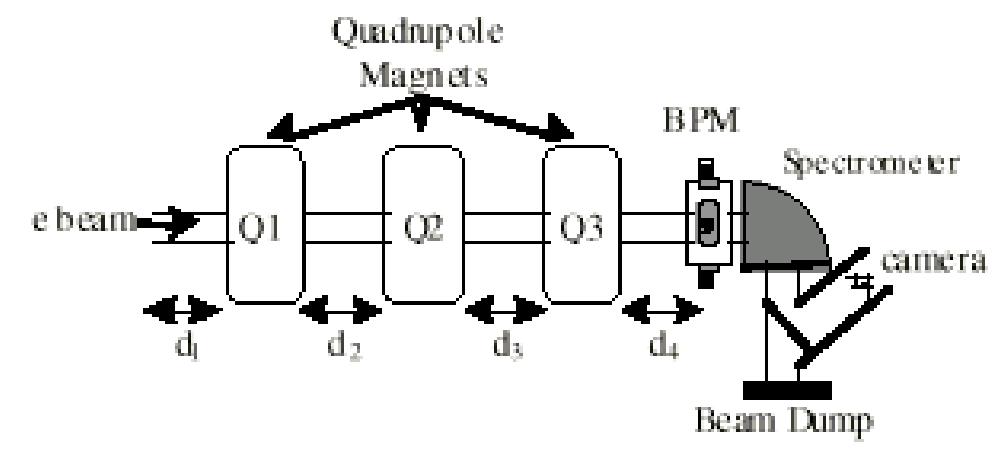

Brookhaven Science Associates

U.S. Department of Energy 


\section{RF Pick up Cavity}

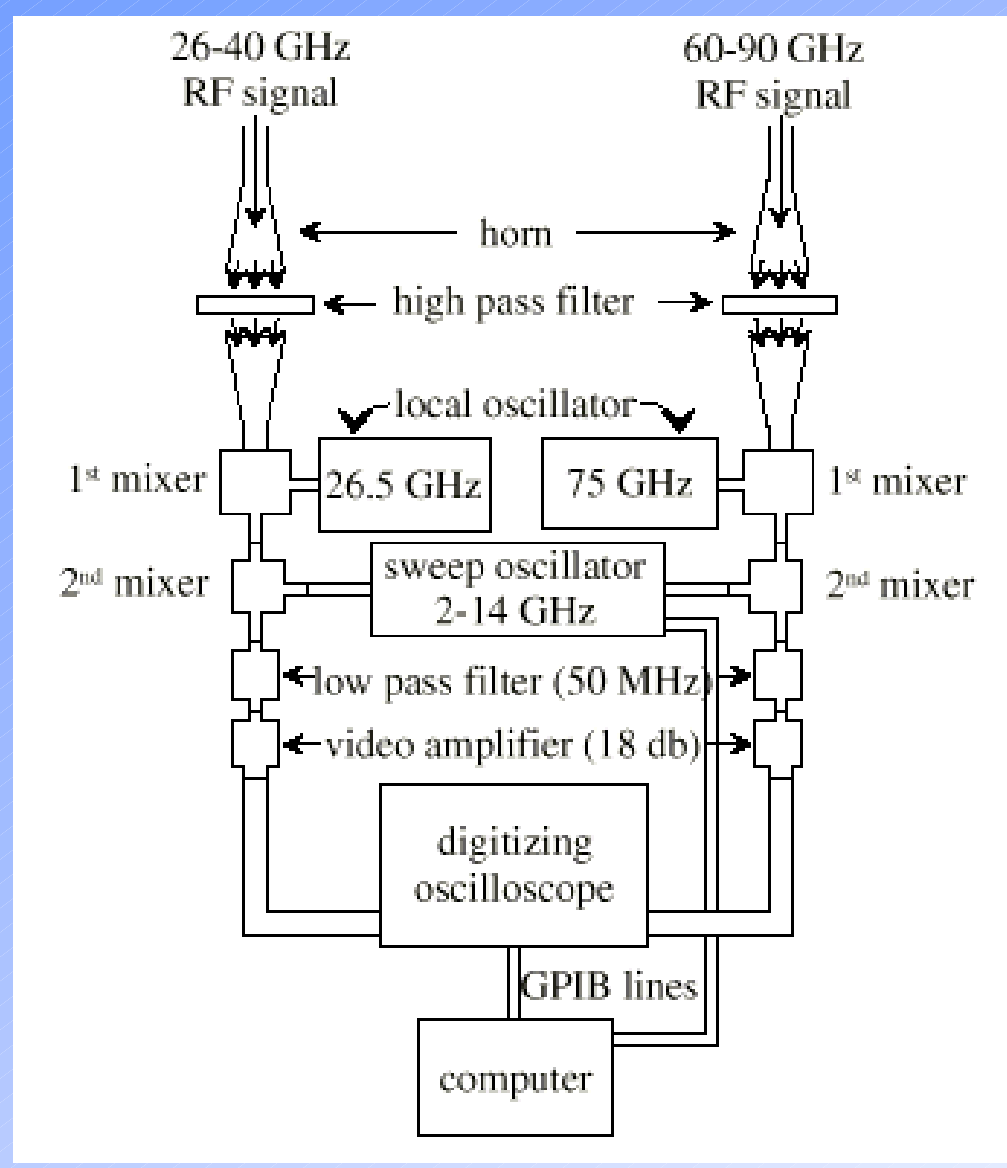

Brookhaven Science Associates U.S. Department of Energy

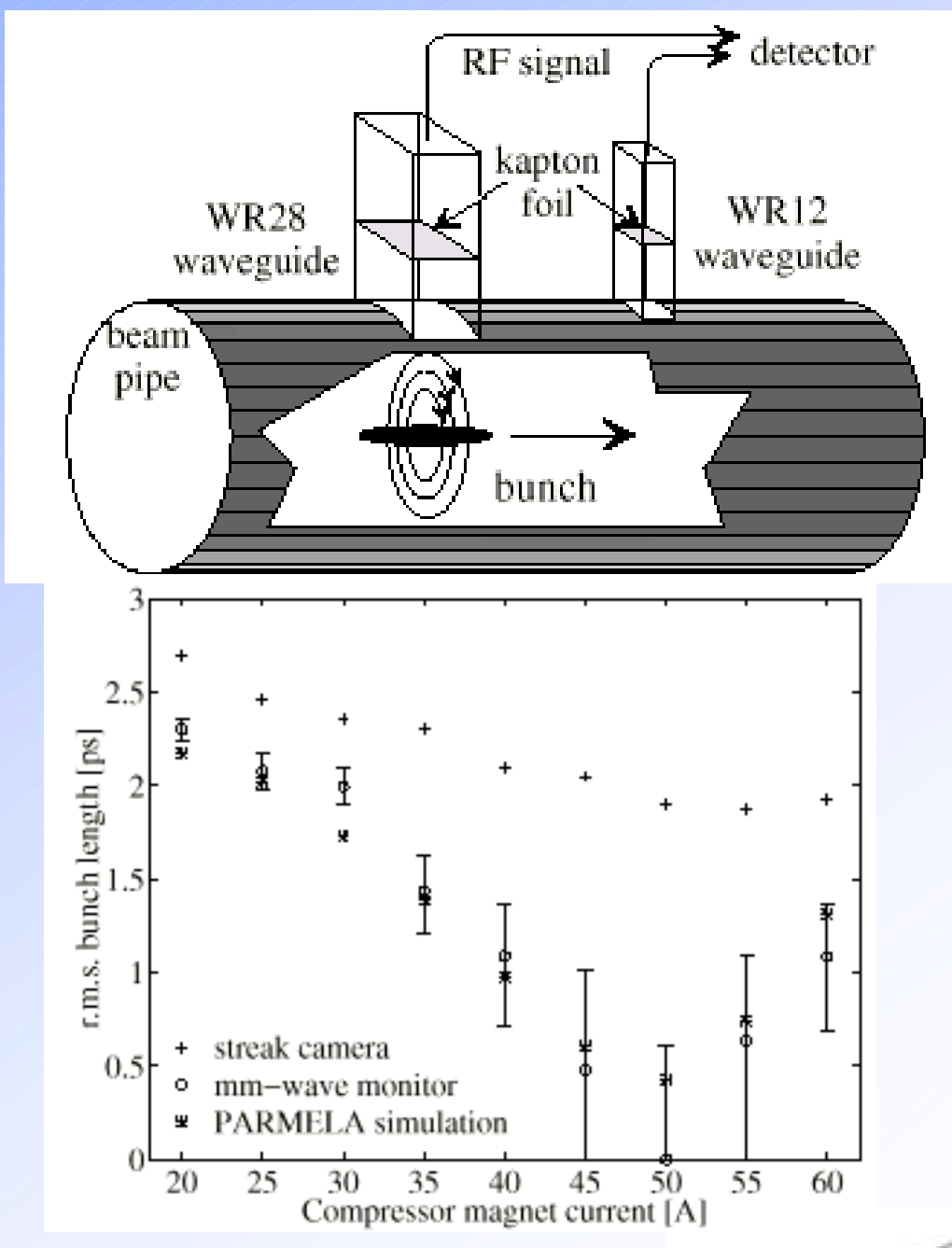

11 


\section{RF Transverse Kicker Cavity}

- Single shot.
- Time domain, and detail
structure, large dynamic range
due to the absence of space
charge effects.
- Self calibration.
- Time Jitter measurements.
- Synchronized with beam.
- Slice emittance measurement
- Longitudinal phase space
measurement combined with
spectrometer
- Cost competitive if you already
have RF source.

X.J. Wang, X-ray FEL and Linear collider
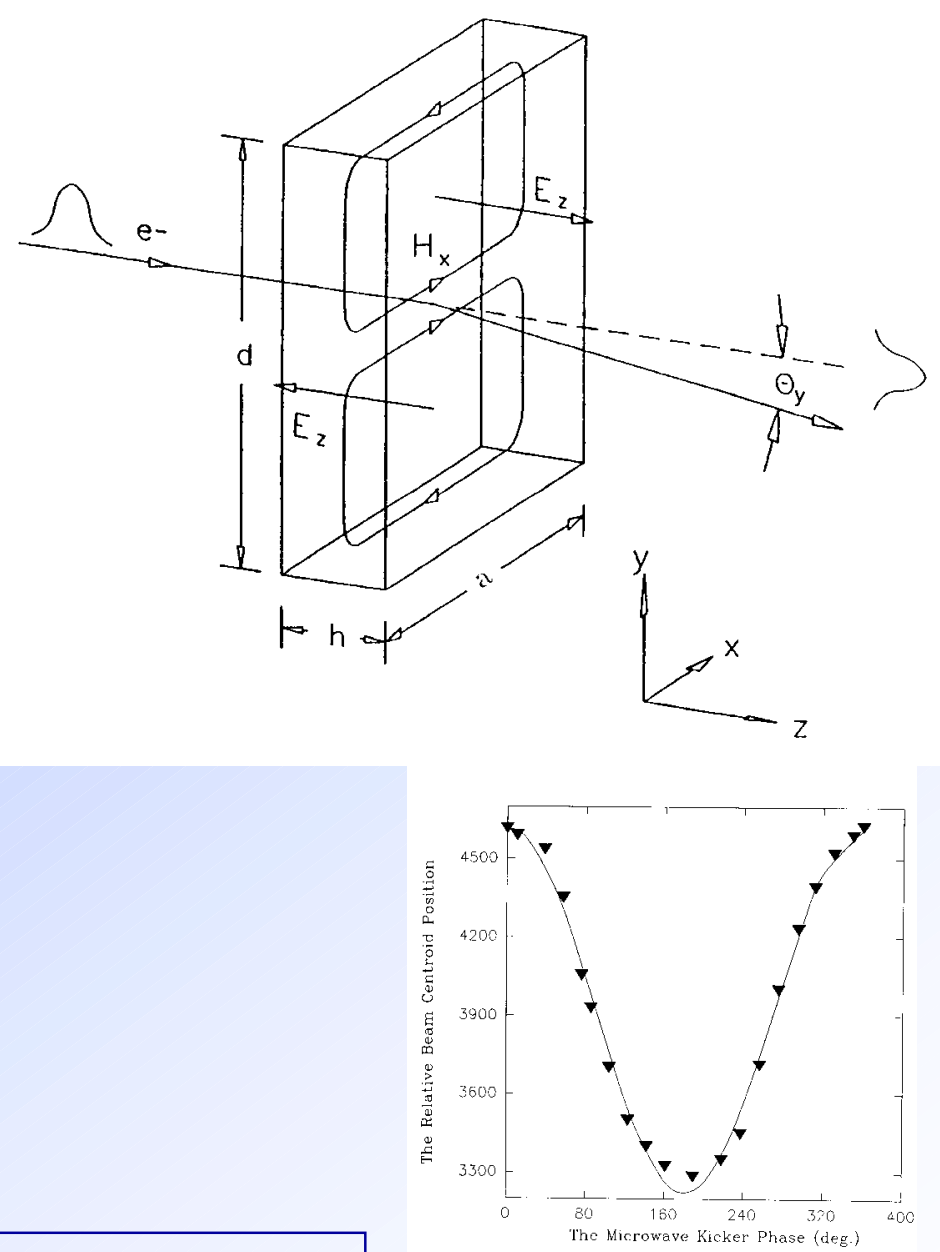


\section{Transverse RF Cavity for Bunch Length and Slice-Emittance Measurements}

\section{(J. Frisch, X.-J. Wang, ...)}
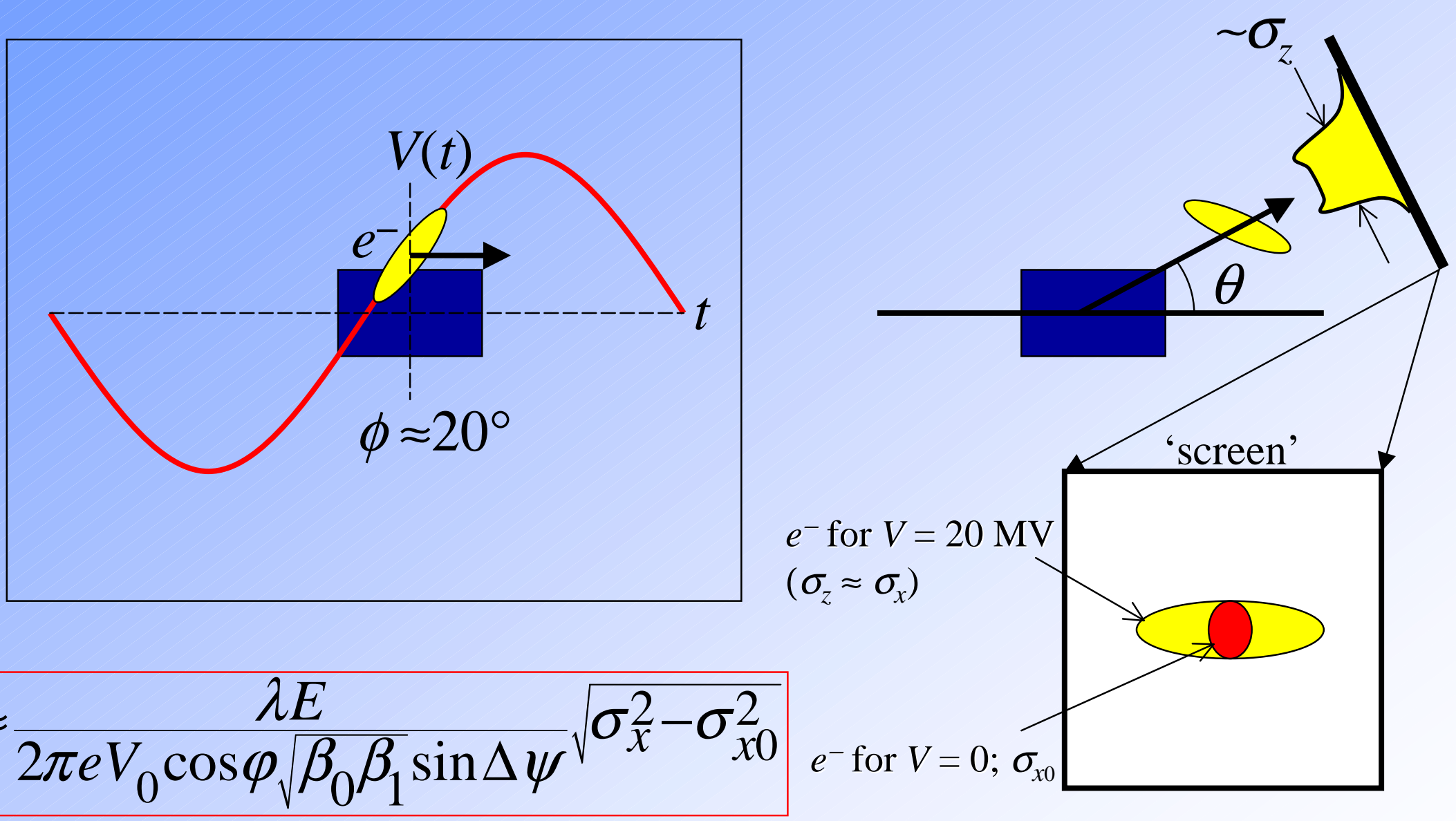

$\sigma_{z} \approx \frac{\lambda E}{2 \pi e V_{0} \cos \varphi \sqrt{\beta_{0} \beta_{1}} \sin \Delta \psi} \sqrt{\sigma_{x}^{2}-\sigma_{x 0}^{2}} e^{- \text {for } V=0}$

$$
V \approx 20 \mathrm{MV} \text { at } \varphi \approx 20^{\circ}
$$

Brookhaven Science Associates

U.S. Department of Energy 


\section{RF kicker cavity for PERL Injector}

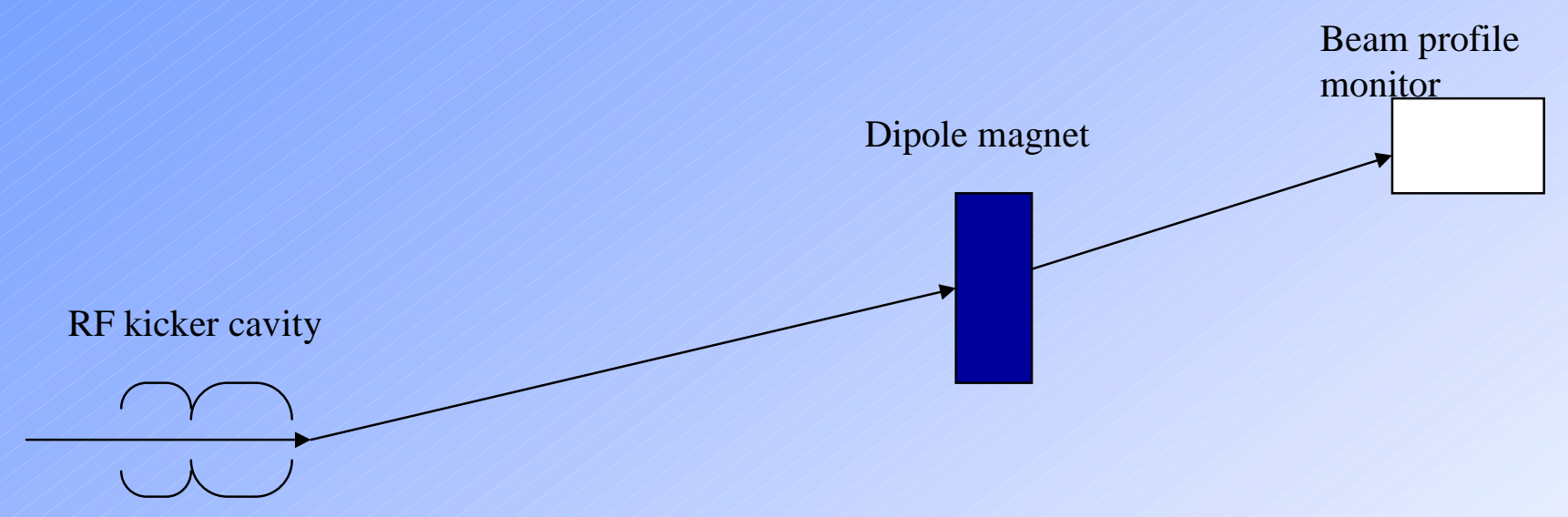

Both energy spread and bunch length. 


\section{Optical transition radiation}

Semi-non-interceptive diagnostics.

Beam Profile

Energy

Emittance

$$
\frac{d^{2} I_{1}}{d u d \Omega}=\frac{e^{2}}{\pi^{2} c} \frac{\theta^{2}}{\left(\gamma^{-2}+\theta^{2}\right)^{2}} \quad \text { Diamond }
$$

Brookhaven Science Associates U.S. Department of Energy
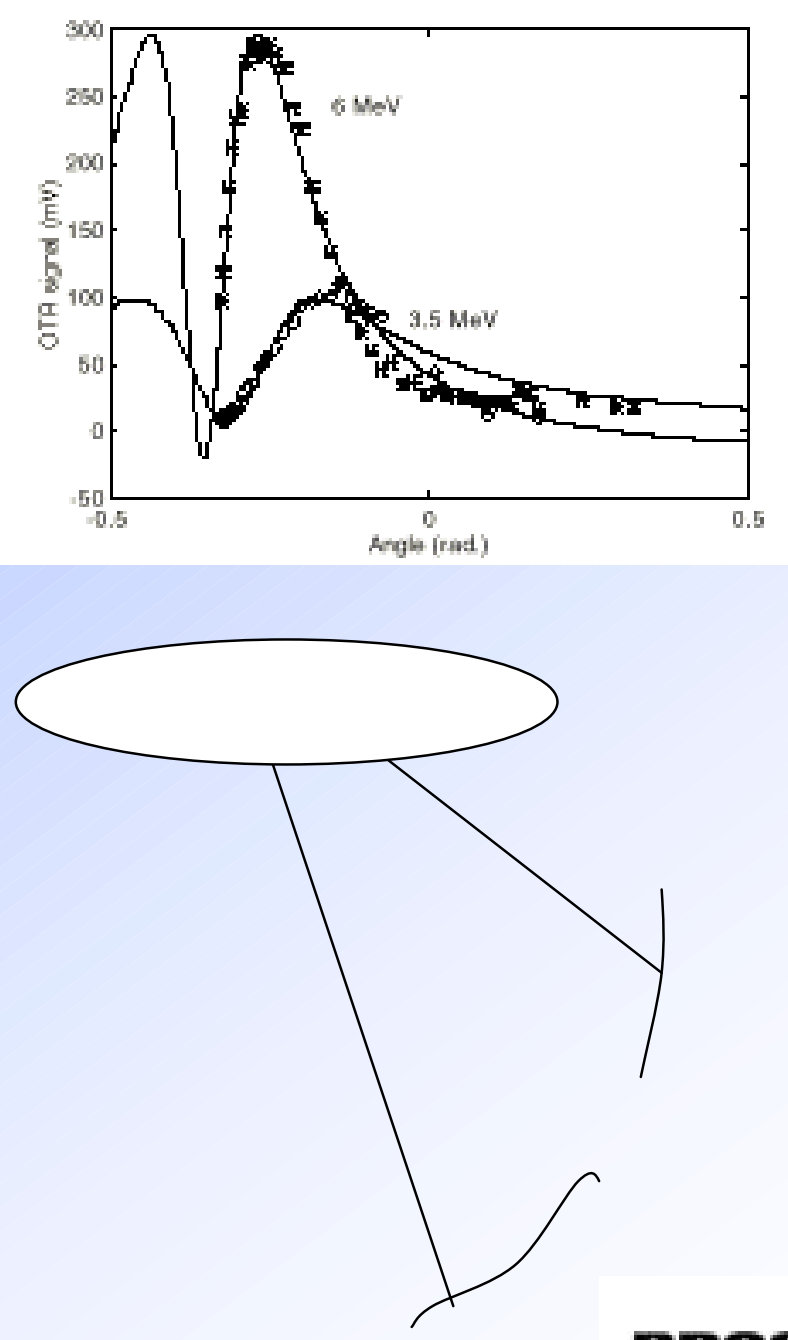

15 


\section{Summary}

Beam instrumentation is the key for performance and stability and reliability. It must be addressed at early stage of R\&D. Many issues are not addressed here, here is a personnel biased experience. 


\section{PERL Injector Workshop Laser and Cathode Working Group Summary Marcus Babzien, Brookhaven Accelerator Test Facility $N S L S, B N L$ Upton, NY 11973}

\section{Introduction}

In order to meet the general charge of developing the optimal electron source for a high average current, low emittance gun, the laser and photocathode must be considered together. The requirements set forth in these proceedings for a user facility specify beam stability and temporal shape, but it is the cathode material that determines the wavelength and power required from the drive laser. These fundamental quantities are the strongest determiners in choosing a laser system for PERL. For this reason, the laser and cathode are considered as one system by the working group. Therefore, design considerations of either the laser or the cathode will have direct consequences on the other.

\section{Cathode Materials}

The first task in developing a high average current electron source suitable for use in a DC or RF gun is to identify the various cathode materials and corresponding properties. These proceedings contain several talks on candidate materials, and a table of the most relevant parameters is shown below. This table provides a starting point from which to choose a cathode and make decisions on the laser systems that may illuminate them. To meet the goals of the meeting, it is necessary to make assumptions based on the experience of the participants, therefore the numbers shown in Figure 1 represent a best guess as to the state-of the-art performance of the different cathodes. Materials at much different levels of development are considered on an equal basis as far as basic parameters are concerned. Further conclusions taking into account the maturity of the different materials are presented at the end of this summary. The materials are broadly grouped into the Tellurides, Antimonides, Cesiated Gallium Arsenide, and a variety of lower quantum efficiency materials including Magnesium, Lanthanum Hexaboride, bonded, or other dispenser cathodes. Next, the required range of wavelengths at which the cathodes operate is given as a usable range, followed by lasers which can provide photons in this range, either directly or via harmonic generation. Typically, the variation in performance over these ranges is considered to be of secondary importance, although some of the wavelength dependence in emittance and quantum efficiency can be useful for optimizing performance. Next, the basic phenomena other than vacuum quality that affect the lifetime of the material are listed, but these are not very material specific. Other issues which deserve consideration are then listed for each material. These were issues that may limit the applicability of a cathode for PERL, but for which no good answers were known. Resolving some of these issues may require research and development. Next, the lifetime as demonstrated at initial fabrication and also more typical operating conditions are listed. Then, considering the wavelength and the PERL $200 \mathrm{~mA}$ current requirement, a the laser power for given quantum efficiency is given as a power*QE product. This demonstrates the basic advantage of the longer wavelength materials, especially GaAs. Expressed this way, an assumption in achievable quantum efficiency directly gives the amount of laser power required at the listed wavelength. 
Some basic conclusions at this point are clear. The lowest quantum efficiency materials clearly involve a much larger effort to meet the PERL requirements than the other classes. This illustrates the tradeoff between cathode and laser, since the R\&D effort required to produce a conventional, multi-100W UV laser system, which is beyond the current state-of-the-art, would be comparable to the effort required to incorporate either a parasitic or dedicated FEL amplifier to drive the photocathode. Such a conceptual design was presented at this workshop by A. Zholents. In addition, problems of laser heating, plasma formation, and cathode degradation may prove difficult to overcome for low quantum efficiency cathodes. As such, they should only be considered if other options fail.

\begin{tabular}{|c|c|c|c|c|}
\hline Type & $\begin{array}{l}\mathrm{Cs}_{2} \mathrm{Te} \\
\text { CsKTe }\end{array}$ & $\begin{array}{l}\mathrm{Cs}_{3} \mathrm{Sb} \\
\mathrm{K}_{2} \mathrm{CsSb}\end{array}$ & GaAs & $\begin{array}{l}\text { Metal } \\
\text { Bonded } \\
\text { LaB6 } \\
\text { Dispenser } \\
\end{array}$ \\
\hline $\begin{array}{l}\text { Usable Photon } \\
\text { Wavelength }\end{array}$ & $260-300$ & $350-600$ & 780 & UV \\
\hline $\begin{array}{l}\text { Laser } \\
\text { Sources }\end{array}$ & $\begin{array}{l}\mathrm{Nd} * 4 \\
\mathrm{Ti}: \mathrm{Al}_{2} \mathrm{O}_{3} * 3 \\
\text { Argon } * 2\end{array}$ & $\begin{array}{l}\mathrm{Nd} * 3 \\
\text { Ti: } \mathrm{Al}_{2} \mathrm{O}_{3} * 2 \\
\text { Argon }\end{array}$ & $\begin{array}{l}\mathrm{Ti}^{\mathrm{Al}}{ }_{2} \mathrm{O}_{3} \\
\text { Diode } * 2 \\
\mathrm{Nd} * 2 \\
\text { Argon }\end{array}$ & Seeded FEL \\
\hline $\begin{array}{l}\text { Lifetime } \\
\text { Limiting } \\
\text { Phenomena }\end{array}$ & All: & $\begin{array}{l}\text { Chemical } \\
\text { preparation }\end{array}$ & $\begin{array}{l}\text { Ion } \\
\text { bombardment }\end{array}$ & $\begin{array}{l}\text { Preparation } \\
\text { technique }\end{array}$ \\
\hline$\frac{\text { Outstanding }}{\text { Questions }}$ & $\begin{array}{l}\text { Usable in DC } \\
\text { gun? } \\
\text { (large thermal } \\
\text { emittance) } \\
\text { Code available? }\end{array}$ & Coating? & $\begin{array}{l}\text { Ion } \\
\text { Bombardment } \\
\text { Limit? }\end{array}$ & $\begin{array}{l}\text { Laser heat } \\
\text { removal? }\end{array}$ \\
\hline $\begin{array}{l}\text { Best QE, } \\
\underline{\text { Lifetime }}\end{array}$ & $\begin{array}{l}24 \% \text { @ prep } \\
2 \%, 1 \text { year @ } \\
10^{-8}\end{array}$ & $\begin{array}{l}14 \%, \text { hours @ } \\
10^{-10}\end{array}$ & $\begin{array}{l}13-15 \%, 1- \\
1.2^{*} 10^{5} \mathrm{C} / \mathrm{cm}^{2} \\
@<10^{-11}\end{array}$ & $\begin{array}{l}0.3 \%, \text { months } \\
\text { @ } 10^{-10}\end{array}$ \\
\hline$\frac{\text { Power*QEE }}{\text { product }}$ & $90 \mathrm{~W} \cdot \%$ & $45 \mathrm{~W} \cdot \%$ & $30 \mathrm{~W} \cdot \%$ & $90 \mathrm{~W} \cdot \%$ \\
\hline
\end{tabular}

The best overall performance at this time is from GaAs, since it has high quantum efficiency, and operates at the longer fundamental wavelengths generated from common lasers. The applicability of this material to an RF gun is in question, primarily because of the high vacuum requirement. In addition, the ion bombardment mechanism that currently limits GaAs lifetime may work differently in an RF gun than DC gun. Therefore, GaAs is best used in a DC gun at gradients around $10 \mathrm{MV} / \mathrm{m}$. In order to extend lifetime further, the vacuum level should be improved approximately one order of magnitude. If this is possible, then a quantum efficiency of $10 \%$ should be sustainable for the several hour lifetime required in PERL. 
The second material that was considered a candidate for PERL is $\mathrm{K}_{2} \mathrm{CsSb}$. It has demonstrated very high quantum efficiency under operating conditions, although with short lifetimes. The major reason that the lifetime was not considered a major flaw was that very little effort has been focused on producing longer lifetime cathodes. The type of improvements reported for CsTe by D. Nguyen at this workshop may be achievable with $\mathrm{K}_{2} \mathrm{CsSb}$, however, this involves R\&D into cathode preparation. Currently, the quantum efficiency that would be achieved in a PERL photoinjector is estimated to be $1 \%$, and this was the design number used in the following sections.

\section{$\underline{\text { Laser Designs }}$}

When developing rough configurations for the drive laser, a target power level is chosen for a cathode based on the quantum efficiency that is likely to be achieved after 24 hours of continuos operation. This means that the injector can be taken out of operation daily to switch either to a fresh cathode in the same injector, or a duplicate injector with a fresh cathode already in place. The final choice will depend on the speed with which a particular cathode can be replaced, the expense of injector duplication, and the impact of daily beam interruption on user experiments. It was assumed that this is the minimum acceptable operating cycle for PERL, but may be too short for some users. This also requires that the laser power compensate for changes in quantum efficiency over the 24 hour operating cycle. Under such assumptions, the laser requirements for the two candidate materials above are 3W at 780nm for GaAs, and $45 \mathrm{~W} @ 350 \mathrm{~nm}$ for $\mathrm{K}_{2} \mathrm{CsSb}$. A further assumption was that temporal shaping should be used to produce a laser pulse with 3 ps rise and fall times, separated by a 10 ps flat period, as shown below:

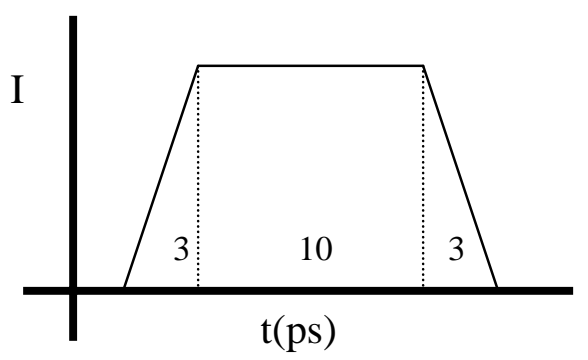

Also implicit in the following discussion is that any laser system will require feedback in intensity, phase and profile in order to meet the long term requirements for PERL. These feedback loops are not shown schematically, and would be expected to correct the long term drift as well as high frequency noise up to their bandwidth limit. The only parameter for which the feedback was deemed a possible weakness was in phase jitter. Although laser oscillators have demonstrated the required $200 \mathrm{fs}$ rms value, no existing system has operated continuously and reliably at that level. Therefore it is considered to be at the limit of what can be achieved. For this reason, this requirement should be studied to determine if it presents a true limit for operation, or if slight degradation in laser performance is tolerable for some fraction of the time. Furthermore, some feedback systems may rely on instrumentation in the accelerator because sufficient sensitivity is not available from laser diagnostics located upstream of the photocathode. 
Feedback is a necessary but not sufficient condition for satisfying the PERL stability requirements. The laser must be designed from the outset for highest possible stability. This includes environmental control for the laser room and all system components, power supplies and electronics. System performance should also rely as much as possible on physical mechanisms that maintain or enhance stability, such as gain or harmonic generation saturation. It is unlikely that a commercial vendor will have the experience necessary to design such a system. Although these considerations are as important as the choice of laser gain medium, they are assumed to be a critical part of the drive laser and will not be covered further.

\section{GaAs Cathode}

\begin{tabular}{|c|c|c|c|c|}
\hline Ti:A12O3 & Temporal & Transverse & Transport + & \\
\hline $\begin{array}{l}\text { CW ML osc. } \\
780 \mathrm{~nm}\end{array}$ & $\begin{array}{l}\text { Pulse } \\
\text { Shaping }\end{array}$ & Shapıng & Diagnostics & $\begin{array}{l}\text { Cathode } \\
3 \mathrm{~W}\end{array}$ \\
\hline $1.3 \mathrm{GHz}$ & $\mathrm{T}=50 \%$ & $\mathrm{~T}=70 \%$ & $\mathrm{~T}=90 \%$ & \\
\hline
\end{tabular}

The first suggestion for a laser is to use a high power Ti: $\mathrm{Al}_{2} \mathrm{O}_{3}$ oscillator directly. A $5 \mathrm{~W}$ oscillator was reported at the workshop, and $\mathrm{GHz}$ repetition rates have been demonstrated, but not simultaneously. It should therefore be a modest extension of existing technology to achieve 10W CW modelocked in a single unit. The short pulses could then be converted to quasi-flattops either by Fourier-plane phase modulation, or direct time-to-space shaping, both of which have been demonstrated to have sufficient control. Some loss is encountered in either scheme, which we estimate as 50\%. A further consideration arises not from the drive laser, but the delayed emission from GaAs that would limit the falling edge of the electron bunch to at least a $10 \mathrm{ps}$ fall time. Following the temporal shaping, a spatial flattop is generated either by random phase masks, aspheric optics, and/or active mirrors. This stage may have transmission up to $70 \%$ if properly designed. Finally, it is assumed that delivery to the cathode will include losses simply due to extended distances or the harsh environment around a photoinjector, as well as multiple splitting for laser diagnostics. This loss is estimated as $10 \%$.

One concern with this simple arrangement is the lack of control over the pulse format because no high power electro-optic modulator exists with sufficient bandwidth to operate at $1.3 \mathrm{GHz}$. This means that the beam current ramp-up required for PERL starting conditions must be accomplished exclusively by changing the laser energy per pulse, not the duty cycle. If this is not acceptable, it would necessitate a more complex scheme using lower power, integrated-optic, Mach-Zender interferometers followed by post-amplification. Also, the PERL requirement for ion-clearing by blanking approximately $100 \mathrm{~ns}$ of the pulse train every 2 us will be difficult with a simple oscillator configuration. It may be possible to develop a high repetition rate Pockels cell for this function, but no participants were aware of demonstrated devices with this capability. 
A second laser option for $780 \mathrm{~nm}$ was identified based on fiber oscillators already demonstrated.

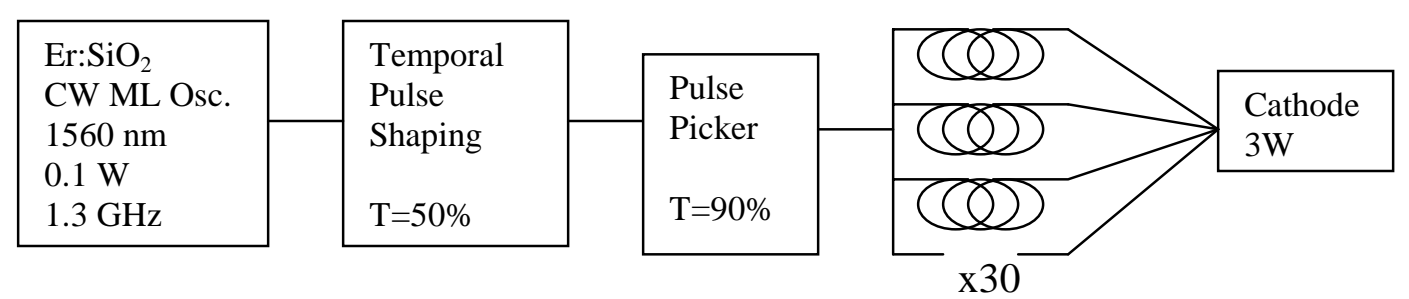

Such erbium-doped fiber oscillators are common in telecommunications applications, and are typically characterized by extreme phase and power stability. $\mathrm{GHz}$ repetition rates are achievable, with up to $100 \mathrm{~mW}$ unamplified power. The temporal pulse shaping could be accomplished with the same techniques as above, and material bandwidth is sufficient for sub-picosecond shaping. There is some concern that shaping may be more complicated because of the non-gaussian gain spectrum when compared with Ti: $\mathrm{Al}_{2} \mathrm{O}_{3}$, and this may necessitate spectral filtering and reduced gain. Following temporal shaping, a GHz bandwidth pulse picker can be used to generate arbitrary trains to satisfy the PERL ramp-up and ion-clearing requirements. An assumption was made that high power fiber amplifiers may not be available, although greater than $10 \mathrm{~W}$ has been demonstrated is research lasers. A conservative estimate of $200 \mathrm{~mW}$ per fiber would require over 30 individual amplifiers, assuming 50\% conversion efficiency from 1560 to $780 \mathrm{~nm}$. This conversion efficiency is routine using periodically poled lithium niobate. In this laser system, the total output of all the amplifiers could be anglemultiplexed onto the cathode. The beam profile achievable with such a configuration may be non-uniform, or depending on the geometry of the electron, may have too large a divergence angle to project to the cathode.

Another fiber-based scheme would start with a similar erbium oscillator, with the output Raman-shifted to $1.06 \mathrm{um}$. Such a system has been demonstrated with up to $1 \mathrm{~W}$, and higher powers are not limited by the fiber amplifier. Hence extrapolating to several watts should not be difficult. This would allow a single, diffraction limited output beam to be used for transport to the cathode.

\section{$\underline{K_{2}} \underline{\text { CsSb Cathode }}$}

The most attractive system for achieving the higher average power in this case may be the Raman-shifted fiber source, as it can be coupled to bulk amplifiers capable of multi-100 W output near $1060 \mathrm{~nm}$. Frequency conversion would likely be the critical factor in deliverable power as efficiency may be limited by a combination of average and peak power crystal damage. At $1.3 \mathrm{GHz}$ and $15 \mathrm{ps}$, the peak power enhancement would be a factor of 50, and very little data is available on damage thresholds in this regime. If it is possible to reach saturated conversion, third harmonic generation of $45 \mathrm{~W}$ should be achievable. Reduced intensity and conversion efficiency could be overcome with higher amplification. Another option is be to use the second harmonic instead of the third. Although the quantum efficiency is about $30 \%$ higher at $350 \mathrm{~nm}$ compared with $530 \mathrm{~nm}$, this may be offset by a higher doubling efficiency. Only by testing the different 
nonlinear crystals involved, and the damage thresholds for the PERL pulse format, will data become available for selecting the best scheme.

\section{Conclusions:}

Because of the relatively small amount of effort that has gone into developing improved lifetime in $\mathrm{K}_{2} \mathrm{CsSb}$, the high quantum efficiency, and visible operating wavelength, this is a very promising candidate for further study. Techniques for extending lifetime are improving operating vacuum in the gun, and using cathode protective coatings. As demonstrated at Los Alamos, large improvements in cathode robustness are possible with a modest research and development effort. Therefore, cathode development appears to be the most fruitful area for further research. Should there be little or no improvement in lifetime, a greater effort could be placed into laser development to reach the necessary power. Finally, within the limitations noted above, the existing performance of GaAs indicates that it may already be a usable photocathode in a DC gun, and represents a fallback option. 


\section{Photocathode and DC Gun Summary \\ Charlie Sinclair \\ Jefferson Laboratory \\ 12000 Jefferson Avenue, Newport News, VA 23606 \\ Tel: 757-269-7679 \\ E-mail: sinclair@jlab.org}

Before discussing DC guns, it is necessary to provide some overview of the cathodes which would be used in these guns (or in RF guns for that matter). The first point is to recognize that only high quantum efficiency photocathodes can be considered to meet the PERL parameters. For any linear photoemitter, the following relation is true.

$$
i(m A)=\frac{\lambda(n m)}{124} \bullet P_{\text {laser }}(W) \bullet Q . E .(\%)
$$

There are three families of practical high quantum efficiency photocathodes - the alkali antimonides, the alkali tellurides, and the III-V semiconductors. The first two of these familes have positive electron affinity, while the III-V semiconductor cathodes are negative electron affinity. The importance of this distinction will become apparent later. One can make a simple summary of these three families, giving the typical operating wavelength, and the product of laser power and quantum efficiency necessary to reach the required $200 \mathrm{~mA}$ specification for PERL. This table makes it very clear that the lasers necessary are very demanding even for a $1 \%$ Q.E. photocathode. Photocathodes with Q.E.'s lower than about $1 \%$ are very unlikely suitable for application in a high average current PERL photoinjector.

\begin{tabular}{|l|c|c|}
\hline Cathode Type & $\begin{array}{l}\text { Operating Wavelength } \\
(\mathrm{nm})\end{array}$ & $\mathrm{P}_{\text {laser }} \times$ Q.E. (W - \%) \\
\hline $\mathrm{NEA} \mathrm{GaAs}$ & 780 & 31.8 \\
\hline $\mathrm{K}_{2} \mathrm{CsSb}$ & 532 & 46.6 \\
\hline $\mathrm{KCsSb}$ & 266 & 93.2 \\
\hline
\end{tabular}

It should be noted that the all of the above lasers are assumed to have an appropriate RF time structure (e.g. $1300 \mathrm{MHz}$ ), and that the $\mathrm{P} \times \mathrm{QE}$ numbers assume that every electron and every photon is useful. Thus, for example, if a Gaussian laser beam is truncated transversely to more closely approximate a "tophat" profile, or if some electrons are removed early in the injector, a higher $\mathrm{P} \times \mathrm{QE}$ product will be required. 
One can inquire about the thermal emittance produced by each of the above cathode families. For our purposes, we write the normalized, rms emittance as:

$$
\varepsilon_{n, r m s}=\frac{r}{2} \sqrt{\frac{E_{\text {thermal }}}{m c^{2}}}
$$

Thus, for example, if a cathode emits electrons from a Maxwell-Boltzmann distribution characterized by a temperature $\mathrm{T}$, the thermal energy in the above expression is simply kT. The above expression is known to give a good description of the emittance from a thermionic cathode.

In the case of GaAs photocathodes, the absorption coefficient for light at $\sim 780 \mathrm{~nm}$ is about $1.4 / \mu$. This leads to a half absorption depth of about $500 \mathrm{~nm}$. Electrons photoexcited into the conduction band of GaAs are very largely thermalized at the bottom of the conduction band by the time they diffuse to the surface of the cathode. Since GaAs is a negative electron affinity cathode, electrons at the bottom of the conduction band may energetically escape the cathode. Thus, the GaAs photocathode should produce an emittance characterized by a thermal energy close to the cathode temperature - i.e. room temperature in most cases. That this is indeed the case has been demonstrated by Bruce Dunham in his Ph.D. thesis, published in the proceedings of the 1995 PAC, p. 1030. Dunham measured that an emittance containing 95\% of the beam from a GaAs phtocathode was characterized by a thermal energy of about $35 \mathrm{meV}$ (n.b. room temperature is 25 $\mathrm{meV}$ ). Dunham's measurements were conducted with a tophat transverse laser profile at the cathode. His measurements were made for a number of spot sizes and illuminating wavelengths. It is worth mentioning that the negative electron affinity $\mathrm{GaAs}$ photocathode is being evaluated for e-beam lithography applications. In this application, the highest beam brightness is very important. A recent paper (Mankos et al., JVST B18, $3010(2000)$ ) reports an effective temperature of $0.05+/-0.02 \mathrm{eV}$, in agreement with Dunham's values. Also, measurements made on the injector for the CEBAF accelerator are in agreement with Dunham's values. In this latter case, the laser has an RF time structure ( $45 \mathrm{psec}$ duration pulses at $499 \mathrm{MHz}$ repetition rate) and a Gaussian transverse profile.

Similar quality measurements of the emittance produced from alkali antimonide and alkali telluride photocathodes have not been made (to the best of this writer's knowledge). However, since these cathodes have positive electron affinity, it is not energetically possible for electrons thermalized at the bottom of the conduction band to escape. Thus photoemission from these materials is from a non-thermal electron 
population. The optical absorption in these cathodes is much higher than in the case

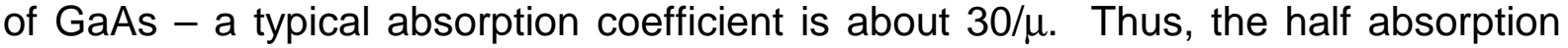
depth is about $23 \mathrm{~nm}$. One would expect that the photoemitted electrons would have a spectrum of energies ranging from about zero up to the energy difference between the exciting photon and the work function. Few measurements of this quantity have been reported, but there are numbers in the literature ranging between 200 and 600 $\mathrm{meV}$ for alkali antimonide cathodes. Alkali telluride cathodes have a smaller electron affinity than alkali antimonides, and thus might be expected to have a somewhat smaller effective thermal energy. At the workshop, numbers like 200 to $300 \mathrm{meV}$ were mentioned.

For PERL applications, the emittance which matters is the geometric emittance at the insertion devices. This can be translated back to the emittance provided by the injector, which in turn is composed of two parts - the thermal emittance from the cathode and the space charge related emittance growth which occurs between the cathode and the beam energy where the electron bunches are sufficiently rigid that further emittance growth is negligible (assuming that effects like wakefields, coherent synchrotron radiation, etc. are limited or controlled). Once one has an injector design and understands the magnitude of the emittance growth, the maximum thermal emittance from the cathode can be specified. This number is directly related to the electric field that must be present at the cathode.

To hold space charge related emittance growth to tolerable levels, one can remove only a fraction of the charge stored at the cathode surface due to the cathode electric field. Thus, given a thermal emittance that cannot be exceeded, one knows the maximum cathode radius which can be illuminated. This, in turn, establishes the cathode field that must be present. The GaAs cathode, having the lowest value for the effective thermal energy, will allow the largest illuminated diameter, and thus may be operated at the lowest cathode fields. The positive electron affinity cathodes, having a higher effective thermal energy, will require a smaller illuminated spot, and thus a higher cathode field, to achieve the same thermal emittance.

At Jefferson Lab, we operate DC photoemission guns with GaAs cathodes for both the CEBAF accelerator, and the FEL. The CEBAF electron source operates at 100 $k V$ with a maximum average current of about $200 \mu \mathrm{A}$, while the FEL operates at 320$350 \mathrm{kV}$ with a maximum average current of $5 \mathrm{~mA}$. Although the FEL gun has been processed to $\sim 550 \mathrm{kV}$, it is operated at lower voltage due to field emission problems. This field emission is due to the fact that the cathode is prepared in situ in the gun. Cathode preparation involves the use of cesium, which lowers the work function of the cathode electrode structure. A gun re-design is underway which will eliminate this problem. An upgrade to the FEL is under development, and will involve 
operation at $10 \mathrm{~mA}$ average current. We are confident that by eliminating the introduction of cesium into the gun structure, we will be able to operate this gun at its design value of $500 \mathrm{kV}$.

The operational lifetime of the GaAs cathodes used in the two guns above is limited only by ion back bombardment. These ions are produced in the cathode-anode gap by the beam, and accelerated back to the cathode, where they cause reduction in the $\mathrm{QE}$ by a variety of phenomena. Given that ion back bombardment is the lifetime limiting phenomenon, it makes far more sense to express the cathode life in terms of the number of coulombs delivered per unit illuminated area, rather than clock hours. Indeed, the 1/e lifetime of a GaAs cathode simply sitting in the static vacuum of one of the nuclear physics guns was measured to exceed 2.3 years. In service, the best $1 / \mathrm{e}$ operating lifetimes we have yet obtained are between 1 and $1.2 \times 10^{5}$ coulombs $/ \mathrm{cm}^{2}$. It should be noted that the ion back bombardment problem will be quite different, and probably much less severe, in an RF gun.

We have demonstrated that with a heat treatment and reactivation, we can fully recover the initial quantum efficiency of an ion-damaged GaAs cathode. How many times this operation can be successfully performed is not well known at present, but it is large. We have achieved further extensions of the practical cathode operational lifetime by illuminating a number of small area spots on a much larger area cathode. In the DC gun case, moving the laser beam spot on the cathode requires only a simple resteering of the electron beam, which is done by a fast, automatic routine on the CEBAF injector. This technique may not be so easy to employ in an RF gun, where one wants to operate with beam on the electromagnetic axis of the cavity.

In contrast to the GaAs cathode, which is formed by adding a single cesium - oxygen or cesium - fluorine monolayer to the GaAs surface, the alkali antimonide and alkali telluride cathodes are stochiometric chemical compounds. It is not unreasonable to assume that they might quite differently under ion back bombardment. Furthermore, since the light is absorbed in such a short depth in these cathodes, most of the damage done by ions is likely to be deeper in the cathode material than the region from which the photoemitted electrons originate. Indeed, Nguyen from Los Alamos reported an extrapolated lifetime of $8 \times 10^{6}$ coulombs $/ \mathrm{cm}^{2}-$ almost two orders of magnitude higher than the best numbers reported for GaAs.

It is possible to make high quantum efficiency cathodes from all three families. At the wavelengths indicated in the table above, quantum efficiencies between 10 and 20\% have been reported for all cathode types. However, only the GaAs cathode has been used in DC guns, while the antimonide and telluride cathodes have been used in RF guns. These cathodes are prepared in chambers external to the RF gun structure, and then inserted into the RF gun with an in-vacuum transfer mechanism. While 
these cathodes have good lifetimes in the static vacuum of the preparation chambers, they all lose quantum efficiency fairly rapidly on insertion into an RF gun. These cathodes appear to stabilize at quantum efficiencies between $1 / 2$ and $2 \%$, at which point they operate stably for extended periods of time. By contrast, the GaAs cathodes reported on are actually formed in situ in the gun, and their dark lifetime in the gun is excellent.

The difference in QE stability is no doubt largely due to the vacuum conditions in the gun. This is one area where a DC gun has an advantage. One has great freedom in choosing the vacuum wall material, the wall location, and the location of ports in a DC gun. In a RF gun, one has a limited choice of wall material, a wall geometry restricted by the realities of a resonant cavity, and great restrictions on the location and size of ports. Thus, it will likely always be true that it is much easier to establish an excellent vacuum in a DC gun, as opposed to a RF gun.

The limiting phenomenon in the cathode field strength and operating voltage of a DC gun is field emission. Field emitted electrons may collect on the ceramic insulator which holds off the primary gun voltage. Unless this charge is drained away, punchthrough of the ceramic may occur. Even if the ceramic is protected from this problem, field emitted electrons striking metallic walls release gases through electron stimulated desorption. Such gases are harmful to the cathode lifetime, either through chemical poisoning of the cathode, or by providing a source of ions.

Recent developments have resolved both of these issues. First, an LBL/Jefferson Lab group has developed a metal ion implantation process which produces a high resistivity sheet resistance on the vacuum surface of large ceramic insulators (ref. F. Liu et al., PAC '97, p. 3752). Ceramics treated this way have been very successfully used in the Jefferson Lab FEL gun. More recently, a Jefferson Lab group has shown that plasma-source nitrogen ion implantation of large area metallic electrodes dramatically reduces the field emission up to quite high fields. Field emission as low as $0.5 \mathrm{pA} / \mathrm{cm}^{2}$ has been observed at DC fields above $25 \mathrm{MV} / \mathrm{m}$ (ref. C. Sinclair et al, abstract submitted to PAC '01). With the benefits gained from these two separate ion implantation processes, it now appears to be within reach to build DC electron guns operating reliably with cathode fields above $20 \mathrm{MV} / \mathrm{m}$.

A note regarding the drive laser for a GaAs gun is worth making. To produce the necessary RF time structure on the laser beam, some form of mode-locking is employed. In most mode-locking schemes, the optical cavity round-trip time must be equal to the desired RF period. At $1300 \mathrm{MHz}$, the resulting cavity length is impractical, leading to designs which use a lower mode-locked frequency followed by complicated schemes to produce the desired $1300 \mathrm{MHz}$ pulse train. There are real issues of long term stability with such systems. Recently, a Ti:sapphire laser mode- 
locked by gain modulation has been developed at Jefferson Lab (ref. Hovater and Poelker, NIM A 418, 280 (1998)). The fundamental frequency of this laser is typically about $225 \mathrm{MHz}$, but it is easy to obtain stable mode-locking at multiples fo the fundamental frequency up to several $\mathrm{GHz}(2.5 \mathrm{GHz}$ has been demonstrated). The output power is independent of the RF frequency, and is quite high. A $2.5 \mathrm{~W}$ version of this laser has been demonstrated at Jefferson Lab, and we believe that this technique can be scaled to about $10 \mathrm{~W}$ without significant problems. We also note that high average power amplification of a high frequency optical pulse train has been demonstrated at the $5.77 \mathrm{~W}$ level (Z. Liu et al., Appl. Phys. Lett. 76, 3182 (2000)). It appears that a $\sim 10 \mathrm{~W}$ average power laser at $780 \mathrm{~nm}$ and $1300 \mathrm{MHz}$ should be well within reach.

Another issue that should receive attention is that of energy deposition in the cathode material. For example, suppose we have a $2 \%$ QE $\mathrm{Cs}_{2} \mathrm{Te}$ cathode illuminated at 266 $\mathrm{nm}$. 46.6 W of laser power are required to deliver $200 \mathrm{~mA}$ average current. If we require a thermal emittance of 0.5 micron, and assume that we have a transverse "tophat" profile beam, the allowable illuminated radius is $1.43 \mathrm{~mm}$. The absorption of $\mathrm{Cs}_{2} \mathrm{Te}$ is about $30 / \mu$, so that half the incident light is absorbed in a depth of only 23 $\mathrm{nm}$. The power deposited in the cathode in this case is over $150 \mathrm{MW} / \mathrm{cm}^{3}$. While it is clear that such a cathode would have to be formed on a thermally conductive substrate, this is a very prodigious power density, raising questions about the durability of the cathode.

In summary, we can say at the outset that the selection of the cathode type will have a very direct bearing on the design of the rest of the system. If a DC gun is chosen, there is at least a good opportunity to create a very excellent vacuum. In an RF gun, creating such a vacuum is more problematic, but it is not yet clear that much attention has been focussed in this issue. The primary problem with DC guns is field emission from the electrode structures. Recent developments in ion implantation have shown considerable promise in creating a highly resistive inner surface on the ceramic insulators, preventing charging from field emitted electrons, and on dramatically reducing the field emission from the electrode structures. It appears that DC electron guns operating with cathode fields in the $20 \mathrm{MV} / \mathrm{m}$ range may be developed in the near future. Such guns would be highly competitive with RF guns.

The GaAs cathode offers the prospect of very low thermal emittance, since the electrons originate from a thermal population at close to room temperature. At some stage in the development of very bright electron sources, the thermal emittance must be considered along with the emittance growth associated with space charge and other effects. Lasers with $1300 \mathrm{MHz}$ RF structure and adequate power to provide $200 \mathrm{~mA}$ average current from a GaAs cathode are only a moderate extrapolation of 
what has already been demonstrated. A GaAs cathode, operated in a state-of-theart DC electron gun is surely a contender as a high brightness, high average current electron source for PERL type applications. 


\title{
Conclusions of the $433 \mathrm{MHz}$ / B-Factory Cavity Based RF Gun Working Group
}

\author{
D.H. Dowell, Chair \\ Working Group Members: R. Rimmer (LBNL), P. Piot (DESY), W. Gai (ANL), J-P. \\ Carneiro (FNAL), I. Ben-Zvi (BNL), X.Y. Chang (BNL), H. Edwards (FNAL) J. Rose \\ (BNL).
}

Since the $433 \mathrm{MHz}$ working group had combined discussions with the $1300 \mathrm{MHz}$ group, this summary includes comments related to the $1300 \mathrm{MHz}$ photoinjector.

\section{Executive Summary:}

The $433 \mathrm{MHz}$ photocathode gun technology is the most advanced of the three approaches discussed in this workshop. Essentially all the PERL photocathode gun performance requirements of high average current operation and beam quality are met either experimentally or by simulation. In addition, the overall architecture of the photoinjector up to injection into the SRF (approximately $25 \mathrm{MeV}$ ) is well-established. Therefore this approach has the lowest technical risk of the three photoinjectors being considered for PERL.

The $433 \mathrm{MHz}$ gun working group, and the workshop participants in general, conclude the single disadvantage of the $433 \mathrm{MHz}$ photoinjector is the high microbunch charge needed to generate $200 \mathrm{~mA}$ of average current. The charge is three times that of the $1300 \mathrm{MHz}$ gun. Combined with the PERL specification for short, sub-ps microbunches, this high charge will strongly radiate in the high-energy bends, leading to significant degradation of the beam quality. However, it should be noted that the 1300 $\mathrm{MHz}$ photocathode gun has been operated only to $1 \%$ duty factor vs. $25 \%$ operation for the $433 \mathrm{MHz}$ gun. In addition, the closely spaced microbunches (769 ps) of the 1300 $\mathrm{MHz}$ pulse train may experience more severe transverse wakes than the more widely separated (2.3 ns) $433 \mathrm{MHz}$ pulse train. These and other issues require further study before deciding which frequency is best for PERL.

A concise list of the working group's recommendations follows:

1. Third-harmonic linearizer is required for either frequency.

2. Develop the high-QE $\mathrm{K}_{2} \mathrm{SbCs}$ photocathode. Incorporate concepts presented in workshop for increasing cathode lifetime.

3. Further investigation of all wakes (CSR and resistive wall) is essential to decide between 433 and $1300 \mathrm{MHz}$ approaches. It is preferable that the wakes be realistically included in the beam transport simulations.

4. Realistic simulation of entire $1300 \mathrm{MHz}$-based system. Include the much lower beam energy out of the cathode cell in the calculation. What can be used for the CW booster and $3.9 \mathrm{GHz}$ linearizer accelerator sections? The single pass beam current is probably too high for a SRF booster.

5. Model energy recovery dynamics. Investigate RF-beam instabilities.

6. Review overall PERL architecture. Compare co-propagation vs. anti-propagation in energy recovery linacs to control beams with large energy differences. 


\section{Photoinjector Architecture}

The photoinjector layout from the gun to injection into the SRF was show by D. Dowell. It consists of the RF gun, a $433 \mathrm{MHz}$ booster, a $1300 \mathrm{MHz}$ linearizer and a chicane compressor. Experimental results demonstrating improved compression with the third harmonic linearizer were shown.

In an earlier session, P. Piot discussed how the linearizer eliminates CSR-induced beam breakup, and pointed out that DESY is interested in collaborations to develop a 3.9 GHz. SRF linearizer cavity.

\section{RF Design}

R. Rimmer presented details of the $476 \mathrm{MHz}$ B-factory cavities and how they could be adapted to $433 \mathrm{MHz}$ for the gun and booster sections. These cavities have already demonstrated $\mathrm{CW}$ operation at the required beam currents, and present little technical risk.

D. Dowell presented details of the Boeing $433 \mathrm{MHz}$ RF gun and APLE (Average Power Laser Experiment) booster cavities. The RF gun has successfully run at $25 \%$ duty factor, but will require some modification for CW operation. The CW APLE cavities are copper-plated aluminum and are available in 3-cell and 5-cell configurations.

A single Tesla SRF cavity can be used for the linearizer.

\section{Photocathode}

The photocathode choice has major implications for the drive laser. A highpower, UV drive laser, as required for $\mathrm{Te}_{2} \mathrm{Cs}$, is considered to be technically risky. GaAs is attractive from the drive laser's point of view, but requires the best vacuum of all the cathodes and has poor temporal response. Therefore the group decided that $\mathrm{K}_{2} \mathrm{SbCs}$ is the only option for PERL.

Consider the following situation for PERL. The $\mathrm{K}_{2} \mathrm{SbCs}$ cathode lifetime decays exponentially. Assume an initial $\mathrm{QE}_{\mathrm{i}}$ of $12 \%$ (14\% has been demonstrated) and that the drive laser power is sufficient to produce the required charge at a $1 \%$ final $\mathrm{QE}_{\mathrm{f}}$. Then the lifetime (LT) needed to operate $\mathrm{T}_{\mathrm{op}}$ hours is given by,

$$
\mathrm{LT}=\frac{\mathrm{T}_{\mathrm{op}}}{\left(\ln \frac{\mathrm{QE}_{\mathrm{i}}}{\mathrm{QE}_{\mathrm{f}}}\right)} .
$$

Therefore 24 hours of operation requires a lifetime of 9.6 hours. Lifetimes of 2.3 hours were demonstrated in the $199225 \%$ duty factor tests at Boeing. Therefore current technology is only a factor of five below PERL requirements, ignoring any improvements in the gun vacuum.

Techniques for greatly improving the lifetime were presented. These include coating with a thin protective layer of $\mathrm{CsBr}$ (presented by D. Nguyen, LANL in an earlier session) and operating the cathode at an elevated temperature to keep it clean. Experiments give a lifetime in excess of 20 hours for a cathode at 120 degrees $\mathrm{C}$. in a poor vacuum, and therefore could easily meet the PERL specification. An automated, multi-cathode system incorporating these ideas should be considered. 
To produce $0.5 \mathrm{nC}$ of microbunch with a $1 \% \mathrm{QE}$ cathode means the drive laser produces approximately 0.1 microjoule per microbunch at a repetition rate of $433 \mathrm{MHz}$ or 43 watts $\mathrm{CW}$ at $527 \mathrm{~nm}$. This should be possible. The drive laser developed by LANL for the 1992 Boeing $25 \%$ duty factor test operated with 0.47 microjoule per microbunch at $27 \mathrm{MHz}$. The 8.3 millisecond macropulse power was 12.7 watts, the average power was 3.2 watts.

\section{Recommendations}

The third harmonic linearizer is required at any photoinjector RF frequency. Detailed simulations of CSR and resistive wall wakes, both transverse and longitudinal, are needed since this will be a major factor in choosing between 433 and $1300 \mathrm{MHz}$ guns. More effort is needed on the $1300 \mathrm{MHz}$ injector to answer questions concerning the availability of a $\mathrm{CW}$ booster and a $3.9 \mathrm{GHz}$ linearizer. The single-pass beam current maybe too high for a SRF booster.

The $\mathrm{K}_{2} \mathrm{SbCs}$ cathode technology should be developed to allow the use of a visible wavelength drive laser. This effort should start soon since the cathode choice places major design requirements upon both the drive laser and the RF gun vacuum. In the longer term, an automated, multi-cathode should be developed.

In conclusion, the $433 \mathrm{MHz}$ photoinjector is the most developed of the three injectors discussed in this workshop. Its sole disadvantage is the $200 \mathrm{~mA}$ average current requires the high-charge microbunches, which strongly radiate in the beam transport system when compressed to sub-ps bunches. 


\title{
Summary of L-band Working Group
}

\author{
Wei Gai \\ $A N L$ \\ Building 360,HEP \\ 9700 South Cass Avenue \\ Argonne, IL 60439-4800
}

We have examined the L-band option for PERL in this working group. Some comparisons were made with $433 \mathrm{Mhz}$ option. There were 4 talks given for preliminary design studies. The summary is given below.

The starting design point is the ANL $1 \frac{1 / 2}{2}$ cell L-band gun. With modified parameters, 1 $1 / 2$ TM02 mode operation and $2 \frac{1}{2}$ cell options were also examined [X.Y. Chang]. From the beam dynamic point view, L-band would provide higher brightness beam at lower charge per pulse which is crucial for beam transport in bends (Coherent Synchrotron Radiation wakefield reduction). CSR can be very severe in beam transport, particularly beam pulse length compression using a chicane as experimentally verified by $\mathrm{Ph}$. Piot. They observed beam energy bifurcation due to CSR at $130 \mathrm{MeV}$. It is believed that the six phase space parameters can be obtained using different L-band options as discussed above.

During the discussion, Bob Rimmer presented their work on the heat load at LBL. He suggests that $100 \mathrm{~W} / \mathrm{cm}^{2}$ is a limit for current operation. However, he also considered a few times of that maybe achievable. Another import result obtained through the discussions, is whether the L-band will be much worse than the $433 \mathrm{MHz}$ structure. Bob Rimmer and Ilan Ben-zvi came to a conclusion that heat generation density in L-band is comparable to that of the $433 \mathrm{MHz}$ using a scaling law.

We have also discussed the liquid nitrogen cooling option for the PERL gun. However, because there is no any experimental data available to date, we have to speculate how to cool the acceleration structure. Two options were discussed: 1) Single phase liquid cooling and 2) Two phases (liquid and gas) cooling. Advantages using liquid nitrogen as coolant are identified: 1) lower the RF power consumption; 2) increased heat conduction; 3 ) improved vacuum condition (no water residues) would improve cathode life time; and 4) sustain higher gradient in the gun than at normal temperature. Due to the attractive of liquid nitrogen option, we recommend some engineering research should be started right way.

Different RF photocathodes were considered, the conclusion is that we already have technologies may be not two far from the PERL requirements. Cathode lifetime and QE can be overcome by using hot standby spares. It was recommended by the working group that a lab should be setup to study the QE issues such as lifetime and vacuum conditions. 
L-band RF power supply for $1-2 \mathrm{MW} \mathrm{CW}$ source is already available at Toshiba. We believe this is adequate for the PERL gun and booster applications.

One concern was raised and discussed but we were unable to get any answer is beam break up in the gun and booster. The average current in the PERL is 20 times higher than TESLA operation, therefore a serious beam break up problem may arise. 


\section{Workshop on Photo-injector for Energy Recovery Linac \\ January $22 \& 23,2001$}

\author{
Charlie Sinclair \\ Jefferson Laboratory \\ 12000 Jefferson Avenue, Newport News, VA 23606 \\ Tel: 757-269-7679 \\ E-mail: sinclair@jlab.org \\ Manoel Conde \\ Argonne National Laboratory \\ 9700 S. Cass Ave. Bldg. 362 \\ Tel: 630-252-6099 \\ E-mail: conde@anl.gov
}

\author{
Alexander Zholents \\ Lawrence Berkeley National Laboratory \\ 1 Cyclotron Road, MS 71-259, Berkeley, CA 94720 \\ Tel: (510)486-7533 \\ E-mail: AAZholents@lbl.gov
}

\section{Max Cornacchia}

SLAC

2575 Sand Hill Rd, Menlo Park, CA 94025

Tel: (650) 9263906

E-mail: cornacchia@ssrl.slac.stanford.edu

\author{
Marty Woodle \\ BNL \\ P.O. Box 5000, Bldg. 725C \\ Upton, NY 11973-5000 \\ Tel: (631) 344-2521 \\ E-mail: woodle1@bnl.gov \\ Thomas Tsang \\ BNL \\ P.O. Box 5000, Bldg. 535B \\ Upton, NY 11973-5000 \\ Tel: (631) 344-2225 \\ E-mail: Tsang@bnl.gov
}

Kwang-Je Kim

APS, ANL

9700 South Cass Ave, Buiding 401/C4265

Argonne, IL 60439

Tel:630 2524647

E-mail:kwangje@aps.anl.gov

James Murphy

BNL

P.O. Box 5000, Bldg. $725 \mathrm{C}$

Tel: (631) 344-5160

E-mail:jbm@bnl.gov

\section{Attendee List}

\author{
Feng Zhou \\ UCLA/BNL \\ B820M, BNL \\ Tel: 631-344-2042 \\ E-mail:zhouf@sun2.bnl.gov \\ John Lewellen \\ Advanced Photon Source, ANL \\ 9700 S. Cass Ave, 401/B2207 \\ Argonne, IL 60439 \\ Tel: 630-252-5252 \\ E-mail:lewellen@aps.anl.gov
}

\section{Philippe-Regis Piot}

Deusches Elektronen Synchrotron DESY-Hamburg

MPY Group,

85, Notkestrasse

D-22603 HAMBURG, DEUTSCHELAND

Tel: +49-40-8998-2756

E-mail: philippe.piot@desy.de

\section{Ivan Bazarov}

CORNELL UNIV.

CHESS Wilson Lab, Cornell Univ., Ithaca, NY 15853

Tel: (607) 255-0678

E-mail: ib38@cornell.edu

\section{Katsuyuki Kobayashi}

Sumitomo Heavy Industries

c/o Aculight Corporation, 11805 North Creek

Parkway

South, Bothell WA 98011

Tel: 425-482-1100, ext. 162

E-mail: kobayashi@aculight.com

\section{Ilan Ben-Zvi}

BNL

P.O. Box 5000, Bldg. 725C

Upton NY 11973-5000

Tel: (631) 344-5143

E-mail: ilan@bnl.gov

\section{David Dowell}

Boeing Physical Sciences Research Center 3116 NW 95th Street, Seattle, WA 98117

Tel: (206)782-6374 (home), (206)544-5409 (work)

E-mail: dowell@eskimo.com 


\section{Dinh Nguyen}

Los Alamos National Laboratory

MS H851

Tel: 505-667-9385

E-mail: dcnguyen@lanl.gov

\section{Marcus Babzien}

BNL/ATF

P.O. Box 5000, Building 820M

Upton, NY 11973-5000

Tel: 631 344-5238

E-mail: babzien@bnl.gov

\author{
James Clendenin \\ SLAC \\ 2575 Sand Hill Road, Menlo Park, CA 94025 \\ Tel: 650/926-2962 \\ E-mail: clen@slac.stanford.edu

\section{Brian Sheehy} \\ BNL \\ P.O. Box 5000,Bldg 725D \\ Upton, NY 11973-5000 \\ Tel: x5491 \\ E-mail: sheehy@bnl.gov

\section{Michael Cole} \\ Advanced Energy Systems Inc \\ 27 Industrial Blvd., Suite E, Medford, NY 11763 \\ Tel: (631) 345-6264, ext. 3004 \\ E-mail: (631) 345-0458
}

\section{Sal Pjerov}

BNL

P.O. Box 5000, Bldg. $725 \mathrm{C}$

Tel: (631) 344-7080

E-mail:pjerov@bnl.gov

\section{Vitaly Yakimenko}

BNL

P.O. Box 5000, Bldg.725C

Upton, NY 11973-5000

Tel: (631)344 7830

E-mail: Yakimenko@bnl.gov

\section{Donald Feldman}

University Of Maryland

Institute for Plasma Research Bldg 223

College Park, MD 20742

Tel: 301-405-8484

E-mail: dfeldman@glue.umd.edu

\author{
Robert Rimmer \\ LBNL \\ MS 71-259 \\ Berkeley CA 94720 \\ Tel: 5104866243 \\ E-mail: RARimmer@lbl.gov
}

\section{John Galayda}

ANL

Building 401

9700 South Cass Avenue

Argonne, IL 60439-4800

Tel:(630)252-7796

E-mail:deputy@aps.anl.gov

\section{Timur Shaftan}

BNL

P.O. Box 5000, Bldg.725C, Upton, NY 11973-5000

Tel:(631)344 5144

E-mail:shaftan@bnl.gov

\section{Wei Gai}

ANL

Building 360,HEP

9700 South Cass Avenue

Argonne, IL 60439-4800

Tel: (630)252-6560

E-mail:wg@hep.anl.gov

\section{Sam Krinsky}

Brookhaven National Lab

P.O. Box 5000, Building 725B

Upton, NY 11973-5000

Tel: (631) 344-4740

E-mail: krinky@bnl.gov

\section{Helen Edward}

FNAL

E-mail: hedwards@fnal.gov

\section{William S. Graves}

BNL

P.O. Box 5000, Building 725D

Upton, NY 11973-5000

Tel: (631) 344-5353

E-mail: wsgraves@bnl.gov

\section{David Robin}

Lawrence Berkeley National Laboratory

1 Cyclotron Road, MS 80-101

Tel: 5104866028

E-mail: dsrobin@lbl.gov 


\author{
Xiangyun Chang \\ IHEP, China \\ Mail Address: P.O. Box 752, Upton, NY 11973 \\ Tel: (631)344-4730 \\ E-mail:address:xychang@bnl.gov

\section{Jean-Paul Carneiro} \\ FNAL \\ E-mail: carneiro@ waldo.fnal.gov

\section{James Rose} \\ BNL \\ P.O. Box 5000, Building 725C \\ Upton, NY 11973-5000 \\ Tel: (631) 344-7079 \\ E-mail: rose@bnl.gov

\section{Frank Stephan} \\ Deusches Elektronen Synchrotron DESY-Zeuthen \\ Photo injector test facility \\ Platanenallee 6, \\ D-15738 Zeuthen, DEUTSCHELAND \\ Tel: +49-33-762-77-338 \\ E-mail: fstephan@mail.desy.de

\section{Triveni Srinivasan-Rao} \\ BNL \\ P.O. Box 5000, Bldg. 535B \\ Upton, NY 11973-5000 \\ Tel: (631) 344-5072 \\ E-mail: triveni@bnl.gov

\section{Steve Milton} \\ ANL,APS \\ Building 401 \\ Argonne National Laboratory \\ 9700 South Cass Avenue \\ Argonne, IL 60439-4800 \\ Tel:(630) 252-9091 \\ E-mail: milton@aps.anl.gov
}

\section{Sandra Biedron}

ANL, APS

Building 401

9700 South Cass Avenue

Argonne, IL 60439-4800

Tel:(630) 252-5703

E-mail: biedron@aps.anl.gov

\section{Xijie Wang}

BNL

P.O. Box 5000, Bldg. 725C

Upton, NY 11973-5000

Tel: (631) 344-5791

E-mail: xwang@bnl.gov

\section{L.H. Yu}

BNL

P.O. Box 5000, Bldg. 725C

Upton, NY 11973-5000

Tel: (631) 344-5012

E-mail:1hyu@bnl.gov

\section{Aaron Tremaine}

UCLA

Department of Physics and Astronomy

University of California, Los Angels

Los Angels, CA 90024

E-mail: tremaine@stout.physics.ucla.edu

\section{Alex Murokh}

UCLA

Department of Physics and Astronomy

University of California, Los Angels

Los Angels, CA 90024

E-mail: alex@ stout.physics.ucla.edu

Juhao Wu,

Stony Brook/BNL

P.O. Box 5000, Bldg. 725C

Upton, NY 11973-5000

Tel: (631) 344-5072

E-mail: jhwu@bnl.gov

John Smedley

Stony Brook/BNL

P.O. Box 5000, Bldg. 535B

Upton, NY 11973-5000

Tel: (631) 344-7865

E-mail: Smedleyebnl.gov

\section{Osgood, Jr Richard M}

BNL

P.O. Box 5000, Bldg. 460

Upton, NY 11973-5000

Tel: (631) 344-3927

E-mail:osgood@bnl.gov

Takahashi, Hiroshi

BNL

P.O. Box 5000, Bldg. 475B

Upton, NY 11973-5000

Tel: (631) 344-4099

E-mail: takahash@bnl.gov

\section{George Rakowsky}

BNL

P.O. Box 5000, Bldg. 725D

Upton, NY 11973-5000

Tel: (631) 344-5298

E-mail: rakowsk1@bnl.gov 